\title{
Home nursing in europe : patterns of professionalisation and institutionalisation of home care and family care to elderly people in Denmark, France, the Netherlands and Germany
}

Citation for published version (APA):

van der Boom, H. C. I. (2008). Home nursing in europe : patterns of professionalisation and institutionalisation of home care and family care to elderly people in Denmark, France, the Netherlands and Germany. [Doctoral Thesis, Maastricht University]. Universiteit Maastricht. https://doi.org/10.26481/dis.20080625hb

Document status and date:

Published: 01/01/2008

DOI:

$10.26481 /$ dis.20080625hb

Document Version:

Publisher's PDF, also known as Version of record

\section{Please check the document version of this publication:}

- A submitted manuscript is the version of the article upon submission and before peer-review. There can be important differences between the submitted version and the official published version of record. People interested in the research are advised to contact the author for the final version of the publication, or visit the DOI to the publisher's website.

- The final author version and the galley proof are versions of the publication after peer review.

- The final published version features the final layout of the paper including the volume, issue and page numbers.

Link to publication

\footnotetext{
General rights rights.

- You may freely distribute the URL identifying the publication in the public portal. please follow below link for the End User Agreement:

www.umlib.nl/taverne-license

Take down policy

If you believe that this document breaches copyright please contact us at:

repository@maastrichtuniversity.nl

providing details and we will investigate your claim.
}

Copyright and moral rights for the publications made accessible in the public portal are retained by the authors and/or other copyright owners and it is a condition of accessing publications that users recognise and abide by the legal requirements associated with these

- Users may download and print one copy of any publication from the public portal for the purpose of private study or research.

- You may not further distribute the material or use it for any profit-making activity or commercial gain

If the publication is distributed under the terms of Article 25fa of the Dutch Copyright Act, indicated by the "Taverne" license above,

Download date: 26 Apr. 2023 
HOME NURSING IN EUROPE 



\title{
HOME NURSING IN EUROPE
}

\author{
Patterns of Professionalisation and Institutionalisation \\ of Home Care and Family Care to Elderly People \\ in Denmark, France, the Netherlands and Germany
}

\section{PROEFSCHRIFT}

ter verkrijging van de graad van doctor aan de Universiteit Maastricht, op gezag van de Rector Magnificus, Prof.mr. G.P.M.F. Mols

volgens het besluit van het College van Decanen, in het openbaar te verdedigen op woensdag 25 juni 2008 I0.00 uur

door

Hannerieke Christina Inge van der Boom 
Promotor:

Prof.dr. H. Philipsen

Copromotor:

Dr. F.C.J. Stevens

Beoordelingscommissie:

Prof.dr. J.P.H. Hamers (voorzitter)

Dr. V. Burau (University of Aarhus, Denmark)

Prof.dr. R. Vos

Dr. J.C.M. Wachelder

Prof.dr. J. van der Zee (Universiteit Maastricht en NIVEL Utrecht) 


\section{Contents}

I A comparative and contextual perspective on home nursing in Europe II

I.I Introduction II

I.2 Background of the study I2

I. 3 The perspectives on home nursing in this thesis I5

I.4 Theoretical perspectives I7

1.4.1 The health sciences perspective I7

1.4.2 The professional perspective - why home nursing? I9

1.4.3 The neo-institutional perspective: home care as a societal sector 2I

1.4.4 A cultural studies perspective: caring cultures from north to south Europe 24

I.5 The countries 26

I.6 A comparative analysis 29

I.7 Research design 30

I.8 Sources 32

I.9 Methods 34

1.9.1 The theoretical perspective functioning as a lens 35

1.9.2 Procedure of analysis and writing 36

1.9.3 Methodological views and considerations 37

I.Io Contents of the book 38

2 Theoretical perspectives on professions and home nursing 40

- Perspectives in the sociology of professions

2.I Introduction 40

2.2 Perspectives in the sociology of professions 40

2.2.1 What 'is' a profession? 42

2.2.2 The process of professionalisation 43

2.2.3 Ideal types and hierarchical relations between professions in health care 44

2.2.4 Abbott's theoretical perspective on professions 45

2.2.5 Developing an interactionist contextual perspective on professions 47

2.2.6 Concluding remarks on the concept 'profession' 48

2.3 Characteristic features of the home nursing domain:

a comparative and contextual perspective on home nursing 49

2.3.1 A special working domain 50 


\subsubsection{Broad sphere of action 51}

2.3.3 Autonomy 51

2.4 The organisation of home nursing work: a generalist perception of care or intra-professional division of labour 53

2.4.1 Intra-professional task differentiation 55

2.4.2 The 'system of professions' - interprofessional cooperation and co-ordination of activities 57

2.4.3 Possible tensions between lifeworld and system values 59

2.4.4 The impact of bureaucracy 6I

2.5 Developing a theoretical framework - the first step $6_{4}$

2.6 The home nursing profession in a changing health care and societal context - Challenges, opportunities and difficulties 66

2.6.1 The impact of a managerial perspective and increasing absence of a professional perspective in health care 66

2.6.2 Demographic changes - the ageing of the population 70

2.6.3 Changing family relations and informal care 73

2.6.4 Cultural diversity 75

2.6.5 Health and elderly policies 76

2.6.6 Differences in health care systems 77

2.7 A comparative and contextual perspective on home nursing 78

3 Overview chapter on home care provision, health care systems and demographic characteristics of the four countries 80

3.I Introduction 80

3.2 Overview of differences in home care provision in Europe 82

3.2.1 Denmark and the Netherlands 83

3.2.2 France, Germany and other European countries 85

3.3 Health systems 87

3.3.1 Health care systems based on social insurance 87

3.3.2 France 88

3.3.3 Germany 89

3.3.4 The Netherlands 92

3.3.5 Health systems based on universal taxes 93

3.3.6 Denmark 94

3.4 Overview of demographic data and key characteristics of each country 95

3.4.1 General demographic data: total area and population characteristics 96

3.4.2 General demographic data: age groups and economic activity 99

3.4.3 Household situation IOI

3.4.4 Chronic conditions and subjective health IO2

3.4.5 Health care professionals IO2

3.4.6 Hospital beds I03 
3.4.7 Social protection IO4

3.4.8 Expenditure on health IO4

3.4.9 Sources of health financing IO4

3.5 Conclusion and discussion I05

\section{Appendices:}

Demographic data and figures on health systems and professionals Io8

Table 1 Demographic data - total area and population characteristics 108

Table 2 Demographic data-Age groups and economic activity Io9

Table 3 Household situation II

Table 4 Chronic conditions and subjective health (\% of 15+ years, 2001) IIO

Table 5 Health care professionals III

Figures Health care professionals - Total number of qualified nursing staff and nursing auxiliaries (per 1000 population, 1996/97-2004) II2

Table 6 Hospital beds II3

Table and Figure 7 Trends in number of hospital beds (1994-2004) II3

Table 8 Expenditures on social protection (2004) II4

Table 9 Contribution on social protection by different stakeholders, 2004 II5

Table and Figure 10 Trends in total expenditure on health

$$
\text { (\% of GDP, } 1975 \text { - 2004) II5 }
$$

Table and Figure 11 Sources of health care financing (percentages) II6

\section{The home nursing domain in Denmark II7}

- The generalist nuRse and community-based Welfare state provision OF INTEGRATED ELDERLY CARE

4.I Introduction: central issues in Danish health care: the role of the state, professionals and the family II7

4.1.1 Structure of this chapter I20

4.2 General background and remarkable demographic trends in Denmark I20

4.3 Principles underlying the Danish welfare state and health care system I23

4.4 Characteristic aspects of the Danish welfare state: public, community-based health care arrangements and maximisation of individual independence I25

4.5 Elderly policies: a radical change from institutional to community-based care I3I

4.6 The overall health care system I34

4.6.1 Decentralised execution of the National Health Security System:

decision making by local authorities $\mathrm{I} 34$

4.6.2 The development of the Danish National Health Security System I36

4.6.3 The financing of health care I39

4.7 A rather low degree of managerial and bureaucratic interference in professional practices $\mathrm{I}_{4} 2$ 
4.8 The history of home nursing $\mathrm{I}_{4} 2$

4.9 Home nursing currently: a generalist nurse I45

4.9.1 The education of nurses; the provision of integrated elderly care 147

4.IO The home care domain; municipal provision of integrated home nursing and home help 148

4.II Conclusion I49

\section{Home nursing in France $I 53$}

- A weak domain in-Between the state, the medical and hospital domain AND THE FAMILY

5.I Four issues characterising the provision of home nursing in France I53

5.2 The characteristics of health care in France 156

5.2.1 The unequal distribution of home nursing services over the country 156

5.2.2 The dominance of medical and institution-based care I59

5.2.3 A cure-oriented attitude towards matters of health and disease $\mathrm{I}_{4}$

5.2.4 The professions against the background of the all-pervading role of the central state in France $\mathrm{I}_{5} 5$

5.3 The home nursing domain: a weak position in a fragmented health system $\mathrm{I} 69$

5.3.1 Hospitalisation à Domicile (HAD) I7O

5.3.2 Service de Soins Infirmiers à Domicile pour les Personnes Agées (SIAD) 73

5.3.3 The growth of the home nursing sector I76

5.3.4 The uneven distribution of services: one of the peculiarities of home nursing in France 178

5.4 The development of home care policies in France I80

5.5 The home nursing profession; short historical sketch I83

5.6 The current home nursing profession $\quad 185$

5.6.1 Legal regulations describing the domain and activities of nurses $\quad$ I85

5.6.2 The organisation and training of nurses 187

5.6.3 The role of independent nurses 189

5.6.4 The role of nurse's aids or auxiliaries I9I

5.6.5 Recent changes in the relation between physicians and home nurses I92

5.7 The importance of the home help domain and care provided by the family I93

5.7.1 Traditional family patterns and values contributing to stigmatisation of professional home care I93

5.7.2 The domain of home help: Aide à Domicile - considerably increasing in size but problems persist ${ }_{198}$

5.7.3 A socio-cultural stigma regarding professional elderly care combated by a holistic perspective 20I

5.8 Conclusion 203 
6 Home nursing in the Netherlands 209

- Changes in health care and society affecting the character

OF THE HOME NURSING PROFESSION

6.I Introduction 209

6.1.1 Structure of the chapter 210

6.2 Current trends in home nursing, health care and society: increasing bureaucracy and standardisation of practices changing the character of professional home nursing 2 Iо

6.2.1 A dense network of home care services 210

6.2.2 The typical organisation of health care in the Netherlands 2I5

6.2.3 The tendencies to organise and bureaucratise daily life in the Netherlands 2I6

6.2.4 Standardisation of the intake: 'objectification' and deprofessionalisation 2I7

6.2.5 Evidence Based Medicine, increasing standardisation, protocols and bureaucracy: the changing character of health care work and professionalism 220

6.2.6 The Dutch 'polder model' 224

6.3 A historical overview of home nursing in the Netherlands 226

6.3.1 The first efforts to establish nursing as a professional occupation 226

6.3.2 The founding of private public health initiatives: the Cross associations 229

6.3.3 Financing of home nursing 23I

6.3.4 Shifts in home nursing tasks; from prevention to home nursing and separate working domains of child care and elderly care 23I

6.3.5 The educational system 235

6.4 Developments in home nursing since the I990s 238

6.4.1 The integration of home care and home help 239

6.4.2 The changing character of home care 24I

6.4.3 The changing relation between formal and informal care 242

6.4.4 The influence of the management state on professional and informal care 245

6.4.5 Worries about current developments in Dutch home care 246

6.5 Conclusion 248

7 Home nursing in Germany 253

- A medically oriented heAlth CARE System AND STRONG RELiAnce ON THE FAMILY

7.I Introduction 253

7.2 The dominance of the medical sector in health care 254

7.3 A strong reliance on the family in the German welfare system 255

7.4 A social insurance system with barriers for community care 258

7.5 German social and health policies regarding long-term care needs 260

7.6 The provision of elderly care in Germany 266

7.7 The development of Sozialstationen 270 
7.8 Educational levels in nursing 27I

7.8.1 Krankenschwester or -pfleger 272

7.8.2 Krankenpflegehelfer(in) 273

7.8.3 Altenpfleger(in) 273

7.8.4 Dorfhelfer(in) 274

7.8.5 Familienpfleger(in) 274

7.9 Client population 275

7.IO Financing of home nursing 276

7.II Funding of the Social Stations 278

7.12 The domain of home help 279

7.I3 Conclusion 280

\section{Conclusion and discussion 285}

- The home nursing domain and profession from a comparative and CONTEXTUAL PERSPECTIVE

8.I Differences in home nursing in Europe the theoretical framework revisited 285

8.1.1 Outcomes of the study - the central part of the theoretical framework 285

8.1.2 Adaptation of the central part of the theoretical framework 289

8.1.3 Outcomes of the study - the middle ring of the theoretical framework 292

8.1.4 Adaptation of the middle ring of the theoretical framework 295

8.1.5 Outcomes of the study - the outer layer of the theoretical framework 296

8.1.6 Adaptation of the outer layer of the theoretical framework 305

8.1.7 A revised theoretical framework of home nursing in comparative and contextual perspective 3 IO

8.1.8 Strengths and weaknesses of the analysis by use of the theoretical framework 3 IO

8.2 Differences in the degree of institutionalisation of the home nursing domain and in caring cultures 3I3

8.3 Significance of this study 316

8.3.1 Contribution to the sociology of the professions: beyond Abbott's network of professions to country-specific constellations in which home nursing is embedded 3I7

8.3.2 Implications for policy and future research 320

References 324

Summary 342

Samenvatting / Dutch summary 353

Dankwoord / Acknowledgements 365

Curriculum Vitae 368 
Chapter 1

\section{A comparative and contextual perspective on home nursing in Europe}

\section{I.I Introduction}

In this book, the profession of home nursing is described from a comparative and contextual perspective. The aim is to gain insight into international differences in home nursing in Europe, as well as the background of these differences. By taking a macro-social perspective and taking the context of the health care systems and the social and cultural background of four European countries into account, this study intends to explain and get insight into the background of these differences.

Currently, in most European countries, the main clientele of home nursing consists of frail elderly and chronically ill people that are in need of long-term nursing care and support to be able to remain living in their own homes. Therefore, this thesis primarily focuses on the provision of home nursing to these particular groups. Major themes are differences in the provision of community-based health care services to elderly and chronically ill people and social, cultural and political views on the way elderly care should be arranged. In some countries, the provision of home nursing care in other task areas and to other client groups is also important, such as home nursing provided in preventive youth health care in the Netherlands, or home nursing provided to the handicapped, but these task areas are only dealt with sideways.

In this book, the relations between home nursing and other health professions and sectors in different European countries are of central importance, in particular the relations between home nursing and the medical domain (physicians such as GPS and the hospital sector), the neighbouring or allied domain of home help (domiciliary care), and the societal domain of informal care, primarily provided by family members and members of the social network, such as neighbours and friends. Thus, the position of home nursing as a community-based health care activity is studied in relation to medical activities and the role of the family in the provision of care to elderly people in four European countries, namely Denmark, Germany, France, and the Netherlands. In addition, European differences in social, cultural and political values and views of particular societies regarding how care to elderly people should be arranged are studied. How are policies and care arrangements designed, so that in one country, elderly people are predominantly cared for by health professionals in their own homes, while in another country, they are they relatively easily admitted into a nursing home, residential 
home, or a medical or geriatric ward in a hospital or residential home? Also, what differences in culture between countries result in diverging roles of family members to care for elderly people? These questions are important to study, as the increase in the number of (very) elderly people, the so-called double ageing of the population, implies that health care systems of all European countries have to respond to the increase in the demand for elderly care now and in the future. In most countries, elderly and vulnerable people are considered as a burden to society and economy, because a large part of the countries' health and welfare arrangements are adapted to their needs. Also, in a period when more and more countries are joining the European Union, a major question is whether health care systems of different countries are converging and whether similar solutions are sought by different European countries to solve the caring needs of elderly and vulnerable people, or whether each country finds its own solutions and devises its own elderly care policies because of substantial social, cultural, political and historical differences.

\section{I.2 Background of the study}

The interest in performing a comparative study on home nursing is partly inspired by results of a study on community nursing that was carried out in the Euregion in I99I, which is the region where the borders of Belgium, Germany and the Netherlands meet ${ }^{\mathrm{I}}$ (Derksen, I99ıа; Derksen, Zee et al., I99ıb; Zee, Kramer et al., I994). The areas of these three countries partly share their historical-cultural background, but are also part of their own national cultures and have different health care systems (Diederiks, Stevens et al., I99I). Three core concepts of community nursing were measured in the same way in the three countries, namely the level of care dependency of the patient, the kind of services or tasks provided by community nurses, and the job interpretation and job satisfaction of nurses. The major conclusion of Derksen et al. was, that apart from differences in legislation and in the way of financing of home nursing that could be attributed to differences in health care and social security systems, also striking differences in community nursing practices were found.

For example, differences in the level and kind of training were found: Dutch community care nurses had followed education specialising into community care, while this specialisation did not exist in Belgium. Also, the size of home

I The Euregion 'Maas-Rhine' is a geographical, economical and cultural closely related area. It consists of two Belgian provinces, Luik and Limburg, the Regierungsbezirk Köln (North Rhine Westphalia) in Germany, and the Dutch province of Limburg. 
nursing teams and the area covered by these teams diverged. In Belgium, teams consisted of 53 nurses, compared to I3 nurses in Germany and II nurses in the Netherlands. The area covered by these teams consisted of $\sigma_{3} 000$ inhabitants in Belgium, 65000 in Germany, and I9 000 in the Netherlands (Derksen, I991a; Derksen, Zee et al., I99Ib). This was reflected in significantly shorter travelling times to patients in Belgium (4 minutes) compared to Germany (about 7.5 minutes).

Also, important differences in the frequency and duration of home visits per day were found: in I99I, a Dutch community nurse made about 8 home visits of half an hour per visit on average, a German nurse about I2 patient visits of about 22 minutes each, and a Belgian nurse visited as much as I5 to I6 patients per day but only spent about I7 minutes per patient visit. These figures were reflected in the time spent on direct patient care: Belgian nurses spent as much as 85 per cent of their time on patients and a marginal amount of time on meetings ( $\mathrm{s} \%)$ and administrative procedures, and German nurses record almost no administrative duties. In contrast, in the Netherlands, home nurses spent 70 per cent of their time on patients, and as much as II per cent on meetings and about 20 per cent on administrative procedures.

Also, striking differences were found in the kind of tasks performed by home nurses in practice:

'Dutch nurses provide significantly more care than nurses in both other countries. Belgian nurses record more domestic activities and German nurses record almost no administrative duties. The largest differences, however, are found in the number of injections (especially for diabetic patients). Dutch nurses record an injection in $16 \%$ of the home visits, Belgian nurses in $29 \%$ and German nurses in $45 \%$. The number and duration of the visits also differs considerably.' (Zee, Kramer et al., I994 p. 798)

Also, differences were found in the degree that nurses considered certain tasks as an important part of their job: Dutch nurses considered preventive and supportive tasks a larger part of their job than Belgian and German nurses. And although German home nurses considered domestic tasks significantly more often as part of their job than nurses in the other two countries, they did not perform these tasks more often in practice.

Another characteristic finding was that Dutch nurses were least satisfied with organisational conditions, while Belgian nurses showed the highest satisfaction with their professional conditions. It would be interesting to investigate the background of these differences. Could these be attributed to relative independence of medical supervision and more diversity in nursing activities in the Netherlands compared to Belgium and Germany? Or for example to a greater burden of respon- 
sibility accompanying larger autonomy and task diversity, or side effects of the reorganisation of home care in the Netherlands at that time? ${ }^{2}$

Apart from differences in the organisation of health care, the study also detected important differences in the living situation of patients: $25 \%$ of the patients lived with their children or other family members in the Netherlands, compared to $32 \%$ in Belgium and 35\% in Germany. However, the availability of informal care did not differ significantly; probably because family members living close to their relatives often provide informal care as well (Dautzenberg, 2000).

Thus, even when comparative research is performed in a relatively restricted area of countries that partly share their language and history and have frequent contacts and forms of co-operation, remarkable differences in the role, tasks and job perception of home nursing are found, as well as differences in patient characteristics, family structures, and relations between different health professions, for example in the form of referral structures. Apparently, differences between countries on the macro-level of health care systems, health and family policies, and social and cultural values regarding long-term care for frail and elderly people are reflected in micro-level community-care practices.

This book departs from the assumption that international differences in home nursing practices and the modalities of nursing exist across Europe. Although societies and societal practices develop and change over the years and a more global culture has developed due to an easier interchange of ideas, knowledge and people between countries, there are assumingly still important differences in national cultures and the way different societies deal with matters of health, disease and dependency and differ in their opinions about the way vulnerable elderly people should be cared for. Fundamental changes take considerable time, and cultural and structural differences between European countries are likely to persist.

This thesis aims to find explanations and backgrounds for these international differences in home nursing practices for elderly people, by positioning these within a broader context and relating them to differences in interprofessional relations, relations between health care sectors, the structures and way of financing of the overall health care system, and differences in social, cultural and political norms and values between countries. The thesis only marginally focuses on the micro-level of the specific tasks performed by home nurses, such as whether there are important differences in the number of injections given to patients, or whether bladder wash-outs or stoma care belong to the task area of home nurses, or whether home nurses in different countries are allowed to perform what kind of technical nursing procedures, such as epidural anaesthesia or catheterisation.

2 A more detailed analysis of these reorganisations is described in the chapter on home nursing in the Netherlands in this book. 
These differences are reported in national and comparative studies focussing on home nursing practices (e.g. Hutten and Kerkstra I996a; Verheij and Kerkstra I992). This thesis, however, aims at uncovering backgrounds of such differences in practices and providing a framework to interpret these differences.

The central issues addressed in this thesis are derived from a macro-social and cultural perspective. Such a broad view on home nursing is fruitful, because currently more practice-based research in nursing exists, performed in the domain of organisational and nursing studies, than sociological and historical 'research of nursing' (Burau, 2000 p. 3). ${ }^{3}$ The first approach is primarily aimed at evaluating and improving current nursing practices and is applied in character, for example studying better or more efficient ways to perform specific nursing practices, while the second approach is more theoretical and reflexive in character and addresses more general aspects of nursing. This thesis follows the second approach, and aims to integrate different perspectives on home nursing, in order to provide a broad, contextual and multi-disciplinary perspective on this profession and the way it is embedded in different kinds of health care systems and societies. The perspectives taken in this thesis are mainly sociological, historical and cultural in character. Also (comparative) policy analysis and organisational perspectives are used, as an important issue for home nursing professionals currently is that most of them are employed in home care or home nursing organisations where organisational and managerial values can come into conflict with professional values (Benson, I973; Mintzberg, I979c; Davies, I983; Los, I997). The different perspectives taken in this book exist next to each other and complement each other, but are not considered as causally related.

\section{I.3 The perspectives on home nursing in this thesis}

Thus, in this thesis, a macro and meso level perspective on home nursing is taken, to analyse international differences in the position of the home nursing domain and the role of the home nursing profession amidst other health care domains and professions and the way its practices are embedded in different cultures. As will be argued in the next chapter, home nursing can be characterised as a broad sphere of action, consisting of two clearly distinguishable fields of activity, namely care provided to 'healthy clients', such as preventive health care (e.g. child care and health promotion), and care provided to different categories of patients, such as short-time nursing care after discharge from hospital, long-term nursing care

3 This argument closely resembles that of Straus, stating that medical sociology should be aimed at 'research of medicine' rather than research in medicine (Straus, I957). 
to chronically ill, disabled and elderly people, and palliative and terminal care to the dying (Kerkstra and Hutten, I996a p. 3). Currently, the core domain of home nursing consists of long-term care for chronically ill and elderly people in most European countries. This field will therefore be focussed on in this thesis.

\section{Levels of analysis and assumptions}

The home nursing profession and its domain are described and analysed at four different levels:

I) The micro level of the domain of home nursing: what kind of home nursing and long-term community-based care and support services for elderly people are provided in each of the countries studied? What international differences in the kind of activities: the 'primary process' of home nursing can be found?

2) The intermediate level of the specific home nursing profession: what is the role and status of the home nursing profession within the health care system of each country? What kind and level of training qualifies for home nursing? What kind of autonomy do home nurses have regarding diagnosis of the patient's needs and the way they perform their nursing activities? What is the intraprofessional division of labour in home care?

3) The meso level of interprofessional relations and relations between different sectors: what are the relations between home nursing and the medical professions or medical sector (GPS, hospitals), the allied domain of home help and the societal sector of informal care?

4) The macro level of the institutionalisation of home nursing as a subsector of the health care system and as a societal domain: what is the position of the home nursing domain as a subsector of health care in relation to other health care domains and informal care? What international differences in modalities of home nursing and home care can be found in different societies and how can these be explained? What impact do diverging socio-cultural and political backgrounds and different health care systems have on the modalities of home care in these countries?

The assumptions behind these questions are that international differences in home nursing practices are interrelated with differences between countries regarding:

I) The kind of welfare state and the kind and range of health care arrangements of a country (Esping-Andersen, I990). These can be recognised in the structure and way of financing of overall health care systems, and in political priorities and policies regarding health care and community-based elderly care;

2) The relation between medical care (cure) and caring and supporting activities (care) in health care systems; 
3) The role of informal caregivers (i.e. mainly family members and neighbours and other members of the social network of people) in the provision of longterm community-based care;

4) Social and cultural norms and values regarding public (formal, professional) and private (family) responsibilities in caregiving.

\section{I.4 Theoretical perspectives}

To gain a broad, integrated view on home nursing, four theoretical perspectives are combined in this thesis. Firstly, a health sciences perspective is chosen, meaning that problems regarding health and disease, becoming older and dependent are studied by looking from multiple perspectives at the way that societies deal with these problems by designing health policies, health care systems and health care arrangements. Secondly, a sociological perspective on professions is used to indicate why home nursing is an important and interesting profession to study. The theoretical arguments behind this statement and the specific characteristics of the home nursing profession and domain will be indicated in the next paragraph and elaborated in more detail in the next chapter. Thirdly, a neo-institutional perspective is used to provide insight in the position of home nursing as a subsector of health care and society. Fourthly, a cultural studies perspective on the way different societies deal with matters of health and disease is used to explain and provide background to international differences in home nursing and care provided to elderly people. Also, specific norms and values and historical backgrounds can explain underlying preferences regarding choices that are made in health policies and regarding health provision in each country.

\subsubsection{The health sciences perspective}

The first perspective that is chosen is a health sciences or public health perspective. This perspective entails that health and illness and the societal institutions involved in dealing with these are studied from an integrated, multi-facetted perspective (FHML, 2006, 2008). Most important is that not a single disciplinary view is chosen to study a problem in health care and society, but multiple scientific perspectives are used to study problems concerned with health and illness. Regarding home nursing, the effects of demographic changes on societies and health care systems, especially the ageing and double ageing of the population are important to study. Especially relevant is the ratio of elderly $(65+)$ and of the very old $(80+)$ compared to the general population. Not all elderly people are of course in need of help and support, but with the increasing age, the likeliness of becoming frail and dependent increases. Also, the increasing prevalence of multiple health problems, 
especially among the very elderly, is reflected in the increasing demand for home care services. Due to the increased life expectancy, a change in the kind of diseases that are treated in health care is taking place. While medical and hospital care primarily focussed on acute diseases, this is gradually shifting during the last decades to the treatment of people with chronic diseases and age-related afflictions. The biological consequences of old age have led to a change in disease patterns among the population that result in changing health care demands. These changes are increasingly recognised by societies and translated in health care policies to deal with the increased demand for long-term care for frail and chronically ill elderly people.

Another development also led to a changing demand for elderly care, namely the change in family patterns throughout Europe during the last decades. After all, when the role of informal care by family members is changing because less family members become available to provide care, and less family members tend to provide this care due to the societal process of individualisation, this leads to an increase in the demand for professional care. Therefore, demographic data on the population structure are studied in each of the countries, as well as the role of informal care and changes in family patterns over time. This thesis studies the way that countries respond to these health needs and foresee in the changing demand for care and support by issuing health policies, by changing and adapting health systems and by offering health care arrangements.

Concerning health policies for vulnerable people, changes in the priorities regarding different subsectors of health care are studied. Especially the balance between the curative domain and the caring domain and shifts in this balance are important. This leads to the study of health care systems and differences in the principles and values underlying the systems in these countries, such as accessibility of different kinds of care by the population and affordability of care by individual clients as well as by society as a whole. Presumably, in some countries medical care by GPS or hospitals is more easily accessible and more affordable than home care. Also, in some countries, governments will emphasise the provision of informal care to the vulnerable, while health policies in other countries prioritise professional home care. Thus, health policies, the structure of health care systems, the relation between different health care sectors, and the choices made in countries regarding the accessibility and affordability of care will be studied. These aspects are closely related to the kind of welfare system of a country and the historical background and development of welfare policies.

Furthermore, the way that societies deal with vulnerable people and the way these are viewed are included in this study, in the sense of societal perspectives regarding the role of elderly and chronically ill people, as well as the kind of arrangements that are available for them. For example, in some countries, elderly people can be valued as wise and experienced and are expected to fulfil an impor- 
tant role in family lives and society, while in other countries, older and vulnerable people can be valued as burdensome. They are considered as dependent on others to provide informal and professional care and support and in need of specifically adapted facilities and housing schemes. As they 'consume' a considerable part of the financial means available for health care and related arrangements, elderly and vulnerable people can be viewed as a costly burden to society. Therefore, the differences in views between countries regarding these groups in the population are also included in this study.

In addition, differences between countries regarding health care practices and the organisation of care are studied. For example, in what way is care organised for individual vulnerable people, who co-ordinates the different kinds of professionals involved, and can individual clients find their way in the health care system regarding the kind of care they are entitled to? Also, the role that is expected of care receivers is studied regarding whether they are considered as independent and autonomous individuals who just need to be supported with facilities to be able to remain living in their own homes, or whether they are considered as dependent and encouraged to enter an institution such as a residential home or nursing home. The differences in views between countries regarding what adequate care for elderly and vulnerable people should entail are central aspects that are studied in this book.

\subsubsection{The professional perspective - why home nursing?}

In the sociology of professions, the medical profession has frequently been described and considered as a 'model' or ideal type for professional development (see for example Freidson, I970a; Hafferty and McKinlay, I993a and chapter two of this book). This thesis, however, aims to contribute to the body of knowledge on professions by focussing on a non-medical profession, and studying whether mechanisms of development and change run the same course as that of a medical profession, or whether there are significant differences. Also, the way of functioning of a non-medical profession amidst other professions and different health care sectors is important to study, as it has been rather neglected that interprofessional relations are different in character when the profession under study does not have a taken-for-granted authority and dominance over other professions, such as the medical profession.

During recent years, the relatively strong focus in the sociology of the professions on the medical profession has gradually shifted to the relation between the medical profession and nursing as an allied, subordinate, or para-profession (see for example Alaszewski, I995; Allen, I997; Fox, I989; Svensson, I996), and to nursing or caring professions as such (see for example Abbott and Wallace, I990; Aiken and Sloane, I997; Brannon, I994; Davies, I995a). Also, in the last decades, 
studies are published that are comparative in perspective, broadening the view on nursing by analysing it within different national contexts. These studies show that the way of functioning of nursing, the content of the work and task area, its relations with other professions, clientele, and the way it is governed, may diverge considerably between countries (Burau, I999b; Hutten and Kerkstra, I996a; Verheij and Kerkstra I992; Zee, Kramer et al. I994).

Most (national) studies on nursing as a profession, however, focus on nursing in hospital settings. There is still relatively little research on the role and function of nurses in other health care domains, especially in community-care settings, such as home nursing. However, in the out-of-hospital domain, the independent task area of home nursing becomes more clearly visible, as will be described in the next chapter. Here, home nursing is not only supportive of medical activities, but also has its own independent domain of caring for and support of vulnerable people to carry out their daily life activities and enhance their quality of life, together with home helps and informal carers. This makes the home nursing domain into an important and particularly interesting professional domain to study. The aim of this study is to gain deeper theoretical understanding of professions in noninstitutional settings.

Also, a central theme in many studies on nursing is the subordinate position of nurses in relation to physicians, while far less attention is paid to other professional aspects. ${ }^{4}$ A study into the domain of home nursing, where nurses work relatively independent and out of the direct influence and sight of physicians, is expected to give more insight in those other professional aspects. Nevertheless, the relation with physicians still remains an important issue, as in some European countries; home nursing clients are only entitled to home nursing when they have a medical referral. This relation will however be different in character than in an institutional setting where physicians and nurses daily work together in multidisciplinary teams. The relative absence of interference of physicians in their work and the fact that home nurses work individually in the homes of patients also implies that their autonomy to decide on the way they perform their tasks is larger than in other nursing domains. Thus, the kind and degree of autonomy of the home nursing profession in comparative perspective will be a major research theme in this book.

Another reason why home nursing is interesting to study is that the societal context, which is considered as one of the key concepts in this thesis, becomes particularly apparent in this domain. After all, home nurses provide their tasks in the private living environment of the patient, where social, cultural and political

4 This is the result of nursing being the 'classic case' of a subordinate profession in relation to medicine (Abbott, I988d). 
norms and values about the expected role of family members in caring for elderly people become visible. The amount of care provided by informal caregivers plays an important part in determining the range of complementary and additional tasks provided by home nurses and other professionals. Thus, home nurses are working at the interface between professional care and informal care, as the domain of home nursing finds itself in an intermediate position between the formal health system and the personal sphere of the client. Also, national health policies regarding elderly people become visible here, that focus on the question whether elderly should preferably remain living in their homes, supported by family members and a range of facilities and professional care, or whether they should move to a home for the elderly, nursing home or geriatric department in a hospital. The home nursing domain therefore functions as a pivoting point between the health care system and the patient and informal carers, and provides insight in the social, political and cultural context of elderly care and the relations between different kinds of care, such as institutional care in homes for the elderly and nursing homes, home help, and informal care.

\subsubsection{The neo-institutional perspective: home care as a societal sector}

The home nursing domain is one of the (sub-) sectors of health care, with as core tasks the provision of care and support to frail elderly people, to enable these to remain in their own homes as long as possible. From a sociological perspective, a sector refers to a domain identified by similarity of service, product or function (Scott and Meyer, I992c p. 137). The boundaries between sectors are defined by their function, not by geographical boundaries, in this case the provision of care to elderly people living in the community. Sectors consist of units, in this case public and private organisations and independent nurses who provide this community-based care. These are interrelated because they have the same function, even though they may be geographically remote. Thus, a home nursing organisation in the North and South of France both belong to same sector and have the same function.

The concept of a societal sector refers to the presence of society-wide structures that are, at least to some degree, functionally differentiated (Scott and Meyer, I992C p. I39). Scott and Meyer (I99I) describe a societal sector as follows:

'(I) a collection of organizations operating in the same domain, as identified by the similarity of their services, products or functions, (2) together with those organizations that critically influence the performance of the focal organizations: for example, major suppliers and customers, owners and regulators, funding sources and competitors.' (Scott and Meyer, I99I p. II7) 
In theory, all the activities associated with a particular function, such as the provision of home care, are performed by a specialised and hierarchically organised set of interrelated organisations performing the same function. However, in practice, also more diffuse social arrangements and health care arrangements exist, such as family members that are performing the same function, or health professionals that belong to another sector who may also provide community-based care. Also, in different countries, the boundaries of societal sectors are dissimilar; for example, a home nurse in Denmark provides a different range of tasks than a home nurse in France and works together with different kinds of other professionals. Thus, a comparative institutional analysis of the home nursing sector will unveil differences in the boundaries and core tasks of the home nursing sector in different countries.

When a societal sector is organised around a core function of society, in this case caring for vulnerable people, it is considered as an institution in the sociological sense (Lammers, Mijs et al., 2oood). ${ }^{5}$ Such a core function consists of activities that are designed to cope with a number of fundamental problems every society has to contend with, and is related to social and cultural norms and values and reciprocal expectations. For example, the state is designed to maintain a certain social order, the church is a societal institution giving meaning to life and death, and health care takes care of the physical and mental vulnerability of the members of a society. In most North, North West and Mid European countries now, the core activities of home nursing, consisting of nursing, caring and supporting frail and vulnerable old and very old people with various activities of daily living in order to enable these to remain living in their own homes, are considered as an important societal task. Thus, in these countries, home nursing can be characterised as a societal sector with a particular function that is important to society, and in this sense, the home nursing sector is a societal institution. This way, this book fits into scientific thinking about institutions from macro sociology, social history, and cultural studies (Powell and DiMaggio, I99I p. 3). In these areas, institutions have always been regarded as the basic building blocks of social and political life and studies in these fields shed light on how meaning is socially constructed.

However, the degree in which the home nursing sector is institutionalised varies between European countries. This thesis analyses these differences in the degree of institutionalisation of home nursing in four countries in Europe in which the home nursing sector is institutionalised to a greater or lesser extent, in the North (Denmark), North West (the Netherlands), Mid (Germany) and Mid to South (France) parts of Europe. In several South European countries, such as Portugal,

5 An institution is often also referred to in the sense of an intramural health care facility, such as a hospital or nursing home. Then the process of 'inclusion by exclusion' takes place; by admission into such an institution, older people are excluded from everyday society. 
Italy or Greece, home nursing hardly exists and can thus not be considered as a societal institution; these countries will therefore not be studied in this thesis. This rudimentary distinction in institutionalisation of the home nursing sector already points to differences in societal constellations and political and cultural notions about what kind of caring arrangements should be provided for elderly people.

The home nursing domain and the organisations in it are situated within an institutional environment, and are influenced in their way of functioning by other societal sectors or domains, organisations, and actors, directly as well as indirectly:

'Institutional environments are, by definition, those characterized by the elaboration of rules and requirements to which individual organizations must conform if they are to receive support and legitimacy. The requirements may stem from regulatory agencies authorized by the nation-state, from professional or trade associations, from generalized belief systems that define how specific types of organizations are to conduct themselves, and similar sources.' (Scott and Meyer, I991 p. I23)

For example, home nursing is influenced by the way of functioning of the hospital sector and medical professionals, the extent to which family members provide informal care to elderly people, the role of the government in health care, the influence of commercialisation in health care, etcetera. By way of this interrelatedness with other societal institutions and actors, the home nursing sector is subject to cultural influences that are partly institutional-bound, and partly also related with certain social classes or groups, certain regions, and society in general.

The norms and values that are adhered to within such an institutional environment make the activities of the organisations recognisable, understandable and acceptable to society. Thus, their existence is legitimised. Scott and Meyer (I99I) hypothesise that "Organizations functioning in sectors that are highly developed both institutionally and technically will develop more complex and elaborate administrative systems and will experience high levels of internal conflict." The reverse hypothesis they state is that "Organizations functioning in sectors that are not highly developed either technically or institutionally are expected to be relatively small in size and weak in terms of their capacity for survival" (Scott and Meyer, I991 p. I26). These hypotheses point to the existence of differences in the extent to which bureaucratic structures exist and interfere in home nursing practices in different countries. This interference is likely to result in tensions between bureaucratic or organisational and professional values, especially when bureaucratic procedures tend to replace professional decision-making. Therefore, international differences in the range and degree of interference of these administrative systems in home care practices will be analysed, which especially seems an issue of concern in home nursing in the Netherlands. 
In this study, international differences in the degree of institutionalisation of the home nursing domain will be explored. For example, home nursing activities can be organised in a homogeneous way, by rather isomorphous organisations operating within clear social or health care structures. However, in countries where home nursing is less established or institutionalised as a societal domain, a variety of professionals, organisations and other forms of elderly care are supposed to exist. These operate within health care structures that form barriers for more coherent ways of provision. Thus in such countries, the same need or function is addressed to by different kinds of services. According to Scott and Meyer (I99I), these dissimilar, but functionally equivalent services belong to the same sector, as the same core function is executed by way of substitution (Scott and Meyer, I99I p. II8-II9). However, their dissimilarity points to a different or lesser degree of institutionalisation of the sector. This degree of institutionalisation of home nursing will be further explored in the subsequent chapters of this thesis, in the sense of the degree in which the organisational or professional activities have become settled and are recognised as performing important tasks in society.

Also, differences in the social status of home nurses, in the sense of the esteem and social appreciation of the activities they perform, will be studied. For example, a society appreciating the more feminine values of caring for people, and aiming at achieving a high quality of life and large amount of independence for elderly people who should be enabled to live in their own homes as long as possible, home nursing activities will enjoy a higher social status than in a society that appreciates masculine, instrumental health care activities that are aimed at curing people from physical or mental disorders. Thus, the social status of the home nursing domain is related with caring cultures and the social esteem of elderly people.

\subsubsection{A cultural studies perspective: caring cultures from north to south Europe}

Many comparative studies on health care state that a North - South axis exists within Europe with different caring cultures, characterising northern countries such as Scandinavian countries, the $\mathrm{u}$, and the Netherlands, as having comprehensive health care systems and welfare arrangements and community care facilities that are available to large groups of elderly, and southern countries that are characterised as having less comprehensive health care provisions and welfare arrangements for elderly people (Esping-Andersen, I990; Giarchi, I996b p. 2832; Mossialos and Le Grand, I999b; Philipsen, I980). ${ }^{6}$ Giarchi (I996) states that

6 Both Giarchi and Philipsen also discern an East - West axis, but this will not be dealt with here. 
since the Reformation and counter-reformation, North and South Europe were split ideologically, and have been entrenched in different religious belief systems for more than four hundred years. In the Protestant North European countries, people reacted differently on developing central states, entrepreneurial activities, and administrative systems, and developed different kinds of health care provisions. Especially in Scandinavian countries, but in other North European countries to a more limited extent as well, family responsibilities to provide care are not included in social policies and welfare systems, and care is predominantly provided by formal organisations and professionals. In South European countries however, informal care provided by family members is strongly entrenched in their health and social care systems and health policies.

The ideal-type classification of countries by Esping-Andersen (I990) points to the same direction (Esping-Andersen, I990). He discerns liberal-democratic type of welfare states in North European and conservative-corporatist type of welfare states in South European countries (including Germany). In the first, rather comprehensive welfare and professional health care arrangements are provided or else guaranteed by the state, while in the latter, the state relies on the principle of subsidiarity and only functions as a safety-net; the community should act first, and the state should only intervene when there is no other alternative (Scharf, i998e p. I30; Giarchi, I996b p. 32; see also the chapter on home nursing in Germany in this thesis).

Philipsen (I980) argues that a pattern of diffusion can be observed from Helsinki in North East Europe to Lisbon in South West Europe, explaining relations between social phenomena by way of cultural diffusion (Philipsen, I980 p. 9). Thus, research into the relation between certain social phenomena should not only look for functional-causal relationships, but also take into account that cultural characteristics diffuse or spread out over a particular cultural area by cultural transfer. Some social phenomena, such as the level of prosperity, are characteristic of modern industrialised societies in general. Other characteristics, however, such as characteristics of health care systems, are related with social and cultural norms and values and the way members of society cope with disease, mortality and reproduction, and are thus sensitive for diffusion. Thus argued, the health systems of neighbouring countries have certain structures in common, that can be explained for a large part by (historical) processes of diffusion. This implies that health care in France has more structures in common with health care in Spain, than with health care in the Netherlands. Also, health care systems of Scandinavian countries are more similar to each other, than the health care system of Denmark compared to that of France. The position of Germany is rather special, as its culture represents cultural influences from North, South, and East European countries; the latter even more so since the unification of East and West Germany, making Germany into a bridging country between eastern and 
western Europe and confronting the Bismarck health care system with elements of the East German and Russian health care systems (Marrée and Groenewegen, I997a).

This North - South gradient is also visible in expectations regarding care provided by professionals and by family members. This thesis will explore whether the general statement that family traditions are fairly strong in a more southern European country such as France, and weakening in North and North West European countries, still holds true. The question whether family care and medical care are the principal sources of care in these countries and professional elderly care has a much lower political priority than in North and North West European countries will be studied.

\section{I.5 The countries}

Four European countries are included in this study. The first country chosen is the Netherlands, as native country of the researcher. Then, three other countries were chosen with crossing borders, to gain insight into differences between countries that are geographically close, but that had made different choices regarding health systems and health arrangements in the past and develop different kinds of health policies for the future. It is interesting to study whether such countries have different caring cultures and different traditions regarding the role of the family in caring for vulnerable people as a result of processes of diffusion, as described in the previous paragraph. After gaining a broad view on home care and the role of informal carers in different countries from the literature, a choice was made between countries that would give insight into differences in caring cultures between North and South Europe and between West and Mid Europe. Also, countries were chosen for content reasons, because these represent a characteristic way of caring for vulnerable and elderly people.

Thus, this thesis departs from the assumption that countries have distinct social-cultural and political identities. Although socio-cultural traditions of neighbouring countries partly overlap and countries are currently subject to cultural influences and large-scale social developments occurring in other European countries, nation-states can nevertheless still be considered as distinguishable entities. This is especially the case regarding matters of health care, as health care systems and health care policies are still devised nationally, although currently, some specific agreements are made on the European level. Also, the line of argumentation of Hofstede (I980) is followed in this thesis, who states that different national cultures can be discerned, as value-orientations regarding ways to cope with health and disease are generally more consistent within countries than between countries (Hofstede, I980a). 
For several reasons, some countries were not included in this study. For example, although being a neighbouring country of the Netherlands, Belgium was not selected. The picture emerging from the literature on Belgium displayed a fundamental lack of homogeneity that is reflected in all areas of life. This is due to the geographical and cultural division into two language-areas, a Dutch speaking Flemish part and French speaking Walloon part. In health care, this is visible in the existence of different health and community care organisations that fulfil similar functions. Also, different umbrella organisations and different ways of data gathering exist that inhibit gaining an integrated view on the modalities of home nursing in Belgium. This country would deserve a more detailed in-depth study into its specific peculiarities that is outside the scope of this study. Another country that is not included is the United Kingdom. Studies on health care and home nursing in particular in the $\mathrm{UK}$ are rather easily accessible for foreigners and a relatively large amount of information is already available.

Some Mediterranean countries, like Spain, Italy and Greece, could not be included due to lack of sufficient data, because professional home care hardly exists in these countries, at least not on a national scale. Therefore, France is included to represent a South European country, although the northern parts of this country can hardly be characterised as such. Nevertheless, the characteristics of the prevailing caring culture in France in which the family plays an essential role is quite similar to caring cultures of other South European countries. The way it diverges of caring cultures in more northern European countries is very interesting to study.

The following four countries are described in this book, because of their specific interests for this study. Firstly, Denmark is included, as representative of a North European (Scandinavian) country and culture. Comparative studies on home nursing and community-based elderly care depict Denmark as progressive in the sense of early and fairly radical policies for elderly people and the existence of a comprehensive range of services and facilities for a substantial number of elderly people (Hutten and Kerkstra, I996a; OECD, I996a). Also, home nursing in Denmark diverges from many other European countries, as it is characterised by a generalist perspective on nursing and as it is provided in an integrated way with other forms of elderly care, community-based as well as institutional-based. This way, traditional boundaries between different forms and locations of care are surpassed. The inclusion of Denmark is expected to add a new perspective on ways in which the domains of home nursing, home help and different other kinds of elderly care can be arranged.

Secondly, the Netherlands is included, as representative of a North West European country and native country of the researcher. As in Denmark, the organisation of home nursing has a long tradition, going back to the late nineteenth century. However, the position of home nurses in society has changed fundamentally; from a local authority alongside the family doctor, working as a generalist nurse know- 
ing the situation of 'her' families, she has become an interchangeable employee of a far more distant home care organisation that operates regionally. This development has had fundamental impact on the character of home nursing work in the Netherlands. Aspects such as rationalisation and objectifying of practices have far-stretching consequences for home nursing as a profession in the Netherlands. Another characteristic of health care in the Netherlands is the absence of a coherent long-term view and a state of continuous turmoil and upheaval because of ongoing reforms and reorganisations during the last decades. The reform of the entire health care system, introduced in January 2006, however remains outside of the scope of this thesis.

Thirdly, France is included in this thesis. As argued above, France is part of the South European, Mediterranean culture that is expected to give more insight in differences in caring cultures between North and South Europe. Also, France is an important country to study, as the provision of community care is quite different in character compared to other European countries, such as Denmark and the Netherlands (Giarchi, I996g; oECD, I996e; Henrard, Ankri et al., I99I; Verheij I996). Several studies state that home nursing is geographically not evenly distributed over the country, and that many elderly in need of care do not receive it, especially in the more remote areas of France. The weak position of home nursing and bad targeting of existing services seem to leave an important role for family members in providing informal care, which will provide more insight into the relation between professional care and family care. Also, France is an interesting country to study, because the provision of home care is not predominantly provided by public or non-governmental home care organisations, such as in many other European countries, but for a substantial part by private, independent nurses. ${ }^{7}$ These selfemployed, independent nurses exist far longer in France than regular home care organisations that have been established as late as i982, and claim to have a quite important position in the provision of home nursing that deserves further study.

Fourthly, Germany is included in this study as representing a Mid European country. Also, several literature sources indicate a quite special position of home nursing and elderly care in Germany. According to the principle of subsidiarity, that also prevails in France, family care is strongly embedded in the German health care system, while community-based forms of care such as home nursing and home help hardly had priority for many years (Scharf, I998c). Medical and hospital care is rather dominant in Germany (Philipsen, I980). This is reflected in home nursing, where the daily work is more concentrating on physical-hygienic and medicaltechnical nursing aspects than on caring and supportive aspects (Derksen, Zee et

7 Also in Germany and Belgium, the provision of home nursing by private nurses is quite substantial (Kerkstra, I996). 
al., ı99ıb; Zee, Kramer et al., I994; Delnoij, ı996a). Even after the introduction of the Pflegeversicherung in I995, which is considered as being quite innovative in character, home nursing is still only reimbursed when a referral from a physician is obtained. Before that, it was not reimbursed at all and the dependence of elderly on their family was substantial. Another reason to include Germany is to study the way this country responds to the ageing of the population, which is highest in the EU (Eurostat, 2005).

Thus, Denmark, France, Germany, and the Netherlands are included in this study (in alphabetical order). These countries are geographically positioned in the North West, Mid and South of Europe, and should provide more insight into different caring cultures from North to South and different ways to cope with an ageing population. As each country has a different social, cultural, historical and political background, this will result in a different constellation of health care arrangements, and consequently, a different position of home nursing in the health care system.

\section{I.6 A comparative analysis}

The countries chosen in this book are particularly suitable for a comparative analysis, as they have a number of major features in common. For example, they all represent the wealthier regions of Europe and all have a parliamentary democracy. All countries have developed a social security system and a health care system during the late nineteenth or twentieth century. Although to different extents, social welfare arrangements exist, guaranteeing every citizen a minimum subsistence level. Also, all countries share the period of post-war reconstruction after the Second World War. Since then, these countries have shared their historical development more than that they have diverged from one another. This is among others shown by their membership in the European Union, which is primarily aimed at forming one large internal economical market, but also stretches out to matters of social security and health care arrangements. Thus, from a very general point of view, these countries share many key characteristics, which makes them suitable for comparison on a more detailed level.

However, despite these similarities, there are important societal, political and cultural differences that influence the conditions under which home nursing is provided in these countries that are interesting to study in more detail. The health care systems of these countries and the social and cultural values that are related to matters of health and disease certainly do diverge from one another. In spite of the fact that these countries are geographically close and have become more and more culturally similar, there are still important differences in value-orientations regarding the way people, and elderly people in particular, should be able to live their lives 
and what kind of facilities should be available to support them in this. The most important and at the same time simple questions are: "Who cares for the vulnerable and elderly? What kinds of care are provided by whom?" For example, should the family provide most of the care, or should this be taken over by professionals? Should elderly predominantly live in institutions, in independent households, or in three-generation families? Are health care policies aimed at enabling vulnerable elderly to remain living in their own homes, supported by an extensive network of community care facilities? Or are health policies aimed at providing elderly care in facilities such as homes for the elderly and nursing homes?

Such social arrangements are related to value-orientations regarding caregiving and the dispersion of health care facilities, and thus with the structure of the overall health care system and policy-making regarding elderly people. By taking these aspects into account in the analysis, the aim is to gain insight into differences in the modalities of the home nursing profession that is embedded in diverging structural and contextual environments, and into the degree of institutionalisation of the home nursing domain in each of the European countries studied. This approach should shed light on whether the central issues or difficulties in home nursing are more universal in character and recognisable in different countries to about the same extent, or whether certain issues are typical for one country, a specific set of countries, or a particular kind of health care system.

\section{I.7 Research design}

This book attempts to perform a contextual analysis of the domain of home nursing by using secondary sources and communication with experts. Generally, existing studies on home care give a detailed account of the situation on the local level, or describe a particular aspect of home care in national or international perspective. This book intends to aggregate and integrate knowledge and views described in earlier studies and policy documents on home care to gain a broad and multifacetted view on the topic. For this reason, no new data were collected by way of e.g. interviews with home nurses in each of the countries under study, and no thick description' is provided such as in ethnographic anthropology, which refers to a rich and thorough description of the research setting or context as well as of the activities and processes observed, in order to understand the cultural meanings attached to these activities (Geertz, I973; Polit and Beck, 2004 p. 435-436).

For this study, no primary data are gathered by for example participant observation or formal interviews, which would require thick description of the participants and their specific cultural context. Instead, an institutional analysis is performed on the basis of different kinds of secondary sources consisting of qualitative as well as quantitative studies and documents. These are analysed and synthesised 
into combined information in which different kinds of knowledge are linked. Most studies used are published between I990-2007, some a bit earlier. These studies offer sociological explanations for certain developments and place these in context. This way, an overview is gained over long-term societal developments. Sources referring to demographic and health care data (European Observatory, Eurostat, OECD, wHO, etc.) are as recent as possible.

This way, a broad overview is gained on the way vulnerable elderly people are valued by different societies and the caring arrangements that are developed. As De Swaan (I988) states in his institutional analysis of the development of welfare states in different countries:

'A book like the present one relies very much on the findings of others. Its strength is not in the discovery of new data, but in the confrontation of developments in succeeding periods, various fields and different countries from which connections and similarities emerge which may have eluded more specialized authors. In the choice of countries for comparison, the availibility of an adequate body of literature on the subject has been the first concern. (...) In the research for this book, the guiding rule has been never to investigate primary sources, to avoid monographs, to rely mostly on studies that reviewed at least one institutional field in one country over an extended period of time, to consult, whenever possible, comparative studies across fields or across countries and always to seek for the social historical or sociological perspective.' (Swaan I988 p. I-2) ${ }^{8}$

The following figure of the research design of this study can be drawn, based on the research questions that are formulated in this chapter (see figure I). The figure displays the way in which the different levels of analysis, the theoretical perspectives, and the kind of sources that are studied should together lead to a broad view on the different modalities of home nursing within Europe.

8 For comparable perspectives, see Scott and Meyer (I99I, I992), Lammers (I983), and Powell and DiMaggio (I99I). 
Figure I Research design

\section{Research Questions}<smiles>C1CCCC1</smiles>

Analytical levels

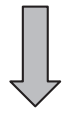

Theoretical perspectives

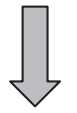

Sources

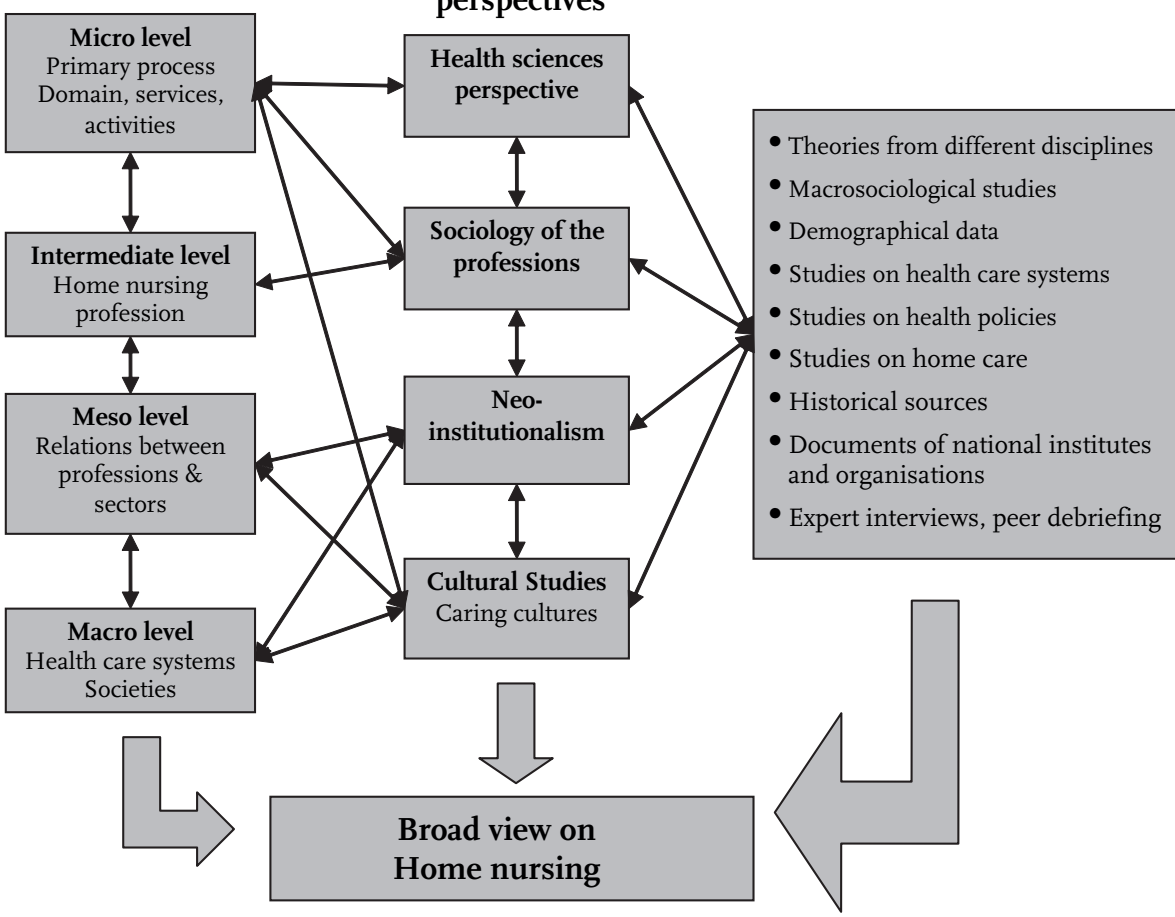

\section{I.8 Sources}

As described in the previous paragraph, the integrative character of this research leads to the analysis of different types of already existing, thus secondary, data and documents with different accounts of various aspects of home care. The following kinds of sources are used:

- Sociological studies on professions and professionalisation, institutionalisation, co-operation and bureaucratisation; to develop the theoretical framework of this study. The way in which these theories are used in this book is described in chapter one and two; 
- Studies on specific countries and by macro-sociological comparisons of different countries; to gain a broad overview of the societal and cultural characteristics and the political climate regarding health care issues, especially regarding the balance between cure and care and towards vulnerable and elderly people;

- Demographical and statistical data; to describe general characteristics of each of the countries and on health care in particular;

- Literature sources on general characteristics of health care systems in Europe and on health policies in Europe;

- Specific and detailed studies on the domain of home care; especially studies focussing on home nursing, home help, elderly care and informal care in different European countries;

- Sources on the history of (home) nursing, elderly care and elderly policies;

- Scientific studies and journal articles were retrieved from the Maastricht University library catalogue and electronic library sources, such as Medline and Pubmed. The following key words were used: home care, home nursing, home help, family nursing, home care services (organisation, administration, legislation, trends), community health nursing (manpower, organisation, administration), community (-based) care, health services for the aged, elderly care, health policy (trends), history of nursing, home nursing history, family care, informal care, etcetera. These keywords produced different amounts and different types of relevant publications about health care, health policies, community care, home-based nursing and informal care on each of the countries;

- Peer debriefing in the form of interviews and correspondences with experts on home care, health professions, and health systems in different countries and scientists of the International and European Sociological Associations (ISA and ESA) studying these fields.

Apart from sources collected through the University Library and electronic databases and the 'snow-ball method' by looking at literature references and sources used in other studies, also reports and publications of national and international organisations, research institutes and Ministries were used that provided information on health care, home care and caring policies regarding vulnerable and dependent people. For example, the who, Eurostat, the oECD, and the European Observatory on health care systems at the European level; CREDES in France; the Danish National Institute of Social Research in Denmark; the Bundesministerium für Arbeit und Sozialordnung and organisations such as Caritas and Diakonisches Werk in Germany; and institutes such as NIVEL, NIZW and LeVv in the Netherlands, regularly publish reports and documents that provided a lot of important and recent information.

Also, documents were collected in the libraries or information centres of the Netherlands Institute for Health Services Research NIVEL (Nederlands Instituut 
voor onderzoek van de gezondheidszorg); the Netherlands Centre for Excellence in Nursing LEVv (Landelijk Expertise Centrum Verpleging en Verzorging); the Netherlands Institute for Care and Welfare NIZw (Nederlands Instituut voor Zorg en Welzijn); all located in Utrecht. In addition, the archives of the National Museum of Nursing and Caring N mvv (Nationaal Museum Verpleging en Verzorging), currently located in Zetten, were visited to collect literature and documents. Its director Mrs Wiegman provided relevant additional oral information as well.

The researcher, native Dutch speaker and also mastering French, German and English, could access studies on the Netherlands, France and Germany and experts in the field in their native tongue. However, due to lack of skills in Danish, only secondary sources on Denmark in English or German could be studied. This was considered as a drawback, but Denmark was considered important for comparative reasons to nevertheless include into the study. To gather additional in-depth information, the Danish Museum of Nursing History (Dansk Sygeplejehistorisk Museum) in Kolding was visited. Its director Mrs Børgsen provided several relevant sources that were not available in the Netherlands, and an expert interview was held with her. Finally, additional articles in French nursing journals were collected at the library of the Nursing Training Institute (Institut de Formation en Soins Infirmiers) in Montélimar. Also, peer debriefing in the form of contacts with several scientists from different countries studying similar fields was used. This communication intended to inform and consult others about the findings and check the validity and reliability of this study.

The theoretical framework and the findings of this study have been presented several times at different scientific audiences at the Maastricht University, the Netherlands Museum of Nursing and Caring (Boom, Philipsen et al., 2004b) as well as at international conferences of the European Sociological Association and the International Sociological Association since 2000 (Boom, Stevens et al., 2000, 200I, 2004a). The period in which home nursing is described in this thesis ends in 2005 , but sometimes references are made to more recent developments when these are considered as specifically important.

\section{I.9 Methods}

The methods used in this book, in the form of use of theory, procedures of data collection, analysis, and writing, characterise this study as a comparative and institutional analysis of the home nursing domain and the way it is embedded in particular societies. To gain such a broad view on home nursing, in which different perspectives are combined and different types of information are integrated, the issues concerned are studied and described in a cyclic way. Instead of following a prefixed, strict order of subsequent steps that is from hypothesis to conclusion, 
as is common in most quantitative studies, the methods of this study can be characterised as a cyclic way of doing research in which different research steps are alternating and co-exist. This means that the style of argumentation in this thesis is cyclic as well, meaning that assumptions, research questions, theoretical concepts and results from quantitative as well as qualitative literature are interconnected, alternated and consequently elaborated upon throughout this thesis. This research process is similar to qualitative studies, of which the designs are generally emergent rather than tightly prefigured (Creswell, 2003 p. I8I; Polit and Beck, 2004 p. I6).

\subsubsection{The theoretical perspective functioning as a lens}

In chapter two, a theoretical framework is built with concepts derived from the literature that are considered important to study to gain insight into the domain of home nursing and adjacent domains. In this thesis, the theoretical framework is used as a 'theoretical lens' or overarching perspective, consisting of topics of interest that guide the data collection and is aimed at attaining a comprehensive view on the phenomenon under study (Creswell, 2003 p. I6, 30 \& I3I; Polit, 2004 p. 29 $\&$ II5-II8). The terms conceptual or sensitising framework or theoretical perspective can be used interchangeably.

The concepts included in this framework arise from macro sociological literature and studies in the sociology of professions and guide the analysis of the texts on caregiving to the vulnerable. The theoretical framework drawn in chapter two is a visualisation of these concepts, facilitating the selection of sources and interpretation of the concepts described, and enabling the researcher to begin to understand how these may be interconnected and mutually influencing developments in different societies. This method is especially useful when dealing with abstract and theoretical studies from the social sciences. It provides a lens that guides the reader and helps to focus on the pre-formulated topics of interest in particular. This kind of theoretical framework should however not be considered similar to models that are used in quantitative studies, that generally indicate causal relationships of dependent and independent variables that are tested through quantitative hypotheses and quantitative methods. The theoretical framework used in this study is proposed as an analytical tool and lens provided by the literature to focus on the most important concepts and the way these are interrelated. It brings order into the complex world without referring to causality.

The theoretical framework acts as a concept map helping to make connections between knowledge gained from previous literature, and new knowledge retrieved during analysis of the documents (Novak, I998; Polit, 2004 p. Iı6; Creswell, 2003 p. $8 \& \mathrm{I} 82$ ). The role of the researcher is then to put segments of knowledge from different studies into a meaningful conceptual pattern. Inevitably, the researcher 
filters the data through a personal lens that is influenced by the personal background and the socio-political, cultural, and historical setting of the researcher that shape this interpretation and render it subjective per definition. The technique of concept mapping enables the researcher to capture the views of different authors into a comprehensive visualised structure. Visual schemes consisting of many facets, such as concept maps or a visualisation of the theoretical framework, aid the researcher to gain a broad view on the complexity and contextuality of societal phenomena. The theoretical framework thus a priori structures the analysis of results. However, it is also part of the final stage of the research. In studies with a cultural theme or a theoretical lens, the theory generally occurs in the beginning part of the study, but can be modified or adjusted at the end of the study, based on the data collected during the study (Creswell, 2003 p. I34). In this thesis, the theoretical framework is revised in the concluding chapter on the basis of the results, to more fully capture and explain the home nursing domain.

\subsubsection{Procedure of analysis and writing}

The following procedure was used during analysis. All books, articles and documents initially selected as relevant for home nursing and the health care and societal context were first read globally. As starting points for the chapters on each country, the most distinguishing features and core problems related to home nursing and health care were selected and highlighted. These striking features were then used as guiding perspectives from which the developments and problems in home nursing were described for each of the countries. Then, closer reading of the literature followed, focussing on the theoretical concepts formulated in chapter two. The relevant aspects regarding these concepts were then included in the chapters on each country. Then, the way these concepts are related to the countryspecific features of home nursing was described by combining and aggregating knowledge and insights from different studies. To provide explanations for these specific features and to gain more insight into the background of the differences between countries, the health care, historical and socio-cultural contexts were studied. This way, different and heterogeneous accounts on home nursing are described in each of the chapters. Thus, this book did not intend to obtain similar and uniform comparative data by laying a systematic grid over home nursing in the countries studied. Then, a different method should have been chosen, for example by approaching experts on home care to provide information according to a fixed format. However, the background and relation with other developments would then not be revealed. The methods used in this study led to quite different accounts on each country, as striking or characteristic issues were specifically sought for in each of the countries. However, this makes comparison of data quite difficult because these are not always comparable. 
As referred to earlier, progression in a study like this closer resembles a circle than a straight line (Creswell, 2003 p. I8I-I9O; Polit, 2004 p. I7 \& 55). This can also be described as a cyclic method of alternation; cycling back and forth from research questions to collection of sources, sifting through the material during analysis, to problem reformulation and back and forth again. Through such an iterative process, the researcher is moving deeper and deeper into understanding the data, finding more information that confirms emerging insights and increasingly able to understand connections between different concepts and describe and interpret the phenomenon under study. Sometimes, during analysis, new concepts or descriptions emerged from the literature on a country. When these were considered very striking or relevant, these were then sought for in literature on other countries as well. Sometimes these were considered equally important, but sometimes appeared to be of minor relevance elsewhere. The issues and concepts emerging during the study that were also considered important are included in the revised theoretical framework described in the conclusion.

Another important part of the research process was peer debriefing; continually discussing the methods and checking the results with colleague researchers in the Netherlands and abroad studying similar fields, and talking to people working in home nursing to check the literature with actual home nursing practices. Generally, the results were strongly recognised and confirmed by experiences of home care managers, home helps and home nurses.

\subsubsection{Methodological views and considerations}

This study is rooted in the social constructivist tradition but at the same time differs from this approach. It shares the search for contexts and the historical and cultural background and the interpretation of the meanings adhered to certain phenomena by interactions of diverse stakeholders. The stakeholders in this study are consisting of important subsectors of society, such as home nursing, home care, medical care, informal care, home care clients, governments, and health policies. Due to the specific societal, political and cultural contexts in each country, the relations and interactions between these subsectors vary, resulting in different national constellations of care to the vulnerable. This way, different societies construct meanings over time regarding the home care domain that are embedded in these specific national contexts. This book tries to uncover these varieties and diverging developments. As described, the divergence between countries is already clear from the different kind and character of sources available. In some countries, meticulous data collection is performed and researchers can easily draw a picture on a specific health care sector. However, in other countries, data collection is scarce, only partly available, or differs in methods and is therefore incomparable to data collected on the same topic in other countries. Also, in some countries, 
accounts on the role of informal care are available at the level of health care practices as well as in governmental policies. In other countries, informal care is hardly mentioned. This obviously leads to different accounts on home care per country and explains why uniform ways of data collection do not fit this thesis. This book makes use of texts that account for the way that societal subsectors function and are interrelated and describe their societal context. This study hopes to gain insight into the way in which abstract and broad societal patterns and structures develop, by analysing meanings and views regarding long-term care for the vulnerable to which societies adhere.

\section{I.Io Contents of the book}

The chapters of this book are arranged as follows. In the second chapter, the theoretical framework that is used in this study is developed. This framework is built up after a discussion of several strands in the sociology of professions and particularly Abbott's perspective on professions, as well as a description of the specific characteristics of the home nursing profession and domain. The different theoretical perspectives described in the first chapter are included in the theoretical framework, resulting in a theoretical lens to study the modalities of home nursing from a comparative and contextual perspective.

The third chapter contains an overview of demographic, health care and country-specific characteristics of each of the countries of this book, providing general background to the subsequent chapters on home nursing in particular countries. These are described and summarised in tables that are included in annexes. To comprehend the chapters on home nursing of each particular country, it is not essential to read this overview chapter, but it includes additional background information that has been helpful to gain a broad view on the specific matters of importance of each of the countries.

Chapter four to seven are empirical chapters on each of the four countries, analysing and describing the home nursing domain and profession and its relations with the medical domain, the role of informal care in elderly care by means of the theoretical framework developed in chapter two. In part, all countries described have to cope with similar problems, such as the ageing of the population, the rising of health care costs and the changes in informal care networks, thus these can not explain differences in home nursing between these countries. However, also issues or aspects of home nursing exist that are characteristic for a particular country and not found to be as striking elsewhere. Therefore, the most distinguishing features of each country related to health care or home nursing practices are selected, as described earlier. These most striking features provide a particular perspective from which the issues are described in each of the countries. First, 
the two most contrasting countries on the North-South axis are described, namely Denmark and France. The chapters on the Netherlands and Germany provide an additional detailed picture of the diverse modalities of home nursing within Europe. Thus, each of the four countries is dealt with in separate chapters on the particular developments and issues regarding home nursing and the particular way it is embedded in the health care systems and the specific social, cultural and political contexts of these countries.

In chapter eight, the concluding chapter, the differences and similarities of home nursing are discussed in comparative perspective, and related to the specific health care and societal context of each country. The theoretical perspective developed in chapter two is used to summarise and compare the different aspects studied in each of the countries. The institutional analysis of home nursing should give more insight into the three aims and research questions formulated at the beginning of this chapter and in the role of home nursing to the vulnerable in contemporary societies. 
Chapter 2

\title{
Theoretical perspectives on professions and home nursing
}

\author{
- Perspectives in the sociology of professions ${ }^{1}$
}

\section{I Introduction}

In chapter one, the three theoretical perspectives adopted in this study were described, namely the professional perspective, the institutional perspective and the cultural perspective on diverging cultures of care within Europe. In this chapter, a theoretical framework is developed, designed to analyse the modalities of the home nursing domain and profession in comparative perspective. In the following chapters, this framework is used to study and describe the general and specific characteristics of home nursing within the context of the health care systems and societal context in Denmark, France, the Netherlands and Germany. The three theoretical perspectives described earlier are included into the theoretical framework.

In the first part of this chapter, the professional perspective is elaborated upon in more detail and different perspectives in the sociology of professions are described. It will be discussed that each of these has its specific shortcomings. Therefore, Abbott's historical-sociological perspective on professions is proposed as a more fruitful approach to study home nursing from a contextual and comparative perspective (Abbott, I988a).

\subsection{Perspectives in the sociology of professions}

In society, numerous activities are performed by professionals. Although a large body of sociological and historical literature on professions exists that is written from different perspectives, relatively few studies focus on the specific dilemmas and difficulties that professionals are confronted with in everyday practice. Many

I The content of this chapter has been discussed in a paper presented at the Fifth Conference of the European Sociological Association, 30. August 200I, Helsinki (Boom, 200I) and a lecture presented at the symposium in the Dutch Museum of Nursing and Caring, 6. October 200I, published in the Dutch journal GeWiNa (Boom, 2004b). 
studies on professions have focussed on ideal type or 'classical' professions that are already well established and generally recognised in society, such as medicine and law. Other professions, with a less clear status and position, have mostly been studied in relation to these dominant professions. This thesis focuses on such a socalled 'subordinate' profession, namely nursing, and more specifically, home nursing. The central aim of this book is to study the specific characteristics of the home nursing profession in each of the four European countries, and to compare similarities and differences in the domain features of home nursing in four different European societies. The interprofessional relations between home nursing and other professions, such as the medical profession, but also home helps, informal carers, and managers of e.g. home care organisations, is an important aspect of the domain features of home nursing and is therefore an important theme in this book.

In general, professional practices consist of problems of individuals or groups of people that are related to important societal values and/or the frailty of human existence. These problems can be solved, cured or relieved by services provided by experts (Abbott, I988c p. 35; Stevens and Philipsen, 2003). Examples of such values or problems are health problems, such as physical or mental illness, dependence or disability (the expertise of health professions), justice (judges, lawyers, solicitors), maintaining social order (the army or the police), but also more 'down to earth' problems such as organisational problems (managers), or designing a house (architects).

Some professions or individual members of a profession work independently, for example GPS, medical specialists and nurses with a private practice, but many professionals are employed in organisations nowadays. In multi-professional or Human Service Organisations (HSOs), characteristic of most health care organisations, different kinds of professionals are working together, and the 'products' consist of services provided to people and not of material artefacts (Hasenfeld, I983a, I992a). Hsos generally exist in the non-profit sector and are related with values such as solidarity and welfare. Providing services to people requires special procedures that are mostly formalised in protocols and professional and quality standards to ensure careful professional practices. However, in multi-professional organisations that are led by professional managers, boundaries between different kinds of professions are sometimes blurring, and professional identities, norms and values can become less pronounced and partly overshadowed by other kinds of values, such as organisational and managerial norms and values (Benson, I973; Mintzberg, I993c). This can lead to tensions between professional standards and organisational standards, for example when a professional wants to offer the best possible care available, but has to compromise because of reasons of efficiency or cost-effectiveness. 


\subsubsection{What 'is' a profession?}

In the sociological literature on professions, a major question has traditionally been how to define the concepts 'profession, professionalism and professionalisation'. One of the dominant perspectives has been the trait perspective, defining specific 'traits' or 'attributes' to construct the ideal-type of a profession. However, there has not been much consensus about the number and kind of traits that should be included (Krogt, I98Ic; Fox, I989 p. 39; Arend, I992; Brint, I993). The reason for this is that according to Abbott (I988), one encounters fundamental difficulties when trying to define a profession precisely and objectively, because it is an ambivalent concept that comprises many different aspects and varies in time and place (Abbott, I988a p. 318). For example, is being a profession determined by years of training? Or by a university degree? Or having a professional association, a scientific journal, or a professional ethical code? Or by the existence of evidencebased practices and thus 'objective, scientific proof' that professional practices are effective? When such classifications are used as standards to evaluate professions objectively, many socially established and culturally recognised professions would be downgraded to mere 'occupations'. ${ }^{2}$ So apparently, there are more aspects to professionalism than those that are tried to grasp in such criteria.

Abbott argues that when describing social phenomena such as professions, a well-known dilemma arises, namely it is hardly possible to formulate a stable, objective and precise definition of culturally charged terms, because their meanings are contingent and fluctuate with their context (Abbott, I988 p. 318). Although the concepts professions and professionalism refer to a long-existing societal structure to organise specific kinds of work, the content of the concept and the actual professional practices provided in specific societies vary according to time and place. As the societal meanings and cultural importance attributed to specific professions vary according to different historical and social circumstances and cultural influences (Siegrist, I990 p. I78-I79), the societal and temporal con-

2 For example, in the Netherlands, political and scientific debates are held about the question whether physiotherapy can be considered as an evidence-based profession, as it has appeared to be difficult to provide 'scientific proof' that physiotherapy practices have favorable effects on patient's complaints. The profession itself claims that the effects of their interventions are difficult to grasp by the scientific methods used in these studies, and that a large part of these effects are attributable to patient-professional interactions that are difficult to measure, and that patients mostly suffer from complaints with multi-factorial causes that do not fit into most scientific designs. However, the lack of clear scientific outcomes of physiotherapy has led to political decisions to restrict health insurance reimbursements for physiotherapy. 
text in which professions and their practices are embedded should be included in accounts on professions.

Thus, the reason why professions and professionalism are difficult to define is that professions are not static, but dynamic, as they are continuously changing due to their interrelatedness with other professions, society and societal developments such as changing health policies and changing cultural views about elderly care. According to Abbott, the elusiveness and dynamism of the concept of professionalism is fascinating in itself and therefore deserves further study. This simultaneously implies that when studying professions, these cannot be separated or viewed apart from the historical, social, and cultural context in which they are embedded. Therefore, the theoretical framework designed in this chapter specifically takes the nation-specific societal and health care context of professions into account.

\subsubsection{The process of professionalisation}

Another major tradition in the sociology of professions; the professionalisation perspective, analyses professional development over time, and is based on the assumption that the process of professionalisation tends to follow a certain characteristic order consisting of successive steps, because professions imitate the structural moves of professions that previously proved to be successful (Abbott, I99I p. 356). Although this perspective does take changes in professions into account and is less static in character than the trait-perspective, there is also no consensus in the literature about the exact sequence of events that professions should pass on their way to develop from a 'mere occupation' to a 'true profession'.

One of the most classical examples of such an order of events was formulated by Wilensky (I964). He identified five stages in the process of professionalisation:

I) The full-time commitment of practitioners to a task that 'needs doing;

2) The founding of a (local and/or national) professional association to promote the interests of the occupation and lay claim on certain areas and functions relevant to that profession;

3) The founding of training schools, in a later stage preferably academic in character;

4) Recognition and protection by the state in the form of credentials, registration and/or licensing (i.e. governmental legislation limiting professional practice to the holders of a mandatory license, in fact resulting in a state-protected professional monopoly); and

5) The establishment of a formal code of ethics to regulate and ensure professional conduct (Wilensky, I964; Abbott, I99I p. 357-358; Brint, I993 p. 26I; Siegrist, I990 p. I80-I8I). 
However, this is only one of several possible lists of characteristic successive developments that can take place within professional groups. Other sequences are possible, and different steps may be formulated. On the whole, such lists are rather arbitrary and say more about the structural characteristics or a possible 'gold standard' for professional development than about professional practices or the importance of such aspects for a particular profession.

\subsubsection{Ideal types and hierarchical relations between professions in health care}

The perspectives in the sociology and history of professions discussed so far mainly focus on theoretical ways to conceptualise professions, and are often based on examples of dominant or 'successful' professions such as medicine and law. There are also many studies on specific settings in health care, such as hospitals. Initially, many of these focussed on the medical profession, as its development over time and the way it legitimised its practices has been considered exemplary for long (Brannon, I994 p. I58). Therefore, the medical profession was considered an 'ideal type' against which other the kind or degree of professionalism of other health professions were evaluated.

Also, many studies focussed on the (hierarchical) relation between physicians and nurses (Aiken and Sloane, I997; Svensson, I996; Allen, I997; Fox, I989). Although nurses have their own working domain, they mostly work in close cooperation with physicians, who generally also have a large influence on the content of their job. Therefore, nurses are not completely autonomous in shaping their role and responsibilities (Schepers and Nievaard, I990). This has often raised the question whether nursing is entitled to the classification of a profession, or whether it is 'only' a semi-profession, para-profession or a mere occupation (Davies, I995b). However, a critique on such a view is that there are no adequate, sound criteria to justify a classification into one of the two categories. There is no sharp distinction between professions and occupations, and the boundaries between both concepts are 'fluid and indistinct':

'Different occupations and clusters of occupations are more accurately described as falling at a certain point along a continuum of professionalism, than as being either professional or not professional in an absolute sense. The appropriate question, then, is how professional one occupational role is compared to another, in relation to certain institutional criteria of professionalism against which both are evaluated.' (Fox, I989 p. 4I)

This implies that the concept of a profession is not a fixed category, and that the degree of professionalism of a specific profession can only be evaluated by comparing it to other professions; either to other, related professions, or to its counterparts in other countries. 
Since the late I960s, a 'paradigm shift' can be noticed in the sociology of professions (Krogt, I98Id p. 58; Fox, I989 p. 40). The one-sided emphasis on formulating certain traits to characterise professions (which had especially been popular in the I950s and early I960s (Brint, I993 p. 262)), or defining several stages a profession should pass in the evolution to its 'ideal state', gradually diminished. Scientific studies were broadened to include the relation between professions and their societal environment. The focus shifted to issues such as power relations between professions (Freidson, I970a, I986a), the relation between professions and the state (Johnson, Larkin et al., I995a; Torstendahl and Burrage, I990a), between professions and the market (Light, I995), and to the interplay between professions among themselves and the social context (Abbott, I988a). Thus instead of formulating essentialist criteria in order to classify a profession into rigid categories of professional and non-professional (with semi- or paraprofessional as hybrid forms), it is has become clear that is more fruitful to acknowledge the dynamic character, heterogeneity and multi-dimensionality of professions and interprofessional relations.

\subsubsection{Abbott's theoretical perspective on professions}

The theoretical perspective of this book is inspired by Abbott's views on professions (Abbott, I988a). For Abbott, of central importance to professions is the concept of jurisdiction over areas of expertise, by which he means the way professions organise and control their work by linking specific knowledge and expertise to certain tasks and responsibilities. ${ }^{3}$ By legitimising professional claims on specific task areas to other professions, the state and the public, they establish their own working domain, in the sense of:

'the legitimation of activities, the right and responsibility to carry out programs of a certain kind, dealing with a broad problem area or focus (...). The possession of a domain permits the organisation (or profession, нв) to operate in a certain sphere, claim support for certain activities, and define proper practices within its realm.' (Benson, I975 p. 232)

On the basis of such a domain, a profession (or in the case of Benson, an organisation) is granted a certain degree of authority, especially when it is a clear domain with activities of high social importance (Ibid., p. 232).

Abbott deliberately discards a precise definition of professions and only describes them very loosely: "professions are exclusive occupational groups applying some-

3 Jurisdiction in this sense is more broadly defined than the more common legal notion of jurisdiction. 
what abstract knowledge to particular cases" (Abbott, I9881 p. 3I8). This definition describes the social status of professions and the central process involved in professional practices in very general terms. In Abbot's view, all occupations that have acquired the status of a profession in society (and are called a profession in common parlance) should be considered a profession. Although its precise meaning is not narrowed down, people generally understand what is meant by the term profession, and therefore need no further specification of what a profession 'is' or represents. Instead, it is more useful to focus on their role and function in society. The definition used in this thesis is based on Abbott's definition, but extended and adapted to the health care context:

'In short, currently one can speak of professionalisation in case of a more or less exclusive occupation that applies more or less scientifically grounded knowledge to more or less specific cases in daily practice by acting more or less methodically with more or less success. In health care one can add: more or less keeping an eye on the interest of the client.' (Boom, Philipsen et al., 2004b p. II7)

Following Abbott's arguments, research on professions should focus on professional practices, and study the content of the actual professional work and the way professions legitimise their jurisdictional claims on specific social problems, rather than on the relatively contingent organisational structure of professions. Furthermore, professions should be considered within a system of interrelated professions (Abbott, I988e). The relations between professions are not stable or permanent, but make up an interacting system or 'ecology' (Abbott, 2004). Within such a system, of central importance are contests over jurisdictions and how those generate a specific division of labour, e.g. by specialisation or subordination. Also, Abbott argues to take the influence of large-scale social developments and contextual factors on professions into account, such as rationalisation, bureaucracy, and the rise of multiprofessional organisations. Influenced by such external forces, tasks are created, abolished, or reshaped, and relations between professions change. Such a broad view on professions provides insight into the role and function of professions in contemporary society and the way knowledge of particular social problems is institutionalised in professions and multi-professional organisations.

These views also closely resemble those of Freidson, who used to be a proponent of the power perspective on professions, but has gradually broadened his views. Similar to Abbott, Freidson stresses the importance of abstract knowledge for professions:

'The only characteristic truly distinctive about the professions is that the market demand for tasks performed by these occupations is channelled through their institutionalized association with higher education. (...) advanced training is 
required for the competent performance of the tasks in question.' (Brint, I993 p. 263)

Also, Freidson's concept of professional power in the sense of 'control over work, based on knowledge monopolies', or 'the ability to control how their work is to be accomplished' (Brint, I993 p. 266), closely resembles Abbott's concept of jurisdictions.

\subsubsection{Developing an interactionist contextual perspective on professions}

Abbott criticises the trait perspective for being too essentialist in character, and the perspective on professions as a more or less fixed 'process of professionalisation' for only focussing on the visible organisational outcomes, such as the founding of a journal or professional organisation. He argues that these lead the attention away from the hidden forces and drives underlying professional practices, that are far more important (Abbott, I99I p. 359; Siegrist, I990 p. I78). Most literature on professions focuses on empirical facts and structural characteristics (in Abbott's terms: 'external appearances'), while the processes and interactions preceding these outcomes (Abbott: the 'intrinsic qualities of professions') are far more important to professions. One can think of competitions between professions to dominate a certain working area; striving for abstract knowledge to be applied in practice; striving for professional autonomy and independence; and legitimising claims on a certain domain to the state, other professions and the public. According to Abbott, these aspects directly refer to what professionals actually do in daily practice, what motivates professions, and what generates changes in professions. For example: "professional interest in knowledge is a conceptual event to which the founding of a journal bears visible witness" (Abbott, I991 p. 359).

Considered from this perspective, the three case studies in the second part of The system of professions were somewhat disappointing. In my view, Abbott's descriptions still more or less revert to a detailed chronological historical description of the founding of professional associations, although admittedly, his accounts display more of the interprofessional competitions and clashes and for example the role of the state in such processes than more traditional accounts on professions (Abbott, I988i, I988j, I988k). However, on the basis of his statements in the theoretical perspective in the first part of Abbott's book, a more rich account on the broad societal processes in which professions are embedded could have been expected.

After all, according to a social constructivist view, the founding of a journal or professional organisation and the precise date of it is not of central importance, but the processes preceding and giving rise to such 'visible organisational outcomes' are more interesting, as these refer to more hidden drives and motivations that underlie and legitimate individual and collective professional activities. These are more ab- 
stract in character than the traits formulated in the trait perspective, and are related to broader societal issues and questions such as 'Why was it considered important by certain members of the profession to found a journal or professional association?' or 'To what societal need did the founding of a journal respond?', for example when support or legitimisation by the state or by other professions could only be accomplished when a profession disposed of a visible organisational structure.

By stating such questions and relating professional activities to societal processes, the 'black box' of the more hidden drives and motivations of professions is opened up and become visible and can be clarified or explained. For example, the current demand for 'evidence based health care' and increasing use of protocols is becoming more comprehendible when it is shown that these are related to specific societal and scientific perspectives, stating that health care practices should be based on convincing scientific evidence that these practices have a beneficial effect for patients, and that they should be based on principles of efficacy and efficiency. After all, when a different scientific and health care paradigm would be dominant in contemporary society, such as a holistic perspective on human beings, for example stressing the connection between man and nature, different scientific principles and different ways to perform health care practices would exist now. Alternative medicine and health practices, fiercely despised by some proponents of the current empiricist scientific paradigm (Renckens, 2004) and not quite accepted by most scientists stressing evidence-based practices, could have had a completely different societal and scientific status. Thus, the societal context is an important source to explain the significance and meaning of certain professional activities or characteristics.

Thus, the account on home nursing in book will display a rich description of societal, cultural, political and health care developments as well, in order to be able to explain processes within home nursing that are intertwined with the broader context in which home nursing is embedded.

\subsubsection{Concluding remarks on the concept 'profession'}

In this thesis, no precise and all-encompassing definition of what a profession 'is' will be formulated, which is based on certain 'professional traits' that would represent the 'essence' of professions, nor the question whether professions develop according to several subsequent evolutionary stages is answered. Instead, this book focuses on the ways in which knowledge and expertise of specific social and cultural problems is organised and institutionalised in a particular domain of contemporary society. For that purpose, Abbott's very general notion of professions is adhered to and extended to the health care context, which is a sufficient base to study the daily activities of professions and to analyse their role in health care and society. Furthermore, it is assumed that the process of professionalisation is not a uni- 
linear, evolutionary process, or a simple collective action by a cohesive group, but a complex and dynamic process in which social and professional forces are interrelated (Abbott, I991 p. 380).

In the next part of this chapter, a theoretical analytical framework is designed, in which the social, cultural, political and historical context in which professions are embedded are explicitly taken into account, as this fundamentally influences the character of the work and the social status of home nursing.

\subsection{Characteristic features of the home nursing domain: a comparative and contextual perspective on home nursing}

In this part of the chapter, the characteristic general features of home nursing are described firstly. These constitute the basic elements of the theoretical framework that is designed. Then, general European trends that are relevant for the home nursing domain and external structures in which home nursing is embedded are described. These are also included in the framework, which is used in the following chapters to gain a contextual view on the modalities of the home nursing profession within different European countries.

Home nursing activities are primarily performed in the domain of ambulant community care, namely in the homes of clients. In most countries, the primary field of activity is the domain of elderly care. ${ }^{4}$ Main activities are the provision of nursing care and support to frail elderly people that are dependent on care by others for their daily activities and in order to be able to remain living in their own homes. Often, home nurses are working closely together with home helps, who concentrate on domestic activities such as cleaning and ironing. In Denmark and the Netherlands, home nursing and home help are integrated into one domain, namely the domain of home care, and activities of both kinds of professionals are provided by one kind of organisation. However, in many other European countries, home nursing is considered as supportive of medical activities and thus as part of the medical domain, while home help is then considered as part of social care. Mostly, this is accompanied by different organisational and financial ways of structuring of both domains.

In many European countries, links are being established between different kinds of care, so that home nursing and for example nursing home or hospital care are working together in order to arrange a seamless network of continuous

4 Only in the Netherlands, home nursing also has an important role in providing preventive Youth Health Care at the so-called consultatiebureaus, providing preventive health care to children aged $\circ$ to 4 . 
care provided by different kinds of professionals around individual patients. This is especially the case in the Netherlands, where this phenomenon is called 'transmural care', and France, where an important part of the home nursing domain is organised by hospitals. These specific differences will be described in later chapters, but this shows that home nursing is not a homogeneous domain of similar services that are provided in all European countries, but indicates that in each country that will be described further on in this thesis, home nursing has a different position in health care and a slightly different domain of activities and different relations with other professionals.

Nevertheless, in the following part of this chapter, generic characteristics of the home nursing domain are described, namely those that distinguish the work of home nurses from that of nurses in other settings, such as hospitals, nursing homes, or psychiatric institutions. These general features have important implications for the activities of home nursing professionals and the content of their job. Also, the aspects that sometimes lead to specific challenges or tensions in home nursing are outlined, as well as the relations with other professions and the position of home nursing in health care and society. Finally, these general characteristics and specific aspects of home nursing are summarised into a theoretical framework. This framework is used in the following chapters to analyse the specific characteristics of home nursing in each of the countries included in this thesis.

\subsubsection{A special working domain}

First, the most distinguishing feature of home nursing is that it is provided in the periphery of health care, instead of in an organisational setting located in the centre of the health care system (Philipsen, I985, 2000). Instead of focussing primarily on diseases or medical disorders, which is a feature of health care activities performed in the medical centre, activities in the periphery are mainly aimed at offering nursing care and social support in case of suffering and complaints, and particularly at providing support in case of dependence and discomfort. Although the underlying disease is generally recognised, it has become a matter of secondary importance in this domain.

'In populations characterised by chronic, recurrent, and/or fatal diseases, curative medicine may have little to offer; yet quality of life can be substantially affected by nursing's emphasis on symptom management and efforts to enhance patients' ability to function in everyday activities of living.' (Aiken and Sloane, I997 p. 207)

While medicine primarily focuses on curative interventions and treatment of (especially acute) diseases, nursing is more strongly oriented toward managing the side 
effects of the illness or treatment, and maintaining or enhancing day-to-day functioning. Thus, nurses do not only have an assisting role by supporting the medical activities of physicians and specialists, but also have a separate, independent role and responsibility of caring for people with health problems and supporting them in their everyday life. While in hospitals, the subordinate role of nurses in relation to physicians may be most striking; in the out-of-hospital domain it becomes clear that they have their own domain and individual tasks that are different in character than those of physicians. In the domain of home nursing, these caring and nursing activities are predominantly performed in the direct environment of the clients, generally in their own homes.

\subsubsection{Broad sphere of action}

One of the most distinguishing characteristics of home nursing compared to nursing in other health care domains is that it is a broad sphere of action. Home nursing consists of several kinds of care, including rehabilitative, supportive, preventive, and technical nursing care, to a wide range of clients with different health problems (Kerkstra and Hutten, I996a p. 3). Traditionally, two clearly distinguishable fields of activity in nursing in the out-of-hospital domain exist. On the one hand, nursing is provided to more or less 'healthy clients', such as preventive health care and preventive mother and child health care, school health nursing, and occupational nursing. On the other hand, several kinds of nursing care are provided to different kinds of patients, such as short-time care in case of incidental health problems, psychiatric complaints, or after discharge from hospital, as well as long-term nursing care to chronically ill, disabled and elderly people, and palliative and terminal care to the dying. In most countries, these different spheres of activity have developed into several more or less separated fields, and have generally resulted in a division between preventive and more medically oriented/technical nursing care (Kerkstra, I990 p. 3). Nowadays, the core domain of home nursing is constituted by long-term care for chronically ill and elderly people in most European countries. Due to demographic and social developments, such as the ageing of the population and the trend to care for people in their own environment as long as possible, this is expected to remain the principal field of activities in the home nursing domain in the future. Therefore, this field is focussed on in this book.

\subsubsection{Autonomy}

Another feature distinguishing home nursing from most other professions is that home nurses generally perform their tasks in the homes of their clients, in the absence of direct interference and close supervision of professionals with a higher 
hierarchical status, such as physicians. This means the work is more autonomous in character than that of nurses working in other domains. Their control over work is relatively high, because they work rather independent of other professionals, while they are in close contact with their clients (Mintzberg, I979c p. 349).

Also, standard procedures and protocols that are increasingly used in health care practices do not always fit these particular circumstances. Therefore, home nursing needs to be tailored to the individual living situation of the patient, which makes it a rather complex activity, requiring a flexible, independent and self-reliant attitude of home nurses. This is even more so because they are a 'guest' in the homes of their patients and need to adapt to the personal living circumstances of each of their clients.

However, as a consequence of the autonomous character of the work and the lack of direct supervision, others, such as physicians or organisational managers, are concerned about their lack of insight in (the quality of) the care delivered, and will try to impose 'objective' standards of care on home nursing work and demand of home nurses to account for their activities. This can especially become problematic when these acts interfere in the way nursing tasks are performed, and when they do not sufficiently take the interests and perspectives of home nurses into account. Another option is that parts of specific professional processes, such as assessment of care needs and the assignment of care to clients, are transferred to bureaucratic bodies, in order to 'objectively' decide on the eligibility to care. An example of this is the emergence of regionale indicatie-organen or regional assessment organisations, assessing the care needs of patients and deciding on the amount and kind of care to be provided in the Netherlands and Germany (Kerkstra and Hutten, I996a; Schneider, Biene-Dietrich et al., I994c; Alber, I996; Kerff, I999). However, this transfer of tasks to organisations has led to a lot of discontent among home nurses:

'Formerly, the home nurse performed the intake. So then the basis was laid for patient contact. But now it is performed somewhere where you don't see it. There are so many subsequent steps before you arrive at the home nurse or district nurse as a patient. Information is often insufficient or distorted. And promises are made, for example by someone of the assessment organisation, that we can't fulfil at all.' (Thiel and Hoven, I999 p. 27-28 focus group interview)

According to Freidson, the increase in external bureaucratic regulation, including review of professional decisions by external agents, poses 'no comprehensive or consistent threat' to the professions (although he admits it does create a degree of external oversight), because the true basis of professional power, namely the basic institutions employed by professions to exercise control over training and practice conditions, remain intact (Freidson, I983 in Brint, I993 p. 270). However, 
in the case of home nursing, this external interference and 'poaching of tasks' or 'invasion' (Abbott, I988; in this case by organisations), weakens their jurisdiction, thereby seriously affecting their autonomy and thus their degree of professionalism.

\subsection{The organisation of home nursing work: a generalist perception of care or intra-professional division of labour}

Several characteristics of the home nursing profession logically follow from the above-mentioned generic features of its domain. Home nursing activities are aimed at providing several kinds of short-time and long-term care and support to different categories of clients, as the care needs of clients are quite diverse and require a wide range of activities. In addition, home nursing also supports family members such as partners, offspring, friends and neighbours in providing informal care. This implies there is a great variety of tasks that are partly overlapping and therefore sometimes result in an unclear division of labour between different levels of home nurses, other health care professionals and informal carers (Cox, Niemantsverdriet et al., I994; Kanters and Smit, I994; Kerkstra, I996b; Philipsen, I987). The broad character of home nursing work also implies that it can be organised according to different (intraprofessional) divisions of labour, that are based on different principles. The preference for one principle or the other is however subject to change and may alternate according to different political or cultural climates and shifting care-giving ideologies (Brannon, I994).

On the one hand, home nursing can be organised from an integrative, holistic perspective on care provision with a generalist conception of tasks. Vocational training in home nursing is then broad and holistic in character, as it focuses on all kinds of nursing tasks. Nurses have an all-round competence and can be employed in all home nursing situations; there is hardly any specialisation into specific nursing areas. However, this implies that all home nurses perform complex as well as basic tasks, and although this makes the work quite varied, it is rather expensive in terms of labour costs.

For the patient, a generalist conception of care generally has advantages, because tasks can (at least in principle) be performed by a single home nurse and thus requires the involvement of only one or a few different professionals. Also, the visiting nurse is familiar with the family situation of the client. This is not only important in the sense of knowing about the availability of informal care, but also to have insight into family relations and for example the kind of emotional support of the client by the family. However, the ideal of 'continuity of care' from the perspective of the patient, in the sense of "interpersonal continuity, the quality and the endurance of relationships between clients and care providers" (Rogers and 
Curtis, I980, in Achterberg, I997 p. I6) seems to be far from day-to-day reality in most European countries. The concept of a home nurse being responsible for only a small number of clients she visits by foot or by bike in her own neighbourhood, and having a rather high social status alongside the family practitioner, as it used to be until some decades ago, is hardly realistic anymore from an organisational point of view (Boom, Philipsen et al., 2004b).

Small, private home nursing agencies may still have personal relations with their clients, but in most 'regular' home nursing or home care organisations, home nurses currently work in teams working in shifts and covering rather large areas, so that organisational schedules determine which home nurse visits which client. For example, in a study on continuity of care performed in the Netherlands, the average client met 16 different (professional and non-professional) caregivers within one year (Achterberg, I997 p. I0). One client even received care from 35 different community nurses within one year, although this is fortunately reported as an extreme exception, as most home nursing organisations try to ensure at least some continuity of care.

Since its initial development, roughly since the last decades of the nineteenth century in most European countries, home nursing has been organised from a generalist perspective emphasising all-round expertise and skills (Kerkstra, I990 p. I). During the last decades, however, several developments have led to pressures on the home nursing domain and have mostly resulted in a different organisation of home nursing work, according to different kinds of divisions of labour (except for Denmark) (Jansen and Kerkstra, I993 p. 4; Kerkstra, I990 p. 2-3). One can think of several of such developments, like demographic trends resulting in an increased demand for home nursing care; epidemiological changes such as the increase in chronic and old-age related afflictions demanding for more skilled, intensive and complex nursing care; technological developments requiring special nursing expertise; fragmentation of care because care is increasingly provided by different professionals; the increasing body of nursing knowledge; and the shortage of nursing staff and dissatisfaction among home nurses about their work and career possibilities, resulting in an early withdrawal from the profession. These developments have resulted in an increased workload and increase in the complexity and number of tasks to be provided by home nurses. This is reinforced by an increased demand for home nursing care due to substitution policies and earlier dismissal of patients by hospitals, and a shift in the characteristics of the client population to frail, elderly people and patients with chronic diseases, both requiring frequent and intensive nursing care.

These developments and pressures have gradually led to a shift in tasks from all-round activities to more instrumental nursing oriented tasks, at the expense of preventive and co-ordinating tasks, and have resulted in differentiation of home nursing practices in most European countries, except for Denmark (Kerkstra, I990 
p. 2; Zee, Kramer et al., I994). This trend is inherent to the growth and development of organisations and professions, the rationalisation of society in general, and the fact that the domain of elderly care has increased enormously in size, so that tasks can more easily divided over a larger number of professionals providing similar tasks (Kanters and Smit, I994 p. 2; Mintzberg, I993d). The rationale of differentiation is that it reduces the overlap in tasks between different professionals and leads to more efficiency, quality of care, and work satisfaction (Jansen and Kerkstra, I993 p. 4; Kanters and Smit, I994 p. 2 and 64). However, the division of work into separate sets of tasks simultaneously reinforces the grip of the organisational management on the work of home nurses and encourages the idea of nursing as providing a 'package of care', subdivided into different functions and 'delivered at the client's home' (Los, I997 p. 722). Consequently, professionals are more and more considered as employees, performing the tasks they have been assigned to by the organisations they work for, than as independent professionals autonomously deciding on the content of their work and the way it should be performed (Ibid.). The gradual increase in the size of organisations during the last decades only reinforces managerial control on professional work. As this development has severe implications for the character of professional work, it will be addressed to specifically in one of the next parts of this chapter.

\subsubsection{Intra-professional task differentiation}

Within professions, tasks can be differentiated or divided in different ways, such as horizontal and vertical differentiation, and specialisation (Jansen, I996; Jansen and Kerkstra, I997a; Kanters and Smit I994; Mintzberg, I993d; Aiken and Sloane, I997; Nijhuis, Vlaskamp et al., 200I). Vertical differentiation means a hierarchical division of labour into different functions and/or different nursing levels, mostly created by separating co-ordinating, complex, and simple tasks, and transferring less interesting ('dirty') work to other health professionals, such as making beds and cleaning tasks to home helps. Nursing work is then divided into different sets of tasks, distinguished by the level and type of education, competence and experience. The range of responsibilities differs per nursing level, and higherlevel nurses have more control over their work compared to lower level nurses. Generally, new functions are created at the top, such as managing, co-ordinating and expert/specialist functions (e.g. team leader, head of staff, care manager), and at the bottom, such as auxiliary nurse. This means that higher nursing levels are becoming more attractive from a career point of view because new possibilities for upward mobility are created (Abbott, I988b p. I06). At the same time, the work of lower level home nurses becomes less varied, which seems to have adverse effects on experienced job satisfaction (Jansen and Kerkstra, I997a). Vertical differentiation is expected to reduce the overlap between different health care profession- 
als, while efficiency increases and labour costs become relatively lower because a higher number of lower skilled, and thus cheaper, personnel can be recruited (Jansen and Kerkstra, I993; Kanters and Smit. I994)5. However, it also implies that home nurses can no longer be employed interchangeably, because it results in different job characteristics for each nursing level (Abbott, I988b p. I06).

Another kind of division of labour is horizontal differentiation, mostly resulting from an increase in nursing knowledge, tasks, or clients. With horizontal differentiation, nursing work is divided over different task areas and nurses specialise by concentrating on a narrower range of tasks, which decreases the variety of work but increases productivity because of increased possibilities for standardisation and work routines (Mintzberg, I993d; Jansen and Kerkstra, I993 p. I2-I3). An example is the division between different task areas, such as Youth Health Care, Mental Health Nursing, Occupational Health Care, Health Promotion, and Elderly Care. Another form of horizontal differentiation is specialisation or task differentiation, often occurring when tasks are so complex that they require special skills or specialised knowledge. This can result from an increase in advanced nursing knowledge, the introduction of medical technologies, or a demand for more complicated forms of care (Abbott, I988a p. 8I, I22). Also, home nurses can follow additional training in a specific 'areas-of-special-expertise', that are mostly arranged according to specific complex problems or illnesses, and become specialist nurses such as AIDs nurse, diabetes nurse, COPD nurse, psychiatric nurse, or care co-ordinator (Jansen, I996 p. 4).

However, there is an inherent dilemma involved in specialisation for the nursing profession, which has led to contrasting theoretical views. On the one hand, specialisation is considered as a means for professionalisation, because it offers a possibility for nurses to make a further career and receive specialised training. It also "allows nurses to develop, demonstrate, and communicate to physicians their superiority in certain important spheres of patient care." (Aiken and Sloane, I997 p. 2I8). The more specialised a professional task is, the more a profession is able to retain control over it and the easier the jurisdiction over its working area can be protected (Abbott, I988a p. 46). This is underlined by a study of the reorganisation of nursing care in a California hospital, showing that reunification of tasks and abolishment of the former division of tasks resulted in work overload due to an overextension of the jurisdiction, which reversed the professionalisation process (Brannon, I994 p. I60).

5 Differentiation of practice is often considered by home nursing organisations as a means to solve budgetary problems, because wages are connected to nursing levels and the ratio of higher skilled, more expensive nurses becomes lower (Kanters, I994). However, the financial advantage is undone in the case of 'qualitative understaffing', when high level nurses perform the tasks of lower skilled personnel (Jansen, I993). 
Conversely, specialisation and differentiation can also be considered as deprofessionalising nursing practice, because its task-oriented perspective leads to fragmentation of care and the parts in themselves become too small a basis for a separate jurisdiction (Abbott, I988 p. Io8). Also, the tasks mostly become more medical in character, which can be considered as reinforcing the subordinate position of nurses in relation to physicians. This is especially the case when tasks that officially belong to the responsibility of physicians are taken over by nurses, (such as giving injections), while physicians remain in control over critical decisions (Brannon, I994 p. I70). ${ }^{6}$ The assignment of less interesting tasks or 'dirty work' of physicians to nurses is generally seen as degradation or 'regression' (Abbott, I98I) from a professional point of view. Remarkably is, however, that it can also be valued positively from a cultural perspective, "in the sense that acceptance of tasks that are viscerally undesirable but socially necessary can enhance the social authority of a profession." (Aiken and Sloane, I997 p. 204-205). Apparently, "the bases on which the public assigns prestige differ substantially from those used within professions. In practice, the public prizes precisely those contacts which professionals want to escape" (Aiken, I997 p. 205, referring to Abbott, I98I). Also, for example in the case of specialisation of hospital nurses into patients with AIDS, taking responsibility over dirty work can open an opportunity for nurses to gain status (Aiken, I997). So it depends on the point of view and context whether specialisation and differentiation is considered as degrading or enhancing the degree of professionalism of nursing.

\subsubsection{The 'system of professions' - interprofessional cooperation and co-ordination of activities}

Another characteristic in which home nursing differs from nursing in other health care sectors is the kind of cooperation with other professionals. Hospital nurses generally work in close cooperation with professionals within the same organisation. Although this makes communication relatively easy, it also highlights hierarchical differences between professionals. Home nurses, however, mostly work as solo practitioners, employed by a home nursing organisation or private practice, or otherwise work in home nursing teams or multi-disciplinary teams. They often communicate and work closely together with general practitioners, nurse practitioners, physiotherapists, social workers, auxiliary nurses and home helps. Also, they currently increasingly work together with intramural professionals, such as spe-

6 In the Netherlands, the Act on Occupations in the Individual Health Care ('Wet BIG') (vws, 2000) legally authorised the performance of medical tasks by nurses (which was called the 'prolonged arm construction'). Professional competencies and responsibilities are now officially acknowledged in such situations. 
cialised nurses (e.g. nurses specialised in CopD or diabetes), nursing home physicians and hospital specialists. In the Netherlands, for example, this development is called 'transmural care', as it crosses the boundaries between different health care domains, which will be described in more detail in the chapter on the Netherlands. But also in other European countries, cooperation between different health care domains and professionals exists. This results in a concerted action of a mix of professionals that are working in different kinds of health care organisations or private practices, each with their own planning and professional standards. This makes communication and joint planning of actions difficult.

However, interprofessional cooperation requires that (formal and/or informal) agreement exists between the different caregivers involved about 'who does what and how'. Or in other words, it requires domain consensus as well as ideological consensus (Benson, I975 p. 235) different care-givers communicate with each other about their tasks and reach joint agreement about each other's role and scope of activities and responsibilities. This means they are operationally gearing their activities to one another, for example by telephone or multidisciplinary consultations. On the other hand, it presupposes joint agreement about the nature of the tasks and the appropriate ways to perform them. Thus, a certain interprofessional division of labour implies that the caregivers involved implicitly or actively agree about one another's basic assumptions and principles. When participants are engaged in highly co-ordinated, co-operative interactions based on normative consensus and mutual respect, an interorganisational network (or in Abbott's sense, a system of interdependent professions) is in equilibrium. Two other dimensions should also be present then, namely positive evaluation of the value of each other's work, and co-ordination of work between different organisations or professions, in order to reach maximum effectiveness and efficiency (Ibid., p. 235-236). However, all four dimensions are not always equally balanced in such equilibrium: although a network may have high consensus on domains and ideologies, it can display a low degree of work co-ordination.

The need for planning and co-ordination of health care activities is considered particularly important when caring for chronically ill, disabled or elderly people, because they are heavily dependent on health care support for their daily life activities and to be able to remain in their own environments. As they mostly suffer from complex or multiple health problems, they encounter a range of different professionals, and although the care provided by different professionals may be efficient and effective when viewed apart, it is fragmented as a whole and requires coopera-

7 See also Cox (I994) en Hermans (2000) for a more applied explanation of Benson (I975). 
tion and co-ordination (Philipsen, I986). Paradoxical, however, is that there mostly is no structured planning of the various activities in these situations. Although a home nurse, general practitioner, social worker, home help manager or informal carer can perform the co-ordination of care (Achterberg, I997 p. 50), in practice there are no formal arrangements regarding who is responsible for this role. In the periphery of health care, it is not evident who should co-ordinate the caring and nursing activities of the different professionals working together (Philipsen, 2000 p. 47). ${ }^{8}$ Some suggest this role should be reserved for patients themselves, but it is questionable whether they are capable of this regarding their health status and dependence. Others suggest that home nurses are most suitable for this role because they have best insight in the patient's situation and his/her needs for care and support, while the general practitioner's view is generally more oriented towards cure and treatment (Philipsen, I986 p. 77). It is suggested that it would be preferable to appoint a co-ordinator per individual client, depending on the kind of problem, the patient's ability to cope, and the composition of the professional and social network (Cox, Niemantsverdriet et al., I994 p. 33). However, the lack of clarity about who is responsible for co-ordination in the home care domain makes the kind of cooperation fundamentally different in character than pre-structured and formalised cooperation between professionals within an organisation such as a hospital or nursing home, and requires more complex ways of communication and ad hoc agreements. Currently, home care organisations in the Netherlands employ 'case managers' that perform this co-ordinating task for individual clients. In France, independent nurses generally claim this role.

\subsubsection{Possible tensions between lifeworld and system values}

Home nurses work in an intermediate position between the institutional environment of health care, and the homely environment of the patient. This may cause problems when the values of these different environments are incongruent or even conflicting. Health care in western, highly industrialised countries has a strong 'systems character': it is highly goal-oriented, specialised and formalised (Habermas, I981 in Philipsen, I988a, I987). Systems are complexes of goal-oriented activities in which criteria of effectiveness and efficiency are guiding principles. During the last decades, systems in health care have increased in size and complexity due to successful specialisation and divisions of labour. Although this has resulted in more adequate ways to deal with many health problems, it has simultaneously led to an inherent value conflict between a fragmented, cure-oriented approach

8 In the Netherlands, both general practitioners and home nurses claim this task (Ravensbergen, I990; Starmans, I989). 
and a more patient-oriented approach, taking the personal illness experiences of patients into account and allowing them to live a meaningful life.

Philipsen (1985, I988, I997) argues, elaborating upon Habermas, that the expressive values of continuity, solidarity, well-being and meaningfulness that belong to the lifeworld of the client are at risk of being 'colonised' by the functional-rational, systems-oriented approach of health care and professional interventions (Philipsen, I985, I988a; Philipsen and Stevens, I997a). This 'lifeworld' can be considered as:

'that aspect of the world surrounding us that is about the communicative content of actions, about the direct experience of the quality of our existence, about meaningfulness, way of living, belonging, in short, where it matters to be at home and feeling home.' (Philipsen, I987 p. I82)

Health care as a social system thus tends to intrude into the lifeworld of the client and instrumentalise, and thereby threatens to destroy, parts of these expressive elements. The impact of health care that is based on instrumental values can especially become problematic when it is provided to clients in their own homes, and when they are suffering from a chronic or degenerative disease that has fundamental consequences for their existence and everyday life.

At the same time, however, a reverse process can take place when the importance of expressive values is overemphasised and the instrumental advantages of health care systems are becoming 'domesticated'. This means the efficient and effective performance of activities such as professional skills and bureaucratic procedures are lost:

'Thus, while a highly "colonized" lifeworld lacks solidarity and meaningfulness and denies the importance of continuity, a "domesticated" system can lose its effectiveness, efficiency and justice.' (Philipsen and Stevens, I997a p. I92)

Particularly in the domain of home care, where home nurses literally step into the home and personal sphere of clients, the boundaries between the instrumental values of health care and the expressive values of the lifeworld are thin, and tensions between the two can become evident. This makes high demands on the professional skills of home nurses who are in an intermediate position between both worlds.

In practice, this means that health care professionals often face the dilemma that the day to day order of 'face work', which is professional, affective neutral (or impersonal) and competent in character, is easily disturbed by 'character work', which is behaviour that is not directly aimed at the specific purposes of health care, but more private in character and involves emotional commitment and engage- 
ment (Philipsen, I988a) and encourages particularism, affective involvement, diffuseness of relations and discretion (Hasenfeld, I983b p. I22). This dilemma is closely related to the character of the nurse-patient relationship: nurses deal with some of the most basic and intimate physical, emotional, and often also spiritual needs of patients, and experience how patients are reacting to and dealing with their illness and treatment (Fox, 1989). Nurses are also in close contact with members of patients' families.

'Under these circumstances, they often become identified with their patients as persons: with their feelings, values, relationships, life histories, and how these bear on what the patient is experiencing. (...) this "commitment to the patient" may engender conflicts of loyalty and role responsibility for the nurse.' (Fox, I989 p. 60-6I) ${ }^{9}$

This commitment to the patient influences the professional relation with the patient, and may affect parts of the effective and efficient performance of activities. The fundamental difficulty for health professionals and more so for professionals working in the homes of clients, is therefore keeping the system characteristics of professional caregiving in balance with the personal sphere or lifeworld or the patient.

\subsubsection{The impact of bureaucracy}

Finally, the increasing systems character of health care and the rationalisation of society in general emphasise administrative and bureaucratic standards regarding efficiency, efficacy and cost-effectiveness, and lately demand for 'evidence-based' health care practices. These trends have a far-reaching impact on the activities of professionals, and consequently also fundamentally affect the content of the work of home nurses. During the twentieth century, there has been a growing trend for professionals to become salaried employees in multi-professional bureaucratic organisations, rather than being self-employed in individual practices (Abbott, I989; Murphy, I990 p. 72). This development is generally referred to as the 'deprofessionalisation' or 'proletarianisation' of professions, which means that 'ordinary' rank and file professionals are loosing their economic independence and the autonomy over their work to organisations, and that their work becomes

9 Although this quote refers to hospitalised patients, the same process takes place (possibly even stronger in character) when caring for patients in their own homes. 
increasingly routine in character. ${ }^{10}$ They are becoming more and more subject to the control and demands of the organisation they are working for (Murphy, I990 p. 72) and are downgraded to employees subject to managerial policies that dictate the terms for their work: the hours, work schedule, wages, and job requirements (Brannon, I994 p. I73).

In itself, bureaucracy is not 'bad', because it is just a way to standardise and formalise competencies, rights, and duties, to guarantee equality and to expel abuse of power, nepotism, and anarchy (Philipsen, I987 p. I85). However, the needs of bureaucratic organisations are by definition not compatible with the need of professions (Benson, I973 p. 38I). "Research indicates that a professional orientation toward service and a bureaucratic orientation toward disciplined compliance with procedures are opposite approaches toward work and often create conflict in organisations" (Blau, I967-68, in Mintzberg, I979c p. 36I). As a consequence, professionals often struggle with a 'double loyalty'; on the one hand they are expected to be loyal to the organisation they work for and its goals and standards, and on the other hand they should remain loyal to their profession and its norms and values or ethical code. However, when the grip of organisations on professionals is becoming too high, their autonomy, independence, and the non-routine character of professional practices are affected, which fundamentally changes the character of their professionalism. However, when bureaucratic and professional elements in an organisation clash, much depends upon the capacity of a professional group to defend its interests and to press for professional roles (Benson, I973 p. 382). The position of professionals in an organisation and the amount of autonomy they are granted also depends on the locus of authority; whether it is concentrated in the administrative part of the organisation (in the case of centralisation of power), or delegated to the professionals themselves (in the case of decentralisation of decision-making powers (Ibid., p. 390). This explains why the relationship between bureaucratic and professional elements may take quite different forms in different types of organisations, countries, or even in distinct periods in the same organisation (Ibid., p. 384). In studying the degree of bureaucratic interference in professional practices, these contingencies should be taken into account.

This increasing interference of bureaucratic procedures in professional work, or 'managerialism' (i.e. the dominance of formal rationality (Dent, I993; Los, I997

Io Murphy (I990) however argues that professional employees are unlike the proletariat in such fundamental ways that the analogy between both conceptions does not hold. He states professions certainly have become bureaucratised, but this "shift from selfemployment to dependent employment, in which professionals' work also becomes effectively subject to management control" should clearly be discerned from the proletarianising of nineteenth century industrial workers (p. 75-76). 
p. 726)), is a general trend in all highly industrialised countries, and is particularly felt in professions in education, health care and social work (Los, I997 p. 709). For example, nursing nowadays involves working with established standards, treatment plans and protocols, by which nurses are evaluated on the basis of formalrational 'systems' criteria of effectiveness, efficiency and quality of care (Philipsen and Stevens, I997a p. 195). However, these are not always congruent with the professional values of home nursing that also wish to take the need for continuity, autonomy, well-being, quality of life, and the personal narrative of the patient into account. However, due to the organisation of work, in everyday practice there is often too little time for home nurses to spend on these more personal needs of patients as well that therefore run the risk of being lost out of sight (Philipsen, I987 p. I85-I86).

'Care for people gets into a tight corner. At least, in my opinion one can't measure everything in minutes. What is paying attention to someone? I have trouble with that. Psychosocial care to people. It already is difficult to grasp: what does it mean then? But on the other hand it is a very important part of the nursing profession. And I think that you can't measure that in minutes.' (Thiel and Hoven, I999 p. 3I interview with a Dutch home nurse)

More generally, the rise of organisations implies that many problems or parts of problems that were under professional judgement before are now becoming subject to bureaucratic rules and procedures (Philipsen, I997b). Tasks are more and more circumscribed in detail and, if possible, standardised (Mintzberg, I993d), so that formal-rational criteria will dominate professional criteria (Los, I997 p. 709). However, this seriously affects professional autonomy and minimises independent decision-making and shaping of activities. Thus, it is questionable whether bureaucratic and rational tendencies are desirable from a professional point of view, and have tended to overshadow independent professional practice:

'The bureaucracy is a thorn in my flesh. And what is worse: when the figures arrive there still appears to be no insight in your work. And you are writing and writing. We have to write down up to five minutes and that takes a lot of time. I do understand that it is required to get insight in things. I agree with that myself. But when I bring my car to the garage, I then get a specified bill and that is good. But that man doesn't have to account for every five minutes for me.' (Thiel and Hoven, I999 p. 30 interview with a Dutch home nurse) 


\subsection{Developing a theoretical framework - the first step}

The particular characteristics of the home nursing domain that have been outlined until now can be summarised into the following theoretical framework, comprising the specific features of the home nursing domain and its relation to other professional domains and larger societal influences:

Figure I Features of the home nursing domain

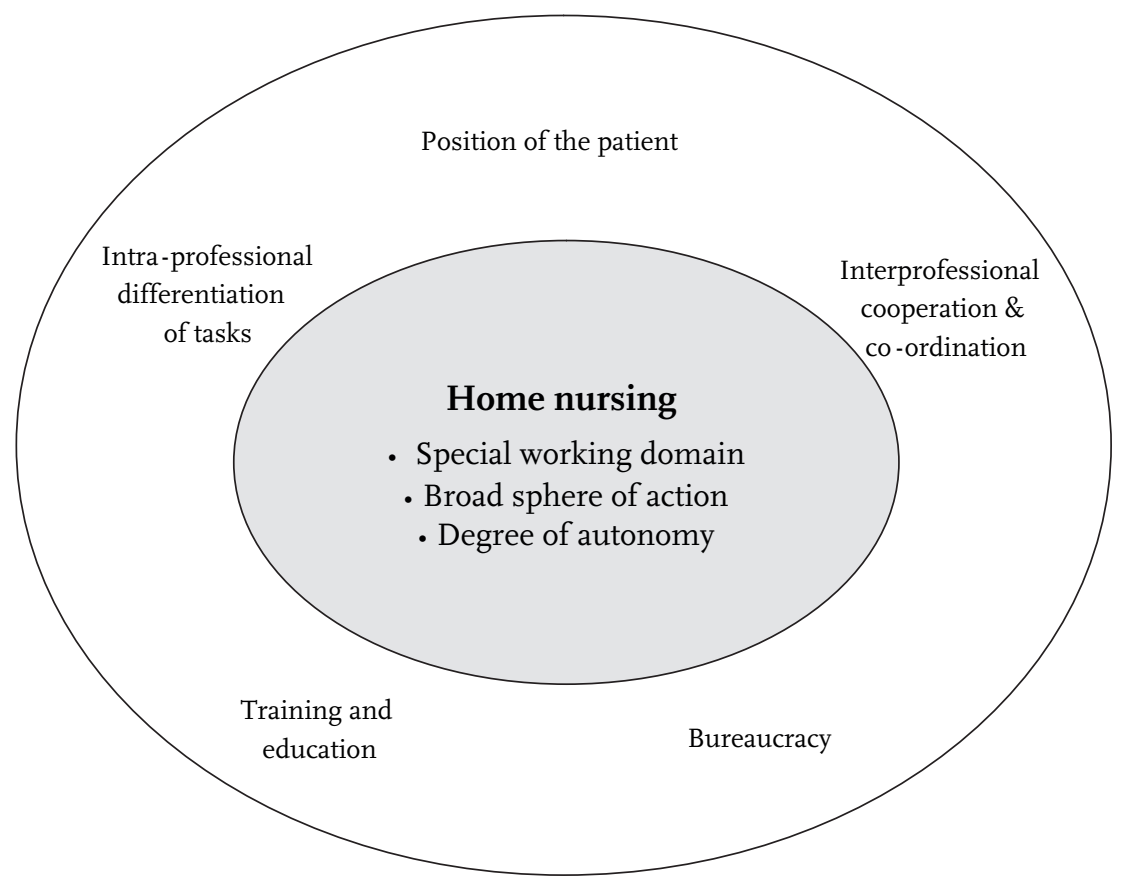

The framework depicts that home nursing is a special working domain, which is situated in the periphery of health care, providing several kinds of care to a wide range of clients with different care needs. In this broad sphere of action, home nursing tasks can be organised according to a generalist or holistic conception of care, according to which every nurse performs all kinds of care to all kinds of clients, irrespective of their age, the complexity of their care needs or level of dependency. Then, there is no intraprofessional division of labour. Or, home nursing tasks are divided according to horizontal differentiation of tasks (e.g. different working areas or client groups) or vertical differentiation of tasks with a differentiation of tasks over nurses with different levels of expertise and training, or complex tasks or care for specific patients are differentiated and assigned to specialised nurses, such as diabetes nurses. 
Also, home nurses often closely work together with other health professionals, requiring at least some degree of domain and ideological consensus about which tasks to be performed, by whom, and how. Interprofessional cooperation also requires that the care provided by different professionals is co-ordinated. However, in practice it is often not obvious who should take this co-ordinating role, that can be performed by the GP, a home nurse, a nurse specialist, a case manager of a home care organisation, or the patient him- of herself. As home nurses are trained in providing long-term nursing and care to frail and dependent people, they are considered to have insight into the specific needs of these people and would be able to take up the co-ordinating role.

Furthermore, the kind and degree of autonomy of home nurses to perform their work according to their own views and standards, and the degree in which they can decide on which tasks to be performed and how, is an important aspect of the profession. The autonomy of home nurses is different and generally larger compared to that of nurses in hospital or other institutional settings; as there is no direct, close supervision by physicians during the execution of tasks in the patient's home. Home nurses, however, can be tied to prescriptions of medical professionals or assignment organisations that decide on the amount and kind of care to be provided to a patient. This external interference into the autonomy of home nurses is different per country.

Another feature of home nursing is that home nurses find themselves in a difficult position between possibly conflicting instrumental and professional values that are part of the instrumental world of health care, such as distance, objectivity, standardisation of care, and efficiency, and the homely environment and personal life sphere of the client, characterised among others by emotional needs and personal attention.

Also, in the daily work, tensions may arise because home nurses are becoming more and more subject to bureaucracy in the sense of rules, regulations and standards that are imposed on the execution of tasks, and the means of control and demands of the organisation or managers they are working for. These external influences on the activities of home nurses may clash with the ambition to work as an autonomous professional and may lead to frustrations on the job because they have to do a lot of paperwork and continuously have to account for their activities to other professionals or their organisation.

The above-mentioned specific features of the home nursing domain are comprised in a theoretical framework that will be used in the next chapters to analyse the modalities of home nursing within different European countries. However, there are also more general aspects that influence the structure and organisation of the home nursing domain and the kind and range of tasks that is provided in each country. Therefore, in the next part of this chapter, these general aspects are described, belonging to the external environment of home nursing. 


\subsection{The home nursing profession in a changing health care and societal context - Challenges, opportunities and difficulties}

In this part of this chapter, the challenges, opportunities for development and difficulties for every-day practice are described, with which the home nursing profession is confronted by changes that are taking place in the external environment. One can think of developments in the societal context as a result of social, political, cultural, and demographic trends, and developments within health care systems, influencing the role and position of home nursing. As home nursing is embedded in health care and society, and both provide the setting within which home nursing is provided, changes external to home nursing can work out two ways. On the one hand, changes in health care or society can have positive influences that result in challenges and chances for development for home nursing, or, on the other hand, these changes can work out negatively, in the sense of resulting in tensions, pressures and dilemmas for home nursing. Thus, the modalities of home nursing are shaped and influenced by external developments that affect the character of the daily work. Therefore, the external environment is integrated in the theoretical framework that is built up further in the following paragraphs.

\subsubsection{The impact of a managerial perspective and increasing absence of a professional perspective in health care}

One of the major current issues for professions in general, including home nursing, is whether they still possess exclusive professional skills and the autonomy to perform these in their professional practices. After all, the central claim of professions is that they possess specific knowledge, skills and/or techniques that are related to a specific social problem, and cannot simply be transferred to other professions, actors, or bureaucratic procedures. Theoretically, the core of professional work consists of three stages, namely the claim of a profession to classify a problem, to reason about it, and to take action on it, or in Abbott's words: to diagnose, to infer and to treat (Abbott, I988c p. 40). ${ }^{\text {II }}$ Although many tasks performed by professions consist of the more common cases, with a simple diagnosis and a purely conventional treatment that can be delegated to subordinates or non-professionals (Abbott, I988c p. 45), for many tasks specific professional knowledge and skills are required. These are acquired (or 'indoctrinated') through a process of several years of training, a period of practical experience, in which the would-be

II Mintzberg describes the same processes in two steps: the 'pigeonholing process' (the task of diagnosing the needs of the client and categorising them so that the standard program to be applied is indicated), and the task of applying, or executing that program (Mintzberg, I979c). 
professional learns to apply abstract knowledge to particular cases, and regular periods of refresher courses (Mintzberg, I979c p. 350). It is not just that professional tasks are complex and require personal judgement, but that particular, contingent cases are evaluated on the basis of an abstract, relatively standardised classification system that professionals are taught during their training period. Thus, diagnosis is a fundamental professional task that is not completely open-ended or dependent of the creativity of the individual professional, but rather circumscribed:

'The pleasure that the good professional experiences in his work is not simply a pleasure in handling difficult matters; it is a pleasure in using skilfully a wellstocked kit of well-designed tools to handle problems that are comprehensible in their deep structure but unfamiliar in their detail.' (Simon, 1977, in Mintzberg, I979 p. 352)

However, these work processes require such an amount of expertise and have such a degree of complexity that these cannot completely be standardised by analysts or controlled by managers, and thus require a relatively large amount of professional autonomy (Mintzberg, I977 p. 352). ${ }^{\mathrm{I2}}$ In this sense, the social status of professionals and the character of their work are not comparable to that of other employed workers:

'Professionals, unlike industrial workers, have salvaged from their new dependency the core of their self-respect and unique status: their technical skills and control of highly specialized knowledge. Professionals thus retain the anomalous authority of craft and expertise, envied and unattainable by all other employees.' (Derber, 1982 in Murphy, I990 p. 74)

In some European countries, however, there are discussions about the question whether the process of diagnosis of the care needs of clients requires specific home nursing skills and professional expertise, or whether this process can equally well, or even better, be performed by outsiders to the profession as well, as in the case of transferring the diagnosis of the care needs of clients to special assessment organisations in Germany and the Netherlands.

Another problem specific for some professions, including home nursing, is that it is difficult to measure the outcomes of their tasks. Currently, managers of home nursing and other organisations where home nurses are working demand insight in and justification of the kind of tasks they perform, how much time each

I2 This complexity and the enormous demand for services both constitute the power of professionals. 
of the tasks take and the efficacy or result of these activities. However, the more instrumental aspects of home nursing, such as performing technical nursing tasks like giving injections, are more easy to measure and register than the more supportive and interpersonal aspects of it, such as the (emotional) support of clients, taking time to listen to their personal experiences and helping them and their relatives and informal carers to cope with the disease, disabilities or increasing dependency. Although the latter are a very important and integral part of home nursing and in general very much needed and appreciated by clients, there is currently less and less time available for these tasks, as these are difficult to circumscribe in concrete activities or exact minutes and are thus difficult to account for to the management of home nursing organisations.

From a theoretical perspective, the more specialised a treatment is, the more a profession is able to retain control over it, while professional jurisdiction over the problem becomes weak and susceptible for invasion by other professions or actors when treatment results are difficult to measure (Abbott, I988c p. 46). Then, others think they can judge upon the tasks performed as well and impose external controls onto these tasks and ask for justifications of the professional activities in terms of managerial or organisational goals. Thus, currently, many professions, especially those that perform tasks that are difficult to measure and of which the results are difficult to circumscribe or account for, are confronted with the problem that other professions judge on their activities. This affects their independent professional judgement and autonomy and thus their professionalism. This is especially the case in countries where home nurses are working in multi-professional organisations with an organisational management and differentiation of tasks. Then, organisational and managerial perspectives overpower the professional perspectives of a particular profession such as home nursing. In contrast, independent, private nurses or nurses working in very small organisations who decide on the tasks to be performed themselves have more abilities to judge upon their activities and remain in control over their professional autonomy.

Also in literature on health care or home nursing, striking is that the activities of professionals mostly remain rather implicit, and specific problems from a professional point of view are hardly discussed. Many studies are written from an organisational and/or policy perspective, taking the activities of professionals more or less for granted. On the one hand, there are (comparative) studies on home care that focus on issues such as the impact of health care reforms, the kind of services provided to elderly people, or differences in the financial and organisational health care structures between countries (e.g. Bertels and Cocquyt, I995a; Evers and Svetlik, I99I; Giarchi, I996a; Hutten and Kerkstra, I996a; Kemenade, I997a; Mossialos and Le Grand, I999a; Nijkamp, Pacolet et al., I99I; OECD, I994a, I996a; Raffel, I984, I997a; Saltman and Figueras, I997a; Schneider, Biene-Dietrich et al., I994a). Most of these studies report the current budgetary problems 
in the home care domain in most West European countries, and the expected rise in the demand for home nursing and other services for elderly people in the near future. However, the impact and consequences of these developments for the home nursing profession are hardly mentioned, although these only add to the already existing considerable shortage of qualified staff in many countries that will undoubtedly increase in the future.

On the other hand, more and more studies are currently written from a clientoriented perspective, and study issues such as the quality of care in relation to the quality of life of patients, continuity of care, coping strategies, and personal networks of elderly or dependent people, or clients' satisfaction with the care provided, e.g. (Achterberg, I997; Courtens, I993; Janssen, I992; Made, Jongerden et al., I994; Mead and Bower, 2000; Smeenk, I998). The increasing popularity of such studies during the last decades may be related to the increasing influence of patient organisations, demanding a more client-oriented approach in health care and more attention for the perspective of patients (Blaauwbroek, I997; Lieshout, I996; Ramakers, I998b). In most of these studies, however, the activities of health care professionals are mainly evaluated from the experiences and expectations of the client and not specifically from a professional point of view.

Thus, a large body of literature on different aspects of health care exists, but many studies written from an organisational, policy-, or client-oriented perspective. Relatively few studies specifically focus on problems or issues that are related to the professional character of the work. Apparently, it is assumed that health care professionals are the natural mediating people between the aims and demands of health care organisations and the health needs of clients, and that the function and role of health care professionals is relatively unproblematic and running quite smoothly. There seems to be no need to explicitly focus on the process and activities in-between providers and clients. However, is this assumption justified?

Since the mid-twentieth century, the social organisation of work has increasingly been determined by organisations that have gradually undermined independent professional practice. Thus, the question is whether professionals still are rather autonomous, or whether they have been degraded to mere employees of more or less bureaucratic organisations: are professionals nowadays still in control of their own practices, or do they just perform the tasks assigned to them by the organisation they work for? When this question is answered positively, this would mean that the division of labour in contemporary society is no longer based on professional task structures, but determined by organisational staffing structures and career patterns of large-scale, bureaucratic organisations where professionals of several disciplines closely work together (Abbott, I989). Consequently, this raises the question whether bureaucracy and deprofessionalisation are destroying the notion of autonomous professions: 
'Does this mean that the production of expertise, like most other modern production, will hereafter be institutionalized in organisations rather than in individuals, that professionalism - the very notion of institutionalizing expertise in individuals - is dead?' (Abbott, I989 p. 280)

As these changes in the organisation of work during the last decades refer to essential qualities of professions, and not just superficial aspects or accidental attributes (Abbott, I988 p. 325), there certainly are questions to ask about the role of professions in contemporary society and about the degree of autonomy and control of professionals over their own work. Professionals themselves often feel as if they just follow orders of other (professional and non-professional) people and caregiving protocols, that both leave little room for individual decision-making (Thiel and Hoven, I999). In the following chapters is therefore studied whether home nursing professionals still hold authority over the process of diagnosing the problem they claim to treat (Abbott, I988c), and can still independently determine the content of their tasks and the way these are performed, or whether professional processes are increasingly being affected by non-professional perspectives, such as organisations or other decision-making processes. However, the dominance and interference of organisations in professional practices will differ per country, and is also depending on the number of home nurses that are employed in organisations or working in independent practices.

\subsubsection{Demographic changes - the ageing of the population}

The dilemmas and difficulties for home nursing as a profession are reinforced by several demographic and social developments that increase the demand for home nursing, especially the increase in the number of elderly people and especially the very elderly among them. On the one hand, these developments imply a greater need for home nursing services, and thus offer chances for development and growth of the home nursing domain, on the other hand, these reinforce already existing pressures on home nursing in most European countries, as the financial leeway for an increase in provision is not increasing to the same extent. For example, in France, the dilemma of home nursing is not so much a shortage of demand, but rather that the demand or need for services is greater than can be fulfilled in practice. As will be shown in the chapter on home nursing in France, this is only partly related to a shortage of home nurses and bad targeting of services, but also to French health policies and infrastructural difficulties in France.

The ageing of the population is a development that is taking place in all European countries, although the specific estimates vary slightly per country. In general, it is estimated that between 1950 and 2050, the proportion of the population aged 65 or over will have more than doubled; from an average of less than 
Io per cent to an average of more than 20 per cent of the total population (OECD, I996b p. I4; Verheij and Kerkstra, I993 p. 54). Factors contributing to this 'second demographic transition' are for example increases in national wealth and personal incomes, reductions in child and adult mortality rates, lower birth rates, investments in health and welfare programmes, and above all, a higher life expectancy. For example, in the Netherlands, the expected life-span was 5I years of age for men and 53 years for women at the beginning of the twentieth century, while these figures have increased to 75 and 8I years in I999 (Ruigrok, 2000; Ruwaard and Kramers, I993; United Nations, 2000). Especially the number of the 'oldest old' among the elderly is expected to increase substantially, which is generally referred to as the 'ageing of the aged' (OECD, I996b p. I4), or 'double ageing' of the population. For example, many European countries are expected to experience an increase of at least 200 per cent in the numbers of those aged 80 or over between ig 60 and 2040 (OECD, I996b p. I5). In the Netherlands alone, the number of people of 75 years and older has increased with $225 \%$ between I950 and I990. At the same time, the number of children and youth has increased with $8 \%$. As is shown in figure 2 , the once pyramid shaped composition of the population structure has changed into an onion-shaped structure.

These demographic changes have profound implications for the home nursing domain, as the prospect of living longer is accompanied by an increase in chronic and degenerative diseases and prevalence of disabling conditions that sharply increase after the age of 75 . The consequent loss of independence regarding daily activities will lead to a corresponding growth in the need for care (OECD, I996b p. I8), especially home nursing, home help and informal care, because the largest proportion of elderly people is growing old in their own environments. ${ }^{\mathrm{I} 3}$ Also, these trends have resulted in a drastic shift in the initial relation between the domain of Youth health care and the domain of Elderly care and care to chronically ill people, in favour of the latter two (Boom, Philipsen et al., 2004b).

However, one should keep in mind that growing old is not by definition synonymous with being disabled or incapacitated; an increasing part of the elderly are considering themselves as being actively pursuing social and leisure activities and greater independence. In France, this is accompanied by the replacement of the notion of old age by the image of the autonomous, actively participating people in their 'troisième âge' (third age) (Henrard, Cassou et al., I990; Henrard, I996 p. 668-69). Namely, although less than Io\% of people of $65^{+}$in the Eu countries

I3 In OECD countries, the proportion of elderly people below the age of 75 who reside in institutions providing long-term care was as low as between 0,4 and I,8 per cent in the early I990 (OECD, I996c). However, after 75, the proportion rises considerably with every five-years age band, and over 80 years of age, the proportion of institutionalised elderly rises to about io per cent. 
are reported as being in good health, the majority of elderly over 75 (70 to 80\%) are not severely disabled or do not suffer from a significant incapacity (Giarchi, I996b p. I7-I8, 2I). Focussing on the problems and incapacities of elderly people endangers the presentation of a one-sided view that ignores that many people are in good health, lead an independent life and bring valuable contributions to society. Nevertheless, the fact remains that a large number of elderly people that does need some kind of family or professional support, although the age of dependence seems to shift gradually to the older age groups. Elderly women over 75 are considered a particularly vulnerable group, as they often suffer from multiple losses, in their family life as well as in their health state (Giarchi, I996g).

Figure 2 Age composition of the Dutch population at January I. I950 and January I. I990 for men and women
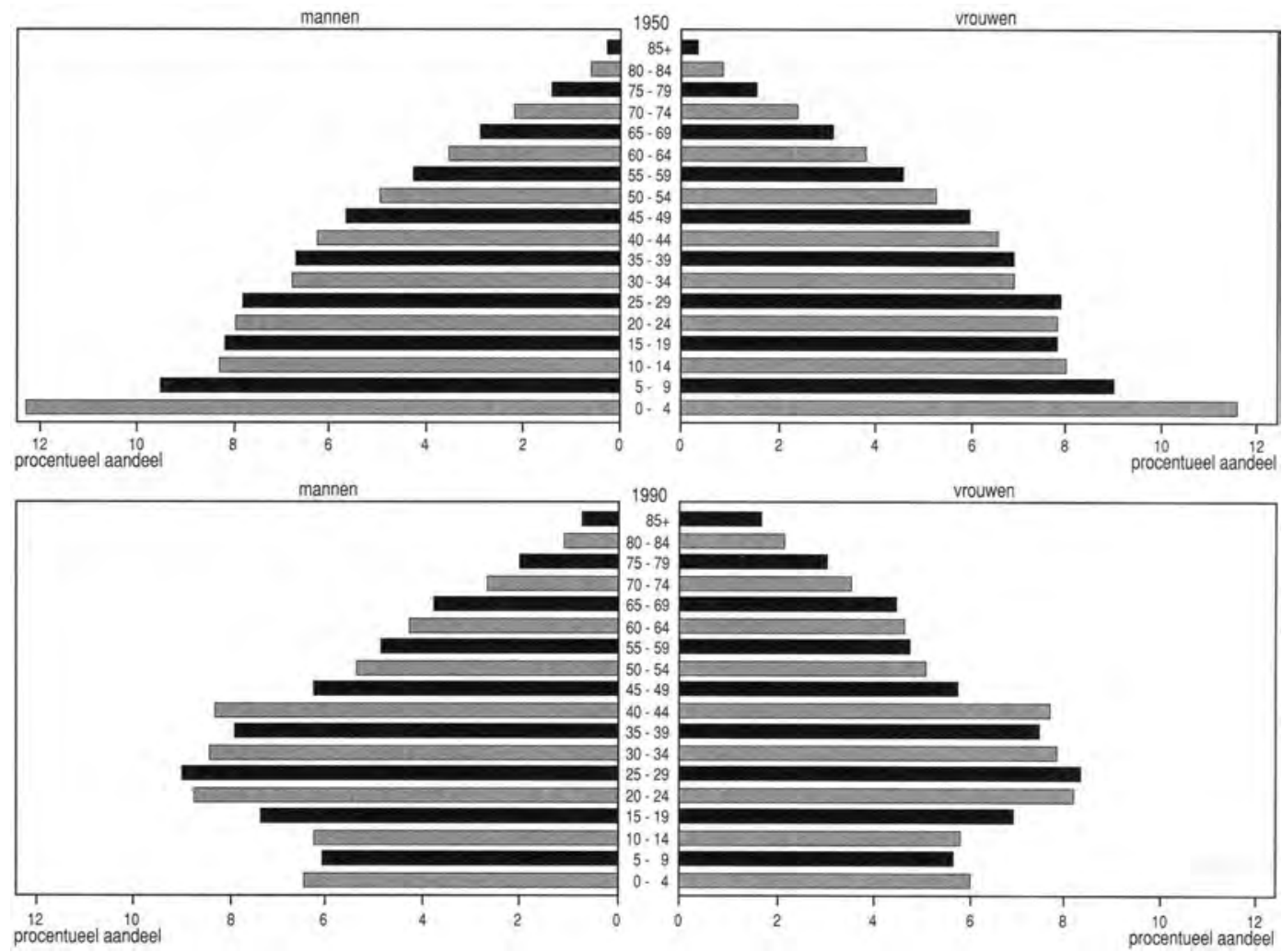

Source: Ruwaard and Kramers, I993. 


\subsubsection{Changing family relations and informal care}

An important social trend further increasing the demand for professional care is that living arrangements of elderly people are changing. Although in many South European countries, such as Portugal, Italy, Greece, Spain and France, the family still functions as a significant support system providing shelter and care, in many North and West European countries a large number of elderly people are living alone or in couples now instead of in three-generation households (Giarchi, I996b p. I8 and 37). ${ }^{.4}$ Paradoxically, this increasing social independence of elderly people is going hand in hand with an increasing dependence of care and support provided by professionals. Namely, the increased intergenerational independence is reinforced by a greater social and geographical mobility and a lesser number of children per family, that have substantially diminished the 'caring potential' of informal care-givers during the last decades in most West European countries. In general, family care is largely provided by younger female relatives, such as daughters and daughters-in-law, most of them between 40/45 and 64 years of age (OECD, I996b p. I9; Dautzenberg, 2000 p. I3). However, due to the increased labour participation of women since the second half of the twentieth century (for example from 49 in I965 to 78 per cent in I988/89 in Denmark (OECD, I996b p. I9)), the availability of informal caregivers has diminished, as well as the amount of time women have left to provide unpaid family care. The vulnerability of available informal care as well as the increasing individualisation in contemporary societies result in an increased demand for professional nursing and caring services. However, the relation between unpaid, informal care, and professional, formal care and cultural and political expectations regarding the provision of informal care by family members differ per country and call for a comparative perspective.

Also, differences in the modalities of home nursing can be traced to diverging opinions between countries regarding the range of responsibilities of family members to provide informal care to elderly and chronically ill people, which results in different relations between formal and informal care. Often, these opinions are underpinned by the 'principle of subsidiarity', traditionally underlying health care arrangements in some countries:

'It means fundamentally that the response to need should first be made within the immediate world of the clients: at first, with family or relatives. Failing these, they are expected to call upon informal helpers, either neighbours or friends.

Should they not be able to provide support, assistance should be sought from

I4 For example, the number of women living alone in Germany is $53 \%$, in the U $\mathrm{k} 53 \%$, and in France 43\% (Giarchi, I996b). 
the voluntary Church or lay organizations. In the event of these agencies of organizations not being able to help, the next step is to approach the welfare organizations and the statutory personal social services. The state is the last port of call.' (Giarchi, I996b p. 32)

This means that the role of the state remains minimal, and only functions as a 'safety net' in case charitable or local institutions and private initiatives are not able to offer sufficient support and the individual in need of help does not receive any support from family or neighbours. In countries where this principle has dominated health care arrangements for a long period, such as France, Germany, the Netherlands, and many Mediterranean countries, the role of informal caregivers has explicitly been interwoven in health care policies (Henrard, I996 p. 672).

In countries adhering to the principle of subsidiarity, moral obligations of family members caring for their frail relatives are supported and promoted by specific health care structures and financial regulations, while health care in general is quite cure-oriented in character. For example, in Germany, there is a strong reluctance of insurance organisations and state politics to extend coverage beyond medical treatment to social care and support (Jamieson, I996). Family responsibilities to provide care have been encoded in legal regulations and are reflected in health care policies (Twigg, I996). Many Mediterranean countries are also considered as "societies in which the family is the primary resource for individuals in all aspects of their lives - economic, social, cultural - and family care of older people is just part of a larger network of mutual opportunity and obligation rooted in the family" (Mestheneos and Triantafillou, I993 in: Twigg, I996 p. 83).

Thus in many countries, care and support provided by family members is considered as 'the informal household production of care', or 'the invisible welfare state’ (OECD, I996b p. I9), substantially complementing professional care provided by the health care system. Many governments explicitly define family care to elderly people as 'the central pillar of care' and thus stress familial obligations to provide care, mostly because of anxiety over public costs. The political assumption is that health care arrangements provided by the state should not be designed to replace informal care, but only be regarded as a supplement to informal care (Giarchi, r996b p. 33).

However, in contrast, in many northwest, Scandinavian countries, health care is considered a public responsibility that should be guaranteed by the state. In Denmark, for example, there is no explicit expectation that families should provide care for elderly parents. As a result, the availability of informal care-givers is not considered in the assessment of care needs of patients, which is common in many other countries (only the presence of a spouse is taken into account) (Jamieson, I99Ic, I996; Twigg I996). Family members do not have any obligations to care, so although families are still close emotionally, they are not the primary source of 
physical care. Thus, although the role of informal care is considered quite important in many, international differences do exist. It seems however that a process of convergence is taking place (Stevens, 2007), because the social and cultural predominance of family traditions and collective values and the availability of informal care-givers is generally diminishing in western societies (Hofstede, I980a), while at the same time the affordability of public health care systems is being discussed in northern Europe.

Thus, the institutional and financial structure of health care, including health policies and social and cultural opinions regarding health care and social services, largely determine the relation between professional and informal care and subsequently, the role of home nursing in society (Jamieson, I996). Esping-Andersen (I990), studying differences between countries regarding welfare state traditions (Twigg, I996 p. 83), identifies three regime types, namely 'liberal' welfare regimes that encourage market provision of services; conservative or 'corporatist' regimes where the family is expected to meet many welfare functions, such as in Austria, France, Germany and Italy; and social democratic regimes mainly associated with North European countries, in which state-provision and financing of services should guarantee availability of and access to a broad range of health care and social services. However, this 'ideal type' kind of classification of welfare states seems to reduce the complexity of everyday reality too much to fit particular countries neatly into one of the three categories. However, the underlying assumption that health care arrangements are intricately interwoven with social, political and cultural assumptions and traditions remains very useful and will be elaborated in our theoretical perspective.

\subsubsection{Cultural diversity}

Another social trend is that in the last decades, the cultural background of clients has become increasingly diverse. Although this cultural diversity has already been familiar in nursing practices in the United States for some time now (and is referred to as 'transcultural nursing' (Leininger, I994 p. 7)), in European countries it is a relatively new phenomenon. Nurses are currently being expected to care for a great variety of cultural groups, which requires that nursing practices can no longer be supplied without taking the values, beliefs and daily living patterns of specific cultural groups into account:

'Nurses are interacting with people from widely different cultures within a short period of time. As a consequence, nurses and other health care providers are expected to know, understand, respond, and interact favorably with strangers from diverse cultural backgrounds in this country and elsewhere. Being helpful to strangers whose values and lifeways are different from those of the nurse 
necessitates a knowledge base of such differences and the creative use of such knowledge. Cultural awareness through knowledge is a critical necessity for professional nurses today and one which is of growing importance. (...) Transcultural knowledge can help the nurse work effectively and comfortably with people of different cultural backgrounds.' (Leininger, I994 p. 7-8)

However, the tensions between the instrumental values of health care and the values of the 'multi-cultural lifeworld' of these patients may be even stronger. This requires specific skills of home nurses in order to be responsive to the particular needs of these clients, and sensitive to their cultural frame of reference. The provision of care in a similar way to all clients alike should especially be avoided in these situations, by adjusting nursing care to the culture-specific circumstances in which it is provided (Ibid., p. Io).

\subsubsection{Health and elderly policies}

Thus, several demographic and societal changes taking place in most European countries influence the demand for home care and thus the possibilities for the home nursing profession and domain to strengthen its position in the health care systems of these countries. However, as we shall see in the next chapters, in general, health policies and financial means that are made available for expansion of home nursing and home help services are still insufficient to meet the increased demand. The shortages in the supply of home care services and shortage of adequate staff in most countries increase the strain and burden on the home nursing profession that already has to cope with several difficulties that result from the dominance of organisational perspectives and the increased bureaucracy and introduction of protocols that have severe implications on the daily work of home nurses.

Apart from these general European trends, there are also country-specific contextual factors that influence the work and position of home nursing, for example the structure of the overall health care system, and, at a macro-level, the specific social, political, and cultural views regarding care for elderly people. As home nursing is interrelated with its societal context, it cannot be isolated or viewed apart from these external aspects. Namely, health care policies, reforms and regulations result in a continuously changing health care environment (Kümpers, Raak et al., 2002). For example, current health policies in many European countries are aimed at substitution, favouring the provision of nursing and caring in people's own environments instead of in hospitals, nursing homes or homes for the elderly. This is also referred to as the ideology of 'ageing in place' (Jamieson, I996 p. 67; OECD, I996c p. 56). The idea is that older people should be able to live as long as possible (or as long as they wish) in their own homes, or at least closely integrated into the community. Essential for this is that next to institution-based care, a wide 
range of ambulant, community-based health and social services should be offered in a variety of settings, such as home nursing and home help, day care facilities, adapted houses, sheltered housing, etc. (Giarchi, I996b p. 30). Substitution is generally politically justified by the aim to curb the ever-increasing health care costs, and the cultural ideology that older people should be able to participate in normal social life as long as possible, by supporting them in their own environments, even when they are very old or have multiple health problems and require intensive or 24-hour help (Giarchi, I996b).

However, the impact and range of health care policies differs per country, as these are embedded into already existing health care structures. For example, in Denmark and the Netherlands, policies aimed at providing care and facilities in the community have high priority, because the number of elderly people living in institutions has been very high since the I95Os (OECD, I996a; Bijsterveld, I996). While in France, in many areas it is still far easier to receive institution-based care in e.g. a residential home for elderly people than to receive home nursing care.

\subsubsection{Differences in health care systems}

Thus, the structure, way of financing and the underlying principles of health care systems are different across Europe, as these have resulted from social, cultural and political choices regarding health care arrangements and have developed over time according to country-specific ways to deal with social and health problems.

Within western Europe, there are two dominant kinds of health care systems, that are historically based on different principles and assumptions (Swaan, I989b; Kemenade, I997a; Schröer, I998; Marrée and Groenewegen, I997b), namely Bismarck systems of social insurance and Beveridge type of national health care systems, with a high level of state involvement. Examples of the first are Germany and the Netherlands, and the latter the National Health Services in Scandinavia, including Denmark, and the Uк (Marrée and Groenewegen, I997b). Although in most countries a mix between public and private welfare arrangements has become customary now, basic differences between both kinds of systems can still be distinguished.

The kind of health care system, including its institutional and financing structure, results in different configurations within which home nursing is provided and thus a different position of the home nursing domain in health care. For example, different referral patterns between countries exist; in some countries, home nursing is directly accessible to clients, but in other countries a prescription or referral by a medical professional is needed first. Also, the entitlements to home nursing care and reimbursement of costs diverge between countries, resulting in different patterns of provision. This implies that the tasks of home nurses and their range of responsibilities diverge between countries, resulting in different modalities of 
home nursing within Europe. In the next chapter, the most important differences in the kind of health care systems between countries will be described further.

\subsection{A comparative and contextual perspective on home nursing}

The recent developments and trends in the health care systems and societies of European countries that have an impact on the home nursing domain and profession are now included in the theoretical framework as well. These can be summarised into the following six aspects, namely demographic changes such as the ageing of the population, changing family relations and patterns of informal care provision, increasing cultural diversity, the absence of professional perspectives, especially because these are overpowered by organisational and managerial perspectives, differences in health and elderly policies between countries, and international differences in the health care systems in which home nursing is embedded. When these are included into the theoretical framework, this results into the following comparative and contextual perspective on home nursing:

Figure 3 A comparative and contextual perspective on home nursing

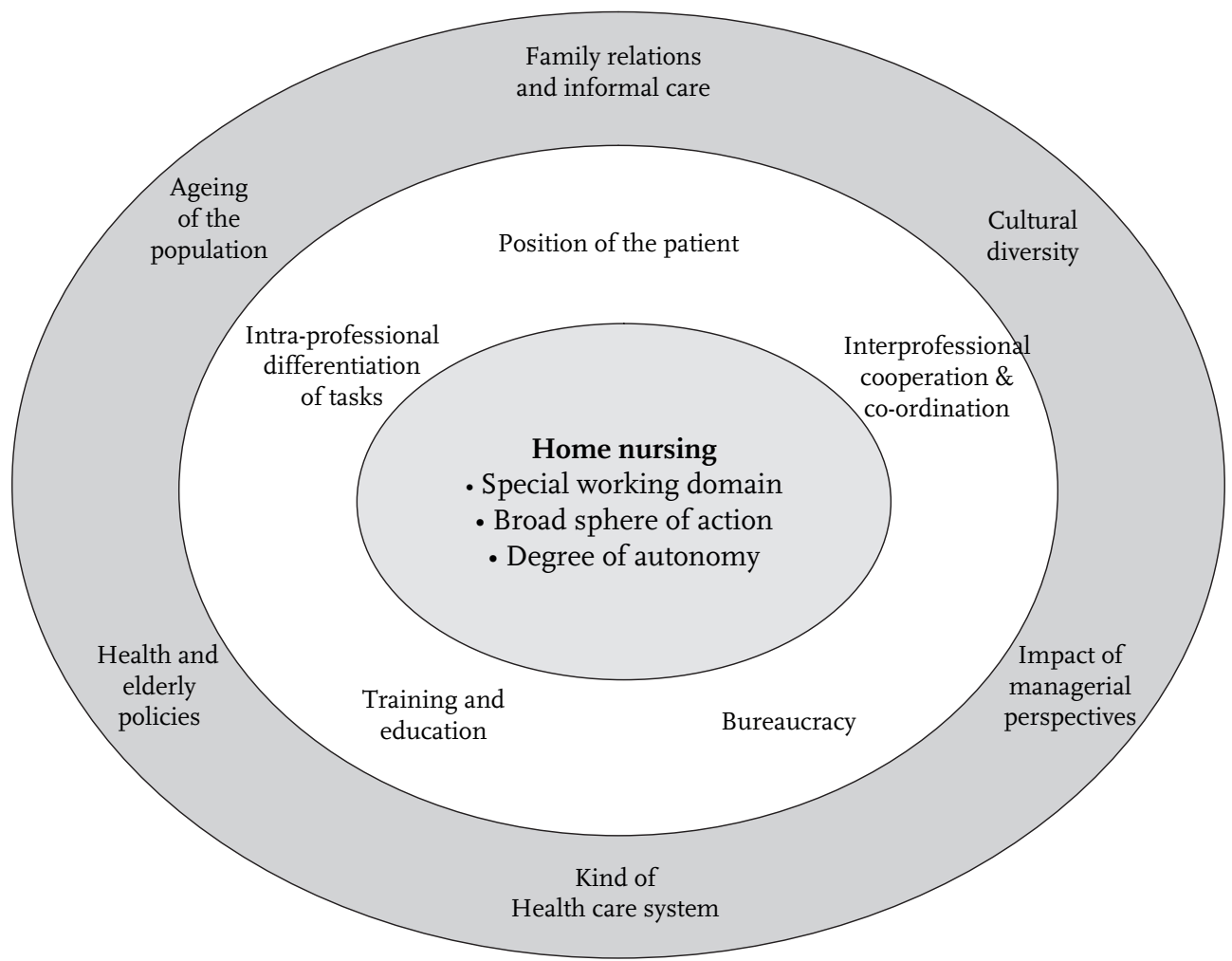


In the inner circle, the general characteristics of the home nursing profession and domain are situated. This inner circle primarily refers to the professional perspective and is used in the next chapters to study the country-specific features of home nursing. The middle circle refers to the professional as well as the institutional perspective and focuses on the degree of autonomy of home nursing, the task division and way of cooperation with other professions. This gives insight into the position of home nursing within the 'system of interrelated professions' and within the health care system. Also, the way that home nursing is embedded in larger societal structures (such as educational facilities, the way a society deals with patients and tendencies to bureaucratise) is focused on. The aspects positioned in the outer ring of the framework are external to home nursing and describe the general societal setting within which home nursing is provided. These aspects depict general societal changes taking place in contemporary European societies, and are intricately linked with societal, political and cultural opinions regarding care provided to elderly and chronically ill people. The outer circle therefore refers to the overall societal context that gives insight into the specific caring culture of a country, by way of the structure of the health care system, specific health care and elderly policies, the role of the family in providing community care and the way that is dealt with the ageing of the population, cultural diversity and the role of managers in health care. Although these circles can be discerned from each other, in society as well as theoretically, these also partly overlap and merge into one another.

In the following chapters, the framework is used to study the modalities of home nursing in comparative perspective, thereby taking the country-specific health care and societal context within which home nursing is provided into account. First, in the next chapter, differences in the health care systems and main social-demographic data are described, to provide general background information to the position of home nursing in each of the countries described in this thesis. 
Chapter 3

\section{Overview chapter on home care provision, health care systems and demographic characteristics of the four countries}

\section{I Introduction}

In this chapter, an overview is provided of general characteristics of each country studied in this book. It provides background information to each of the following chapters in which the countries are described separately. It is not the intention to provide a standardised comparison for benchmarking reasons, but to provide a rich description of the main characteristics and features of each country. Sometimes, the fact that data are not comparable or available is significant because that indicates for example that that particular aspect is considered less important than other aspects in that specific country. In many respects, the countries are similar because they are all situated in Western Europe and comparable in wealth and economic status. Also, all four countries have social security schemes and health care schemes that guarantee the population a minimum subsistence level, proper housing and sufficient means in case of sickness, disability, old-age, unemployment or other hardships. However, despite these similarities, political choices that underlie these schemes and the historical development of the particular schemes of each country are different and relevant for the subject of study.

Another similarity is that certain demographic changes are taking place in all European countries. Namely, in all countries, life expectancy has increased during the last decades, combined with fertility levels that have decreased below the reproduction level. This results in an EU population that is becoming increasingly older. This demographic ageing means that the share of older people is increasing compared to the share of those of working age ( $15-64$ years). For example, between I993 and 2003, the share of those aged 65 and over in the total population rose by about 2 to 3 per cent in Eu countries. It is obvious that these demographic trends will have economic and social consequences in a number of areas, including health care systems and health care provision.

However, the pressure of the ageing population is felt in different degrees in different countries, because there are marked differences regarding the percentage of the population being older than 60 or 65 years of age between countries. In 2004, Italy, Sweden, Greece, Belgium and Germany had the highest shares of people aged 65 and over (Eurostat, 2005). Also, figures on the proportion of elderly 
people compared to those of working age ( $15-64$ years) differ per country. Therefore, it is useful to gain a general overview of the most characteristic differences in the population of the four countries under study.

Firstly, an overview is given of the size of the home nursing sector in each of the four countries and the percentage of the population covered by home nursing and home care services. Unfortunately, it is difficult to find recent and reliable nationwide data. Often, data are dated, unavailable, or only refer to specific regions, cities, or initiatives.

Secondly, even when countries would be quite similar in many respects, their health systems may be quite different and result in different patterns of health care provision and a different balance between cure and care. Therefore, main features of the health care systems are described. A short outline is given of the two basic models of health care systems in Europe; namely the Beveridge public health system and the Bismarck social insurance system. In the four countries chosen, both types of health care systems are represented: in France, Germany and the Netherlands, health care has largely been based on a social insurance system until recently. One can argue, however, that the Dutch health care system is more or less mixed: the AwBz regulation closely resembles a national tax system, and about $40 \%$ of the population was covered by private health insurance. In January 2006 , a new health care system was introduced covering the entire population with a basic insurance. In France, important changes have also taken place since the Juppé reform of I996 and the introduction of a general income tax (CSG) that fundamentally affected the character of the health system. Namely, since I998, the employee contributions, characteristic of a social insurance system, have largely been replaced by this tax. Employers' contributions however still exist. The French health system can currently be characterised as a mixed system. Denmark has a public health system that is based on local taxes. In this chapter, a short description of the health care system of each country is given, to gain insight in the balance between the home nursing sector and other subsectors of health care, especially hospital care and medical care. After all, the balance between the caring and curing sector is important to study, because it sheds light on national priorities in health care and may thus explain existing differences in the modalities of home care between countries.

Thirdly, an overview is given of the most important demographic data of each country, such as its geographical size, the number of inhabitants, the population density, and the share of elderly people in the total population. In the consequent chapters of this book, the way that particular countries are preparing their health systems regarding the ageing of the population will be described in-depth. Also, data on the level of economic activity and family structure are presented. The fact whether many families are still living in three-generation families or in small family units or single households is relevant regarding the availability of informal care and thus the demand for professional home care. 
Fourthly, main differences are described regarding health care professionals, such as the total number of physicians and nurses per country and the rates per I $0 \circ \circ$ population. Also data on the number of hospital beds per I o०० population are presented. These figures also provide insight into the balance between cure and care in the health systems of each country.

Finally, insight is provided into the spending on social protection and the different sources of financing of health care in the different countries. At the end of this chapter, these data are summarised in tables and figures.

\subsection{Overview of differences in home care provision in Europe}

In Europe, large differences exist regarding he provision of home care. Of many countries, it is quite difficult to find recent, equivalent, complete and comparable data, especially in countries where home care has a low priority. In many countries, data concerning home nursing as well as home help are accumulated or no clear distinction is made between these services in the figures provided. This makes it difficult to draw a clear and comparable picture of each country.

In three north west European countries; namely Denmark, the Netherlands and the $\mathrm{UK}$, the provision of home care (i.e. home nursing and home help) has a long historical tradition that goes back to the last quarter of the nineteenth century or even earlier (Kerkstra and Hutten, I996a; Mossialos and Le Grand, I999b; Kemenade, I997a; Schneider, Biene-Dietrich et al., I994a). In these countries, the provision of home care has established itself as an important sector of health care next to other health care domains. However, this does not imply that there are no problems concerning home nursing and home care in these countries. It only indicates that the problems or difficulties are different in character than in countries where home care is a less well-established sector of health care. The following chapters will show that a different balance between cure and care results in different kinds of problems for home care. In all countries, home care is currently mainly provided to elderly people (Kerkstra and Hutten, I996a). The amount of care provided to different age groups of elderly people varies; in Denmark, the 'youngest' subgroup of elderly people $\left(60-65^{+}\right)$receives a quite large amount of home care, while in the other countries, home care is predominantly provided to the oldest age groups, especially those over 70 or 75 years of age (Jamieson, I996). In Germany and the Netherlands, home help was originally targeted at families with younger children, to support the family when the mother was ill or hospitalised, but services are re-targeted towards elderly people now. 


\subsubsection{Denmark and the Netherlands}

The home care sector is most comprehensive and differentiated in Denmark: a variety of services for elderly people exists and a relatively large percentage of the population makes use of them, not only elderly people (Zee, I996). Danish policies have stressed for some decades now that older people should be able to live in their own homes as long as possible, supported by health care and social services, and other facilities for elderly people (Mossialos and Le Grand, I999b p. 33; O ECD, I996d). The high political and societal priority of elderly care is reflected in the expenditures on health care: namely, I $8 \%$ of the total expenditures on health care are spent on caring activities in Denmark, compared to $15.4 \%$ in the Netherlands and 9\% in Germany (Schneider, Dennerlein et al., I992).

In Denmark, home care services are quite evenly spread over the country, and the sector is so large that a relatively large amount of people below 65 years of age are provided with services as well (Jamieson, I996). About $7.3 \%$ of the total population aged $65+$ received home nursing in I99I, of which more than $70 \%$ were over 67 years of age (Zee, I996). In I992, $20.5 \%$ of the people of 67 and over (67 is the pension age in Denmark and other Scandinavian countries) received home help services, ranging from $15 \%$ of those aged between 70 and 74 and as much as $61 \%$ of those over 85 (OECD, I996d).

In Denmark and the Netherlands, home nursing and home help are integrated into one domain. This means that both kinds of activities are generally provided by the same home care organisation. In the Netherlands, this is the result of new home care policies since the I990s; before that, home help belonged to the domain of social care and was supervised by social workers (Swagerman, I997). In most other countries, home nursing and home help are still separately provided by different kind of organisations and financed differently as well. Generally, in those countries, home help belongs to the social services (and not to health care) and is mostly organised by the local authorities (Kerkstra and Hutten, I996a).

In the Netherlands, the percentage of elderly people living in institutions, such as nursing homes and homes for the elderly, is quite high compared to other countries. Since the I950s especially (e.g. since the I950 Law on the post-war reconstruction of the Netherlands), housing policies have been aimed at building specialised housing facilities for people over 65 years of age (bejaarden), with or without physical disabilities. For long, Dutch policies and arrangements facilitated the admission of elderly people to these special elderly institutions. For example, according to the $1_{9} 65$ Social Security Law, admission into special elderly institutions was facilitated by the state that took over admission costs when necessary (Bijsterveld, I996 p. I66-I73). One of the aims of this policy was to stimulate elderly people to move from family houses to special elderly facilities, because of the large need for family homes for younger people and families in the I950s. As 
a result of this trend, the percentage of Dutch elderly living in institutions is still quite high compared to other European countries. However, since recent decades, long waiting lists for residential facilities exist, so that only very old and dependent people can be admitted. Also, in current policies it is no longer thought desirable to stimulate older people to move to elderly facilities without necessity. Therefore, Dutch policies are now aimed at supporting elderly people to remain living in their own homes, supported by home care services and informal care provided by family members and neighbours or friends. Due to these developments, an extensive system of residential as well as home care facilities currently exists for elderly people in the Netherlands. In Denmark, new legislation was introduced in I987, in which a quite radical position was taken (Vallgårda, Krasnik et al., 200I p. 56). According to the Act on Housing for Older and Disabled persons, only independent dwellings should be built, and residents in apartments for elderly people were put on an equal footing with all other elderly people living in their own dwellings and should not resemble institutions (Plovsing, I992 p. I4; Rostgaard and Fridberg, I998b p. 93). The objective of the National Commission on Ageing was that the housing function and service function were separated, and the 'package' of care that was included for residents of nursing homes was abolished. Since then, nursing homes have been considered as ordinary housing, and new nursing homes were no longer allowed to be built (Plovsing, I992 p. I7; Ribbe, Ljunggren et al., I997 p. 8; Vallgårda, Krasnik et al., 200I p. 56). Traditional nursing homes were closed at the rate of about Io\% per year, and home care policies are given a higher priority. Only elderly in such a bad state of health that they cannot remain living in their own homes, not even with social and health assistance offered by the municipality, are admitted. Since the I987 Act, only so-called apartments for the old are allowed to be built, that should resemble independent dwellings instead of institutions. Security should be part of daily life, and it should be possible for each individual to summon help and care at short notice from the apartment in case of illness or accidents. Thus, Denmark takes a quite specific and radical stance regarding elderly people, in which 'ageing in place' is an important assumption and in which elderly remain integrated into society whenever possible.

In I992, about $5 \%$ of the total Dutch population received home nursing care (Kerkstra, I996b). This high percentage is due to the amount of care provided to mothers with young children aged o to 4 at the Consultatiebureaus (baby welfare centres), that accounts for as much as $40 \%$ of the home nursing care provided. Of the other age groups, mainly elderly people receive home nursing. Although the percentage of the population receiving home care decreased from $7 \%$ in I990 to $5 \%$ in I992, the number of visits per client increased considerably: from 8.9 in I9 85 to I5.4 in I992. This is probably due to the closer targeting of services to specific groups of elderly people, in order to prevent institutionalisation into nursing homes and homes for the elderly. In I99I, $4 \%$ of the Dutch population (mainly 
elderly people) received home help, but long waiting lists existed for many years, due to substitution policies, budget deficits and shortage of personnel (NRC Handelsblad, I999). However, during the last few years, many changes have taken place in home care as well as in the overall health care system.

\subsubsection{France, Germany and other European countries}

In many other European countries, especially in Central and South Europe, the home care sector is still relatively small in size and home care services are quite unevenly distributed over the population. This is especially the case in countries such as Italy and Greece, where home care is hardly part of health care services, but to some extent also in France and Germany (Kerkstra and Hutten, I996a; Mossialos and Le Grand, I999b). In these countries, medical activities and hospital services have a dominant position in the health care system. Also, informal care is quite important. Home nursing and home help services have been developed relatively late or are still being developed and thus still form a relatively small subsector of health care that is rather overshadowed by the curative sector. In France and Germany, a specific problem is that the population is not evenly covered by home care. Especially in rural areas, gaps in the network of services exist and the supply of services provided is less uniform than in other European countries (Verheij and Kerkstra, I993).

Especially in France, large differences exist in the availability of home care between regions. Two-thirds of the services are located in the greater Paris area, while in some areas home care is hardly provided or not provided at all (Kerkstra and Hutten, I996a p. I5; ofCD, I996e; Verheij, I996; Verheij and Kerkstra, I993). Overall levels of provision are low (Jamieson, I996). For example, in I991 only $0.15 \%$ of the total population aged 60 years and over received home nursing, and only $0.6 \%$ of those aged 65 and over, while the average level of dependency of clients is high (Verheij, I996). Thus, the availability and accessibility of services is not guaranteed in all regions in France (Jamieson, I99IC). It is estimated that

only Io\% of those dependent for Activities of Daily Life (ADL-activities) receive organised home nursing in France (Verheij, I996). However, since the I980s, the provision of both home nursing and home help has become a priority of the central and local governments (OECD, I996e). Several policy reports have been issued since the Ig6os that were aimed at improving the independence of older people by supporting them in their own homes. The number of home nursing organisations and hours of home nursing provided have increased considerably since then. However, severe problems regarding the provision of home nursing still exist, such as lack of planning and co-ordination of services, inconsistent policies regarding benefits and co-payments, waiting lists, and unbalanced geographical provision (Ibid., p. I38). 
Regarding home help, problems similar to home nursing exist. The most important problem seems to be to target the available resources to the right people: "only $35 \%$ of those in need actually receive home help, and of those receiving home help only 60\% need this service" (Henrard, I991, in Verheij, I996 p. I28). In I988, only $3.5 \%$ of the population aged 65 and over received home help. $70 \%$ of the clients are over 70 years of age. During recent years, the provision of home help has also increased considerably; from only $3 \%$ of the elderly people in i980 to $7 \%$ in I990. However, the average number of hours supplied is quite low, namely less than 3 hours per week. Another problem is that as much as 725 municipalities were still uncovered by home help services in I993 (against 4293 in I983) (Verheij, I996). This indicates that in spite of serious political efforts to improve homebased services, severe problems currently exist regarding home care in France.

In Germany, home help is only part of health care when it is provided in addition to home nursing to heavily dependent clients; in other cases it is considered as social care. No clear overall statistical data are available of the population covered by home care services in Germany. This is partly due to lack of adequate information systems for collecting such data, and partly due to considerable regional variations in service provision (Jamieson, I99IC). It is estimated that in the late I970s, only about $1.5 \%$ of those aged $65+$ received organised support services at home, which may have increased to about $3 \%$ in the early I990s. However, strong reservations regarding these figures should be held in mind, as these are not completely reliable (Dieck, I99I).

The provision of home help services is also quite low in Germany: less than $5 \%$ of the elderly receive home help (according to Jamieson, this figure is even lower: under $3 \%$ (Jamieson, I99IC)). This contrasts sharply with Belgium, France, the Netherlands, and the United Kingdom, where between 6 and Io\% of the elderly receive home help services. In Denmark the figure is even higher than io per cent (OECD, I996C). This indicates that in Germany, the health care system is more hospital-based and cure-oriented than home-based or care-oriented.

Opinions about home care in Belgium diverge: some authors state that it is a reasonably well established subsector of health care (Kerkstra and Hutten, I996a), while others state that it is still rather overshadowed by the medical and hospital sector (Mossialos and Le Grand, I999b). Thus, in many European countries, and especially in Mediterranean countries, elderly people in need of help are heavily dependent on medical services and facilities and rely on family members or acquaintances for informal help, and not on institutional kinds of elderly support.

Remarkable is that in countries where the home care domain has a rather weak position in the health care system that is more curative-based than care-oriented, such as Belgium, France and Germany, home nursing is provided by a relatively large number of independent nurses working on a fee-for-services basis in small private practices (Kerkstra and Hutten, I996a; Mossialos and Le Grand, I999b). In 
Belgium, for example, independent nurses cover as much as about 40 per cent of the market. In contrast, in countries where the home nursing sector is quite well established within the health care system, such as Denmark, the United Kingdom and the Netherlands, home nursing is mainly provided by regular facilities such as local public or semi-public organisations instead of by private nurses or commercial organisations.

\subsection{Health systems}

In the following part, a description is given of the major characteristics of the health systems of the countries described in this book. In general, there are two ideal types of funding health care: an insurance system, which can be subdivided in social and private insurance, and a national health system, funded by local or national taxes (Kemenade, I997a; Kerkstra and Hutten, I996a; Mossialos and Le Grand, I999b; Swaan, I989b; Marrée and Groenewegen, I997b). In practice, the funding of health care is more or less mixed in all countries. In France, Germany and the Netherlands, health care has long been mainly financed by social insurances, while health care in Denmark is mainly financed by taxes. Currently, more than half of the sources in France also come from a tax, the CSG. Systems vary in the parts of the population that are covered, in levels and kinds of payments, in the services included and excluded and the nature of legal constraints. Also the degree of government influence on insurances and on decision-making in health care varies per country.

In both kinds of systems, individual clients can opt for private insurances, as a supplement to social insurance as well as tax based health insurance, to cover the costs of additional or special health services. Premiums of private insurances (capitation) are mostly related to individual risks, such as age, sex, and state of health or occupation. Capitation can be calculated per individual or per risk group. The private insurance package can be combined with co-payments, benefit limitations and exclusions. The amount of government control in private insurances varies, but is generally rather limited.

\subsubsection{Health care systems based on social insurance}

A social insurance health care system is the oldest kind of collective insurance against health care costs and its nation-wide scale originates from the late I880s in Germany. A social insurance system is characterised by: a compulsory health insurance system, paid by employers and employees; a public and self-control system; and public and non-profit delivery. The part of the population that compulsory insurance applies to is established statutorily and therefore differs per country. 
Premiums (payroll taxes) are usually based upon (income and risk) solidarity and mostly not or only limited, on individual risk factors such as age or lifestyle. The height of the premiums can be calculated in various ways and can be fixed by governmental or non-governmental agencies. The level of premiums can differ per social group. In the Netherlands, a social insurance system existed until January 2006 that was characterised by a split between people insured under the compulsory Sickness Fund and people that were privately insured. The most far-reaching social insurance system is a traditional national insurance, covering the whole population with a wide range of services, with an income-related premium, noncompetitive providers and a reimbursement system by which costs for health care services are remunerated to clients afterwards when they send in their bills to the insurer. But there are also social insurance systems with limited benefit packages that only cover a limited group of people and are supplemented by private insurance schemes. In most European states, a mix of social and private insurance in different combinations exists.

\subsubsection{France}

In France, until recently, the majority of health care for the compulsorily insured was financed by social insurance contributions of the statutory health insurance Assurance Maladie, namely about 74 per cent (Mossialos and Le Grand, I999b). The system evolved from mutual benefit associations that proliferated during the Igth and 2oth centuries (European Observatory on Health Care Systems, 2007a). A compulsory health insurance system was established for low-earning employees in industry and business in I930, covering as much as two-thirds of the population by I939. National social health insurance was introduced after the Second World War. In I974, coverage was expanded to cover the entire population, but only since I999 coverage is extended to all people residing in France. The French health care system is gradually becoming more decentralised to the regional level, but at the same time, a shift in power has been going on from the health insurance funds to the state.

An important recent reform since I996 (the Juppé reforms) is the replacement of wage contributions of employees by a tax on income, thus increasing the role for the parliament in setting expenditure targets and policy directions. Also, annual Acts on Social Security Funding that are issued since I996 set the national ceiling for health insurance expenditures to control costs and set budgets that are determined through negotiations between professionals and health insurance funds. The French health care system is regulated by two main kinds of players: the state (The National Assembly, the government and ministries) and the statutory health insurance funds. Local communities also play a small role in regulating the system. The statutory health insurance system is composed of three main insur- 
ance schemes, supervised by the Ministry of Social Security; the general scheme (Regime General), the agricultural scheme and the scheme for non-agricultural self-employed people. The main scheme covers about $84 \%$ of the population, the agricultural scheme $7.2 \%$ and the latter $5 \%$ of the population.

Affiliation to the Assurance Maladie is through different schemes, determined by an individual's socio-professional category. The main scheme, the Regime General, is mainly financed by payroll contributions paid by employers (I2.8\%) and employees (6.8\% of their gross salaries), but the employees' contributions are currently replaced by a national tax that is dependent of income. A large part of the population takes additional Voluntary Health Insurance that complements the compulsory insurance and also covers co-payments. Three types of additional insurance exist: the Mutuelles, mutual organisations of which $87 \%$ of the population are voluntary members, contributing to about 5 per cent of the financing of health care; provident associations; and private for-profit commercial insurance companies, contributing to about 5 per cent of the financing. The Mutuelles are supplementary insurance funds that coexist with private for-profit insurers, although they cover different benefits. Within the Assurance Maladie scheme, patients have freedom of choice in using health care services, but they contribute a significant proportion to covering the cost of care themselves in the form of co-payments (the ticket modérateur). In I995, as much as Io per cent of the French population received completely free health care because they could not afford the usual co-payments. Patients have free choice of public ( $25 \%$ of the hospitals) or private hospitals $(35 \%$ of these are non-profit, $40 \%$ is for-profit) and of general practitioner.

The fundamental change in the system that was introduced by the replacement of the employees' contribution by the national income tax; the Contribution Sociale Géneralisée (CSG) makes the current French health system into a mixed health system. Elements of the social insurance system still exist in the form of employers' contributions, but the general tax that accounts for about $40 \%$ of the financing of health care introduces an important element of a public health system. An important problem in the French health system is the geographical disparities in the distribution of health professionals (European Observatory on Health Care Systems, 2007a). Also, the system is very complex in structure. Among other things, the unrestricted freedom of patients and providers and retrospective payments result in high levels of expenditure. Reforms are introduced, but some are however impeded by persistent poor relations between the state and the insurance funds.

\subsubsection{Germany}

The German health care system originates from the late nineteenth century and has evolved from the reforms introduced by Bismarck in the I88os. The health 
system of the former East Germany (GDR) was quickly transformed in the I990s according to the treaties of unification to adopt the statutory health insurance system of West Germany (European Observatory on Health Care Systems, 2007c). Since then, most formal differences between the eastern and western part have gradually been adjusted. The leading reform principles after unification have teen to reduce structural east-west differences and to strongly contain costs in all health care sectors. At the same time, in de last decade of the 2 oth century, statutory long-term care insurance was introduced to better meet the health needs of the population.

A fundamental characteristic of the German health care system is the sharing of decision-making powers between the federal government, the Länder, corporatist organisations of sickness funds, physicians and dentists as well as other legitimised civil society organisations. The national level (Federal Ministry for Health and Social Security, Federal Assembly, Federal Council) is responsible for passing health reforms regarding statutory insurance and is thus largely restricted to providing a regulatory framework within which the provision and financing of health care services take place. The Länder are responsible for planning inpatient capacities and financing investments in hospitals, nursing homes and social care institutions. The corporatist level is represented by the non-profit, quasi-public sickness funds and regional associations of physicians and dentists that treat sHI-insured patients. In addition, there are a vast number of organisations that represent professional and manufacturers' interests and welfare organisations.

The German health system is characterised by a decentralised system of mandatory Social Health Insurance ( $\mathrm{sHI}$ ) according to the Bismarck model: multiple competing sickness funds exist, and there is a mix of private and public providers. Before 2006, this was also the case in the Netherlands. The health care system is executed by social sickness funds, the Gesetzlichen Krankenversicherung or G KV, which are independent, non-profit organisations (NGOS) that also have regulatory, managerial and planning competencies. The sickness funds are the collectors, purchasers and payers of statutory health and long-term care insurance. Due to many mergers, their number decreased from more than I200 in I993 to 292 in 2004. In 2002, social insurance financed about 65 per cent of health care (Busse and Riesberg, 2004). Revenue collection and micro charging levels are free from governmental interference as this is in the hands of the sickness funds.

The majority of health care for the compulsorily insured is financed by social insurance contributions. Eighty-eight per cent of the population are covered by SHI by membership of the sickness funds; 78 per cent of them are compulsory insured, and Io per cent join the scheme voluntarily. Contributions for sH I are not dependent on risk and dependent of income level, and have been shared fiftyfifty by employers and employees since I949 up till 2004. From July 2005, the share of employees is larger, namely $54 \%$, compared to $46 \%$ paid by employers. 
Rates between sickness funds vary, but the average contribution rate was $14 \%$ of gross income in 2004. Since I996 almost every SH I-insured person has the right to freely choose between sickness funds, while funds are obliged to contract with any applicant. There is a risk-spreading scheme to compensate for differences in need for care and morbidity. In addition to this scheme, three kinds of coverage co-exist; private health insurance, taken by $6 \%$ of the population, free governmental schemes for specific groups in the population that cannot pay the sHI or other insurance $(2 \%)$, and $4 \%$ of the population receive governmental schemes because they are ineligible to take G KV cover as Beamte or civil servants, such as police officers, university professors and public officials. These Beamte enjoy an advantageous special governmental scheme. Only 0.2 per cent of the population chooses not to be covered by any insurance at all. These figures indicate that the social insurance scheme enjoys a high degree of acceptance within the German population, probably because the package of benefits covered by sH is very comprehensive. The possibility of steep rises in premium levels and the obligation to insure each additional family member under private insurance are however important drawbacks of the system. ${ }^{\mathrm{I}}$ Mandatory members of sickness funds pay a certain level of their income as co-payments, but lower than the amount that voluntary members have to pay.

The provision of health care is of a 'payment in kind' or third party payer principle. Patients have free choice of family doctor among sickness funds. GPS do not have a gate-keeping role, as medical specialists are directly accessible by the population, which is an essential characteristic of German and French health care that diverges from Denmark and the Netherlands, where GPs do have a gate-keeping role. Ambulatory care is almost exclusively delivered by strictly regulated private for-profit providers, but hospital care is delivered by a mix of public and private providers. Most acute hospitals are enlisted in 'hospital plans' and thereby regulated and financed basically by the same mechanisms regardless of ownership. Patients have free choice of public or private hospitals; about half of the hospital beds are publicly owned; the other half are mostly owned by non-profit private hospitals. There are small waiting times and people have equal access to services. An important weakness of the system however is its fragmentation across sectors and the pressure of the contributions on the income of the population.

I According to table 5, 7.4 per cent of the German health care is financed by Voluntary Health Insurance (vHI). As in the Netherlands, this kind of insurance substitutes for, and is mutually exclusive from, the statutory health insurance scheme. The kind of coverage and payment system of vH I companies can vary per country, although trends indicate that luxurious care provisions are more and more replaced by budget plans. 


\subsubsection{The Netherlands}

In the Netherlands, until January 2006, when a fundamental change in the health care system was introduced, the health care system was mainly financed by a mixture of social and private insurance (Mossialos and Le Grand, I999b). Historically, the Dutch health care system is different from many other European countries, because it largely originated through the efforts of voluntary non-governmental organisations (NGOS) (European Observatory on Health Care Systems, 2007d). This is why almost all hospitals are private, non-profit establishments. Another important feature is its long history of consensual processes of continuous consultation, discussion and policy debate. Most reform decisions have focussed on increasing competitiveness and difficulties in introducing effective market competition while maintaining solidarity and financial accessibility. As in Denmark, the system of primary health care is strong and well developed, which contributes to a feeling of satisfaction about the system of the Dutch (and Danish) population.

In 2002, social insurance contributions accounted for about 75 per cent of the total health care budget (about 35\% was financed by the sickness insurance, and about $39 \%$ by the AwBz, see further on). Only 6 per cent was financed by taxes, and I4 per cent by Voluntary Health Insurances (private insurance). About 60 per cent of the population was covered by statutory social insurance provided by the Ziekenfonds (Sickness Fund) (Keuning, I997 p. 29); namely employees and their families earning below a certain wage level (of about $€ 30000$ in 2000 (Gilles, Kesteloot et al., 200I)); people drawing a welfare allowance (e.g. the unemployed, disabled, widows); and pensioners who were insured by sickness funds before they reached the age of 65 . The sickness fund premiums were partly paid by employers $(4.95 \%$ of the wage level) and employees $(2.85 \%)$. The premiums were paid to the Sickness Fund Council and distributed over the sickness funds, of which there were about 45 that mostly worked regionally. The package of health care covered was quite large. Most of these sickness funds still exist as commercial insurance companies now and are providing basic or additional health insurance under the new insurance scheme.

The remaining 40 per cent of the population exceeded the income ceiling for social insurance and were encouraged to join a voluntary health insurance scheme. These people were free to choose their own health insurance company (there were about 80 of them) and could choose between different packages of care covered. Premiums varied per health insurance company and the amount of money the insured person was willing to pay. Only one per cent of the Dutch population (mainly the very wealthy) chose not to be covered by insurance at all, which is no longer possible in the new system.

Apart from the sickness fund and private insurance scheme that existed until 2006 , and also under the current new health care system, the entire Dutch popu- 
lation is covered against heavy medical risks of serious or long-term afflictions by compulsory premiums that are paid in a national insurance scheme, regardless of income, namely the Algemene Wet Bijzondere Ziektekosten (Exceptional Medical Expenses Act, which is a general act regarding special and costly health care costs) (Meulen and Burg, I998; Keuning, I997 p. 30). Examples of these so-called 'uninsurable risks' are medical and pharmaceutical care, nursing home care, psychiatric care, mental health care and home help. As the amount of health care costs paid by the Aw Bz is larger than the amount paid by the sickness and private insurances, it can be considered as a very essential element of the Dutch health care system, almost comparable to a universal tax system.

Since 2006, the Dutch health care system has changed fundamentally with the introduction of a universal basic insurance. Plans for such a universal insurance scheme have already been proposed in I990, the 'Plan Simons'. Simons, state secretary of the Department of Health Care at the time, proposed to reform the health care system fundamentally, in order to make the insurance structure more transparent and better organised (Keuning, I997 p. 28). The sickness fund and private insurances were to disappear, while the AwBz would be extended to a broad basic insurance scheme, covering most health care facilities. The rightwing government that came into power in 2002 introduced this compulsory basic insurance scheme in January 2006, among others to reduce unnecessary bureaucratisation and inefficiency in the health care system (Raad voor de Volksgezondheid en Zorg, 2002 p. 25). The system is to be carried out by private health insurance companies that operate under market competition. The full consequences of this new type of insurance system for providers, insurers and patients are not quite clear yet.

Most of the acute hospitals in the Netherlands are non-profit and owned by intermediate non-governmental organisations, and patients have free choice among those hospitals approved by health insurance or government. Free choice of a general practitioner is restricted to twice a year under the health insurance scheme. The GP has a gate-keeping role, as in Denmark, and restricts access of patients to hospital and specialist care and other health care facilities.

\subsubsection{Health systems based on universal taxes}

A tax based national health care system is the other major kind of health system. In such a national system, health care is financed by taxes and executed by the government. Every citizen has the right to obtain the care available, and - in theory there is total equity of care. That means that all kinds of care are accessible to all kinds of people, regardless of age, sex, income, ethnic background, occupation, or other potentially distinguishing criteria. Expenditure is fixed by annual parliamentary budget negotiations. The u K National Health Service ( $\mathrm{NHS}$ ), originating from 
I942, is the most familiar example of such a system. The trademark of systems such as the NHS is the provision of health care by state-run organisations and financing via public funding, such as local or national taxes. National systems can be combined with parallel private insurance schemes or co-payments by clients (e.g. for prescriptions in the U K). Denmark also has a national health system, which is mainly organised and financed locally.

\subsubsection{Denmark}

In Denmark, 82 per cent of the total expenditure on health care is financed through general taxation (Mossialos and Le Grand, I999b; Christiansen, Enemark et al., I999; Vallgårda, Krasnik et al., 200I). However, in contrast to the $\mathrm{U}$ K health system that is mainly financed by national taxes, the principal source of funding in Denmark is local taxation, namely about 62 per cent. Only 20 per cent are national taxes that are used to finance the highly specialised National Hospital, and to supplement the budgets of counties and municipalities in the form of state block grants. About I6.5 per cent of total health expenditure is financed by clients in the form of co-payments. Pensioners, low-income families and the disabled are either exempted from co-payments (categories vary between municipalities) or pay reduced co-payment rates. There are no co-payments for most non-clinical services, only for physiotherapy, dental care and spectacles, and drugs. About $30 \%$ of the population purchases Voluntary Health Insurance (private insurance) that is mainly used to cover co-payments. vHi has become more and more popular due to the perceived shortcomings of the health care system, especially the waiting lists. However, vH I only contributes for less than 2 per cent of health care financing.

The Danish health care system is mainly public and decentralised. The budgets, and hence health care activities as well, are the responsibility of the counties and municipalities. However, the state government has some power to control the overall budget and growth rate, but plays a relatively limited role in health care; its main functions are to regulate, coordinate and provide advice by policies and legislation.

Danish health care is characterised by the absence of major reforms since the establishment of the national health system in I973, although some cost-containment measures were taken (Christiansen, Enemark et al., I999; European Observatory on Health Care Systems, 2007b). Health care was a major issue during the $200 \mathrm{I}$ elections, which was largely due to media interest in waiting lists, but national and local reforms in the last decade have focussed on increasing productivity and quality and reducing waiting lists and attempts to improve coordination between the different administrative levels of the health system. The degree of satisfaction with the health care system is high in Denmark, and 
strong political and public commitment to the universal health system based on equal access for equal need and health care provided free at the point of use remains strong.

GPS and practising specialists are privately organised and financed through the Public Health Insurance that is managed by the counties. GPS act as gatekeepers to hospitals and specialist treatment, and in principle, patients have to be treated at the lowest level of care (cost-wise) at which effective treatment is possible. Choice of family doctor is free for Group Two insured (only 2.5 per cent of the population), while Group One insured (97.5 per cent of the population) can change their family doctor once per six months. Most of the hospitals are publicly owned by the counties (over 90 per cent), which are also responsible for the financing of hospitals. When they exceed their budgets, they have to stop intake from waiting lists during the last months of the year. Since October 1992, patients have been given a degree of freedom to choose the hospital of their choice, also when it is situated outside the home county, but a referral from a GP is needed. This introduced an uncontrollable element into the counties budgets, as the home county has to pay the county in which the patient is treated (in practice, it mainly concerns acute care). Since I993, counties have gradually introduced contracts with individual hospitals, e.g. about maximum waiting times. GP and hospital care is free for all Danish citizens, but patients in need for basic hospital treatment are not allowed to be treated in specialised departments of university hospitals.

Municipalities finance and are responsible for out-of-hospital care, such as home nursing and home help, health visitors (including pre- and postnatal home visits), and other preventive services, such as the municipal and school dental service.

\subsection{Overview of demographic data and key characteristics of each country}

In the following part, a comparison is made of the four countries in this book, namely Denmark, France, Germany and the Netherlands. The figures presented and compared give an overview of differences and similarities regarding the general background of these countries. The data described in the following paragraphs are summarised in tables that are presented in the appendices at the end of this chapter. When possible, comparable and recent data are presented. However, due to uneven availability of data, different data presented by different institutes, different definitions that countries use to describe and measure certain phenomena, and sometimes unavailability of data of similar years, accurate interpretation and comparison of the data is sometimes difficult and conclusions are drawn with some caution. 


\subsubsection{General demographic data: total area and population characteristics}

The first important difference regarding Denmark, France, Germany and the Netherlands is their marked difference in size (see table I). France is one of the largest countries in the $\mathrm{EU}$, with a total area of more than $630000 \mathrm{kms}^{2}$ (Eurostat, 2007a). Although the size of Germany (after the unification) is almost half of that of France, with an area of almost $360000 \mathrm{kms}^{2}$, it counts more inhabitants, namely 82.5 million people. Germany has the largest population of the 25 countries in the EU; I8\% of the EU population lives in Germany, followed by France, where $13.5 \%$ of the EU population lives. Together with the $\mathrm{U}$ K and Italy, these four countries comprise $57 \%$ of the Eu population (Eurostat, 2005). In contrast, Denmark and the Netherlands are quite small countries that are about one-tenth of the size of France and Germany. The area of Denmark counts $43000 \mathrm{kms}^{2}$, and the Netherlands about 4I $500 \mathrm{kms}^{2}$. If only land area is counted and lakes and rivers are excluded (which is used to calculate the population density), the area of the Netherlands is only 34 ooo square kilometres.

Although the size of Denmark and the Netherlands is comparable, there are significant differences in population density (Eurostat, 2007a). Denmark's total population counts 5.4 million people, compared to I6.3 million in the Netherlands. With a population density of I25 people per $\mathrm{km}^{2}$, Denmark is one of the least densely populated countries in Europe, after France that only counts 80 people per $\mathrm{km}^{2}$. The Netherlands is the most densely populated country in the Eu and counts as much as 482 people per $\mathrm{km}^{2}$. Its capital Amsterdam is the largest city, with more than I million inhabitants, but the Randstad-area in North and South Holland can actually be considered as one large city area counting 6 million inhabitants, with cities melting into one another (Eurostat, I999, 2007a; United Nations, 2000). The rest of the Netherlands is also very densely populated. Thus, living space is quite scarce in the Netherlands. In Germany, the population density is about average in the EU with 23I people per $\mathrm{km}^{2}$, but there are also some very densely populated areas with agglomerations of cities, such as the industrial area in the southwest, the Ruhrgebiet. The capital of the country, which is Berlin since the unification, has 3.4 million inhabitants but is not the largest German city. There are several large cities, such as Essen (almost 6.5 million inhabitants), and Hamburg.

The percentage of the population living in urban areas is comparable in Denmark (85\%), Germany (87\%) and the Netherlands (89\%), but considerably lower in France (75\%). The Danish capital of Copenhagen counts I.3 million people, which is as much as one fifth of the total population. In France, about $20 \%$ of the total population is living in Paris and its surroundings, the Île de Paris, namely II.3 million, of which 9.5 million live in the city of Paris. This implies that other areas in Denmark and France are quite scarcely populated, especially when one considers that the average population density of both countries is already quite low; 
namely I25 and 80 people per $\mathrm{km}^{2}$, respectively. Especially in France, contrasts between urban and rural parts of the country are sharp: the Ile de Paris is highly urbanised, and there are several other large cities, while some other parts of the country are very scarcely or almost unpopulated, such as the causses in mid-France. Nine-tenth of France's total area consists of farmland and forests, and as much as $93 \%$ of its 'communes' are classified as rural (Giarchi, I996g p. 209). Almost half of the communes have fewer than 300 inhabitants. Many of these rural and scarcely populated areas suffer from lack of jobs, economical activities and social and health services. As the younger generations have moved to more urban areas for work, the family network has fallen apart. Ambulant services for elderly people are often unavailable or even non-existent in these areas; thus many elderly people live in social isolation and are deprived of formal and informal help. As will be argued in the chapter on home care in France, the uneven availability of provision and support across the country is a particular problem.

In Denmark, the total population increased with 0.3 per cent between I995 and 2000 (United Nations, 2000), and is expected to increase to 5.6 million people in 2025, but decline to the current 5.4 million again in 2050 (Eurostat, 2007a). In the Netherlands, population growth has been $0.4 \%$ between I995 and 2000, and is expected to increase with another I.I million to I7.4 million people, but no further growth is expected between 2025 and 2050. In France, the annual growth rate was also $0.4 \%$ between 1995 and 2000 , and the population change was still positive in 2005 , but the total population is expected to decrease from 62.9 million in 2005 to 61.7 in 2025 . In Germany, the total population has remained almost stable between 1995 and 2000 with a growth rate of only 0.I\%. The population change has currently become negative because of the low birth rate of I.3 children per woman, which is not supplemented by migration. By 2025 , the total population is expected to remain at the current 82.5 and then decrease to 74.6 in 2050 . This has to be considered against the background of the share of people over 65 years of age in Germany that is highest in the EU, which means that the pressure of the ageing population is most urgent in Germany.

Finally, figures are presented of the percentage of non-nationals in the four countries and of the number of asylum applications. After all, cultural diversity is one of the aspects included in the theoretical framework; care workers such as home nurses and home helps who provide home care, are increasingly meeting clients of different cultural backgrounds, especially in the larger cities. The figures on migrants should however be interpreted with extreme caution, as countries use different definitions of migrants and asylum seekers and produce different migration statistics, and several corrections and recalculations during recent years make the overall picture unclear (UN F PA, 2000). Although the exact figures or estimates are not reliable, general trends and differences between countries can nevertheless be described. 
In Denmark, the percentage of non-natives is quite low, only 4.7\% (I998) (Eurostat, I999). Many asylum-seekers, about 23\%, come from Somalia. The number of asylum applications has increased from 5 I00 in I995 to Io 300 in 2000 , but has decreased sharply to 2 Ioo in 2005 (Eurostat, 2007a). The policy concerning foreign immigrants currently is quite restrictive in Denmark and meets severe criticism in the EU. In the Netherlands, that has long been know for its tolerance and advocacy of the multi-cultural society, the climate regarding non-natives has changed considerably in recent years, in society as well as in politics, as discontent about the number of foreigners asking for asylum has increased. The percentage of non-Dutch inhabitants is quite low, as in Denmark, namely only $4.3 \%$ (I998), compared to 9\% in Germany (I997) and II.0\% in France (I982) (Eurostat, I999). A considerable part of the population consists of people from the former Dutch colonies Indonesia and Surinam that already came to the Netherlands in the I950s and who were accepted without problems by Dutch society and who integrated quite smoothly. In the I960s and I970s, many guest workers from Italy, Turkey and Morocco came to the Netherlands because of a lack of Dutch employees. Many of these were employed in unskilled or lowly skilled jobs, such as in the mines, cleaning services or garbage collection. Their children or grandchildren (second or third generation Turks or Moroccans) are reasonably well integrated into Dutch society now, although their social and economical situation is generally less favourable than that of Dutch natives. Currently, the largest categories of non-Dutch nationals are people from Turkey and Morocco. Since I997, an increase in immigration has taken place of Dutch nationals from the Antilles and Aruba, as well as of non-nationals war refugees from Iraq and Afghanistan. Since I995, the number of asylum applications has increased from 29300 to 43900 in 2000 (Eurostat, 2007a). Thereafter, a steady decline has occurred to I2 300 in 2005 (provisional number) ${ }^{2}$. This indicates a stricter policy by the Dutch government since the 2Ist century, about which opinions diverge widely in Dutch society.

The percentage of non-nationals in France is quite high with II per cent (I998) (Eurostat, I999), but a large part of the total number of foreigners come from one of the many former colonies of France, such as Algeria, and also from other European countries, especially Spain, but also pensioners from the U $\mathrm{K}$ and the Netherlands. Until the early 2 oth century, France had the reputation of being most open to immigrants, but tensions between different population groups and incidents of racial discrimination do exist and regularly lead to clashes in suburban areas with a high percentage of immigrants living in deprived conditions. The number

2 Several corrections on data have been performed in recent years, making the overall picture not quite clear (UNFPA, 2000). 
of asylum applications has more than doubled in ten years, from 20400 in 1995 to 42600 in 2005 (provisional number) (Eurostat, 2007a).

In Germany, 9.0\% of the population is of non-German origins (I997) (Eurostat, I999). The most important homelands of non-nationals are: Turkey (28.6\%), the former Yugoslavia (9.8\%), and Italy (8.3\%). Since 1997, migration patterns of non-nationals have changed and diverge of that of other European countries: a significant drop in arrivals (mainly of civil-war refugees and asylum-seekers) and a steep increase in departures took place as a result of increased emigration of non-nationals (I4\%), especially people returning to the former Yugoslavia. This resulted in a decline in net immigration and added to the overall decline of the total population. These trends are reflected in the number of asylum seekers; in I995, the figure was as high as I27 900, which declined to 78600 in 2000 , and further dropped to 28900 in 2005 (provisional number) (Eurostat, 2007a).

\subsubsection{General demographic data: age groups and economic activity}

Demographic data on the different age groups in Denmark, France, Germany and the Netherlands display the following picture: Germany is 'suffering' the most of the ageing population, as well as in percentages as in absolute numbers (table 2) (Eurostat, I999, 2005, 2007a). About I3 million people are 65 years or older in Germany, which is a staggering number compared to the 2 million in the Netherlands and less than one million in Denmark in France. This group represents I $8.6 \%$ of the total German population, compared to the EU average of 16.5 and I4. 0 in the Netherlands, I5.0 in Denmark and I6.4 in France. The consequences for health care facilities will especially be felt in the coming years, as the percentage of those of 80 years and older (4.3) is not very different from that of Denmark and France (4.I/4.5), but the high figure especially concerns the age group of those of 65 to 79 years, namely I4.3 per cent in Germany, compared to I0.9 to II.9 in Denmark, France and the Netherlands. When this age group gets older and older, co-morbidity and dependency will become more prevalent, thus the demand for formal and informal care is expected to increase significantly in the coming years. When one considers that as much as $4 \mathrm{I} \%$ of the German people over 65 years of age are living alone (I992), this also indicates a serious problem for health and social care.

In addition, Germany not only has the highest share of older people, but at the same time the lowest share of people aged o to I4 years, namely only I4.5\% of the total population, compared to the EU average of $\mathrm{I} 6.5$, and I8.5 to I8.8 in Denmark, France and the Netherlands. Thus the term 'double ageing' definitely applies to Germany. Also, the fertility rate is lowest in Germany of the four countries, namely I.4, while 2.I are considered as the replacement level in the EU. The EU figure is I.5, but the figure is higher in the Netherlands (เ.7), Denmark (เ.8), and France (I.9). 
Another specific characteristic of Germany is the uneven balance between men and women. In all countries, the number of women exceeds that of men. The EU average is I05 women per Ioo men, and all four countries are not far from that. However, when looking at the percentage of men and women in the higher age groups, a different picture emerges. Namely, in 2000 only ig per cent of those over 60 years of age were men and 26 per cent women in Germany (United Nations, 2000). In the other countries, the figure is also higher for women than men, but not so wide apart as in Germany. It seems plausible that the Second World War had a deep impact on the considerably lower number of men in this age group.

In the Netherlands, the population age structure diverges somewhat from that in the other countries, as the population is relatively young: $86.0 \%$ is between $\circ$ and 64 years old and only $\mathrm{I}_{4} .0 \%$ is 65 years or older (compared to $15.0 \%$ in Denmark, I6.4 in France and I8.6 in Germany (2005). The lower percentages concern all age groups: $60+, 65+, 75+$ and $80+$.

The population in Europe has been characterised by high life expectancy at birth, which has increased by eight years for both sexes over the last forty years (Eurostat, 2005 p. 79-80). Although life expectancy is six years higher for women than men, this difference is starting to decrease because life expectance of men has increased more than that of women during the last decade in most European countries. The increasing life expectancy, combined with the decrease in fertility, results in a population that is becoming increasingly older.

France has one of the highest life expectancies in the European Union, namely 77 years for men (the EU average is 75, as in Denmark) and 84 years for women (the highest in the EU) (Eurostat, 2007a). Denmark is one year below the Eu average of 80 years for women. The difference in life expectancy between men and women in France is widest in Europe.

The fertility rate for the EU as a whole is I.5 children per woman since I994, while the French rate is I.9, which comes close to the replacement level of 2.I. This also accounts for the relatively high growth rate of the population of $0.4 \%$ during I995 to 2000 in France. Fertility rates are lowest in Germany (I.4) and explain the negative growth of the total population.

Regarding economic activity, differences between countries are large. Employment rates are highest in Denmark (76\% of those of working age, 2005) and the Netherlands (73\%) and lowest in Germany (65\%) and France (63\%) (Eurostat, 2007a). France and Germany also have the highest unemployment rates, namely around io per cent, compared to less than 5 in Denmark and the Netherlands. In Denmark, the share of economically active women is highest of the four countries. In the Netherlands, as much as three quarters of the women work part-time and until a few years ago, only 45 per cent had jobs at all. Only io per cent of working 
women work $80 \%$ to full time. This fits the Dutch culture since the I950s that is still present, according to which it was a sign of wealth and religious values when families could afford to stay home with the children and the men provided for the family's income. Currently, also a lot of Dutch men work part-time (23\%). In France, it is not very customary to work part-time.

The standard of living in Denmark is among the highest in Europe. Taxes on income and wealth are also very high, namely more than 30 per cent, compared to IO to I2 per cent in the other countries. But this figure is somewhat misleading, because contributions to health care financing are paid in the form of taxes in Denmark and is thus included in the high figure, while it is paid in the form of social insurance contributions in the other countries. Nevertheless, the high figures for economic activity of men and women in Denmark are necessary for families to be able to afford the high taxes and extensive welfare and health care system.

\subsubsection{Household situation}

In order to gain insight in the availability of informal care, and on the increasing individualisation of society during several decades now, figures on household or family situation were gathered and on the proportion of elderly living with their offspring (table 3). Recent and comparable data were however difficult to obtain. In France, only I3 per cent of households consist of one adult living alone (2000), which is almost similar to the Netherlands (I4), but lower compared to Germany (I6) and Denmark (I7) (Eurostat, 200Ia). The traditional family with two adults with dependent children is dominant in France, namely $43 \%$ and much higher than in the other three countries (34 to $38 \%$ ). The percentage of elderly of $65+$ living with their offspring is also higher than in the other European countries, and was still $17 \%$ in 1990 . However, figures are not really comparable because they refer to different years, and those of Denmark concern elderly of 70+. Remarkable is the high percentage of elderly of 70+ that are living alone in Denmark, namely more than 50 per cent, which is far higher than in the other countries.

The data that are available seem to confirm the hypothesis that people in southern European or Mediterranean countries and in Germany are more often still living in three generation families than people in the north of Europe (Twigg, 1996; Giarchi, I996a). In North West Europe in particular, few elderly people are living with their children: in Denmark only $4 \%$ of those over 70, in the Netherlands only $8 \%$ of those over 65 (OECD, I996b). As these figures concern the late I980s, more recent figures are expected to be even lower. However, conclusions about the availability of informal care should not be drawn too easily, as the absence of large families does not simultaneously imply the absence of informal caregiving; considerable amounts of care are provided when families live nearby or family members have easy access to each other (Twigg, I996; Dautzenberg, 2000). Of 
equal or more importance is the kind of welfare tradition of a country: in social democratic regimes, mostly present in North and North West Europe, shared values are held that care should primarily be provided by the state, while in South European countries and countries with conservative welfare regimes, such as Germany, the family is considered as an important source of help and support (EspingAndersen, I990).

\subsubsection{Chronic conditions and subjective health}

Another remarkable difference between the four countries concerns the (subjective) values that people attribute to their health (table 4) (Eurostat, 2007a). Namely, as much as $76 \%$ of those of I5 years and older rate their health as good to very good in Denmark and $72 \%$ in the Netherlands, compared to only $58 \%$ and $47 \%$ in France and Germany. In Germany, as much as I9\% of the population aged I5+ rate their health as bad to very bad, which is two to three times higher than in the other countries. The percentage of people being hampered by chronic conditions is lowest in Denmark, for men as well as women.

\subsubsection{Health care professionals}

Table 5 presents data on health care professionals. Some figures are given in manyears (Full-Time Equivalents) and are thus corrected for professionals working part-time; some figures are absolute numbers and therefore not corrected, but concern more recent years. Different pictures emerge when describing the number of physicians that are licensed to practice (including those who are licensed but do not work as a physician), economically active physicians (including those who may work in different jobs), or practicing physicians. Namely, in the fist case, Denmark would have 4.0 physicians per I 000 population, but in the latter, 2.9 per I 000 population. In the Netherlands, also more physicians are licensed (3.5 per I ०००) than practicing (I.9 per I ०००, I999). This figure is far lower than in France (3.0) and Germany (3.4). Unexpectedly, the number of practicing physicians in Denmark is not so different from figures of France and Germany, while the latter two countries are known for their high density of physicians (Eurostat, 200Ia, $2007 \mathrm{~b}$; Vallgårda, Krasnik et al., 200I). The number of GPS do however confirm that picture; in the Netherlands, there are only 0.4 GPS per I o०० population, in Denmark 0.6, in Germany I.I, and in France I.5. However, as in Denmark as well as in the Netherlands, GPS are gatekeepers for specialist medical care, one would expect that in these countries, the proportion of GPS would be higher. It could be that in these smaller countries, distances to GPS are shorter and GP practices may thus have more patients per GP, explaining these lower figures. Also, there is a shortage of physicians in the Netherlands. 
Regarding nurses, the figures do confirm the hypothesis that Denmark and the Netherlands are countries with a more favourable caring culture than France and Germany: in Denmark, there are II.० nurses per I o०० population, and in the Netherlands even I4.O. Thus, it could also be the case that nurses have taken over parts of the job of GPS, such as nurse practitioners that have become more prevalent in the Netherlands in GP practices. In France and Germany, there are 7.I and 7.5 qualified nurses per I ooo population. Date on the number of second level nurses (auxiliaries) confirm the picture that in France, especially many second level nurses are working in health care and their numbers have increased considerably, namely 7.4 per I ooo population, compared to 4.7 in Denmark and only 2.2 in Germany. Unfortunately, the figure of the Netherlands is not available. In both figures following table 5, trends over time (I996/97-2004) in the total number of qualified nursing staff per I ooo population and nursing auxiliaries are depicted. These pictures also show clearly that their numbers are higher in Denmark and the Netherlands than in Germany and France, but it also shows that the number of nurses has remained about stable, while the number of second level nurses has increased at a fast rate in France and Denmark but remained stable in Germany. It thus seems that Denmark and France have responded to the increasing demand for caring staff, especially by increasing the numbers of second level nurses. It also explains the current feeling of a 'crisis in nursing' (Pflegenotstand) in Germany (Scharf, i998e).

\subsubsection{Hospital beds}

Figures on the number of in-patient and acute care beds confirm the impression that France (7.8 and 3.9 per I ooo population) and Germany $(8.6 / 6.4)$ are more medical and cure-oriented than Denmark (4.0/3.3) and the Netherlands 5.0/3.I per I ००० population). In addition, France has I.5 and Germany 2.I 'other beds', such as in specialist hospitals (Eurostat, 200I, 2007). Germany and the Netherlands also have a high number of beds in nursing and residential care facilities, namely almost 8 per I 000 population in Germany (I999) and I4.4 in the Netherlands, while France has I.3 long-term nursing care beds per I $0 \circ \circ$ population. These figures are unavailable for Denmark, confirming the earlier mentioned fact that Denmark radically changed long-term elderly care facilities from residential to ambulant facilities. In all four countries, trends in the number of hospital beds between I994 and 2004 indicate a decrease in the number of hospital beds, which was most strong in Germany. This can be explained by the curb in health care costs due to deteriorating economic circumstances all over Europe. 


\subsubsection{Social protection}

Social protection encompasses all actions by public or private bodies to relieve households and individuals of risks or needs that are associated with among others old age, sickness, childbearing and family, disability, unemployment. Social benefits are direct transfers in cash or kind by social protection schemes to households or individuals to relieve them of the burden of such needs (Eurostat, 2005 p. I33). Table 9 shows that expenditures on social protection are about equally high in the four countries. The figure for France is highest (31.2\% of the Gross Domestic Product) and the Netherlands lowest, with $28.5 \%$ of GDP (Eurostat, 2007). Per head of the population, Denmark spends most, namely in 200 Euros, compared to around 8 ooo in the other three countries. In all countries, the largest share of the total benefits is spent on old age, for example on old age-pensions, disability pensions, and early retirement benefits; namely 36.2 to 42 .I per cent; sickness and health care come second; family and children (Denmark, France) or disability (Denmark and the Netherlands) come third.

Social protection schemes are financed in different ways, for example by social contributions paid by employers and employees, or by the government, and by other sources such as interests. In the EU, about $39 \%$ of the social protection was financed by employers, $36 \%$ by the governments, and $22 \%$ by the protected persons themselves (Eurostat, 2005 p. 137). However, the countries each display quite different pictures. For example, the government pays 63.5 per cent of the social protection in Denmark, compared to 35 per cent or less in the other three countries. In France, the employers pay the largest share in the form of social contribution (45.5\%). In Germany, employers and the protected persons themselves contribute most, as in the Netherlands (2004).

\subsubsection{Expenditure on health}

Trends in the total expenditure on health in the last two decades, as a percentage of GDP (I975 - 2004) show fluctuations in each country. Denmark has remained between 8 and 9 per cent of GDP, France's expenditures rose sharply from $6.4 \%$ in I975 to I0.5\% in 2004 (OECD, 2007). Germany's expenditures rose from $8.6 \%$ of GDP in I975 to equal the level of France in 2004 (I0.6\%), while the level in the Netherlands fluctuated between 6.9 and 9.2 per cent.

\subsubsection{Sources of health financing}

Finally, sources of health financing are described. However, figures have changed fundamentally for the Netherlands and France because of the recent restructuring of their health systems. In both countries, social insurance contributions by 
employees have been replaced by a general scheme covering the entire population. In the Netherlands, employers' contributions have also been replaced, but these still exist in France and contribute for more than 50 per cent to the total sources of health care. Taxation is now the most important source in France (almost $40 \%$ of the total sources) and has been for long in Denmark because of the national health system based on local government taxes ( $82 \%$ of the total sources of health care). In the Netherlands, a large share of the sources is paid under the AwBz scheme (Exceptional Medical Expenses Act) that covers the population for high unaffordable risks. Out-of-pocket payments by the population are lowest in the Netherlands. Figures on France are unclear; all sources indicate that out-of-pocket payments are an important source of financing, but these are not included in the tables. Therefore, the total number of sources in France exceeds roo per cent.

\subsection{Conclusion and discussion}

In conclusion, this chapter has shown that the health systems in the four countries under study display a range of differences regarding their historical development, current structure and way of financing, and the health care arrangements offered. For example, the number of hospital beds, physicians and nurses per I ooo population differ per country. Also, demographic data regarding the age composition of the population, population density and population growth (or decrease, such as in Germany) and family structures differ between countries. Thus in each country, the health care system is embedded in the specific socio-cultural and political context and historical traditions and developments. Therefore, the conclusion is that no one health care system is similar to that of another country; each country seeks and has sought different ways to provide for the needs of the population. Therefore, this book aims at providing rich, qualitative descriptions of the health care systems and the domain and profession of home nursing, to value each country in itself and consider what these differences in health care systems and national contexts mean for the provision of home care and the profession of home nursing.

Also, several quantitative studies exist that judge different health care systems by developing indicators or frameworks to measure their quality or performance. For example, the Netherlands Institute for Health Services Research NIVEL regularly performs such studies, and bases its comparisons on detailed empirical data of each country, often gathered via key informants. The wHo has also undertaken a large-scale comparison of health systems in the world in the World Health Report 2000 (wHO, 2000). It is however difficult to compare health care systems that are essentially different.

In the wHo report, the performance of the health systems of I9I countries in the world is assessed by using a framework assessing three fundamental goals of 
health systems; namely I) improving health; 2) enhancing responsiveness to the expectations of the population; and 3) assuring fairness of financial contribution (wHo, 2000 chapter 2; Murray and Frenk, I999, 2000). Regarding the first goal, the wHo considers the average levels of population health as well as inequalities in the distribution of health among the population important.

Regarding the second goals, responsiveness of the health system, the degree to which a population's expectations are met are examined, in the sense of how clients should be treated by providers of prevention, care or other services. The third goal is fair financial contribution. According to the wно, a fairly financed system ensures financial protection for everyone (wHo, 2000 p. 35). Risks should be distributed according to ability to pay rather than the risk of illness.

The outcomes of the comparisons performed by the wHo show that it is extremely difficult to compare health care systems by using quantitative calculations and developing indicators to judge the quality of their performance. This can be illustrated by the ranks given by the wHo to the four countries studied in this thesis. For example, the overall performance of the French health system is ranked first of I9I countries in the world and ranked fourth on performance on the level of health (wHo, 2000 p. I53). This high ranking is explained by the high life expectancy and high health care expenditures (Navarro, 2000). However, the performance of the French health system regarding accessibility and equal distribution of care can be severely questioned, especially regarding community care services, as will be described in the chapter on France in this book. Other south European countries with high life expectancy rates, such as Italy and Spain, were also ranked high, namely second and third of the igi countries in the world. However, these health care systems have seldom been considered as models of efficiency and effectiveness before. For example in Spain, the presentation of the wHo-report paradoxically coincided with demonstrations against the health care authorities against the 'intolerable' situation in the health care system (Navarro, 2000). So the wно should investigate how the first indicator should be changed in order to get more representative outcomes.

According to the same report, Denmark has the worst health care system in the EU. It ranks as low as 34th on overall health system performance and 65 th on performance on the level of health. However, many other sources state that the Danish health care system functions very well. Danish health care is characterised by a high level of services that are affordable for the entire population, and a broad range of well-distributed facilities that are of a very high standard compared to most other European countries (Zee, I996). Also, the equity of the Danish system is high in terms of access to general practitioners, hospital care, and different forms of community care. National surveys point to a high level of satisfaction with the health care system by the population (Vallgårda, 2002). One of the reasons for the low rankings of the Danish health care system could be that life expectancy did 
not increase as much as in other western European countries in the last decennia, but this is not sufficient to explain the bad overall ranking. ${ }^{3}$ This is also due to factors external to the health care system, among others to the drinking and smoking behaviour of the Danish population resulting in higher morbidity and mortality at earlier stages in life.

The Dutch health care system is also ranked quite low of the European countries, namely I7th on overall health system performance and igth on performance on the level of health. Germany is ranked 25th and 4Ist respectively, but arguments for these ratings and the differences between these are not explained. The wно does not evaluate, discuss or comment on the rankings of the countries at all and does not relate the outcomes to other sources. This can be considered a major drawback of the 2000 World Health Report.

The wHo report has met criticism in the literature (Vallgårda, 2002; Navarro, 2000; Williams, 200I). Namely, the wHo ratings are for an important part based on the assumptions that the health status of the population is largely to be measured in terms of (disability-adjusted) life expectancy and mortality rates (Vallgårda, 2002 p. I), and that health care activities are very effective in reducing mortality and morbidity. Higher investments in health care per person would then drastically diminish the disease burden in the world. Possibly, for a rough insight into differences in health systems between well-developed North or North-West European countries and former East European countries, South American or African countries, the wHo framework and calculations work reasonably well. However, for comparing countries that are geographically close, the comparison leads to strange results. One of the main points of criticism on the wHo report is that figures on health care expenditure, high life expectancy and mortality rates are poor criteria to measure the quality and performance of health care systems and effectiveness of medical activities, because many other factors also influence mortality rates (Navarro, 2000; Williams, 200I). The wно largely ignores the influence of social, cultural, economic and political circumstances and social protection schemes in a country on health and disease. Also, many health care activities are not so much aimed at prolonging life and ensuring a disability-free life, but at improving the quality of life and caring and supporting people to maintain their everyday activities. The quality and performance of these activities is however not taken into account in the wHo report.

This thesis is based on the assumption that an international comparison of health care systems needs to be based on other instruments and criteria and differ-

3 Between 1970 and 1996 it only increased with 2 years for men, which is low compared to other north European countries, e.g. 4.4 years in Norway and 4.3 years in Sweden. However, since I995, average life expectancy has increased faster than elsewhere with another I.7 years. 
ent kinds of data. Therefore, health care systems and the performance of subsectors of health care focussing on the vulnerable and elderly, such as home nursing, home help, and informal care, are studied within the national socio-cultural and political contexts, as these contexts profoundly influence health care structures and can explain international differences. This can lead to different descriptions per country, in which the country-specific background is taken into account.

\section{Appendices: \\ Demographic data and figures on health systems and professionals}

Table 1 Demographic data - total area and population characteristics

\begin{tabular}{|c|c|c|c|c|}
\hline & Denmark & France & Germany & Netherlands \\
\hline Total area $\left(\mathrm{kms}^{2}\right)$ & $43098^{b}$ & $632834^{b}$ & $357027^{b}$ & $\begin{array}{r}41526^{b} \\
\text { (land area: } 33.783 \text { ) }\end{array}$ \\
\hline Total population (millions, 2006) & $5.4^{b}$ & $62.9^{b}$ & $82.5^{b}$ & $16.3^{b}$ \\
\hline Share of the EU-25 total population & $1.2 \%$ & $13.5 \%$ & $17.9 \%$ & $3.5 \%$ \\
\hline $\begin{array}{l}\text { Population density } \\
\text { (persons/kms², 2004) }\end{array}$ & $125^{b}$ & $80(2003)^{b}$ & $231^{b}$ & $482^{b}$ \\
\hline Urban population & $85 \%^{a}$ & $75 \%^{a}$ & $87 \%^{a}$ & $89 \%^{a}$ \\
\hline $\begin{array}{l}\text { Population of the capital } \\
\text { (millions) }\end{array}$ & Copenhagen $1.3^{\mathrm{g}}$ & île de France $11.3^{b}$ & $\begin{array}{r}\text { Berlin } 3.4^{b} \\
(\text { Essen: } 6.5)^{g}\end{array}$ & $\begin{array}{r}\text { Amsterdam } 1.1^{\mathrm{g}} \\
(\mathrm{N}+\mathrm{S} \text { Holland } 6.0)^{\mathrm{b}}\end{array}$ \\
\hline $\begin{array}{l}\text { Projected population } 2025 \text { and } 2050 \\
\text { (millions) }{ }^{1}\end{array}$ & $5.6 / 5.4^{b}$ & $61.7 /-^{c}$ & $82.1 / 74.6^{b}$ & $17.4 / 17.4^{b}$ \\
\hline $\begin{array}{l}\text { Annual population growth rate } \\
(1995-2000)\end{array}$ & $0.3 \%^{a}$ & $0.4 \%{ }^{a}$ & $0.1 \%{ }^{a}$ & $0.4 \%^{\mathrm{a}}$ \\
\hline $\begin{array}{l}\text { Natural population change } \\
(x>000,2005)^{2}\end{array}$ & $9^{b}$ & $275^{b}$ & $-144^{b}$ & $52^{b}$ \\
\hline Population change $(x>000,2005)^{3}$ & $16^{b}$ & $368^{b}$ & $-63^{b}$ & $29^{b}$ \\
\hline$\%$ of non-nationals 4 & $4 \cdot 7^{\mathrm{d}, \mathrm{e}}$ & $11.0^{\mathrm{h}}$ & $9.0^{\mathrm{d}, \mathrm{f}}$ & $4.3^{\mathrm{d}, \mathrm{e}}$ \\
\hline \multicolumn{5}{|l|}{ Asylum applications ${ }^{b}$} \\
\hline 1995 & 5100 & 20400 & 127900 & 29300 \\
\hline 2000 & 10300 & 38700 & 78600 & 43900 \\
\hline 2005 (provisional number) & 2100 & 42600 & 28900 & 12300 \\
\hline
\end{tabular}

Sources: a. United Nations, 2000 p. 21, 55, 69, 75, 135, 197; b. Eurostat, 2007a; c. UNFPA, 2000 p. 85-86; d. Eurostat, 1999 p. 237-261; e. 1998; f. 1997; g. 1995; h. 1982.

1 Projected population data need to be interpreted with caution: different institutes and different estimate scenarios present different figures.

2 Natural increase or decrease: the difference between the number of live births and the number of deaths in the year.

3 Sum of the natural increase and net migration.

4 Countries differ in the way they produce migration statistics and who they consider to be a migrant or asylum seeker. The data should be interpreted with caution, and variation in data sources and definitions result in problems when comparing. 
Table 2 Demographic data - Age groups and economic activity

\begin{tabular}{|c|c|c|c|c|c|}
\hline Population & $E U$ & Denmark & France & Germany & Netherlands \\
\hline Aged 0-14 (1998, x 1000$)$ & & $952^{b}$ & $11180^{b}$ & $13098^{b}$ & $2883^{b}$ \\
\hline Aged 0-64 (1998, x 1000$)$ & & $4503^{b}$ & $49556^{b}$ & $69091^{b}$ & $13545^{b}$ \\
\hline Aged 65+ $(1998, \times 1000)$ & & $792^{b}$ & $917^{b}$ & $12966^{b}$ & $2110^{b}$ \\
\hline Aged $80+(1998, x>1000)$ & & $207^{b}$ & $2197^{b}$ & $3028^{b}$ & $493^{b}$ \\
\hline \% aged 0-14 (2005) & $16.5^{f}$ & $18.8^{\mathrm{g}}$ & $18.5^{g}$ & $14.5^{g}$ & $18.5^{\mathrm{g}}$ \\
\hline$\%$ aged $0-64$ (2005) & & $84.9^{g}$ & $83.6^{\mathrm{g}}$ & $81.4^{g}$ & $86.0^{\circ}$ \\
\hline$\%$ aged $65+(2005)$ & $16.5^{f}$ & $15.0^{g}$ & $16.4^{g}$ & $18.6^{g}$ & $14.0^{g}$ \\
\hline$\%$ aged $65-79(2005)$ & $12.5^{f}$ & $10.9^{g}$ & $11.9^{g}$ & $14 \cdot 3^{g}$ & $10.5^{g}$ \\
\hline$\%$ aged $75+$ & & $7.0^{e}$ & $6.6^{\mathrm{e}}$ & $6.5^{\mathrm{e}}$ & $5.7^{\mathrm{e}}$ \\
\hline $\begin{array}{l}\% \text { aged } 80+(2005) \\
=. . \% \text { of } 65+\end{array}$ & & $\begin{array}{r}4.1^{\mathrm{g}} \\
25.7^{\mathrm{a}, \mathrm{d}}\end{array}$ & $\begin{array}{r}4.5^{g} \\
26.6^{a, c}\end{array}$ & $\begin{array}{r}4 \cdot 3^{\mathrm{g}} \\
25 \cdot 2^{\mathrm{a}, \mathrm{c}}\end{array}$ & $\begin{array}{r}3.5^{\mathrm{g}} \\
23.1^{\mathrm{a}, \mathrm{c}}\end{array}$ \\
\hline$\%$ aged $60+(2000$, men / women $)$ & & $\begin{array}{l}17 / \\
23^{\mathrm{e}}\end{array}$ & $\begin{array}{l}18 / \\
23^{\mathrm{e}}\end{array}$ & $\begin{array}{l}19 / \\
26^{\mathrm{e}}\end{array}$ & $\begin{array}{r}16 / \\
21^{\mathrm{e}}\end{array}$ \\
\hline Women per 100 men (2005) & $105^{g}$ & $102^{g}$ & $106^{g}$ & $104.4^{\mathrm{g}}$ & $102^{g}$ \\
\hline $\begin{array}{l}\text { Life expectancy (in 2004) } \\
\text { (years, men / women) }\end{array}$ & $75 / 80^{g}$ & $75 / 79^{f}$ & $77 / 84^{g}$ & $76 / 81^{g}$ & $76 / 81^{g}$ \\
\hline Total fertility rate (2005) $^{\prime}$ & $1.5^{\mathrm{g}}$ & $1.8^{\mathrm{g}}$ & $1.9^{g}$ & $1.4^{\mathrm{g}}$ & $1.7^{8}$ \\
\hline $\begin{array}{l}\text { Employment rate } \\
\text { males / females }(2005)^{2}\end{array}$ & $\begin{array}{r}63^{f}(2003) \\
71 / 55\end{array}$ & $\begin{array}{r}76^{g} \\
80 / 72\end{array}$ & $\begin{array}{r}63^{g} \\
68 / 57\end{array}$ & $\begin{array}{r}65^{g} \\
71 / 60\end{array}$ & $\begin{array}{r}73^{g} \\
80 / 66\end{array}$ \\
\hline $\begin{array}{l}\text { \% of persons employed part-time } \\
\text { (total, men/women, 2006) }\end{array}$ & $17^{f}(2003)$ & $\begin{array}{r}24 \\
13 / 36^{g}\end{array}$ & $\begin{array}{r}17 \\
6 / 31^{g}\end{array}$ & $\begin{array}{r}26 \\
9 / 46^{g}\end{array}$ & $\begin{array}{r}46 \\
23 / 75^{g}\end{array}$ \\
\hline $\begin{array}{l}\text { Unemployment rate (\% of the } \\
\text { econom. active pop., 2005) }\end{array}$ & $9^{f}(2004)$ & $4.8^{g}$ & $9.5^{\mathrm{g}}$ & $11.2^{\mathrm{g}}$ & $4.7^{g}$ \\
\hline $\begin{array}{l}\text { Taxes on income, wealth, etc. } \\
\text { (\% of GDP, 2005) }\end{array}$ & $13^{\mathrm{g}}$ & $30.6^{g}$ & $11.4^{g}$ & $10.2^{g}$ & $11.6^{8}$ \\
\hline
\end{tabular}

Sources: a: OECD, 1998 chapter Demographic references; b: Eurostat, 1999; c. 1996; d. 1994; e: United Nations, 2000 p. 21, 55, 69, 75, 135, 197; f. Eurostat, 2005; g. Eurostat $2007 \mathrm{a}$.

1 The mean number of children that would be born alive to a woman during her lifetime if she were to pass through her childbearing years conforming to the fertility rates by age of a given year. It is therefore the completed fertility of a hypothetical generation, computed by add-ing the fertility rates by age for women in a given year (the number of women at each age is assumed to be the same). The total fertility rate is also used to indicate the replacement level fertility; in more developed countries, a rate of 2.1 is considered to be replacement level.

2 Employment rate: percentage of those 15-64 years of aged that are employed. The employed persons are those aged 15-64 over, who during the reference week did any work for pay, profit or family gain for at least one hour, or were not at work but had a job or business from which they were temporarily absent. 
Table 3 Household situation

\begin{tabular}{|c|c|c|c|c|c|}
\hline & & Denmark & France & Germany & Netherlands \\
\hline $\begin{array}{l}\text { \% of households with } \\
\text { children 0-17 (2001) }\end{array}$ & & $27^{d}$ & $31^{d}$ & $25^{d}$ & $28^{d}$ \\
\hline $\begin{array}{l}\text { Average number of } \\
\text { persons per private } \\
\text { household (2003) }\end{array}$ & & $2.2^{d}$ & $2.4^{d}$ & $2.1^{d}$ & $2.3^{d}$ \\
\hline \multirow{6}{*}{$\begin{array}{l}\% \text { of persons living } \\
\text { in private households } \\
(1996)\end{array}$} & $\begin{array}{l}\text { One adult living } \\
\text { alone }\end{array}$ & $17^{c}$ & $13^{c}$ & $16^{c}$ & $14^{c}$ \\
\hline & $\begin{array}{l}\text { Lone-parent with } \\
\text { dependent children }\end{array}$ & $3^{c}$ & $4^{c}$ & $3^{c}$ & $2^{c}$ \\
\hline & $\begin{array}{l}2 \text { adults with } \\
\text { dependent children }\end{array}$ & $36^{c}$ & $43^{c}$ & $34^{c}$ & $38^{c}$ \\
\hline & $\begin{array}{l}3 \text { or more adults } \\
\text { with dependent } \\
\text { children }\end{array}$ & $7^{c}$ & $8^{c}$ & $7^{c}$ & $7^{c}$ \\
\hline & $\begin{array}{l}2 \text { or more adults } \\
\text { without dep. } \\
\text { children }\end{array}$ & $36^{c}$ & $32^{c}$ & $40^{c}$ & $38^{c}$ \\
\hline & Total & 100 & 100 & 100 & 100 \\
\hline $\begin{array}{l}\text { Decrease of the \% of } \\
\text { elderly }(65+) \text { living with } \\
\text { their offspring }\end{array}$ & & $\begin{array}{r}28(70+, 1962)^{b}- \\
4(70+, 1988)^{a}\end{array}$ & $\begin{array}{l}24(1975) \text { - } \\
17(1990)^{\mathrm{a}}\end{array}$ & $\begin{array}{r}14 \\
(W-G e r, 1987)^{a}\end{array}$ & $\begin{array}{r}12(1979)- \\
8(1987)^{\mathrm{a}}\end{array}$ \\
\hline $\begin{array}{l}\% \text { of elderly }(65+) \text { living } \\
\text { alone }\end{array}$ & & $\begin{array}{l}35(70+, 1962)- \\
53(70+, 1992)^{a}\end{array}$ & $\begin{array}{r}22(1962)- \\
28(60+, 1990)^{a}\end{array}$ & $\begin{array}{r}36 \text { (W-Ger, 1975) - } \\
41 \text { (Unif. Ger, 1992) }\end{array}$ & $\begin{array}{l}27(1981)- \\
31(1986)^{\mathrm{a}}\end{array}$ \\
\hline
\end{tabular}

Sources: a: OECD, 1996b; b: Plovsing, 1992 p. 11; c: Eurostat, 2001a p. 84; d: Eurostat, $2007 a$.

Table 4 Chronic conditions and subjective health (\% of $15+$ years, 2001)

\begin{tabular}{|c|c|c|c|c|c|}
\hline & & Denmark & France & Germany & Netherlands \\
\hline \multirow[t]{6}{*}{ Self perceived health } & Very good & 41 & 9 & 8 & 17 \\
\hline & Good & 35 & 49 & 39 & 55 \\
\hline & Fair & 18 & 34 & 34 & 23 \\
\hline & Bad & 5 & 4 & 15 & 4 \\
\hline & Very bad & 1 & 4 & 4 & 1 \\
\hline & Total & 100 & 100 & 100 & 100 \\
\hline \multirow{2}{*}{$\begin{array}{l}\text { Hampered in daily } \\
\text { activities because of } \\
\text { chronic conditions }\end{array}$} & Males & 4.7 & 9.5 & 8.9 & 6.6 \\
\hline & Females & 7.2 & 11.3 & 11.4 & 10.5 \\
\hline
\end{tabular}

Source: Eurostat, 2007a. 
Table 5 Health care professionals

\begin{tabular}{|c|c|c|c|c|c|}
\hline & & Denmark & France & Germany & Netherlands \\
\hline \multirow[t]{2}{*}{$\begin{array}{l}\text { Physicians / doctors licensed to } \\
\text { practice (2004) }\end{array}$} & Absolute number & $\begin{array}{r}21756^{a} \\
(2003)\end{array}$ & & $388201^{a}$ & $56540^{a}$ \\
\hline & per 1000 population & $4.0^{a}$ & & $4.8^{a}$ & $3.5^{\mathrm{a}}$ \\
\hline \multirow[t]{2}{*}{$\begin{array}{l}\text { Economically active physicians / } \\
\text { doctors (2004) }\end{array}$} & Absolute number & $\begin{array}{r}18450^{a} \\
(2003)\end{array}$ & $207736^{a}$ & $306435^{a}$ & \\
\hline & per 1000 population & $\begin{array}{r}3.4^{\mathrm{a}} \\
(2003)\end{array}$ & $3.4^{\mathrm{a}}$ & $3.7^{\mathrm{a}}$ & \\
\hline \multirow{2}{*}{$\begin{array}{l}\text { Practicing physicians or doctors, } \\
\text { seeing patients in a hospital, } \\
\text { practice or elsewhere (GPs and } \\
\text { specialists) }\end{array}$} & $\begin{array}{l}\text { Persons; man-years (FTEs, } \\
\text { different years) }\end{array}$ & $\begin{array}{l}15336^{a} \\
(2003)\end{array}$ & $171807^{d}$ & $\begin{array}{r}279722^{a} \\
(2004)\end{array}$ & $30316^{a}$ (1999) \\
\hline & per 1000 population & $\begin{array}{r}2.9 \\
(2003)\end{array}$ & $3.0^{b, c}$ & $\begin{array}{r}3.4^{a} \\
(2004)\end{array}$ & $\begin{array}{r}1.9^{a} \\
(1999)\end{array}$ \\
\hline \multirow[t]{2}{*}{ GPs } & $\begin{array}{l}\text { Persons, man-years } \\
\text { (FTEs) }\end{array}$ & $3313^{e}$ & $86858^{d}$ & $86389^{d}$ & $6814^{e}$ \\
\hline & per 1000 population & $0.6^{e}$ & $1.5^{d}$ & $1.1^{d}$ & $0.4^{\mathrm{e}}$ \\
\hline \multirow{2}{*}{$\begin{array}{l}\text { Total number of qualified } \\
\text { nursing staff (2003) }\end{array}$} & Number & $59410^{a}$ & $423431^{a}$ & $618518^{a}$ & $226200^{a}$ \\
\hline & per 1000 population & $11.0^{a}$ & $7.1^{a}$ & $7.5^{a}$ & $14.0^{a}$ \\
\hline \multirow{2}{*}{$\begin{array}{l}\text { Total number of nursing } \\
\text { auxiliaries (second level nurses, } \\
\text { 2003) }\end{array}$} & Number & $25543^{a}$ & $438697^{a}$ & $183159^{a}$ & \\
\hline & per 1000 population & $4.7^{\mathrm{a}}$ & $7.4^{a}$ & $2.2^{a}$ & \\
\hline
\end{tabular}

Sources: a: Eurostat, 2007b; b: Eurostat, 2001a p. 71, 73; c: 1999; d: 1996; e: 1995; f: 1994; h: Vallgårda, Krasnik et al., 2001 p. 61. 
Figures Health care professionals - Total number of qualified nursing staff and nursing auxiliaries (per 1000 population, 1996/97-2004)

Total number of qualified nursing staff per 1000 population $(1997-2004)$

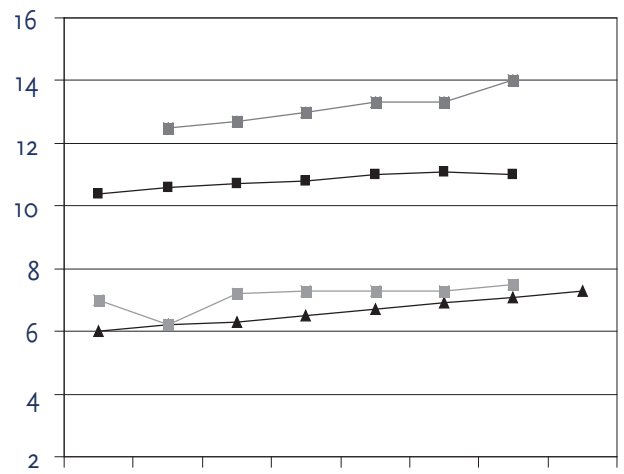

- Denmark

$\rightarrow$ France

$\rightarrow$ - Germany

- Netherlands

$19^{9^{1}}, 9^{9^{0}}, 9^{9^{9}} 2^{0^{0}} \nu^{0^{0}} 2^{0^{0^{2}}} 2^{0^{0^{3}}} 2^{0^{0^{x}}}$

Total number of nursing auxiliaries per 1000 population $(1996$ - 2004)

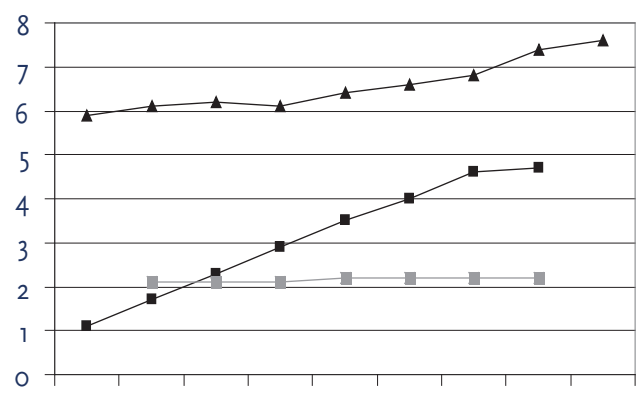

- Denmark

$\neg$ France

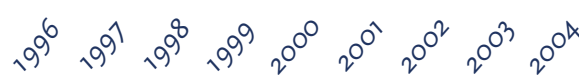


Table 6 Hospital beds

\begin{tabular}{|c|c|c|c|c|}
\hline & Denmark & France & Germany & Netherlands \\
\hline Total number in-patient care beds (2003/04) & $21480^{b}$ & $465758^{b}$ & $707806^{b}$ & $81360^{b}$ \\
\hline Number per 1000 population (2003/04) & $4.0^{a}$ & $7.8^{\mathrm{a}}$ & $8.6^{\mathrm{a}}$ & $5.0^{\mathrm{a}}$ \\
\hline Total number of acute care beds $(2003 / 04)$ & $17692^{b}$ & $234150^{b}$ & $531333^{b}$ & $49986^{b}$ \\
\hline Number per 1000 population (2003/04) & $3.3^{b}$ & $3.9^{b}$ & $6.4^{b}$ & $3.1^{b}$ \\
\hline $\begin{array}{l}\text { Total number of long-term nursing care beds (excl. } \\
\text { psychiatric beds, 2004) }\end{array}$ & & $79989^{b}$ & & \\
\hline Number per 1000 population & & $1.3^{b}$ & & \\
\hline $\begin{array}{l}\text { Total number of other beds (specialty hospitals, etc. } \\
2003 / 04 \text { ) }\end{array}$ & & $92235^{b}$ & $176473^{b}$ & $3086^{b}$ \\
\hline Number per 1000 population (2003/04) & & $1.5^{b}$ & $2.1^{b}$ & $0.2^{b}$ \\
\hline Total number of nursing and residential care beds & & & $\begin{array}{r}645456^{b} \\
(1999)\end{array}$ & $\begin{array}{r}229420^{b} \\
(2001)\end{array}$ \\
\hline Number per 1000 population & & & $\begin{array}{r}7.9^{b} \\
(1999)\end{array}$ & $\begin{array}{l}14.4^{b} \\
(2001)\end{array}$ \\
\hline
\end{tabular}

Sources: a: Eurostat, 2007a; b: Eurostat, 2007b.

Table and Figure 7 Trends in number of hospital beds (1994-2004)

\begin{tabular}{|c|c|c|c|c|}
\hline & Denmark & France & Germany & Netherlands \\
\hline Total number (1994) & & 523242 & 790851 & 83402 \\
\hline per 1000 population & & 9.1 & 9.7 & 5.4 \\
\hline Total number (1996) & & 508075 & 783631 & 81610 \\
\hline per 1000 population & & 8.7 & 9.6 & 5.3 \\
\hline Total number (1998) & 23883 & 508046 & 762596 & 80875 \\
\hline per 1000 population & 4.5 & 8.7 & 9.3 & 5.2 \\
\hline Total number (2000) & & 494464 & 749473 & 82475 \\
\hline per 1000 population & & 8.4 & 9.1 & 5.2 \\
\hline Total number (2002) & 22742 & 480479 & 731919 & 80435 \\
\hline per 1000 population & 4.3 & 8.1 & 8.9 & 5.0 \\
\hline Total number (2004) & 22225 & 465758 & 707806 & \\
\hline per 1000 population & & 7.8 & 7.8 & \\
\hline
\end{tabular}

Source: Eurostat, 2007b. 
Hospital beds per 1000 population (1994 - 2004)

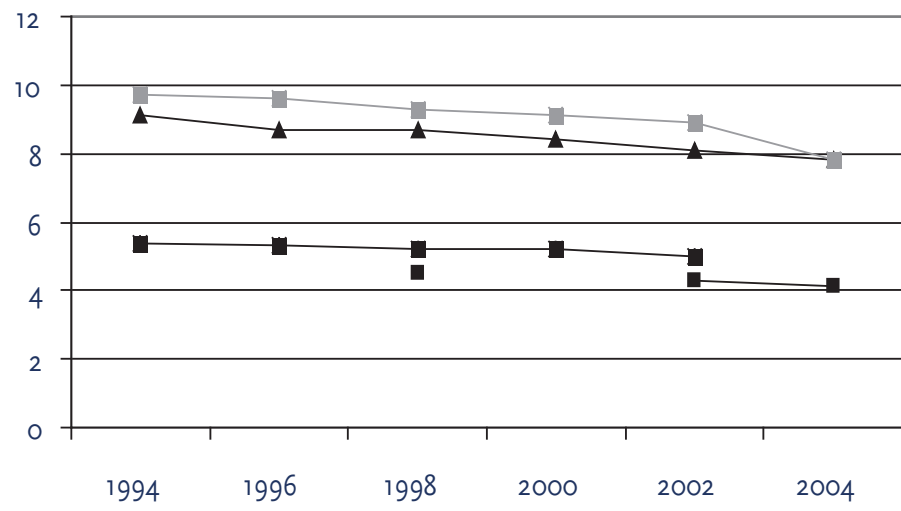

Table 8 Expenditures on social protection (2004)

\begin{tabular}{l|r|r|r|r|r|} 
& Denmark & France & Germany & Netherlands \\
$\begin{array}{l}\text { Total expenditure on social protection } \\
\text { (\% of GDP) }\end{array}$ & 30.7 & 31.2 & 29.5 & 28.5 \\
\hline $\begin{array}{l}\text { Total expenditure on social protection per head of } \\
\text { the population (in Euros) }\end{array}$ & 11200 & 8300 & 7900 & 8600 \\
\hline $\begin{array}{l}\text { Social benefits by function } \\
\text { (\% of total benefits) }{ }^{2}\end{array}$ & Sickness/health care & 20.6 & 30.0 & 27.2 & 30.4 \\
\hline & Disability & 13.9 & 5.8 & 7.7 & 10.9 \\
\hline & Old age & 37.2 & 37.1 & 42.1 & 36.2 \\
\hline & Survivors & 0.0 & 6.5 & 1.4 & 5.4 \\
\hline & Family/children & 13.0 & 8.5 & 10.5 & 4.8 \\
\hline & Unemployment & 9.5 & 7.8 & 8.6 & 6.3 \\
\hline & Housing & 2.4 & 2.9 & 0.8 & 1.3 \\
\hline & Social exclusion, n.e.c. & 3.5 & 1.5 & 1.7 & 4.7 \\
\hline & Total & 100.0 & 100.0 & 100.0 & 100.0 \\
\hline
\end{tabular}

Source: Eurostat, 2007a.

1 Expenditure on social protection contains: Social benefits, which consist of transfers, in cash or in kind, to households and individuals to relieve them of the burden of a defined set of risks or needs; Administration costs, which represent the costs charged to the scheme for its management and administration.

2 Social benefits consist of transfers, in cash or in kind, by social protection schemes to households and individuals to relieve them of the burden of a defined set of risks or needs. 
Table 9 Contribution on social protection by different stakeholders, 2004

\begin{tabular}{|l|r|r|r|r|}
\hline & Denmark & France & Germany & Netherlands \\
\hline Employers' social contribution & 10.2 & 45.5 & 36.3 & 34.0 \\
\hline Social contribution paid by the protected persons & 19.6 & 20.6 & 27.6 & 34.7 \\
\hline General government contribution & 63.5 & 30.4 & 34.5 & 19.2 \\
\hline Other & 6.7 & 3.5 & 1.7 & 12.1 \\
\hline Total & 100.0 & 100.0 & 100.0 & 100.0 \\
\hline
\end{tabular}

Source: Eurostat, 2007a.

Table and Figure 10 Trends in total expenditure on health (\% of GDP, 1975-2004)

\begin{tabular}{|l|r|r|r|r|}
\hline & Denmark & France & Germany & Netherlands \\
\hline 1975 & 8.7 & 6.4 & 8.6 & 6.9 \\
\hline 1980 & 8.9 & 7.0 & 8.7 & 7.2 \\
\hline 1985 & 8.5 & 7.9 & 9.0 & 7.1 \\
\hline 1990 & 8.3 & 8.4 & 8.5 & 7.7 \\
\hline 1995 & 8.1 & 9.4 & 10.1 & 8.1 \\
\hline 2000 & 8.3 & 9.2 & 10.3 & 7.9 \\
\hline 2004 & 8.9 & 10.5 & 10.6 & 9.2 \\
\hline
\end{tabular}

Source: OECD, 2007.

Expenditure on health

(\% of GDP, $1975-2004)$

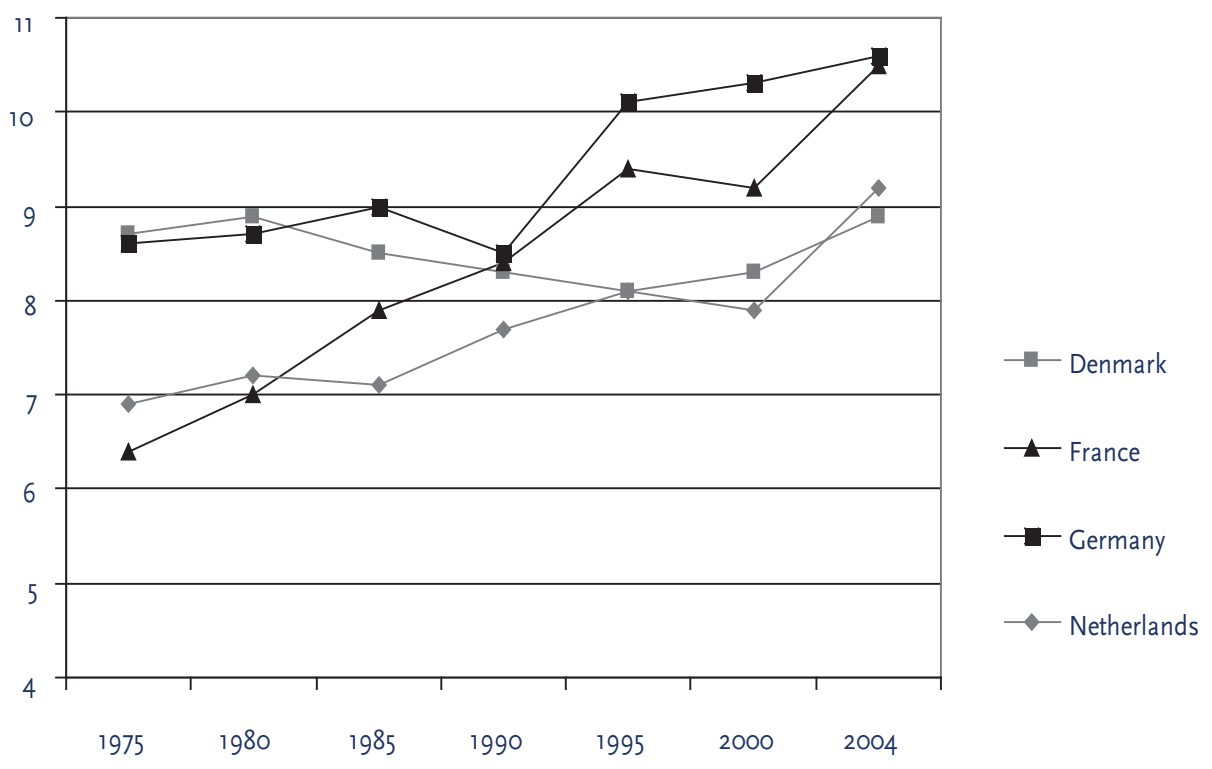


Table and Figure 11 Sources of health care financing (percentages)

\begin{tabular}{|c|c|c|c|c|c|}
\hline \multirow[b]{2}{*}{ Taxation } & \multirow{2}{*}{$\begin{array}{r}\text { Denmark } \\
(1999) \\
82.0^{b}\end{array}$} & \multicolumn{2}{|c|}{$\begin{array}{r}\text { France }^{1} \\
(1995 / 2000)\end{array}$} & \multirow{2}{*}{$\begin{array}{r}\text { Germany } \\
(2002) \\
7.8^{\mathrm{d}}\end{array}$} & \multirow{2}{*}{$\begin{array}{r}\text { Netherlands } \\
\text { (2002) } \\
5.6^{\mathrm{e}}\end{array}$} \\
\hline & & $0.8^{a}$ & $38.7^{c}$ (CSG) & & \\
\hline Social Insurance & - & $\begin{array}{r}73.9^{a} \\
\text { (of which } \\
\text { employers about } \\
63 \text { ) }\end{array}$ & $\begin{array}{r}54.5^{c} \\
\text { (of which } \\
\text { employers 51.1) }\end{array}$ & $67.5^{d 2}$ & $\begin{array}{r}74.5^{\mathrm{e}} \\
\text { (of which } 35.6 \text { is ZFW } \\
\text { and } 38.9 \text { is AWBZ3) e }\end{array}$ \\
\hline $\begin{array}{l}\text { Voluntary / private Health } \\
\text { Insurance (VHI) }\end{array}$ & $1.5^{b}$ & $\begin{array}{r}10.9^{f} \\
\text { (1990, incl. } \\
\text { mutuelles) }\end{array}$ & $12.8^{f}$ & $8.4^{d}$ & $14.3^{\mathrm{e}}$ \\
\hline Out-of-pocket payments & $16.5^{b}$ & $13.9^{a}$ & $10.2^{f}$ & $12.2^{d}$ & $5.8^{e}$ \\
\hline Other & & $-{ }^{a}$ & $6.8^{c}$ & $\begin{array}{r}4.1^{\mathrm{d}} \\
\text { (employer) }\end{array}$ & \\
\hline Total & 100.0 & 99.5 & 113.0 (?) & 99.9 & 100.2 \\
\hline
\end{tabular}

Source: a: Mossialos and Le Grand, 1999b; b: Vallgårda, Krasnik et al., 2001; c: Sandier, Paris et al., 2004; d: Busse and Riesberg, 2004; e: Exter, Hermans et al., 2004; f: Bellanger, 2004.

1 From different sources is does not become completely clear how current figures should be interpreted. All sources mention the existence of $\mathrm{VHI}$ and out-of-pocket insurance but these are not always mentioned in the tables presented. Possibly, this confusion is due to the resent fundamental changes in the health financing in France. Namely, the most striking difference in health insurance funding in France between the 1990 and 2000 is the substitution of most employee payroll contributions by a tax (CSC; Contribution Sociale Géneralisée). Of the 'other' sources of finance, 4.9 of the 6.8 is compensation of the health insurance funds by the state for the loss of contributions because of state policy decisions.

2 Of the $67.5 \%, 56.9$ is statutory health insurance, $7 \%$ is statutory long-term insurance, 1.8 is statutory retirement insurance, and 1.7 is statu-tory accident insurance.

3 ZFW = Sickness Fund Act; AWBZ = Exceptional Medical Expenses Act.

Sources of health finance (percentages)

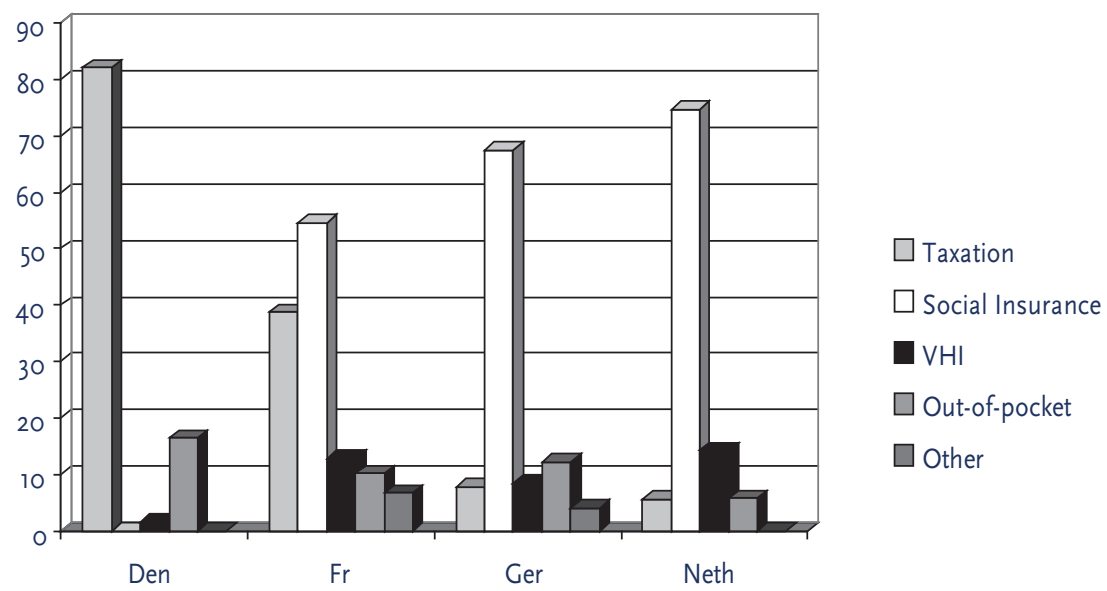




\title{
The home nursing domain in Denmark
}

\author{
- The generalist nurse and community-based welfare state \\ provision of integrated elderly care
}

\section{I Introduction: central issues in Danish health care: the role of the state, professionals and the family}

In Denmark, a dense network of home nursing services exists across the whole country. Equity regarding access to services is an important value of the Danish health care system, also regarding services for elderly people. Compared to other European countries, three aspects regarding the provision of health care are characteristic for Denmark. Firstly, although Denmark has a national health system (NHss; National Health Security System), the provision of health care is strongly decentralised; most services are provided and financed at the local level of counties or municipalities (European Communities and wHo, I997; Vallgårda, Krasnik et al., 200I). The government only provides an overall legal framework and is able to influence the provision of care or the setting of priorities by local governments and councils only indirectly, for example by providing extra earmarked grants to counties to spend on specific health care services, such as specific kinds of surgery of which waiting times need to be diminished. Also, mechanisms have been gradually introduced by which influence on the provision of care can be exerted by the Danish population. For example, one is now free to choose to be treated in the hospital of ones own choice across the whole country. The far-stretching decentralisation and underlying democratic principles of the health care system in Denmark contrasts sharply with the situation in most other European countries, where the national government is more directly regulating the financing and organisation of health care and is also often involved in the provision of health care services, such as in the UK.

A second typical aspect of Denmark is the culturally and politically accepted principle that people do not have a formal obligation to care for elderly or chronically ill people; the general opinion prevails that the welfare state should guarantee that these people are cared for by publicly provided health and social care. This is embedded in the structure of the Danish health care system, in which general accessibility and availability of health care services are main principles. It also results in a higher social status of health professionals compared to other coun- 
tries: as family members, neighbours and friends are not expected to feel obliged to provide informal care to elderly or chronically ill people; state provision of professional services is seen as a right for all and the activities of health professionals are valued more highly than elsewhere (Jamieson, I99Ic). This contrasts sharply with the situation in France, where it is generally accepted that elderly and chronically ill people heavily depend on their family members to provide informal care. French people hesitate to call in professionals, as they experience that as intrusion of strangers into the personal life sphere. ${ }^{\text {I }}$ Whereas the state as well as the population in France consider the provision of professional health care services mainly as a supplement to already existing informal care, opinions in Denmark are almost the reverse; it is generally accepted that offspring does not and should not take a major role in providing care for elderly parents (Jamieson, I99Ic).

The strong reliance on professional care is characteristic of health care provision in the Scandinavian countries and is intimately linked with social-democratic traditions and values, according to which the welfare state takes over direct responsibility of caring for children, the aged, and the helpless. Individual independence is maximised, even when this implies a heavy financial burden to society (EspingAndersen, I990). Especially in Scandinavian countries, social policies are formulated and welfare systems are designed in which policies regarding families are not tied to community care. This contrasts with policies regarding the role of family members in Central Europe, such as Germany and south European countries such as France and Spain, where family care is very important (Giarchi, I996b).

In Denmark, family responsibilities to care and provide assistance are for an important part taken over by professionals working in the public sector, so that citizens can pursue their own careers and personal values. For non-Scandinavian people, the meaning of these cultural values is difficult to grasp. For example, these do not imply that Danish people are indifferent regarding the welfare and well-being of their elderly parents, frail family members, friends or neighbours; it only means that caring and nursing are regarded as activities belonging to the domain and responsibility of health care professionals and that next-of-kin are alleviated from family responsibilities in order to provide for their own family income. For example, in contrast to many other European countries, the availability of family members or neighbours that are able to provide informal care is not assessed in the assignment of home nursing in Denmark; only the presence of a spouse is generally assessed during intake procedures (OECD, I996d). In contrast, in most non-Scandinavian countries in Europe, assumptions that informal carers will provide a substantial amount of care are formalised in assessment procedures and restrictions in the entitlement to and reimbursement of professional care. For example, in Germany,

I See the chapter on home nursing in France in this book. 
the Netherlands and the United Kingdom, the role of the family is intrinsically interwoven in the structure of health care; informal and professional care are intended to complement each other and the availability of informal care is explicitly assessed in the assignment of caring services. In France, the central role and responsibilities of the family are even more strongly entrenched in the health care system and time and again emphasised by the central government. In Scandinavian countries, however, the health care system and health professionals are the first port of call to provide sufficient elderly care. Against this background, it is not surprising that the social status of the caring and nursing professions in the health care system of Scandinavian countries is higher than in other European countries.

Related to this is the third characteristic aspect of Danish health care, namely the affordability of services, which is shared with other Scandinavian countries as well. Tax rates in Denmark are fairly high, among others to afford the comprehensive health care system and welfare state facilities. The standard of living in Denmark is among the highest in Europe; Denmark is one of the ten richest countries in the world in terms of GDP per head (European Communities and wHo, I997 p. 8). In most families, both husband and wife work to provide for the family income; the percentage of women participating in the labour market is considerably higher in Denmark than in other non-Scandinavian countries, namely $72 \%$, compared to 57 to $66 \%$ in the other countries described in this thesis (Eurostat, 2007a). Many women in Denmark work full-time. $46 \%$ of the working population is female, which is the second highest percentage in the Eu after Finland. As much as $67 \%$ of the total working population is working in the service sector, which is a very high figure. The percentage spent on social security is one of the highest in the $\mathrm{EU}$, and much efforts have been undertaken to facilitate the lives of working parents, such as subsidies to day care institutions, extensive maternity leave and parental leave. These figures point out that the social and political choice for a comprehensive welfare state system and public health care system demands for high financial efforts and a high level of labour market participation of the population. The existence of public instead of family responsibilities to provide care and help culturally legitimises the abstinence of the population to care for elderly and dependent people. It fits the social choice to maximise individualism, without leaving elderly people uncared for.

Thus seen from a comparative viewpoint, the most striking characteristics of health care in Denmark are:

I) A comprehensive national health system that is provided by the welfare state and (financially) supported and approved by the population. Equal access to all kinds of health care and social services is a central principle, which is ensured by the local availability of services provided by municipalities (strong decentralisation) and democratic structures levelling financial inequalities; 
2) The socio-cultural and political principle embedded in the health care system that Danish citizens are relieved from family responsibilities to care for elderly, dependent and chronically ill people. Care to vulnerable people is considered as belonging to the domain of the health care system and should be provided by health professionals working in public health care facilities;

3) During recent years, community-based care has a strong priority over institutional care and should preferably be provided in an integrated way.

\subsubsection{Structure of this chapter}

In this chapter, first a description is given of general characteristics of Denmark and its population and the main structure of the health care and welfare system, as these provide the background to understand the structure of the home care domain. The Danish health care system is more familiar to the Scandinavian health care systems than to the health care systems of the other countries described in this book. Especially the role of family members is quite different in Denmark; these do not have such a central role in supporting frail elderly people than elsewhere. The way this is supported by societal and cultural structures and developments is described.

Also, government policies regarding elderly care are described, to gain insight in the quite radical position that has been taken since the early i980s and make it understandable why home nursing and home help are not only provided in the homes of clients, but also in nursing-home like facilities. Then, a more detailed description is given of the way health care and social care services are organised, namely in a highly decentralised way by local municipalities. The principles underlying this system and the way of financing are described to understand the general acceptance of the rather encompassing health care system in Denmark.

Then, a short description of the history of the nursing profession is described, indicating that it is a rather homogeneous profession with a very high degree of organisation compared to other countries. The chapter is finished with a description of the current home nursing profession that can hardly be separated from home help services because of the far-reaching integration of both domains in Denmark.

\subsection{General background and remarkable demographic trends in Denmark}

Denmark is a small country with 5.4 million inhabitants that live rather concentrated in urban areas (85\%). One-third of the population is concentrated in the Copenhagen region that counts I.3 million inhabitants (Eurostat, 2007a). Other cities in Denmark are of medium size. The average population density is low, 


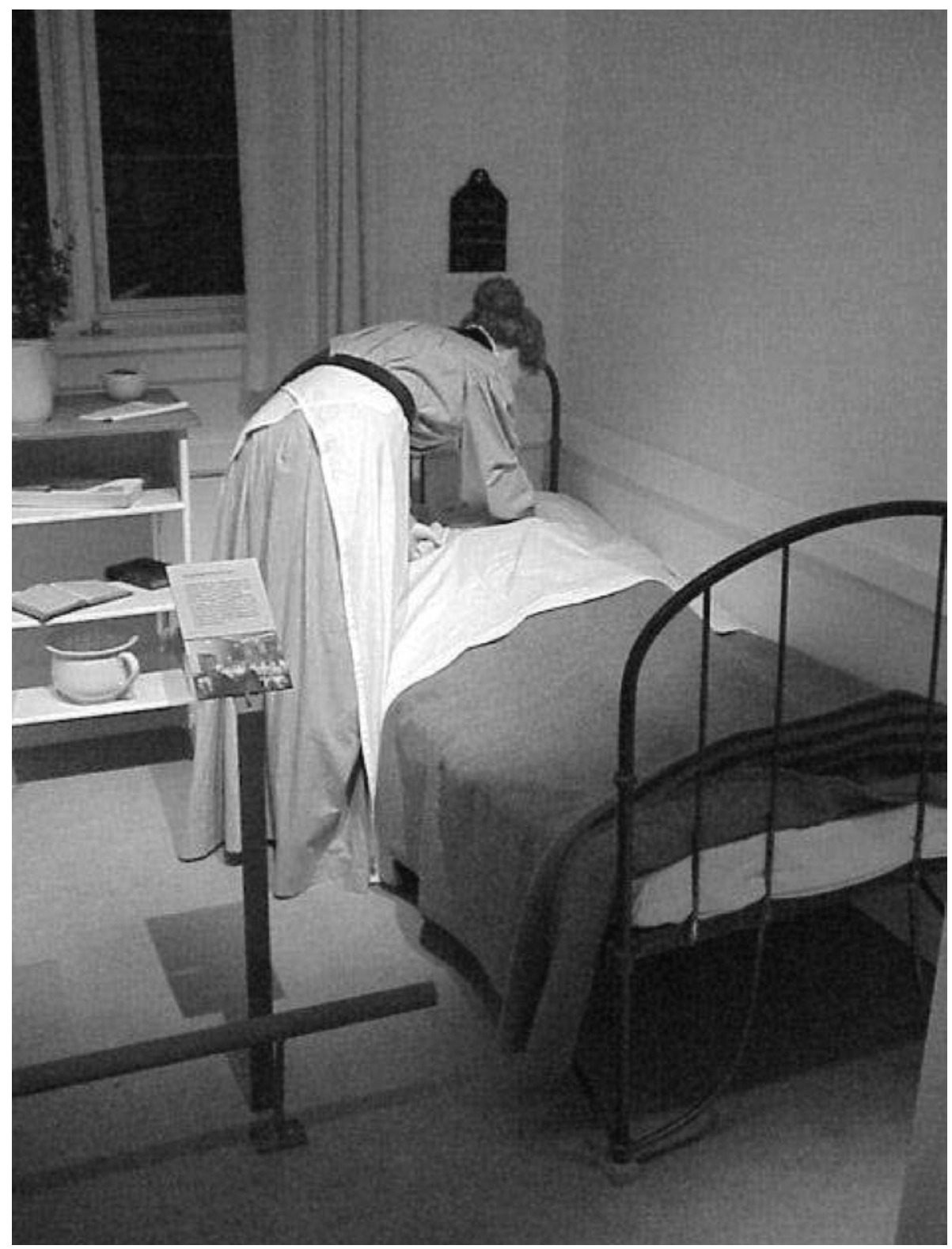

Picture I A Danish home nurse - Scene in the Danish Museum of Nursing History in Kolding, Denmark (Dansk Sygeplejehistorisk Museum, 2004a)

namely I25 people per $\mathrm{km}^{2}$, contrasts quite sharply with the Netherlands, that is about similar in size as Denmark but has three times as many inhabitants (482 per $\mathrm{km}^{2}$ ). Thus apart from the capital city, the country is rather thinly populated. 
The Danish population is quite homogeneous, both ethnically and culturally (Vallgårda, Krasnik et al., 200I p. I-3). Denmark has a modern market economy and a high standard of living. Differences between the rich and the poor are smaller than in many other countries, as redistribution of the wealth of individuals is a main political objective and socially accepted value. The role of the state, especially at the municipal level, and of other public authorities is quite strong regarding the provision and regulatory control of comprehensive services for citizens.

Denmark has a long tradition of public welfare provision and decentralised welfare administration (Ibid., p. Io, 24). In the eighteenth and nineteenth centuries, towns and counties were responsible for poor relief and health care. Although the central state laid down the guiding principles, local authorities carried out most welfare measures, which is currently still the case. Danish welfare politics in general, and health care politics in particular, are characterised by consensus regarding basic institutional structures. Since the Second World War, political parties of all signatures have continued to support the idea that access to health care should be independent of the ability to pay or the place of residence of citizens.

Until the I970s, the medical profession strongly influenced health care politics. The medical profession was very homogeneous, as only one medical school existed in Copenhagen between I838 and I936. Only two other medical schools were established during the twentieth century; namely in Århus in I936 and in Odense in I966. Since I920, almost all Danish physicians have joined the Danish Medical Association DMA that participated in most government committees on health care. Since the I970s, debates were held about health care matters, as political interests in health care increased and the influence of the medical profession declined. Nevertheless, the DMA has been influential for long and the medical profession can be regarded more as part of the state rather than as a policy-making body external to the state. Nurses have been organised since 1899 and have also been represented in government committees (Ibid., p. I2). The proportion of nurses being organised is very high compared to other countries, namely $95 \%$ of them is a member of the Danish Nurses' Organisation DSR (Balslev Wingender, I999 p. I2).

Denmark has been a forerunner in the second demographic transition that is still taking place in many European countries ${ }^{2}$ (European Communities and wHo, I997 p. 6). As a result of rising divorce rates, low marriage rates, and birth rates below replacement level, household size and family structure are changing at a faster rate than in most other European countries. For example the average of $\mathbf{2 . 2}$ persons per household is smallest in the Eu after Sweden. Life expectancy rates also diverge from other European countries and increase less than in other Euro-

2 The first demographic transition refers to the increase of the population because mortality figures dropped below birth figures. 
pean countries. The average life expectancy in the EU is now 75 for men and 80 for women. That of Denmark is 75 and 79, compared to $76 / 77$ for men and 8I/84 for women in France, Germany and the Netherlands (Eurostat, 2005, 2007a). One of the reasons for the lower life expectancy of females is that Danish women have adopted male behaviour patterns since the ig6os. For example, it is the only country in the EU where there is almost no difference in smoking prevalence according to gender (Ibid., p. 25). The life style of women has gradually become almost similar to that of men; Denmark is a very emancipated country. ${ }^{3}$

\subsection{Principles underlying the Danish welfare state and health care system}

In social democratic regimes, such as in Scandinavian countries, principles of universalism and state dominance prevail (Henrard, I996 p. 672). That means that rights, services and benefits are made available to all, irrespective of differences in socio-economic status, and are emancipated from tasks that were traditionally provided by the family. The aim of policies in these kinds of regimes, that are typical for Scandinavian countries, is to maximise individual independence rather than family dependence. This contrasts sharply with policies in many south European countries, where family solidarity is stressed and caregiving by family members is encouraged in state policies.

Since the ig6os, there have been considerable efforts to build an elaborate welfare state system in Denmark, in which social benefits are not only considered as a safety net, but also contribute to the general welfare of the population and aim to reduce economic inequalities among the population. The Danish welfare state is built on the political objective to ensure good living conditions for all groups of the population and to redistribute most of the wealth to society, as in Sweden.

Denmark resembles the other Nordic countries in basing social services on the principles of universalism and high standards of care, meaning that a relatively large proportion of children and older people receive services that are provided in a highly professionalized setting (Rostgaard, I998). Politicians as well as the general population support this. To ensure that services are provided according to need, a local approach to service delivery is chosen. Thus, the municipalities provide services. In contrast to other Nordic countries, however, Denmark favoured services over cash benefits, which is why financial support for informal carers has played a

3 According to Hofstede (I980), Denmark is a feminine country, which makes it easier for women to adopt male lifestyle patterns than in countries with a strong masculine culture, such as many Mediterranean countries (Hofstede, I980e). 
minor role, and the family has not been integrated in the care system as a source of help. Informal care is only of supplementary nature because of the extensive scale of formal care provision. Only during recent years, the large role of spouses in the provision of informal care is gradually receiving more attention, and some support schemes have been set up now.

This contrasts sharply with the situation in the Netherlands, where informal carers are included in the formal caring system as if they were professionals, as is described lengthier in the chapter on the Netherlands in this book. In Denmark, basic care for older people and child day care are primarily regarded as the responsibility of the public domain and public provision, and are only marginally supplemented by informal and voluntary care. ${ }^{4}$ In contrast, in many other European countries, care for elderly people and child care are considered as primarily belonging to the private domain and family members should take up a large part of this care. Only when informal carers are unavailable or unable to provide care, people should turn to professionally provided care. This is especially the case in countries relying on the principle of subsidiarity. The more southern a country in Europe, the stronger the shift in balance between the private and public domains can be recognised. Also, social choices regarding these domains are related to the political regimes of countries: in countries with a conservative tradition, family solidarity regarding children and elderly is stressed, while in countries with a social-democratic tradition, these tasks are generally considered as belonging to the public domain of professionals and the welfare state (Esping-Andersen, I990).

Regarding health and social care provisions, the public sector is by far the most important in Denmark. The principles of the Danish welfare mix consist of:

I) Universal entitlements, as every citizen is entitled to services, social facilities, and social security benefits;

2) Public financing of welfare services by direct and indirect taxes. In Denmark, measures taken by the government are generally considered as the best way to guarantee a high level of economic security and a high level of entitlement to facilities for the entire population;

3) Redistribution by levelling income differences by social payments;

4) Priority setting regarding facilities for children and elderly people;

4 Some provisions have been introduced, for example a care leave introduced in I990, that grants an allowance equivalent to the maximum home help wage, to enable someone to care for a terminally ill person (Rostgaard, I998 p. 93 \& I02). Also, under the Social Service Act that has been implemented in 1998 , older people can choose an informal carer to carry out the domestic part of the home help service. As in other Nordic countries, the municipalities can employ family members as staff in the home help services, but this option is seldom used. 
5) Decentralised provision and financing, and only central governmental control by providing overall legal frameworks;

6) Strong powers to the local authorities, as these are responsible for the implementation of national social policies at the local level.

The Danes are quite proud of their welfare state, although they have to pay a high level of taxes. About 30\% of the GDP is spent on social protection (social welfare and public health) (Plovsing, I992 p. 8; Eurostat, 200Ia). Compared to other European countries in the EC, Denmark spends a larger proportion of the social expenses on social services, especially for the elderly and children, while other countries are using considerably more on cash benefits. Denmark, similar to other Scandinavian countries, has developed its public sector to a high extent; about 30\% of the Danish labour force is employed in the public sector, out of which almost two-thirds are working within the social, health and educational sectors.

Nevertheless, more than 300 voluntary organisations exist, that participate in the provision of care for children and elderly people, and are considered as a supplement to public provision (Rostgaard, I998 p. I03). About 35\% of the national voluntary organisations provide institutional care, and 70\% offer care and help in the periphery, about one-third target on older people. For example, the organisation 'Selvejende Omsorgsorganisationers Sammenslutning', owns one-third of all nursing homes and service housing in Denmark, often those facilities that were established with a Christian background or other ideology. There are also pensioner's organisations, such as the Elder Mobilisation Project, to encourage mutual assistance among older people. The (local and national) governments mostly support these 'help to self-help' organisations. For example, the Social Development Program introduced in I998, made subsidies available for older people and their associations to carry out local initiatives, and the Social Service Act stressed the importance of enforcing the individual influence on social services, and the necessity to create coherence and use early preventive measures (Ibid., p. I35). These principles have encouraged new initiatives, such as the preventive visit that elderly are offered yearly, and the establishment of Senior Citizens Councils. Thus, the voice and influence of elderly people regarding the provision of elderly care and their possibilities for self-determination are considered as very important in Denmark.

\subsection{Characteristic aspects of the Danish welfare state: public, community-based health care arrangements and maximisation of individual independence}

As in all Scandinavian welfare states, people in Denmark are aware that they can count on the state for essential services such as education, health and social care 
and assistance. These are supposed to be of high quality and uniformly available to all citizens, regardless of their socio-economic status. There are several reasons that are specific for Denmark that may explain the strong development of the welfare state and the direct interference of the local government in the provision of public health and social facilities. Firstly, changes in family structures have been considerable in Denmark between the ig6os and the i980s/I990s, and have been more far-reaching than in other European countries, see table I.

Table I Shifts in the household situation of elderly people in four European countries

\begin{tabular}{|c|c|c|c|c|}
\hline \multirow[t]{2}{*}{$\%$ of elderly of $65^{+}$} & \multicolumn{2}{|c|}{ Living alone } & \multicolumn{2}{|c|}{ Living with offspring } \\
\hline & $1960 s$ & $1980 / 90 s$ & $1960 \mathrm{~s}$ & $1980 / 90 s$ \\
\hline Denmark (70+) & 35 & 53 & 28 & 4 \\
\hline France & 22 & 28 & $\begin{array}{c}24 \\
\text { (I975) }\end{array}$ & I7 \\
\hline Germany & $\begin{array}{c}36 \\
\text { (I975, } \\
\text { W-Ger) }\end{array}$ & $\begin{array}{c}4^{\mathrm{I}} \\
\text { (Unif. Ger) }\end{array}$ & - & 14 \\
\hline The Netherlands & $\begin{array}{c}27 \\
\text { (I98I) }\end{array}$ & $3 \mathrm{I}$ & $\begin{array}{c}\mathrm{I} 2 \\
\text { (I979) }\end{array}$ & 8 \\
\hline
\end{tabular}

Sources: Plovsing, I992 p. II; OECD, I996b; Eurostat, 200Ia p. 84; Platz I990.

The table clearly indicates that the proportion of elderly living alone is considerably higher than elsewhere, and Denmark has been a forerunner in this respect: in the I96os, already $35 \%$ of the elderly were living alone. Since then, it has become more and more customary for elderly people to live alone in Denmark: more than half (53\%) of the elderly of 70+ are living alone in the I980s/I990s, compared to 28 to $4 \mathrm{I} \%$ of those of $65+$ in the other countries. Elderly living with their offspring have become rather an exception in Denmark: while in the I960s, about $28 \%$ of the Danish elderly lived with their children, their number has decreased to $4 \%$ in the I980/90s (Plovsing, I992 p. I2; OECD, I996b). Compared to the figures of the other countries, this clearly is a very low percentage.

The low number of elderly cohabitating with their offspring and the high number living alone are both aspects of family life that are closely related to the demand for care, as the largest proportion of professional home care is received by elderly living alone in independent households. Thus, the living arrangements of elderly people since the ig6os are such that they encourage the demand for professional home care. Trends indicate that family structures in other European countries are following this living pattern; home care organisations should be prepared to account for this additional demand for professional care in the near future.

Secondly, a substantially higher percentage of Danish women has been economically active since 1995 , namely $72 \%$, compared to 57 to $66 \%$ of the women 
in other European countries, see table 2. Also, women in Denmark mainly work full-time, while in Germany almost half of the women work part-time (46\%) and in the Netherlands even 75 per cent (Rostgaard and Fridberg, I998b p. Io9; Eurostat, 2007a).

Table 2 Trends in labour force participation by sex, \% of the population (I995-2005)

\begin{tabular}{|c|c|c|c|c|}
\hline & \multicolumn{2}{|c|}{1995} & \multicolumn{2}{|c|}{2005} \\
\hline & Men & Women & Men & Women \\
\hline Denmark & 75 & 62 & 80 & 72 \\
\hline France & 64 & 47 & 68 & 57 \\
\hline Germany & 72 & 48 & $7 \mathrm{I}$ & 60 \\
\hline The Netherlands & 70 & 45 & 80 & 66 \\
\hline
\end{tabular}

Sources: United Nations, 2000; Eurostat, 2005, $2007 \mathrm{a}$.

Especially younger women are participating in the labour market to almost the same extent as men, and in contrast to other European countries, there is no difference between married and unmarried women in Denmark. One of the greatest social changes in the last decennia has been that the phenomenon of the housewife is disappearing in Denmark. In contrast, especially in the Netherlands, the family structure of men providing for the family income $180 \%$ of the men are economically active and $3 / 4$ of them work full-time) while many women choose to remain fully or part-time at home to care for their children is still quite strong, despite an increase in female labour participation and an increase in child care facilities in the last few years (Rostgaard, I998 p. 3I2-3I7). In contrast, in Denmark, policies have aimed at providing sufficient child care and elderly care facilities to enable women to participate in the labour market and to fully or partially relieve them from caring for their children and parents. According to Rostgaard (I998):

'Provision of care for children and older people is generally abundant, ensuring that parents can participate in the labour market and that older people receive the necessary help independent of their family. Having expanded in both volume and relevance as part of the consensus policies which have been the common model for policy making for a number of years, social services now constitute an important element of the Danish welfare model.' (Rostgaard, i998b p. 87)

Especially since the mid-I96os, during the 'second stage of the welfare state' that was characterised by an economic boom and almost full employment, public involvement in the production of services, especially for children and older people, increased. This "paved the way for what has been seen as a new contract between women and the state, whereby the concept of motherhood was renewed, legiti- 
mising women's take up of paid employment." (Siim, I993 in: Rostgaard I998b p. 9I). As most of the informal care for elderly people (apart from spouses) is provided by female family members and especially daughters and daughters-in-law (Dautzenberg, 2000; Potting, 200I) help from family members has become less available in Denmark. Instead of regular help with tasks in the home, help from family members and relatives has become more episodic and advisory in nature, and for a large part concerns help with the organisation of formal care or practical help such as laundry or minor repairs (Rostgaard, p. I36).

Thirdly, there is no legal duty of children to care for their parents in Denmark (Plovsing, I992 p. IO). There only exists an obligation to support one's spouse and children under the age of I8. In Denmark, children primarily help their parents in short term acute situations and play an important role in mobilising public services on behalf of the elderly.

'Informal care given by the family or by organizations through volunteers does not play such a big part in Denmark as the formal care, according to investigations (Platz, I990). In the Danish Welfare State there is a broad consensus about the principles that the elderly have a right to aid from the public, when need arises. The aid is open for all, and it is important to notice the general lack of stigma attached to receiving health and social services. The Danish system of service provision enables the elderly and their relatives to be independent of each other, as help with activities of daily living tends to be obtained as formal care through public services.' (Plovsing, I992 p. I5)

However, although families are thus not formally obliged to care for elderly people, this does not imply that no help is offered to them by family members; it only means that the assistance is voluntary and intended to supplement public provisions. However, this does not imply that older Danish people are without contact with family members and relatives. Although the percentage of elderly $(60+)$ reporting they had daily contact with their children is lower than the EU average (44\%), many of them confirm they had contact with their relatives in the previous weeks. Moreover, the same survey found older Danes to be least lonely: less than $5 \%$ said that they often felt lonely (Walker, I993 in: Rostgaard, I998). Informal care thus plays a less prominent role in Denmark than in most other European countries, where family members are encouraged to provide informal care by health care policies and regulations regarding the entitlement to care. Especially the contrast with France is quite sharp, where the family is the 'natural' and first port of call for help and assistance (Giarchi, I996g p. 207-208).

Fourthly, changes in the health care system and institutional care have increased the need for social services for elderly people, as hospitals are discharging old patients earlier now and places in nursing homes have been reduced consider- 
ably in Denmark (Plovsing, I992 p. I2). As these developments are combined with the political aim to improve the social conditions of elderly, this has led to an increase in social expenses for the elderly (pensions, institutional care, home care and rent subsidies), namely from 8\% of the Danish GDP in I976 to II\% in I989. In this period, expenses on institutional care have decreased considerably, from $23 \%$ in 1976 to $15 \%$ in 1989 , while expenses on home care and rent subsidies have increased relatively slowly, namely from 9\% of GDP in I976 to II\% in I989. When pensions and subsidies are excluded, and focussed is only on care for elderly people, expenditures on home help increased rose from $0.7 \%$ of GDP to I.I\%, as these were expanded in the light of the de-institutionalisation policies, whereas expenditures on nursing homes decreased from I.8\% to 0.8 of GDP (however, as the GDP has risen with about $4 \%$ a year during the I990s, net expenditures on elderly care have increased).

The next diagram (figure I) shows the current distribution of expenditures on the main social services for elderly people $(67+)$ :

Figure I Net-expenditures on main social services for older people $(67+$, I994)

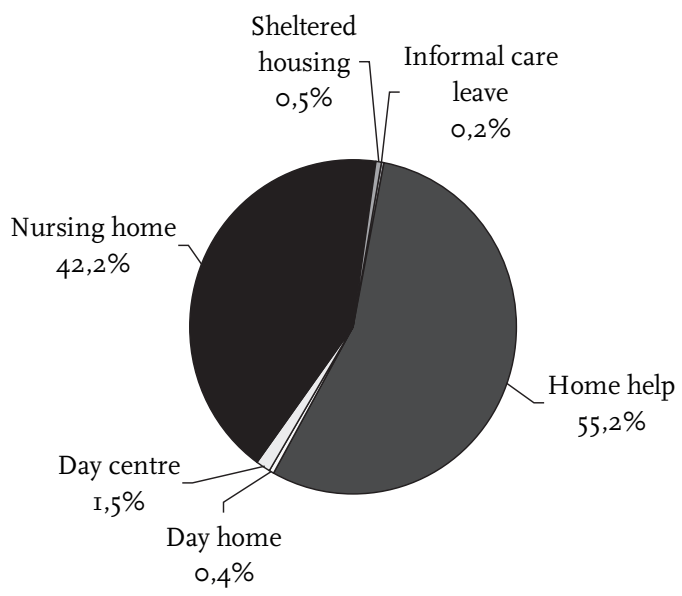

Source: Rostgaard, 1998 p. 97.

Expenditures on leave for informal carers are almost negligible, and also expenditures on day homes, sheltered housing and day centres are very low. A very large part of the costs is still spent on nursing homes (42\%), but this is not surprising as despite substitution policies, a considerable number of elderly (especially those that are very old) are still living in nursing homes in Denmark, and nursing home care is very expensive as it concerns high levels of care. In Denmark, the ratio of staff and nursing home residents is I:I (Rostgaard and Fridberg, I998b; Plovsing, I992 p. I7). The largest proportion of expenditures concerns home help, namely 
$55 \%$. Rostgaard probably means home care consisting of both home nursing and home help, as home nursing is not mentioned separately.

In the next diagram (figure 2), the use of services in percentages of those over 67 years of age is depicted:

Figure 2 Use of main social services for older people $(67+$, I996)

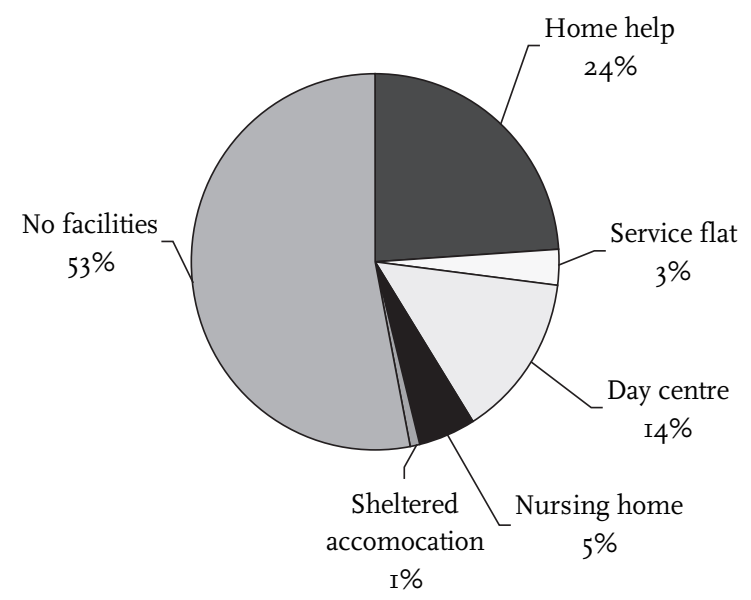

Source: Rostgaard, p. I33.

The figure shows that more than half of the elderly seem to cope without making use of special elderly facilities or social care. Also, although home nursing care is responsible for more than $40 \%$ of the expenditures, this concerns only $5 \%$ of the elderly, while much more use is made of far cheaper facilities such as day centres (I4\%) and home help (i.e. home care, 24\%). Also, subsidised transport schemes are offered, and provisions such as meals-on-wheels, snow clearing, gardening and extra house cleaning. Day homes, accounting for $0.4 \%$ of the expenditures, are available for elderly that remain living at home but are in need for more care than home help can offer, and the care level resembles that of nursing homes. Day homes, however, are more like recreational centres that offer activities such as language courses.

The combination of the four factors described in this paragraph: many elderly people living alone; a high participation of women in the labour market; priority of professional over informal care in the health care system; and emphasis on community-based instead of institutional care; leaves room for a substantial amount of ambulatory care provided by professionals and financed by a public health care system, that is devised by the national government but executed at the local level. The Danish population generally supports the comprehensive welfare state arrangements, by which others than the family should provide care and assistance, espe- 
cially to elderly people and children. These tasks have been made the responsibility of the public sector, where many Danish women find employment.

\subsection{Elderly policies: a radical change from institutional to community-based care}

Danish policies regarding social and health care for elderly people diverge from policies in other countries (Vallgårda, Krasnik et al., 200I p. 56). In most countries, policies aim to reduce institutional care, such as hospital and home nursing care, by care provided in the community, such as home nursing or home help. Policies in Denmark are however going much further than that and can be considered as quite radical. Since the I970s, care policies for older people have mainly aimed at providing care in the home instead of in an institution (Rostgaard and Fridberg, I998b p. I34). In I972, the Social Assistance Act was introduced, which underlined the municipal obligation to organise long-term home help, and which prioritised nursing homes over other forms of institutionalised elderly care, such as residential homes or homes for older and sick people. State subsidies were only given to nursing homes that became reserved for those with the most pronounced needs.

In 1979, the Danish government appointed a National Commission on Ageing, which formulated an elderly philosophy or 'Ageing Package' that was supported by all political parties (Plovsing, I992 p. I3; Ribbe, Ljunggren et al., I997 p. 8). Its principles stated that living conditions of elderly people should be organised as such to enable:

I) Continuity in life; institutionalisation should be prevented when possible and elderly should be able to remain living in their own homes as long as possible;

2) The highest possible self-determination or autonomy, meaning that elderly should be able to choose for themselves how they are taken care of by the possibility of choosing between a number of public services according to their individual needs and wishes;

3) Promoting self-help and the use of own resources, meaning that public aid should primarily be given according to the principle of help-to-self-help, by supporting the activities set up by the elderly and their associations.

From the I950s until the early I970s, large numbers of nursing homes were built, and institutional social and health care expanded considerably, as 'growth without policy' prevailed. However, since the late I970s, this has been curbed into a decrease in the provision of institutional care, especially nursing homes, and a deliberate increase in the provision of home care in accordance with the abovementioned targets. 
In I987, new legislation was introduced, in which a quite radical position was taken (Vallgårda, Krasnik et al., 200I p. 56). According to the Act on Housing for Older and Disabled persons, only independent dwellings should be built, and residents in apartments for elderly people were put on an equal footing with all other elderly people living in their own dwellings and should not resemble institutions (Plovsing, I992 p. I4; Rostgaard and Fridberg, I998b p. 93). The objective of the National Commission on Ageing was that the housing function and service function were separated, and the 'package' of care that was included for residents of nursing homes was abolished. Residents living in special dwellings for elderly people are now able to choose the services they would like to receive, just as noninstitutionalised elderly, and only receive home services when needed, instead of being provided with a fixed package of care. Following this, nursing home residents now also receive the full amount of their pensions (Rostgaard and Fridberg, I998b p. I35).

These so-called 'integrated centres for the old' are part of the policies for the future regarding housing for elderly, and are established in new buildings or re-built former nursing homes. Their form varies, but the idea is that a centre with common facilities is attached to a group of independent elderly apartments, for example with guard, common premises, a cafeteria, bookstall, library and workshops. In this formula, the housing and service function is separated.

Since I987, nursing homes have been considered as ordinary housing, and new nursing homes were no longer allowed to be built (Plovsing, I992 p. I7; Ribbe, Ljunggren et al., I997 p. 8; Vallgårda, Krasnik et al., 200I p. 56). Traditional nursing homes were closed at the rate of about I०\% per year, and home care policies are given a higher priority. Only elderly in such a bad state of health that they cannot remain living in their own homes, not even with social and health assistance offered by the municipality, are admitted. These elderly are entitled to a 'package' of housing facilities and care of a high standard, such as a single room with bathroom facilities, attendance, care, 24-hours a day assistance, nursing, occupational therapy, technical aid and medicine, hair dressing, etc. In addition, there were several kinds of other housing facilities for elderly people before i987, such as sheltered housing, service flats, public flats for pensioners, etc. Since the act however, only so-called apartments for the old are allowed to be built, that should resemble independent dwellings instead of institutions. Security should be part of daily life, and it should be possible for each individual to summon help and care at short notice from the apartment in case of illness or accidents. However, a large problem is the insufficient number of the 'apartments for the old'. In many municipalities, there are large waiting lists for these apartments and for admission in nursing homes.

As a result of these policies, the number of people in nursing homes has decreased considerably since I987. Protected housing schemes now provide 
services according to the individual's needs. Formerly, each nursing home was assigned its own physician, treating all the inhabitants. Nowadays, however, inhabitants of nursing home-like facilities are individually registered with a GP. This development was accompanied by a large increase in the number of home nurses and home helps employed by municipalities. Because of the many technological improvements, provision of acute assistance both day and night became possible; thus many home care organisations now provide 24 -hours home care. ${ }^{5}$ Thus, as a result of deliberate political decisions that initially tried to abolish the existence of nursing home facilities in Denmark, the nursing home sector has diminished considerably in size. In I982, $6.7 \%$ of the elderly of 67 years and older were living in nursing homes (about 43500 people) and in I987 6.I\% (4I 400 people), which further decreased to $4.6 \%$ in I996 (32 0oo people of $67+$ ) (Rostgaard and Fridberg, I998b p. I49-I50). Especially the number of very old people that are living in a nursing home decreased considerably: in I982, still $20 \%$ of those of 87 years and older were living in a nursing home, compared to $18 \%$ in I987 and only II.9\% in I996. It is advocated that elderly remain living in their own homes when possible, supported by professional help when needed, and live in apartments that are specially adapted for elderly (where help is also offered). An important measure that ensures that elderly can remain in their own homes is the pensioners' rent subsidies (Plovsing, I992 p. I9), entailing that pensioners only pay $15 \%$ of their income on rent, if they do not have supplementary income to the old age pension paid by the Danish state. The state finances $75 \%$ of this subsidy, the municipalities $25 \%$.

However, despite the fairly radical de-institutionalisation policies a comparison between countries shows that the percentage of Danish elderly residing in nursing homes is still rather substantial, namely 4\% (Ribbe, Ljunggren et al., I997 p. 6). The number of elderly living in institutions is still more than $15 \%$ in Denmark, compared to Io in the Netherlands, and only 4 to $6 \%$ in France (when hospitals providing long-term are included).

5 Also in the Netherlands, many home care organisations offer 24-hour assistance; many have extended their services after the merger of home help and home care organisations in the I99os. 


\subsection{The overall health care system}

\subsubsection{Decentralised execution of the National Health Security System: decision making by local authorities}

Denmark has a National Health Security System (NHSS) that is publicly financed and strongly decentralised. The Ministry of Health is the principal authority regarding the health system and is responsible for the general legislative framework (European Communities and wHo, I997 p. 37). The National Board of Health, falling under the Ministry, has an administrative, advisory and supervisory role. The execution of health care services is however strongly decentralised since i970; most administrative powers and governmental responsibilities are delegated to the local authorities, as the state aimed at bringing decision making closer to the people. The way in which the health sector should be organised and which services should be provided is not specified in national legislation. Thus, county and municipal authorities have great independence regarding decision making and design of their health and social policies and rather free to prioritise their expenditures (Vallgårda, Krasnik et al., 200 p. I6). They are only limited by governmental legal regulations that ensure the social rights of citizens and governmental limits on expenses (Plovsing, ı992 p. I6).

In I970, major reforms were implemented in Denmark's public administrative structure by reducing the number of counties from 25 to I4 and the number of municipalities from over I300 to 275 , to ensure that they would be sufficient in size and capacity to handle welfare arrangements. This contrasts sharply with France, where many of the 36000 communes are too small to guarantee the provision of sufficient services (Kemenade, I997e p. 59). The I6 authorities in Denmark (I4 counties and the cities of Copenhagen and Fredriksberg) and the communities fall under the authority of the Ministry of Internal Affairs and are responsible for the provision and financing of health care, social services, infrastructure and education. Their independence is established in the Constitution (Vallgårda, Krasnik et al., 200I p. 5), and they are granted the right to collect taxes in order to finance their activities. Most counties and municipalities have a committee on health and social affairs that is responsible for their tasks under the Public Health Insurance Act, and a hospital committee responsible for running the hospitals. Sometimes these committees are merged into a health committee. Both receive part of the total annual health care budget of the counties or municipalities.

The counties own and run hospitals and prenatal centres, and finance GPS, specialists, physiotherapists, dentists, and pharmaceuticals through the National Health Security System (N Hss) (Vallgårda, Krasnik et al., 200I p. 20). Health care accounts for about $70 \%$ of the county council's budget. Most hospitals, namely $90 \%$, are publicly owned by the counties. Each county has at least one central hos- 
pital and a number of minor hospitals. There are only roo to 200 hospital beds in private for-profit hospitals, which is less than I\% of the total number (Ibid., p. 55). Since October I992, patients with a medical referral for hospital care are free to choose for a hospital of the suitable level of specialisation in the whole of Denmark. The county the patient is residing should pay this. It is expected that this will result into increased cooperation between hospitals across the country, more similarity in availability of medical technologies and specific diagnostic and curative activities, and a decrease in differences in waiting times.

The municipalities are responsible for health services such as nursing homes, home nurses, health visitors, municipal dentists and school health services, and social services including social welfare allowances, care for vulnerable elderly, handicapped and chronically ill people, and community mental health centres. When local care is not available temporarily and people can for example not be discharged from hospital, then municipalities have to pay the extra hospital costs. This principle encourages them to provide sufficient services.

Although officially, there are no conditions regarding who provides the services, public provision by health professionals has a strong tradition in Denmark is generally considered as being the cornerstone of the welfare state in relation to basic problems, whereas in the Netherlands the family is considered as the cornerstone of society and of health care provision. This difference clearly results in different choices regarding the structure and financing of the health care system. Thus, most of the services in Denmark are publicly provided and the municipal health authorities primarily employ health professionals. The National Association of Local Authorities and the different professional organisations negotiate on their fixed salaries and working conditions (Vallgårda, Krasnik et al., 20oI). Private provision by non-profit, independent (selvejende) providers does exist; these negotiate with one or several municipalities to provide their services on a contract basis. The municipality reimburses their net running costs. Despite modernisation strategies of the non-socialist government regarding welfare state arrangements in the I980s, the traditional ideological opposition to private for-profit organisations providing core social services still remains strong. During the I980s and I990s, the concepts market provision and privatisation of social services were put on the political agenda several times as being more economical, effective, flexible and humane, compared to the generally 'wasteful, ineffective, bureaucratic and possessive public provision' (according to the non-socialist government). This image was however rejected again and again by the Social Democratic Party that feared a decrease in the quality of care and increase of social inequality. The I998 Social Service Act facilitated private, for-profit provision of care by allowing that a nonpublic provider could perform the cleaning tasks of home care, but most users are reluctant to choose for a private provider. 
The decentralised system is designed to ensure that sufficient services are provided locally, because municipalities have insight in the needs of the local population and have the responsibility to finance and provide the services needed. Decentralisation makes coordination of services easier, as individual municipalities are responsible for financing and provision of all local home care and institutional care. However, local variations in health and social care provided exist because of differences in resource allocation and prioritisation between municipalities; some have very elaborate services of high quality, others provide services of a lower standard (Vallgårda, Krasnik et al., 200I p. 25-29; Plovsing, I992 p. I6).

Although policy-making and decision-making in Danish health care are delegated to local levels to an extent that is incomparable to most other European countries, the state does exert control over the health care sector. For example, as all education of authorised health professionals, except chiropractors, is public, the government controls the number of health professionals and the content of their training and qualifications (Vallgårda, Krasnik et al., 200I p. 23). Also, the annual national budget negotiation is increasingly used by the central government to influence the development of the health sector and to set the overall economic framework, for example by highlighting priority areas and giving earmarked grants to municipalities to reduce waiting lists of specific treatments. Also, the government tries to influence the provision of care by the counties and their way of setting priorities by guaranteeing for example waiting times and demanding that the counties provide certain treatments. The counties frequently express dissatisfaction with these procedures, as it reduces their autonomy and conflicts with the fundamental principle of decentralised health care in Denmark. Thus, despite the decentralised financing and provision of health care since I970, the state still (and increasingly) desires to intervene in the development of health care.

\subsubsection{The development of the Danish National Health Security System}

The Danish health system is cherished by the population as one of the most public systems in Europe. There is hardly any market competition compared to other European countries; competitive structures are rather rudimentary and these have no priority in Danish politics, as competition is not considered as a desirable principle in health care provision. This is shared with other Scandinavian countries.

Health insurance gradually developed since the second half of the nineteenth century, when funds were established by guilds to provide their members with financial assistance (Vallgårda, Krasnik et al., 200I p. I2). Artisans and other groups followed by setting up funds to prevent workers becoming dependent on poor relief in case of ill health. In 1892 , an act was passed ensuring that the state would subsidise insurance schemes, although it was feared that these would reduce philanthropic support (Vallgårda, 200I). Initially, the majority of health insurance 
scheme members came from the lower classes. In I900, only $20 \%$ of the population was covered. In I929, the Radical Liberal government was replaced by a Social Democratic and Liberal Democratic coalition government, that held power until I940 and established a tradition of public welfare provision by breaking with the previous periods of non-intervention. The structure of the welfare system was however criticised for its complexity, inefficiency and lack of clarity. The number of people covered increased from $42 \%$ in I942 and 90\% in I973. By this time, contributions could almost be considered as a universal tax. As the Social Democratic government preferred a tax-based system, the insurance schemes were abolished and replaced in I973 by a more homogeneous national health system covering the whole population and guaranteeing equal access to benefits.

Regarding elderly care, Denmark has a long tradition. As early as I889, a specific policy for older people was formulated: the Old Age Assistance (Alderdomsunderstøttelse) that guaranteed income or services in kind to those of 60 years and older (Rostgaard and Fridberg, I998b p. 89). Municipalities were obliged to establish asylums for poor older people, that were mostly set up in poor-houses and work-houses, where residents had to conform to institutional life and give up most of their independence and earn their living through work, even the very old. Only older people that were able to remain in their own homes 'escaped' from institutionalisation and could receive rent support, meals, fuel or cash. The Assistance policy intended to greatly improve the social standards of elderly people, but most of the harsh living conditions were maintained. In I922, old age assistance was reformed, and a regular old age pension was introduced for those of $65^{+}$, and new types of old age homes were built, with no strict working conditions accompanying residence anymore. Facilities in Denmark went much further than elsewhere; in larger cities, special town areas were even built for very old people needing medical care, such as 'The Old People's Town' in Copenhagen and Århus. After the Second World War, facilities diversified. Many elderly people were able to live independently, while the number of very old people in need of care and support increased as life expectancy increased. Thus, old age homes were gradually transformed into nursing homes with higher levels of care, and expanded in number in the I950s and I960s. Their standard was generally very high compared to that in other countries, as most of them had single rooms with an individual bathroom, and residents were invited to participate in decision-making. Public financing of child and elderly care facilities increased (Rostgaard and Fridberg, I998b p. 90). After the introduction of the national health system, the state required that municipalities with more than 4000 inhabitants should build residential homes and establish individual old age housing (Aldersrenteboliger) for retired elderly people. This was heavily subsidised by the government.

In I973 and again in I979/80, cuts in expenditures were necessary due to economic problems, and the comprehensiveness of the social welfare system, that is 
so characteristic for Nordic health care systems, was questioned (Rostgaard and Fridberg, I998b p. 92). It was no longer considered legitimate to endlessly provide more resources for welfare problems. During the ig80s, the non-socialist Danish government stated that the ideology of the welfare state had to be changed, as the system proved to be too costly and ineffective (Rostgaard, I998 p. 92), and the objective was that public expenses should not increase (Plovsing, I992 p. 2I). The already existing policy of de-institutionalisation of handicapped and elderly people was further encouraged, as well as the use of private resources in day care for children and elderly facilities. However, provision remained public, as privatisation of services has never been an option in Denmark.

Since the start of the National Health Security System in 1973, the system is characterised by the absence of major reforms (Christiansen, Enemark et al., I999). Compared to other Nordic countries, the 'modernisation of the welfare state' was taken up by introducing some gradual adaptations (Rostgaard, I998 p. 94-95). For example, some cost-containment measures were taken: GPS now act as gatekeepers to hospitals and specialist treatment, and patients should be treated at the lowest level of care (cost-wise) at which effective treatment is possible. A cooperative initiative between the state government and the local authorities has produced annual budgets that ensure an overall ceiling on expenditures of the municipalities and counties. Also, several measures have been taken to limit the costs of pharmaceutical products used by patients and discourage medical consumption of specialist and hospital care. Characteristic of Denmark is that the expansion of services continued, but now against a backdrop of tighter economic means, better targeting of resources and focus on development of quality, thereby taking limited financial resources into account. Time and again, discussions are held about introducing co-payments for home help, to keep up with the increasing demand for care. This is however opposed to by a large part of the population, as it breaks with the principle of universal access and free or only moderate co-payments for services.

Currently, the Danes have to choose between two health plans (European Communities and wHo, I997 p. 38; Vallgårda, Krasnik et al., 200I p. 29). About 98\% of the population is belonging to Group I, which provides free physician and hospital care, but limits access to care by assigning each person to a local GP who acts as a gatekeeper to the rest of the system and refers to hospital and specialist treatment and physiotherapy. Patients may change their GP once in six months. The number of patients registered per GP is fixed through negotiations between the Organisation of General Practitioners, that is part of the Danish Medical Association, and the NHSS committee (Ibid., p. 40). The distribution of GPS nationally is quite even and is about 0.6 per I 000 inhabitants. A small part of the population, only I.7\%, opts for Group 2 insurance, and is allowed to visit any GP and specialist without a 
referral, but has to pay co-payments for all medical services and pharmaceuticals, except hospital care.

Although the entire population is covered by the NHSS, a part of the population opts for additional voluntary health insurance to cover the costs of treatments that are only partially reimbursed or do not fall under the public health system, such as private hospitals, dentists and spectacles. This kind of insurance is becoming increasingly popular: in $1998,28 \%$ of the population was additionally insured, most of them by the mutual (non-profit organisation) Sygeforsikringen Danmark, dominating the market with a share of $96 \%$. Most people opt for voluntary insurance because of the fear of long waiting times and 'poor service' in public hospitals. The expected increase in the number of people that choose for additional voluntary insurance, is considered as a threat to the willingness of people to contribute to the public health care system which may undermine the equity in access to health care, as not everyone can afford additional health insurance. For the same reason, private hospitals are a controversial issue that is hotly debated in the Danish parliament. Others regard these as a good and innovative supplement to the public sector.

\subsubsection{The financing of health care}

In Denmark, more than 80 per cent of the total expenditure on health care is financed through general taxation; 20 per cent are national taxes and about $6_{3}$ per cent are local taxes (primarily on income (8I\%) in real estate (6\%)) (Mossialos and Le Grand, I999b; Christiansen, Enemark et al., I999). I7\% is financed through user charges and insurance premiums. This is different from the UK health system, which is primarily financed out of national taxes. Pensioners, lowincome families and the disabled are either exempted from co-payments or pay reduced rates. Individuals such as chronically ill people can ask the National Board of Health for exemption of charges for pharmaceutical care.

In contrast to other European countries that were confronted with ever-rising health care costs, in Denmark health care expenditures fell during the period between I980 and I992 and dropped below the Eu average. Expenditures on hospital care declined since I980 and amounts spent on ambulatory medical care and medication hardly increased. The diagrams in figure 3 and 4 clearly show the priority that is given to nursing care; the proportion of money spent on nursing care, namely I $8 \%$, is considerable higher than the Eu average of $9 \%$ and highest in the EU together with Sweden and the UK. Expenditures on ambulatory medical care are almost half the EU average, probably because most people need a referral for medical specialist care, unlike in Germany and France, where people have direct access to private specialists. 
Figures 3 and 4 Proportions spent on different health cae sectors in Denmark and the EU Health care expenditure in Denmark

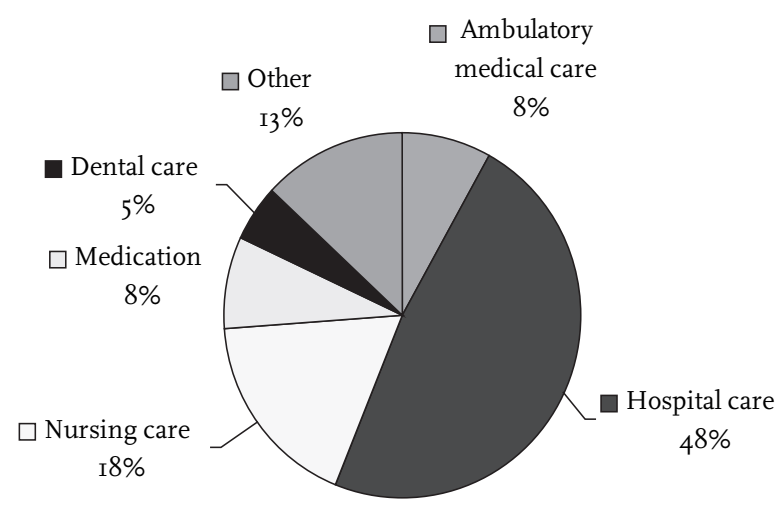

Health care expenditure in the EU

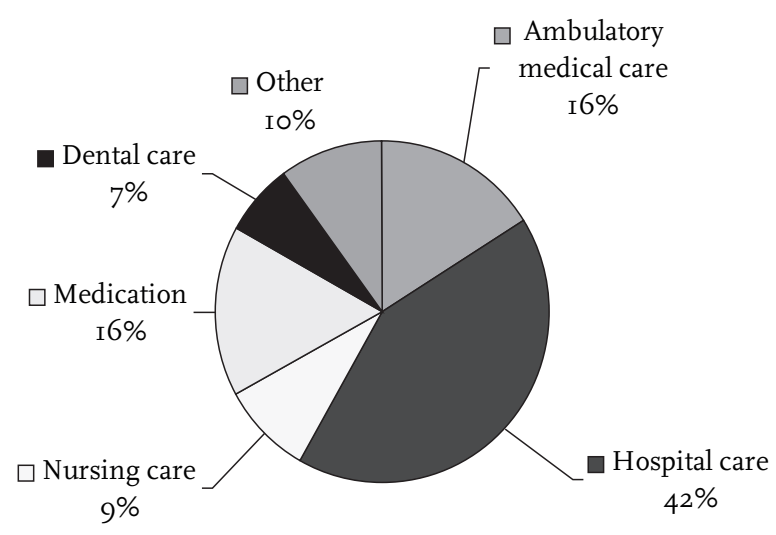

Source: Vallgårda, 200I.

Yearly, national budget negotiations take place between the Ministry of Health, the Ministry of Finance and the county and municipal councils, represented by the Association of County Councils and the National Association of Local Authorities (Vallgårda, Krasnik et al., 200I p. 7I). In these negotiations, agreements are made such as the maximum level for county and municipal taxes; the level of state subsidies to the counties and municipalities in the form of general grants; redistribution of finances aimed at equalisation to compensate for variations in taxes between counties and municipalities (accounting for age distribution, the number of single parents and single people of $65^{+}$, the number of foreign citizens, those without educational qualifications, unemployment rates, housing standards and 
the number of inhabitants in socially deprived areas (Indenrigsministeriet, I998, in Rostgaard, I998)); and the size of extraordinary earmarked grants for specific areas needing additional resources.

Health professionals in Denmark that are employed by the municipalities, such as nursing home staff, home nurses, health visitors and municipal dentists, are paid a fixed salary. The health system is structured as such that it is an incentive for GP activity, intending to prevent unnecessary referrals to hospitals and specialist treatment and to provide GPS with economic security. As in the Netherlands, Danish GPs have a central, gatekeeping role in health care. Of the total number of physicians in Denmark, 23\% work as a GP. They derive almost all of their income from the NHss, according to a scale of fees agreed by the Organisation of General Practitioners and the NHss Committee. Medical specialists also derive almost all their income from the NHss, as $98 \%$ of the population is Group I insured. Very few physicians are employed in the private for-profit sector, which contrasts strongly to France and Germany, where many physicians have a private practice or work in private hospitals.

Municipalities fully cover the costs of home help, and 95\% of nursing home costs. Nursing home residents themselves pay the remaining 4 to $5 \%$ of the costs. They only pay for the services needed and not for the entire package available, which is common in most other European countries (Rostgaard and Fridberg, I998b p. 98). Co-payments for day centres and sheltered housing facilities are about one-fifth of the total costs, see table 6 .

Table 6 Sources of financing of elderly care $(67+$, I994), percentages

\begin{tabular}{lccc}
\hline & State & Local authorities & Clients \\
Nursing homes & - & 95 & 5.0 \\
Home help & - & I00 & - \\
Day centres and homes & - & 77 & 23 \\
$\begin{array}{l}\text { Sheltered housing } \\
\text { (excl. celdreboliger) }\end{array}$ & - & 80 & 20 \\
Home care allowance & IOO & - & -
\end{tabular}

Source: Rostgaard, 1998 p. 98.

Thus, the state and local authorities pay the largest part of elderly facilities. Municipalities receive reimbursement of these costs from the state for $12 \%$ of expenditure, through various subsidies, block grants and equalisation grants. The subsidies are mainly used to guide local policy and finance extra costs for the expansion of services; for example in I993/94, the state financed 50\% of the increase in municipal costs for elderly services and $30 \%$ for child day care, mainly to reduce local unemployment and waiting lists. 


\subsection{A rather low degree of managerial and bureaucratic interference in professional practices}

In many European countries, health care professionals and patients often complain about bureaucracy and the dominance of economic targets in health care. In Denmark, the situation seems rather the reverse; the role of managers in health care organisations is far more limited than in most other countries. Throughout the twentieth century, health care professionals played an important role in the organisation and execution of health care activities and were also influential regarding health care policies, especially the medical profession. The trust in health care professionals and their social status seems to be higher than in many other European countries, which can be recognised in the larger degree of autonomy and less interference of managers and bureaucratic procedures. However, gradually, this seems to be changing. Since the I980s, more attention for management and public health skills is rising (Vallgårda, Krasnik et al., 200I p. 62). An increasing number of economists, professional managers and lawyers are employed in health administration, and more and more health professionals are following postgraduate management courses. Many health professionals are critical of this trend, stating that economic and management targets have become more important than quality of care. In most other European countries, the existence of managers in health care organisations has been considered 'normal' for many decades now. Apparently, Danish health care professionals have successfully delayed the increasing interference of managers in health care for a long time, ensuring rather autonomous professional practices. As administrative expenses are moderate in Denmark, this indicates that the extent to which management procedures intrude into daily health care practices is presumably still more limited than in other countries. Nevertheless, Danish health professionals resist bureaucratic interference in professional practices, as in other European countries.

\subsection{The history of home nursing}

As in the United Kingdom and the Netherlands, nurses in Denmark were already organised at an early stage: in I899, the Danish Nurses' Organization DSR (Dansk Sygeplejeråd) was established, following the example of the British Nursing Association (Balslev Wingender, I999 p. I2). The Dutch Nursing League (Nederlandsche Bond voor Ziekenverpleging) was established in I893. Membership of the DSR is voluntary, but the number of nurses that join the nurses' organisation is traditionally very high in Denmark. For example, currently 7300 nurses, or 95\% of all Danish nurses (including students and pensioners) are a member (Dansk Sygeplejeråd, 2004 b). The four-leaf clover emblem, symbol for membership in the DNo, has also 
become symbol of the trained nurse, and 'the pin' (see figure 5) is an important reason why nurses become a member (Balslev Wingender, I999 p. 8).

Figure 5 Picture of 'the pin', indicating membership in the Danish Nurses' Organisation

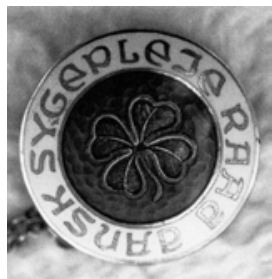

Source: Stallknecht, I999 p. 6.

Next to that, every nurse training school and training hospital has its own emblem as well; nurses meeting each other often look at each others emblem first to see where they are trained (see figure 6 and 7 as examples; but there are far more of these emblems) (Stallknecht, I999). ${ }^{6}$

Figure 6 Emblems of nursing schools in Thisted and Viborg, 1958 - I975 and I975- now

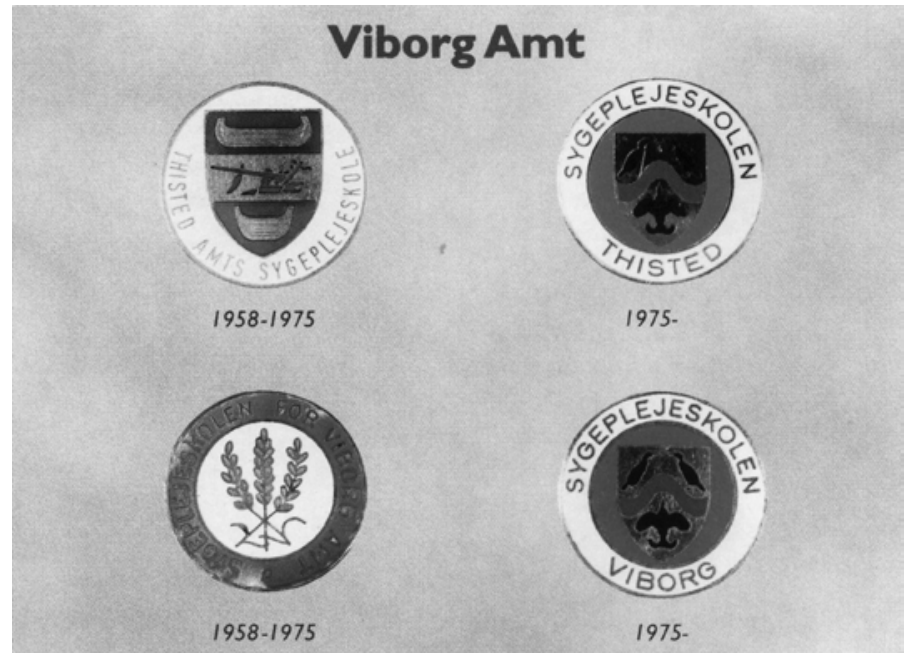

Source: Stallknecht, I999 p. 24.

6 Confirmed in the interview held in April 2002 with the manageress of the Dansk Sygeplejehistorisk Museum (Danish Museum of Nursing History) in Kolding, Denmark, Mrs Inger-Marie Børgsen. 
Figure 7 Emblems of the 'private' (Deaconess and Catholic) nursing schools, I942 - I991

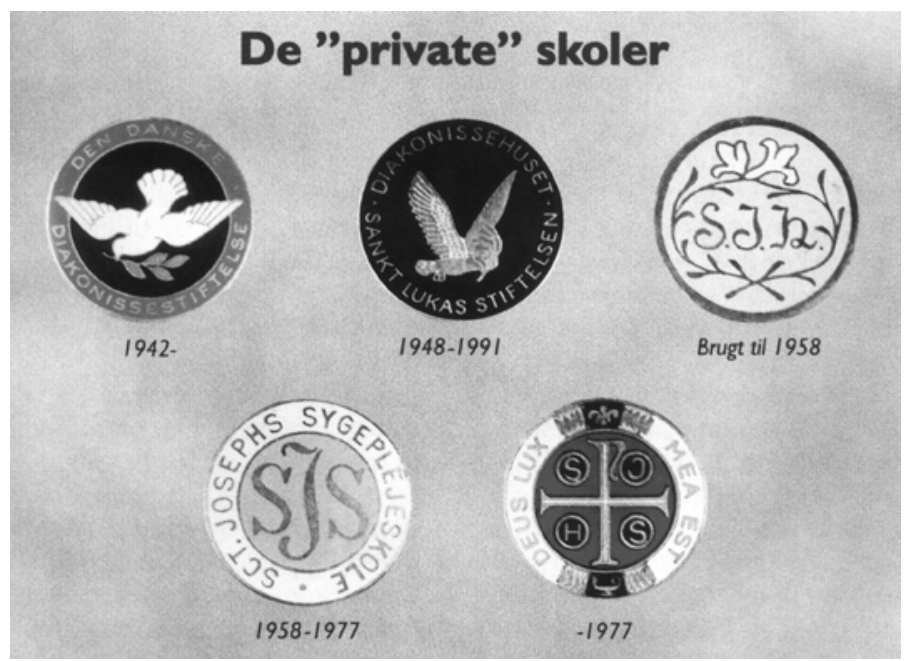

Source: Stallknecht, I999 p. 27.

The 'private', confessional Deaconess and Catholic nursing schools started very early: the Danish Deaconess Foundation (Danske Diakonissestiftelse) started their nursing training in 1863 , and the Catholic St. Lukas Foundation (Sct. Lukas Stiftelsen) followed only one year later, in I864 (Ibid., p. 26). In I936 opened the St. Josephsister- and nursing school in Copenhagen (Sct. Joseph søsterne en sygeplejeskole i København).

In I899, the Danish Nurses Organization (DNO) was established by nurses from the General Hospital in Copenhagen and Charlotte Norrie, who participated in the International Women's Congress in London in I899 (Ibid., p. I2).7 At the English and Irish matrons' conference of that congress, Ethel Bedford Fenwick spoke out for the international organising of nurses, and Charlotte Norrie was appointed to the provisional committee that was to draft the constitution for the new International Council of Nurses (ICN). Shortly after her visit to London, the Danish Nurses' Organisation was established. The overall goal was to raise the social status of the nursing profession through better education, working and living conditions, and to promote the professional interests of nurses, by trying to influence and control the development of nursing. The effort of making nursing into an independent, professional discipline is one of the three themes than runs throughout the one

7 Charlotte Norrie also became the first (though controversial) president of the DNO (Balslev Wingender, p. 13). 
hundred year history of the DNo. The second theme in the history of the DNo is the aim to assemble all Danish nurses into one organisation. For this, the structure of the organisation was adapted throughout the years to the major political goals of the profession and the members' demands for co-determination. The third theme is the struggle for improved wages and working conditions. An important aspect of this is the development of the D No from merely being a professional organisation to also being a trade union. "Central to this development is the confrontation with the attitude that nursing is a vocation, a confrontation which, on a small scale, began to emerge in the I930s" (Balslev Wingender, I999 p. 8).

Between I899 and I933, nurses struggled for consolidation of nursing as a profession, and the DNo struggled for consolidation of becoming the organisation of the nurses. In I899, it still was a small, elite association, but its aim was to become a prominent and widely respected organisation. In I933, the Danish Parliament adopted legislation that authorised the nursing profession. From that period, the organisation and nurses struggled for improved wages and better working conditions, and the dno and employers entered into the first collective agreements. The concept of nursing as a vocation and the traditional way of life for nurses were gradually rejected, and the first steps of creating a trade union were taken. Several topics, such as the education of nurses, salaries and working conditions, were heavily discussed, but only temporary resolutions were found in the years leading up to ig6o. In the period between I960 and I999, the dno obtained negotiating rights for all Danish nurses, and 'attempted to "walk on two legs", a professional association and a trade union leg' (Balslev Wingender, I999 p. 8). Also, currently, as in many other European countries, one of the main problems is a shortage of nurses.

\subsection{Home nursing currently: a generalist nurse}

In Denmark, the home nurse is a central person in case of illness of an elderly person (Plovsing, I992 p. I9). She not only offers assistance and nursing, but also estimates the need for technical aids, or transfer to an apartment for the old or nursing home. This means that Danish nurses have more decision power and autonomy compared to their colleagues in other countries, where often medical professionals (Germany, France) or 'objective assessment organisations' (the Netherlands) determine the need for home nursing and the tasks to be performed. Home nursing is provided without co-payments and in practice, most clients have a referral from a GP although they officially do not need it. ${ }^{8}$ This indicates the strong gate-keeping role of the GP in the Danish health system. A home help and the local home help

8 Kroneman, 2006. 
manager assess the need for home help and decide on the amount of help to be provided (Rostgaard, p. I39).

Since I996, municipalities are obliged to conduct a preventive home visit to people of 80 years and older, since I998 this was extended to people of $75+$ as well, according to need, but at least twice a year (Rostgaard and Fridberg, I998b p. I44). These visits should allow the elderly person and the person paying the preventive home visit (forebyggende hjemmebesøg) to evaluate the need for help and care, to ensure that elderly make use of their own resources, maintain their functional abilities as long as possible and enhance their social network. Visits can also be paid to elderly in nursing homes, and in exceptional cases when a spouse died, or in case of serious illness or discharge from hospital. This preventive 'check for home care is typical for Denmark, as in the other countries, patients have to contact health care services such as home nursing or home help themselves. Often, a medical professional is involved in this process, such as a GP. Thus, these health care systems are demand-led. In the Danish system, primary health care facilities and prevention play a large role, and because these are organised and provided at the local level, insight in the local situation of people in need of services is tried to be guaranteed. The assumption is to prevent people from entering the (mostly more expensive) medical sector and institutions by providing help at an early stage in the community.

At the national level, the association of communities (KL, Kommunerne Landsforening) negotiates with the nurses' association about general guidelines for the ratio of the number of head nurses, nurses and assistant nurses at community level (Zee, I996). However, communities are not obliged to adopt these guidelines literally.

Home nurses and assistant nurses provide home nursing services. There are no specific nursing grades or levels of expertise distinguished, and there are no separate lists of tasks specified for nurses or assistant nurses; all home nurses provide simple as well as complex tasks and earn the same salaries (Zee, I996 p. 87; Børgsen, 2002, April). This is an important difference compared to other countries, where differentiation of tasks is an important organisational principle. Maybe, because of a relative lack of managerial interference and a rather strong position of health professions, no differentiation of tasks has been introduced; also because a generalist conception of care and continuity of care are important principles in Denmark. However, Denmark also has specific problems regarding nursing: many nurses complain about their salaries and the lack of career possibilities, as there are no different nursing levels. Home nursing tasks include usual activities, such as giving drugs and injections, wound care and health promotion. Nurses mostly care for patients that are discharged from hospital. As the length of hospital stays decreases, these patients often still need intensive care and treatment. Assistant nurses perform a full range of nursing and home help activities for the chronically 
ill, especially when that reduces the number of professionals involved for clients. Continuity of care is thought an important principle in Denmark.

\subsubsection{The education of nurses; the provision of integrated elderly care}

Since the introduction of de-institutionalisation policies, increasing efforts in the I980s tried to integrate both sectors and to offer coordinated care, by combining home nursing and home help (Rostgaard, I998 p. I34). A seamless package of care, offered to all groups of elderly people, was aimed at. Thus, of the 275 municipalities, 236 have set up integrated staff units with both home nurses and home help. In the other 39 municipalities, staff may also work in integrated teams, but formally, they still each belong to their own professional group. Following this, staff now provides care, regardless of where the elderly person lives, whether this is at home or in an institution. Currently, however, the recruitment of nurses is a serious problem in Denmark, as in most European countries (Plovsing, I992; Zee, I996; Balslev Wingender, I999).

Denmark is the only country where home nursing comes most closely to a holistic conception of nursing, in contrast to other European countries with a detailed division of labour, such as the Netherlands and the United Kingdom (Raalte, Kuiperij et al., I999). After nurses had at least two years of practical experience in institutional nursing, community nursing organisations provide additional training into home nursing.

In I99I, a new staff educational reform has been introduced, to support the reshaping of the ageing care system (Plovsing, I992 p. 23; Zee, I996). This has resulted in a basic education for all kinds of jobs within the care sector. After the basic training, one can specialise in various directions, such as assistant nurse, nurse, therapist, etc. For example, for assistant nurses, a combined education programme with home helps exists since I99I: the first year is shared, after that a further I, 5 years of training qualifies for assistant nurse. This reform is intended to support a development that already started in many municipalities, namely the total integration of institutional care and home care. Staff can now provide care and support to all local elderly, irrespective of whether they live in nursing homes or in their own homes or in dwellings specially adapted for elderly people. The idea is that each professional should partly work in an institution and partly in the homes of elderly, to obtain a more flexible use of staff resources (Boll Hansen and Platz, I99I).

This policy is more radical than in other European countries: in France, Germany and the United Kingdom, social care and health care for elderly people are separated organisationally and financially, while in the Netherlands, policies in the I990s have aimed at integration of home help (social care) and home nursing (health care), but institutional care and home care are still separate health care sectors, although co-operation between both is increasing (so-called 'transmural care'). 
Also, since the I990s, most municipalities have established 24-hour-a-day home help and home nursing, that consists of an emergency scheme that enables people to summon help day or night, and a visiting scheme according to which assistance is given by fixed day and night routines, also after normal working hours (Plovsing, I992 p. 20; Rostgaard, I998 p. I35). This service may reduce the demand for places in nursing homes, encourage earlier discharge from hospital and may prevent hospitalisation on social grounds. Especially in cities such as Copenhagen, the 24 hours-a-day home care cars are driving to and fro (Børgsen, 2002, April). This indicates that in Denmark, the domain of home care to elderly people can be considered a societal subsector that is widely accepted by the population and supported by health care policies.

\section{Io The home care domain; municipal provision of integrated home nursing and home help}

In Denmark, the same department of the local community provide home nursing (Hjemmesygepleje) as well as home help services (Hjemmehjolp) (Zee, I996 p. 87). National legislation determines that all 275 municipalities should have a district nurse service and home help service with skilled staff (Plovsing, I992 p. I9). This differs from the situation in Germany and France, where home nursing belongs to the domain of health care and home help services belong to social care. As these each fall under the responsibility of different authorities and executing bodies, these are generally separately provided. The situation in the Netherlands however resembles that in Denmark, as home nursing and home help have been integrated and are provided by a local home care organisation (Swagerman, I997). In Denmark, apart from home nursing and home help, the municipalities also finance, coordinate and administer care arrangements such as centres, technical aids and meals-on-wheels, help with gardening and snow clearance (Plovsing, I992 p. 20). Municipalities support activities that aim to keep elderly active and healthy, such as pensioners' clubs, high-school education, picnics, visits to theatres, travels, etc.

Home help has been free of charge for people with low incomes and those in need of six hours or more weekly since its introduction in I973 (Rostgaard and Fridberg, I998b p. I42). People with higher incomes had to pay co-payments. Since I989, long-term home help became free for all, which is considered as one of the virtues of the Danish system. ${ }^{9}$ However, the Association of Municipalities states that co-payments for certain categories of home help may be reintroduced,

9 In 1992, co-payments were reintroduced, but this was withdrawn after five months after much criticism (Rostgaard, I998 p. 93). 
as the demand for home help increases because of the substitution of institutional care by home-based care and the double ageing of the population. As mentioned before, during the I980s, the Danish government stated that public expenses should not increase (Plovsing, I992 p. 2I). However, this appeared to be difficult as the number of older people increases and more elderly remain living in their own homes. Many municipalities had to economise as the total number of elderly people receiving home help increased. Although the proportion of elderly receiving home help remained the same between I980 and I989 (I0\% of the elderly of $67-69$ years of age and more than $60 \%$ of the elderly of $85+$ ), fewer hours of help were provided per family. Also, the range of tasks have been decreased slightly, as less assistance is currently offered with tasks such as cleaning, cooking and shopping. Instead, more frequent, but shorter visits, and nursing assistance have received a higher priority. Also, municipalities have tried to organise the home helpers in self-governing groups, that are together responsible for a group of older people. These experiences show more effectiveness, but break with the principle that the same home help when possible should support elderly.

\section{II Conclusion}

The characteristics of home nursing in Denmark are summarised into the contextual theoretical framework (see figure 8). The inner circle characterises the home nursing domain in Denmark, which has a long tradition dating back to the last decennia of the nineteenth century, as in the u k and the Netherlands. Remarkable compared to most other European countries is, that professionals are the primary source of care, and that informal care plays no major role. This can be considered as the Scandinavian model of health care, whereby the welfare state guarantees the provision of care to vulnerable groups such as children and elderly people. Family members are alleviated from the responsibility to provide informal care. Regarding nursing, efforts have already been undertaken early in the twentieth century to consider nursing as an independent profession. The autonomy of nurses is large compared to other countries. They perform the assessment of the client's needs themselves and generally perform the care needed themselves as well. Denmark favours generalist provision of care; continuity of care by having one single home nurse or nursing assistant perform different kinds of tasks is an important principle. Local municipalities that are legally obliged by the national state to provide long-term care provide home care. These have to ensure general accessibility and availability of care to vulnerable groups. Nurses are employed by municipal public elderly care services, that provide care in the homes of clients as well as in institutions, such as nursing homes and integrated centres for the old and protected housing facilities. The total integration of care to elderly people, wherever they live, 
Figure 8 Contextual framework of home nursing in Denmark

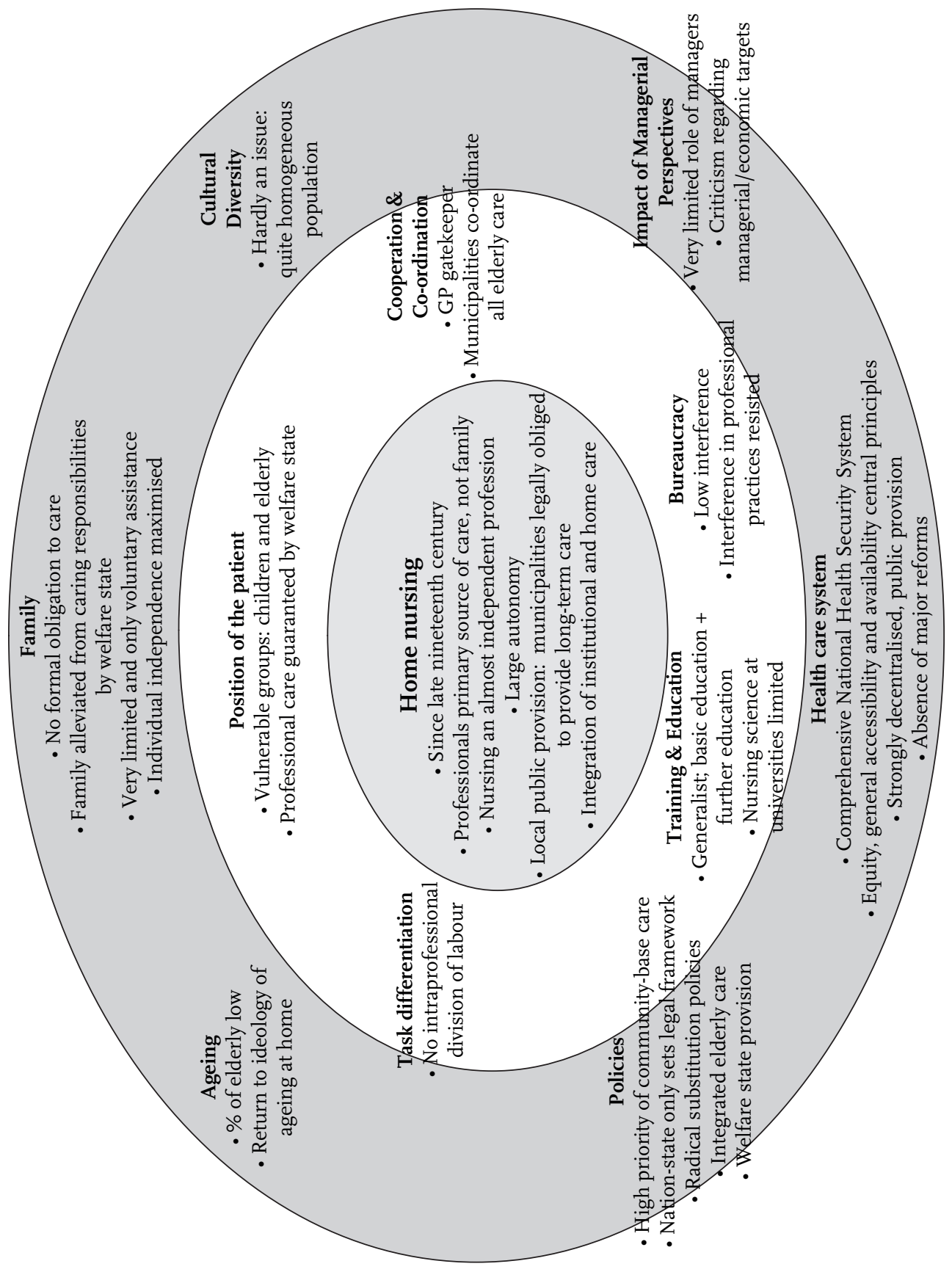


has become an important principle in Denmark since the late i980s, that started with the policy intention to abolish all nursing homes.

The middle circle of the framework characterises the organisational aspects of home nursing and the relation with other organisations, professionals, bureaucratic procedures and the patient. Elderly people and children are defined as vulnerable groups in Denmark, for which extensive professional facilities are guaranteed by the welfare state. The GP acts as a gatekeeper; hospital and specialist care is only accessible after referral by a GP, as in the Netherlands. As all kinds of ambulant as well as institutional care for the elderly are organised by the local municipalities, these co-ordinate all elderly care, irrespective of where clients live. Nurses are employed by the municipalities and provide elderly care in the homes of clients, special apartments for elderly people, as well as in residential homes and nursing homes, wherever they are needed. This is considered and described as the 'total integration of elderly care' and is unprecedented. Nurses receive a generalist basic education, which is the same for auxiliaries and nurses. They can specialise after that. Nurses perform all kinds of nursing tasks and division of tasks is uncommon; nurses and assistant nurses perform the same kind of tasks. Continuity of care for the patient is an important principle in Denmark, which means in practice that first level nurses also perform basic tasks such as hygienic care, allowing that the same home nurse mostly performs the care provided to the patient. Thus, no intraprofessional division of labour exists; first level nurses perform the same tasks as assistant nurses. There is low interference of bureaucratic rules and procedures in caregiving, and professionals who strongly believe in professional autonomy resist such interference.

The outer layer of the framework represents the contextual environment of home nursing at the level of society. The number of elderly people in Denmark is lower than in most other European countries. Current policies emphasise the ideology of ageing at home, striving for the situation in which elderly are predominantly living at home or in adapted apartments for elderly people and supported by sufficient health care facilities. The idea is that elderly remain a part of society in this way. Striking regarding Denmark is the divergent role of the family regarding elderly care. Apart from spouses, family members have no formal obligation to care. The welfare state guarantees sufficient elderly care and extensive facilities, maximising individual independence. This factor also explains the high level of labour participation of women in Denmark; on the one hand because families need an extra income to be able afford the high social security contributions and taxes, on the other hand because they are free to strive for their personal career. Thus, the welfare state alleviates family members from caring responsibilities. Thus, care by family members is very limited and only voluntary in nature. It is mostly only episodic and advisory in nature, for example to mobilise public services. 
The health care system is characterised by a comprehensive National Health and Security System, where equity, general accessibility and availability are central principles. This fits the strong social-democratic and welfare state tradition in Denmark that is highly supported by the population. The provision of care is strongly decentralised; the local municipalities provide home care services. The majority of the health care, hospital care as well as elderly care, is publicly provided. Only a very small minority is provided by private organisations. Major reforms were hardly issued, resulting in a stable health care system.

Health care policies regarding elderly highly prioritise community-based care. The nation-state only sets the legal framework; decisions are made at decentralised levels by counties in case of hospitals and municipalities in case of home care. Since the late I980s, radical substitution policies were designed, intending to abolish all nursing homes, which came from the ideology to enable people to grow old in their own environment and remain a part of society. However, the aims appeared too radical. Nevertheless, home care is high on the policy agenda in Denmark. Another important development is welfare state provision of home care by the local municipalities; thereby realising integrated provision of elderly care.

As Denmark is a quite homogeneous country and the percentage of non-Danish inhabitants is relatively low compared to other European countries, cultural diversity hardly plays a role. The role of managers in health care is also very limited in Denmark. Efforts are undertaken to educate professional managers in health care, but medical and nursing professionals object to managerial interference and are quite critical regarding the trend to set economic targets on caregiving. 
Chapter 5

\title{
Home nursing in France
}

\author{
- A weak domain in-between the state, \\ the medical and hospital domain and the family'
}

\section{I Four issues characterising the provision of home nursing in France}

In comparison with other countries, four issues are typical for the French home nursing situation. Firstly, home nursing is not equally available in all areas, as there are large regional differences in the distribution of home nursing services (Verheij, I996 p. I23). Moreover, in some of the more remote parts of the country, home nursing services are not available at all. The unequal availability of the population to home nursing services is the most striking aspect of home nursing in France, especially compared with countries such as Denmark or the Netherlands, where dense networks of services exist covering the whole country.

Secondly, health care in France is quite oriented towards institution-based structures. Generally, the family is considered as the first port of call, as well as neighbours or friends. However, when this informal care network is insufficient or unavailable, for example because a lot of younger people has left the rural areas, assistance is generally sought at the local ambulatory public or private medical and social facilities, such as GP or specialist practices (médécins en ville), or luncheon clubs serving meals for elderly people. When these are not able to provide sufficient support, people rather turn to institutionalised forms of care, such as homes for the aged (maisons de retraite or retirement homes), or long-stay hospitals, than to home nursing care. Retirement homes generally have medical care sections to provide for elderly with high care needs; and hospitals in France generally have long-stay wards to care for elderly people with long-term care needs (Giarchi, I996b; Ribbe, Ljunggren et al., I997). The concept of nursing homes that are especially designed for elderly with high care needs is familiar to most other European countries, but does not exist in France (Ribbe, I997; see further on in this chapter).

I I would like to thank my mother, Inge van der Boom-Keemink, and her friend Christel Gallifet for their kind efforts they have undertaken to collect a significant number of articles in French journals on nursing that were unavailable in the Netherlands, during the exceptional heat of August 2003. In addition, I would like to thank Mme Christine Gans, documentaliste of the Institut de Formation en Soins Infirmiers at Montelimar, for her help. 
The question is why elderly people and their families less easily turn to services provided in the homes of patients, such as home nursing or home help. On the one hand, there is a strong reluctance of the population, and especially of elderly people, to accept 'strangers' such as caring professionals into the private sphere, as these are felt to intrude into the personal sphere of life of the patient. The private sphere is cherished as a personal environment where non-family members or very close friends do not easily get access. This feeling is far more prominent in France than in the other countries studied in this dissertation, and may thus be considered as typical for France. On the other hand, it is far easier to organise and finance institutionally based care than community-based care, because of problems regarding the availability of home nursing care and the structure of the health care system that makes it easier to have an elderly person admitted into an institution.

Thirdly, health care in France is quite medically oriented. During the twentieth century, the professionalisation of French home nursing seems to be considerably hampered by the dominance of cure over care in the health care system. GP and specialist care, the hospital sector and pharmaceutical sector have a much larger priority than ambulatory nursing and caring services provided in the community. Co-operation between physicians (GPS, hospital and private physicians) and home nursing services is reported to be difficult because the need for co-operation is not always recognised by physicians (Verheij, I996 p. I23). Hospitals rather organise home-based hospital services themselves (HAD, see further in this chapter), than work together with community nursing services or private home nurses (infirmières libérales). Co-operation with local home help services seems to be less problematic or even very fruitful (Verheij, I996 p. I23); this might be because these belong to the social service sector and are thus not considered as competitors of health care services.

Fourthly, the role of the state is quite strong compared to other countries: the central government as well as the local governments have a large say in all kinds of aspects of daily life. In general, matters are centrally organised and from there transferred to the periphery (Galès, I999 p. 96). Throughout the twentieth century, the French economy followed the trajectory of Taylorisation, which gave rise to the pyramid-shaped centre-periphery model. Namely, in France, (economic) developments follow a hierarchical logic: first Paris, then followed by the regional capitals, which also reflects the country's political structure. The unequal distribution of virtually all kinds of services (transport facilities, social, economical and health care services) can be traced back to the industrial revolution, when France very quickly became characterised by strong economic centralisation. Business, capital and qualifications came to be concentrated in the Paris region that fully benefited from the transport revolution. During many ages, Paris has also been the cultural and intellectual centre of France, which is referred to as the "tout Paris" 
phenomenon: "Paris is the centre of national intellectual life in France to an extent inconceivable in the United States, Italy, Britain, or Germany" (Krause, I996e p. I68), and this is the case regarding virtually all societal matters in France. However, the farther things move away from the centre, the more difficult it becomes to get them organised properly, due to unavailable resources or (infra-) structural problems in the more remote and rural areas of France. Although other countries have strong capitals as well, such as the u k or Denmark, in these countries these function less as a centralistic force from where all societal matters are organised. Especially in Denmark, the government has decided for strong decentralisation of policy- and decision-making powers.

Since the Ig6os, a counter-movement began in France in which the regions were given more economic and political priority, known as decentralisation politics (Galès, p. 96-97), supported in the I960s and I970s by the regionalism of left-wing and middle-class people that promoted the rural areas as a 'refuge' from the over-crowded cities. Nevertheless, the decentralisation policies were only partly successful, as they were accompanied by an increase in government intervention in social and technological matters, and because the attraction of the Ile de France (the region of Paris) for economic, cultural, political and infrastructural activities remained considerable. For example, during the ig80s, despite the efforts to decentralise, the Paris region absorbed most of the economic growth, especially regarding employment, which is also described as 'metropolitanisation'. The government's aims to redistribute geographical inequalities in wealth, especially via social security and public employment was not very successful, as the situation in the regions remained lagging far behind the more favourable situation in the Paris region.

Although France is one of the oldest centralised states in Europe and has done persisting efforts to homogenise, it remains a social and political construction that is more characterised by persistent diversity between regions and localities than by cultural homogeneity (Galès, I999 p. IOI). Several sociological studies have demonstrated a surprising stability in behavioural patterns in a large number of localities and regions during several centuries, especially regarding family structures, religious practice and political preferences. Harismendy (I999), when asked whether French society is very different now from what it was forty years ago, argues that the traditional opposition between the north and west of France and the south of France has now been replaced by a split between rural France and areas surrounding small towns on the one hand and large cities like Paris on the other hand (Harismendy, I999 p. 83). Thus, the policies and rules designed at the level of the central or even regional state often do not correspond to the realities of everyday life far away in the periphery:

'In the Paris basin national strength is absolutely dominant; since the nineteenth century families are small, individuals more mobile than elsewhere and 
neither religion nor political parties wield any particular power over them. This region, and only this region, corresponds fairly well to the idea of a homogeneous France. Beyond this, regional or local strength begins to make itself felt, in Brittany, in the east, to the south of the Massif Central and in the Basque country; ...this has been progressively associated with Catholicism. In these already peripheral regions, a different style of society has persisted up to the present.' (Le Bras, I986, cited in Galès, I999 p. I04)

The force of traditional family structures is still very much felt in social and economic life in these regions, although the extreme parochialism of French society has weakened since the I96os, among others by increased mobility. But nevertheless, next to the bulwark of the central state extending all over the country, a second bastion exists in France, namely that of the family. Family life is considered as a separate sphere of life, independent of and almost impenetrable by the public sphere.

\subsection{The characteristics of health care in France}

\subsubsection{The unequal distribution of home nursing services over the country}

One of the most striking aspects regarding home nursing in France compared to other countries is the uneven availability of home nursing services across the country (Verheij, I996; Giarchi, I996g; oECD, I994d). Due to the uneven distribution of people over very densely populated areas such as Paris and its surroundings on the one hand, and very scarcely populated areas in the middle and south of France on the other hand, the network of social, economical and health care services is unequally distributed as well. Community-based services such as home nursing and home help are unequally available across the country; in remote areas of France these are sometimes non-existent. In these areas, younger people have often left to more urban centres for work, leaving older generations behind with declining social and family networks, sometimes resulting in social isolation. Their situation is then worsened by the lack of social and health care facilities in their living environment and housing facilities that are not suitable considering their needs or increased fragility. For example, one-third of the elderly live in older or substandard houses; facilities such as an indoors toilet and central heating are not standard ${ }^{2}$

2 A quarter of the elderly do not have an indoors toilet, only $46 \%$ of them (39\% of those of $85^{+}$) have central heating (Giarchi, I996) 
(Giarchi, I996g p. I97). Namely, the tendency of older people is 'to stay where they are', even when facilities are not corresponding to their needs.

In countries such as the Netherlands and the United Kingdom, networks of voluntary religious and charitable organisations have developed during the nineteenth and twentieth century in such a strong way that they provided for a considerable part of the care to vulnerable people. In the u $\mathrm{k}$, informal and voluntary carers are recognised by the government and in the health system. They are officially recognised in the Carers (Recognition and Services) Act I995 as fellow-client and supported in the form of respite care arrangements, day centres and support, information, training and advise by professionals (Kremer, 2000 p. 56). Also in the Netherlands, support of voluntary and informal carers is increasingly taking place. Former private non-governmental organisations (mostly of religious signature) providing home nursing all across the Netherlands, such as the Cross Organisations, have been officially integrated into the health care system since the I990s. In France, similar powerful voluntary or private initiatives do not exist (Giarchi, I996g p. 2I0; Pomey and Poullier, I997 p. 52). The general feeling, in politics as well as society as a whole, is that the responsibility to provide care and support primarily lies with the family ${ }^{3}$, or when informal care is unavailable, with the state (i.e. the local or central authorities). Considering the poor supply of home care services across the country, and the virtual absence of voluntary organisations filling this gap, a large amount of care and support to elderly people must come from family members and other informal carers but remains rather invisible, as family matters in France are largely kept in the private sphere. As voluntary carers and informal carers are not very well organised, as elsewhere in Europe, and not officially recognised for their important role in caring to vulnerable people, they remain out of sight of the public domain. It is therefore difficult to gain insight into the amount of care provided by the informal care network in France. In Denmark, adhering to the Scandinavian caring perspective, there are also no extensive voluntary care provisions, but the state guarantees individual people the right to professional care and sufficient availability of care for the whole population (Kremer, 2000 p. 45).

In France, the strong hold of traditional family values can also be recognised in the relatively large number of three-generation families in France. Compared to other European countries, many French elderly are living with their offspring; in I975 almost a quarter (24\%) of those of $65+$ (Eurostat, 200 Ia p. 84 ), and still I7\% in I990 (OECD, I996b). This figure does not give an indication of the amount of

3 Not only 'in natura' but also financially: in France, all social assistance payments (except subsidies for home help since I977) are subject to the obligation alimentaire: a legal requirement for families to meet the living costs of their elderly relatives if they can. This obligation goes back to the Code Napoléon that was introduced at the beginning of the nineteenth century (OECD, 1996 p. I35) 
family care provided, as living apart does not imply the absence of family support; the concept of 'cohabitation of family members in the neighbourhood or nearby' would better indicate the amount of informal care provided (Henrard, Ankri et al., I99I p. II3). However, the amount of informal care provided is not only influenced by vicinity, but by several objective and subjective factors that are related in a complex way (Thomas, Chanoin-Merlet et al., 2002; Dautzenberg, 2000). Apart from family members living nearby, the availability of home help is also important, as it supports the family and delays their 'breaking point' and therefore postpones the institutionalisation of the vulnerable person (Thomas, 2002 p. I04I). In France, as both home help and home nursing are not available everywhere, the (often invisible) burden on family members may be large, and when these are not able to provide care, the step to institutionalisation of elderly is small. There are more safety-nets for vulnerable people and their families in other countries, where informal carers are supported by health care and societal forms of support, networks of voluntary care exist and home care is more widely available.

It is however difficult to draw conclusions on the relation between the amount of formal and informal care provided. On the one hand, it might be that due to strong family values and family solidarity and the culture that care and support is first and foremost sought in the private sphere in France, the home nursing and caring domain has never developed or even never has had to develop in such as strong way as in north and west European countries, that have chosen for different principles underlying their caring systems. This may explain the relatively low amount of home-based care provided in France and the relative weakness of the caring domain in comparison to the medical and hospital domain. However, on the other hand, it might also be the reverse, namely that despite several efforts of the central government to promote home-based care in the last several decades, the home care domain remains underdeveloped and unfit to provide for the needs of the elderly population in France and lags behind the demand for care. Because of the absence of a network of voluntary and charitable organisations providing for vulnerable people, the family has no choice but to take care of their older relatives themselves, as no other safety net is available. Where public provision of care is absent, independent nurses (infirmières liberales) are filling this gap, providing their private nursing services in the homes of patients on a fee-for-service basis. The costs of private nursing care are reimbursed by the Assurance maladie when there are no 'regular' home nursing services available in that area. The relatively large amount of home nursing services provided by these independent nurses indicate the niche that is left by 'regular' home nursing services in France. This gives the independent nurses a quite special and important position, that they use, as we shall see further on in this chapter, to lay claims on specific central professional tasks such as the co-ordination of different forms of care for vulnerable clients living in their own homes. 


\subsubsection{The dominance of medical and institution-based care}

The second issue that is related with the weak position of the home nursing domain is that the French health care system is quite oriented towards medical and institution-based care. The medical sector (care provided by GPS, medical specialists and hospitals) clearly has priority over the nursing and caring sector, which is not only reflected in gaps in the provision of home nursing, but also in the relatively high number of physicians (G PS and specialists), and hospital beds per I 000 population compared to other European countries. For example, the number of hospital beds is about 8 per I ooo population, as in Germany, compared to 4 to 5 per I $0 \circ 0$ in Denmark and the Netherlands (Eurostat, 2007b). The health care system is organised in such a way that medical and hospital care is very easily accessible to patients; they are allowed to visit the physician of their choice as often as they wish (and are also free to consult several physicians about one complaint) and have direct access to private specialists and all types of hospitals (Kemenade, I997e p. 63). Most specialists that are employed in hospital also run a private practice. The number of hospital beds is generally considered as oversized, but several plans issued by the central government to reduce the number of beds met strong resistance by the responsible regional authorities. Medical costs have always been easily reimbursed by the sickness insurance scheme, without questions about appropriateness, and consumption is hardly limited because of the almost universal coverage of costs by the insurance system, as the ticket modérateur or co-payments are usually covered by the additional insurance through mutuelles or private insurance.

In contrast, ambulatory health services are not directly accessible, as a prescription of a physician (in case of home nursing and home help) or social worker (for home help) is needed. The financial and organisational structure of the French health care system is such that it is far easier to get medical help from physicians or specialists, to be hospitalised or to be admitted in a home for the elderly, than to receive home-based care (OECD, I996e). During the last decades, the boundaries of the health insurance system have been redefined, and the traditional role of social assistance and being a mere safety net only to provide for those with insufficient funds and no family network has been restated to cover a wider range of home and institutional care costs. Nevertheless, it still remains difficult to organise homebased care and receive reimbursement of the care provided, as the payments for care have developed in an incremental way. This has resulted in a very complex and overlapping set of provisions, in which all the main social insurance schemes, local and central governments, and private obligations under family law are involved:

'The diversity of the sources for care funding in France make it necessary for the elderly person and their family to negotiate with a wide range of policy actors to assemble an "income and costs package". In particular, it appears to be 
an extremely complex operation to obtain all the necessary resources to support an elderly person in a well-adapted home with adequate income and appropriate health and social care. By contrast, entry to a long-stay ward or retirement home is simplicity itself. The sheer complexity of support for home care costs, coupled with the uncertainty whether all of the components of the package can be assembled, must be an inhibiting factor to choosing home care alternatives when making decisions about home care'. (OECD, I996e p. I35)

Thus, older people in France and their formal and informal carers are caught in the middle of what must be the most complex system of administering health and social care in Europe (Giarchi, I996g p. I99; OECD, I994d; OECD, I996e; Pomey and Poullier, I997; Dumons and Pollet, I999). The provision of health care is highly fragmented in France, as there is a public sector, a private non-profit and a private for-profit sector, with services that belong to the health care system and to the social services. Most of the care is covered by the insurance system or the additional insurance, but sometimes pension schemes or state allowances pay for the care provided (for example for home help, when people and their families are not able to pay the costs themselves). The complexity of the system and the diverse actors and payment schemes involved puzzles the users and often also bewilders the providers of care (Giarchi, I996g p. 210). Especially regarding extramural care for elderly people, a micro-system or infrastructure of care exists, involving public and private services belonging to health care, social care and community services (e.g. luncheon clubs, télé-alarm services). As different payment schemes are involved, it is difficult for older people and their families to gain insight in the possibilities for care, and sometimes, financial burdens falling on elderly or their families are high, for example, when they have to pay for the board and lodging accommodation in retirement homes or shelters themselves.

Another problem is that care for the elderly by social and health institutions is not coherent, especially as the differences between the regions are noticeable and because planning at the national and departmental level or co-ordination at the local level are almost or entirely non-existent (Pitaud, Vercauteren et al., I99I p. I). Policies that have been aimed at transforming the almost non-existing homebased care structures into an infrastructure of home care services enabling elderly to remain in their homes within several decades seems to be only inadequately reached, especially considering the limits set on expenses on health care and social services in the same period. Moreover, the more action is undertaken, the more needs are revealed that remained invisible before. Until now, the existing services do not seem to be able to meet the needs of the population effectively and efficiently (Henrard, Ankri et al., I99I).

Thus, the health care structure entails barriers for the use of home-based care that do not exist regarding ambulant medical and hospital care and long-stay 
facilities. Nevertheless, it is estimated that about $97 \%$ of the health expenditures of elderly people are met by the statutory health insurance scheme, as the costs of e.g. home nursing services are reimbursed (OECD, I996e p. I34). Social care, including home help, board and lodging costs of retirement homes and long-stay wards, is paid by the Aide Sociale budgets of the Departments when elderly and their families do not have sufficient funds to pay for these services. ${ }^{4}$ When their income is higher than the social assistance ceilings, social care is financed by pension schemes.

Consumption of medical care is also encouraged by the quite large number of physicians in France (as in Germany) compared to other European countries. The physician-patient ratio, which had been one of the lowest in Europe with less than 45 oo or only 0.9 physicians per I 000 population in I955, increased rapidly to one of the highest, namely from I.8 in I976 (85 000) and 3.0 in I999 (almost I72 000), see figure I (oECD, I998; Eurostat, 200Ia; Krause, I996a). The enormous increase was primarily due to the (temporary) abolishment of the numerus clausus in I968.

Figure I Increase in the number of physicians I900-2000

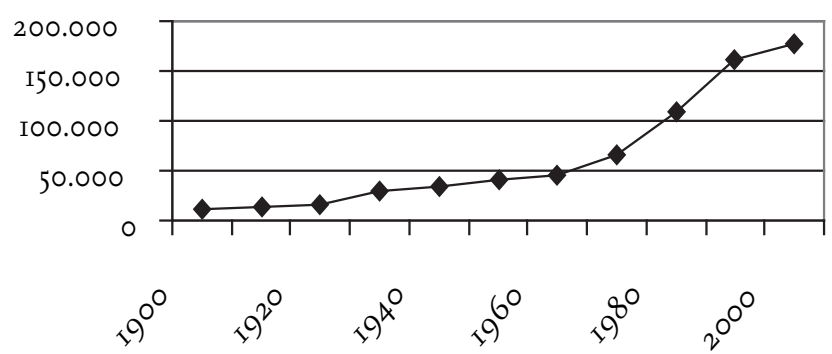

Source: Krause, I996a.

Although the numerous clausus was reinstalled only a few years later, the nature of the medical marketplace had changed permanently as there was an enormous increase in physicians entering medical practice since the I970s, and their number is not expected to decrease until about 2030. Currently, especially the number of GPS is high compared to other countries: almost 87 o०० or I.5 per I ००० population (comparable to Belgium), compared to 0.4 in the Netherlands and 0.6 in Denmark and the UK (OECD, I998).

4 These Departmental social assistance allowances are subject to the obligation alimentaire, which means that the family is to pay for social care, unless a means test demonstrates the family's ability to pay the costs of their elderly relative's maintenance and housing. 
Major reorganisations in the French health insurance and hospital system in the I96os had created a larger number of salaried positions in the state hospitals and thus a greater need for physicians, and many specialists entering the health care market established their own private practices. Because of this, medical services (GP and specialist care) are found even in the more remote communities of France, to an extent that is unknown in other European countries. ${ }^{5}$ In the late I970 however, positions became unavailable in French hospitals and specialist practices, and a large part of the physicians that graduated in the I980 have had to enter general practice. Due to this flooding of the GP market, it is estimated that one out of four GPS will eventually have to leave medical practice or will barely make enough money to cover their retirement payments.

The developments described here have led to a significant change in the character of the French medical profession during the twentieth century:

'In I930 French medicine was essentially autonomous and committed to a feefor-service system. By the late ig8os, it had become a profession in which $64 \%$ were on salary, and were thus largely under the control of nonphysician administrators. A high-flying elite of state-based hospital-university professors and a second high-flying elite of specialists in private practice in major metropolitan centers, (...) has to be compared with the mass of the profession, working in hospitals and clinics, on salary, for minimal wages, and the mass solo and smallgroup practitioners in the countryside, many of whom, like their brethren in the non-university hospitals and clinics, are also barely members of the French middle class.' (Krause, i996e p. I39)

This might be one of the reasons, next to differences in the attitude of the French regarding matters of health and disease, and differences in the structure of the health care system, why French GPs are quite eager to subscribe drugs to patients: it is one of the ways to supplement their rather low income (Gruyter, 2003). Deschepper et al. hypothesise that they might also easily give in to patients' wishes in order not to endanger good relations with them as they only have a relatively small number of patients in their practice (Deschepper, Stichele et al., 2002).

In the Netherlands, where a strict numerus clausus has restricted the number of medical students during many years, the situation is almost the reverse and has resulted in a shortage of physicians. The number of GPS is far lower and GP practices are much larger than in France. Most Dutch G Ps complain that they have

5 Nevertheless, as all kinds of facilities and services in France, physicians are also unevenly distributed over the country. The national figure is about 3 per I o०० population, but it varies between I.9 and 3.I per area, and is even as high as 7.0 in Paris (Pomey, I997 p. 56-57). 
more patients than they can cope with rather than too few, and that their work load is too high (Berg, Bakker et al., 2003). A Dutch GP deals with I92 patient contacts a week (an increase of 33 contacts or about $20 \%$ since I987), but at the same time, they work fewer hours a week: from 53 hours in I987 to 44 hours a week in 200 I. This implies that they spend less time per patient, as patient contacts are limited and preferably dealt with by telephone instead of appointments in the GP practice or house calls, and many patients are treated in the (newly developed) GP posts in hospitals at weekends, and more and more tasks are delegated to assistants and other supporting staff (Berg, Bakker et al., 2003 p. 1055-56).

In France, in contrast, the situation is rather the reverse and GPs spend a lot of time on their patients and try to establish a long-lasting professional relation with their client. The fee-for-service reimbursement and the increase in the number of physicians have provided powerful incentives for growth in the volume of certain kinds of medical care, especially prescriptions and diagnostic tests (OECD, I994d p. 52). It seems likely that physicians pursue a target income and aim to increase the volume of services per physician. Thus, an overpopulation of physicians can significantly increase the health-care costs of a society, the introduction of several measures to control health care costs in the ig80s and 9os by the state ${ }^{6}$ (Krause, I996 p. I38; Lancry and Sandier, I999 p. 458). In France, state regulatory bodies as well as the Sécurité Sociale system interfere in the medical profession and further weaken its social-economic position and professional autonomy.

The emphasis on medical and institution-based care in the French health care system is also reflected in the organisation of home nursing: France is the only country (until recent developments elsewhere) where two different kinds of home nursing exist next to each other: Service de Soins Infirmiers à Domicile pour Personnes Agées (ssiad or Home Care Services for Elderly People), which is comparable to home nursing in most other European countries, and Hospitalisation à Domicile (HAD or Hospitalisation at Home), which is far more medical in character and generally organised by hospitals. The latter is mostly offered to people remaining in their own homes but in need of complex medical or technological care and falls under the hospital law. This distinction in home nursing does not exist in other European countries; generally all home care is organised by ambulant home care organisations, also rather complex care. In the Netherlands for example, nurses that have specialised in specific areas of expertise mostly provide this kind of care.

6 E.g. the introduction of treatment guidelines for physicians (RMO's: Références Médicales Opposables) was intended to prevent duplication of services or prescription of inappropriate services; the introduction of a carnet de santé (medical records in the form of a small booklet carried by patients) was to avoid conflicting and redundant prescriptions and limit the access to multiple physicians; and a prospective target growth rate was set to limit the growth in fees and prescriptions by non-salaried physicians. 


\subsubsection{A cure-oriented attitude towards matters of health and disease}

A third aspect that may explain the rather weak position of home nursing in the French health care system is that the French have a rather medical, cure-oriented attitude towards matters of health and disease. In general, they adhere to a medical definition of diseases, asking and expecting to be 'healed' by medical interference and drugs. The role of GPS, hospitals and private medical specialists in the health care system is large in France. Stevens and Diederiks (I995), studying health cultures of five European countries, state that "People in France are characterised by a strong curative orientation, a high degree of health uncertainty and a weak charity orientation" (Stevens and Diederiks, I995 p. 82). ${ }^{7}$ Sickness is generally regarded as a physical condition, and according to Payer (I988), often considered as an affliction of the liver, the notorious crise the foie (Payer, I988).

In other European countries, the population generally takes a more reluctant stance, and mostly people adhere to the strategy of nursing one's illness when they are feeling ill, because they feel the illness must run its course and the body should be given time to recuperate by itself, so there is no need to influence this process with medical means. In countries such as Belgium and France, people generally do not follow the strategy to 'wait and see', but visit a physician or pharmacist in an early stage and expect to be prescribed drugs such as antibiotics (Deschepper, Stichele et al., 2002). This is also encouraged and made possible because of the rather high density of physicians in both countries compared to other European countries.

These differences in patterns of medical consumption can partly be explained by structural differences in the health care and social insurance system; in the Netherlands for example, it is accepted by most employers and employees that people remain absent from work for one or a few days when they are not feeling well, while in Belgium, Germany or France, a physician's note is required to be allowed to stay at home, instantly 'medicalising' the affliction (Deschepper, Stichele et al., 2002 p. I853). But apart from such structural differences, health-related value orientations, or in other words, the cultural and societal norms, values, ideas, habits and customs concerning issues of health and illness of a particular society, may explain a large part of international differences such as the far higher number of prescriptions in France compared to other countries and the large number of pharmacies all over the country. French physicians, and GPS in particular, prescribe more drugs per visit ( 3.2 items) than their counterparts in other countries, especially anti-depressives, vasodilators and antibiotics. Also, the use of antibiotics is highest in the EU (37 defined daily doses per I oo० people per day, I997) in

7 A curative orientation signifies the value that is attached to the technological, cure oriented development of modern health care. 
France, compared to e.g. 27 in Belgium and 9 in the Netherlands (Cars, Moelstad et al., 200I). This large variation in medical consumption among EU countries is unlikely to be caused by differences in the frequency of bacterial infections, and thus more likely to be traced back to physicians' and patients' attitudes to antibiotics, as well as differences in historical backgrounds, cultural and social factors, and structural disparities in health care and social insurance systems (such as a higher number of physicians per I oo० population in some countries).

Payer (I988) states that French physicians are eager to prescribe certain medications to patients, even when there is no proof that it has any effect: "We're not certain that they don't work", and "it proves that the doctor is doing something for the patient. When a French doctor writes a prescription, there's always a part that is aimed at raising the morale." (Payer, I988 p. 4I-42). Pharmaceutical expenditure (prescribed and over the counter) represents $18.4 \%$ of the total expenditure for medical goods and services in France, even though drug prices are usually lower in France than in other countries (Lancry and Sandier, I999 p. 460-46I). It is an important contributor to the high amount of money that is spent on health care in France, almost IO\% of the GDP, compared to e.g. 6.5\% in Denmark and 6.9 in the u k (Mossialos and Le Grand, I999d p. 46-47).

\subsubsection{The professions against the background of the all-pervading role of the central state in France}

The fourth aspect that is characteristic of France is that the state is an important centre of power regarding all aspects of French life (Krause, I996e). It stands at the centre of the society and makes many decisions that in other countries, such as the United States or Great Britain, are made in the private sector. This has its origins in the period of the absolute monarchies in France, when a corps of bureaucrats consisting of lawyer-magistrates and tax collectors that was gathered around the monarch, fanned out over the countryside and increased the grip of the central state on all organs of local government and many aspects of what elsewhere would be considered as private business (Ibid., p. I23). After the 1789 Revolution, this system was not affected fundamentally, because the absolutist state took the place of the absolute monarchy and all matters remained under supervision (tutelle) of the administration. Although different political regimes succeeded each other during the nineteenth and twentieth century, these always had to be considered against the steady background of the central state with its bureaucracy.

In France, the state required far greater authority over the professions than e.g. the English or American state could or did (Abbott, I988g p. I58). Although professions in all countries turn to the state to seek protection when they make jurisdictional claims, what distinguishes the French situation is that the French state was already powerful when professions started to organise themselves. Consequently, 
it does not only have a say in professional jurisdictions, but exercises control over professional structures such as organisations as well. Next to that, the state is a planner and distributor of services (enforcing a certain level of medical services in more rural areas or enforcing advocates to deal with unattractive legal cases), referee in professional disputes, and an important actor in setting prices. Most physicians' charges are set by the state; price agreements (conventions) are made between the sickness funds (Caisses d'Assistance-Maladie) and the syndicats, which are union-like collective-bargaining agents of the medical profession that communicate with state bodies (Abbott, p. I6I-I62). This situation is similar to that in Germany.

Krause (I996) states that professions have a second-class status and limited rewards in French society (Krause, I996e p. I25). Since Napoleonic times, university education has been considered as second-rate to the applied and non-theoretical education that was given in the grandes écoles that prepared for state service in the French administration. These 'grandes écoles' (Ecole Polytechnique, Ecole des Ponts et Chaussées (bridges and roads), Ecole des Mines, Ecole Normal supérieure, and Ecole nationale d'administration) educate most of the French governing elite. The better students of these schools make a career in the upper levels of the French state or find their way into the private sector, to become chief executive officers of most of the semi-public and private large corporations in France. France is the only country where engineers graduating from these Ecoles stand at the top of the professional prestige ladder since the nineteenth century, as they were responsible for the development of the infrastructure of France and taking up positions as 'ingénieurs d'Etat'. The training of high-status professionals was largely monopolised by these Grandes Ecoles (Abbott, I988h p. 200).

It is embedded in the French educational system that professional education trained in universities and elsewhere is defined as socially second-rate. In addition, French universities are considered as conspicuous by the state as they are too questioning and leftist in tone. ${ }^{8}$ Krause (I996) argues that the split education system and the different values attached to this (highly valued education taught at the Grandes Ecoles, versus lower status university and other education) is accepted in France because of the typical acceptance of the French of hierarchical relations and their general acceptance of the large role of the state in their life, of which a sense of inevitability is communicated to French children during their school period (Krause, I996 p. I25). The state's legitimacy is taken for granted and the

8 During most of the nineteenth century, universities in the German sense did not exist: they had been destroyed by the French Revolution (Krause, I996 p. I63; Abbott, I988 p. I99). The system that replaced them consisted of a national system of lycées and university faculties. Until now, the system of grandes écoles remains separate from the universities, and the dominance and elitism of the first puts the universities in a peculiar position that is typical for France. 
typical response to the state is not to participate in it, but to remain quite passive. However, from time to time large-scale resistance to the state is also common in French history, of which the revolutions are the most evident examples, but also more recent massive demonstrations against state measures substantially frustrating French societal life, such as strikes by school teachers, truck drivers blocking motorways, or all train traffic coming to a stand-still.

In France, professions are classified as 'interest groups', in a somewhat negative sense. While the state is said to represent the 'general interest', the professional ordres (for professional discipline and control) and the syndicats (professional bargaining associations negotiating with the state on the fees charged for professional services) only represent the interests of their members. Associations, trade unions, and political parties are considered as primarily defending their own interests, and their actions were always considered as competition to the central government, intending to decrease its power (Pitaud, Vercauteren et al., I99I p. 49). During the Second World War, the power of the unions was destroyed and 'ordres' or corporatist alternatives to professional interest groups were set up, that were to bargain with the state and 'police' the professions. For example, the ordre for the French physicians, more or less replacing the dismantled French doctors' bargaining union Csm F (Confédération des Syndicats Médicaux Français), was to monitor ethical conduct and strictly enforce the fee-for-service system and installed as the ethics and licensing organisation of the profession. It was set up with a highly hierarchical structure, with local, regional and national ordres, and a central ordre in Paris. Medical representatives of several ministries are given seats on the national order that all practicing physicians in France must join and support. However, it represents the conservative opinions of the national and regional leading members far more than those of 'ordinary physicians' it supposedly represents. About one-third of physicians is quite critical of the ordre and would like its function and structure to be reformed. In I98I, Mitterrand attempted but failed to abolish the ordre. Thus, compared to other European countries and the us, the role of the French state is much more pervasive, even regarding the medical profession, which is generally one of the most autonomous of all professions:

'The role of the state is all-pervasive in French medicine, from overall control of the tutelle over the ordre, to the financing and running of the Ministry of Education (which controls the medical Faculties), to the payment for most of the services, to the shaping of the market and the development of new methods of cost control in state-funded research bureaus and in organisations of the state itself.' (Krause, I996e p. I39)

Until the Mitterrand government in I980, the French governments were uniformly rightist and overwhelmingly statist. Under De Gaulle and his successors, 
the power of the state was identified with the good of France, which was to provide employment for all. The increasingly centralised and powerful French state worked together with capitalist forces in state-concerted economic planning, to plan the 'future of France'. Professional groups were not considered as major actors in this development, but as more or less irritating factors impeding the progress of France. Due to their union-like background and self-interested attitude, the ordres have always remained somewhat suspect in the view of the state.

The role of the state is also quite strong in the health care system. The health care system may be considered as quite free because practising physicians and patients have considerable autonomy. In the I920s, the principles of the médecine libérale were laid down, that are characterised by free choice of physician (GP and specialists) by the patient, freedom of prescription and location by the physician, fee-for-service payment by the patient without interference of a third party, and confidentiality. At the same time, however, some aspects of the system are highly and centrally regulated by the government or by the insurance system that is supervised by the government (Poullier and Sandier, 2000 p. 899), as the health care system falls under the regulatory authority of the French government, that intervenes directly in the production and financing of health care services (Giarchi, I996g p. I94; Kemenade, I997e p. 59, 65-66). It controls relations between institutions financing care, health care professionals and patients, in the face of general health and economic imperatives.

At national level, the Ministry of Social Affairs and the Ministry of Health are the most important actors regarding the health care system, and within these the Directorate of Social Security, of Hospitals and of Health. There is also a State Secretary for Elderly People. Recent structural health care reforms, especially the Plan-Juppé of I996, are intended to increase the grip of the state on national health insurance matters and expenditure, despite the officially decentralisation policy for all health services since i982. The Juppé-Plan, highly controversial and having provoked social turmoil at the end of I995 (Lancry and Sandier, I999 p. 463), includes measures aimed at the improvement of quality and safety of care and a reduction of costs and the introduction of a complete coverage of the population by the Assurance maladie.

The ever-rising health care cost poses the government for considerable problems, especially regarding the national deficit. The rising costs are due to a tension that is present in the health care system. Namely, in I945, the French attempted to put Beveridge principles into practice with Bismarck tools: a compulsory national health insurance system (that was already partly implemented in I928 to cover wage-earners against major risks) with universal coverage and health care that is considered as a 'right', was tried to combine with a pluralist system of provision and fee-for-service payment for most of the medical care (Dumons and Pollet, I999 p. 224-225; OECD, I994d p. 5I). This results in a costly combination, as both contain 
incentives to expand medical care. Co-payments, generally intended to limit health care consumption, do exist, but the ticket modérateur is mostly entirely or for a large part covered by the mutuelles, by which most people are additionally covered.

The government has exercised strong controls over fees and prices, but only weak controls over the volume of inputs and activity (OECD, I994d p. 5I). During the last several decades, health care expenditure in France has been higher than in other countries, and the government hardly seem to be able to limit these expenses, as the system can be characterised as mainly 'demand-led'. There is a considerable freedom of choice for consumers and a corresponding high level of autonomy for providers. This means that there is much consumer-led competition in the system, private and independent providers (physicians, hospitals, home nurses) are responsive to patients, and consumer satisfaction is high (Ibid., p. 52). As the population is nearly universally covered by health insurance, there is no disincentive for patients to restrain their initial demands for care. Consequently, France has above-average levels of consultations with physicians, prescribed pharmaceuticals, acute hospital beds, and acute hospital admissions. Also, there are several persisting difficulties in the health care system, such as the inefficiency of (public hospital) services, the inability to diminish the number of hospital beds regionally, and deficiencies in the availability of ambulant long-term care. Unlike in other countries, the latter has even been rationed, although the supply of care is not keeping up at all with the growing needs of the ageing population.

\subsection{The home nursing domain: a weak position in a fragmented health system}

In France, the health system is rather fragmented and can be characterised by a dichotomy between the medical domain that is managed by the national government, and the social domain, that is managed by the local governments. This results in a complex split in institution-based elderly care as well, because many elderly are cared for in hospitals with long-term departments, which are medical institutions, but also in welfare institutions that fall under the local social services, such as retirement homes. The board and lodging facilities are then paid by the elderly person, the family, pension schemes, or in some cases by state allowances. Elderly people residing in the medical ward of a retirement home because they are in need of intensive or complex medical care however fall under the medical sector and the costs are financed by health insurance (Ribbe, Ljunggren et al., I997). A physician advising the sickness insurance funds determines admission into the medical care section of these homes.

Regarding home nursing, the same split in the system can be recognised. Namely, there are two kinds of home nursing services in France, that are financed 
and administered separately and are considered as different kinds of services: Hospitalisation à Domicile (HAD; Hospitalisation at Home) and Service de Soins Infirmiers à Domicile pour Personnes Agées (SSIAD, SIAD, SIADPA; Home Nursing Services for Elderly People) (Verheij, I996; Com-Ruelle, Midy et al., 2000).

HAD and SSIAD are two different forms of home nursing that have been established as separate services several decades ago, falling under different domains and each with its own aims. had services are considered as medical services as these were set up as out-reach services of hospitals that fall under hospital law. HAD services concentrate on people with severe conditions that would otherwise be cared for in hospital. In that sense, it is different from SSIAD services and the way ambulant home nursing is provided in other European countries, as HAD is much more specialised and medical in character than SSIAD. In the domain of HAD, nursing is primarily conceptualised as a profession supporting medical and hospital activities, and not so much as an independent profession with an own job description and task domain. It is typical for the fragmentation and medical character of the French health care system that two different types of home nursing exist, one primarily organised by hospitals and quite medical in character and one comparable to ambulant home nursing provided elsewhere, primarily targeting on vulnerable people needing care and support in order to remain living in their own homes.

\subsubsection{Hospitalisation à Domicile (HAD)}

Hospitalisation at Home (HAD) started in Paris (Artot, I989). In I956, the first HAD trials began, and in I960, an agreement was signed between the social security offices (that reimburse health expenses in France) and the Health and Social Security services of Paris. At the same time, two private services were developed in the Paris region. In this period in other European countries, nursing home facilities were built to care for heavily dependent people, primarily elderly people. Nursing homes are institution-based care facilities, providing high levels of care as well as room and board, including professional support with activities of daily living and morbidity, psychosocial care, personal care, and paramedical care. Until now, France has no nursing home facilities similar to these (Ribbe, Ljunggren et al., I997), but has developed other structures to provide high-level professional care to the same target group, consisting of frail elderly people with chronic diseases and/or disabilities (mental, physical, or both). This concerns about $2 \%$ of the French elderly. These are primarily cared for in long-stay hospitals, medical care sections of retirement homes and by HAD services. Demented elderly people are usually placed in psychiatric and long-stay hospitals. About $4 \%$ of French elderly live in retirement homes or private commercial residences, and as much as $94 \%$ of the French elderly of 65 years and over live in their own homes, which is high compared to other European countries (see table I). 
Table I Percentage of people of $65+$ living at home and in institutions

\begin{tabular}{lcccc}
\hline Place of residence & Denmark & France & The Netherlands & $\begin{array}{c}\text { The United } \\
\text { Kingdom }\end{array}$ \\
$\begin{array}{l}\text { Own home, independently (with } \\
\text { informal and/or formal care, incl. }\end{array}$ & 85 & 94 & 90 & 93 \\
$\begin{array}{l}\text { domestic help and home nursing) } \\
\begin{array}{l}\text { Residential homes, homes for the aged, } \\
\text { old people's homes (low levels of care) }\end{array}\end{array}$ & $10.5^{\mathrm{a}}$ & 4 & 6.5 & $3.5^{\mathrm{b}}$ \\
$\begin{array}{l}\text { Nursing homes (high levels of care) } \\
\text { Hospitals (intensive medical care) }\end{array}$ & 4 & - & 2.5 & 2 \\
\hline
\end{tabular}

Source: Ribbe, I997.

${ }^{a}$ Including some sheltered housing and other special dwellings for elderly

${ }^{\mathrm{b}}$ Including some young disabled

The HAD service care for extremely serious and complex patients in their homes, thereby relying on the resources of the public (state-owned) hospitals, that were the prime mover of the HAD development, and supported by the central government. Nowadays, there are 68 HAD structures offering 4000 'places' (bed equivalents) that are unequally distributed over the country (Com-Ruelle, Midy et al., 2000 p. 32). These fall under the hospital law of I99I. The HAD sector is thus rather small compared to the 47000 sSIAD-places in I992. Nevertheless, reimbursement of the costs of HAD services was about three times higher than SSIAD services, indicating that care provided under HAD is much more expensive because of its more medical-technological and specialist character.

The HAD structure closely resembles the development of transmural care and high-level supplementary home care in the Netherlands during the I990s, to bridge the gap between community-based care ('extramural care') and hospital care ('intramural care') (Linden, I997b). The philosophy behind HAD and transmural care are however different, as with transmural care, already existing forms of care are rearranged or substituted by providing them in non-institutionalised settings, e.g. by providing ambulant specialised nursing care instead of specialist hospital care or bringing medical technologies into the homes of patients. HAD, however, has especially been created as an extension of hospital care to serve a closely defined part of the population in need of high levels of care, at a time when other facilities for these people were still absent.

Initially, HAD had been intended as a substitute for hospitalisation, because a patient in need of hospital care was provided with high-level medical care in his/her own home. In I960, the idea was that the ability to recuperate would be much enhanced in the patient's own environment. After the first experiments were considered successful, a large number of similar services were started in the prov- 
inces, cities and more rural areas (e.g. in the areas of Lyon, Nice, Pau, Grenoble). About $45 \%$ of these are provided by the public sector and $55 \%$ by private HAD services (Giarchi, I996g p. 20I). In I974, the state set minimum standards in a convention that were to be met by all new HAD organisations.

Since the I970s, HAD was promoted because of the worries over the continuous increase in health care costs, that forced governments to develop alternatives to the expensive and often inefficient care provided in the public hospitals. However, the creation of patient places under the HAD scheme was not successful in reducing the number of beds in 'traditional' hospitals, because this was resisted by the regional authorities responsible for hospital care (Pomey and Poullier, I997 p. 58). According to the 1974 Convention, costs per day should remain at least at half the costs of medical-hospital services (Artot, I989). In practice, the costs lie at about one-third.

Nowadays, HAD benefits from new technological developments, because medical equipment that could formerly only be installed in hospitals has been reduced in size and its user-friendliness has increased considerably, so that they can be transported to the patient's home now, such as continuous chemotherapy (more than half of the HAD patients suffer from cancer (Com-Ruelle, Midy et al., 2000)). The future of HAD structures is difficult to predict: it can be extended to more serious cases, especially in the field of cancer treatment and palliative care, or focus at less severe cases and serve as 'intermediate' service for patients that still need medical care but no longer have to stay in hospital.

HAD can be provided to patients that were hospitalised in a state owned hospital, or a private hospital when approved by the insurance scheme, or on GP request. A nurse then pays a diagnostic visit to the patient and determines whether the patient's condition and situation is suited to receive care under HAD at home. The prescribed nursing care consists of very intensive or highly technical nursing care. With HAD, physicians practicing in the community (médécins de ville) can now take care of their own patients whose severity of the illness goes beyond their 'normal' competence, because of the support by the hospital. For example, the Paris HAD co-operates with 3000 community physicians (presumably GPS as well as private specialists) that are offered extra training. The patient is regularly visited by his or her own GP, who also supervises the HAD care provided. In case of a change in the patient's situation, he or she can return to the hospital for consultation and reassessment of the treatment. Once the patient has been accepted for HAD, he or she receives daily care by a multi-disciplinary team, consisting of home nurses, nursing auxiliaries and home helps, dieticians, and social assistants (Delaunay, Teletchea et al., I996; Artot, 1989). These work together and co-operate with independent physicians and hospital professionals. The continuity of care provided by the different professionals in the own environment of the patient is considered as substantially contributing to their quality of life. 
Most of the nurses performing HAD services are employed by these structures, although independent nurses are also hired. When these need training to perform specific technical activities, the HAD structure organises and pays for that. Nursing auxiliaries also have an important role in the provision of HAD, and work in close co-operation with nurses. They are not allowed to perform (medical) nursing activities, not even under delegation. Their activities are restricted to personal hygienic care, observation and prevention, confusingly referred to as 'nursing' in France (Com-Ruelle, Midy et al., 2000 p. 33). Currently, the complementary role of nurses and nurse's aides and the more prominent role of aides are being discussed by the profession and at governmental level now, as nurses' tasks gradually shift towards co-ordination of care, while nurse's aides more and more perform care at the bedside. A revision of the décret de competence, i.e. the legal regulations regarding the competences and the activities nurses are allowed to perform, including a possible increase in salary, is under construction now.

\subsubsection{Service de Soins Infirmiers à Domicile pour les Personnes Agées (SIAD)}

The kind of home nursing that was set up in the form of community-based care, emphasising the provision of care to elderly and vulnerable people to enable them to remain living in their own homes and which is comparable to home nursing provided in other countries is called Service de Soins Infirmiers à Domicile pour les Personnes Agées, or siad (sometimes also called ssiad or siADPA) (Com-Ruelle, Midy et al., 2000 p. 3I). This service falls under the Ministry of Social Affairs and National Solidarity, which is responsible for the elderly. Home nursing was not established in France until I98I. In the I975 law on social and socio-medical institutions, home nursing services were foreseen, but were officially recognised as late as I98I. Until then, home nursing had been a small, entirely self-employed service belonging to the private sector. In I98I SSIAD regulations were specified for the distribution of nursing care and 'nursing' (i.e. personal hygienic care, observation and prevention (Com-Ruelle, Midy et al., 2000)), primarily to dependent elderly persons:

'Les services de soins infirmiers à domicile (SSIAD) (...) créés en mai I98I, ils délivrent aux personnes âgées maladies ou dependants, sur prescription médicale, les soins infirmiers médico-techniques ou d'hygiène assurés par des professionels et aides-soignants. Leur financement relève de l'Assurance maladie sous la forme d'un forfait journalier.' (CREDES, 2002)

Also, the objectives and operating conditions were defined:

'The objective assigned to them is to prevent, postpone, or shorten stays in hospital and in socio-medical establishments (governed by the hospital or social 
sector). Their principal function is to give nursing and personal care (by carrying out specific care that enables people to perform basic tasks of daily living). The care should be more continuous and better co-ordinated than that provided on a fee-for-service basis.' (Henrard, Ankri et al., I99I p. I08)

Home nursing in France is organised by public and private bodies and different types of providers, such as a local social service centre of a municipal authority, a health centre, municipal nursing care centre, a retirement home, or a home help organisation (Henrard, Ankri et al., I991 p. I08). There is much variation in the kind of services delivered; sometimes a whole package is offered, including mealson-wheels, télé-alarm and maternity care, sometimes only home nursing (Verheij, I996 p. I20). The geographical scope of these organisations varies considerably; some cover the entire Department, some only cover the local municipality. A medical prescription is needed for reimbursement. General physicians write nine out of ten prescriptions, compared to one by specialists. Remarkable is that a relatively small part of the population benefits from nursing services; namely in I992, $5 \%$ of the population consumed as much as $82 \%$ of the expenditures on nursing (CREDES, I998).

A large problem in France is that the need for care does not guarantee the provision of services:

'If it is estimated that about 500.000 people alone are dependent just for the activities of everyday life, then about Io per cent benefit from the service, which necessarily implies that independent nurses, and especially the informal support of family and the neighbourhood, play a predominant role.' (Henrard, Ankri et al., I99I p. I09)

The beneficiaries can be divided into three groups, namely Io\% that only require very little help with basic activities, but having psychiatric problems or being incontinent, 54\% more serious cases that require partial help with basic activities, and 36\% that are very limited in their abilities and need total help with all the basic activities. Whether home nursing care is prescribed or not depends on the knowledge of the existence of these services of physicians, elderly people and their families, and the local presence and availability of these services (Henrard, Ankri et al., I99I). This diverges from the situation in other European countries, where home nursing is considered as a major, well-established sector of the health care system providing for the needs of elderly and vulnerable people (except maybe for Germany, where there are also large difficulties regarding availability and reimbursement).

One of the largest problems in France is thus a large mismatch between people and home care services: some elderly receive services they do not need, while other, 
often heavily dependent people receive no home care at all (Henrard, Ankri et al., I99ז; Verheij, I996; Colvez and Ridez, I996):

'On estime que 20 à $30 \%$ des personnes servies sortent de leur domicile sans aide et n'ont donc que des incapacités modérées, tandis que près de la moitié (47\%) des personnes confines au lit ou au fauteuil ne bénéficient d'aucune aide.' (Colvez and Ridez, I996 p. I64)

Other studies confirm this picture (Curtis and Bucquet, I987; Aublet-Cuvelier, Barberger-Gateau et al., I99I). This implies that a substantial part of the services provided do not reach those who need them most. Especially the high amount of older women living alone is a matter of great concern, as this is the most vulnerable group in need of home care. In France, about 30\% of the women aged 65-69 and $48 \%$ of those $80-84$ are living alone. This implies an enormous number of people; namely 2.7 million of elderly women over 60 years of age are living alone in France, and I.I million women of 75 years of age and over. In I995, France counted about 8 million people of 65 years and over, of whom 700 ooo were heavily dependent (Com-Ruelle, Midy et al., 2000 p. 3I and 84). The number of men is only one-third to one-fifth of this number. About 430000 elderly people are cared for in their homes. Their average age was 8I years (I988); 80\% was 74 years or over. Thus the client population of home care is quite old, as elsewhere in Europe. Among those receiving help, a quarter lives alone, a third live with their partner, and a third with a younger person. Although the home nursing domain has increased considerably in size during the last decades, the 56650 places provided are far from enough to meet the existing need for care; these do not even provide for one-tenth of the estimated need.

Initially, the establishment of home nursing services was resisted by independent nurses (infirmières liberales), which are private nurses working on a fee-forservice basis. They opposed to the idea that salaried nursing managers of organisations were given responsibility for co-ordinating nursing care services for individual clients, as they considered themselves as the most appropriate actors for this task (Henrard, Ankri et al., I99I p. I0I). They feared their position and the autonomy they had built up in the local community would be threatened by the establishment of regular home nursing services with employed nurses. However, as we shall see later on, the knowledge of independent nurses about local community-care services is insufficient to legitimately claim this co-ordinating role (CREDES, 2002). Nevertheless, the role of independent nurses is substantial in France. Their large number and their strong competitive position due to the fact that they operate in a niche that is left by the insufficient availability of regular home nursing services, make them into important actors in the provision of home care. Verheij estimates that as much as $69 \%$ of the total home care services are 
provided on a private basis (Verheij, I996), for a large part by these independent nurses. This differs from elsewhere in Europe, where private provision is only marginal (The Netherlands, UK) or almost non-existent (Denmark).

\subsubsection{The growth of the home nursing sector}

The home nursing domain has increased enormously in size during the last decades. In I98I, it only offered 3000 places (i.e. bed equivalents). In I984, the 650 home nursing organisations already offered 22200 places, signifying an enormous growth within a few years. These employed I 400 salaried nurses and 3500 auxiliaries, while as much as 3500 independent nurses had an agreement with a care service to provide services on a fee-for-service basis (Henrard, Ankri et al., I99I p. I09). Thus, initially, as much as half of the care provided was performed by independent nurses. There were however major geographical disparities: about one-third of the Départements had a capacity of less than 3.5 places for I oo० inhabitants of 75 years of over, which is almost negligible, while twelve Départements benefited from a capacity of more than ro places for I ooo people of 75 or over. The national average was 5.9 places, or 0.7 nurses or aides per I 000 people of $65+$ or I.4 per those over 75 years of age.

In I996, the home nursing domain had increased further in size, and all 56650 places offered by the I 547 organisations were fully deployed. Despite this incredible growth, the needs of the elderly population were still not sufficiently met (CREDES, 2000; Verheij, I996; OECD, I996e p. I37). In table 2, the increase of the sector is summarised:

Table 2 Increase of the home nursing sector between I98I and 1996

\begin{tabular}{lrrrrr}
\hline Year & 1981 & 1984 & 1987 & 1990 & \multicolumn{1}{c}{1996} \\
Number of places & 3000 & 22200 & 28200 & 40000 & 56650 \\
Number of organisations & & 650 & 780 & I I 99 & I 547 \\
\hline
\end{tabular}

Sources: Henrard, I99I; Verheij, I996; CREDES, 2000.

The role of home nursing is however still rather marginal. Table 3 shows that the number of people receiving home help is about ten times higher than those receiving home nursing: 7 beneficiaries of $65+$ receive home help and io of $75^{+}$, compared to 0.6 of those $65+$ receive home nursing and $1 \%$ of the elderly of $75^{+}$.

Thus, the role of home help is far larger than that of home nursing and appears to be an important source of support for elderly people in France. Therefore, the domain of home help is also dealt with in this chapter. 
Table 3 Percentage of beneficiaries of home help and home nursing services by age, I9 88

\begin{tabular}{lcc}
\hline Age groups & Home help & Home nursing \\
$60-64$ & 0.4 & 0.05 \\
$65-69$ & 3 & 0.1 \\
$70-74$ & 4 & 0.2 \\
$75-79$ & 8 & 0.5 \\
$80-84$ & I2 & I \\
$85^{+}$ & I4 & 2 \\
Total $65^{+}$ & 7 & 0.6 \\
Total $75^{+}$ & I0 & I.0 \\
\hline
\end{tabular}

Source: Henrard, Ankri et al., I99I p. 323.

However, even more important is the role of the family and neighbourhood. Table 4 and 5 give an indication for the large amount of support by the social and family network. It also shows that care provided by a professional or volunteer is especially offered to elderly people living alone. The care that is not available from an in-living relative is then taken over by professional or volunteer care. When elderly are living with spouses or other family members, then the percentage of professionals or volunteers is only 28 to $35 \%$. In most cases, professional care, family care and care by a friend or someone from the neighbourhood is overlapping and elderly receive support from multiple sources.

Table 4 Percentage of people of 65 and over, having one (or several) disabilities without being confined to bed, according to the kind of support received

\begin{tabular}{lcccc}
\hline Source of help & \multicolumn{2}{c}{ Whole population of $65^{+}$} & \multicolumn{2}{c}{ Population of $65^{+}$living alone } \\
& Ile de France & Rhône-Alpes & Ile de France & Rhône-Alpes \\
Professional or volunteer & 28 & 35 & 40 & 50 \\
Informal care, total & 94 & 93 & 88 & 78 \\
Of which: & $7 \mathrm{r}$ & 75 & 49 & 52 \\
- member of the family & 23 & $\mathrm{I} 8$ & 39 & 26 \\
- neighbourhood/friend & &
\end{tabular}

Source: Henrard, Ankri et al., I991 p. 324.

The figures also suggest that in the Rhône-Alpes region, the infrastructure of care is such that a relatively high amount of professional care is provided to elderly people compared to other regions. This points to the regional difference in caregiving patterns in France. 
Table 5 Percentage of people aged 65 years or over, living at home and confined to bed or armchair, according to the kind of help received

\begin{tabular}{lccc}
\hline Source of help & Hte. Normandie & Ile de France & Rhône-Alpes \\
& $(1978-79)$ & 1982 & $(1986)$ \\
Professional or volunteer & 4 & 26 & 45 \\
Member of the family & 82 & 95 & 86 \\
Neighbourhood/friend & I4 & 5 & 8 \\
\hline
\end{tabular}

Source: Henrard, Ankri et al., I991 p. 323.

\subsubsection{The uneven distribution of services: one of the peculiarities of home nursing in France}

The largest problem regarding home nursing in France is the difficulty of realising an equal availability and distribution of services over the different regions. The city and region around Paris, the Île the France, is best provided, whereas other parts of the country have far less provided with home nursing services. The problem is especially large in the more remote and rural parts of the country. This has to do with the structure of France, geographically as well as politically and socio-economically. Namely, the population itself is unequally distributed over the country. The city of Paris counts as much as 9.5 million inhabitants (United Nations, 2000), while the rest of the population is unevenly distributed over other parts of the country. Twenty-five per cent of the French population lives in non-urban areas. The country is administratively divided into 96 Départements or districts that are grouped into 22 regions. Nine-tenth of France still consists of farmland and forests, which contrasts sharply with the highly urbanised Paris area and growth poles of regional development, such as Lille, Bordeaux, Lyon, Marseille (Métropoles d'Equilibre, intended to create a greater balance between Paris and other cities) (Britannica, I992). The infrastructure of major highways and railroads, most of them leading back and forth to Paris, the low number of navigable waterways, 9 and the Massif Central, a vast plateau of about $86000 \mathrm{kms}^{2}$ (about one-sixth of the country's area) form structural as well as natural obstacles hindering easy access to and transport between different regions and larger towns.

As much as $93 \%$ of the 36000 communes or municipalities are classified as rural (Kemenade, I997e p. 59; Giarchi, I996g p. 209). Almost half of the com-

9 Most large rivers, such as the Loire, Rhône and Rhine are of more use for power generation, the winning of sand and gravel, and the use cooling water for atomic power stations than for commercial navigation, due to rapid flow or dangerous currents (Britannica, I992). Most rivers are rising substantially and sometimes flooding in winter or spring due to heavy rainfall and melting ice and snow and thus unsuitable for infrastructural purposes. 
munes have fewer than 300 inhabitants. Since the I96os, groups of between I5 and 20 communes have been amalgamated administratively under a market town to form a viable service unity (Britannica, I992). However, the figure of I 547 home nursing organisations at a total of 36000 (or about I 800 larger groups of) communes indicates that by far not every commune or even group of communes disposes of a local home nursing organisation. Clients thus have to turn to an organisation farther away, with obvious consequences regarding their accessibility by home nurses.

Specific for France is that since the mid-nineteenth century, there has been a marked depopulation of remote, mostly mountainous, rural areas, and an increase in urban growth. For example, between the I86os and I970s, the rural population decreased by more than one-third. While the growth and fertility rate of urban areas increased because of the younger age of the urban population and because immigrants primarily settled in the cities, the rural areas were left with an ageing population and low birth rates. France has one of the lowest population densities in Europe, thus the social network to rely on in these areas is thin and the elderly population is quite vulnerable. Many elderly people have become physically as well as socially isolated, as their family and social network is declining, and social, economic, and medical facilities are difficult to be maintained in these sparsely populated areas. These people mostly hardly have access to professional services, as the delivery of health care is quite difficult and expensive in such poorly resourced parts of the countryside (Giarchi, I996g p. 206). For home care professionals, the distance between their clients is incomparable with the situation in e.g. The Netherlands that has a high population density, flat landscape, and high number of Home Care organisations and employed professionals. Their clients are generally within easy reach and often still visited by bike. The situation in France is totally different. Even when distances in kilometres are reasonable, the geographical characteristics of the country make it very difficult to reach elderly people in remote and/or mountainous areas and provide them with sufficient help. Whereas in the Netherlands, a part of the very dependent client population is visited several times a day, this is not possible in France. Therefore, great concern is expressed within governmental departments over the one-third of the older French population who live in these areas.

Thus, one of the major problems is that home nursing services are quite unevenly distributed over the country and sometimes not available at all. According to Verheij (I996), two-thirds of the services are located in the Paris area where about one-fifth of the total French population is residing (Britannica, I992). Thus, there is a quite uneven balance between the Paris region and the rest of the country. In some of the most remote rural areas, where extramural services such as home nursing are non-existent, the local authorities are presented with the situation that the social and health needs of elderly people are not met. France, lacking a rich 
heritage of voluntary or charitable activities, unlike for example the United Kingdom, the situation of these vulnerable people is worsened (Giarchi, I996g p. 210; Pomey and Poullier, I997 p. 52). However, more recently, some voluntary, mostly humanitarian groups have come to existence, such as older people that work for older people (Giarchi, I996 p. 206).

Although the ageing of the population is common to other European countries, it is of considerable social and economic importance in France. It also has a long tradition, as it has already been felt since the early igth century because of the low birth rates and the long life expectancy. Therefore, the French government has stimulated the founding of families by special subsidies and tax benefit schemes for many years, and the French fertility rate of I.7 is now one of Europe's highest (together with the UK and only recently Denmark) (Eurostat, I999; United Nations, 2000; Harismendy, I999):

'France has been concerned with its low birth rate for years and worries that in a few generations there will be no more French men and women. The French birth rate is currently below the replacement level, (...), but it is higher than the birth rate in West Germany (...) and in Britain (...). Perhaps the incentive given to pregnant women and young families - totally free medical care plus allocations familiales, periodic payments for each child regardless of income - have had their effect.' (Payer, I988 p. 48$)^{\text {Io }}$

Nevertheless, the unbalanced age structure of the population and the steady increase in the proportion of elderly people clearly puts a strain on the working population to provide pensions, medical and social services, and retirement housing (Britannica, I992).

\subsection{The development of home care policies in France}

Regarding home care policies, France also takes a rather specific stance. Firstly, the central state takes a large role in devising health care structures and health care policies (Henrard, Ankri et al., I99I p. 99). Secondly, in these structures and policies, the importance of the family is emphasised again and again. Already since the I96os, elderly policies have been developed in which the importance of maintaining elderly people in their homes, for a large part supported by their fam-

Io See also Harismendy, I999. 
ily, was stressed: 'La politique de maintien à domicile des personnes âgées dépendantes' (Clément, I993 p. I40; Colvez and Ridez, I996 p. I64).

Until the Ig6os, there were no specific policies for older people as such. The majority of elderly people still lived with their families. Only assistance of the poor (mainly elderly, isolated people) in cash or in kind was provided. From these help schemes, some charitable organisations developed, initially financed by the departmental social aid and since the late I950s by the primary sickness benefit funds. Sickness insurance and old age insurance were then financed by a common fund that was part of the general security system. A complementary benefit was added to the care provided for physicians and home help tasks. Home help was then considered as auxiliary function to nursing and aimed at avoiding hospitalisation.

In 1962, the Laroque report Politique de la Vieillesse, Rapport de la commission d'étude des problèmes de la vieillesse was issued by the Commission for the Study of the Problems of Old Age, installed by the Fifth Republic and chaired by P. Laroque, one of the founders of the Social Security. In this report, also referred to as 'Policy for the elderly', social policies for elderly were outlined that should enable older people to remain in their own homes. In French policies in the I960s and 7os, home nursing and home help have been proposed as an alternative for hospitalisation and admission to retirement homes (Colvez and Ridez, I996). The notion of 'old age' was replaced by the notion of 'troisi me ge' (la troisième âge) (Henrard, Cassou et al., I990; Henrard, I996) and aimed at replacing the social image of elderly people as being in need of support, passive, excluded and dependent, by the image of becoming active, oriented towards leisure activities, and maintaining relations between generations (Clément, I993). This new vocabulary about elderly stressed the possibility of pursuing social and leisure activities and greater independence. Old age itself ('quatri me ge') was postponed to a later age, and acquired a purely negative image and was confused with incurable illness.

According to these policies, a package of measures and services should be developed to ensure the 'social integration of elderly people' and avoid the admission into institutions of people whose autonomy was affected. Housing problems were to be solved, social activities proposed, and help with domestic tasks and health problems should be offered. By this development, home helps differentiated from being mere nurse's auxiliaries, assisting with nursing tasks and paid by sickness benefit, to professionals independently performing supporting activities in order to prevent institutionalisation of elderly people (paid by the old-age insurance schemes). In I967, home help came to be financed by the old-age insurance that was separated from the sickness benefit.

Proposals of the i962 Laroque report were incorporated in the sixth and seventh governmental five-year plans for economic and social development (I970-74 
and I975-79). The aim to prevent institutionalisation of Ioo o०o elderly people was formulated by a scheme of solving housing problems, offering home nursing care and help with domestic tasks, and social activities such as senior's clubs in 322 areas. The social participation of retired people and the improvement of their homes became compulsory for these areas. The responsibility for the choice of services was left to the local authorities; while the financing was shared between the state, benefit offices and the local communities. Due to the financial crisis affecting the expenditures on social services of the local governments, the cheapest services reaching a maximum of people were chosen, namely primarily clubs, cultural and physical activities, and information campaigns. Regional sickness benefit offices hesitated to finance health care services in the home because of the resistance of independent nurses.

However, reports issued in later years, such as the report Vieillir Demain (Growing old in the Future) of I982, stated that the policy aims were not achieved sufficiently yet. Although the number of elderly that lived in with their children was diminishing at that time, the report nevertheless stressed the importance of continuing relations between generations. Keeping elderly in a family environment should be facilitated and a complementary relationship between relief provided by professionals, volunteers and family members should be developed. In i98I, a specific ministerial structure was created to implement the proposed policy and home nursing was officially recognised, thereby enabling their rapid expansion. Home nursing services were intended to help the family or neighbourhood to take a dependent elderly person in their homes and look after them in their day-today life, supported with some nursing care, thereby avoiding separation through hospitalisation or institutionalisation. Home nursing was to enforce relations at neighbourhood level, and was integrated into the general societal scheme advocated by the central government of taking responsibility for elderly people. About 500 posts for local service co-ordinators were created. These were to improve the links between home care services and institutions (OECD, I996e p. I38), but after I983, the financing of these posts was transferred to the local level and only a few of these were continued because most municipalities had to reduce social expenditure. Emphasis was then put on neighbourhood services (solidarities) (Henrard, Ankri et al., I99I). Remarkable is that in France, home care facilities were closely linked with family structures and considered as a complement to care provided by family members.

The I982 Braun report (Braun later became the Minister for Elderly People) reaffirmed the priority of soutien à domicile and proposals were issued for the implementation of home-care policies and promotion of maintenance within families. In I986, a governmental circular again promoted family caring arrangements by suggesting the development of home-based alternatives for hospitalisation. Although the wish to curb hospital expenses was the main motivation, it 
was wrapped in the vocabulary of the value of the family environment. The intention was to shift financial and personnel sources to socio-medical institutions and intermediary forms of care, like day hospitals (shelters) and nursing services, thus facilitating the 'maintenance of contact with the family'. However, these policies had too little effect:

'nearly 30 years after the Laroque report, 'maintenance in the home' had not given rise to the implementation of a co-ordinated global policy. Successive surveys show an increase in the number of people living in institutions, partly linked to the quantitative insufficiency of means of help at home.' (Henrard, Ankri et al., I99I p. I02)

Whereas the establishment and development of structures for home nursing was opposed to by independent nurses, home-based services were not very active themselves in advocating policies for elderly people for a long time. Only in the course of the rg8os, the 'National Union of Associations for Care and Services in the Home' has become aware of its role as partner and creator of socio-medical policy together with financing bodies and locally elected representatives. The French population itself never acted on the problems of maintaining elderly people in their homes, probably because this was primarily considered a private matter and not an issue for public debate. Nevertheless, due to continued policy efforts to expand the domain of home care, also in France currently home care services exist, providing services in the homes of patients/clients and offering services that are accessible for elderly people in the neighbourhood, such as eating a meal in a luncheon club. However, the regional availability of services remains a bottleneck, and needs are still not sufficiently met. This implies that a large amount of care is expected from family members and informal carers.

\subsection{The home nursing profession; short historical sketch}

Until well into the first half of the twentieth century, nursing was strongly subordinate to the medical profession and associated with a religious calling, like elsewhere (Clément, I993; Piatkowski, I994; IFSI, 2003). From the beginning of the nineteenth century until the First World War, the image of woman performing caring activities began to emerge, including the right of training. However, specific knowledge to care for people was not obligatory at that time, in the hospitals as well as in the homes of patients. In I902, the role of nurses is described in a circular, and the first nursing school is established in Paris: the école d'infirmières at the hospital Salpétrière (Drouet, I995). Hospital physicians tried to appoint more competent staff. The activities of nurses were still purely executive and aimed at 
supporting medical tasks. During the First World War, the role of nurses and their skills became more and more recognised by the population.

In I922, a state nursing certificate was officially recognised by decree (Brevet de capacité professionelle) that allowed nurses to use the title of Infirmière diplomée d'État, in I938 followed by a State Nursing Diploma (Diplôme D'État d'infirmier). Since I946, this diploma is legally required to perform nursing activities and can be acquired after two years of training. Non-qualified persons were allowed after examination to perform limited nursing activities or work as an auxiliary. In I95I, the first nursing school for nurse co-ordinators was established by Decree and in I958 it issued its first certificates.

In I978, the role of nursing was reformulated. Instead of being purely subordinate to the medical profession, it became recognised as a profession with an independent job description and domain. Although its activities are closely related to the activities performed in the medical domain, its independent tasks and activities are more and more recognised. The task description of nursing is further developed in more scientific terms.

In the I980s, several laws and decrees were introduced that officially recognise and circumscribe the role, activities and training of nurses, such as the I980 law on nursing practices, or the I984 Nomenclature générale des Actes Professionels (NGAP; a list of professional activities that was established by decree) (CREDES, 2002). In I999, the Ministry decided to renew the nomenclature of nurses to account for new kinds of activities of independent nurses, for example regarding new developments in the domain of pain care, palliative care, and intensive home care. The list is to evolve and reckon with planning and co-ordination of activities around the patient's bedside, and not only be a list linking activities with tariffs. The debate about this is initiated by the Projet de Soins Infirmiers (PSI), as the execution of this project requires more (diagnostic and evaluative) skills of nurses that take over these tasks from physicians.

In February 2002, the renewed version of the décret de competence (firstly established in I993) was introduced, describing the role and tasks of the nursing profession. Currently, the role of the nursing profession in the French health care system is being transformed, due to several developments identical to those in other countries, such as a changing demand (qualitatively and quantitatively; a greater demand for care than cure, and the increase in the number of elderly), medical developments (technological as well as mentally, modifying the supply of care and medical practices), and the increasing health care costs that demand for a more efficient organisation of care provided by the health care system. Also, the competencies of nurses are expanding because of their increased technical expertise. Consequently, the role of nursing is shifting towards more responsibilities for nurses and a new kind of relation with physicians. These developments are reflected in the regulations concerning delegated tasks: e.g. in the domain of 
pain care, protocols for delegated tasks have been adjusted by enlarging the competencies and responsibilities of nurses. ${ }^{\text {II }}$ They have been given more autonomy to perform certain tasks described in the protocol, without the need to refer to a physician. Recent developments aimed at modernisation of the executing nursing staff are the revision of the decree of competence, the Project de Soins Infirmiers (PSI; nursing project, that is described further on in this chapter) in the private sector, and the development of nursing protocols in the hospital sector.

\title{
5.6 The current home nursing profession
}

\subsubsection{Legal regulations describing the domain and activities of nurses}

In France, the role of nursing is strictly defined in the statute of the paramedical professions and the décret de compétence that was revised in 2002 (Com-Ruelle, Midy et al., 2000 p. I3). In the diverse articles of the Code de la Santé publique or Public Health Act, the domain of activities of the nursing profession is described:

\begin{abstract}
'Est considerée comme exerçant la profession d'infirmière ou d'infirmier toute personne qui donne habituellement des soins infirmiers sur prescription ou conseil médical, ou en application du rôle propre qui lui est dévolu. L'infirmière participe à différentes actions, notamment en matière de prévention, d'éducation de la santé et de formation ou d'encadrement.' (Com-Ruelle, Midy et al., 2000 p. 29)
\end{abstract}

To this role belongs nursing care that is aimed at supporting people to care for themselves and partly or completely compensating for a loss or reduction of autonomy. The role of nurse's aides (aides soignants) is also specified and is restricted to personal hygienic care, observation and prevention, confusingly described as 'nursing' in French, ${ }^{\mathrm{I} 2}$ and tasks supporting activities of daily life. The role of these nursing auxiliaries in the provision of care is very large in France and increasing,

II The latter consist of activities that are prescribed by physicians but performed autonomously, activities prescribed by physicians under the condition that a physician can intervene every moment, and technical acts in which nurses are only allowed to participate in the presence of a physician (Com-Ruelle, 2000 p. 29).

I2 The use of the term nursing is quite confusing as in other countries, this refers to the nursing profession in general. However, the concept of 'nursing' used in the CREDES report refers to tasks of personal hygiene, observation and prevention. 
especially in the expanding domain of social care, where they support elderly and handicapped people to maintain their activities of daily life.

According to the Code de la Santé publique (Public Health Care Act), only nurses with a State Nursing Diploma (Diplôme d'État Infirmier) obtained at an Institute for Nursing Education (IFSI; Institut de Formation en Soins Infirmiers) that is acknowledged by the Ministry of Health, or an equivalent diploma, are allowed to perform nursing activities (Com-Ruelle, Midy et al., 2000 p. 27). Since April 2000, this right is issued per individual nurse by the Prefect of the region after advice of a regional committee. After that, the nurse is registered in the Direction Départementale des Affaires Sainitaires et Sociales (DDASS) in the category concerned (infirmier exerçant à titre liberal, infirmier salarié du secteur public, du secteur privé ou de secteur psychiatrique, infirmier specialisé; nurse working independently, salaried nurse working in the public sector, private sector, or psychiatry, specialised nurse).

Rights and duties and general conditions of professionals fall under the Code de la Santé publique and the Code de la Sécurité sociale (and in certain cases the Code du travail or the Code pénal). The wages of nursing professionals are related to the national expenditures on care and fall within the framework of the Act on the Financing of the Social Security (Loi de Financement de la Sécurité sociale) (Com-Ruelle, Midy et al., 2000 p. 23). Expenditures on ambulatory nursing care were about ig billion French francs in 1998 and 20 billion in I999, which was about $2.1 \%$ of the total medical consumption in France. This indicates an increase of $5 \%$ of expenses on nursing and a volume growth of $4.2 \%$, which can partly be contributed to increased siad activities. Independent nursing activities, that fall under the Convention Nationale des Infirmiers ( $\mathrm{CNI})^{13}$ account for about 7.7 billion francs. Between I995 and I998, reimbursements on nursing care (both salaried and independent sector) have increased with $2.1 \%$ per year.

The Convention Nationale des Infirmiers states that independent nurses are paid on a fee-for-service basis according to the nomenclature of professional activities, that distinguishes between personal hygienic care (Actes Infirmiers de Soins) and technical nursing activities (AMI; actes medico-infirmiers). AIs activities account for just over half of the nursing activities performed in I997, namely $64 \%$ of the expenditures on independent nursing care. The sickness insurance (Assurance maladie) reimburses all or part of the care provided to the patient according to codes that correspond to fixed tariffs. To prevent an increase in costs, quotas limit activities of independent nurses. When nurses exceed this limit, they have to pay part of their income back to the sickness insurance. This limits the volume of

I3 The CNI is agreed upon by the liberal professions and the bodies of the Sickness insurance. 
their income and is intended to ensure that infirmiers liberales work under circumstances that are compatible with providing quality of care.

\subsubsection{The organisation and training of nurses}

As elsewhere, three main categories of nursing professionals can be distinguished in France (Com-Ruelle, 2000, introduction):

- Infirmiers specialisés; specialised nurses working in hospitals, management or education;

- Infirmiers qualifiés; qualified nurses, also described as infirmier diplômé d'Etat (state certified nurse) or infirmier de premier niveau (first level nurse);

- Infirmiers non qualifiés; non-qualified nurses, also described as infirmier de second niveau, auxiliaire, assistant or aide-soignant (second level nurse, auxiliary, assistant or nursing aid).

Education takes between one year for aides-soignants and auxiliaries de puériculture (nurse's aides and child care auxiliaries) and five years for specialised nurses such as infirmiers anesthésiste (anaesthetic nurses) (Com-Ruelle, p. 27). The basic diploma of general nurse (soins infirmiers généraux) takes three years of in-service training in an Institut de Formation en Soins Infirmiers (IFSI), with 2240 hours of theoretical courses and 2275 hours of practical training. ${ }^{\mathrm{I}}$ After that, further career possibilities are found in staff or educational functions, child care (with one year of additional training), operating staff (9 months additional training) or anaesthetic nurse (24 months additional training). During the last decades, nursing education in France has become longer and more demanding due to medical and technical developments and the increase in nursing and coordinating tasks. Students entering nursing education currently have a higher educational level; $94 \%$ of them have finished secondary education.

There are several professional federations (syndicats) and associations. Generally, each nursing category has its own organisation, such as a national federation for operation nurses, health education nurses, etc. For example, the Fédération nationale des infirmiers libéraux (FNI; national federation of independent nurses) negotiates about the national regulations for independent nurses with the Sickness insurance (Assurance maladie). However, the capacity of nurses to represent themselves or to act as a powerful united group is doubted in the literature, as they are hardly represented at the level of official bodies, especially the State and the Social Security, but also in hospital boards. At the Ministry of Health, there is no ministerial manager or department specifically responsible for nursing issues,

I4 In Com-Ruelle p. I35-136 this is described in more detail 
although a number of nurses have an advisory function for the Minister. Nevertheless, as in Germany, the nursing profession in France is locally represented in every Direction Régionale des Affaires Sanitaires et Sociales DRASS (Regional Bureau of Social and Health Affairs), that has an office of the Conseiller Technique Régional en Soins Infirmiers (Regional Technical Nursing Council). The Regional DRAss bureaus report to the regional prefect, and are responsible for the delivery of all kinds of health services, prevention and local health promotion, by planning public hospital facilities and health and social facilities via annual budgets and revision of the health care map (that was established in the Hospital Law of I970 (Giarchi, I996g p. I99) that is based on the regional and local needs (Kemenade, I997e p. 59). This way, nurses do have a voice in the way ambulatory care, hospital treatment and prevention are organised at the local level.

In France, there were more than 420000 infirmiers diplômés d'Etat (general and specialised nurses) in 2003 , corresponding to 7.I nurses per I 000 population (Eurostat, 2007b). As much as Io to $15 \%$ of these were working as independent nurses in the private sector in 200I (Eco-Santé, 2003). The total number of nurses in France is comparable to Germany, but much lower than in Denmark and the Netherlands, that have II.O to I4.0 nurses per I oo० population. However, the total number of second level nurses (auxiliaries) is much higher in France (7.4 per I ooo population in 2003) than in other countries; Germany only had 2.2 auxiliaries per I 000 population and Denmark 4.7 (Eurostat, 2007b).

Figure 2 Division of professions working in the French health care system

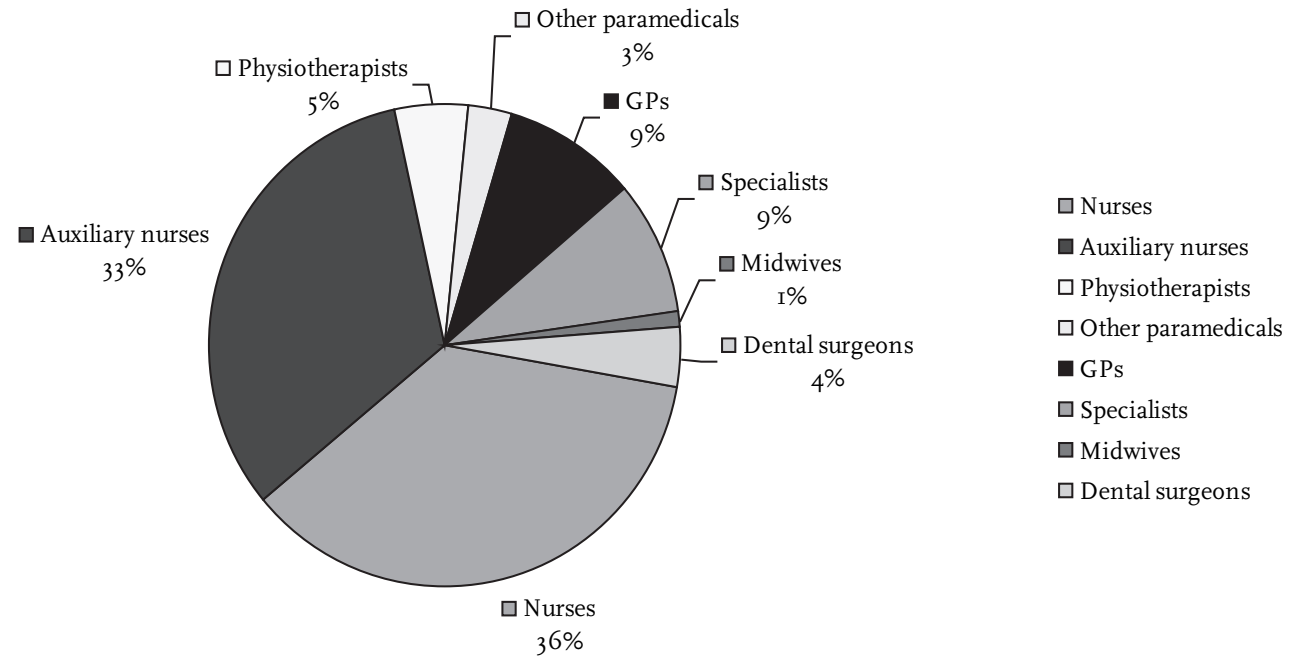

Source: Com-Ruelle, 2000 p. 20. 
Since I97I, the nursing profession experienced a steady growth: in I980, there were about 245000 nurses compared to 360000 in I998, which is an increase of $38 \%$. The number of nurses will further increase in the coming years, but at a steadier rate, as a fixed quota of students entering nursing education (I999) has been determined per annum. However, it is difficult to foresee whether the number of nurses entering the health care system will be sufficient to provide for the needs of the ageing population due to a lack of detailed studies determining the need for nursing care (Com-Ruelle, p. 23).

As in other countries, the nursing profession is the largest profession in the health care system (see figure 2).

As in other countries, French nurses are working in public and private health care organisations such as hospitals, HAD or SIAD organisations, retirement homes, health centres, school health care, or in the independent sector. The largest number of nurses, $59 \%$ of the total nursing FTEs, is working in hospitals.

\subsubsection{The role of independent nurses}

One of the most remarkable aspects compared to other countries, is that independent nurses are providing a substantial part of home nursing services in France. The independent, private sector has developed in an earlier stage than the public home nursing sector that was established in I981, and has become a sector of considerable importance. In this private sector, individual nurses (infirmières libérales) work in private practices (cabinets de ville) and perform nursing tasks that have been prescribed by medical professionals, similar to the regular services. They work from their offices, providing ambulant care, and in the homes of their clients (à domicile), both on a fee-for-service basis. Most of them hardly co-operate with the regular SSIAD services, as these are their competitors and they consider the employed home nurses as intruding and breaking down their relatively wellestablished local practices. The mean age of the client population of independent nurses high, namely 79 years. Many of those suffer from a chronic dependency and about half of them have cognitive or psychological problems. $37 \%$ are heavily restricted in their autonomy (having problems with dressing, personal hygienic care and food intake). As much as $28 \%$ are almost or totally dependent and not able to move themselves in their homes.

The independent nursing sector has developed more strongly than the public nursing sector: in $19838.6 \%$ of the nurses worked in the independent sector, which has increased to $16.8 \%$ in I998. The density of independent nurses is higher in the southern half of the country, while they are not very well represented in the Île-deFrance. Thus, in areas where regular public and private health care structures such as hospitals, HAD en SIAD organisations are relatively well available, such as in the Paris region, the independent nursing sector is not very extended. In contrast, in 
areas where the population is less covered by regular health care structures, the independent nursing sector is more developed. This suggests that the infirmières libérales provide their services in the niche that is left by the health care system. This explains why the independent nursing sector is primarily more strongly developed in those areas that are not very well covered by the regular health care system.

In France, the independent nurses are considered to perform the role of co-ordinator of medical, nursing and social services (home help and other social support services) to elderly people in their own homes. They have claimed to represent their patients and their families during many decades. It is stated in the literature that they are in a privileged position compared to employed home nurses, as independent nurses are well acquainted with the individual situation of their clients, as are assumed to have knowledge of the local situation and the local health and social care services. Their co-ordinating role is expected to be further developed during the coming years. However, recent studies show that the legitimacy of this claim can be questioned. Namely, in 200I, the Minister of Labour and Solidarity declared the wish to clarify the ways of co-ordination between independent nurses and the SOcial services (CREDES, 2002). To study this, a survey was held in 200I by CREDES, and independent nurses were asked to evaluate current co-ordinating practices. The study showed that independent nurses, as the patient's co-ordinator of care, contact 2.6 actors per patient on average; primarily the family $(86 \%)$ and the treating physician $(63 \%)$, and in about half of the cases, the local home help service. Social services and social workers are reported to be difficult to co-operate. After contact is established, the local home help service and the family (in almost 6o\% of the cases) mostly provide care. The SSIAD service is only contacted in $20 \%$ of the cases, as these are saturated and are mostly not able to offer any care. Nevertheless, when they are contacted, they do provide care in $40 \%$ of the cases. In $14 \%$ of the cases no solution is found after the independent nurse has contacted several professionals and services, because of lack of finances, unavailability of staff, or refusal by the patient or the family. This means the patient is then left without the care needed.

Remarkably, according to the survey, the most important problem regarding the co-ordinating role of independent nurses is that they know too little about the local health and social care facilities:

'La complexité du secteur de l'aide à domicile, y compris au niveau local, rend la demarche parfois malaisée. Un infirmier sur dix déclare de ne pas connaître les attributions des intervenants sociaux dans sa zone géographique; cette situation est cependant moins marquée en zone rurale.' (CREDES, 2002 p. 3)

Thus, the knowledge they are assumed to have about local health and social structures and professionals is inadequate and should be improved before they can legitimately claim their co-ordinating role. 
Also, the study also showed that independent nurses primarily focus on nursing tasks. While nationally, employed nurses perform about 30\% d'Actes infirmiers de Soins, referring to basic nursing tasks, $16 \%$ of the independent nurses perform $80 \%$ of these tasks. They also provide slightly more medical nursing tasks such as injections and wound-dressings than average. A large problem for patients is that independent nurses generally do not want to perform hygienic care (washing and dressing), as they do not consider these as their responsibilities. These are left to auxiliaires de vie, family members, or a hygienic nurse. Domestic tasks are mostly also left to aides ménagères (home helps) or auxiliaires de vie. Compared to other European countries, this only seems logical, as hygienic and domestic tasks are generally considered as not belonging to the domain of home nursing care. However, in France, this means that hygienic and domestic needs of patients are often not met. While in other countries, other professionals take up these tasks, for example nurse auxiliaries or home helps, in France, these professionals are not always available due to severe shortages in services in many regions. Thus, when independent nurses are refusing to perform these tasks, this leads to very difficult situations for many clients of whom most of them are dependent for washing and dressing. Therefore, current debates and discussions on nursing are focussing on the competencies and division of tasks among the different home care professionals in France.

\subsubsection{The role of nurse's aids or auxiliaries}

In I999, there were about 330000 nursing auxiliaries or nurse's aids (aides-soignants; ziekenverzorgsters) (Com-Ruelle, p. I9). Their number has even increased more rapidly than that of first level nurses, namely with $53 \%$ between I980 and I998. These aids primarily benefit from the expansion of the social sector, of which home help services are also a part. To compare: during the same period, the number of hospital professionals has not increased. The role of auxiliaries is very important in the provision of care in France. They provide most of the care, namely $73 \%$. Auxiliaries are not allowed to perform nursing activities, not even delegated tasks (Ibid., p. I4). The official assessment of the patient's situation after a medical referral is however performed by a nurse. These use standardised assessment forms on which the level of dependency of the client is indicated (Verheij, I996 p. I22). Table 6 shows that as much as $80 \%$ of the staff of home nursing organisations consists of aides soignants: of the staff total of I9 400 more than I6 000 are nurse's aids.

The share of first level nurses (that are trained for at least three years) in the provision of services is $\mathrm{I} 6.2 \%$ (I998). They primarily perform coordinating tasks and technical activities on prescription, such as injections or wound dressings. The increase in nursing services of 44\% between I99I and I996 (37\% in FTE's) is 
primarily due to the growth of activities of nursing auxiliaries that increased with $42 \%$ in FTES.

Table 6 Personnel of home nursing services compared to the number of independent nurses

\begin{tabular}{lcc}
\hline & Salaried personnel & $\begin{array}{c}\text { Independent nurses } \\
\text { (hired on a fee-for-service basis) }\end{array}$ \\
Co-ordinating nurses & I 930 & I9 350 \\
Nurses IDE & I 450 & \\
Nurse's aids & I6 020 & 22050 \\
Total caring staff & I9 400 & \\
Administrative staff & 2 I50 & \\
\hline
\end{tabular}

Source: SIAD, I996; Com-Ruelle, Midy et al., 2000 p. 32.

Nursing aids are not allowed to be salaried by a physician or to work independently in a private nursing practice; according to the I997 Convention Nationale des Infirmiers, they are only allowed to perform their activities on a salaried basis, for example in hospitals or in had or SIAD organisations. About $68 \%$ of them work in the public sector. Most of the nurse's aides (64.4\%) work in health care organisations, while an increasing number of them are working in the expanding social sector (e.g. home help), from $12.5 \%$ in 1983 to $29.2 \%$ in 1999 .

\subsubsection{Recent changes in the relation between physicians and home nurses}

A recent development in the provision of nursing care is the Projet de Soins Infirmier (PSI; Nursing Care Project), indicating a change in relations between physicians and nurses (CREDES, 2000; Com-Ruelle, Midy et al., 2000 p. 3I). Customary in France is that physicians, mostly GPS, prescribe nursing care and thus assess and diagnose the patient's need for care. When care is assigned according to the PSI procedure however, the GP prescribes PSI, and then a nurse is given Io days to present a Nursing Plan, in which the social and health status of the patient is evaluated on the basis of I4 basic needs and the kind and frequency of care needed is described. The plan then has to be signed by the prescribing GP. This procedure transfers the diagnostic role from physicians to nurses and thus indicates a significant shift in relations between them. As is stated in the theoretical chapter, this increases the degree of professionalisation of home nurses, as performing diagnosis is one of the essential characteristics of a profession.

However, the PSI project is still very much discussed, because it crosses the borders of the domain of health care and that of social care and thus has financial consequences. Independent nurses, that have a substantial role in home nurs- 
ing, oppose to the PSI project as they refuse to provide personal hygienic care to people with a stable health situation. They consider this the responsibility of aides soignants (assistant nurses) or professionals working in the social sector such as home helps. In the social sector, changes are also imminent because the state increasingly recognises the important contribution of the social sector in supporting older people in their own homes. Therefore, the responsibilities and competencies of aides soignants are being revised.

Since 2002, the PSI project has been replaced by the DSI: la Démarche de Soins Infirmiers (CREDES, 2002). The DSI intends to enable the maintaining at home of 350000 (temporarily or permanently) dependent people, irrespective of their age. After medical prescription, the independent nurse plans the activities needed by the client (living at home or in a shelter service) and proposes her plan of action to the physician. The nurse provides the care needed to maintain, compensate or restore the client's autonomy, formulates an individual care plan that is aimed at supporting the client to take up his/her own life, sometimes with help of several kinds of professionals, and evaluates the patient's (medical) condition and the care provided.

\subsection{The importance of the home help domain and care provided by the family}

\subsubsection{Traditional family patterns and values contributing to stigmatisation of professional home care}

The following part of this chapter describes the typical role of the family and the domain of home help in France. These are very important in the provision of care to elderly people living in their own homes, as the home nursing domain is rather weak and leaves gaps in the provision of care, that are partly filled by home help and especially by informal care. One of the reasons for the large role of informal care is that in France, the importance of family solidarity and intergenerational relations is considered as a given by the population as well as the government. As a result of this shared value and tradition, elderly people first turn to their partner and offspring for support, and only when help from both is not available, they turn to others, such as more remote family members, or members of their social network. Only when these are not available as well, they turn to professionals, but very reluctantly:

'Selon le modèle 'hiérarchique compensatoire', la personne âgée demande un soutien d'abord à son conjoint, à default à ses enfants: ce n'est qu'en l'absence d'aide possible de l'un ou de l'autre qu'elle se tourne vers d'autres members de la 
famille, voire vers un réseau de voisinage ou amical, ou encore vers les services spécialisées.' (Clément, I993 p. I40)

The health care system is structured according to the same principle, namely the principle of subsidiarity, that states that family members are the first port of call, and public services should only provide services when no other forms of care are available (see also the theoretical chapter of this thesis). This contrasts sharply with countries with a national health system and strongly developed welfare state, where values are adhered to that 'the system' (mostly the public health system, such as in the UK and Denmark) should primarily mobilise and guarantee sufficient forms of care. Informal care is then considered as a supplement to formal care, but not emphasised as primary source of care, such as in France. Especially regarding the appeal made on the family, the North - South difference in caring values that is described in the introduction can be recognised.

One of the reasons for the large role of the family is that traditional family patterns, such as living in three-generation families, are rather persistent in France. Especially in rural areas, cohabitation of parents and siblings is still rather common. The modernisation of family patterns that is taking place everywhere in Europe as well as in the more urban areas of France hardly seems to have penetrated in these areas. For example, in five rural cantons in the Haute-Garonne as much $46 \%$ of the parents are living with their siblings in agricultural families, compared to $21 \%$ in non-agricultural families (Clément, I993 p. I4I). As much as $73 \%$ of the siblings are living with or in the vicinity of their parents, compared to $40 \%$ in non-agricultural families. These are high percentages compared to urban areas and family patterns elsewhere in Europe, where cohabitation is less frequent and many sons and daughters have moved farther away for work. ${ }^{15}$ Then, the provision of informal care is more difficult or not possible at all and elderly people have to look for other kinds of care:

'M. Roland (76 ans, ancient ingénieur) dit de son fils et de sa belle-fille: "Ils sont très gentils (...) Si j'avais besoin d'eux, ils viendraient. Ils prendraient l'avion jusqu'à T. et le taxi jusqu'ici." Mais aussi: “C'est certain qu'ils ne viendraient pas pour me soigner au lit tous les jours. A 700 kilomètres, ce n'est pas possible. C'est leur vie, c'est la nôtre." C'est donc quasiment en terme de rupture que l'éloignement est vécu.' (Clément, I993 p. I43)

I5 Especially in north and north west European countries, cohabitation has become rather an exception than a customary family pattern. 
This quote shows, which is confirmed in several studies, that geographical distance of offspring is an important predictor for the availability and amount of informal care provided (e.g. Dautzenberg, 2000). Thus, it is assumed that the provision of informal care is higher in rural areas, where the majority of siblings are living with or in the vicinity of their parents. However, cohabitation is not always motivated by the wish to facilitate informal care. In these rural milieus, cohabitation is often motivated by economic reasons:

'... la cohabitation avec les parents a un caractère d'évidence pour nombre d'agriculteurs. Garder les parents âgés lorsqu'on hérite de la maison fait partie de la tractation économique, même s'il n'est pas du tout certain que la situation soit aujourd'hui bien vécu, dans le sense où cette pratique constitue un cas de plus en plus isolé dans une société qui choisit d'autres options.' (Clément, I993 p. I42 and I45)

Cohabitation is also not always the most comfortable situation for all persons concerned. Especially caring relations with the in-living daughter-in-law are often reported as difficult by the elderly parents: "la belle-fille, et parfois la fille, apparaissent comme concurrentes sur le terrain domestique, la belle-fille peut être perçue comme 'l'étrangère', la fille peut plus ou moins répondre aux attentes" (Clément, I993 p. I50). Thus, the daughter-in-law and sometimes the daughter are considered as intruders in the private household. But when the daughter-in-law is already considered as a 'stranger', this is even felt more strongly towards home care professionals. Indeed, studies show that French elderly people have strong sentiments regarding household and personal matters, explaining for a large part the reluctance of elderly people and their family members to call in professional help. This diverges strongly from the other European countries described in this thesis, where professional help is far more accepted and people often even feel they are entitled to professional care, as they pay for it by insurance contributions and taxes. Again, the kind of health care system (based on welfare state principles or, conversely, on the principle of subsidiarity), and traditional values shared by the population regarding the role of professional care and the role of the family, are important indicators that explain this diverging attitude between European countries. It appears that in France, receiving home care, and especially home nursing, still bears a stigma and is associated with elderly that are left in the cold by their family and social network. Thus, a strong moral obligation is felt by family members to provide care, which is also stressed again and again by politicians. For example, during the exceptional heat wave in the summer of 2003 , a lot of societal and political upheaval arose because almost I5 ooo excess deaths were reported, especially among (very) elderly people (Douste-Blazy and Falco, 2004). This initially led to a political reaction accusing people who went on holiday to leave 
their elderly parents behind and uncared for. Such a reaction would be unthinkable in Scandinavian countries, where professional home care is guaranteed and people are alleviated from caring responsibilities to family members (except from spouses). In 2004, the Plan canicule (heat wave plan) was presented by Douste-Blazy, minister of health and social protection and Falco, delegated minister for elderly people, introducing measures of prevention and intervention in case of future heat waves. The national heat wave plan describes the responsibilities of central, departmental and local governments, institutes such as Météo France, emergency services and the media and demands the development of local safe-guard systems for elderly people living in institutions as well as in independent households. The plan describes four alert levels; level one stands for alertness (la Vigilance), level two is a state of emergency (l'Alerte), and level three is the level where different kinds of interventions are planned (l'Intervention), see figure 3:

Figure 3 Organisational scheme of the third level of the Plan canicule

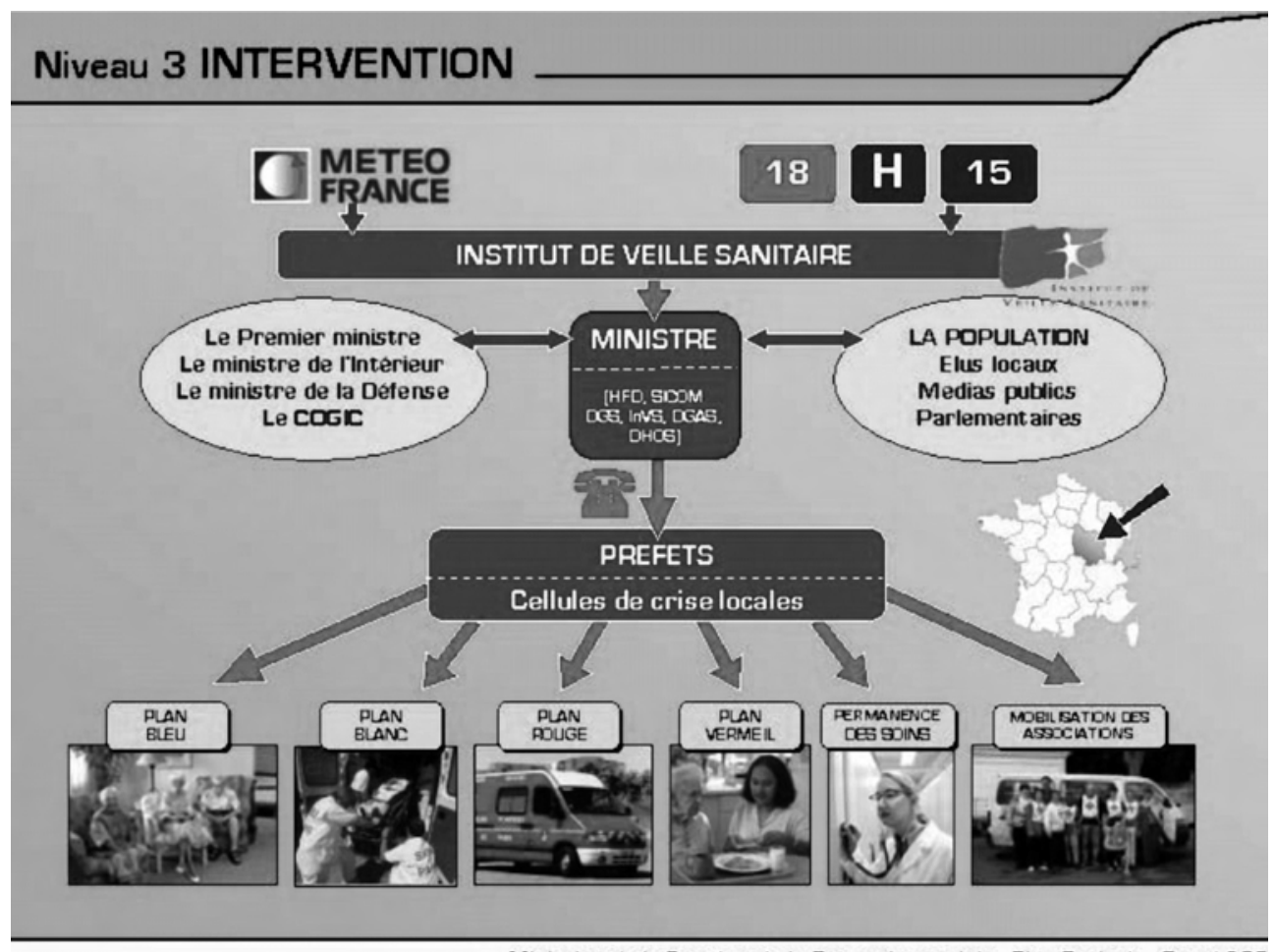

Ministère de la Santé et de la Protection sociale - Plan Canicule - 5 mai 200

Source: Douste-Blazy and Falco, 2004 p. I4. 
Figure 4 Leaflet of advice to elderly people in case of extreme heat or heat wave

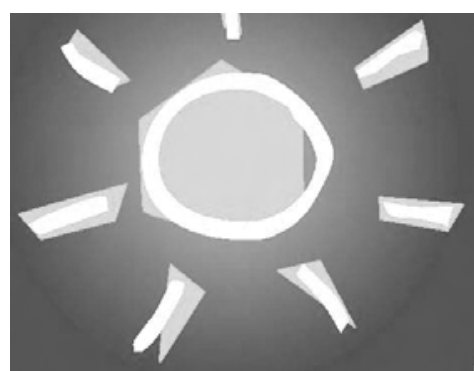

\section{En période de fortes chaleurs ou de canicule}

\section{Personne âgée}

Je mouille ma peau plusieurs fois par jour tout en assurant une légère ventilation et ...
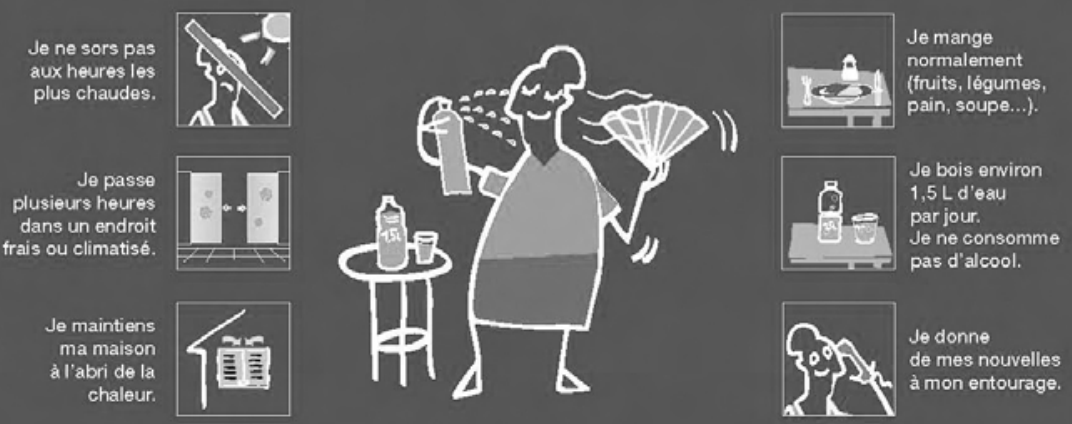

\section{Enfant et adulte}

Je bois beaucoup d'eau et...

Je ne fais pas d'efforts physiques intenses.
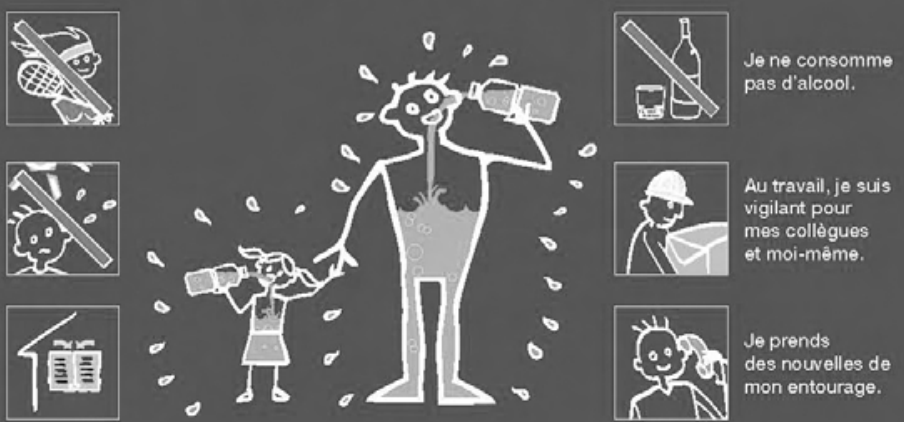

Je ne reste pas en plein soleil.

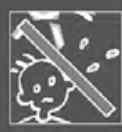

Je maintiens

ma maison àl'abri de la chaleur.
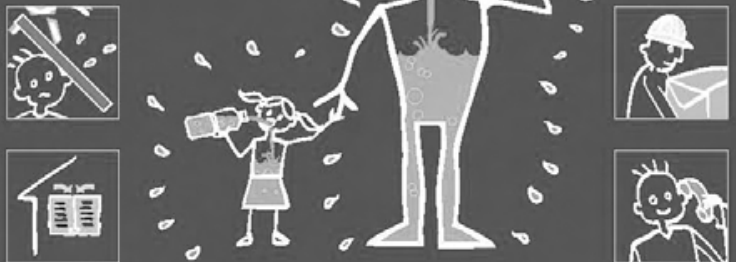

Au travail, je suis vigilant pour mes collègues et moi-même.

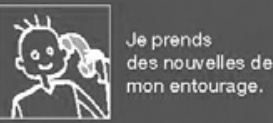

En cas de malaise ou de coup de chaleur, j'appelle le 15.

Pour plus d'informations : 0800066666 (Appel gratuit) www.sante.gouv.fr/canicule • www.meteo.fr ou 3250 (0.34erminute) 
The fourth level (la Requisition) is catastrophic and requires requisitioning of transport means by the state and mobilisation of the army. When the minister of health and social protection demands the prefects of the Departments to issue level three, four plans come into action: the Blue plan (mobilisation of retirement homes), White plan (mobilisation of hospitals and emergency services), Red plan (mobilisation of civil security and fire brigade) and Gilded plan (mobilising volunteer associations for isolated elderly people) and permanent GP care. Since the Plan canicule, all municipalities have had to establish a safeguard system that comes into action on orders of the Department prefect. For example, Alès, one of the pilot cities since 2003, has developed a network of voluntary voisins-tuteurs, literally translated: neighbour guardians, who are activated by the local Solidarity service of the CCAS (Centre Communcal d'Action Sociale) during a period of difficult climatic circumstances (Editor, 2005). Currently, all local municipalities have such a volunteer network, generally co-ordinated by a municipal co-ordinator, that daily contacts elderly people and offers preventive advice how to deal with the heat to those elderly who are registered under the Plan canicule, see figure 4 (Ancenis, 2007; Lieshout, 2006). When an elderly person is identified as being in need of extra care, home care professionals can be asked to provide extra care. Remarkable is thus that although the plan is issued at central government level and legally installs responsibility at the Departmental and local municipal level, the system is generally executed by volunteer neighbour guardians. This again emphasises the tendency in France to organise care and support to elderly people on the level of networks of informal care instead of on the level of professional care, even in the case of climatic emergency situations.

\subsubsection{The domain of home help: Aide à Domicile - considerably increasing in size but problems persist}

One of the important sources of help next to the family is the domain of home help: Aide à Domicile. The first home help services started around I955 in France (Henrard, Ankri et al., I99I p. I04). In the following years, a variety of services for elderly people were created, and the amount of home help provided quadrupled from I8.4 million hours in I960 to 72 million in I989 (Colvez and Ridez, I996 p. I64).

In the early I990s, there were about 4 ooo home help facilities provided by the municipalities in 90\% of the French districts, which employed 70 o०० to I0० ००० home helps (aides ménagères) (Giarchi, I996g p. 205). Home help belongs to the social services, as many other facilities for elderly people in France that has a strict division between health care and social services. People are only entitled to home help on prescription of a physician or local social worker (Henrard, Ankri et al., I99I p. 205).

However, despite thirty years of elderly policies that are aimed at maintaining elderly people at home and a significant growth of home care services, several 
problems persist, that act as barriers for the further development of this sector (Henrard, Cassou et al., I990; Clément, I993 p. I40). Firstly, the services provided (home nursing as well as home help) are still far by insufficient for the number of people in need of care, and there is lack of coordination of the different services provided. Secondly, politicians at state and municipal level and managers of home care organisations have too little insight in the kind of services that people with a loss of autonomy need, in the success factors for maintaining them at home, and in the way social and family support to elderly people functions. Each municipality has its own policy and there is no regional planning of services. This has led to a serious mismatch of people and services in France, as some people that receive services do not need them, while many heavily dependent people receive no home care services at all. Therefore, the burden of care on informal carers, and especially family members, is substantial. Thirdly, the financing of home help is quite complicated, as a network of actors is involved (see figure 5). Also, clients are contributing for an increasing part themselves, although they do receive exemption of charges of the Sécurité Sociale and tax reductions.

Figure 5 Picture of the different financers of home help

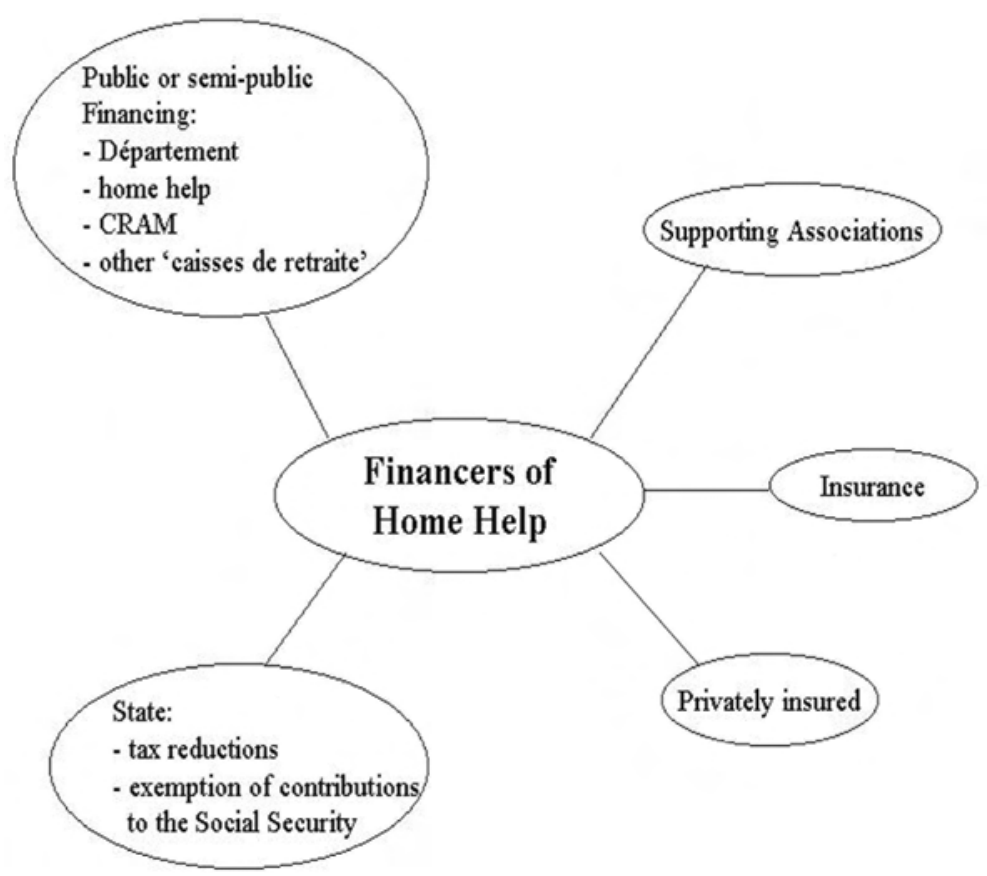

Source: Champarnaud, 1996 p. $22 .^{16}$

I6 CRAM stands for Caisse regionale Assurance maladie 
As mentioned, an important problem regarding home help is that the targeting of services should be improved considerably. Similar to home nursing, many elderly in need of services do not receive them (Henrard, Ankri et al., I991; Verheij, I996; Colvez and Ridez, I996). A study performed in the I980s identified that of the people living alone with disability in the regions of Haute-Normandie, Lille and the Île-de-France, only $21 \%$ received home help. Of those receiving home help, only $49 \%$ were in need of services, while they received as much as $52 \%$ of the volume of help provided (Curtis and Bucquet, I987). This is confirmed by more recent studies, among others by the PAQUID project, a prospective study to determine the potential need for home help. It found that in the Département Gironde, only $45 \%$ of those receiving home help belong to the target group, and only $49 \%$ of those in need of help (living alone and being dependent for at least one of 5 instrumental Activities of Daily Living) were receiving care (Aublet-Cuvelier, Barberger-Gateau et al., I99I). These findings strongly indicate that a quite large group of people that receive home help do not actually need it, while others in need of services because they are dependent or suffer from disabilities do not receive services. Especially in rural areas, a high level of needs is detected that is not met.

Studies also show that receiving home help services is not related to (or even negatively related to) physical incapacities (Colvez, I996 p. I69-I70), while this is an important indicator in other countries. Age, the level of income, subjective experienced health status and incapacities for household tasks were. The factor income level shows the unequal distribution of home help services: people above a certain income tax level hardly receive any home help. Especially people below a certain threshold that are also exempted from co-payments, have easy access to home help services. The steep increase of tariffs above this threshold clearly acts as prohibiting factor for the use of home help services. This partly explains the bad targeting of services to heavily dependent people; income is a more important indicator than physical incapacities.

Recently, this mismatch of people and services is recognised and efforts are made by the state and the Sécurité Sociale to rationalise services for dependent people, among others by using instruments to measure the level of independence of elderly people, as well as the level of resources and support provided by the client's environment. However, for this, radical changes are required in current home help practices and intake procedures that determine the attribution of care to clients, which is a process requiring considerable time and effort. In the Netherlands, this process has been commenced with the implementation of the standardised indication procedure performed by the RIO that determines the amount and kind of care each client is entitled to. 


\subsubsection{A socio-cultural stigma regarding professional elderly care combated by a holistic perspective}

The image arising from the literature is that the domain of home help is socially and culturally more accepted than the home nursing domain in France. In the way of communication to their clients and potential clients and their family, home help services show awareness that their entrance into the private domain of people is a sensitive matter and may be considered as intrusive and interfering (Champarnaud, I996). The wish to keep personal matters private and the feeling that professionals are 'strangers' intruding in the private sphere of the family seems to be more pronounced among the population regarding home nursing than home help: it is more easily accepted by elderly people and their family members to call in support by home help professionals for household tasks, than calling in nursing professionals to provide personal care.

As argued, state policies, the structure of the health care system, and traditional family values in France clearly emphasise that elderly people should preferably be cared for by family members rather than by professionals. The home help domain reacts on the rather strong societal and cultural stigma associated with professional help by emphasising that they support and mobilise the already existing family and social network and not only provide for the objective needs of the clients by providing concrete services, such as help with domestic tasks or meals-onwheels services, but also take the personal situation and complexity of the patient environment into account.

A clear example is the way the activities performed in the ADM R-project (Aide à Domicile en Milieu Rural), which is home help especially aimed at the rural population, are described. ADM R professionals emphasise that they have a holistic perspective (une approche globale) and take the entire situation of the client into account:

'Il suffit d'aller au domicile d'une personne âgée pour lui prodiguer des soins (...) pour s'apercevoir que tout est important: l'aménagement et l'entretien de son environnement, les liens avec son entourage, ses besoins de relations.' (Champarnaud, I996 p. 2I according to an ADMR home care nurse)

They realise that health problems of patients not only ask for medical or technical answers, but also affect the entire life of patients. They aim to mobilise the family and social network and try to ensure continuity of care by co-ordinating the different medical and social activities involved, ensure access of patients to health care services, promote the patient's autonomy, and have a signalling function. They especially claim to respect the personal 'projet de vie', lifestyle and individual choices of their clients. The following quote of the French umbrella of home help organisations Adessa, illustrates the vocabulary used: 
'Vivre chez soi. Garder ses repères. S'occuper de sa famille. Entretenir son domicile. Bénéficier de soins. Veiller à l'éducation de ses enfants. Maintenir ses liens sociaux. Poursuivre ses activités. Etre accompagné dans ses déplacements. Compter sur un soutien moral. Ne pas se sentir seul. Telles sont les aspirations qui peuvent être celles de chacun d'entre nous, à tout âge de la vie. Que nous soyons parent, enfant, retraité, actif, sans emploi, malade ou tout simplement débordé, nous cherchons tous, à un moment donné, pour soi ou pour un proche, une aide pour mieux vivre à domicile.' (Adessa, 2004)

A very romantic image of the life of elderly people is presented, which is strongly linked to the societal image of French elderly people that are still fully participating in social and family life and is fully in accordance with the policy vocabulary on the 'troisième age'.

However, this stance not only characterises the home help domain, but can also generally be recognised in caring professions in France. For example, an aidesoignant à domicile (nurse auxiliary) of a Hospitalisation à Domicile service (Santé Service at Puteaux) describes his professional task in the following way ${ }^{\mathrm{I}}$ :

'soigner, aider, soulager ceux que la maladie à frappés, car il faut garder à l'esprit que la maladie est synonyme de souffrance et de déchéance, à la fois physique et morale (...) il est l'élément essentiel de nos rapports avec les patients qui sont, avant tout, des êtres humains. Enfants ou vieillards, ils sont riches d'un passé de bien-portants, ce qui leur confère, de notre part, un profond respect de leur dignité humaine.' (Delaunay, Teletchea et al., I996 p. I6)

Most professionals in France emphasise above all the personal contact with their clients and the family, as mutual human beings; they describe the provision of care and support to in terms of 'un relation de confiance' or 'une véritable communion d'âmes' (a relation of trust or a true unity of souls). They emphasise that even activities that seem to be insignificant can be regarded as very important for the client:

'La simple toilette peut paraître, pour certains, un acte inodin; (...) J'ai remarqué, en maintes occasions, qu'après une toilette bien faite, le malade $<<$ pomponné>>, coiffé, se sent revigoré et retrouve, vis-à-vis de lui-même et de son entourage, toute sa dignité.' (Delaunay, Teletchea et al., I996 p. I6)

I7 HAD services primarily care for people of different ages that are very ill, such as cancer patients, people with AIDs or multiple sclerosis. 
An independent nurse, who has worked in a hospital before, realises now she works independently that the technical activity she performs only constitutes part of the care, and talks in the same terms:

'C'est là que le mot <<accompagnement>> prend toute sa dimension. En effet, les patients demandent de plus en plus une écoute, pas n’importe laquelle, une écoute attentive qui m’incite à repenser chaque soin, à l'adapter selon la personne, son mode de vie, ses besoins réels, ses désirs, donc à respecter son identité et ses choix. Cela nécessite d'être à côté d'elle, jamais en avant, jamais en arrière.' (Delaunay, Teletchea et al., I996 p. I9)

Taking time to listen to and accompany the patient is also considered very important:

‘.., cela me laisse la possibilité de me consacrer davantage aux demandes de la personne. Je prends le temps de me s'asseoir auprès d'elle, de l'écouter, de lui parler. (...) Laccompagnement est, en fait, tout le chemin parcouru auprès du patient et avec lui. Durant ces soins, je dois rester cette tierce personne à qui l'on peut se confier, qui ne juge pas, qui sans remplacer la famille ou le conjoint garde sa place auprès de la personne malade.' (Delaunay, Teletchea et al., I996 p. I9)

Thus, remarkably, in contrast to other countries, most home care professionals in France do not emphasise their relation with the client as being objective, efficient, or that they provide care according to certain professional, quality or ethical standards. Instead, the human aspects of caregiving seem to be much more pronounced in France than in other countries, possibly out of the respect for the precariousness of intruding as a 'stranger' into the personal sphere of the client. In north and northwest European countries, other values predominate, such as professional skills and knowledge, or organisational values such as efficiency and cost-efficiency.

\subsection{Conclusion}

The characterisation of home nursing in France is summarised in the contextual framework developed in the second chapter (see figure 6). Regarding the inner circle, the domain characteristics, it is remarkable that the establishment of home care organisations took place much later in France than in other European countries. Regular home care organisations, the Service de Soins Infirmiers à Domicile pour les Personnes Agées (SSIAD), have been established since I98I, while in 
Denmark, Germany and the Netherlands, home care initiatives already existed a century earlier. Another variant of home care in France, Hospitalisation à Domicile (HAD), has already existed since I956. HAD is organised by hospitals to provide home care to extremely serious and complex patients, thereby relying on the resources of the hospital. HAD is far more medical-technical and specialist in character than SSIAD; and HAD nursing care is purely considered as an extension of medical care and nurses thus clearly have a subordinate role. Next to regular home nursing organisations such as SSIAD and HAD, a large private nursing sector has existed for long, existing of independent nurses with a private practice that provide a substantial amount of home nursing care and can be considered as a major player on the health care market. These have filled the gap or niche in the health care market that was left by regular services.

SSIAD services have increased at a fast rate since the I980s to more than I 500 organisations providing care all over France nowadays, but their capacity is still insufficient and the needs of many elderly people are still not met. Another important problem in France is the large regional and local differences in availability of home care. Especially in remote and rural areas that are not very densely populated, home care and other social services are generally completely absent. Also, when services are available, a mismatch of people and services due to bad targeting results in people receiving care that hardly need it, while others in need do not receive services at all. Therefore, the availability and provision of home care services is not adequate in many regions in France. Especially when family members are not available as informal carers well, the situation is far from adequate and should receive more attention of local and national policy makers.

Until well into the twentieth century, nursing was considered as a subordinate profession in France. In I978, however, the role of nursing was reformulated and recognised as a profession with an own job description and domain. Although its independent tasks and activities are more and more recognised, nursing in France is still considered as subordinate to the medical profession.

An important finding in this study is that home care professionals display a different attitude regarding clients and appreciate different professional values than their counterparts in other countries. Especially in the Netherlands, systems values such as efficiency, rationality and bureaucratic procedures have a large impact on caregiving, resulting in a certain distance and neutrality between home nurses and clients. Although personal contact with clients is considered important, it is always overshadowed by lack of time and the need to carry out certain procedures, such as professional protocols or bureaucratic rules. However, in France, home care professionals (home nurse as well as home helps) display values in the caregiving process that are more closely linked to the lifeworld of the client. For example, they take a holistic view regarding the personal, often complex, situation of the client, emphasise the personal relation with their clients, supporting them in their 
individual way of living (their projet de vie), as well as stress the support they offer the already existing informal care network. Human values such as relieving the suffering of clients, helping them to remain able to participate in family life and keeping their human dignity are prominent in their vocabulary about their work. Nurses stress the fact that professionals as well as clients are both human beings and therefore need their support and trust and company, which can be expressed in small activities, such as combing the client's hair, see to the patient's well-being, and listening to the patient. Instead of a rather neutral relation of professional and client, a very personal and engaged stance is taken.

Regarding the middle part of the framework, the organisation and training of home nursing and the relation with other actors, the large role of second level nurses and independent nurses is striking. They predominantly provide nursing care. Especially independent home nurses, working from private practices, have a strong position in home care and claim co-ordination of services provided to clients. However, studies show that their knowledge about local professionals and services is often insufficient. Also, most independent nurses do not want to provide domestic and hygienic activities, but when a second level nurse home help is not available, the client remains deprived of the proper care needed. So although independent nurses fill an important gap in the provision of ambulatory care, their role is not sufficient to fulfil all the needs of clients and their lack of knowledge of the local situation does not make them ideal persons to take up the coordinating role for their clients. Further, cooperation between health professionals and coordination of different services is almost non-existent.

Training of nurses in France consists of three nursing levels and is taught at state training institutes (IFSI). Debates are going on in France about the role and responsibilities and professional profiles and task domains are being reformulated now. As already described, in home nursing interpersonal relations with patients are more prominently visible than administrative rules and procedures. Most vulnerable groups in France are elderly and the very elderly without offspring living nearby. Especially in remote and rural areas, these often live in social isolation and deprived circumstances. In most cases, when there is no family nearby or family members are no longer able to care for their elderly relatives who are becoming very dependent, residential care is chosen. Elderly homes in France generally have a medical ward for elderly with multiple severe health problems, comparable to nursing homes in other countries. Home care professionals are very often not called in because they are not available locally and also because these are considered as strangers intruding in the private environment of the elderly person.

This can also be recognised very strongly in the outer ring of the theoretical framework, focussing on the societal and health care context. Namely, a stigma is attached to ambulant care in France; people receiving home care are in need 
Figure 6 Contextual framework of home nursing in France

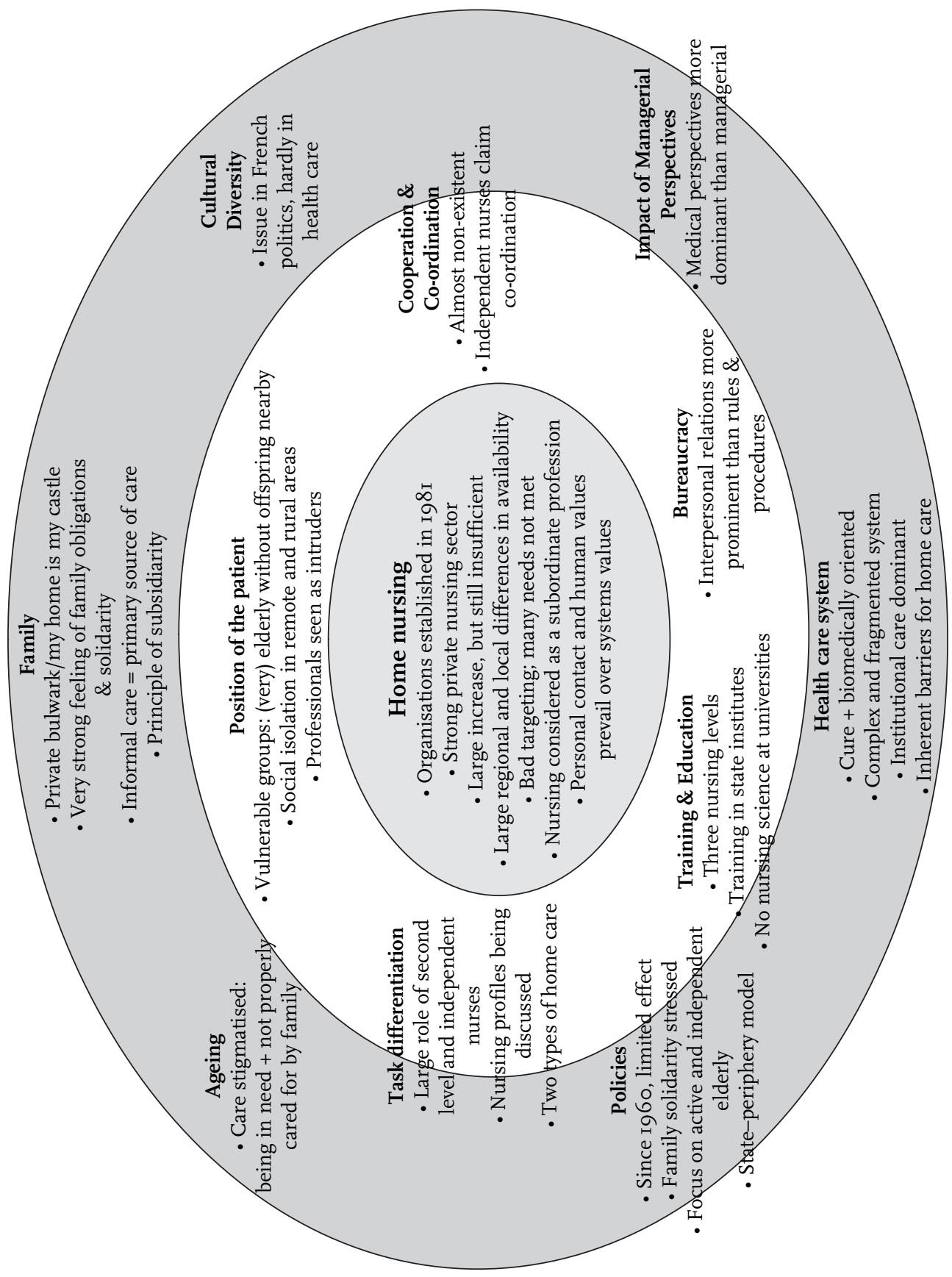


and dependent, and not properly cared for by their family. This stigma withholds people from asking professional home care. Therefore, many elderly turn to their family and social network for help. The national heat wave plan, Plan canicule, also departs from this idea by installing local systems of neighbour guardians (voisinstuteurs) to check on vulnerable elderly regularly during periods of extreme heat in summer, when family members are absent due to the holidays. Although the plan describes responsibilities of the national, Departmental and local governments, the execution of the plan completely relies on local networks of volunteers and only in case of level three and four on mobilisation of professionals.

The health care system in France is, as in Germany, strongly cure-oriented and the bio-medical perspective prevails. Hospital and specialist care and institutionbased facilities are given priority over ambulant care. The GP does not function as a gatekeeper for medical care, as hospitals and private specialists are directly accessible to patients, but patients in need of home care do need a referral from a physician. Nursing homes as such do not exist, but elderly and very elderly people in need of complex care are staying at a long-stay ward in hospitals, or in special care wards in retirement homes. The complexity of the reimbursement system, the fragmentation of the health care system and the lack of availability of services are important inherent barriers that prevent people from arranging long-term ambulant care such as home nursing.

Policies regarding elderly were already issued since the ig6os, but have been limited in effect. An enormous growth in the domains of home nursing and home help has taken place since then, but is still insufficient to provide for all the care needs. Also, policies have not concentrated on the most vulnerable groups of elderly, because there the responsibility of the family is heavily stressed, but on those elderly that are still rather active. The notion of the troisième âge (third age) was introduced, focussing on social and leisure activities for active and independent elderly, such as senior clubs. There is an imperative of social participation of retired and senior people over 55 years of age. However, services for vulnerable groups of dependent and very elderly people, the quatrième âge (fourth age), have had far less priority and are insufficient. In all policies and societal and health care structures, the dominance of the central state can be recognised. Most activities are centred in the Paris region and spread to the periphery. This can also be recognised in home care; the Paris region has far more home care facilities than more remote regions. Also, a large difference exists between large city areas and rural areas. In the latter, home care organisations often do not even exist and the density of independent nurses is also very low, meaning that many elderly people that are living in these deprived areas have caring needs that are not met, especially when relatives are not available as well. The current socio-economic and logistic infrastructures in France still enforce this situation, resulting in large contrasts between densely and scarcely populated areas. 
Cultural diversity as an issue hardly described in literature on home nursing. Although it is an issue in French politics, it hardly is in health care. The impact of managers and bureaucratisation are also issues that hardly play a role in France; the role of physicians is far more prominent and also the lack of accessibility and availability of home nursing in many regions are more pressing issues. Medical care by GPS and private specialists and hospital care have priority in France, as well as care provided by family members and other informal caregivers. When the lack of care to (very) elderly people is discussed in politics, the family is being blamed for not taking up their responsibilities, instead of that the health care system or politics are being blamed for not offering sufficient home care and professional support. This stance diverges very strongly of other European countries. The family is considered as a private bulwark: 'my home is my castle', including far-stretching responsibilities towards one-another. Very strong feelings of family obligations and family solidarity exist, stressed by the state and in health care policies. Informal care is very strongly considered as the primary source of care. Although the principle of subsidiarity in France, meaning that the family is the first port of call in case of care needs, is also underlying the German and Dutch health care system, its ideology is far more extended in France. Namely, the family is not only the first port of call, but in many cases also the only port of call. The state does not guarantee caring facilities for vulnerable people, such as in Denmark and to a lesser degree in the Netherlands, but makes strong appeals to the family to take up their caring responsibilities. 
Chapter 6

\title{
Home nursing in the Netherlands
}

\author{
- Changes in health care and society affecting the character \\ of the home nursing profession'
}

\section{I Introduction}

The history of home nursing in the Netherlands starts in the I880s with the founding of non-governmental local and regional Cross Associations, as in Denmark. Since then, these have formed a dense network of home nursing services across the country, functioning at the village and neighbourhood level. As Dutch society was developing into different confessional pillars at that time, the local cross associations fell under a national Cross association of a particular confessional signature, namely the general Green Cross, the Roman-Catholic White-Yellow Cross or Protestant-Christian Green-Orange Cross Associations. The history and tasks of these local organisations will be described further on in this chapter.

The last decades of the twentieth century can be characterised as a period of changes in health care and society that also affected home nursing. Since I990, the cross associations providing home nursing and the home help organisations have been clustered into larger home care organisations, falling under the general umbrella-organisation Landelijke Vereniging voor Thuiszorg (LVT; National Home Care Association, recently changed into 'Z-org, sector organisation for care employers'). Instead of relatively independent local organisations with a specific signature and knowledge of the local community, home nursing now became part of a larger organisational system providing different kinds of professional community care. As will be described further on, this changed the character of home care and made it easier for societal and health care developments to change the character of home nursing practices as well.

Furthermore, since the I970s, several plans have been issued by the government proposing a fundamental change of the health care system by introducing market elements and competition into the system. Also, it will be argued that the increasing rationalisation and modernisation of society also has a fundamental impact on current health care practices, such as the increasing use of procedures, protocols and the movement of Evidence Based Practices. From a professional

I Parts of this chapter have been published in Boom, 2004b. 
perspective, it becomes problematic how, when and in what degree such developments interfere in professional autonomy and decision-making. An example that will be described in more detail in this chapter is that the process of diagnosis of the patient's care needs is no longer performed by a home nurse but currently performed by an intake officer of an indication organisation. This chapter will show that the current 'objective' and standardised assessment procedure can be considered as deprofessionalisation of the home nursing profession at the work floor.

\subsubsection{Structure of the chapter}

In the first part of the chapter, the home nursing domain and the specific problems regarding home nursing in the Netherlands are described and related to more general trends and changes in Dutch health care and society. Then, an historical overview of the development of the home nursing domain and the different task domains is provided, and the current educational system with different vocational levels. Then, developments in the home nursing domain and the character of home care provision since the I990s are described, as well as changing relations between home nursing and informal care. The chapter will end with current issues in Dutch home care, especially focussing on the current approach of home care that is more and more considered as a marketable commodity, encouraged by national policies. In the conclusion, the specific situation of home nursing in the Netherlands is summarised in terms of the theoretical framework.

\subsection{Current trends in home nursing, health care and society: increasing bureaucracy and standardisation of practices changing the character of professional home nursing}

\subsubsection{A dense network of home care services}

In the Netherlands, a quite substantial part of the population receives home nursing care per year, compared to other European countries, namely $5 \%$ of the total Dutch population (Kerkstra, I996b). However, this figure is somewhat misleading compared to home nursing provided in other countries, because home nursing has differentiated into two separate domains during recent years; namely the domain of elderly care and the domain of youth health care. In the latter, as much as $40 \%$ of the total amount of home nursing care is provided in the 'consultatiebureaus' (infant welfare or well-baby centres) to children aged o to 4 years of age.

Home nursing care is quite evenly distributed over the country and mostly provided by local and regional home care organisations under the umbrella of 
national Cross associations such as the Green or White Cross (Kerkstra, I996b); see map I. Most of these regular organisations provide integrated care since the I990s, such as home nursing, specialised nursing care, maternal care, home help and social care, and have become larger and larger due to mergers with other local home care organisations.

Map I Number of regular home care organisations per municipality in the Netherlands, I999

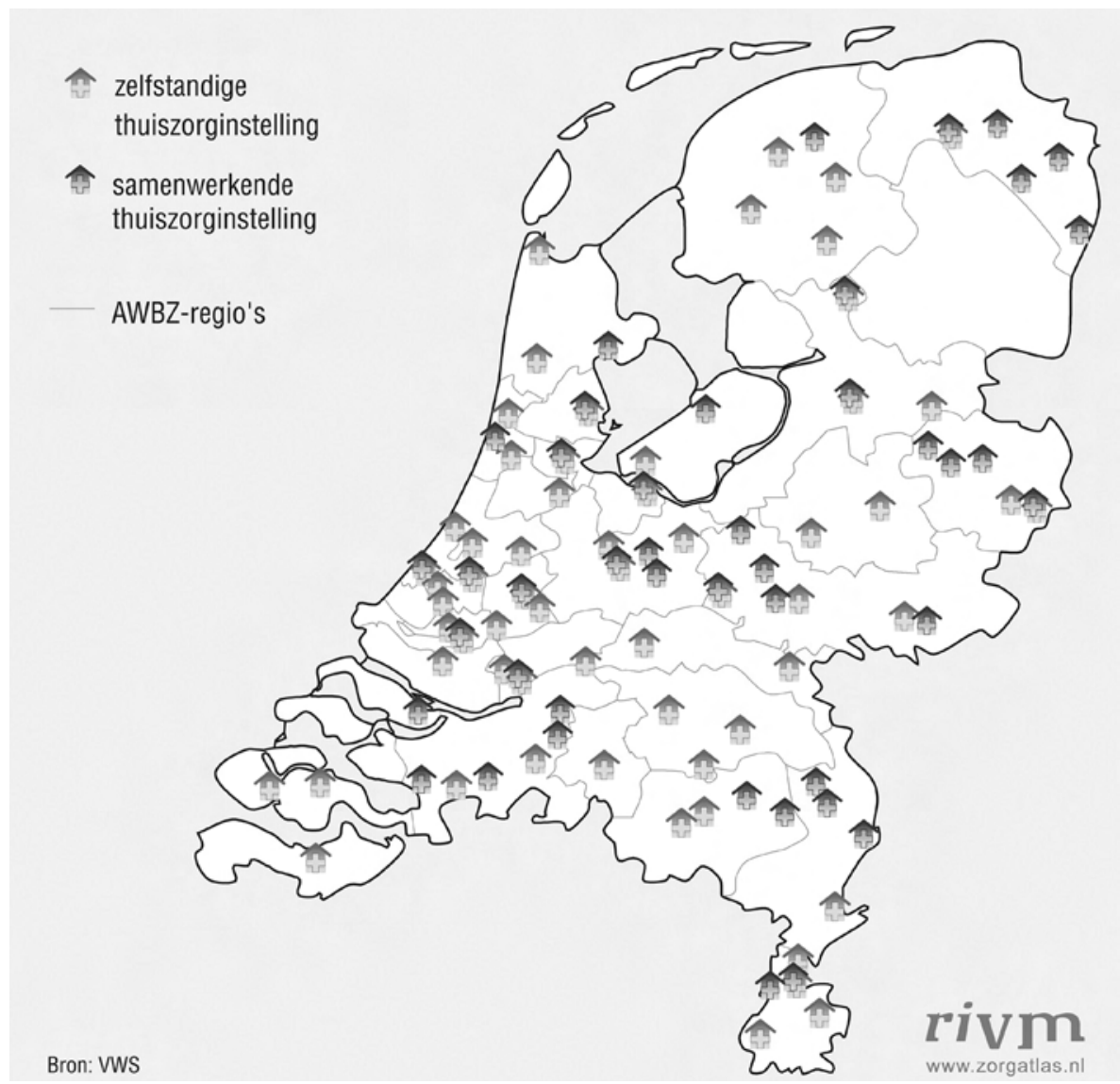

Source: Giesbers (RIVM), 2002a.

Opinions in the Netherlands prevail that care should be equally and easily accessible to everyone and affordable by all. Formerly, clients only paid membership contribution to the local Cross association and were then fully reimbursed for the home care provided, but since 2004, the level of co-payments has considerably been increased by the government. The level of co-payments now depends on the 
level of income and is tied to a maximum (www.cak-bz.nl; www.thuiszorg.nl). Also, clients have to have a so-called 'indication' in order to be entitled to home nursing and home help. The largest amount of costs is reimbursed by the health insurance of clients, or by government regulations such as the AwBZ (Algemene Wet Bijzondere Ziektekosten), that insures people against uninsurable health care costs.

Since the r88os, home nursing has predominantly been organised by local Home Care or Cross associations. For long, home care has been a monopoly of these organisations, except for a small number of nurses working on an individual basis for the rich who could afford to pay for that care at home. Since the I990s, almost all home nursing and home care is provided by 'regular home care organisations'; these are non-governmental, non-profit organisations mostly originating from the traditional private local cross associations. These organisations are in recent years also seen as 'private'; they can go bankrupt and mergers are judged by the Dutch 'Anti-Trust Board'. From January 2007 onwards, domiciliary care that used to fall under the AwBZ, and support of informal and voluntary care will come to fall under the wмо (Wet Maatschappelijke Ondersteuning; Societal Support Act) (Ministry of Health 2006). According to the wmo, the Ministry of vws (Public Health, Welfare and Sports) transfers responsibility over all local social support facilities to the municipalities. The aim of the wmo is to increase the participation of people in society and to ensure a decentralised, coherent and integrated support system. The municipalities are free to develop their own local policy, based on the needs and structure of the population. When people are not able to participate in society with or without the help of voluntary of informal carers, the municipality guarantees support. In the wmo, former regulations such as the Welzijnswet (Welfare Act), Wet voorzieningen gehandicapten (Act on facilities for the handicapped), several subsidies that fell under the Aw Bz (such as support of informal care, services that are provided in residential facilities, domiciliary care), and Public Mental Health Care are bundled to ensure coherent local policies concerning societal support and civil participation. The municipalities will contract out support facilities such as domiciliary care and invite local organisations to tender. This implies that home care organisations are no longer free to offer domiciliary care themselves but have to tender for contracted care at the municipalities. Long-term caring and nursing facilities remain under the AwBz.

Since the mid-I99os, as a result of national policy promoting privatisation, next to the regular home care organisations, home care came to be provided by commercial organisations and private, independent nurses that founded their own home nursing practice. In I999, there were 2I7 commercial home nursing organisations, most of them situated in the provinces North Holland (35), South Holland (32) and North Brabant (4I), ranging from 'one-man or one-woman' private practices to large commercial organisations and intermediary offices (see map 2). There is no cross association or commercial organisation in the northeastern prov- 
ince of Drenthe at all, because of the failure of an early experiment with a commercial organisation. Home care in this province is provided by organisations situated in the four neighbouring provinces of Groningen, Flevoland, Overijssel en Gelderland.

Map 2 Number of private home care organisations per municipality in the Netherlands, 1999

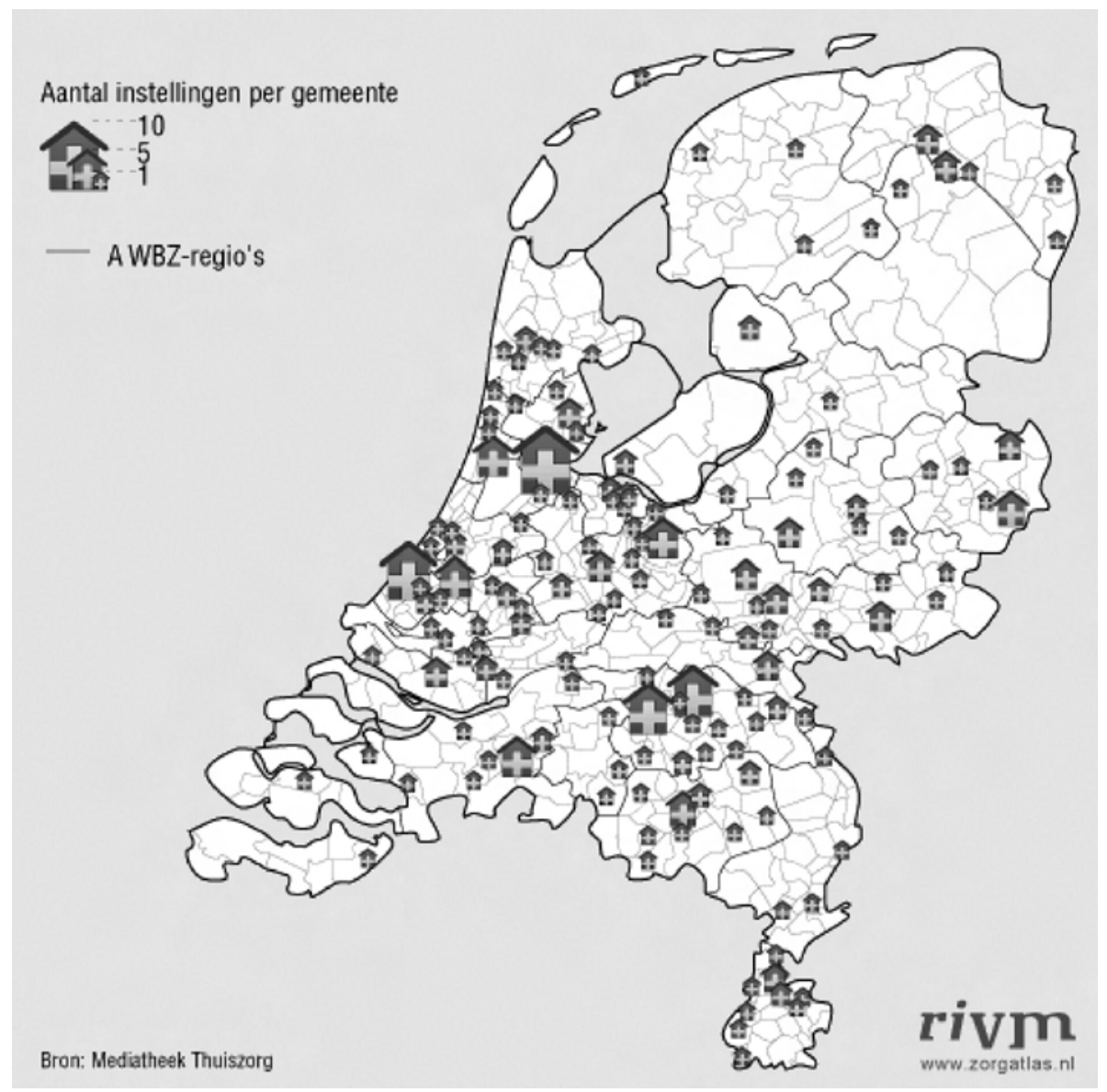

Source: Giesbers (RIVM), 2002d.

Thus, although a modest trend can be recognised regarding the development of private, commercial organisations, especially in the larger cities in the Netherlands, this remains rather marginal as the largest amount of home nursing services remains to be provided by non-governmental home care organisations originating from the traditional private, non-profit cross associations. 
This contrasts strongly with the situation in Belgium and France, where private nurses cover a very substantial part of the home nursing market; even up to about $40 \%$ of the total amount of home nursing provided (Kerkstra and Hutten, I996a; Mossialos and Le Grand, I999b). The mean national amount of hours of care provided per I 000 population in the Netherlands is 2300 (with regional variations between I 830 in middle region of Holland to 2720 hours in the city of The Hague) (Giesbers, 2002b). ${ }^{2}$

In general, the Dutch attitude towards caring is quite favourable. There are many facilities for people in need of care and support, such as homes for the elderly, senior apartments with collective facilities for elderly people, nursing homes, and a rather dense network of home nursing and home help services. This is complemented by regulations that favour the provision of informal care by family members, neighbours and friends, which will be described further in this chapter. Also, caring is considered as an independent professional activity with its own specified and circumscribed domain, and not only as supportive of the medical profession. This contrasts with countries such as France and Germany, where caring is more considered as being an extension of medical activities (Zee, Kramer et al., I994). In these countries, medical-technical skills and curative services are more prominently present in home nursing practices than the more caring and supporting aspects of nursing, such as informing, educating and providing supportive kinds of care. ${ }^{3}$

Nevertheless, although there is a relatively favourable caring culture in the Netherlands, there are certainly serious problems regarding the provision of home nursing. One of these is the continuous sense of 'crisis' in home care due to government measures and staffing problems, and other problems can be traced back to far-reaching bureaucracy and increasing use of protocols that seriously affect

2 The need criterion for care was calculated in a report by PricewaterhouseCoopers as an instrument to decide on access for home care organisations to the market by the Ministry of vws (Giesbers, 2002c). The calculating model includes population prognosis, the work areas of home care organisations, the number of patients per age group (I995), agreements on production (I997/98), realisation figures (I995/97) and home care policy measures (I998). The need criterion was not adequately met in mid Holland and the province of Flevoland, but new home care organisations were allowed to be established there.

3 For example, the number of injections given by home nurses is much higher in Belgium and Germany than in the Netherlands: in I99I, Dutch nurses gave injections in I $6 \%$ of the home visits, compared to $29 \%$ among Belgian nurses and $45 \%$ among German nurses (Zee, I994). However, these data must be interpreted with caution as the mean age of Dutch clients was about 5 years younger than German clients and physical dependency of Belgian clients was higher, indicating a difference in (age-related) health status and thus in care-dependency. 
independent performance of professional activities. The idea of crisis is increased by seeing the ageing of the population as a burden and menace to the future.

\subsubsection{The typical organisation of health care in the Netherlands}

Another important development in the Netherlands is the continuous reorganisation of the organisation and financing of the health care system. For example, in January 2006, a new basic insurance was introduced for the entire population, abolishing the existing distinction between private health insurance and those insured by the compulsory Sickness Fund. People can choose to be insured by an insurance company of their own choice, and can opt for private insurance packages additional to the basic insurance (Ministry of Health, Welfare and Sports, 2006). Until 2006, the Dutch health care system was characterised as a Bismarck social insurance system primarily funded by social health insurances and for about 20 per cent by private funding, especially private health insurances (Maarse and Okma, 2004b). Currently, people take a compulsory basic insurance with a private health insurance company of their own choice and can opt for additional insurance packages. The insurance companies operate under market competition and have to ensure that people are provided with care (in Denmark, care providers have to guarantee the provision of care).

The vast majority of health care providers are private non-profit institutions. Most hospitals, nursing homes, homes for residential care and home care organisations are organised by private non-governmental organisations. This is an inheritance of the past, as until well into the twentieth century, the Dutch government preferred a non-interventionist approach regarding matters of health, disease and poverty. The state government as well as the local governments primarily left the founding of health care facilities to combat unhygienic circumstances and infectious diseases to private charitable initiatives. In most other European countries, charitable initiatives remained an unorganised matter, rather without obligations (Adriaansens and Zijderveld, I98I p. 23). In the Netherlands, from the I880s onwards, cross associations vigorously took charge of public health care throughout the whole country by founding a vast network of local, provincial and national cross associations. This intermediate level of private organisations taking care of public health care matters is typical for the way in which the Dutch health care system is organised. It diverges from other European countries, where such initiatives were taken over by the government, such as in the u K, or remained rather unorganised, as for example in France. In concordance with the division of Dutch society into different religious and political pillars since the late nineteenth century until far into the twentieth century, the private cross associations represented and emphasised their own religious signatures and the specific values and interests of their own religious pillar. This pillarisation ('verzuiling') is an important and typical 
characteristic way in which Dutch society has been organised for long and can still be recognised today. Numerous private social, cultural, educational, welfare and health care organisations have shaped the modernisation process in the Netherlands for many decades and have been incorporated in a complicated way into the current welfare state and health care system (Adriaansens and Zijderveld, I98I).

\subsubsection{The tendencies to organise and bureaucratise daily life in the Netherlands}

Next to the strong historical development of private initiatives on the intermediate level of organisations, other aspects are also typical for the way that matters are dealt with in the Netherlands. During recent decades, tendencies are strong to organise and pre-structure matters in a top down way, also called managerialism, and to deal with things in a bureaucratic way by designing and referring to rules and regulations and administrative procedures. Efficiency, rules and principles, universality and impersonality are key concepts of managerialism (Traynor, I999 p. I4). Traynor argues that managerialism finds its roots in the scientific management movement advocated by Taylor at the beginning of the twentieth century, and that the current managerial talk in health care of "self-motivation, autonomy, excellence and closeness-to-the-customer can be understood as a rhetorical mask for the same Fordist drive for deep and penetrating control of the workforce by management (and ultimately by government)" (Ibid., p. I4). As will be argued further on in this chapter, the control of the management in health care organisations extends far into the autonomy and professionalism of health care workers. Managerialism is related to the growth of bureaucracies in modernised societies, as a way to organise matters. After all, the fragmentation resulting from increased task differentiation and division of labour is tried to be halted by regulations that are designed by managers to coordinate the activities of the different professionals and organisations involved. Thus, in itself, bureaucracy is useful. It regulates relations and contacts between people or their social institutions in an impersonal and just way. However, when rules have become ends in themselves instead of mere means and proliferate such that an efficient and transparent way of dealing with matters is hampered. When this 'displacement of goals' occurs, rules tend to become dysfunctional and result in 'over-bureaucracy' (Lammers, Meijs et al., 2000a p. 88). Then, the more negative sides of bureaucracy become apparent, next to the positive. Organisations for instance tend to generate their 'own' goals that overshadow the initial goals. Also, the initial interests of the members of the organisation are then replaced by the interests of the organisation itself, which is also known as 'emancipation of means' (Ibid., p. 89).

These adverse effects of bureaucracy cannot only be recognised in daily life in the Netherlands, but is also strongly felt in all health care sectors. Several problems regarding home nursing that are often mentioned in the literature and in public 
and political discussions about home care, such as long waiting lists, shortage of staff, lack of co-ordination between professions and organisations, discontent of home nurses about working conditions and career possibilities, high absenteeism, and a high amount of nurses that untimely leave the profession, can be related to the tendencies to over-organise and over-standardise matters in the Netherlands. Kerkstra (I996) suggests that the extensive organisation of many aspects of Dutch society might be traced back to the relative scarcity of living space, requiring detailed ways to regulate social life (Kerkstra, I996b p. 216).

\subsubsection{Standardisation of the intake: 'objectification' and deprofessionalisation}

Some of these adverse consequences can be seen in home nursing. Firstly, parts of the job that have always belonged to the core of the home nursing profession have been transferred to external bodies and actors, thereby affecting the character of home nursing activities and the degree of professionalism. Namely, others than home nurses nowadays tend to decide on the conditions under which home nursing is provided. For example, since I997, the access to care facilities, and thus also the entitlement of clients to home nursing care, is decided on by a special organ, the Regionale Indicatie Organen (RIOs, or regional assessment organs) (Tjadens and Duijnstee, 2000). Since January 2005, their function has been taken over by the CIz (Centrum indicatiestelling zorg), deciding on the entitlement to care by patients within the AwBz framework (Giesbers, 2005). ${ }^{4}$

Since their establishment in I997, the RIOs were given authority to determine whether a client is entitled to care, to what kind of care (provided by what kind of professional), and decide on the total amount of care that is reimbursed by the health insurance. This procedure has been introduced to ensure objective, independent and standardised judgement of the clients' situation, irrespective of contingent regional circumstances and/or the personal preferences of the intaker, and to improve quality of care by enabling better tailoring of care to the individual needs of clients, especially when it is provided by different professionals or organisations (Lyke and Toren, I996; Donker, I997; Nijenhuis, I997a).

The establishment of the Rios had different effects. Namely, firstly, the assessment of the clients' situation came to be performed by an external decisive organ with special professional 'intakers' that have a more detached, bureaucratic perspective instead of a caregiving perspective. As 'officials' they represent the values of the bureaucratic setting they are working in, and perform their task on the

4 Since 2005, the CIZ (Centrum indicatiestelling zorg) executed the indication of the AW BZ and for about half of the Dutch municipalities. Since 2007, municipalities have taken over the indication under the wmo framework. 
basis of detailed specific procedures and rules. The decoupling of the processes of intake (according to Abbott: the diagnostic phase) and actual provision of care (the therapeutic phase) has seriously affected the degree of independence, responsibility, and autonomy of home nurses and has led to a lot of discontent among home nurses in the Netherlands:

'When I chose for home nursing, many tasks were included. All of them have successively been taken away. Sometimes that is quite good, for example the split between Youth Health Care and Elderly Care. But the intake, I thought it was very good that we did that ourselves. It was our competence; we had insight in the situations.' (Thiel and Hoven, I999 p. 27-28 focus group interview)

'In the past, I could agree on things with people myself (...) Now I come to these people and just have to execute like some kind of Dumbo and keep my mouth shut for the rest (...) Because of the central process of indication, in my opinion our profession has just been eroded. It truly is a sad story.' (Lyke and Toren, I996 p. 29)

Secondly, it has led to further bureaucracy, because with the separate intake procedure, another layer has been introduced in the caregiving process. It is accompanied by special procedures and forms requiring transfer of information between different kinds of professionals, and between home care organisations and the assessment organ. This new overhead structure has enlarged the distance between the home care organisation and patients (Tjadens and Duijnstee, 2000 p. II8):

'People of all sectors have put forward that bureaucracy has increased. More forms have to be filled out and organisational tasks take more time. Especially in home care many objections have risen regarding the increased bureaucracy.' (Thiel and Hoven, I999 p. 30)

'I stumble onto the bureaucracy. The mass of paperwork has increased enormously.' 'There is a whole bureaucracy and that is clear then. But two months later it changes again and then you need a different form or a different daily round. You also mix up all rules. Sometimes you think: well ok, now I know. But you forget. You confuse all old rules again with the new rules.' (Thiel and Hoven, I999 p. 30 focus group interview with home carers)

Therefore, it is argued that the trend to objectify and standardise the assessment of the clients' needs, has resulted in deprofessionalisation of the home nursing profession in the Netherlands, by transferring an essential part of the professional 
caregiving process to non-practitioners (Donker, I997 p. II0). Whether this has indeed led to more effective caregiving remains to be questioned, because after indication, the option that the care provided is continuously adapted to the clients' changing situation by the professional during the caregiving process is virtually ruled out. It is often complained about that the process of indication between the initial need and application for professional care and the moment that the actual caregiving is provided takes too long. When the home nurse actually starts to provide the care the client is entitled to according to the RIO/CIZ, the clients' situation and health status has often changed in the meantime, so that the care is not adequately fitting his or her situation anymore (Donker, I997). Adjustment of caring activities to the new situation has to be requested officially at the indication authority again, which is thus a lengthy, bureaucratic and inflexible process.

Another serious criticism is that the care assigned by the RIO is a sum of minutes that are calculated and added on the basis of a fixed list of needs and corresponding professional activities, but it is the question whether the outcome is congruent with the actual needs of clients. For example, dressing wounds, providing drugs or determining the glucose level, giving eye drops, washing the client, lifting him or her from bed and accompanying the client to the toilet and bringing him or her to a chair each take a certain number of minutes. Also, helping with cleaning is calculated in minutes. These are added and then divided over professionals with different levels of training and tasks, mostly a home nurse and a nurse auxiliary or home help. Consequently, the client is cared for and supported by different professionals coming at different times during the day or week. So it often happens that the home nurse comes in the early morning to provide drugs, while the auxiliary or home help comes at a later hour for the other tasks. Although planning bureaus of home care organisations currently try to send the same professionals each time, this is mostly not possible all the time because of different working schedules. This has resulted in a situation that is far from ideal for clients and incomparable with the situation several few decades ago, when the local home nurse one was familiar with provided generalist care.

Many managers of home care organisations, professionals, clients and scientists are quite critical about the establishment of the RIOs:

'The indication for home care is so variable per week and even per day, that a global indication doesn't work. Of course giving account afterwards is necessary, care can be protocolised and you have to be transparent. (...) With the RIOs, we threw away millions that have finally been withdrawn from direct care in the end. Therefore, I say: reverse that interference, at least for home care. (...) Reduction of the care to work consisting of minutes is horrible.' (Verseput, 2005 p. 28 from an interview with the former director of Home Care organisation Nieuwe Waterweg Noord) 
'That system of expressing care in measured minutes should go into the rubbish bin. Of course the home care organisation should know how much time is needed on average, but people have to be able to deviate from that. Caregivers shouldn't have the feeling that they have stolen ten minutes of time when they stay longer at a patient. What has priority, should receive attention, and that is to be judged by the carers themselves. (...) Care is not a product.' (Grypdonck, 2005 p. 44)

Grypdonck (2005) worries about the current situation in home care, where organisations determine the amount of minutes a home nurse, nurse auxiliary or home help is allowed to visit to a patient. She argues that care too closely resembles factory work and nurses have far too little say in their own work, and pleas for the development of business principles that are based on caring concepts instead of industrial concepts.

\subsubsection{Evidence Based Medicine, increasing standardisation, protocols and bureaucracy: the changing character of health care work and professionalism}

The professional autonomy of home nurses is also affected by related trends. Namely, a current trend in health care in general, not only in the Netherlands but also in most developed countries, is that more and more guidelines for practices and protocols are developed. During the last several decades, the leeway for home nurses in daily practice and the freedom to make independent decisions based on professional expertise, skills and experience have become more limited by an increasing use of guidelines and standardised protocols in nursing (Philipsen, 2006, I997b). This fits the recent developments of Evidence Based Medicine (Е В $)$ ) and Evidence Based Practices in health care that are based on 'objective scientific proof that things (i.e. drugs, therapies, health care procedures) work': "The assumption of Евм is that scientific medical knowledge is primarily associated with established outcomes from patient related research, and that guidelines are the best vehicle for introducing these scientific insights into medical practice" (Vos, Willems et al., 2004 p. I68). Horstman (2004) argues that Е в $\mathrm{M}$ fits processes of objectification and depersonalisation that have been taking place during twentieth century society and have fundamentally affected the character of professionalism (Horstman, 2004). Expertise of professionals, that was strongly qualitative in character and based on their training and specific, context-bound experiences, has gradually been replaced by a different kind of professionalism that is less tied to the individual professional:

'The trust in experts as a person, as belonging to a specific scientific elite, made room for trust in their instruments - statistics, standards and explicit, procedural methodical rules. The pressure of justification in the public domain thus 
led to de-personalisation and increased anonymity of expertise, the less personal the better! While before, personal insight, experience and wisdom counted as sources for 'objectivity', objectivity in the 2oth century is more and more associated with figures. After all, what is more neutral, detached or objective than figures?' (Horstman, 2004)

Obviously, the use of protocols and Evidence Based Health Care are intended to enhance the scientific character of health care practices and thereby improve these (Vos, Houtepen et al., 2002). For certain professions, it is used as a way to increase their degree of professionalism and the legitimacy of their practices. For example, Dutch insurance physicians make use of protocols to legitimise their decisions regarding people's inability to work, that are not so much medical or objective, but more deliberative or moral in character (Meershoek, I999; Berg, Horstman et al., 2000). Also in physiotherapy, guidelines and protocols for treatment are increasingly being developed during recent years to enhance the scientific character of this profession (Bie, 2003).

Horstman (2004), however, argues that for some professionals, the application of procedures and scientific evidence leads to a decrease of professionalism. Formerly, the public trusted the individual professional on the basis on his acquired skills and experience, using these to judge the specific situation of the individual client (Abbott, I988a; Philipsen, 2006). This 'personal expertise' has gradually been replaced by the expectations of clients that professionals apply their standardised knowledge in a proper way and according to the latest procedures, so that they receive proper and just treatment regardless of their specific situation by no matter which professional. The special character of expertise of individual professionals then makes way for the performance of activities by the 'average professional: "When figures and standards become decisive (...), experts and professionals are made comparable, measurable and to be monitored". Then, the social status and individual reputation of the individual professional is no longer important and professionals become interchangeable. This makes it easier to employ professionals in organisational structures where they can work in shifts that suit the organisation. Also, outsiders to the profession, especially managers and policy makers, can exert more control over their job.

In the Netherlands, current home care practices are guided by standardised protocols, application of guidelines, and extensive registration of activities now, that generally have few openings to react on the specific situation of the individual client. These developments now tend to prevail over the professional and personal aspects of caregiving. Thus, the effects of justification and increasing scientific character of health care practices are that the professional character of the caring professions work has diminished, while it has increased the professional character of the medical professions. Namely, care belongs to the so-called 'orphaned' 
fields in health care, where "it is much more difficult to define and collect the right evidence because of: the long period of time of the disease course; the poor or non-availability of control groups; the interplay of different interventions at the same time; and many other factors which show how complex it is to expand the Евм methodology in these areas" (Vos, Willems et al., 2004 p. I67). The specific situation of home care clients often suffering from multiple and chronic health losses makes the application of caring protocols difficult. The context in which care is provided is incomparable with the care provided in clinical settings where EBM is more easily developed and applied. Grypdonck (2005) that a better training of nurses and auxiliaries would make many protocols superfluous:

'Protocols are attractive because these are a very good management instrument. These provide the management large possibilities for control. And these exist because otherwise, people do not know what to do. It would be better to train them properly.' (Grypdonck, 2005 p. 43)

Grypdonck argues that guidelines give direction and summarise current knowledge. They should however be used in a flexible way and encourage professionals to think and interpret the specific situation by applying their knowledge and experience, instead of to prescribe what should be done.

Also, during the last decades, the character of home nursing work has changed because people external to the profession more and more control the way home nursing is provided. Namely, work content and pace are both highly determined and steered by the management and planning departments of home care organisations (Pool, I997a). These nowadays demand that home nurses account for their activities in written documents and meticulously write down all their activities, among others because of the need to ensure kwaliteitszorg (quality of care) according to the Quality of Care Institutions Act (Kwaliteitswet Zorginstellingen) (Pool, I997a p. 212). As a result, many home nurses, as many other Dutch health care professionals, complain that they loose a lot of time with filling out forms and schedules, which prevents them from devoting time and personal attention to their patients (Thiel and Hoven, I999). This affects the fundamental values of nursing work, as quality of care is often described by professionals as well as clients in terms of 'being able to devote sufficient time to patients':

'Timewriting is not ridiculous, because our activities have a business-like aspect and I think it is absolutely good that this has come up. Yes, but there are so many activities that can absolutely not be just classified in codes. Thus I just put an awful lot under care coordination. Otherwise I am busy filling in these working lists at least an hour a day. Well, I prefer spending that time to patients.' (Thiel and Hoven, I999 p. 29) 
'A visit by a home-care nurse is estimated in time and may take a set amount of minutes which should preferably be expressed in technically performed activities. Patients and nurses on the other hand, emphasize that it is especially the non-technical issues of care, which are essential. (...) For them quality is synonymous with available time. Clients say: "A good nurse is a nurse who has time for her patients" and subsequently a client remarks: "But they are so rushed. They don't take time to have a talk, to have a cup of coffee. So I chuck up what's bothering me and I cry silently for the umpteenth time" (...) The tension between economic and humane goals, which plays a part both in nursing and caring, is seen by nurses and carers as one of the main problems of their profession.'

(Pool, I997a p. 208)

This lack of time results in a lot of discontent among nurses about their work, as they are not able to provide the kind of care they would like to, resulting in burnout feelings and discouragement to remain working in the profession in the long run. For example, absence through illness was as high as $10.4 \%$ in home care in I999, which is much higher than figures of other societal sectors (Stevens, Mathijssen et al., 200I p. II). Also, a high number of nurses and home helps are leaving the caring sector: in I994, I6.9\% of executive staff (Windt, Calsbeek et al., I997). Exit interviews indicate that this could have been prevented in $50 \%$ of these cases when labour circumstances had been better (Stevens, Mathijssen et al., 200I p. II). Working pressure and the lack of possibilities for personal development were mentioned as important factors. Although every organisation of course needs managers, the problem in the Netherlands is that these are too unfamiliar with the content of the care provided:

'Maybe the sad thing of managers in the Netherlands is that they are so little content oriented. Internationally, the idea that management can be isolated from the content has already been abandoned. After all, it makes quite a difference whether you manage people that work with ill people, or people that manufacture bottles. The manager of a home care organisation should be a scientifically trained nurse. His way of thinking should start from care.' (Grypdonck, 2005 p. 43)

However, the current abundance of rules, regulations, procedures and formalised ways of communication are more and more closely resembling activities that are performed in machine bureaucracies, where relatively simple and repetitive tasks only require a minimum of skills and education than complex activities of professionals working in human service organisations that require a relatively high amount of expertise, abstraction, specific skills, training and experience (Mintzberg, I979b; Hasenfeld, I983a; Abbott, I988c; Hasenfeld, I992a). However, health care activities concern people with individual, situation-bound, and often complex 
needs and problems, and are not provided to 'standardised patients' to which protocols can just be applied. Currently, the idea that health care activities fundamentally different in character than activities performed in bureaucratic organisations has sometimes been pushed to the background. Standardisation of health care activities has fundamentally altered the provision of health care in the Netherlands.

\subsubsection{The Dutch 'polder model'}

On a more general, societal level, a specific aspect of Dutch culture can be recognised in these trends, namely the 'polder model'. This culture is typified by lengthy consultation processes, deliberations, finding solutions for problems by formalising them, installing special study committees, attending meetings, writing policy papers, and inventing detailed procedures and standards for action, instead of leaving matters to professionals and executive staff to decide on the basis of their specific expertise and skills or their authority. The democratised way of decisionmaking and deliberation in the Netherlands makes it difficult to realise short-term actions on important societal matters, as a range of different actors are engaged in such processes: politicians, counsellors and (legal) advisors, scientists, officials of various institutes and organisations, etc. It certainly is praiseworthy that many stakeholders are involved and have a say in deliberation processes, but it impedes the pace of decision-making and slows the implementation of planned changes down.

A clarifying example of the inertness of processes of change in the Netherlands is the restructuring of the health care system. Already in I974, the Structuurnota Gezondheidszorg (Health Care Master Plan) was issued that aimed at restructuring health care, because the continuous expansion had led to unbalanced growth of facilities, fragmentation, and a disproportionate increase in costs (Adriaansen and Laan, I994c; Keuning, I997; Maas and Mackenbach, I995; Ministry of Health, 2006). Since then, other governmental plans were issued, such as the Dekker Plan in I987 and the Simons Plan in 1992 that aimed at changes and modifications in health care as well. With the Dekker Report Bereidheid tot Verandering ('Willingness to Change'), plans were launched to introduce elements of market competition into health care that should act as incentives to increase efficiency and to bring the supply of services and demand for care more in concordance. This caused quite a lot of turmoil, as the Dutch health care system was not market-oriented at all at that time. Next to deregulation of governmental interference, substitution of intramural care (hospital, specialist care, nursing homes) by ambulant forms of care such as home care and informal care, the Dekker Report also proposed to introduce a new basic insurance system. The restructuring of the insurance system was taken over by state secretary Simons during the next governmental period, and became known as the Simons Plan. The idea was to 
extend the AwBz, so that almost the whole insurance package of the Sickness Fund insurance should be covered by the new compulsory insurance system. The basic insurance is to cover the entire Dutch population, and overcome the distinction between private and Sickness Fund insured people. People can opt for additional insurance by private insurance companies. A new element of the basic insurance system is that insurers statutory have to accept and care for their clients and that individual premium differentiation (for the basic insurance) is forbidden. The government compensates people with low incomes and pensioners. However, the restructuring proposals affect the roots of the Dutch insurance system and welfare state and have resulted in many political discussions. It touches on such fundamental values and historically developed structures that it was debated about for long, without concrete results. In May 2003, almost thirty years after the initial proposals, the second Balkenende cabinet agreed in the coalition agreement to introduce the compulsory standard insurance for the entire population. In June 2005, the Minister of vws set the date of introduction of the basic insurance at January 2006. However, many implementation problems and uncertainties were expected and the exact consequences for different stakeholders (GPS, patients, insurance companies) are unclear yet.

At the level of home nursing, the unrest and continuous reforms in health care results in complaints and dissatisfaction of home nurses about the way they have to work. They have to obey organisational and administrative directives at the expense of independent decision-making and time spent on personal attention to patients. Because of this, a number of them leave their profession untimely, rates of sickness absence are high, and other nurses try to escape from this by starting their own private home nursing practice in order to have a larger say in their own working conditions.

During recent years, the Dutch consensus-oriented and tolerant 'talking culture' has met serious criticism by the general population, because it was gradually felt that this 'polder model' led to escapism of responsibilities and inertia regarding decision making by the government, policy makers, managers and organisations at all societal levels (Schilstra, 2002; Wever, 2002; Wijers and Graaf, 2002). Also, a gap between the government and the population became apparent. It was felt that important issues are not dealt with properly because of long and non-transparent processes in which the initial goals and aims become troubled by a proliferating web of rules and regulations that continuously led to new rules and regulations instead of to clear actions. In different societal sectors, this non-solving strategy of evading problems by defining new rules and regulations is felt to obstruct the course of things and hampering (especially new) developments (Schuijt, 2003). Since then, politics and society are confronted with feelings of scepticism and dissatisfaction of the population due to feelings of lack of security, waiting lists in health care, delays in investments in infrastructure, bureaucracy, and decreasing tolerance. 


\subsection{A historical overview of home nursing in the Netherlands}

The history of nursing as a profession and as a way to earn one's living dates back to about the last two or three decades of the nineteenth century. In the Netherlands, the nursing profession has remained in the shadows of the medical profession for long and remained closely associated with religious and feminine values until well into the twentieth century. This is said to have hampered the development of nursing into an independent profession with a separate task domain in the Netherlands (Wiegman, I996; Goelabdien, 1996). However, this process is common to the development of nursing in other European countries. In Denmark, the U K and the Netherlands, it was recognised earlier that nursing is a profession requiring specific training and expertise than in countries such as Germany and France, where nursing has been considered for a far longer period as a profession primarily supporting the activities of medical professionals.

\subsubsection{The first efforts to establish nursing as a professional occupation}

The evolvement of professional home nursing is linked to the historical development of hospital nursing, as home nurses were initially trained in hospital in the Netherlands. It is also intertwined with the history of the Cross associations (Jamin, I999a). In I878, the first education in nursing was founded by the Amsterdam department of the North Holland White Cross association, to professionally educate lay (i.e. non-confessional) middle class nurses to care for hospital patients as well as patients in their own homes. These nurses should gradually replace the lower class non-trained hospital staff ('oppassers' or keepers) that were primarily responsible for feeding the patients and keeping order at the wards (Boom, I997). However, during the second half of the twentieth century, the character of the hospital changed from a place primarily providing long-term elementary care and a proper diet to poor patients from the lower classes, into the 'modern' hospital as we know it now, namely a place primarily providing acute, short-term medical curative treatment to patients from all social classes. Hospital physicians and surgeons more and more criticised the way the untrained staff took care of patients and had an increasing need for staff that was more skilled and educated in nursing and caring skills. Hospital directors, generally the head physicians, were strong proponents of proper vocational education for nurses and many hospitals started nursing education to professionalize nurses and 'liberate' nursing from the image of charity (Wiegman, I996).

In I890, eight hospitals offered nursing education, namely Amsterdam (4), Rotterdam (2), Groningen, and Leeuwarden. In Amsterdam, the hospitals closely worked together with the White Cross association. By then, I75 nurses, of whom 60 were students, received free theoretical education taught by the physician- 
director of the hospital, and a small yearly salary. They lived in the hospital during their education period. In exchange for their education and housing, they worked at the hospital departments, under supervision of the head nurse who provided practical nursing training. To become a certified nurse, students had to pass a final exam after two or three years. They were then allowed to work as a hospital nurse or home nurse. In spite of the initial efforts to establish nursing as a profession for girls from the higher classes, more and more students from lower social classes were admitted to the training because of the increasing demand for nurses. This need was due to the continuous expansion of hospitals and the gradual opening up of the labour market for middle class girls. After their graduation, many so called 'lady-nurses' from the higher classes left the hospitals to start working as a private nurse, as working circumstances in hospital nursing were not very attractive due to long working hours and many tiring and lowly skilled household tasks such as scrubbing floors..$^{5}$ Also, many of them stopped working because of marriage. Most nurses from the lower classes remained working in the hospitals. Thus, despite the intention of hospital physicians to enhance the professionalism of nursing, the social status of hospital nursing remained rather low, and the social gap between nurses and physicians remained large (Wiegman, I996).

In I893, the educational system was adopted by the newly founded Nederlandsche Bond voor Ziekenverpleging (Netherlands Nursing League). The board of the League, consisting of five physicians and the lady-nurses Anna Reynvaan and Jeltje de Bosch Kemper, advocated educational uniformity across the country, and represented the interests of hospitals and nurses. However, as the League found considerable support among hospital physicians and directors, nurses did not have a great say in the League. After I900, when the League had founded a national decentralised network of sub-departments, many hospitals became a member of the League and had their nursing staff professionally trained according to League standards. In I907, the White Cross, that had remained an independent training institute, also joined the League because the number of students had risen beyond their capacities. Nursing education then became a purely in-service training offered in hospitals.

In I907, a uniform educational system and exam were established, and in I92I, the system and nursing certificate received the status of state education and thereby became legally protected by the Ministry of Labour. In the I92I law, that came into force in 1924 , a differentiation in nursing was introduced by the distinc-

5 Hospital nurses largely received their payment in kind, such as food, accommodation and medical care; only a small part was paid in cash (Goelabdien, I996 p. I7-I8). They generally worked in shifts of II to I3 hours a day and were seldom allowed a day off, and were therefore hardly able to lead a private life. 
tion between the 'A-education' which stands for general nursing education, and the 'B-education', psychiatric nursing (Goelabdien, I996 p. 2I). In I928, separate additional registrations of the nursing certificate were officially acknowledged, such as home nursing (wijkaantekening) and maternity care (kraamaantekening).

Remarkable and quite typical for the Netherlands, in contrast to for example the $\mathrm{UK}$, is that nurses did not actively take the professionalisation of nursing into their own hands but left the further development of nursing to physicians, that consequently had a large say in the education of nursing and the content of the job:

\begin{abstract}
'Although from that moment, the profession indeed disposed of the most important attributes for professionalisation, such as an own journal (I89o), a league (I893), a uniform educational system (I907), textbooks and legal recognition (I92I), the essential feature of professionalisation, namely control of the occupational group itself over its own professional development, was lacking.' (Wiegman, I996 p. I02)
\end{abstract}

The professionalisation of nursing was also hampered by the division of Dutch society into different religious pillars. Confessional hospitals initially did not join the Nursing League and their educational programme, but educated their nurses themselves according to an educational model that was thoroughly based on religious principles. Also, it focussed on practical skills and not so much on theoretical knowledge. A nurse trained in a confessional hospital or institution did not receive a diploma, but was judged on the basis of personal qualities such as devotion, tact, love and patience. For example in Protestant hospitals, leading a religious life and servitude were considered more important qualities for nurses than expertise and professionalism. The thoughts underlying the education of Roman Catholic nuns was characterised in a similar way, and was inspired by a religious charitable life, the image of caring as a vocation, accompanied by personal qualities such as self-sacrifice, unlimited availability, and serving love. However, this distinction between confessional and general nursing education did not hold for long. Complaints about confessional nurses increased because differences between confessional nurses and nurses trained by the League increased. When the League established a national registration system for certified nurses, more and more confessional hospitals and organisations became a member of the League and restructured their training according to the League model.

Since I9००, another nursing association was founded, namely the Nederlandsche Vereeniging tot Bevordering der Belangen van Verpleegsters en Verplegers 'Nosokómos' (Netherlands Association promoting the Interests of Female and Male Nurses), that often clashed with the League. In I909, Nosokómos joined the International Council of Nurses (ICN), which was a disappointment for the League, as only one national organisation could become a member. However, Nosokómos received 
more support abroad than in the Netherlands, where it was rather controversial and found too radical, as it was considered too closely associated with the socialist and feminist movement. Most nurses were forbidden to join Nosokómos by their superiors, who feared (organised) labour unrest, because of general dissatisfaction among nurses about the long working hours and low salaries. ${ }^{6}$ Nosokómos, that strived for the development of nursing as an occupational group that stood up for its own rights and a social status equal to that of physicians, often clashed with the interests of the League and the confessional hospital staff, that still considered nursing as a calling and an assisting occupation, subordinate to the medical profession. In I928, Nosokómos dissolved itself (Goelabdien, I996 p. 2I). In the same year, the more broadly oriented Nationale Bond van Verplegenden (National League of Nurses) was founded, that strived for the improvement of the social status of nurses, among others by improving the educational system. However, strategic issues, internal struggles and the organisational fragmentation kept the nursing occupation divided and prevented the unification into a single, powerful association, which slowed the professionalisation of nursing in the Netherlands down (Goelabdien, I996; Wiegman, I996). Since the founding of the Dutch Nursing League in 1893 , several occupational organisations and associations were founded, merged and were dissolved again. At the beginning of the twenty first century, over 60 organisations exist that represent nursing and caring occupations (Pool, I997a p. 209), currently converging in the Algemene Vergadering van Verpleging en Verzorging AVV (General Council of Nursing and Caring).

\subsubsection{The founding of private public health initiatives: the Cross associations}

As described before, until well into the twentieth century, the Dutch government remained at a distance regarding the regulation of health care matters. Only general laws and supervising organs were introduced, such as the Geneeskundig Staatstoezicht (State Medical Control) and the I872 Wet op de Besmettelijke Ziekten (Law on Infectious Diseases) (Adriaansen and Laan, I994C). However, this law was hardly executed, for a large part due to insufficient funds. The state government as well as the local governments primarily left the founding of health care facilities to private initiatives. For this reasons, hospitals as well as home nursing organisations were founded by private initiatives. In 1875 , the first department of the White Cross was founded in the Dutch province of North Holland by physician and medical inspector Penn and Allebé, secretary of the medical council (Jamin, I999a). They were

6 Although the I9I9 Labour Act regulated workdays of 8 hours and 45 hours a week for workers, this did not apply to nurses. A separate regulation for nurses was introduced with the Nursing Act in 1929, regulating workdays of Io hours and 55 hours a week, which did not improve the working conditions of nurses very much. 
inspired by the work of the Red Cross (founded in I859) and strived for bundling of local independent and fragmented initiatives that tried to prevent and fight epidemic diseases into an organisation of non-confessional signature. Already in I878, a training programme for nurses started in co-operation with a hospital in Amsterdam.

The White Cross expanded quickly to 6I departments and ro ooo members in I900. The cross associations considered as their main task the improvement of the health status of the population and the prevention of epidemic diseases by improving personal hygiene and living conditions. After I9IO, cross associations were also founded in the province of South Holland, under the name of Green Cross (Adriaansen and Laan, I994C). This name, also signifying a non-denominational, neutral signature, was adopted by cross associations founded in other provinces in later years, and was adopted by the White Cross in North Holland as well. In I9ı6, Roman-Catholic White-Yellow Cross associations were founded by the bishops of the cities of Breda and 's-Hertogenbosch, who held the opinion that health care could not be separated from religion. This opened up opportunities for Catholic girls to become active in nursing or maternity care. In I938, the League of Protestant-Christian Home Nursing Societies was founded. Since the I850s, sisters of Deaconess Houses had mostly provided nursing in the homes of Protestant patients (Stolk-van Delen, I983; Adriaansen and Laan, I994c). The need for an own Protestant nursing organisation was hardly felt until well into the twentieth century.

In I94I, home nursing was provided by I 350 local cross associations that had more than 850000 members. Every local organisation had its own board of directors, consisting of people of different backgrounds. All boards had a physician as advisor, and confessional organisations also had a parish priest or vicar as board member, to monitor whether the activities of the organisation and the home nurses were in line with religious beliefs. At that time, three national cross associations existed: the general Green Cross (of which the North Holland White Cross was a member); the Catholic White-Yellow Cross; and the Protestant-Christian OrangeGreen Cross. Thus, despite the intentions of the founders of the first White Cross association, the cross associations developed in line with the religious pillars of Dutch society. The domain of home nursing experienced a steady growth during the twentieth century: in 1898 , home nursing was only provided in 40 places, which increased to 740 in I923 (Jamin, I999 p. 60). In I94I, there were I 800 home nurses working in the Netherlands, which increased to about 3000 in 1957 and more than II 000 in I995 (Nivel, nzi et al., I997a). Thus, despite its private local origins, the cross associations succeeded in establishing a dense network of home nursing organisations across the entire country, similar to the public provisions in the U K and Denmark. 


\subsubsection{Financing of home nursing}

Initially, the activities of the cross associations were financed by subscription fees of members, voluntary contributions, subsidies or grants, gifts and legacies (Adriaansen and Laan, I994C). As these funds were not sufficient to pay for the costs of the local organisation, they were dependent of supplemental funds from the municipalities. The national cross association was subsidised by the Prevention Fund of the national government, as the cross associations performed activities that contributed to the improvement of the public health situation. The provincial authorities subsidised activities such as the consultatiebureaus (maternity care) and tuberculosis care.

Nowadays, home nursing is financed by the provision General home care of the AwBz (Tjadens and Duijnstee, 2000). Home care organisations receive a budget, which is based on production and tariff agreements. Until recently, home care was free of charge for clients, who only had to join a Cross association at a small membership fee. Nowadays, the government and insurance companies fund home care. To be entitled to a certain hours of home care, clients need an indication of the RIo/CIZ and pay co-payments that are based on income, age and household situation. The introduction of this measure has met serious opposition by both home care organisations and clients, and has resulted in a decrease of $7 \%$ of the amount of care provided since the beginning of 2003, as many clients feared they would not be able to pay the co-payments (Landelijke Vereniging voor Thuiszorg 2004). Many people that were entitled to home care before do not receive an indication for care now and have to find different solutions, for example by involving informal caregivers. People in need for long-term home care, longer than three months, can apply for a Personal Budget (PGB; Persoons Gebonden Budget) that provides them with money to pay for services of their own choice, including informal care. According to the new Health Insurance Act introduced in 2006, only reimbursement of maternity care is falling under the basic compulsory insurance; people have to opt for an additional insurance package in order to be reimbursed for home care. However, reimbursement is tied to a maximum; people do need an indication and need to pay co-payments.

\subsubsection{Shifts in home nursing tasks; from prevention to home nursing and separate working domains of child care and elderly care}

In the late nineteenth century, home nursing work largely concentrated on the prevention of epidemic diseases, and improvement of hygiene in living circumstances (Jamin, I999a). Tasks then primarily consisted of disinfection of clothes and furniture, loan of equipment such as beds and nursing equipment and stretchers for 
transportation of sick people, hygienic education of the population, prevention of child mortality and diseases such as tuberculosis, cholera, typhoid and smallpox, and provision of home nursing care and maternity and child care. In I899, the White Cross began to provide home nursing activities. Other local organisations and Protestant Deaconesses also provided care for sick people in their homes. In addition to the vocational training in the hospitals, training courses were offered in the first decades of the twentieth century, such as a degree in tuberculosis control when monitoring of tuberculosis patients was assigned to home nurses, and special courses in infant care, mental health care and rheumatoid care. In I920, these additional degrees were officially acknowledged and resulted in a special home nursing qualification.

During the development of home nursing, the content of the work gradually changed. As living and working conditions of the population slowly improved and medical knowledge of diseases increased, the emphasis on the prevention and spread of epidemic diseases declined in favour of caring for people that were discharged from hospital but still needed nursing care, home care for patients suffering from chronic diseases, and maternity care (Adriaansen and Laan, I994C). Tasks of home nurses shifted from general preventive measures to direct patient care in the homes of families and preventive education of individuals and small groups. In the I950s, home nurses had the following broad and generalist task description: caring for ill people in their homes, managing the loan of nursing equipment, make house calls to promote child hygiene and to combat tuberculosis, organise sessions in consultation bureaus for child care, tuberculosis, and specific chronic diseases such as rheumatoid arthritis, perform social hygienic activities, keep an administration of the activities, and perform other tasks assigned to the home nurse by the board of the local cross association. Although the local home nurse worked in close co-operation with the local general practitioner and had to report to the board, she was able to perform her job rather autonomously and had a rather high social status in the area she worked in.

As described, mother and child care also became an important sphere of activity of home nurses during the course of the twentieth century in the Netherlands (Jamin, I999b). Traditionally, lower class mothers and their newborn babies were cared for by untrained dry-nurses ('bakers'), who often held traditional and prejudiced views that lacked basic medical knowledge. Around the turn of the twentieth century, progressive physicians wanted to change this situation by advocating preventive activities and pleading for the development of professional care to reduce infant mortality. Initially, cross associations gave advice to parents about child care and provided preventive child care when paying home visits to families. However, this was thought insufficient to guarantee good mother and child care and reduction of child morbidity and mortality. Structural professional care, support and advice by specially trained maternity nurses or aids during the first days after birth 
was thought essential to lay the foundation for a proper care and upbringing by parents and improvement of the health status of children.

In the mid I930s, cross associations became to play a leading role in the further development of mother- and child care (Jamin, p. Io6; Adriaansen and Laan, I994C). They provided a special training course for dry-nurses, and in some large cities, schooling facilities for maternity care were founded. These maternity carers were assigned to special mother- and child care organisations and provided care in poor families, or worked privately. They thus withdrew themselves from the influence of the cross associations, who considered this as an undesired development. Consequently, in the I950s, a national network of maternity centres (Centra voor Kraamzorg) was founded by the cross associations, where maternity nurses were trained and worked under supervision of a head teacher. Although maternity care has differentiated into a separate working domain now, most home care organisations still offer maternity care next to home nursing and home help. In addition, private organisations for maternity care exist.

Next to maternity care for mothers and new-born babies, a large number of mother- and child care facilities (consultatiebureaus or infant welfare centres) was founded by cross associations since I90I, to monitor the development of babies and young children, see table I:

Table I Increase in the number of consultatiebureaus in the Netherlands

\begin{tabular}{lcl}
\hline Year & Number & Details \\
I901 & I & The Hague \\
I929 & 250 & Increase of about Io per year \\
I935 & 750 & Tripled in 6 years \\
I950 & I 472 & Doubled in I5 years \\
I970 & 3000 & Doubled in 20 years \\
200I & I 400 & Decrease because of reorganisation \\
\hline
\end{tabular}

Source: Lieburg, 200I.

These centres provide information to parents, execute the state vaccinate programme, and have a signalling function in timely detecting physical or psychological disorders. Generally, these bureaus are employed by a physician and a home nurse and several volunteers. Nowadays, the whole country is covered by a rather dense network of local consultatiebureaus that provides the majority of parents with their services, see map 3.

However, the number of infant welfare centres is slightly declining during recent years (Verheij, Boven et al., 200I). A recent development is that increased co-operation is taking place between the well infant welfare centres and the Municipal Health Authorities (GGD; Gemeentelijke Gezondheids Diensten), to offer inte- 
grated youth care and close the gap in care provided to children aged o to four (provided by the consultatiebureaus) and those five years and older (provided by the GGD) in the Netherlands.

Map 3 Number of infant welfare centres per municipality, I999

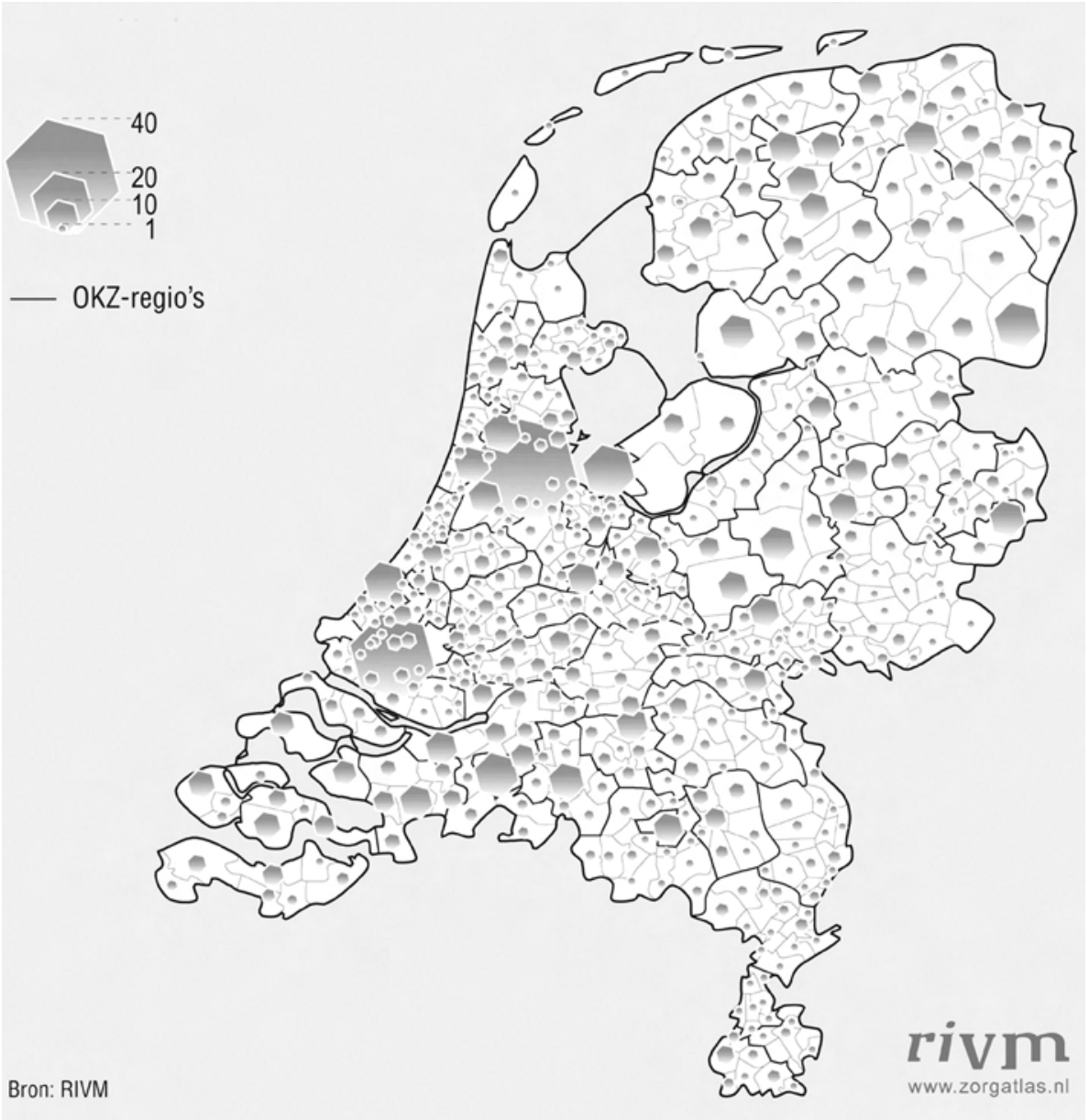

Source: Boven (RIVM), 2002.

Next to mother and child care, elderly care has become the second important sphere of activity of home nursing. Due to the double ageing of the population, the majority of home nursing care is currently provided to the vulnerable in society, namely dependent elderly and very elderly people and people with chronic diseases. In the present educational system, future home nurses choose between the domain of Youth Health Care and Elderly Care, as home nursing has differen- 
tiated into two different working domains. This poses problems for former nurses re-entering the labour market; as these have been educated in the former generalist educational system, they have to be retrained first before they can apply for a job in home nursing.

Nowadays, tasks of home nurses primarily consist of providing nursing in the homes of clients that are dependent because of a (chronic) disease or handicap and terminally ill people, providing support by giving information, advice and instruction, providing psycho-social support, performing medical-technical nursing activities, co-ordinating care provided by different health professionals, and develop (multidisciplinary and transmural) care networks (Stuurgroep Wijkverpleging met toekomst I999a). This indicates that during the last decades, the co-operation with other health professionals has increased. Some of the tasks formerly performed by home nurses, such as the loan of equipment, is now performed by home care organisations, the care for families and new-born babies in their homes has differentiated into a separate working domain and has been transferred to the domain of Maternity Care and Youth Health Care, and more simple caring tasks and basic personal care are currently performed by nursing aids and home helps. This indicates that the task description of Dutch home nurses has been narrowed down from generalist to primarily focussing on tasks that require a higher level of education and skills, such as co-ordinating tasks, increased cooperation with other professionals, and when performing care in the homes of clients, concentrating on medical-technical nursing tasks. Also, differentiation took place and most home care organisations now employ specialised nurses that specifically care for patients with diabetes or COPD. These nurses have had additional training into these differentiations.

\subsubsection{The educational system}

In I997, a new educational system was introduced (Nijhuis, Vlaskamp et al., 200I). In I996, the report Gekwalificeerd voor de toekomst (Qualified for the Future) was issued by the Ministry of vws and of oc\&w (vws and ocw, I996). The report announced the introduction of a new educational system for all nursing levels, auxiliary nurses, home helps and geriatric helps, and other health professionals providing care. The in-service nursing and caring education in specific institutions such as general or psychiatric hospitals, nursing homes, or home care organisations has been abolished, and nurses now receive a broad training at independent training institutions, the Rocs (Regional Educational Centres). During their training, students follow a traineeship or work in one or more care organisations to receive practical training on the job. After the generalist basic training, students choose a differentiation into a specific category of patients that amounts to about onefifth of the study and consists of a theoretical and practical part. Students that are 
graduated can continue their education by following education to become a nurse specialist ${ }^{7}$, specialised nurse ${ }^{8}$ or study for additional grades in caring, that have been established in the 2000 National Regulation Additional Nursing Education; an agreement between the National Association of Hospitals NvZ, Association of Academic Hospitals (vaz) and the General Meeting Nursing and Caring (Avvv) (NVZ, vAz et al. 2000).

The educational system now distinguishes between the profession or occupation of nurse, carer and helper (Nijhuis, Vlaskamp et al., 200I). The qualification structure, determining the demands and qualifications per diploma, is divided into level I to 5; level one is the helping auxiliary, level two the helper (helpende), level 3 nursing aid (verzorgende), level four the nurse with intermediate vocational education (м во; Middelbaar Beroeps-Onderwijs, verpleegkundige), and level five, nurses with higher vocational education (н во; Hoger Beroeps-Onderwijs, verpleegkundige). With this, the educational system is in accordance with the directives regarding the recognition of vocational education of the EU (vws and ocw, I996 p. I5-I6). The differences between the levels are determined by:

I) the level of responsibilities;

2) the level of complexity of the caring situation, and

3) 'transfer'. This latter indicates the degree in which the professional is able to apply his or her knowledge and skills in different situations.

Regarding the level of responsibilities, level 2 carers only have the responsibility to perform tasks. Level 3 nurse auxiliaries are also able to draw up and evaluate care plans for individual patients. Level four nurses, confusingly called 'second level nurses' (verpleegkundige van het tweede niveau) plan and perform care, and are in addition able to co-ordinate and organise care provided to individual clients by different professionals. Level five nurses that are actually called 'first level nurses' (verpleegkundige van het eerste niveau), also have a consulting and model function. This means the nurse has an advisory role to other professionals regarding all phases of the nursing process, and supports others in complex situations where standard procedures do not apply. Level five nurses also - at least in theory - have a co-ordinating function regarding the content of the care: she decides on the interventions to be performed, in what order, and by what kind of professional. However, this is contrary to current home nursing practice in the Netherlands, where home nurses are dependent of the indication advisor that has taken over

7 Nurse specialists are e.g. psychiatric nurses, diabetes nurses, COPD nurses.

8 Specialized nurses are IC nurse, Ic pediatric nurse, IC neonatology nurse, pediatric nurse, obstetric and gynecology nurse, oncology nurse, emergency care nurse, dialysis nurse. 
these tasks that include the first two or three steps of the basic nursing process. It is clear that this interference seriously undermines an important part of the professionalism of home nurses.

Regarding the level of complexity, a similar subdivision can be made to distinguish the different levels. The criteria of complexity are based on the degree in which the tasks are based on application of routines and standard procedures in predictable situations (level 2), a combination of procedures (level 3), or whether the professional works in a quickly changing situation where new procedures, flexibility and efficient strategic thinking is demanded. This can be visualised in the following way:

Figure I Level of complexity of the tasks performed by different nursing and caring levels

\begin{tabular}{|c|c|c|c|c|}
\hline \multirow[t]{2}{*}{ Vocational level } & \multicolumn{4}{|c|}{ Degree of complexity } \\
\hline & Routines & $\begin{array}{l}\text { Standard } \\
\text { procedures }\end{array}$ & $\begin{array}{l}\text { Combination } \\
\text { of (standard) } \\
\text { procedures }\end{array}$ & New procedures \\
\hline \multicolumn{5}{|l|}{ Level 2, helper } \\
\hline \multicolumn{5}{|l|}{ Level 3, nursing aid } \\
\hline \multicolumn{5}{|l|}{ Level 4, nurse } \\
\hline Level 5, nurse & & & & \\
\hline
\end{tabular}

Source: vws, I996 p. I9.

The third criterion indicates the degree in which the knowledge and skills of the carer are context-bound, namely only applicable in a limited number of specific situations (level 2), or occupation-specific, namely applicable in diverging situations, or whether the professional is able to transfer his or her knowledge and skills to other situations, that are very different and often multi-disciplinary in character and demand for analytical and reflective thinking, see figure 2.

According to the educational system, nurses are educated as a generalist who should be able to have a comprehensive view of the entire care situation and consequently act on it. Considering the degree of education and experience, home nurses are functioning at level five (Stuurgroep Wijkverpleging met toekomst I999a). Co-ordination of care provided by different professionals in the patient's home and coaching of nursing aids and caring staff are important tasks. According to professional directives, level four and five nurses should be able to perform the nursing process independently, which consists of anamnesis, diagnosing, drawing up a nursing plan, providing nursing care and evaluation of the care provided. 
Figure 2 Indication of the level of transfer of skills of the different educational levels

\begin{tabular}{l|c|c|c|}
\hline Vocational level & \multicolumn{3}{|c|}{ Skills } \\
\cline { 2 - 4 } & Context-bound & Occupation specific & $\begin{array}{r}\text { Independent of } a \\
\text { specific profession }\end{array}$ \\
\hline Level 2, helper & +++ & + & + \\
\hline Level 3, nursing aid & ++ & +++ & ++ \\
\hline Level 4, nurse & + & +++ & + \\
\hline Level 5, nurse & & +++ & ++ \\
\hline
\end{tabular}

Source: vws, 1996 p. 20.

Both level four and five nurses, and part of the level 3 nurse auxiliaries fall under the Act вIG (Individual Health Care Professions Act) that executes the registration of a number of health care professions and legally protects their title (2005) (vws, 2000). ${ }^{9}$ The principle of the Act is that the practice of medicine is open to all, but it also states that some procedures may only be carried under specified circumstances. The intention of the Act is to promote quality of care and protect patients against malpractices. All health care professionals are now allowed to perform so-called 'reserved procedures'. This means that certain medical activities, such as giving injections, that were often executed by nurses in practice although they were officially not allowed to perform these (because only medical professionals were legally entitled to perform medical activities), are now approved to be performed by competent nurses when authorized by a physician, dentist or midwife. Supervision or possible intervention is no longer required.

\subsection{Developments in home nursing since the $1990 \mathrm{~s}$}

Apart from the major change of the educational system of the nursing and caring professions, another important change has taken place in home nursing in the I990s in the Netherlands. Namely, home nursing and home help have been integrated into one domain, namely the domain of home care (Swagerman, 1997). Before I990, home nursing and home help were provided separately, by a varied patchwork of more than 300 local and regional, confessional and public organisa-

9 Professions falling under the Act BIG are pharmacists, physicians, physiotherapists, health care psychologists, psychotherapists, dentists, midwives and nurses (www.bigregister.nl) 
tions, foundations and associations, which provided their services on a small scale. Home nursing, infant and child health care, maternity care and lending of medical and nursing aids such as raised beds and crutches, were still primarily provided by local or municipal cross associations. There were only a handful of commercial home care organisations and there was hardly any competition.

\subsubsection{The integration of home care and home help}

In I990, the Centrale Raad voor Gezinsverzorging (Central Home Help Council) and the Nationale Kruisvereniging (National Cross association) merged into the newly founded Landelijke Vereniging voor Thuiszorg (LVT; National Home Care Association). Nowadays, the Netherlands is one of the few European countries where home nursing and home help are integrated into one domain, together with Denmark (Zee, I996; Swagerman, I997; Adriaansen and Laan, I994d). Many independent small home nursing and home help organisations have merged into larger (sub-) regional organisations providing 'integrated care', which means that home care is offered as one single package by the same organisation. Depending on the kind of domiciliary care required, a home nurse, home help or maternity nurse is sent to the client. The first integrated care organisation started to provide its services in Hengelo, in the Dutch northeast region of Twente, in I992. Since then, about I30 regional integrated home care organisations have been founded. Most of them provide home nursing, home help, infant welfare care and maternity care and work on a non-profit basis. Also, an increasing number of organisations have been founded that work on a commercial basis since the introduction of market elements in health care (see map I). These organisations are represented in the national umbrella organisation LVT and have to be certified.

Thus, since the late I980s and beginning of the I990s, the Dutch landscape of home nursing has changed from primarily small-scale, local organisations that were mainly working on a non-profit basis, into more large-scale regional, integrated and competitive organisations. These are often cooperating with other kinds of health care services to form integrated or 'transmural' care networks (Meurs, I989; Linden, I997b; Kümpers, Raak et al., 2002). This has been stimulated by the government and by health care providers themselves, to unify and simplify the provision of care that had become increasingly non-transparent and difficult to manage because of far-reaching fragmentation. The aim was to develop a more client-oriented approach ('zorg op maat') and to provide care that is more tailored to the individual needs of the client, by toppling the health care organisation from supply-oriented to demand- or patient-oriented. This approach has led to many mergers and care innovation projects ('zorgvernieuwingsprojecten') during the last two or three decades. 
This process has taken place in two different forms; firstly, regional care networks have developed by means of integration, alliance, or merger of different health care organisations and actors (such as insurance organisations) in the same region, which is in fact decentralisation; and secondly, by the development of the concept of 'transmural' care (transmurale zorg). According to this concept, intramural and extramural forms of care are integrated or working closely together, to bridge the existing gap between general primary care and specialised hospital care in the segmented Dutch health care system. The National Council for Public Health Care defines it as such:

'Transmural care encompasses those kinds of care which are tailored to meet patient's needs. It is provided by caregivers from primary and hospital teams on the basis of coordination and cooperation, with shared responsibility and specification of delegated responsibilities.' (Smeenk, I998 p. I5)

Caregivers should be enabled to supply the care required regardless whether the patient is hospital or community-based regardless of the boundaries between primary and hospital care. For example, specialised nurses such as diabetes nurses, (partly) take over tasks of hospital physicians to improve continuity of care for chronically ill patients and enhance cooperation between different providers of care (Vrijhoef, 2002). In the us and $\mathrm{uk}$, this concept of care is generally described as integrated care as well.

Although these trends have many positive effects and especially improved accessibility of health care facilities for clients because they are offered different kinds of care by one counter or central organ ('I loket'), there are also several serious drawbacks, because the ever-changing health care landscape is very fragile because it still lacks durability, the regional networks obtain a monopoly position that rather serves market interests than patients' interests, and the existing bureaucratic structures proliferate rather than are being abolished because of different funding structures or budgets and complex regulations (Kümpers, Raak et al., 2002 p. 352). Also, it has become difficult for clients to keep track of all these changes, especially because they find themselves in a particular fragile and dependent situation when they are suffering from poor health (Philipsen, I985, I986). When they can't see the wood for the trees anymore, case-managers are taking over on the patient's behalf to ensure they receive the kind of care they need. Although this certainly contributes to better tailoring of care to the individual needs of patients, it also creates another intermediate level within health care and thus makes communication processes more complicated.

Another recent innovation in Dutch health care that was introduced under the umbrella of tailored care, is the 'persoonsgebonden budget' or PG B; a personal budget that clients can apply for when they are dependent of care for at least three months, 
in order to be able to buy the kind of care that fits their particular situation most. The PGв was introduced in the Netherlands in 1996, and has multiple positive aspects for clients as well as providers of care, ${ }^{\text {Io }}$ but is also seriously hampered by bureaucratic procedures (Brok, 200I).

\subsubsection{The changing character of home care}

Simultaneously with these organisational changes in the I990s, the character of home nursing and home help gradually changed, and its charitable character and non-profit ideology was gradually transformed into a more businesslike and nononsense approach, considering nursing and caring as marketable commodities instead of as special activities requiring a personal and individual approach to clients. These changes took place at four different levels. Firstly, at the administrative, managerial level, as many managers of home care organisations became 'commercial' managers with a policy or marketing background, instead of people with a nursing background. Secondly, at the professional level, the nursing profession differentiated into different hierarchical levels of nurses and carers, each with their own tasks and responsibilities. Nurses no longer provide generalised or 'holistic' care, but specialise in different kind of work areas, client groups or diseases (vws and ocw, I996). In home nursing, the social status of nurses and the content of the work also changed considerably:

'The first home nurses were independent, creative, innovative sturdy females (tantes) that entirely went their own ways in the rural areas. They kept their ears to the ground. That made home care into a mobile, creative organisation. These aspects have always been the strengths of Dutch home care, all foreign countries used to come to and have a look. I wish they remain that in the current large organisations. Because of the increase in scale, that mobility and flexibility is endangered. Home nurses always were en route. In the nineteenth century by foot and on horse, skates, later on the Solex or by Volkswagen. And they always were very creative. (...) In hospital (...) a nurse had to obey the physician. She especially shouldn't show that she knew more than he did. But in the districts and rural areas, home nurses ruled.' (Wiegman, 2005 p. 3I-32)

Thus, the rather large autonomy and considerable social status of home nurses, that still existed in the second half of the twentieth century, have largely faded away

Io For example, clients can now decide themselves whether they apply for care provided by an organisation, a professional working in a private practice, or an informal carer; they have more say in the kind of care and the conditions under which care is provided, and they are now able to pay informal carers. 
and have been undermined by developments in society and health care since the I990s (Boom, Philipsen et al., 2004b).

Thirdly, the training system changed. The educational level of caring staff has become lower because of changes in the educational system. Only level five nurses are trained in schools of higher vocational education, while nurses of other levels are trained in secondary education that is provided by a Regional Training Centre (ROC; Regionaal Opleidingscentrum) (Nijhuis, Vlaskamp et al., 200I). Thirdly, at the level of patient care, nursing became more medically-technically oriented, more standardised, protocolised and bureaucratised, leaving less room for personal attention to clients (Thiel and Hoven, I999). Fourthly, the changing character of home nursing is also felt at the level of informal and voluntary care.

\subsubsection{The changing relation between formal and informal care}

Informal care can be characterised as being non-organised, unpaid care that originates from social relations (Lyke, 2ooob p. I35). In the Netherlands, caring for some one else has been considered for long to be a private, taken-for-granted activity that goes without saying (Tjadens and Duijnstee, 2000). The family was and still is considered as one of the main pillars of society in the Netherlands, in politics as well in the private domain. Compared to other countries, a large number of Dutch women do not participate in the labour market, because a relatively large amount of them stay at home or work part-time to be able to provide care for their family members; children as well as elderly. The government has stimulated for long that women had an important task in the home and the male head of the family should provide for the family income. This was further emphasised by the head figures of the religious pillars of Dutch society at the time. This traditional division of family tasks can still be recognised in the relatively low percentage of Dutch women working; 66\% of the women are working, of whom $77 \%$ work parttime (Eurostat, 2007a). Especially the burden of care on middle-aged women, the so-called 'sandwich generation', is high, because these often not only care for their husband and children, but also for their parents or parents-in-law, other family members or neighbours or friends (Dautzenberg, 2000). Currently, about II\% of the total population of 16 years and older provides informal care to a person in need of support (Tjadens and Duijnstee, 2000 p. I08).

Since the I970s, informal care, in Dutch described as 'mantelzorg', signifying a 'warm cloak of comfort', has increasingly been recognised and described as the 'unseen, unheard and unnoticed' foundation of care that is one of the pillars of the Dutch welfare state (Lyke, $2000 \mathrm{~b}$ p. II2). However, a change has taken place during the I990s, as informal carers and voluntary workers are increasingly described in economical and social-political terms, and considered as cheap labour that relieves the burden of care from professional health care workers. The government states 
in policy reports that professional care should only complement already existing informal care, instead of vice versa (Tjadens and Duijnstee, 2000). This is however a specifically Dutch way of thinking, that may be related to the long tradition of charity and private, often confessional, caring initiatives. The current government views emphasise that care should primarily be a matter of the family and social network, only to be supplemented by professional care when necessary. This attitude is still closely connected with the principle of subsidiarity that also underlies health and welfare systems of countries such as Germany and France and many Mediterranean countries. However, in Scandinavian countries, about the opposite way of thinking can be discerned. In these countries, professional care is guaranteed by the public health system for those in need of care and support. Therefore, women have been able to pursue their own careers, and labour participation of women is far higher in these countries than in the Netherlands.

Since the I990s, the Dutch government not only stimulates informal care, but also defines what should be considered as 'good care' and how an informal carer should act to be a successful carer (Lyke, $2000 \mathrm{~b}$ p. I49). By describing in policy documents what informal care ideally should entail, informal care becomes formalised and proto-professionalised: "While policy makers initially began to describe (informal) carers as ideal and indispensable part of the caring system, in the present caring policy they especially connect them to the duty to care and their shortcomings in that" (Lyke, 2000b p. 135-136). Their tasks have thus been integrated in the caring system and apart from granting them official appreciation, they are also made indispensable. Although Dutch policy makers consider imposing a duty to care is impermissible, opinions nevertheless prevail that all possible available informal care can and should be used.

Even put stronger, the availability of informal carers has almost become a prerequisite for receiving professional care (Londen, 2005). While in earlier days, a mix was presupposed between self-care, informal care and professional care, the values of professional care have become overpowering. Feelings of love, solidarity and belonging together, that used to be important motives for providing informal care, have become duties and a plight that are regulated and more or less demanded by the current values and entitlement rules:

'Factually, informal care is not-formally-arranged care. Devotion, willingness to make sacrifices, compassion, solidarity, ideology are motives here, and these represent important societal values (...). It is indeed not wrong when formal care hopes, or even counts on the availability of informal care. However, a too large societal pressure, I would almost say "anti-social" pressure is put on informal care when the policy makers (the designers of the system) ánd the formal (professional) carers take for granted that that informal care should be present.' (Londen, 2005 p. I7-I8) 
These assumptions, that have become inherent in the Dutch caring system and are sustained by professionals and intake advisors who decide on the entitlement to professional home care, strongly contrast with societal values and welfare state arrangements in Scandinavian countries. For example in Denmark, people do not have a formal obligation to care for their family members except for spouses, and the availability of informal care is not weighed in the assignment of home care. Opinions in Denmark prevail that offspring will not and should not take any major role in providing care for their elderly parents; state provision of professional services is seen as a right for all, and welfare state arrangements, including health care services, are shaped according to that cultural assumption (Jamieson, I99IC). However, opinions in most other European countries prevail that support should primarily be sought in the private domain and welfare arrangements should only provide for professional care where informal care is unavailable (Giarchi, I996b).

Van der Lyke (2000) describes that different vocabularies exist in the Netherlands on informal care. This contrasts sharply with Germany, where strong appeals are made to informal carers as well, but who are not described in specific terms in health policies. In the Netherlands, a social political vocabulary exists in policy papers, labelling informal care as labour activity performed in the private sphere that is comparable to professional care provided by the labour market. The patient and his or her social network become objects that can and should be trained and 'professionalized' and the duty of individual members of society to sustain the health care system is emphasised (Lyke, 2000a). In scientific literature on informal care, a psychological vocabulary is used, referring to care in the same terms as labour performed in the public sphere and labelling informal carers as potential patients. Based on the assumptions inherent in this vocabulary, informal carers are offered advice how to preserve their own health and well-being, for example by participating in support groups, following training courses in e.g. rational-emotive training, lectures about caring for patients with specific diseases such as diabetes or rheumatoid arthritis, and being offered technical nursing equipment to facilitate caring activities. The idea is that this kind of support might decrease the risk of stress, burnout and overburdening due to the demanding character of informal care. Informal carers themselves make use of a romantic vocabulary, referring to the social relations between the different people involved, such as the patient, GP, home help, home nurse, hospital specialists, and other family members. In this vocabulary, the uniqueness and ambiguity of the situation, communication, empathy, personal attention and interdependence of the people involved are stressed. Van der Lyke states that informal carers are increasingly described in economical terms in policy and science documents as people who need to be trained to provide care in an almost rational, professional way, while the social and emotional aspects of informal care are marginalised. 
Since I997, the availability of informal carers is officially weighed in the assessment of the clients' need for professional care by the RIOs. The introduction of the personal budget PG B, with which individual clients receive money with which they can pay the carer of their own choice, fits this way of thinking perfectly. According to PGB procedures, informal carers are considered as health care employees that are being paid for their services. In this sense, informal care is 'professionalized' and transformed into a basic part of the health care system. The PG в budget, allowing for financial compensation of informal carers, also transforms their activities into 'work' that can be defined in economical terms and subjected to bureaucratic procedures, instead of leaving it a matter of personal devotion to a person that is emotionally close.

\subsubsection{The influence of the management state on professional and informal care}

Van der Lyke (2000) argues that the changing character of informal care and the rationalisation of professional care, by emphasising rationality, procedures, protocols, methodical thinking and professional distance can be related to the changing character of the Dutch welfare state into a management state, in which economical notions such as efficiency and effectiveness have become dominant (Lyke, 2000a p. I37). Mutual solidarity and social security, that were considered fundamental aspects of the Dutch welfare state, are being replaced by economically defined goals and efficiency, to make optimum use of financial and personal resources and prevent unnecessary waste of means in times of scarcity. This no longer primarily applies to the business sector, but is currently also found appropriate for societal sectors where other values used to prevail. Van der Lyke states that because of this, the relation between the public and private domain has become more complex and less transparent. Namely, on the one hand, the government withdraws from the private domain by transferring means, choices and duties of the state to the private domain; namely to families and informal carers. On the other hand, this shift of responsibilities is accompanied by increased control, regulation and intervention and disciplining of carers into 'rational workers'. The pressure on informal carers thus increases, despite apparent government withdrawal, as its power and concepts have been dispersed over different actors and instruments:

'The government has imposed the management of care organisations to account for their 'core-business' to their financers, the insurers. They have to communicate on which product they prefer their accounts to be settled, which 'indicators' they use and how they want to monitor and improve the quality of their product. To do this, the managers of home care organisations in turn ask caring professionals to record what kind of care they provide, to whom, how many hours they 
spend on that and the result of their intervention, preferably by means of figures of performance standards. In specific, this means that since the development of the management state, caring professionals need to determine caring results, label time, and thus keep a thorough administration. As professional carers are only allowed to come when informal carers are unavailable, informal carers are also requested to provide insight into their actions. So for example, assignment advisors ask carers to account for when and how much care they (can) give. (...) Informal care is increasingly expressed in terms of time, money and result.' (Lyke, 2000a p. I5O-I5I)

However, this tendency is also severely criticised by those who argue that professional care is fundamentally different from informal care:

'Informal care or family care in my opinion is a sign of meaningful time; not a sign of performance time. Informal care concerns in the essence personal commitment, active attention, emotional ties and not providing a caring performance as quickly and efficient as possible (...). When [an informal carer in the caring process of professionals] is scheduled professionally, her meaningful time is transformed into performance time. And that is not even the worst, the worst is that those who do that are not aware of what they are doing, think it is all the same.' (Kunneman, I995)

\subsubsection{Worries about current developments in Dutch home care}

To summarise, during the I990s, a period of 'commercialised' home care was introduced, with key words such as efficiency, efficacy, flexibility of budgets, staff, and supply of services, quality of care, cost-effectiveness, competition, etc. etc. On the one hand, these developments may indeed have resulted in improvements in home nursing, because a more varied package of care is offered now, services have become more targeted to the direct needs of patients, and home care organisations have gained better insight into the costs of the actual care provided now. On the other hand, these developments have led to over-regulation and over-standardisation of care, at the expense of attention to the more personal and individual needs of clients, and also the degree in which nursing and caring professionals are currently able to perform their job on the basis of their professionalism, knowledge, experience, and skills.

Thus, currently, home nursing in the Netherlands is suffering from increasing bureaucracy, the changing character of the welfare state into a withdrawing management state, and the changing character of professionalism from personal expertise to procedural and scientifically objectified, but impersonal expertise. This process is however accompanied by worries by those who criticise the business- 
like approach in social sectors such as health care, welfare and education, arguing that the social and humane aspects of these activities are more and more being neglected.

Especially regarding primary care, that should have a low threshold for clients, a business-like approach may be harmful because of the risk that certain categories of people are 'forgotten' or left into the cold, because they are not able to organise the care they need themselves. The GP, home nurse and home help are professionals that have relatively easy access to these people. For example, Verseput, former home care organisation director, believes that although there certainly are categories of people that prefer to arrange their own care needs, the average home care client is fundamentally different. She advocates the redevelopment of integrated, ambulant primary care organised at small-scale district-level that can easily reach different kinds of vulnerable people:

'The category of elderly people - I sometimes refer to as the "grey-on-travels wave" - that have good pensions and independently buy the care needed themselves, will increase, but it will never become the largest category. People are getting older and older and I always see a turning point when they are no longer able take their own lives into their hands. They are really not waiting then to choose between ten insurance packages or surf on the Internet to find out who provides the best and cheapest care and where. I notice an increasing social isolation: children living far away or people without offspring, neighbours that are no longer available, elderly that are afraid to open the door after six o'clock in the evening. Come along to certain neighbourhoods of Schiedam, the impoverishment behind the front doors, rubbish bags at inner courtyards that "walk away" because rats are in them... The RIAGG doesn't get there, because the client doesn't have a referral for help, the GGD (municipal health authorities) also doesn't know it anymore. And who remains coming? That is that home nurse or home help. I keep persisting that the actual commercialisation is not a good concept for primary care.' (Verseput, 2005 p. 29)

Others state that a wrong image exists among politicians and policy makers of the client population of home care organisations, namely those who are hardly in need of care or quite capable to arrange the care they need. They therefore see no harm in their measures:

'Sometimes it seems as if a taboo is being placed on being handicapped or ill and becoming older lately. When I see those advertisements in the media and everyone who is just talking about the independent elderly person who is constantly on journey as it were, who does all kinds of nice things and can manage himself fantastically. But that high-spirited and healthy elderly person is not the 
target of home care. The moment one gets ill and handicapped, one just is less independent.' (Pool, 2005 p. 93)

Although efficiency, quality and patient-oriented care are slogans of Dutch home care, at the same time very distressing things are happening in direct patient care, for example when the media reports professionals have tied up handicapped or elderly people because there is no proper care available. Pool (2005) questions the excuse of professionals who say there are no other solutions under the current stranglehold of tight time schedules. He states they are then hiding behind their management and planning department and run away from their responsibilities:

\footnotetext{
'Why do I see a documentary on television recently about demented elderly that are tied up at home, because there is no supervision? That surely is completely unacceptable? You cannot say at the same time that you guarantee quality of care. I know home care is under pressure, but you can wonder why nurses obviously allow such situations to happen. As a professional, you then have to notify someone very strongly somewhere, make protests within your organisation. I can imagine that you find yourself against a wall, but it is a matter of civilisation not to allow such situations under any circumstances. One can also choose not to provide home care in such a situation and look for a different solution; that is quality.' (Pool, 2005 p. 93)
}

Thus, there are serious worries about the current situation in home care in the Netherlands that can be attributed to the continuous rationalisation and budgeting of health care since the I990s. Instead of the patient, often managerial perspectives of turnover figures, efficiency measures and benchmarking are on top of the agenda of home care organisations.

\subsection{Conclusion}

The situation regarding the provision of care to vulnerable people in their homes in the Netherlands, especially elderly and chronically ill people, is summarised in the contextual framework of home nursing below (see figure 3). For long, Dutch home nursing has been one of the forerunners in Europe, together with Denmark en the uk. Home nursing officially came into existence with the founding of the Cross associations that started in 1875 and experienced a rapid growth that resulted in a dense network of NGOs across the country within a few decades; the local cross associations of different religious signatures. Care by cross associations has always been accessible to everyone in the Netherlands, provided they became a member of the local cross association for a very low amount of money. Since recently, ac- 
cessibility has become more limited due to intake procedures restricting access to those with an indication and because of co-payments.

The cross associations have been amalgamated into the Dutch health care system by firstly the introduction of the AwBz, abolishing the cross associations, and secondly the mergers of home nursing and home help organisations into largescale home care organisations that provide care services on a market basis since the I990s. Care has become a commercialised product and the character of home nursing has changed considerably during the last two decades: home nurses are no longer rather independent professionals working under the flag of local cross associations in their own neighbourhoods, but have become employees of largescale regional home care organisations. In these organisations, managerial and administrative values and measures, such as time-writing and efficiency, have come to the fore more and more, at the expense of professional autonomy and caring concepts such as devoting time and personal attention to patients. Instead of the home nurse with local authority next to the family doctor, who provided care in various cases because she was familiar with the specific situation of her clients and worked as a generalist, she has become an exchangeable professional working according to the procedures and time schedules of the home care organisation and strict differentiation of tasks. This development fundamentally changed the character of home nursing in the Netherlands and can be characterised in this respect as deprofessionalisation. These developments have caused a lot of discontent among home nurses in the Netherlands. In the I99os, the aim of health care policies was integration of home nursing and home help provided by the same home care organisation, but since January 2007, home help falls under the wмo (Wet Maatschappelijke Ondersteuning) and under municipal policies, comparable with social services in many other countries.

Regarding the second ring of the theoretical framework, describing the organisation and training of nurses and the relations with other professionals and patients, striking in the Netherlands is the strong task differentiation, attached to different nursing levels. This results in fragmentation of caregiving and loss of continuity of care for the patient. Until well into the twentieth century, home nurses were educated as generalists and performed a wide range of tasks in the homes of their patients. Nowadays, home nurses travel to quite a number of patients a day to perform a limited set of well-circumscribed tasks and providing these according to a fixed number of minutes that have to be registered administratively. Since I997, in-service training in hospitals and other health care institutions has been replaced by vocational training in regional training centres (ROCS), next to higher professional education (нво-v). Nursing is divided into five different levels, after which nurses can specialise in for example diabetes nurse, nurse practitioner, etc. Guidelines and protocols are an important part of nursing training. Also, nursing science is taught and studied at university level. Regarding cooperation and 
co-ordination, the GP has an important role in Dutch health care and acts as gatekeepers, as in Denmark. Often, the GP also co-ordinates care that is provided by different kinds of professionals. Co-operation over the boundaries of care, with intramural as well as ambulant services in transmural care chains is an important development in the Netherlands. Here the role of specialised nurses and nurse practitioners is also quite important, that fulfil a bridging function between the different parts of the care chain. The attuning of the different caring activities to each other is quite complex and is accompanied by complicated communication processes and transfer of patient records.

A very important aspect in the Netherlands is bureaucracy. In all matters of social life, bureaucracy plays an important role. This tendency comes from the wish to deal with matters in an objective, equal and fair way, without being influenced by personal preferences or prejudices. The intake procedure by indication organisations is an example of this; the idea is that people with similar care needs receive the same kind of care, irrespective of whether they live in the North or the South of the Netherlands. However, these kinds of procedures require a lot of administrative work, which is often tedious and delaying in nature. Although bureaucracy is mostly associated with efficiency, in health care practices the administrative procedures are generally hampering professional practices and limiting professional autonomy. Tasks have to be registered and performed according to the rules and regulations of the health care organisation and procedures issued in health care, but this takes valuable time that cannot be spent directly to patients. Especially time-writing care in minutes is a pressure point in home-care. In addition to increasing managerial bureaucracy, nursing practices are more and more described in nursing protocols that also diminish the autonomy and leeway of nurses by creating a professional bureaucracy. These protocols are valuable because these ensure quality of care and accountability in nursing, but at the same time, adaptation of caring work to the specific circumstances of the patient is becoming more difficult. The patient is therefore often seen as a 'victim' of the bureaucratic and systems character of health care. Due to the schedules of the home care organisation and strict division of tasks, patients often receive care by many different home care professionals and have to explain their specific situation over and over again. Also, the administrative registration of tasks takes valuable time of the care provided by professionals, mostly at the expense of personal attention to the patient. Home nursing come, perform their tasks, register them and have to hurry to the next patient. Talking to the patient and checking on his or her well-being or situation has to be done in the meantime. There is hardly any time to signal changes in the patient's situation. Even if there would be time, the home nurse would not be able to adapt the care provided to the changed situation, because that is the task of the indication officer. Currently, new developments are proposed whereby the indication office will be replaced. 
Regarding the outer ring of the framework; the context in which home nursing is embedded, one can say that the percentage of the population above 65 years of age is about average. The number of institutionalised elderly living in residential homes or in nursing homes is still quite high. This results from the tendency after the Second World War to build special institutions for elderly people. Currently, long waiting lists prevent elderly from entering these homes; only the very old and very dependent are admitted. Substitution policies since several years try to replace institutional care by home care. In this sense, the Netherlands has also adopted the European trend to let people grow older in their own environments. Older people and other vulnerable groups such as children are considered as a (financial) burden to society. Welfare state arrangements exist, but not as extensive as in Denmark. Regarding policies, it is striking that policies are not stable but are changed every few years. Periods of budget cuts are interchanged with periods in which home care receives extra money. An important change in home care has been the introduction of co-payments. Equity and accessibility are strongly emphasised in policies as well as systems to ensure quality of care, such as the Kwaliteitswet Zorginstellingen (Quality Act Care Institutions), introduced in I996.

The overall health care system has changed fundamentally from a Bismarck system to a basic insurance system covering the entire population, executed by commercial insurance companies since January 2006. Similar to health policies, the health care system is also confronted with continuous reforms and the constant introduction of new measures, which affect the stability of the system. In contrast with Germany and France, where medical curative care has priority, in the Netherlands cure and care are relatively independent domains. The value of both domains to enhance the health and quality of life of the population is recognised. Like in Denmark, care is considered as an important domain of activities. The government favours competition in health care, thereby promoting the idea that care is a marketable product. Therefore, the impact of managerial perspectives in health care is high. Managers have a large say in all aspects of caregiving and managerial values, such as efficiency and efficacy and accountability by registering all activities prevail over professional values. Cultural diversity, which is much discussed since the last decade, is considered as a burden but also as a challenge. Especially in the larger cities, home nurses have to take diverging cultural backgrounds of clients into account. The role of the family is rather peculiar in the Netherlands. Informal care is considered as an indispensable cornerstone of health care. The availability of informal carers, not only of spouses such as in Denmark but also of siblings and sons or daughters in law, is assessed during the indication procedure. When these are available, professional care is not granted. Informal care is thus a duty in the Netherlands, which is formalised in procedures. Informal carers can be paid from a personal budget of the elderly person, so that a relative can be hired to provide care instead of a professional. At the same time, these are proto-profes- 
sionalised by offering courses. However, in many cases, providing informal care is felt as a burden. Daughters and daughters-in-law who care for their elderly parents feel a lot of pressure and exhaustion, especially when they also have a job and/or children. As in Germany and France, the principle of subsidiarity is underlying the Dutch health care system; therefore a strong moral appeal is done on family members to provide informal care.

Figure 3 Contextual framework of home nursing in the Netherlands

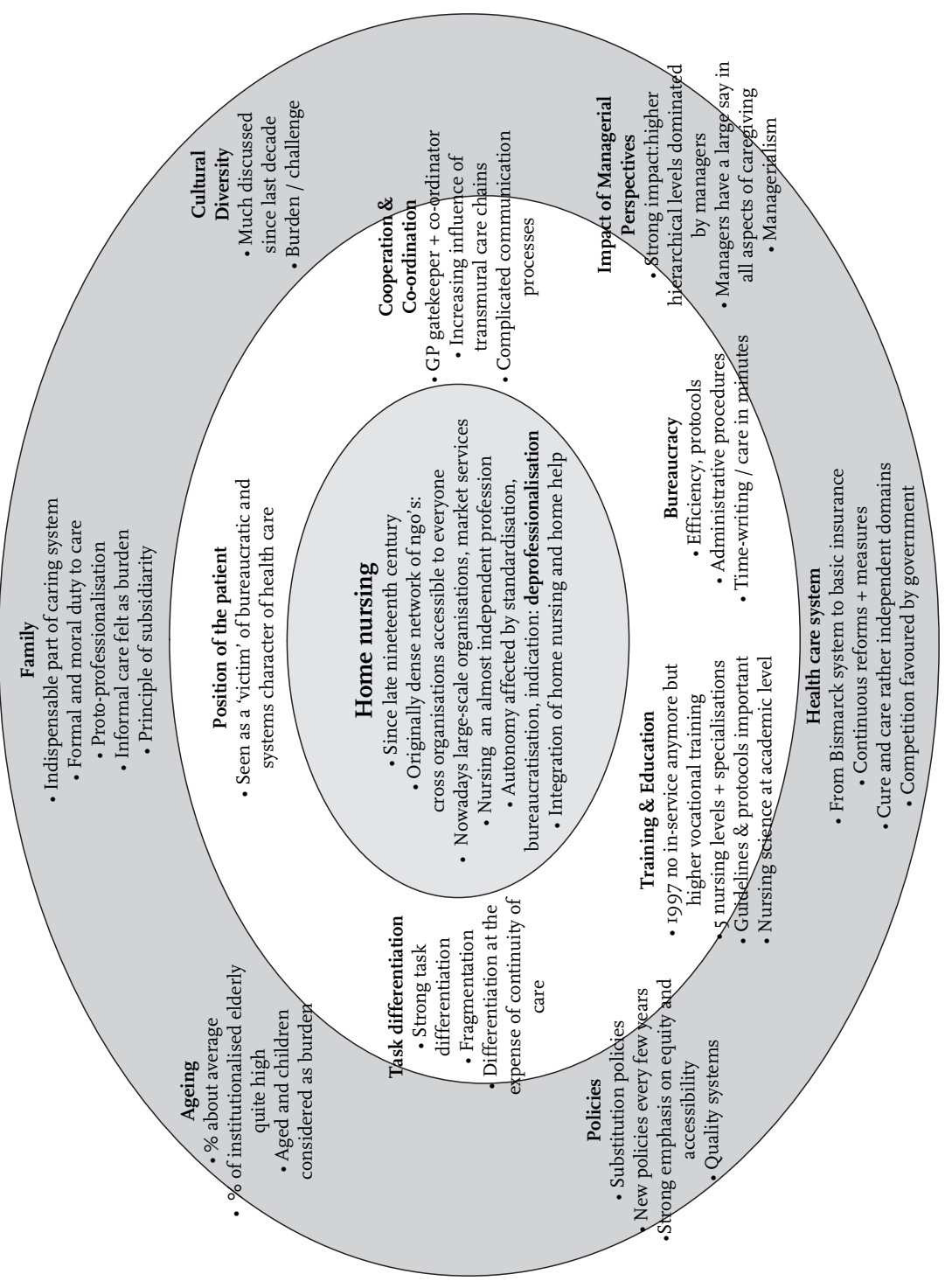


Chapter 7

\title{
Home nursing in Germany
}

\author{
- A medically oriented health care system \\ and strong reliance on the family
}

\section{I Introduction}

'Despite the stated preference of German social policy for community rather than institutional responses to care problems, when examining formal services, it is evident that there continues to be a structural imbalance which favours institutional care. Moreover, it is an imbalance which appears to be increasing.' (Scharf, r998e p. I34)

'Die Gesundheitsversorgung ist in Deutschland stark am medizinischen Modell ausgerichtet, wodurch andere Formen der Sorge lange Zeit gesellschaftlich eher vernachlässigt wurden. Das gegenwärtige Interesse an Sorge steht in Deutschland mit der Tatsache im Zusammenhang, daß die deutsche Gesellschaft eine “vergreisende” ist.' (Schnepp, 200I p. II)

These quotations illustrate that the German health care system is quite medically and technically oriented, and that medical care and hospital traditionally has had priority over other forms of care. Next to cure-oriented activities in health care, the reliance on the family to provide care and support is strong in Germany. The domain of professional caring activities, such as home nursing, has encountered financial as well as structural barriers for a long time.

Another striking aspect of German society and health policies is that elderly are considered as a problematic societal group. For a long time, they have been a somewhat neglected group that were to cope with their health and financial problems themselves or within their families. Compared to many other European countries, Germany was quite late with developing policies targeting on elderly people in the community. Current social and economic policies are largely responsible for a pessimistic view on elderly: 'The language has become littered with terms which portray the ageing process as a burden' (Scharf, I998b p. I), such as Überalterung (over-ageing) and Altenlast (burden of the elderly). The situation of elderly and elderly policies are however gradually receiving more attention in health policies and by the scientific discipline of (social) gerontology, that studies the specific situation of elderly from multiple perspectives. Although other European countries 
are confronted with the same demographic situation, doubts whether the national economy is able to provide sufficient resources to cope with the increasing health and social care needs of elderly people is most pronounced in Germany (Scharf, I998b p. I4).

The problem of the double ageing of the population in the $\mathrm{EU}$ is largest in Germany, as the number of elderly and very old people is highest (Eurostat, 2007a). Namely, I8.6\% of the population (as much as I3 million people!) is aged 65 years of over, compared to $14.0 \%$ in the Netherlands (just over 2 million people) and I5.0\% in Denmark (less than one million people). The number of people between 65 and 79 years of age is also highest in Germany, namely I4.3\% Also, the number of elderly of $65^{+}$that are living alone is particularly high in Germany, namely 4I\% (I992) (Giarchi, I996h; OECD, I996b). This puts strains on the provision and availability of home care, as single elderly people, especially very old women, are the category of clients that are most in need of home care services. For many decades, however, caring and supporting activities that are performed in the 'periphery' of the health care system did not have a very high priority in Germany, compared to medical and cure-oriented activities performed in the 'medical centre' of health care. ${ }^{\mathrm{I}}$

\subsection{The dominance of the medical sector in health care}

One of the characteristics of the German health care system is the strong position of physicians. Namely, in Germany, as in France, the number of GPS and hospital beds per I ooo population is quite high compared to the Netherlands and Denmark, see table I.

Table I Number of GPS and hospital beds per I ooo population

\begin{tabular}{lcccc}
\hline Number per 1000 population & France & Germany & The Netherlands & Denmark \\
GPS & I. $5^{\mathrm{f}}$ & I.I $^{\mathrm{f}}$ & $0.4^{\mathrm{g}}$ & $0.6^{\mathrm{g}}$ \\
Hospital beds $^{\mathrm{b}}$ & 9.0 & 7.0 & 5.0 & 4.6 \\
\hline
\end{tabular}

Sources: b: Eurostat, 200Ia p. 7I, 73; OECD, I998 chapter Medical resources and activity, year: f. 1996 , g. 1995 .

In the first two countries, the number of GPS per I 000 population is I.I - I.5 compared to $0.4-0.6$ in the latter three countries. The number of hospital beds varies between 7 and 9 beds per I oo population and $4.6-5.0$ per I 000 inhabitants

I For an elaboration of the concepts 'periphery' and 'medical centre', see chapter two of this thesis and Philipsen, 2000. 
respectively. This indicates that the medical sector is quite dominant over other health care activities in the systems of France and Germany compared to the other two countries.

This can also be recognised in nursing, which is not considered as an independent activity in Germany, but primarily as an extension of medical activities and complement to medical or hospital care. Nursing tasks are more medical-technical in character than in other European countries (Derksen, I991a; Derksen, Zee et al., I99Ib; Zee, Kramer et al., I994). Until I995, for example, the activities of nurses were only reimbursed when they were medical-technical in character. In this sense, nursing is more considered as an allied profession than as an autonomous profession with an independent task domain in Germany: 'Die Pflege hat sehr stark darunter gelitten, dass sie als "Assistentzberuf” der Medizin definiert wurde' (Schleich, 2004c). This contrasts with Denmark, where the nursing profession is generalist in character, and non-technical values such as providing support and empathy are also considered as very important aspects of nursing (Børgsen, 2002, April). Also, in Germany, procedures determining whether people are entitled to home nursing services are largely controlled by physicians: a medical referral is needed to receive reimbursement for nursing care (Delnoij, I996a), and the insurance committees deciding on the assignment of nursing care is staffed by physicians. Sometimes, physicians in some regions are not willing to cooperate with the Sozialstationen (Social Stations or social centres) providing home nursing and home help (Dieck, I99I). Thus, in Germany, the medical sector has a stronger position in the health care system than the nursing sector, and home care has not had a very high priority in German health policies for a long time.

\subsection{A strong reliance on the family in the German welfare system}

The dominance of medical care over nursing and supportive care is strongly related to the way health care is organised in Germany. Germany is a Federal Republic consisting of I6 Länder, 5 of which were incorporated in I990, when the former German Democratic Republic (East Germany) was united with the Federal Republic (West Germany) (Rostgaard and Fridberg, I998d p. 485). Each of the Länder has its own legislation. ${ }^{2}$ Germany is a democratic and social federal state, often characterised as a conservative and corporatist type of welfare state:

2 This federal structure results in different cultures and values per region and a different functioning of health care facilities. Also, it results in difficulties to gain access to nationwide figures of health care activities. 
'conservative because the role and responsibilities of the family are stressed in the German welfare state, and corporatist because implementation of the "social state" is carried out by non-government insurance associations and large associations of independent welfare providing service agencies, within the framework set out by legislation and public authorities.' (Rostgaard and Fridberg, I998d p. 485-486)

The principle of the social state is not just directed at the government; citizens are expected and obliged to practice individual and collective self-help and social solidarity. Traditional family values are strong in Germany, and there has been a moral obligation (and until the introduction of the Pflegeversicherung in 1995 a legal obligation as well) for family members to provide informal care to older and indigent people. As in many European countries, especially those in central and south Europe, the emphasis on family responsibilities regarding elderly is reflected in the role of the state in health care. In terms of health and social policies, this implies that welfare and social care is governed by the principle of subsidiarity. This means that the community should act first, and that the state (whether at national, regional or local level) should only intervene when there is no alternative. It should not offer services if another organisation can perform the task satisfactorily on the state's behalf, and only function as a safety net (Scharf, I998e p. I30):

'It means fundamentally that the response to need should first be made within the immediate world of the clients: at first, with family or relatives. Failing these, they are expected to call upon informal helpers, either neighbours or friends. Should they not be able to provide support, assistance should be sought from the voluntary Church or lay organisations. In the event of these agencies of organisations not being able to help, the next step is to approach the welfare organisations and the statutory personal social services. The state is the last port of call.' (Giarchi, I996b p. 32)

Care is primarily left to family members, but this is not explicitly articulated in family policies. There is no unequivocal vocabulary about family care, as is the case for example in the Netherlands, where home care policies formulate informal care (mantelzorg) as a necessary and important complement to professional care. Also, the availability of informal carers is explicitly weighed in the assessment of the need for home care by the regional assessment organisations (RIOS) in the Netherlands. In Germany, however, the ambiguity of the conceptualisation of informal care is reflected in the lack of consistency in scientific literature, as it is referred to as Familienpflege, häuslicher Pflege, häuslicher Versorgung, Home-Care, Angehörigenpflege, Tochterpflege, naturwüchsiger Pflege, and Laienhilfe (Schnepp, 20or p. 8). The 
individuals providing informal care are also described in different terms. ${ }^{3}$ Informal carers themselves mostly motivate their caregiving as something natural, a normative and moral 'internal sense of duty', a feeling to have to meet the other's expectations, and feelings of reciprocity: "Derjenige, der Sorge erhalten hat, sorgt für denjenigen, der für ihn gesorgt hat” (Schnepp, 200 p. Io) (He/she who has received care, cares for the ones that cared for him/her).

In accordance with the principle of subsidiarity, the provision of social and welfare services is left to various non-governmental and non-profit associations that traditionally have had a strong element of voluntary work. These are organised in six major associations that co-operate within an umbrella organisation and have become large professional organisations (employing more than one million people in different services). German policies for long-term care of frail elderly people reflect the belief in family solidarity. Public initiatives to support the provision of professional care have developed slowly, and policies have aimed at reinforcing family care. Benefits in kind for professional care provided in the community have been developed alongside development of cash benefits for family members that provide the care. As a result, the vast majority of benefits paid for long-term care of older people are cash 'payment-for-care' benefits, and only small proportions of older people receive care from professional home care services. Thus, mostly, help and support is sought within families. In the Netherlands, a similar development has taken place in the form of personal budgets (PGBS) that can be assigned to people in need of long-term community care (Ramakers, I998a). Patients now have a larger say in determining the kind of care they want to receive and can choose between professional providers or pay informal carers. The presuppositions behind these regulations are however quite different in both countries; in the Netherlands, the independence of clients is increased as the relation with their informal or professional carer becomes more on an equal footing, as the care receiver becomes their 'employer'. In Germany, however, the benefits largely reinforce the existing tradition that people first and foremost tend to their family to provide informal care.

This situation contrasts with Denmark, where health care is primarily a welfare state matter and provision is predominantly public to relieve family members from caring responsibilities. Also, the Danish nursing sector is defined as having an important independent function alongside the medical sector, while the nursing sector is rather overpowered by the medical sector in Germany.

3 Such as Pflegeperson, informeller Pflegeperson, pflegenden Angehörigen, pflegenden Familienangehörigen, Familialen Helfern (Schnepp, 200I, p. 8). 


\subsection{A social insurance system with barriers for community care}

The health care system in Germany is financed by a social insurance system that has its origins in the late nineteenth century (Mossialos and Le Grand, I999b). The role of the government is largely restricted to providing a regulatory framework within which the provision and financing of health care services take place. The execution of the insurance scheme is executed by intermediate, non-governmental organisations, the health insurance associations. There are as much as 800 of these independent and non-profit social sickness funds (Gesetzlichen Krankenversicherung or GKV). ${ }^{4}$ Health care is provided by several different welfare organisations, many of these are of religious signature, and private providers. This pluralist way of organisation makes the health care system quite fragmented.

Medical treatment has already been an insurance-based and legally enforceable right for a long time; nursing care however only since the introduction of the Pflegegesetz in I995. Within the insurance framework there is a strict hierarchy of service entitlement, ranging from medical treatment, specialised nursing, basic nursing, and home help as a supplement to nursing. The health care system has inherent barriers for the provision of long-term ambulant care, while access to physicians and hospitals is easy. For example, people can consult specialists directly, while in the Netherlands, a referral from a GP is needed. Also, German patients have free choice of family doctor among sickness funds.

As France and the Netherlands that also have an insurance based system, the majority of health care is financed by social insurance contributions. In Germany, $90 \%$ of the population falls under the insurance scheme, $75 \%$ compulsorily as their incomes fall below a certain level, and I3\% are voluntary members (Greiner and Graf v.d. Schulenburg, I997 p. 80; Mossialos and Le Grand, I999b). People with higher incomes can also choose for a private insurance company. 60 per cent of the privately insured people, that is 6 per cent of the total population, are ineligible to take gkv cover because they are Beamte (civil servants) such as police officers, university professors and public officials. Two per cent of the population is covered by free governmental health care. Only 0.3 per cent of the population chooses not to be covered by any insurance at all. These figures indicate that the social insurance scheme enjoys a high degree of acceptance within the German population. Competition between funds will increase in the near future, which is already the case in the Netherlands, where most insurance organisations are forprofit insurance companies.

4 The number of sickness funds is decreasing: there were still I22I in I993, but many local funds have merged (Greiner, I997 p. 8I). 
For people that are not labelled as 'ill' in the medical sense, but 'merely' as disabled or in need of help, some coverage for long-term care at home was introduced with the I988 Health Insurance Reform Act, but both the amount of care and the period of time covered were still severely limited. A more radical solution has been sought with the introduction of a compulsory insurance scheme for long-term care for the entire population, namely the Pflegegesetz in I995. ${ }^{5}$ This is considered as a major innovation in the German health care system in the literature and in politics. However, this law, designed to cover the risks of long-term care, is still not adequate, as only specific types of care are covered. Until I995, people in need of long-term care have had to rely on the social welfare system, which is a highly selective, means-tested system operating on the basis of the principle of subsidiarity, which means that only when no family members were available to provide care or to pay for professional care and patients were not able to pay themselves, they were entitled to social welfare arrangements. This had a stigmatising effect on these people. Although the entitlement to long-term home care has been problematic for a long time, and in some ways still is because entitlement regulations are very strict, home care has been fairly successful in providing short-term care after medical treatment, and enabling earlier discharge from hospital. Thus, the characteristics of the health and insurance system in Germany resulted in the fact that tasks of home nurses are more medical-technical in character than in other European countries, and home nursing is primarily considered as an extension of medical care. Other aspects of nursing are less pronounced in Germany.

The situation in Germany is very different compared to for example Denmark, one of its neighbouring countries. Not only the health systems are different in character, but also the choices in health policies, as value-orientations regarding matters of health care and family responsibilities rather diverge. In Denmark, debates have already been held about long-term care since the I970s, and fairly radical policies favouring home care over institutional care have been introduced at that time. These have resulted in coherent policies at the lowest local level regarding long-term care for elderly people, and the availability of a variety of services enabling elderly people to remain in their own homes as long as possible, supported by adapted houses, professional home nursing and home help, and a range of other facilities. In Germany, however, debates held in the mid I990s, still focussed on the preceding question whether and how and to what extent long-term care should be financed. This reluctance to entitle people to home care services has resulted in a further medicalisation of care in Germany. Because a medical

5 For a long time, the state has been reluctant to extend social welfare entitlements, while at the same time, insurance organisations were very reluctant to extend coverage beyond medical treatment. Therefore, the introduction of the Pflegegesetz took some decades. 
referral is needed for home care, and only the more nursing-technical activities are covered under the Pflegegesetz, organisations providing home care services tend to medicalise their services (for example, by shifting provision from personal care and household tasks to more specialised nursing tasks, such as giving injections, or blood transfusions).

\subsection{German social and health policies regarding long-term care needs}

The concept of Health Insurance funds originated from the Social Insurance Act, passed by the Bismarck administration in I88I, introducing compulsory insurance for low-paid industrial workers (Delnoij, I996a; Swaan, I989b). This can be considered as the beginning of the modern German welfare state. Before that, since the mid-nineteenth century, there had already been some regulations in Prussia for industrial workers, such as coal miners, to protect them from acute dangers associated with work, thereby guaranteeing employers an adequate supply of workers (Scharf, I998c p. 25). That such policies were adopted at all can be attributed to the desire of the predominantly conservative politicians to overcome some of the worst side effects of Germany's rapid industrialisation. Also, there were some very powerful employers (mainly industrialists) that provided some kind of social benefits to their employees, such as housing, education, and limited pensions, primarily to pre-empt state intervention into their affairs and guarantee a steady labour supply. However, the level of support was still very limited. The emphasis in Germany continued to be placed on self-help, upon getting people back to some form of work so that they could look after themselves. Local authorities were responsible to provide welfare assistance to individuals so that they could help themselves, which is still the case in contemporary Germany. Social welfare arrangements for the non-working part of the population were dealt with by local churches, communal bodies and private charity associations.

The key elements of Bismarck's social insurance legislation were intended to protect individuals against the social risks associated with ill health (I883), industrial accidents (1884) and invalidity and old age (1889). Their function was to provide limited compensation to industrial workers who were forced to withdraw from the labour market temporarily or permanently. At the same time, it was an attempt to divide workers according to social class and de-politicise the emergent social democratic movement. Cover did not extend to 'less politically dangerous social groups', such as white-collar workers or the self-employed, as these were less 'dangerous'.

Since the I880s, there has been a remarkable degree of continuity in policy in Germany (Scharf, I998c p. 24). The principal elements of Bismarck's social policies been preserved until the I990s, and have even been reinforced by recent leg- 
islation. It is rather remarkable that in spite of the enormous political, social and economic upheavals experienced by Germany more than a century, such as the collapse of the German Empire and Weimar Republic (I918-I933), World War I, the rise and fall of National Socialism, the Second World War, and the division (I949) and reunification (I990) of East and West-Germany, these have not altered the fundamental structure of Germany's social policy regime. Each political period and government has left its own mark, but German social policies are still characterised by the following principles:

- It is an employment-based insurance rather than a universal insurance scheme. This means that non-working groups, such as pensioners and the unemployed, do not have a high priority in the benefits provided;

- The social insurance system is funded by contributions of employers and employees, and is not administered by the state such as a public system, but by intermediate, non-governmental agencies; the insurance organisations (as in the Netherlands and France);

- Two principles are underlying the social insurance system, namely that of contribution and equivalence. The contributory principle means that there is hardly any direct taxation (such as in Denmark and the $\mathrm{uk}$ ), but primarily contributions of employers and employees. According to the equivalence principle, benefits are linked to an individual's earnings and contribution record. The system thus attempts to compensate employees for lost income rather than redistribute benefits according to social need. The focus on labour activity results in a difficult position of non-working groups in society, such as pensioners and the unemployed, that had to turn to social assistance when they could not pay for services themselves;

- The principle of social self-administration requires that the representatives of employers and employees work in co-operation to administer the social insurance schemes;

- The combination of compulsory insurance and the organisational diversity of insurance bodies led to competition between insurance providers, as in the Netherlands (Schmitt, I988 in Scharf, I998 p. 27-28).

In the I920s, improvements in social policies contributing to the situation of elderly were introduced, despite serious economic crises. For example, in the I920 2 pensions were reformed, and in I924 the poor relief was replaced by a system of social assistance (Sozialfürsorge). Its purpose was to produce a qualitatively different type of social support for poor people, by establishing a basic right to state support at times of acute need and to promote the social integration of people who fell upon hard times, thereby trying to shift away from established, stigmatising patterns of thinking that held individuals personally responsible for finding themselves in a state of poverty. However, it failed in its attempt to release poor people 
of the stigma of dependence on state support, not in the least because of the strict means-testing of benefits. This stigma, also attached to the current Sozialhilfe, still affects the provision of social assistance benefits in Germany. While in Denmark it is common practice to apply for social assistance and professional care and support under the public health system, in Germany, this is accompanied by stigma because it is considered and institutionalised as being the last available alternative, and signifies that self-help and support from the family and neighbours has failed.

After I949, older people in East Germany (the German Democratic Republic) were marginalised and apart from artificially low housing and fuel costs, came to benefit from social policies after the reunification in I990 (Scharf, I998c p. 35). In West Germany (the Federal Republic), the Allies tried to introduce a Beveridge national health system after the Second World War, but did not succeed. In the period after the war, the German government strived for restriction of social expenditure to control the inflation and promote economic growth. Only since the economic miracle (Wirtschaftswunder, from the mid I950s until the latter half of the I960s), funds were sufficient to introduce substantial improvements in the welfare state, such as the I957 Pension Reform Law. This was considered necessary, as in I955, 5.5 million pensioners were living below the poverty line, with pensions of little more than $30 \%$ of average earnings. The reform removed a substantial proportion of older people from acute economic difficulties.

In I96I, the Social Assistance Act (Bundessozialhilfegesetz) was introduced, but the treatment of older people under this law has been largely unfavourable, as support for age-related physical or mental difficulties is only possible after means-testing. This means that only when people or their family are not able to pay for the care they need themselves, they are supported by social assistance. Thus, instead of giving elderly people entitlements to receive professional care and support, which is common in most other European countries, the notion in Germany that elderly are a social problem because they need social assistance is reinforced. The persisting emphasis on the principle of insurance instead of the less developed principle of provision (Versorgungsprinzip) tends to work to the disadvantage of people that do not or no longer have access to the labour market. The effect is that more and more people, including significant numbers of older people, are made dependent of forms of social assistance instead of being reimbursed for home care services that are reimbursed by the health insurance system.

Since the late ig6os and I970s, economic problems prevented further social policy development. The problems of older people were however noted at ministerial level; in I970 a small department Altenhilfe Probleme der älteren Generationen (elderly support problems of the older generations) was established within the Federal Ministry of Youth, Family and Health (Bundesministerium für Jugend, Familie und Gesundheit). However, this did not lead to the fundamental rethinking of social 
policy such as for example in the United Kingdom in this period. ${ }^{6}$ The unification of East and West Germany in I990 highlighted problems that were already present. From then on, an official social ageing policy is gradually developed, but still rather reluctantly. In I99I, a Federal Ministry for the Family and Senior Citizens was established (Bundesministerium für Familie und Senioren) to address the situation of elderly people. In I994, however, the Ministry was enlarged to a Bundesministerium für Familie, Senioren, Frauen und Jugend, thus spreading the priorities over more areas and making it less likely that a coherent, structured elderly policy could be developed.

Currently still, older people's interests are frequently ignored in the policy-making process (contrary to Denmark), especially when these conflict with interests of more powerful social groups, such as employers or employees (Scharf, I998c p. 44). Although the increasingly difficult situation of people requiring long-term care and the burden on informal carers was already debated since the I970s (Bundesministerium für Arbeit und Sozialordnung, 1997), it was not until I994 that the Federal Parliament (Bundestag) finally approved legislation that was to entitle people to reimbursement of long-term care, namely the Pflegeversicherung. According to Scharf, "The delay in the care legislation can be regarded as an indication of the low status accorded to people requiring care and to informal carers within a social welfare system that is so clearly oriented towards the labour market" (p. 45). Schnepp states that:

'Wenn Angehörigenpflege gegenwärtig in Deutschland im Mittelpunkt politischen Interesses steht, so kann dies auf die Einführung des sogenannten Pflegeversicherungsgesetzes zurückgeführt werden. Dieses Gesetz bietet den Rahmen für Angehörigenpflege, häusliche Pflege und professionelle Pflege im häuslichen Bereich. (...) Die Diskussionen über dieses Gesetz sind nicht neu, sie wurden bereits vor 20 Jahren geführt (Meyer, 1996). Ausgangspunkt führ diese Diskussionen war die Absicht, Pflegebedürftigkeit besser Abzusichern und die Position der pflegenden Angehörigen zu stärken, da die belastenden Konsequenzen der Angehörigenpflege bekannt waren. Außerdem wollte man gesellschaftlichen Entwicklungen, wie Individualisierungstendenzen und der Abnahme primärer sozialer Netze, vorbeugen. Gleichzeitig bestand die Absicht, die bisherigen Säulen der Sozialversicherung durch eine zusätzliche Versiche-

6 For example, the uk White Paper Caring for people (Department of Health I989) displayed an encompassing view on community care, stating that 'Community care means providing the right level of intervention and support to enable people to achieve maximum independence and control over their own lives. For this aim to become a reality, the development of a wide range of services provided in a variety of settings is essential' (Giarchi, I996 p. 30). 
rung zu entlasten. Insgesamt sollte es zu einer neuen Kultur des Pflegens kommen. Diese Forderung kann sich wohl nur auf die Politik selbst beziehen, da es immer eine Kultur des Pflegens in lebensweltlichen Bereich gegeben hat.' (Schnepp, 200I p. II)

The Pflegeversicherung, the long-term care insurance executed under the Pflegegesetz of I994 and part of the social health insurance system, is compulsory for the entire population and paid for by employers and employees. It has the following characteristics:

- A preference for community-based care over institutional care;

- A limited choice between domiciliary and institutional care provisions;

- People receiving care have to contribute in the form of co-payments if their means are sufficient;

- It intends to upgrade social status of care professionals;

- Informal carers are provided with social security;

- A preference for more flexibility in care services (Scharf, I998 p. 46)

The care reform represents a significant step forward in terms of a provision for elderly people, seeking to overcome problems associated with the provision of long-term care. As a result of the ever-increasing numbers of elderly people and changing health and social policies regarding elderly in other European countries, public values and expectations in Germany have gradually changed as well. The public opinion that there is a crisis in nursing (Pflegenotstand) has led policy-makers in Germany to reconsider existing arrangements for elderly people in need of care and support. Greater emphasis is now placed upon promoting services that enable older people to remain living independently. For example, the i984 amendment to the Federal Assistance Act formally prioritised the provision of ambulatory care (i.e. community care) over institutional care (Scharf, I998e p. I49):

\footnotetext{
'Sozialgesetzbuch xi 『3: Vorrang der häuslichen Pflege: Die Pflegeversicherung soll mit ihren Leistungen vorrangig die häusliche Pflege und die Pflegebereitschaft der Angehörigen und Nachbarn unterstützen, damit die Pflegebedürftigen möglichst lange in ihrer häuslichen Umgebung bleiben können. Leistungen der teilstationären Pflege und der Kurzzeitpflege gehen den Leistungen der vollstationären Pflege vor.' (Schleich, 2004a).
}

Thus, the emerging preference in Germany for community rather than institutional care, which has been evident in social policy statements for some years now and is gradually finding its way into legislation, closely mirrors current policy reforms in many other European countries, such as the United Kingdom and the Netherlands. 
However, the Pflegegesetz is also criticised, because it reinforces the dependence of people requiring care upon their families, as care allowances that are paid are so low that these are insufficient to purchase professional care. "This effectively leaves care in the hands of relatives, encouraging families to persevere with caring responsibilities even when institutional provision may be more appropriate" (Scharf, I998c p. 46; Theobald, 2003). Thus, the long-standing emphasis in Germany on individual and family solutions to common social risk factors regarding health and social needs perpetuates. Scharf also states "there still appears to be a long way to go before the services on offer to older Germans match the stated policy preferences" (Scharf, I998d p. II4). As community care is still weakly organised and unevenly distributed in Germany, and informal care and support mechanisms are under increasing pressure, a structural imbalance and tension in care provision exists which still tends to favour institutional responses to the health and social care needs of elderly people. For example, a substantial proportion of elderly people receiving hospital care do not necessarily require this kind of care, or remain longer in hospital because of shortcomings in other spheres of Germany's health and social care system (Scharf, I998e p. I20). Evers (I995) also states that:

'Die Privilegierung von Gesundheit gegenüber Hilfe und Pflege verweist auch auf kulturelle Werte und Deutungsmuster, aufgrund derer es gesellschaftlich weithin akzeptabel erscheint, nahezu jeden Preis für den lebensverlängernden Fortschritt zu zahlen, während immer noch allzu oft der "Notgroschen" des Ersparten nur zögernd für Pflegeleistungen locker gemacht wird.' (Evers, I995) ${ }^{7}$

The strict separation of (curable) health from (incurable) social care needs lies at the heart of the problems associated with the organisation and funding of social care and support. While the health insurance scheme will fund the treatment of older people's medical conditions when they are considered as curable or likely to respond to treatment, it will not meet the costs of caring for older people whose ailments are regarded as simply being characteristic of old age frailty. Thus, for example medication for dementia patients is reimbursed, but costs for supervision of these patients are not. Before the Pflegeversicherung, forms of support for elderly people were mostly paid by themselves or by their families, or when their means were lacking, by local authorities (social assistance). As the costs of professional social care were high, most recipients were quickly reduced to the (socially stigmatised) status of social assistance recipients.

As the shift from institutional care to community-based care in policies in Germany is fairly recent compared to other European countries, and the period

7 In Schnepp, 200I p. I2. 
in which elderly were not considered as a vulnerable group that deserved special public and government attention, it will still take considerable time before the new ways of provision are consolidating. As is shown further in this chapter, some transitions are already taking place; remarkably however, not so much from institutional to community-based care, but within different kinds of community care. $^{8}$

Also regarding the living environment of elderly people, Germany is less far than most other countries studied in this dissertation. For example, sheltered housing schemes (betreutes Wohnen) that have existed in other European countries for several years now are still regarded as innovative in Germany. Also, many houses need to be adapted to the needs of elderly people, to enable older people to remain living in their own homes (Scharf, I998d p. III).

\subsection{The provision of elderly care in Germany}

In Germany (counting 82.5 million people), about 7 million people are not able to care for themselves any more and need some kind of support. About I.5 million people are cared for in their homes, and 570 ooo live in institutions (Schleich, 2004a). The situation in many nursing homes in Germany is critical, and these do not have a very good reputation (Delnoij, ı996a). In many of these, nursing does not meet the legal standards:

'Immer wieder decken die Medien Pflegeskandale auf, berichten von untragbaren Zuständen in Pflegeheimen. Überforderte Pflegerinnen und Pfleger kämpfen selbst mit gesundheitlichen Problemen und wechseln oft nach wenigen Jahren den Beruf.' (Schleich, 2004a)

In the Netherlands, similar problems are reported in the media. In both countries, people in nursing homes are sometimes only fed and washed because of lack of personnel; also called 'pyjama days', or in German 'Satt-und-sauber-Pflege':

'Der Pflegenotstand in Deutschland besteht zunächst einmal in einem Personalmangel: Wir haben einfach zu wenige Arbeitskräfte, insbesondere in der stationären Altenpflege. Zusammen mit ihrer unzureichenden Ausbildung führt das in Einzelfällen zu Pflegefehlern. Die Menschen bleiben in den Betten lie-

8 Especially from more traditional forms of care (Gemeinde- und Haus- und Familiepflegestationen) to more innovative forms of care such as Sozialstationen and Mobilen Sozialen Hilfdiensten. 
gen, werden nicht mehr aktiviert, die zwischenmenschliche Kommunikation liegt brach und die Ernährung ist unzureichend.' (Schleich, 2004b)

Figure I (below) indicates the division of costs over different types of social services and cash benefits for people of 65 years and older:

Figure I Division of costs over different types of social services and cash benefits (people 65+)

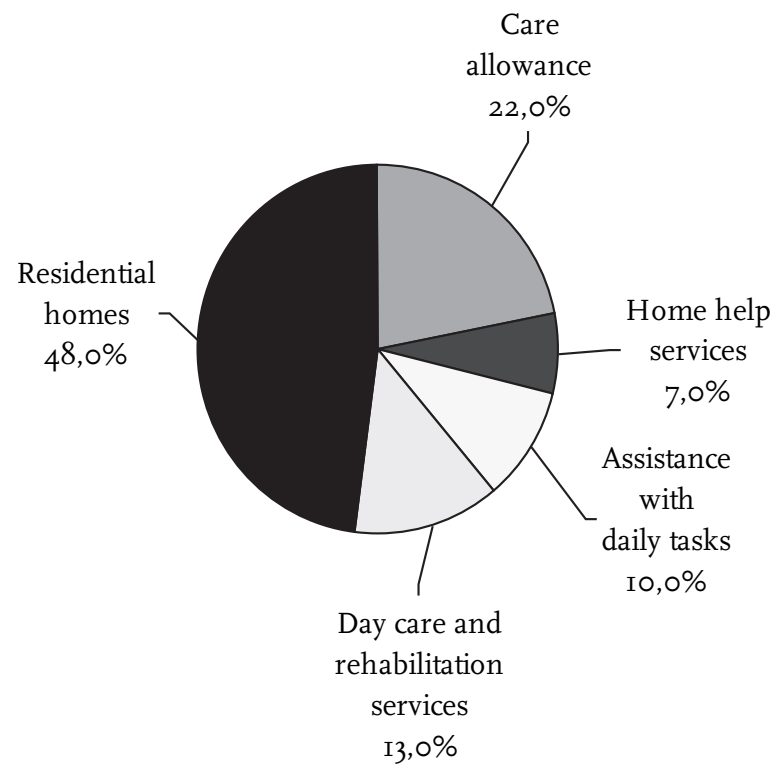

Source: OECD Social expenditures database I998, in: Rostgaard, I998d p. 494.

The German system of care and welfare is characterised by an institutionalised form of co-operation between the public bodies engaged in the field of care and various (private initiative) welfare associations. During the past thirty years, the so-called Sozialstationen (Social Stations or social centres) have become the most important community-based organisations providing home nursing, home help and other services as well (Rostgaard and Fridberg, I998d p. 530).

Several types of organisations are providing ambulatory care for the elderly and other categories of clients; the Ambulante sozialpflegerische Dienste (organisations for ambulatory social and nursing care) (Delnoij, I996a; Rostgaard and Fridberg, I998d). These can be divided into:

- Gemeindekrankenpflegestationen, only providing (specialised) home nursing;

- Haus- und Familiepflegestationen, the 'traditional' home help services providing domestic care and family support; 
- Dorfhelferinnenstationen, providing housewife relief services;

- Sozialstationen, (social centres or Social Stations) which are integrated organisations for home nursing and home help and also providing other ambulatory services;

- Mobilen Sozialen Hilfsdienste, only providing cleaning, meals-on-wheels and other kinds of help at home for elderly.

Comprehensive and regular data on the number of organisations providing ambulatory social and nursing care are only available for services run under the auspices of welfare associations, the so-called Freie Wohlfahrtspflege. These provide social support on a non-statutory, non-profit basis. These consist of six national umbrella organisations:

- Diakonisches Werk (Protestant);

- Caritas (Catholic);

- Arbeiterwohlfahrt;

- Zentralwohlfahrtstelle der Juden in Deutschland;

- Deutsches Rotes Kreuz;

- Deutscher Paritätischer Wohlfahrtsverband (Delnoij, I996a; Derksen I991a.

Table 2 Number of home-based care services and employed persons under the Verbänden der freien Wohlfahrtspflege

\begin{tabular}{|c|c|c|c|c|c|c|c|c|c|c|c|}
\hline & \multicolumn{2}{|c|}{$\begin{array}{c}\text { Social } \\
\text { Stations }^{1}\end{array}$} & \multicolumn{2}{|c|}{$\begin{array}{c}\text { Home } \\
\text { nursing }\end{array}$} & \multicolumn{2}{|c|}{$\begin{array}{c}\text { Domestic } \\
\text { work }^{3}\end{array}$} & \multicolumn{2}{|c|}{$\begin{array}{c}\text { Dorf- } \\
\text { helferinnen }\end{array}$} & \multicolumn{2}{|c|}{$\begin{array}{l}\text { Mobile home } \\
\text { services }\end{array}$} & $\begin{array}{c}\text { Total } \\
\text { number }\end{array}$ \\
\hline & $N r$. & Empl..$^{5}$ & $N r$. & Empl. & $N r$. & Empl. & $N r$. & Empl. & $N r$ & Empl. & Empl. \\
\hline \multicolumn{12}{|c|}{ West Germany } \\
\hline 1984 & I 542 & I4 309 & 2248 & 3658 & 729 & 7891 & $4 \mathrm{I} 2$ & 607 & 252 & I 859 & $\begin{array}{llll}5 & 183 & 28 & 324\end{array}$ \\
\hline 1990 & $2 \mathrm{I} 4 \mathrm{O}$ & 2I 492 & I 585 & 2902 & $87 \mathrm{I}$ & $44 \mathrm{I} 7$ & 463 & 659 & 729 & 4798 & 578834268 \\
\hline 1993 & 2403 & 30344 & I 066 & 3057 & 562 & 5452 & 433 & 927 & 892 & I 8 I3 & $5356 \quad 41593$ \\
\hline \multicolumn{12}{|c|}{ East Germany } \\
\hline 1993 & 666 & 5899 & 6 & $\mathrm{I} 2$ & 6I & 972 & 3 & 53 & 158 & I 279 & 894 \\
\hline \multicolumn{12}{|c|}{ Whole of Germany } \\
\hline 1993 & 3069 & 36243 & I 072 & 3069 & 623 & 6424 & 436 & 980 & I 050 & $3 \circ 92$ & 625049808 \\
\hline 1996 & 3749 & 54656 & 63I & 2315 & 599 & $38 \mathrm{I} 2$ & 430 & $82 \mathrm{I}$ & I 403 & 3696 & $68 \mathrm{I} 265300$ \\
\hline
\end{tabular}

Source: Rostgaard, I998 p. 531.

${ }^{\mathrm{I}}$ Sozialstationen

${ }^{2}$ Gemeindekrankenpflegestationen

${ }^{3}$ Haus- und Familienpflegestationen

${ }^{4}$ Mobilen Sozialen Hilfsdienste

${ }^{5}$ Full time and part time employed. 
Table 2 shows the number of services provided by the organisations falling under the Freie Wohlfahrtspflege and the number of persons employed in these organisations (Rostgaard and Fridberg, I998d). The services provided are not especially targeting on elderly people, but are working with all categories of clients in need of their services. The actual provision of care, however, differs from state to state and details of the organisation of home care vary greatly throughout the country. It is difficult to find information about the number of service providers outside these welfare organisations. ${ }^{9}$ Regarding the social centres, more than $82 \%$ operated under these organisations, $7 \%$ had mixed ownership and only $7 \%$ were run by the municipalities.

In I996, the Freie Wohlfahrtspflege were running a total of 6 8I2 Ambulante Socialpflegerische Dienste, employing 65300 persons (full-time or part-time). This is an increase of more than 30\% since I993, when there were 6250 organisations and 49808 employees. The number of Gemeinde- und Haus- und Familie-pflegestationen has decreased with 30\% between I990 and I993, while Sozialstationen and the mobile home help services have increased in number. There is a trend in the last years that the social centres are offering the more diverse kinds of care to different categories of clients, and are developing into more comprehensive care organisations. The number of agencies that only provided domestic work and help (home help) also decreased in recent years, at the favour of the social centres, mobile home help services and also recently, private service providers. Although the market for home nursing is still dominated by non-profit organisations, in recent years a growing number of nurses have decided to work free-lance of have developed for-profit nursing organisations (especially in some bigger cities, private services working on a commercial basis cover about $40 \%$ of the market).

'War die Pflege früher vornehmlich in der Hand gemeinnütziger Organisationen wie Caritas, Rotes Kreuz, Innere Mission, Arbeiterwohlfahrt oder Malteser Hilfsdienst, so sind in den letzten Jahren immer mehr private Anbieter auf den Markt getreten. Den gemeinnütziger Sozialstationen kann meist eine längere Erfahrung und eine gute Ausbildungsstruktur Ihrer Beschäfgigten nachgesagt werden, die sie allerdings auch vergleichsweise hoch bezahlen müssen. Die neuen privaten Dienste dagegen sind mit Ihrem Personal flexibler und daher oft Preisgünstiger, was sich manchmal auf die Qualität der Mitarbeiter auswirken kann.' (zDF-online Ratgeber, 2000)

9 Statistics on the number of providers will supposedly improve after the introduction of the social care insurance scheme, as the services have to be approved by the care insurance funds. 
In general, however, the home care market hardly seems to work as a market because recipients of benefits in kind lack the information about alternatives and about the quality of care (Rostgaard, I998 p. 532).

\subsection{The development of Sozialstationen}

Thus, traditionally and nowadays still, but at a much smaller scale, home nursing was provided by the Gemeinde-krankenpflegestationen. This kind of care is however increasingly taken over by the Sozialstationen. These can be considered as a very important development in the provision of community care. The first Sozialstation was established in I970 in Rheinland-Pfalz. Since then, they have been established all over Germany, in the former Eastern Ländern as well since I990. In the state of Bremen, the services are not provided by Sozialstationen but by Dienstleistungszentren (service centres). The specialised medical nursing provided by the social centres is financed by health insurance; the clients that are fully or partly covered by health insurance and sometimes by social assistance finance the other kinds of care. The new care insurance scheme benefits are the most important source of income of the social centres. The financial situation of the centres however varies according to difference in subsidies that are granted for different activities by the Länder and local authorities. In I996, the 3749 Sozialstationen in Germany employed 54656 (full-time and part-time) employees. A typical Sozialstation employs 8 to Io nurses, either generalists or nurses specialised in care for the elderly. Volunteers and nursing aides, who perform basic nursing procedures and home help services, assist them. The size of the population served by a Sozialstation varies between 12000 and 50000 , depending on its size and the degree of urbanisation. Most patients are visited once a day on workdays, some of them twice or three times a day. An average house call only takes about 20 minutes.

Two case studies show there seems to have taken place a shift in the focus of activities of the Sozialstationen (Dieck, I991; Verheij and Kerkstra, I992). They were intended as institutions for short-term intervention and acute nursing care, but in fact have become long-term care facilities: about $90 \%$ of their clients are chronically ill. Most of their clients are old and very old people. The case study performed in Berlin also showed that $57 \%$ of people with a high level of need did not receive any home care.

At the time of the introduction of the Pflegeversicherung, the Bundesministerium für Arbeit und Sozialordnung (Federal Ministry for Labour and Social Affairs) stated that there were several problems regarding the Social Stations:

- Sozialstationen take over patients from informal caregivers at a very late stage, so that their need of care is so high that they will be admitted to a nursing 
home. Had the necessary ambulatory care been available earlier, they could have stayed at home;

- Outside working hours, care is provided to a very limited degree only. Also the competitive behaviour of ambulatory care providers prevents agreements between providers to provide 24 -hours' service.

The Berlin case-study also mentions that other health care and social institutions are not very much aware of the range of services the Sozialstationen offer. Besides that,

- Coordination of services is minimal, because the actual condition of the client is unknown until the first visit;

- Timing is poor, for example because hospitals discharge patients on Friday afternoons, resulting in problems for the Social Stations;

- The job definition is too strict; low-status and low-income tasks have priority over actual nursing tasks;

- GPS are often unwilling to co-operate;

- Care allocation often is arbitrary and bureaucratic;

- Training standards are low, specifically among home care assistants.

Whether these problems are representative for other Social Stations and federal states is not clear, however. Nevertheless, the introduction of the Sozialstationen since the I970s seems to be rather successful, as the number of specialised home nursing services run by confessional welfare associations decreased at a fast rate. While in I970, there were still 8.700 of these in West Germany alone, this decreased quickly to 2248 in I984 and I 066 in I993. The I 072 Gemeindekrankenpflegestationen still existing in the whole of Germany in I993, employing 3 o69 employees, further decreased to only 63I in I996, employing 2 3I5 people in total. In the same period, the number of social centres managed by welfare associations, increased from one in I970 to I 542 in I984 (West-Germany), 3 o69 in I993 and 3749 in I996 (Rostgaard and Fridberg, I998d).

\subsection{Educational levels in nursing}

There are three major differentiations in nursing, primarily education for nursing in institutional settings. A specialisation into community nursing exists, but is not compulsory for working in home nursing; only a minor part of the nurses working in the community is qualified as a Gemeindekrankenschwester (Derksen, I991a). This is quite different from the situation in the Netherlands, where home care organizations employ different levels of (specialized) home nurses and home helps, but almost no other kinds of nurses. In Germany, however, a greater variety 
of professionals is employed in organizations that provide nursing and care in the homes of people. The following three nursing levels, described in the I985 Gesetz über Berufe in der Krankenpflege and Ausbildungs- und Prüfungsordnung für die Berufe in der Krankenpflege, are distinguished in Germany:

- Krankenschwester or -pfleger: nurse generalist with three years of education. Many of them have specialised as a community nurse (Gemeindekrankenschwester) in one additional year of part-time study that can be entered after working in the profession for at least two years;

- Krankenpflegehelfer(in); auxiliary nurse, one year of education;

- Altenpfleger(in), specialised in care for the elderly, two or three years of education.

\subsubsection{Krankenschwester or -pfleger}

Krankenschwester or pfleger have followed three years of education at Krankenpflegeschulen that are part of most hospitals (Diakonisches Werk der EKD, 2004a; Caritas Soziale Berufe, 2004a). Nurses at least have had i 600-2 Ioo hours of theoretical training and $2500-3000$ hours of practical training, that is closed with a state examination. People already qualified for specific health professions are allowed to follow a shorter training course. Students can choose between four working areas for their practical training:

- General medicine and nursing of elderly people;

- Surgery;

- Gynaecology or Urology, Infant Care;

- Psychiatry, Child Care and home nursing.

They work in hospitals, sanatoria, nursing homes and homes for the elderly and home nursing (e.g. in the Sozialstationen and Diakoniestationen) and for example in health offices. Main tasks of nurses are:

'die kontinuierliche, verantwortungsbewusste Beobachtung und qualifizierte, geplante Pflege und Versorgung der Kranken, die Durchführung von ärztlichen Verordnungen, Assistenz bei ärztlichen Maßnahmen der Diagnostik und Therapie, Anleitung und Anregung der Patient/innen zu gesundheitsförderndem Verhalten, Anleitung und Beaufsichtigung von Krankenpflegeschülern/-schülerinnen und anderer Mitarbeiter/innen auf der Station, Organisations- und Verwaltungsaufgaben, Kontaktpflege mit Patient/innen und ihren Angehörigen und die Zusammenarbeit mit anderen Gesundheits- und Sozialberufen.' (Diakonisches Werk der EKD, 2004a) 
This quotation underlines that the tasks of nurses are primarily considered as derived from medical tasks in Germany, while in countries such as Denmark, the Netherlands and the $\mathrm{u} \mathrm{K}$, an important part of nursing tasks are considered to belong to a separate domain that is specifically nursing in character.

There are several specialisations in nursing in Germany, such as anaesthesia nurse, intensive care nurse, surgery nurse, preventive nurse, psychiatric nurse, nursing management, education of nurses, home or community care nurse ( $\mathrm{Ge}$ meindekrankenschwester).

\subsubsection{Krankenpflegehelfer(in)}

The training for the differentiation of Krankenpflegehelfer(in) is one year (Diakonisches Werk der E KD, 2004a). It consists of at least 500 hours of theoretical training and a practical training of at least I Ioo hours. The diploma is received after a state exam. These auxiliary nurses are qualified to work in hospitals, sanatoria, nursing homes and homes for the elderly and home nursing (e.g. in the Sozialstationen and Diakoniestationen). Their tasks consist of nursing and caring of patients and assisting tasks. There are almost 35 nursing schools in Germany, many of these are intern, organised in (religious) hospitals or organisations of religious signature, such as the Diakoniewerk or Evangelischer Johannesstift. ${ }^{\text {. }}$ The average number of places per year is 20-25.

\subsubsection{Altenpfleger(in)}

In Germany, Altenpfleger (elderly nurses) also work in home nursing (Diakonisches Werk der E KD, 2004b; Caritas Soziale Berufe, 2004b). In August 2003, the regulations that fell under the responsibility of the individual Länder were made uniform by the introduction of the Altenpflegegesetz. ${ }^{\text {II }}$ The training, mostly three years in all Länder ${ }^{\mathrm{I} 2}$, will from then on take three years in the whole of Germany. Theoretical training at an Altenpflegeschule of at least 2 ooo hours takes turns with long periods of practical training of $2200-2500$ hours in total. The curriculum changes regularly because of the changing needs and living circumstances of elderly people,

Io For example the Diakoniewerk München-Maxvorstadt or the Schule für Krankepflegehilfe im Ev. Johannesstift Berlin.

II This law also lays the foundation for innovation of Altenpflege as a profession. It suffers form a very low social status, and the professionals working with very old people are often overburdened and have too little knowledge of the specific problems of these people. The law intends to make the occupation more attractive, and sets national standards for the essential competences that are needed (Schleich, 2004).

I2 In some of the Länder, the training takes two years, with one additional practical year. 
changes in nursing and medical knowledge and changes in health policies. Altenpfleger work in institutional facilities such as nursing homes, psychiatric clinics, hospitals with a geriatric department, or in ambulant elderly care such as a Social Station or Diakoniestation or day care facilities. Their task is to care for the physical and metal well-being of elderly people, by performing nursing and domestic tasks, social care and giving advice. When they are employed by a religious organisation, religious care for the soul is included as well. There are several possibilities for further specialisation in elderly care and management. The training into Altenpflegehilfer(in) (elderly nurse assistant) takes one year.

\subsubsection{Dorfhelfer(in)}

Also in Germany, a specific occupation exists in home care, namely Dorfhelfer(in) (Caritas Soziale Berufe, 2004c). These provide help in families when someone should take over the tasks of the housewife or farmer $(\mathrm{m} / \mathrm{f})$, by taking care of the children and the (farming) household. Education takes one to two years of theoretical and practical training. There are three training schools in Germany with 66 places per year. Mostly, these 'village helpers' are employed by social care organisations (Wohlfahrtsverbande); church and community care organisations, Diakonieund Sozialstationen, farming organisations and Dorfhelferinnen organisations. The tasks consist of household activities, nursing, supporting and child-raising tasks in farming families. In other countries, such a specific occupation does not exist; home helps probably mostly execute these activities.

\subsubsection{Familienpfleger(in)}

Another occupation employed by the Social Stations and the organisations falling under the Wohlfahrtsverbande is Familienpfleger(in). For a large part, these work in families and are responsible for the household and/or the children. Training takes three years, of which the last year consists of working in practice. This kind of differentiation probably is most identical to home help in the Netherlands (gezinszorg) in the second half of the twentieth century, when home helps mostly worked in families with children to assist the mother when she was ill or was not able to cope (Vulto and Morée, I996). However, since the I990s, home help has become primarily an occupation performing household tasks.

A quite important legal distinction is made in Germany between nursing activities that are characterised as Grundpflege, which consists of helping with basic needs and adl-activities, and Behandlungspflege, that consists of specialised, technical nursing procedures. Krankenschwester carry out both kinds of services, Altenpfleger and Krankenpflegehelfer only provide Grundpflege. 
In the ambulant mobile services, originally a large part of the staff consisted of young men who had chosen to do community work instead of military services, and voluntary workers. Because of this, these services were able to offer home help at a very modest price. However, currently these organisations have become more staffed with professionals and are offering comprehensive services, resembling the social centres.

Generally, there is a shortage of (qualified) community nurses in Germany, except for Berlin. In I990 there were about Io ooo community nurses. Throughout the country there seems to be much variation in nurse-to-population ratios.

\subsection{Client population}

It is hard to gain insight in the number of recipients of home care and home help provided by one of the services because of lack of data, although registration has improved since the introduction of the Pflegeversicherung. It is estimated that two million Germans receive nursing care under this insurance (Bundesministerium für Arbeit und Sozialordnung, I997; Schleich, 2004a). About 570 o00 people receive institutional care, while as much as I.5 million people receive care by one of the $\mathrm{I} 3$ 000 ambulant services. Only I0० 000 of these receive care from private providers, the rest is provided by the non-profit ambulant services. A large part of the clients are over 80 years of age and suffers from multiple health problems. Social and nursing services for the elderly are however still considered to be inadequate to meet the demand (Rostgaard and Fridberg, I998d p. 522).

Care provided by family members or neighbours is by far the most important; informal caregivers support $90 \%$ of those in need of care. $9 \%$ of those in need do not receive any professional or informal care at all. A survey carried out in I991/92 stated that almost $8 \%$ of all elderly of 65 years and older living in private households were in need of care, and another $12,5 \%$ were in need of support with domestic tasks. However, it is estimated that about $3 \%$ of the population of 65 years and older receive home care, and between one and three \% receive home help, which clearly is not sufficient (Rostgaard and Fridberg, I998d p. 532). Scharf (I998) also states that only one-third of older people who require care and live at home make use of professional services, whilst this figure falls to one-tenth for those who need some form of domestic support (Scharf, I998e p. 135). At the same time, the proportion of people aged 65 and over living in some form of residential or nursing home increased from $4.6 \%$ to $6.8 \%$ between I985 and I992. This means that there are more elderly living in institutions in Germany than elderly receiving home nursing or home help, and that the provision of organised forms of elderly care is far inadequate to meet the demand. The appeal of elderly to family members and other informal caregivers is therefore substantial. 
Most contacts are initiated by the patient or the family or neighbours, but also by GPS or by the hospital. Officially, assessment should be carried out by a physician for reimbursement. Assessment forms, which are used in all Sozialstationen, are therefore adapted to doctor's orders. In practice, however, doctors work in close cooperation with the head nurse of the Social Station. She also decides upon the level of expertise that is needed and who is going to deliver the care. The nurse serving the specific area in which the client lives normally delivers care. Assignment procedures however differ per Station: some centres try to ensure continuity of care, other prefer staff rotation or group caregiving.

\section{I0 Financing of home nursing}

The German system is based on the principle of subsidiarity: no formal services are offered when informal service is provided. If formal services are needed, the family has to pay for them (unless they are covered by health insurance). When they are unable to pay, formal providers (social welfare) take over the costs.

Before I989, nursing services were only provided under health insurance as a complete substitute for hospital care or if it enabled an early discharge (Delnoij, I996a). The federal nursing law (Sozialgesetzbuch, 『37) distinguished two types of benefits:

- Grundpflege: basic nursing in the form of basic personal care; a 'Kann-Leistung' considered as an 'optional' service;

- Behandlungspflege: specialised nursing; technical nursing procedures supporting medical treatment; an 'Ist-Leistung', considered as a 'necessary' service.

This means that basic nursing is only covered by health insurance when specialised nursing is provided as well. Specialised nursing is seen as an adjunct to medical treatment. According to the same law, $\mathbb{3} 8$, home help (Haushaltshilfe) is only provided and reimbursed in addition to specialised nursing in families with a child under twelve years of age.

In addition to this, since I989, patients living alone and needing intensive nursing care (Schwerpflegebedürftige) are entitled to a certain number of home visits per month. The service can include both basic care as well as homemaking services. Instead of services, it is also possible to apply for a cash benefit when relatives are providing the care. Apart from that, based on $\rrbracket_{5} 6$ of the law, informal caregivers (when they have cared for at least I2 months) can apply for a professional nurse during their own vacation. Nursing care under the health insurance system is generally offered for a period of four weeks. 
In January I995, the Pflegeversicherung was introduced. It is financed out of premiums that are paid for $50 \%$ by employees and $50 \%$ by employers. To obtain consent from employers, one national holiday was cancelled in I995. Since April I995, home nursing is reimbursed, and from July I996 onwards the benefits package also includes long-term nursing care at home. Publicly insured will be covered by their Statutory Health Insurance funds, while privately insured we be obliged to take out a Pflegeversicherung with their insurance company. People who are (partly) incapable of ADL-activities for at least six months, due to physical or mental illness are eligible for care and reimbursement.

The long-term care legislation distinguishes between three levels or categories of care:

- Level I: erheblich Pflegebedürftige, who need help with at least two or more activities of daily living (ADL) in the sphere of personal hygiene, feeding or mobility, at least 90 minutes each day, of which more than 45 minutes should be spent on meeting personal care needs. In addition, they require home help services several times a week. Benefits consist of professional home care that is tied to a maximum, or cash benefits with which a patient can buy his own home care.

- Level iı: Schwerpflegebedürftige: people who need help with ADL-activities at least three times a day and regularly need home help services. The help must be required for at least 3 hours a day, with more than 2 hours being spent on carerelated tasks;

- Level iri: Schwerstpflegebedürftige, people with the most acute care needs, who require constant, round-the-clock help with ADL-activities and regular help with household tasks. Help must be needed for at least 5 hours a day, of which at least 4 hours must be care-related tasks. Service benefits (home care or nursing home care) are tied to a maximum, as well as cash benefits (Delnoij, I996a; Scharf, I998e p. I2I-I22).

In 1997, of the people receiving care under this scheme, 43.8\% (more than 500 000) people were categorised as level I, receiving $400 \mathrm{DM}$ in cash or nursing activities with a maximum of $750 \mathrm{DM}$; about the same number of people were categorised as level II, namely $43.6 \%$ (more than 500 ooo people), receiving $800 \mathrm{DM}$ in cash or nursing activities with a maximum of I 800 DM; and I2.6\% (almost I50 000 people) were classified as level III, receiving I 300 DM in cash or nursing activities with a maximum of 2800 DM (Bundesministerium für Arbeit und Sozialordnung, I997). Table 3 indicates percentages of recipients over different forms of care. 
Table 3 Recipients of social care benefits, in percentages, I997 (first half year)

\begin{tabular}{|c|c|c|c|c|}
\hline & Care-level I & Care-level II & Care-level III & Total \\
\hline \multicolumn{5}{|l|}{ Home care } \\
\hline $\begin{array}{l}\text { Home help / care from } \\
\text { professional services }\end{array}$ & 8 & 7 & 5 & 7 \\
\hline Payment for care & 67 & 56 & 37 & 57 \\
\hline Combinations & 7 & II & I4 & IO \\
\hline Holiday relief & O.I & 0.2 & 0.3 & 0.2 \\
\hline Short term care & 0.3 & 0.4 & 0.4 & 0.3 \\
\hline Partial residential care & 0.2 & 0.3 & 0.3 & 0.3 \\
\hline Residential care & I7 & 25 & 43 & 25 \\
\hline Total & I00 & I00 & IOO & IOO \\
\hline
\end{tabular}

Source: Rostgaard, I998 p. 527.

Before its implementation, the Arbeiterwohlfahrt foresaw some problems regarding the Pflegeversicherung: the maximum amounts set by the government are too low compared to the average real costs of nursing care. Even with these benefits, it will not be possible for many patients with low incomes to afford the costs of care (Greiner and Graf v.d. Schulenburg, I997 p. 94). Many patients will still be dependent on public social assistance, although the Pflegeversicherung was intended to diminish this dependency. Besides that, the overall expenditure cap imposed on the new care insurance could have a negative effect on the quality of care (Delnoij, I996a; Rostgaard, I998). The medical advisors of the Health Insurance funds (Medizinischen Dienst der Kranken- und Pflegekassen) assess the patients' need for care and determine the category they belong to. There are however serious doubts about the ability of medical professionals to assess the patients needs for nursing care, as they not always have insight into the need for caring and supportive kinds of care and care for dementia patients, being experts on 'cure' rather than on 'care'. However, the German government is not very susceptible for this criticism (Schnepp, 200I p. I2).

\section{II Funding of the Social Stations}

The success of the Sozialstationen is mainly due to the financial promotion of them by several states and health insurance programs. They receive about $65 \%$ of their income from the Health Insurance funds (fee-for-service), I0-20\% from taxes, I0\% from out-of-pocket payment (fee-for-service), less than Io\% by umbrella organisations and less than $5 \%$ by private insurance companies (fee-forservice). 


\section{I2 The domain of home help}

Home help services are provided by the 'traditional' Haus- und Familienpflegestationen (623 in 1993) and by the 'new' Sozialstationen. The Mobilen sozialen Hilfsdienste (I050 in I993) do not provide traditional home help services, but additional services, mainly to the elderly, such as meals on wheels and cleaning services. They enable elderly to stay at home instead of being institutionalised. Apart from salaried employees, also a large number of volunteers is working in the Mobilen sozialen Hilfsdienste, as well as Zivildienstleistenden (objectors to military service). Only about half of the employees is specifically educated in ambulatory social or nursing care.

The clients of home help services consist of families with children as well as elderly people. Clients of the Mobilen sozialen Hilfdienste are mainly elderly and very old people, $3 / 4$ of these are women. About $40 \%$ is disabled and have a Schwerbehindertenausweis. The majority of the clients receive help for more than 6 months, $60 \%$ were visited 5 times a week, and another $20 \%$ three to four times a week.

The tasks of the home help services are diverse. The Haus- und Familienpflegestationen and Sozialstationen primarily provide temporary care for families or individuals at home; including basic personal care, pedagogical and psychological care, and homemaking services. The Mobilen sozialen Hilfsdienste provide information and advice regarding social and other ambulatory services, homemaking tasks such as cooking, dishes, tidying up, Betreuungsdienste (consisting of company, shopping, walking, accompany clients to the doctor, administrative help). About $70 \%$ of the mSHS also provide basic nursing care, only $38 \%$ provide shopping and cleaning services.

The provision of home help services is also based on the principle of subsidiarity. Clients have to pay for help themselves, and only if they or their children cannot afford it, social welfare will take over the costs. Under certain conditions, home help is covered by health insurance in addition to ambulatory nursing care. Categories that are entitled to home help are households with as child under the age of twelve or a handicapped child, households where the mother is hospitalised or has recently been discharged and no other family member can take over. Remarkably, elderly are not the target group for home help, which is quite different compared to other European countries.

Since 1982, home help for elderly can be partly reimbursed under the health insurance, when these are also receiving technical nursing care. Also the I99I category of Schwerpflegebedürftige of the Pflegegesetz entitles them to basic nursing and home help.

Funding is a main problem regarding home help services. Mobilen Sozialen Hilfsdienste do not have a solid financial base but depend heavily on volunteers, participants in unemployment programmes and Zivildienstleistenden. The staff 
of the MSDS is sometimes financed by a state budget, sometimes added by municipal funds. The Dienste are also sometimes financed by municipal funds, in the form of a global budget, or based on the number of employees, output (hours of service provision) or a percentage of the total costs. Also, fees per hour are charged to patients for the services provided, that are either paid by the clients themselves, or by social welfare or health insurance. The percentage of costs covered by fees varies enormously: some MSDS are covered for 60\%, some for less than 10\% by fees for service.

\subsection{Conclusion}

The contextual analysis of home nursing in Germany is summarised in the theoretical framework that was developed in chapter two of this book (see figure 2). It shows that the provision of ambulant care to elderly and vulnerable people in Germany is accompanied with several problems of a different character. Regarding the inner circle of the domain characteristics, one can say that home nursing has its roots in the Diakonisches Werk of religious nurses, already in the nineteenth century. However, this has remained the initiative or religious organisations at local levels for long, without becoming a dense national network of home nursing organisations such as in the Netherlands. One of the reasons is the lower homogeneity of Germany due to the different federal states (Länder). Striking is that peripheral forms of care traditionally have had low priority in German health care for a long time compared to forms of care that are provided in the medical centre of health care, such as medical and cure-oriented activities. Also, the development of nursing and home nursing into an independent profession with an autonomous domain of activities has long - and still is - been hampered by the idea that nursing is a subordinate profession, supporting the medical profession. Nursing is still primarily considered as an extension of medical activities and complement to medical and hospital care, instead of a separate profession with its own task definition. Another important finding is that home nursing is weakly organised and fragmented over different kinds of organisations and professions, and that it is unevenly distributed over the country. In institutional nursing homes as well as in ambulant nursing, a sense of 'crisis in care' is felt, because of a substantial lack of manpower and lack of adequate funds and elderly policies, resulting in many nursing homes and home care organisations not meeting the legal quality standards of care.

The middle circle of the framework is about the way home nursing work is shaped and about the relations with other professionals, fields and patients. As in most European countries, single elderly people are the most vulnerable group. In Germany, single elderly women and very elderly women constitute the most 
vulnerable groups that are predominantly in need of home care services. The loss of lives of men in the First and Second World War and the longer life expectancy of women in general contribute to the fact that higher numbers of single elderly women live in Germany compared to other European countries. The training of home nurses is considered rather insufficient, especially because it is only offered in-service and mainly focussed on institutional forms of care. Nursing science at universities is still rather limited.

Health care in Germany is rather medically oriented and a strict separation of health and social care exists, which is also reflected in task differentiation because of the legal distinction between Grundpflege, helping with basic needs and adlactivities, and Behandlungspflege, which consists of specialised, technical nursing procedures. Krankenschwester carry out both kinds of activities while Altenpfleger and Krankenpflegehelfer only provide Grundpflege. Regarding co-operation and coordination, one can say that the influence of physicians at all levels of health care is important. For example, procedures determining whether people are entitled to home nursing care are largely controlled by physicians: a medical referral is needed to receive reimbursement for nursing care, and insurance committees deciding on the assignment of nursing care is staffed by physicians. Sometimes, physicians in some regions are not willing to cooperate with the Sozialstationen (Social Stations or social centres) providing home nursing and home help. Although the introduction of the Pflegegesetz in I995 has been a great improvement for the home care domain, its disadvantage is that only technical nursing activities and care to very dependent people with complex health needs are covered. Physicians mainly prescribe medical-technical activities, and home care organisations tend to medicalise their nursing services by giving priority to specialised nursing over supportive tasks. Co-operation between different kinds of professionals and organisations is hardly taking place because of the boundaries between the different health and social domains, the influence of physicians and the fragmented provision of home care that is provided by many different kinds of organisations and professionals. Bureaucracy, which is a very important problem in the Netherlands, does not seem to be such a problem in Germany. The influence of physicians and the feeling that the country is not ready for the amount of elderly people with care needs are more prominent.

Regarding the outer circle of the framework, the socio-cultural, political and health care context, striking for Germany is that traditional family values are still very strong. On the one hand, this may be the result of the legal responsibility of children to care for their elderly parents or to pay for professional or institutional care. On the other hand, it may also be reinforced by the inadequacy of elderly facilities to meet the demand for care. Many families are reluctant to send elderly family members to nursing homes because of the impression that quality standards are not met. The reliance of elderly people on their family members and 
Figure 2 Contextual framework of home nursing in Germany

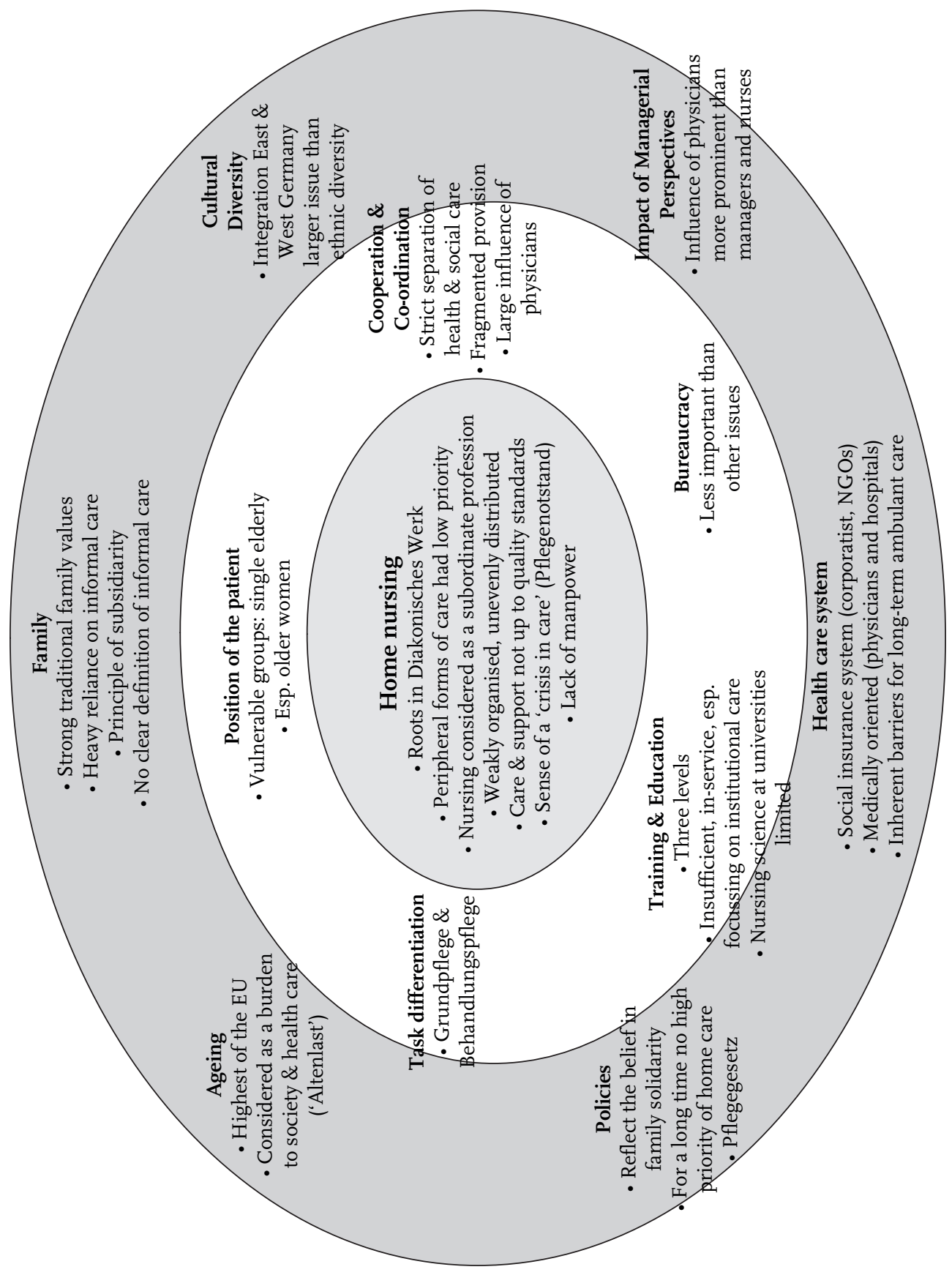


other informal caregivers is therefore substantial. The strong traditional family values are further reinforced by the principle of subsidiarity underlying the health care system, namely that in case of need, vulnerable people first and foremost have to turn to their family members for support and that the state is the last port of call. This contrasts strongly with the Scandinavian model where the welfare state ensures care for all. However, although informal care thus plays a very large role in Germany, a clear conceptualisation of informal care in health care policies does not exist, in contrast to for example the Netherlands and Denmark. Informal care is not defined or described clearly and thereby not officially incorporated in the health system, but nevertheless, the reliance of elderly people on their family is very large.

The ageing of the population is strongly felt in Germany. With a figure I8.6\% elderly of 65 years and older in the total population, which is almost 13 million people in absolute numbers at a total population of 82 million people, the pressure of how to care for elderly people and how to finance sufficient facilities is strong. This is reinforced because the health care system has always been quite medically oriented and the provision of home care to the vulnerable has not had a high priority for a long time; for many decades a strong preference has existed for institutional forms of elderly care, such as residential homes and nursing homes. Due to lack of facilities and because there is no homogeneous nation-wide organisation of ambulant long-term care, the ageing of the population is clearly felt as a burden to society and health care and the services available are far insufficient to fulfil all the needs. However, gradual changes have become visible in policies in Germany as well, among others because of the changing health and social policies regarding elderly people in Europe who are put higher on the agenda, as well as the burden of the increasing number of elderly people in Germany and the sense of crisis in nursing (Pflegenotstand) and the problems associated with the provision of long-term care to elderly people. The introduction of the Pflegegesetz in I995, after a long period of debates, has been an important development, but its emphasis lays on the more complex cases needing substantial or even constant, round-theclock, care and support. Thus, in the German health care system, care provided by physicians and hospitals is predominant and inherent barriers for long-term ambulant care exist.

In Germany, the social welfare system is clearly oriented towards the labour market. The health care system is for example paid by social insurance contributions paid by employers and employees. The social status of non-working people, such as pensioners, informal carers, disabled and very old people in need of care and support, clearly has had low priority for a very long time. Finally, cultural diversity and the impact of managerial perspectives are subjects that are not very prominent. The integration of East and West Germany is higher on the agenda than ethnic diversity, although Germany counts many people from foreign coun- 
tries, such as Turkey. It seems that the predominance of medical perspectives over nursing perspectives and the rather long neglect of the needs of the elderly and vulnerable are more problematic for the development of home nursing into a separate, independent domain. 
Chapter 8

\title{
Conclusion and discussion
}

\author{
- The home nursing domain and profession from \\ a comparative and contextual perspective
}

\section{I Differences in home nursing in Europe - the theoretical framework revisited}

In chapter two of this book, a comparative, contextual framework was developed as an analytical tool to study home nursing in different European countries. A range of aspects that were described as important in the literature on professions, home nursing and related professions, health care systems and political and socio-cultural differences between countries were included in the framework. In this chapter, the outcomes of the analyses of home nursing in Denmark, France, the Netherlands, and Germany performed in this book are described and compared on the basis of the theoretical framework. Also, aspects of the theoretical framework are adapted and new aspects are included when the findings pointed to their relevancy. Then, the strengths and weaknesses of the framework are evaluated. Finally, the implication of this study for the theory of professions, and more specifically for Abbott's perspective of professional networks (i988), is described. An elaboration of this theoretical perspective in the form of the concept of linked ecologies (Abbott, 2005) is proposed as a better way to conceptualise professional life and professional developments in current societies.

\subsubsection{Outcomes of the study - the central part of the theoretical framework}

The central part of the framework represents three specific characteristics of home nursing, namely that it is a special working domain, situated in the periphery of health care and providing care and support to people with health problems to enhance or maintain their day-to-day functioning. This central characteristic was indeed found in all countries studied. In Denmark and the Netherlands, the independent role and responsibility of home nurses is more recognised than in France and Germany, where nursing is still considered as a profession being subordinate to the medical professions and where the tasks of (home) nursing are for a large extent derived from or extensions of medical activities. This is especially visible in France, where another home nursing domain exists next to a home nursing domain that is comparable to home nursing elsewhere, namely HAD (Hospitalisa- 
tion à Domicile), which is organised by hospitals and where caring tasks are just supportive of medical tasks. In Germany, since the Pflegegesetz that has been introduced in I995, only clients who are very dependent for at least six months and need care every day are eligible for care and reimbursement. As the health care system is medically oriented, peripheral forms of care such as home nursing had a low priority in Germany for long, resulting in a weakly organised domain with services that are unevenly distributed over the country. Also in France, the health care system is oriented towards medical and institutional care. Although a large increase in ambulant home nursing and home help services has occurred since the I980s, these are still insufficient to provide sufficient care to vulnerable people. Even more than in Germany, large local differences in availability of services exist and the care needs of many (very) elderly people are not met. This situation is worsened by the fact that services provided are badly targeted, so that some times very dependent people do not receive care while others who are in better health do receive services. In Denmark, nursing is considered as an almost independent profession with a large autonomy. The independent role of home nursing in supporting people with health problems is clearly recognised and found important next to the medical domain. Health policies and municipal responsibilities are aimed at providing sufficient care to vulnerable and elderly people to enable them to remain living in their own homes as long as possible or to live independently in special apartments adapted for older people. In Denmark, as in other Scandinavian countries, the welfare system is arranged thus that professionals are the primary source of care, not the family. Therefore, the provision of care and support by home nurses, auxiliaries and home helps is more accepted by the population than in Germany and south European countries where the health care systems are more medically oriented and an important role is expected of informal carers to provide care and support to vulnerable and elderly people.

The second main characteristic is that home nursing is a broad sphere of action providing several kinds of care to a wide range of clients with different care needs. Currently, in all countries studied, the core domain of home nursing is constituted by long-term care for chronically ill and elderly people due to the ageing of the population and the trend to substitute institutional care in hospitals, nursing homes and homes for the elderly by community-based care provided in the homes of the clients. In Denmark, all kinds of elderly care are integrated and provided by the local municipalities, ensuring a broad package of care and support services, also including meals on wheels, gardening, and snow clearing services. Also, preventive tasks still play a large role in Denmark, because home nurses have an important signalling function. They regularly visit very elderly people to monitor their situation and signal any deterioration in their independence and day-to-day functioning and determine whether they are in need of home care. In non-Scandinavian countries, preventive nursing care hardly has any priority and care is aimed 
at those already in need of services. In the Netherlands, home nursing has been an independent domain since the I88os, but has been integrated with home help in the I990s. Currently, large-scale organisations provide a range of services to vulnerable and elderly people, consisting of home nursing, home help, specialised nursing such as diabetes care, and often also ironing and gardening or odd jobs in the house on a market basis. The package of care is not homogeneous because home care is not publicly provided, as in Denmark. In the Netherlands, a societal midfield (maatschappelijk middenveld) of non-governmental organisations (NGOS) has existed for long that fulfilled health care functions that were executed by public organisations in other countries, especially those with national health systems such as in Denmark and the u k. The Dutch NGos consisted of local cross associations who were able to decide on the services offered themselves, resulting in still existing regional differences in the packages of care. In the last decades, many changes in home care have taken place in the Netherlands, changing its character and resulting in a more market-like environment under which these organisations have to operate. For example, the introduction of a new law in 2007, the wmo, placed home help under the responsibility of the municipalities and home care organisations and cleaning services are now competing for contracts to be able to provide services. Thus, the financing of home nursing and home help are separated now. Another important field of activities that has traditionally been part of the home nursing domain in the Netherlands consists of mother and child care in the well-baby centres (consultatiebureaus). This has developed into a separate field in the last decades. Whereas home nurses used to perform all kinds of tasks because she was trained as a generalist nurse visiting families in her own neighbourhood and provided care to mothers and babies as well as ill family members of all ages, nowadays nurses have to choose between the fields of elderly care and child care during their training. Preventive well-baby care is generally still offered by home nursing organisations but is performed by different home nurses than those providing elderly care. Maternity care that has also belonged to home nursing for long has developed into a separate field of activity and is currently generally offered by independent for-profit maternity care organisations. In Germany and France, care for infants and babies is generally performed by physicians such as GPS and paediatricians and home nursing is exclusively focussing on vulnerable and (very) elderly people living in their own homes. Home help is considered as a different domain than home nursing and is financed differently. Therefore, independent home nurses in France refuse to perform hygienic care (washing and dressing), as they do not consider these tasks their responsibilities. These are left to auxiliaires de vie, family members, or a hygienic nurse. Domestic tasks are mostly left to auxiliaires de vie or aides ménagères (home helps). Sometimes, when these are not available, personal care and support with household tasks are not provided at all. 
The third central assumption of the theoretical framework was that the degree of autonomy of home nurses is a central characteristic of home nursing, distinguishing it from other nursing professions. Namely, as they perform their tasks in the homes of their clients, no direct interference and close supervision of professionals with a higher hierarchical status exist, for example by a head nurse or a physician. This implies that the home nurse is in charge herself and the work is more autonomous in character than that of nurses in other domains, for example in hospitals, where intra- and interprofessional hierarchies exist. In principle, this holds true for the countries studied in this thesis. It is assumed that home nurses in Denmark work rather autonomously as home nursing is considered to be an independent profession. Danish home nurses perform the intake as well as provide the care. Also, they are trained to work all-round, as continuity of care for clients is considered very important in Denmark and home nurses perform a wide range of different tasks for individual clients. In France and Germany, home nursing is considered as a subordinate profession and generally others than home nurses decide what kind of care should be provided, such as the medical advisors of the Health Insurance funds (Medizinischen Dienst der Kranken- und Pflegekassen) who assess the patients' need for care and determine the category under the Pflegegesetz they belong to in Germany. In France, independent nurses working from their private practices probably have more autonomy than employed home nurses who have to work according to the rules and procedures of the SSIAD organisations. A new development in France is the Projet de Soins Infirmiers (PSI). According to the PSI procedure, the GP prescribes PSI and then a nurse draws up a Nursing Plan, in which the social and health status of the patient and the kind and frequency of care needed are described. The plan has to be signed by the GP. This procedure changes the relation between GPS and home nurses, as the diagnostic role of physicians is granted to home nurses, which allows them more professional autonomy to decide on the care to be provided and to assess the situation of the client themselves. Thus, the professional autonomy of (independent as well as employed) home nurses can be considered somewhat larger in France than in Germany.

In the Netherlands, a different situation was found. No direct supervision of home nurses by health professionals with a higher hierarchical status is executed during the work, but a different kind of supervision and interference appeared to fundamentally affect the independence and professional autonomy of home nurses. Currently, home nursing work is increasingly steered and monitored by managers, planning bureaus of home care organisations, and officials of separate organisations that perform the intake procedure. Home nurses have become employees of home care organisations and meticulously have to register their activities per minute to account for and give insight into their work to the management of the home care organisation. Also, while home nurses used to perform the intake of clients themselves, currently the assessment of the clients' eligibil- 
ity to care and the kind and amount of care they need is performed by a separate organisation, formerly the RIO, now CIz (Centrum Indicatiestelling Zorg). However, as Dutch health care is continuously confronted with reorganisations and new policy proposals, this procedure is also under consideration of being changed. Dutch home nurses work in shifts and their work is divided according to task differentiation, so that each nurse visits a large number of clients per day and only performs a limited set of tasks in line with her level of training. Also, personal attention to patients is generally overshadowed by administrative procedures that have to be performed and the very tight time schedule of home nurses. Thus, currently, especially in the Netherlands but for example also in the $\mathrm{uk}$, the autonomy of home nurses is not so much affected by interprofessional relations with physicians, but by procedures designed by managers and officials that intend to monitor the activities of home nurses. In Denmark, this managerial and bureaucratic interference is criticised and halted by health care workers who do not accept any interference in their professional work.

\subsubsection{Adaptation of the central part of the theoretical framework}

Thus, the three central characteristics of home nursing that were identified in the beginning of the study appeared to be useful analytic concepts to identify differences in home nursing in Denmark, the Netherlands, France and Germany. However, other aspects also appeared to shed light on international differences that could be included into the central part of the framework. Firstly, the period of establishment or history of home nursing appeared to be important for the development of home nursing and its establishment as a separate domain alongside the medical domain. Namely, home nursing has been able to develop into a strong health care sector and almost independent profession in Denmark and the Netherlands and also the uk, where it was established in the I880s. However, in France, regular home nursing organisations, the SSIAD services, were only established by decree in I98I and before that, the care needs of vulnerable and elderly people were met by informal carers and independent, private nurses who were not organised collectively. Although the home nursing domain has increased considerably in size since the I980s, it is still far insufficient to provide for all the care needs in France.

Secondly, the characteristic that it is a special working domain falls apart into two sub-characteristics, namely whether home nurses are considered as having an independent role and responsibility, such as in Denmark and the Netherlands, or whether their activities are considered as an extension of or supportive of medical activities, such as in France and Germany. While in the first case, home nursing can be considered as an independent subsector of health care with a subsequent higher social status; in the second case, home nursing has lower priority than medical and curative care and thus a rather low social status. 
Thirdly, the characteristic of home nursing as a 'broad sphere of action' is undergoing marked changes. In Denmark, home nurses still work all-round and have a generalist profile, and perform preventive as well as nursing and supporting tasks to people of different ages. In the Netherlands, home nurses have also been working all-round for almost a century. However, currently, they have to choose to specialise in elderly care or youth health care during their training. Elderly care and Youth health care, the latter consisting of preventive parent and child care in the well baby centres (consultatiebureaus) and maternity care, have differentiated into separate spheres of activities. Preventive care tasks (Health Promotion and Education) are only a very minor part of the job and only performed by level five nurses. The all-round character of Dutch home nursing is currently also being affected by the development of the concepts of integrated or 'transmural' care (transmurale zorg), care chains and disease management. According to these concepts, the caring process of individual clients, often those suffering from a particular disease such as diabetes or rheumatoid arthritis, is then subdivided into several smaller parts, the chains. These are each executed by health care professionals of different organisations and health care sectors. This way, integrated or 'transmural' care networks are created according to which both intramural and ambulant health professionals are working closely together and share responsibilities. The idea behind these concepts are to bridge the gap between general primary care and specialised hospital care and to better tailor the care to the individual needs of patients (Meurs, I989; Linden, I997b; Kümpers, Raak et al., 2002). For example, specialised nurses such as diabetes nurses, (partly) take over tasks of hospital physicians to improve continuity of care for chronically ill patients and enhance cooperation between different providers of care (Vrijhoef, 2002). However, drawbacks of these developments are that another intermediate level within health care is created with complicated communication processes and that home care is fragmented into smaller parts, leading to further horizontal differentiation and restriction of the generalist profile of home nurses.

The characteristic of being a broad sphere of action is also affected by changes in the client population. In Denmark, home nursing is still aimed at those people in the population that are dependent of care; families as well as elderly people, irrespective of their age. This has also been the case in the Netherlands for many decades. However, currently home nursing in the Netherlands predominantly focuses on specific groups of vulnerable people in need of long-term ambulant care, especially (very) elderly people who need care and support with their daily life activities to be able to remain living in their own homes. Also chronically ill patients, patients with multiple diseases, the handicapped, and dying patients are important client groups receiving home nursing. This is similar to France and Germany, where the main sphere of activities of home nurses also mainly consists of care to (very) elderly and chronically ill patients. Tasks of home nurses are gener- 
ally restricted to medical-technical nursing tasks, such as wound dressing, giving injections, changing stomas, etc, because only these are reimbursed by the health care insurance. Whereas in Denmark, home nursing still has a generalist profile and in the Netherlands other spheres of activities such as well-baby care still exist, the core part of home nursing in Europe is currently constituted by care to the vulnerable and (very) elderly. Therefore, broad sphere of action is better changed in the framework into main sphere of activities: caring for the vulnerable and (very) elderly.

Fourthly, domain size and local or regional availability of services appeared to be important aspects that characterise the home nursing domain in countries. This appeared to be problematic in Germany and especially in France, where home nursing is not available at all in some remote areas. In contrast, the home nursing domain is extensive in size in Denmark and the Netherlands, where the local availability of services is guaranteed by the welfare state (Denmark) or the insurance companies (the Netherlands). At first sight, the accessibility of services all over the country seems to be related to the early establishment of home nursing, but this does not hold true for Germany, where home nursing also has early origins, but where a strong development of the home nursing domain was probably prevented by the lack of national unity during the twentieth century and the low priority of ambulant caring services for long. Also, the fact whether home nursing is of public, non-governmental or religious character does not predict a strong or weak development of the home nursing domain over time. Namely, home nursing in Denmark is public in character but the domain size and availability of services is about comparable to the Netherlands where home care is executed by non-governmental cross associations mostly with a religious background. In Germany, home nursing has its origins in the Diakonisches Werk of the Deaconesses' houses that also existed in the Netherlands, generally a Deaconesses' hospital that educated deaconesses to become nurses who also provided nursing in the homes of patients. However, although the history of the Diakonisches Werk in Germany is dating further back than that of the cross associations in the Netherlands and both kinds of associations have a religious signature, the contemporary modalities of home nursing in Germany and the Netherlands are very different. In the Netherlands, a rather strong home nursing domain exists in the form of a dense network of regional home care organisations that provides a substantial package of care of good quality all over the country, while in Germany, local differences in availability and quality of services exist and the home nursing domain is quite weakly developed because it has been overshadowed by the priority of curative care in the health care system.

Finally, the degree of autonomy did appear to be important aspect to gain insight into the character of home nursing in different European countries. However, not only the question of how much autonomy home nurses have to execute their work is important, but also what kind of autonomy they have and which aspects affect their 
autonomy. For example in the case of the Netherlands, the professional autonomy of home nurses is not so much affected by physicians but by indication officials and by managers of home care organisations. This external interference leads to a lot of discontent of home nurses about their working conditions, as administrative procedures are more and more prevailing over the caring aspects of the work.

\subsubsection{Outcomes of the study - the middle ring of the theoretical framework}

The middle ring of the theoretical framework was used to analyse how international differences in home nursing in Europe are shaped by differences in the organisation and training of home nurses, the relations with other organisations and professionals, the particular position of the patient in each country, and the way that bureaucracy affects home nursing work.

Firstly, regarding the position of the patient, one can say that this appeared to be a useful aspect of the framework, giving insight into differences between countries. Although the kind of patients that home nursing focuses on is about similar, their situation differs per country and they have different attitudes towards home care. In Denmark, the welfare state defines children and elderly people as vulnerable groups in need of care and support and guarantees professional care to these groups by making municipalities legally responsible to offer extensive child and elderly care arrangements. In Germany, single elderly are considered as a vulnerable group, and especially older women. Because of the difference in life expectancy between men and women, worsened by the effects of both World Wars in Germany, a large number of elderly women are widowed and live in single households. Home care is not always available due to differences in regional availability, and when it is available strict entitlement rules exist so that only severely dependent elderly receive home care. The health care system relies on the principle of subsidiarity and the duty to care of family members, meaning that only when the family is unable to provide care the Pflegeversicherung is responsible to offer care. Therefore, many vulnerable people are deprived of home care and rely on the willingness of their family members to provide care and support. In France, the health system is also based on the principle of subsidiarity and the social network of people is considered as the main port of call for informal care. However, many younger people have left their region for work, so that elderly and very elderly people, especially those living in remote and rural areas where the population is scarce, live in social isolation. As these regions also suffer from lack of sufficient health care and social facilities, many of them are deprived of the care they need. A specific feature of people in France is also that they consider professionals as intruders into their private sphere, thus even when care is available locally the reluctance to apply for care is large. In the Netherlands, patients are entitled to care and support when they receive an indication for care when they suffer from 
particular chronic diseases or other clear health problems, but only when informal care is not sufficiently available. Then, the provision of care is guaranteed by the health system (when waiting lists are no hindering factor), but clients have to contribute in the form of co-payments. However, as a result of the many procedures that have to be followed before and during the provision of care, clients consider themselves as 'victim' of the bureaucratic and systems character of health care. Formal regulations are being placed above the humane aspects of caring in the current Dutch health care system. This contrasts to France, where professionals try to place personal contact and human values above systems values and bureaucracy in their contacts with clients.

The training and education of home nurses is different in the four countries studied. Denmark is the only country where nurses are trained as generalists and where different nursing levels do not exist. Home nurses work all-round and are employed by the municipalities and provide care irrespective of where they live, in their own homes, homes for the elderly or special elderly apartments. No intraprofessional division of labour exists in home care except from first level (home nurse) and second level nurse (auxiliary). Each client is preferably cared for by a single nurse because continuity of care is considered very important. In France, three levels of training exist for nurses, but as specialised nurses almost exclusively work in hospitals, home care nurses are generally first and second level nurses, as in Denmark. They are trained in state nursing institutes. Second level nurses perform the bulk of the home care provided; only a fraction of the nurses employed in SSIAD services are first level nurses. Next to that, a quite large number of first level nurses work from independent private practices. The role, kind of activities and training of each health professional is strictly described by decree in the décret de competence, but their profiles are currently being discussed and reformulated. In the Netherlands, strong vertical and horizontal task differentiations exists, resulting in fragmentation of the care provided. Nursing is divided in five vertical levels, each with its own task description and level of training, from intermediate (м во) to higher vocational education (нво). In addition, nurses can further specialise in specific fields, such as diabetes nurse or rheumatoid arthritis nurse, which is called horizontal differentiation because nurses of the same level of training focus on particular fields of activities. These specialised nurses are generally also working in home care organisations. This means that clients in need of different kinds of care can be visited by several home nurses of different levels per day or per week, as each professional only performs a limited set of activities. Continuity of care for patients has made way for far-reaching division of labour in the Netherlands, which suits large-scale organisations from a financial point of view because nurses are paid according to their educational level. At several Dutch universities, scientific study of nursing practices is performed to establish 'best practices'. Academic study of nursing also takes place in Germany but on a more limited scale. 
In Germany, three nursing levels exist. Nurses are trained in-service, generally in hospitals and can choose between four working areas during their training, of which psychiatry, child care and home nursing is one. First level nurses who worked for at least two years can further specialise into community nursing with a part-time training. Second level nurses or auxiliaries only have one year of training. Also, elderly carers work in home care. Regarding task differentiation, a quite important legal distinction exists between nursing activities that are characterised as Grundpflege, which consists of helping with basic needs and adl-activities, and Behandlungspflege, that consists of specialised, technical nursing procedures. Krankenschwester (first level nurses) carry out both kinds of activities; Altenpfleger (elderly carer) and Krankenpflegehelfer (nurse auxiliary) only provide Grundpflege.

The aspects cooperation and co-ordination received rather limited attention in the literature, except from the Netherlands where these concepts are often discussed. Presumably, this is because far-stretching division of tasks in health care exists in the Netherlands, rendering proper cooperation between different health care professions and co-ordination of the different kinds of care provided to individual patients important. Increasing cooperation between hospital specialists, GPS and nurse practitioners, home care nurses and nurse specialists require proper coordination and attuning of activities. The person taking responsibility for this can differ per situation; sometimes the client or a family member takes up this role, sometimes the GP, and sometimes a nurse practitioner or nurse specialist. Also, a special function is created to fulfil this co-ordinating role and steer the complex communication processes for the client, namely that of case manager employed by a home care organisation. In Denmark, cooperation and co-ordination is not very often discussed because the municipalities provide and thus co-ordinate all home care provided locally. In France, independent nurses claim the role of care co-ordinator, but they often lack the knowledge of the local availability of services. Cooperation between different professionals is generally almost non-existent. In Germany, cooperation between different health care professionals also hardly exists, on the one hand because physicians sometimes refuse to co-operate with home nursing organisations, and on the other hand because a strict separation exists between different kinds of care. Medical care, nursing, and social care are different domains that are reimbursed according to different schemes, which results in fragmented provision and lack of cooperation. Thus, cooperation and co-ordination have proven to be valuable concepts that give insight into different mechanisms and ways of organisation of care in the health care systems that were studied.

Remarkably, the concept bureaucracy also primarily appeared to be important in the Netherlands. In Denmark, bureaucracy is not very influential and health professionals resist its interference in professional practices. The trust in professional expertise is rather high. In France, bureaucracy also hardly plays a role in nursing practices (unlike in other social domains, where it can be extensive!). As care is 
stigmatised and people are reluctant to call professionals in for help, interpersonal relations and building up trust between caring professionals and patients during the caring process are more prominent than bureaucratic rules and procedures. However, the way that health and social care are administered is very complex and form barriers for people and their families to apply for home care. Also in Germany, bureaucracy seems to be less important than other issues, for example the influence of physicians in health care. In contrast, bureaucracy is a hotly discussed issue in home care in the Netherlands. Not only arranging home care is a complex matter, as in France, but also the provision of home care is stacked with administrative procedures such as time-writing. All activities that are performed at each patient need to be meticulously written down and counted in minutes and signed by both home nurse and client. As a Dutch home nurse reported, sometimes an elderly client finding his or her glasses to be able sign for activities the home nurse performed takes more time than the activities themselves and brings the home nurse into time problems because of the tight working schedule. Every day, she visits quite a number of patients where she performs certain tasks that have to be accounted for meticulously to the home care organisation. Also, home nursing practices are more and more circumscribed in nursing protocols that diminish the possibilities for home nurses to adapt the care to the specific situation of the client. This is aggravated by the indication system that strictly describes the care to be performed during the intake procedure and home nurses are not allowed to deviate from this when she thinks it necessary. Then, a re-indication procedure needs to be started that takes several weeks. Thus, home nurses in the Netherlands are not able to adjust quickly to the changing situation of clients. These aspects fundamentally affect the work of home nurses and have led to a lot of discontent among them. Thus, bureaucracy has shown to be an important concept to gain more insight into the specific character of home nursing within European countries.

\subsubsection{Adaptation of the middle ring of the theoretical framework}

Regarding the middle ring of the theoretical framework, all concepts turned out to be useful and important as analytical tools to study differences in the modalities of home nursing in Europe. As far as can be decided, no central aspects were missed that should be added. Only a minor adaptation of this part of the framework is proposed. Task differentiation appeared to be directly related to the level of training of nurses in the countries studied, except for Denmark where vertical and horizontal task differentiation do not exist. Elsewhere, second level nurses trained at intermediate vocational level are only allowed to perform a certain limited set of tasks, while first level nurses who are trained at higher vocational level perform more complex and medical-technical nursing tasks and also fulfil co-ordinating and planning roles. Specialised nurses only focus on patients with a particular 
kind of chronic disease. Thus, the aspects Task differentiation and Training and education can be combined in the new framework into Levels of training and task differentiation.

\subsubsection{Outcomes of the study - the outer layer of the theoretical framework}

In the outer layer of the theoretical framework, aspects are included that represent the socio-cultural, political and health care context in which home nursing is embedded. As the main clientele of home nursing consists of (very) elderly and vulnerable people, an important aspect influencing the priority and growth of the home nursing sector is the ageing of the population in each country. In all European countries, the ageing of the population is a recurrent theme in health policies. In Denmark and the Netherlands, the percentage of the population aged $6_{5}$ years and over is below the $\mathrm{EU}$ average of $\mathrm{I} 6.5$ per cent and about average in France (Eurostat, 2005). The percentage in Germany is far above the Eu average, namely I8.6 per cent. The number of people between $6_{5}$ and 79 years of age in Germany is highest of the EU, namely almost $15 \%$ of the total population. Therefore, worries about the ageing of the population particularly exist in Germany, not only because of the numbers of elderly (almost I3 million people are currently over 65 years of age), but especially because of the expected increasing need for elderly care in the coming decades when the age group of 65 to 75 years will grow older and become more dependent and develop chronic or multiple diseases. Another reason for worry is that the percentage of elderly people in Germany is disproportionate with the number of young people. ${ }^{\mathrm{I}}$ Due to this unbalanced composition of the German population, the ageing of the population is considered a threat to society and the health care system in particular. As home care did not have a high priority for a long time in German health care, shortages in provision are expected as well as shortages of staff.

European countries react with different policies on the ageing of the population. In Denmark, enabling people to grow old in their own homes, which is called the ideology of 'ageing in place', has a high priority for several decades now. Since I987, radical substitution policies were introduced, and since then the number of elderly in nursing homes has been considerably reduced. New nursing homes were no longer allowed to be built. The welfare state has set the legal framework for the local provision of all kinds of community-based elderly care, such as home nursing, home help and a range of other facilities such as meals

I In Germany, only I4.5 per cent of the population is between 0 to I4 years of age, compared to 18.6 per cent of people over 65 , whereas in Denmark, France and the Netherlands the proportion of those between $0-\mathrm{I} 4$ is higher (I8.5 - I8.8\%) than that of people over 65 years of age (14.0-16.4\%). 
on wheels, snow clearance and help with gardening and shopping. Growing old and vulnerable is a recognised and accepted part of society in Denmark for which the welfare state guarantees sufficient care and support. In most other European countries, however, age and frailty is considered as a burden to society. This is also the case in the Netherlands, although the percentage of people over 65 years of age (I4.0\%) is lower than elsewhere in Europe, so the 'problem of the ageing society' is less pressing in the Netherlands than is suggested in policies and the media. The number of elderly people in residential homes (verzorgingshuizen) and nursing homes is very high in the Netherlands since the I950s, thus substitution policies have set the agenda for several decades now to replace institutional care by home care. However, every few years, new health care policies and budgetary measures are introduced resulting in an instable situation in elderly and home care. Since January 2007, municipalities contract out domiciliary care according to the wMo (Wet Maatschappelijke Ondersteuning; Societal Support Act). This implies that home care organisations have to tender for contracted care at the municipalities. This is leading to uncertainties and even bankruptcies of home care organisations, for example when municipalities choose for cleaning companies to perform household tasks in the homes of elderly people instead of for home help organisations with qualified personnel offering more expensive contracts. This development has affected the provision of integrated care by home care organisations that had been aimed at since the I99os. Although a wide range of home care services is offered in the Netherlands, accessibility criteria are strict and entitlement to care depends on the availability of informal carers, the insurance package, and the kind and amount of care needed according to the indication.

In Germany, the ageing of the population is clearly considered as a burden, partly because of their numbers, and partly because of the lack of availability of sufficient and suitable institutional and home care facilities. Care and support services are not up to quality standards and a considerable lack of qualified nursing personnel exists, which has led to a sense of crisis in nursing (Pflegenotstand). For a large part, this has resulted from the low priority of home care in health care policies for a long time. The Pflegegesetz introduced in I995 has brought improvement by providing an insurance framework for the provision of long-term nursing care, but in general, the health care system is still quite medically oriented. This is related to the strong belief in family solidarity on which the health care system is based. People have a legal duty to care for their elderly parents in Germany, in the form of informal care or payment of elderly care facilities. In France, family solidarity is also emphasised strongly, but in a more romantic way than in Germany. The private sphere of the family is considered as a shelter from the public sphere and family bonds and mutual obligations between generations are stressed in health care policies and by the government. Since the ig6os, several elderly policies have been issued, but these had limited effect, partly because of the 
dominancy of cure and institutional care, and also because these policies focussed on the wrong group, namely elderly who are still active and independent and pursue leisure activities, the so-called troisième âge, by promoting the local setting up of activities for seniors and pensioners, such as luncheon and cinema clubs. In a sense, these policies reflect a positive and romantic image of elderly people, who are still integrated into society and fully active in social and family life. Very elderly people, the quatrième âge, acquired a purely negative image of being heavily dependent, socially isolated and often suffering from a terminal disease. Since the I980s, more emphasis was laid on home care by establishing the SSIAD services to complement the independent nurses providing home care from their private practices. Since then, the home care domain has increased considerably in size, but the number of services is still far insufficient and these are too badly targeted to provide for all the care needed in France. A serious problem is also the state-periphery framework that is found throughout French society and which is also recognisable in home care: the closer one lives to the Paris region and growth poles of regional development where the highest percentage of the population lives, such as Lille, Bordeaux, Lyon, and Marseille, the larger the availability of home care services. However, these urbanised areas contrast sharply with the nine-tenths of scarcely populated farmlands and forests in France. Over 90 per cent of the municipalities are classified as rural, and these are often deprived of sufficient social-economical and community-care services. An example of the way policies work out according to the state-periphery framework is the Plan canicule (heat wave plan) that was issued in May 2004, less than a year after the summer of 2003 when almost I5 000 excess deaths were reported due to the heat, especially among (very) elderly people (Douste-Blazy and Falco, 2004). The Ministry of Health and Social Protection decides on the basis of information of the weather Institute Météo France and the Institut de Veille Sanitaire (Institute monitoring the sanitary situation) when the level one state of alertness passes into a state of emergency (level 2) or of interventions (level 3). Then, the prefects of the Départements are informed, who order the local safeguard system to come into action. Under to the 'gilded plan' of the level of interventions, elderly people who live in independent households are visited or phoned regularly by a local network of volunteers, called neighbour guardians, to check on their health situation. These elderly people are described as isolated and left behind in the summer months by family members who leave for a holiday. This vocabulary stigmatises elderly people living in their own homes as being pitiful because they are left uncared for, as well as accuses family members that they do not fulfil their obligations towards their elderly family members.

The former example shows the marked differences between countries regarding socio-cultural and political views on the role of the family towards frail elderly family members. This aspect of the theoretical framework appeared to be very important and is related to traditional socio-cultural values, but also to the role of 
the central state, reflected in health care and elderly policies, and to the kind of health care system in a country. In France, very strong feelings of family obligations and family solidarity exist, among the population as well as in politics. Both consider informal care the primary source of care for frail elderly people. This also underlies the health care system in the form of the principle of subsidiarity. People first and foremost have to mobilise sufficient support from their social network and family. When this is not available and elderly people are not able to live with their family members, a place in a retirement home is applied for. The health care system is so complex and fragmented, that

'it appears to be an extremely complex operation to obtain all the necessary resources to support an elderly person in a well-adapted home with adequate income and appropriate health and social care. By contrast, entry to a long-stay ward or retirement home is simplicity itself. The sheer complexity of support for home care costs, coupled with the uncertainty whether all of the components of the package can be assembled, must be an inhibiting factor to choosing home care alternatives when making decisions about home care'. (OECD, I996e p. I35)

Also, a hindering factor for calling in home care professionals is that home care bears a stigma in France, indicating that the elderly person is in need and not properly cared for by the family. This is reinforced by the feeling that professionals are considered as intruders into the private sphere of the home as a private bulwark. Although in Germany, for example, traditional family values are also strong, in France strong feelings of 'my home is my castle' fortify the private sphere against intrusion by the public sphere. This also applies to home nurses. As said, in Germany also a strong reliance on informal care exists by way of the principle of subsidiarity underlying the health care system, next to the legal responsibility of children to care for their elderly parents or to pay for professional or institutional care. However, although informal care thus plays a very large role in Germany, a clear conceptualisation of informal care in health care policies does not exist. The exact role of informal care in the provision of care is not defined or described clearly, but nevertheless the reliance of elderly people on their family is very large. This contrasts strongly with Denmark, where people are alleviated from caring responsibilities by the welfare state and no formal obligation to care for elderly family members exists. Only the presence of a spouse is considered in the assessment of the need for home care. Individual independence is maximised and people are encouraged by the system to pursue their individual careers. Labour participation of men and women is therefore very high in Denmark. The disadvantage of the extensive caring arrangements guaranteed by the state is however the expensiveness of the system, which forces married men and women to work to be able to afford the high taxes. Informal care in Denmark is only voluntary in nature and 
generally limited to support in short-term acute situations and in mobilising public services on behalf of the elderly:

'In the Danish Welfare State there is a broad consensus about the principles that the elderly have a right to aid from the public, when need arises. The aid is open for all, and it is important to notice the general lack of stigma attached to receiving health and social services. The Danish system of service provision enables the elderly and their relatives to be independent of each other, as help with activities of daily living tends to be obtained as formal care through public services.' (Plovsing, I992 p. I5)

The situation in the Netherlands lays in-between Denmark and France/Germany. The principle of subsidiarity also underlies the health care system, defining informal care as an indispensable part of the caring system, as in France and Germany. The availability of informal carers, not only of spouses but also of siblings, sonsin-law or daughters-in-law, is assessed during the indication procedure. When these are available, a person in need of care is not entitled to professional care provided by a home nurse or home help. Informal care is thus a formal as well as a moral duty in the Netherlands. Elderly people who received an indication for care, meaning that they are entitled to care according to the intake official, can apply for a personal budget ( $\mathrm{PGB}$ ) with which they can hire a home care professional, a family member or someone from the social network to provide care. In a sense, informal carers are proto-professionalized in the Netherlands by this system. This is reinforced by courses offered by home care organisations to informal carers as if they were employees. In many cases, providing informal care is felt as a burden. Daughters and daughters-in-law who care for their elderly parents feel a lot of pressure and exhaustion, especially when they also have a job and/or children. Generally, they feel they have no choice because of their formal and moral duty to care.

The role of the family in the countries studied is strongly related to the kind of health care system. In Denmark, the central state established a National Health Security System (NHSS) in I973. The provision of health care is strongly decentralised; most services are provided and financed at the local level of counties or municipalities. The government only provides an overall legal framework and is able to influence the provision of care or the setting of priorities by local governments and councils only indirectly. County and municipal authorities thus have great independence regarding their health and social policies and are rather free to prioritise their expenditures. Total equity, general accessibility and availability of health care services are main principles in the Danish health system, which means that rights, services and benefits are made available to all citizens, irrespective of differences in age, sex, income, ethnic background, occupation, or other 
potentially distinguishing criteria. Also, the fact that vulnerable people (except children under I8) are not dependent on their family members for sufficient care and support is an important characteristic of the Danish health care system. This means that the provision of home care is guaranteed and that sufficient caring professionals are available to provide the care needed. Denmark and the Netherlands both have a high number of nurses, namely II.० and I4.० per I ००० population, compared to only 7.5 per I ooo population in Germany and 7.I in France. This indicates that cure and care are almost independent domains with separate task definitions existing alongside one another in Denmark and the Netherlands. An important difference between the health care systems of these countries is that in the Netherlands, continuous reforms, laws and measures are proposed and introduced, resulting in a very instable and unclear situation in health care. In contrast, major reforms have been absent for many decades in Denmark. Another difference is that Denmark has a national health system, while the Dutch health care system has long been based on the Bismarck social insurance system financed by compulsory contributions paid by employers and employees and based on the level of income. About 6 o per cent of the population was covered by statutory social insurance provided by the Ziekenfonds (Sickness Fund), primarily those with salaries below a certain level. Historically, the Dutch health care system has been different from many other European countries, because it largely originated through the efforts of voluntary non-governmental organisations (NGOS) such as the cross associations providing home care. Since January 2006, the Dutch health care system has changed fundamentally with the introduction of a universal basic insurance, compulsory for all and executed by private health insurance companies that operate under market competition. The full consequences of these major reforms are not fully crystallised out yet.

Germany still has a social insurance system that originates from the late nineteenth century. The health system of the former East Germany (GDR) was quickly transformed in the I990s according to the treaties of unification to adopt the statutory health insurance system of West Germany. A fundamental characteristic of the German health care system is the sharing of decision-making powers between the federal government, the Länder, the corporatist, quasi-public and non-profit sickness funds, and regional associations of physicians and dentists. The sickness funds are the collectors, purchasers and payers of statutory health and long-term care insurance and are thus rather powerful. The health care system in Germany is quite medical-curatively oriented and care provided by hospitals and physicians have had priority over long-term home care for long. On the one hand, specialist care is directly accessible by patients and consulted quite easily and often, while on the other hand access to home care is restricted because a medical referral is needed and only those with considerable care needs are entitled to reimbursement under the Pflegegesetz. 
In France, until recently, the majority of health care for the compulsorily insured was financed by social insurance contributions of the statutory health insurance Assurance Maladie. A fundamental change in the system was introduced by the replacement of the employees' contribution by the national income tax; the Contribution Sociale Géneralisée (CSG), which transformed the current French health system into a mixed health system. Elements of the social insurance system still exist in the form of employers' contributions, but the general tax that accounts for about $40 \%$ of the financing of health care introduces an important element of a public health system. An important problem in the French health system is the geographical disparities in the distribution of health professionals and especially the uneven availability of home care services across the country. In some regions, home care is not available at all. Remarkable is that regular home care services were only established since I98I; until then, home nursing was only provided by independent nurses. This resulted in a cure and bio-medically oriented health care system in which hospital care and institutional facilities dominate over homebased care. Elderly are primarily cared for by family members or in residential homes. As nursing homes do not exist in France, both residential homes and hospitals dispose of long-stay wards where intensive medical care is provided to heavily dependent elderly people. In the health care system, a micro-system of care exists, involving public and private services belonging to health care, social care and community services (e.g. luncheon clubs, télé-alarm services). The complexity of the system and the diverse actors and payment schemes involved puzzles the users and often also the providers of care. Thus, the French health care structure entails barriers for the use of home care that do not exist regarding ambulant medical care, hospital care, and long-stay facilities.

The last two aspects of the theoretical framework did not appear to be as important as thought beforehand. Namely, in Denmark, France and Germany, the impact of managerial perspectives was not much of an issue in health care. In Denmark, the role of managers is still very limited and health care professionals have successfully criticised the setting of managerial and economic targets until now. In Germany and France, the influence of physicians and dominance of medical perspectives in health care are more prominent issues than perspectives of managers and nurses. Only in the Netherlands, 'managerialism' is an issue in health care, referring to strong tendencies to organise and pre-structure matters in a top down way and to deal with things in a bureaucratic way by designing and referring to rules and regulations and administrative procedures. Efficiency, rules and principles, universality and impersonality are key concepts of managerialism. Managerialism is related to the growth of bureaucracies in modernised societies in order to organise the complex division of labour and task differentiation. When care is fragmented into small parts provided by different kinds of professionals and organisations, which is clearly the case in the Netherlands, regulations are 
designed by managers to coordinate the activities of the different actors involved. In itself, bureaucracy is useful, because it regulates interpersonal communication in an impersonal and just way. However, when rules have become goals in themselves and proliferate to such an extent that an efficient and transparent way of dealing with matters is hampered, they tend to become dysfunctional and result in 'over-bureaucracy' and 'over-regulation' of matters. Several problems regarding home nursing that are often mentioned in the literature and in public and political discussions about home care, such as long waiting lists, shortage of staff, discontent of home nurses about working conditions and career possibilities, high absenteeism, and a high amount of nurses that untimely leave the profession, can be related to the tendencies to over-organise and over-standardise matters in the Netherlands and the too large impact of managers and other hierarchical levels on the content and way of performance of home nursing work. Home nurses and home helps strongly feel that their professional autonomy is affected by the tendencies of managerialism and bureaucracy in Dutch home care.

Cultural diversity was expected to be an important aspect in contemporary home nursing, as the populations of European countries have become more and more mixed and multi-cultural by people immigrating from former colonies, asylum seekers and refugees from war regions and repressive regimes, and guest workers and their families. Especially in the larger cities, home nurses are currently often providing care to people with different cultural backgrounds. According to Leininger (I994), this would require that they should be trained to take the values, beliefs and daily living patterns of specific cultural groups into account:

'Nurses are interacting with people from widely different cultures within a short period of time. As a consequence, nurses and other health care providers are expected to know, understand, respond, and interact favorably with strangers from diverse cultural backgrounds in this country and elsewhere. Being helpful to strangers whose values and lifeways are different from those of the nurse necessitates a knowledge base of such differences and the creative use of such knowledge. Cultural awareness through knowledge is a critical necessity for professional nurses today and one which is of growing importance.' (Leininger, I994 p. 7-8)

It was assumed that awareness of the cultural diversity of clients would be an important issue in contemporary home nursing. However, although immigration politics and cultural differences in society are hotly discussed issues in France and the Netherlands and the integration of people with different backgrounds is considered as a burden and challenge to society and politics, cultural diversity appeared to be a hardly discussed issue in home care as yet. De Veer (200I) concludes that only a few local and national studies are currently being performed in 
the Netherlands to gain more knowledge about the specific home care needs of immigrants:

\begin{abstract}
'National data about the use of home care by immigrants and the care needs of immigrants are not (yet) available. From the diverse, especially local, studies performed does emerge that foreign elderly people hardly use home help or home nursing. The inventory shows that a diversity of activities has been started to actually reach this group. (...) More information is needed about the exact care needs of immigrants.' (Veer, Francke et al., 200I p. 23-24)
\end{abstract}

The few studies performed report that elderly immigrants make less use of home care than native Dutch people (Foets, Lucht et al., 2005; Janse, 2006). Possible reasons mentioned are: unfamiliarity with home care or ignorance of its existence by immigrants; the large role of family care in many foreign cultures; immigrants may be hesitant to apply for home care because of the lengthy and complicated indication procedure; lack of money to pay the co-payments; home care is possibly not well-attuned to the expectations of non-native people; or because they fear that their customs or habits will not be respected by home care professionals (Veer, Francke et al., 200I). Instead of a macro study like this book, scientific study on a micro level would probably yield more results regarding the question whether home nurses are sensitive regarding cultural differences and whether they have sufficient knowledge of foreign cultures. In-depth interviews with home nurses and their clients would provide more insight in their experiences and the difficulties or challenges they are confronted with while providing care to non-native people. Voortman (I999) for example found that none of the home nurses of a north east Dutch home care organisation who daily provide care to (mainly Iranian) refugees and asylum seekers had received any kind of information or coaching on nursing to people with a different cultural background (Voortman, I999).

In Germany, the integration of East and West Germany has been a larger issue in politics and health care than cultural diversity. In Denmark, immigration politics are very strict, resulting in a quite homogeneous population. As a result, cultural diversity is also not very much an issue in Danish health care. Thus, hardly any insight was gained in this book regarding the challenges posed to home care by the increasing cultural diversity of the population. Nevertheless, it remains an aspect deserving more in-depth scientific research in the near future. More knowledge should be gathered on how to lower the barriers to apply for home care of immigrants with care needs. Also, the surplus value of different kinds of local initiatives, such as home care organisations for Muslims (Elsevier, 2007) or local information and support centres for non-native people in need of care (Zorgmediatheek, 2006), need to be studied regarding their effectiveness to reach elderly immigrants. 


\subsubsection{Adaptation of the outer layer of the theoretical framework}

The aspects included in the outer layer of the theoretical framework have been found to be very important for explaining differences in home nursing between the four European countries studied. Although the ageing of the population is a trend that is found all across Europe, the way it is experienced is unique for each country. In some countries, such as Denmark, growing older is an accepted part of society and a range of facilities is available to support elderly people to remain living independently as long as possible. In contrast, growing older in Germany and the Netherlands is considered as a burden to society and the health care system, while the image of elderly people in France focuses on those who are still quite active and fully participating in social life. French elderly and very elderly who need care and support when they have become dependent because they suffer from health problems are stigmatised as isolated and not properly cared for by their family.

The health and elderly policies in countries, the role of the family and the kind of health care system have also shown to differ considerably in each country and have appeared to be important in shaping the modalities of home nursing. Namely, the way that health policies formulate the role of informal care in health care and the amount to which health care structures rely on informal care for a large part determine the role of the family and social network and thereupon also the role of home care professionals in caring for frail elderly people. For example, in Denmark family members are alleviated from caring responsibilities because health policies of the welfare state have resulted in health care structures that are able to guarantee sufficient professional care and support to those in need of care. In contrast, the role of informal care is defined as very important or even essential in the Netherlands, France and Germany. In those countries, the principle of subsidiarity underlying the health care systems reserves a large role for informal care because the family and social network is the first port of call in case of needs and dependency. In the Netherlands, informal care is considered an important complement to professional care. The entitlement to professional care is formally weighed by an indication procedure in which the availability of informal carers is an important factor. However, when the need for care has been established, the indication entitles a person to call in professional care that is sufficiently available throughout the country. A national network of cross associations has existed for long that currently exists in the form of non-governmental home care organisations that fulfil a public function. In France and Germany, the role of the family is indispensable because home care is not sufficiently available in all regions. In France, this is due to the lack of socio-economic and health care facilities in large, scarcely populated areas because matters in France are organised according to the state-periphery framework. In the Paris area, the density of home care facilities is much larger than in rural areas, where home care is sometimes not available at all. In Germany, the 
legal responsibility of siblings to care for their elderly parents and the health care system that has been medically oriented for long constitute barriers for long-term ambulant care. The low priority of home care, the quite medical definition of nursing care under the Pflegegesetz, the lack of qualified manpower and the weak and fragmented organisation of home care are important factors that have resulted in a weakly organised and unevenly distributed home care domain. This leaves many informal carers no choice than to care for elderly people themselves.

Thus, the ageing of the population, health and elderly policies, the kind of health care system and family relations and informal care have proven to be very important aspects giving more insight into the modalities of home nursing in Denmark, France, the Netherlands, and Germany. The impact of managerial perspectives particularly only appeared to be an aspect of considerable importance in Dutch home care. As yet, it is not much discussed in the other three countries. However, literature suggests that it is an issue in the U $\mathrm{K}$. Therefore, it is suggested to maintain this aspect in the theoretical framework as it is a central issue in Dutch home care and possibly also in other European countries. As it is closely related to bureaucracy, it has much to recommend to combine both as bureaucracy and the impact of managerial perspectives in the middle ring of the framework, but the trend of managerialism is taking place on a different societal level. Bureaucracy in the theoretical framework refers to the degree to which home nursing work is subject to bureaucratic rules and procedures and the amount to which these affect the professional autonomy of home nurses. Managerialism, on the other hand, refers to the influence of professionals of a higher hierarchical level, generally managers, on the way that home nursing is performed. For example, by way of the systems and procedures they design to monitor the activities and performance of home nurses to let them account for their work. In a sense, this is a positive trend because the quality of care provided to patients is guarded. However, the autonomy of home nurses to independently arrange their work based on their specific professional knowledge and experience is severely affected when the control of managers over home nursing work becomes too large.

Although not much insight was gained into the aspect of cultural diversity, it remains an important aspect deserving more specific scientific research because European societies are increasingly becoming more culturally diverse. Findings suggest that the percentage of immigrant elderly people using home care services is considerably lower than that of native people, while their care needs are presumably about equal or even larger due to their generally lower social-economical status which is associated with a less favourable health situation. Also, it is stated that current home care services are insufficiently aware of cultural and religious differences that also touch on home care tasks such as washing a person, administering medication, involving informal carers, and expecting an active attitude of the patient. However, sensitivity to cultural differences poses a problem in health 
care. After all, one of the important achievements of modern health care is that care is provided according to universalistic principles. This means that health care is provided to people without regard to persons, thus irrespective of their age, health status, socio-economic background and ethnic background. This is probably easier in medicine than in home care, where home nurses and home helps are providing care in the homes of clients, where differences in beliefs and customs become more visible. As was stated in the second chapter of this book, the tensions between the systems values and universalistic principles of health care on the one hand and the personal situation and specific way of life of clients on the other hand is a particular characteristic of home care. Therefore, it is sometimes difficult for home nurses to find a balance between these two spheres and not to become too involved in the personal situation of clients and on the other hand not having a too detached professional attitude. This dilemma for home nurses is similar in all European countries.

The findings in this book point to the inclusion of a new aspect into the outer layer of the theoretical framework, namely the central state. The views of the central state regarding social relations and the role of the health care system in caring for vulnerable groups appeared to be very important. Both shape the health policies and health care structures that promote or even guarantee the provision of professional home care on the one hand, or form barriers for the development of the home care domain. In Denmark, the welfare state only provides the legal framework of health care and the financing of provision of care are decentralised to the local level. Municipalities have a legal obligation to guarantee professional care and the state grants people the freedom to pursue individual careers by alleviating them from caring responsibilities for their elderly family members. This shapes a favourable climate for the provision of professional home care. In France and Germany, however, the central state supported the development of health care systems that are based on the extensive provision of elderly care by the family and social network. Both health care systems are cure oriented and have given priority to hospital and medical care for long, neglecting the importance of sufficient home care facilities for the increasing numbers of elderly people. They also omitted to react on societal changes, for example changing family patterns during the last decades during which it became less customary to live in three-generation families. Elderly people more and more live in independent households and geographic mobility of siblings has increased considerably, thus diminishing the safety net for elderly people who are confronted with health problems and are in need of care and support. Also, due to the ever-rising health care costs and stronger cure orientation of hospitals, patients are discharged in an earlier stage, when they still need intensive home care. These developments have resulted in an increased need for home care facilities. However, the states and health care systems of France and Germany did not responded sufficiently and early enough, resulting in a current 
lack of availability of services, lack of manpower, a weak organisation of the home care domain and structural barriers in the health care and reimbursement system for the provision of home care. In the Netherlands, the situation is different. Originally, home care has very well been organised by the dense network of cross associations that has been set up since the i88os. Home nursing has developed into a rather well established domain in society and home nurses had a rather high local social status alongside the GP because both worked together in families in their own neighbourhood and she provided all-round care to young and old. Home nursing was generally available, directly accessible to clients apart from a very low membership fee to the local cross organisation, and fully reimbursed by the health insurance. However, continuous health care reforms that have been issued since the I970s resulted in a very different situation now. The health care system has been fundamentally reformed and is now based on a compulsory insurance for all, executed by insurance companies working under market competition, and many plans, measures, budgetary cuts and changes in policies have taken place in the last decades. Local cross associations have made way for large-scale regional home care organisations and general accessibility has made way for a bureaucratic system of care needs that are determined by a strict indication procedure. Also, the provision of informal care has been integrated into the caring system and has become an indispensable part of elderly care of which the availability is assessed in the indication procedure. Family members currently not only have a moral duty to care, but also a formal duty to care. Thus, the central states have shown to be very powerful actors in shaping the circumstances and structures within which home nursing is provided. Central states turn out to be especially powerful in creating the conditions under which the home nursing domain can flourish or remain or become a marginal health care sector that is not able to meet the demand for care of vulnerable societal groups.

Another aspect that emerged as important in characterising differences in the context in which home nursing is embedded is the difference in health-related values between countries. Namely, the balance between medical-curative care provided by physicians and hospitals on the one hand and nursing care and support provided in the domain of home care on the other hand appeared to be highly influenced by the perspectives on how to deal with matters of health and disease in countries. For example in Germany and France, the dominance of biomedical perspectives has resulted in medical-curative care overshadowing ambulant longterm care in the health care system and prevented a strong development of the home care sector: "Health care in Germany is strongly oriented to the medical framework, having resulted in a kind of neglect by society of other forms of care for a long time" (Schnepp, 200I p. II). Throughout the twentieth century, caring and supporting activities performed in the 'periphery' of the health care system did not have a very high priority in Germany, compared to medical and cure-ori- 
ented activities performed in the 'medical centre' of health care (Philipsen, 2000; see chapter 2 for an elaboration on this). This is similar in France, despite several policy plans on elderly that have been issued since the I96os.

In France and Germany, people have a rather medical, cure-oriented attitude towards matters of health and disease. In general, they adhere to a medical definition of diseases, asking and expecting to be 'healed' by medical interference and drugs. This is encouraged because in Germany and France, an employee needs a physician's note to be allowed to stay at home in case of illness, instantly medicalising the affliction. In contrast, in the Netherlands, most employers accept that people remain absent from work for one or a few days when they are not feeling well and causes of illness are more sought in psychosocial factors than in biomedical causes (Deschepper, Stichele et al., 2002 p. I853). In other European countries, for example in the Netherlands, people generally take a more reluctant stance towards illness and adhere to the strategy of letting it run its course and giving the body time to recuperate by itself, without wanting to influence this process with medical means. In countries such as France, Germany and Belgium, people generally do not follow the strategy to 'wait and see', but visit a physician, specialist or pharmacist in an early stage and expect to be prescribed drugs such as antibiotics (Deschepper, Stichele et al., 2002). Therefore, a large variation in medical consumption among EU countries exists; for example, the use of antibiotics in France is highest of the EU and French physicians, especially GPS, prescribe more drugs per visit than their counterparts in other countries (Cars, Moelstad et al., 200I). These differences in patterns of prescription and medical consumption are unlikely to be caused by differences in health status of populations but more likely to be traced back to differences in physicians' and patients' health-related value orientations, or in other words, the cultural and societal norms, values, ideas, habits and customs concerning issues of health and illness. This dominance of biomedical perspectives in Germany and France can also be recognised in the health care systems of these countries that are more cure oriented than care oriented and where institutional and medical care receive priority over ambulant and nursing care. The domains of cure and care seem to be more in balance in Denmark and the Netherlands. These cultural as well as structural differences regarding matters of health and disease have thus formed barriers for the development of the home care sector in France and Germany, while these favoured the development of home care into an extensive and easily accessible domain in Denmark and the Netherlands. 


\subsubsection{A revised theoretical framework of home nursing in comparative and contextual perspective}

Concluding, the following revised theoretical framework is proposed to explain the modalities of home nursing in comparative and contextual perspective (see figure I).

Figure I A revised theoretical framework of home nursing in comparative and contextual perspective

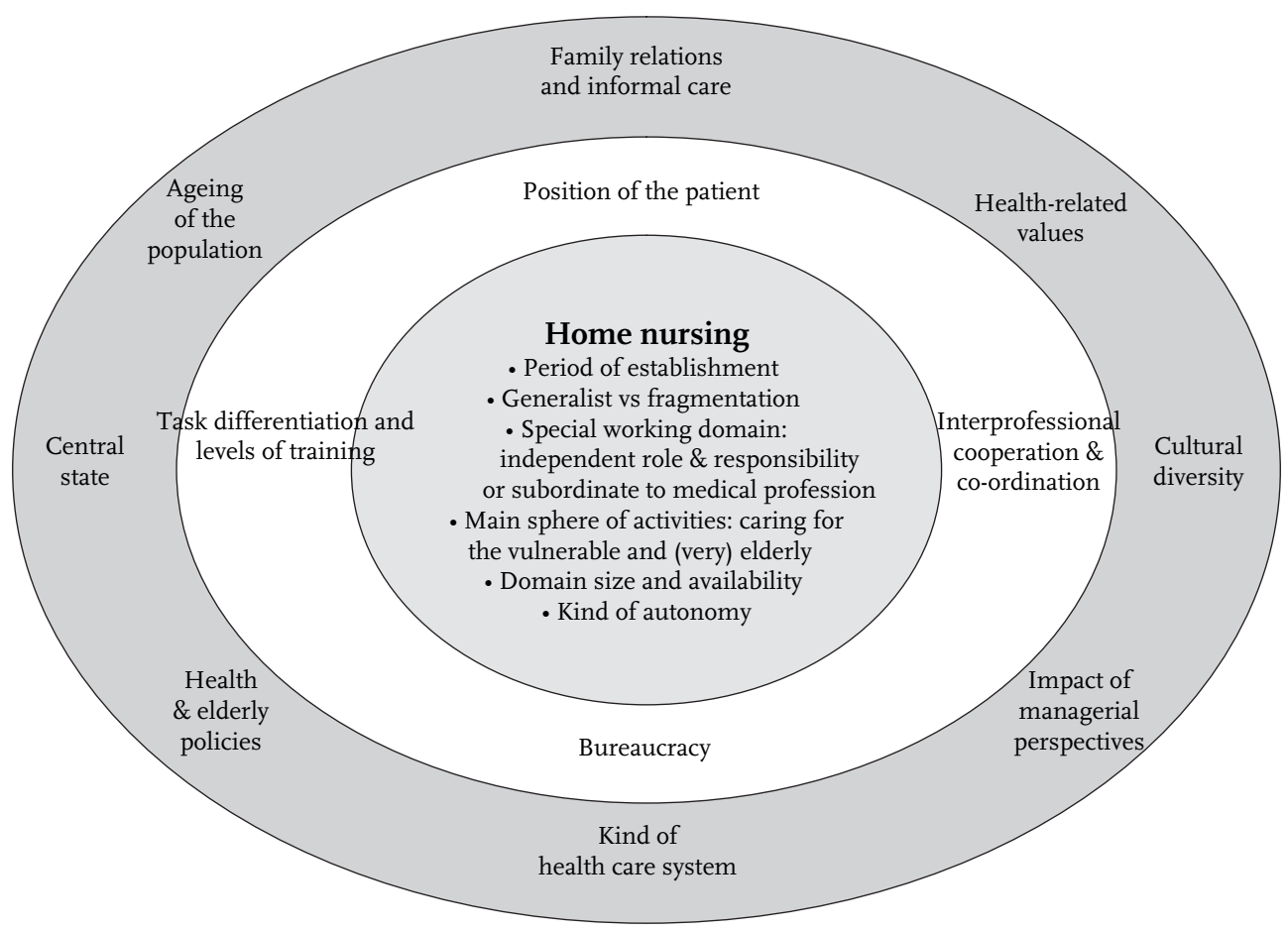

\subsubsection{Strengths and weaknesses of the analysis by use of the theoretical framework}

The analysis of the domain and profession of home nursing in this book by use of the theoretical framework has some strengths and weaknesses that go hand in hand. The strength of the analysis according to the theoretical framework is that it encompasses many aspects at the level of home nursing itself as well as the intra- and interprofessional level and at the level of health care systems and the socio-cultural and political environment. By using the framework as an analyti- 
cal tool, a broad and comprehensive picture of the modalities of home nursing could be drawn, resulting in an elaborate study on home nursing in four European countries. Per country, a specific picture of the particular characteristics of home care and the health care and societal context in which it is embedded was drawn. Not only four quite different descriptions of home nursing resulted from this, but also explanatory backgrounds emerged from the study to interpret the differences between these modalities. The analysis shows that one aspect is not decisive for particular problems in home nursing in a specific country, for example the proliferation of managerialism in the Netherlands, but that many aspects play a role in shaping home nursing and that many aspects are interwoven. Only when all aspects are studied in relation to each other, a coherent view on the current modality of home nursing arises. However, this also implies that this modality is contingent and fluent in time, because when a particular aspect of society or health care changes over time, it influences other aspects that are included in the framework. This leads to a different constellation in which home nursing is embedded and gradually to a different modality of home nursing. The pace of change can however differ per country, according to the stability or instability of social and health care structures and speed of cultural and political changes. For example, this book shows that Dutch society is rather in turmoil in the last few years, as well as the health care system that is fundamentally changed and reorganised. The health care system is also being changed in France, resulting in fragmentation of care and difficulties for clients to gain an overview of the diverse regulations. In contrast, the socio-cultural climate and health care system seem to be rather stable in Denmark. The favourable attitude towards care provided by professionals for many decades now, the autonomy granted to health care professionals and the priority of community-based care in health policies have led to a stable position of home care in the health care system. This way, home care is institutionalised to a higher degree in Denmark than in the other countries studied. This shows the contingency of the current modalities of home nursing; after all, if the attitude of the central state or the population would have been different, or if the historical development of home nursing or the health care system would have been different, the current modality of home nursing in that particular country would be different now as well. Therefore, this study presupposed that home nursing could only be described properly when considering it within the broader context of the network of professions, the health care system and society.

The tendency of the framework to be all encompassing has also shown to be a major drawback during this study. After all, taking so many aspects into account implies not only an in-depth study of home nursing itself, but also of many other aspects, such as professional theory, the character of health care systems, the role of informal care in countries, cultural differences regarding matters of health and disease, the attitude towards vulnerability and old age, the role of the state, 
managerialism and bureaucracy. The all-embracing character of the study and the accompanying complexity to draw an integrated picture of all the different aspects related with home care largely explains the lengthiness of the period in which this study was performed. The aim of this study has never been to study only one or a limited number of specific aspects of home nursing from a micro perspective, but the intention has been from the beginning to draw a broad, general picture of home nursing and all relevant related aspects from a comparative, cultural studies' perspective. The outcomes would enable drawing conclusions on the backgrounds of these differences and generating theoretical ideas about international differences in the modalities of professions and the institutionalisation of home nursing as a professional domain.

Another drawback of the framework is that it includes many interrelated aspects, while it is not entirely clear how these aspects influence each other, how strong these influences are, or in what direction the influence is exerted. The assumptions that aspects are interrelated and developments in societal spheres influence each other were more important in this study than how they are interrelated exactly. As the descriptions concern societal developments taking place over long periods of time and resulting in certain specific current constellations of home care domains, the strength and direction of the relations cannot precisely be determined and are just described and interpreted.

Another possible criticism of this study could be that the descriptions of home nursing in the four countries lack uniformity because no specific research tool was developed, such as a standardised questionnaire to be used similarly in all countries. Instead, the assumption of this study was that the country-specific situations in which home nursing is embedded are essentially different and that home nursing should be studied in its multiformity. Therefore, the theoretical framework was developed in which many factors were included to enable the international comparison of home nursing from a broad and contextual macro-sociological perspective. The differences in the socio-cultural and political backgrounds and in the health care systems were taken as vantage points and resulted in countryspecific and therefore rather different rich descriptions of the modalities of home nursing and the contexts in which it is embedded. The incomparability of the different national situations was taken as an advantage rather than a drawback and resulted in quite different characterisations of home care in the four European countries. Rather than comparing apples and oranges, the different kinds of fruits were tasted in turn and their specific characteristics and qualities were separately valued and interpreted by using the aspects of the theoretical framework. This led to rich descriptions of the country-specific constellations or 'linked ecologies' (see further on) in which home nursing is embedded. 


\subsection{Differences in the degree of institutionalisation of the home nursing domain and in caring cultures}

This book departed from the assumption that international differences in home nursing practices and the modalities of nursing exist across Europe. Despite increasing tendencies in Europe to develop common frameworks for societal and health problems and the increasing interchange of ideas, knowledge and people between European countries, important differences were found on all levels studied between the four countries. Marked differences between national cultures have been found regarding the way these societies deal with matters of health, disease and dependency and the way they differ in their opinions about how elderly and vulnerable people should be cared for. Instead of finding increasing convergence within Europe regarding elderly care, it appeared that each country has found its own solutions and devises its own elderly care policies because of substantial social, cultural, political and historical differences and fundamental differences in health care systems. These differences have been found at all four levels of analysis defined in chapter one.

Firstly, at the micro level of the domain of home nursing or the primary process was found that differences exist in the kind of activities of home nurses. In Denmark, home nurses perform all kinds of tasks due to their all-round profile, while in the Netherlands, a strict task differentiation exists and tasks are divided according to nursing levels. In Germany, first level home nurses provide relatively more technical nursing tasks because nursing is considered as an extension of medical care and because of the strict entitlement rules under the Pflegegesetz. In France, independent nurses predominantly provide medical-technical nursing tasks and refuse to perform caring and supporting activities because they do not consider that as belonging to their job. Also, an additional domain of home nursing exists in France in the form of HAD that is quite medical in character and substitutes for hospital care for patients who need complex and specialised long-term care.

Secondly and thirdly, at the intermediate level of the home nursing profession and meso level of interprofessional relations, marked differences were found in the role and status of home nurses and their position within the health care system of each country. In Denmark, the independent role and task definition of home nurses is generally accepted and it is customary to call in health care professionals to care for elderly and vulnerable people. Family members are alleviated from caring responsibilities by the state and health care system. Therefore, the professional autonomy and social status of Danish home nurses is rather high. In the Netherlands, the autonomy and social status of home nurses used to be higher than it is now. The proliferation of bureaucracy, legal and organisational rules and procedures and the fragmentation of the profession into different nursing levels have affected the autonomous and all-round character it used to have. Also, the strict 
intake procedure has limited the accessibility of home care to the population, for example because the availability of informal carers restricts access to home care. The formal and moral appeal on people to care for their elderly family members has increased during the last decades. The rather high social esteem of local home nurses who personally knew the situation of many families in her neighbourhood has been replaced by a new system of interchangeable home nurses working according to organisational schedules and strict task descriptions that are drawn up by others from outside the profession. This has obviously affected the social status and autonomy of home nurses and has led to a lot of discontent among home nurses about their working conditions. The climate regarding the provision of care that was quite favourable traditionally has changed in a negative sense in the Netherlands during the last ten to twenty years.

In France and Germany, home nursing is considered as subordinate to the medical profession and operating in the periphery of health care. Care provided by physicians and hospitals is dominant and receives priority by the state and in the health care system. Strict and sometimes complicated rules exist regarding entitlement to home nursing and the principle of subsidiarity underlying the health care system of both countries form important barriers for the use of home care. The important role of the family to care for their vulnerable family members is heavily stressed in both countries; in France a predominantly moral appeal is done on the family, accusing them of leaving elderly in the cold, while in Germany a legal and financial plight exists to care for elderly family members. Due to these circumstances, calling in professional home nursing is restricted and the social status of home nurses is rather low compared to the social status of physicians in both countries.

Fourthly, important international differences were also found regarding the macro level of the institutionalisation of home nursing as a subsector of the health care system and as a societal domain. As we have seen in this book, the core function of home nursing as a societal institution to care for (very) elderly and vulnerable people is strongly related to social and cultural norms and values and reciprocal expectations of state, professionals, clients and families. These strongly differ per country and have supported or hindered the institutionalisation of home nursing as a societal sector throughout the twentieth century. For example in Denmark, the welfare state, municipal responsibilities, health policies prioritising ambulant elderly care and cultural values favouring professional care over informal care have enable a strong degree of institutionalisation of home nursing. In contrast, health policies favouring medical and institutional care in France and Germany, supported by political and cultural views on the strong role of the family in caregiving to elderly people has hindered the development of a strong home nursing sector and thus prevented the institutionalisation of home nursing in society. This has led to current problems in both countries to cope with the double ageing of society 
and the ever-increasing costs of health care. The Netherlands take in a position inbetween; in many ways the position of home nursing has been as favourable as in Denmark for a very long time, but the marked differences in the health care systems with different views on the responsibilities of the welfare state have resulted in a lesser degree of institutionalisation of home nursing in the Netherlands. However, the degree to which the caring domain is considered to have a separate task definition alongside the medical domain is larger than in France and Germany.

This is in line with the way that Scott and Meyer (I99I) discern different degrees of institutionalisation (Scott and Meyer, I99I). Namely, in countries where home nursing activities are organised in a homogeneous way by rather isomorphous organisations operating within clear social or health structures, the degree of institutionalisation of home nursing is higher than in countries where a variety of professionals, organisations and other forms of elderly care exist that operate within health care structures that form barriers for more coherent ways of provision and where the same function is addressed to by different kinds of services. On the basis of this can be concluded that of the four countries studied, the degree of institutionalisation of the home nursing domain is highest in Denmark and lowest in France and Germany. The Netherlands takes in a special position, because although a nation-wide network or isomorphic home care organisations exists, these do not operate any longer under clear health care structures because the health care system is under continuous reconstruction and reform. Also, the proliferation of managerial perspectives has affected the professional autonomy of home nurses and form barriers for the further institutionalisation of the domain of home nursing.

The cultural studies perspective of differences in caring cultures between North and South Europe displays an almost similar picture. According to the North-South axis described in chapter one, different caring cultures can be discerned within Europe. On the one hand, northern European countries such as the Scandinavian countries, the UK and the Netherlands are characterised as having comprehensive health care systems and extensive welfare arrangements and community care facilities that are available to large groups of elderly. Especially in Scandinavian countries, family responsibilities are not included in social policies and welfare systems and formal organisations and health professionals predominantly provide care. On the other hand, south European countries are characterised as having less comprehensive health care provisions and welfare arrangements for elderly people (Giarchi, I996b; Mossialos and Le Grand, I999b). Also, informal care provided by family members is strongly entrenched in their health and social care systems and emphasised in health policies. These assumptions are congruent with the ideal type classification of welfare state regimes by Esping-Andersen (Esping-Andersen, I990). North European welfare states are then typified as corresponding to the liberal-democratic ideal type where rather comprehensive welfare and professional 
care arrangements are provided or guaranteed by the state, and South European welfare states, as well as Germany, are typified as conservative-corporatist. In the latter, the state relies on the principle of subsidiarity and only functions as a safety net; the community should act first and the state only intervenes when there are no other alternatives.

The existence of different caring cultures and welfare state regimes is confirmed in this thesis. The comprehensive health care arrangements and provision of care to elderly people that is primarily performed by professionals in Denmark are consistent with the typification of a northern caring culture of a liberal-democratic welfare state. On the other hand, the caring culture in France and Germany can be characterised as southern and conservative-corporatist in which the principle of subsidiarity underpins the health care arrangements; the role of the state is minimal and the role of family members in caring for the vulnerable is large. It also becomes clear why the Netherlands take in a mid-position: one would characterise the caring culture as northern and quite favourable for the development of the home nursing domain, as in Denmark. However, the Dutch state has adhered to the principle of subsidiarity for a long time as well, expecting a large role of family members, volunteers and charitable organisations. This also explains the founding of non-governmental cross associations since the end of the nineteenth century that have been the exclusive providers of home nursing for more than a century in the Netherlands.

The process of cultural diffusion (Philipsen, I980) explains that socio-cultural norms and values and the way that members of society cope with matters of health and illness are subject to cultural transfer. This explains why the health care arrangements for elderly people are entirely different in Denmark compared to France. Both countries are geographically rather far apart and processes of cultural diffusion hardly take place, apart from general trends taking place all over Europe. The way that elderly care is arranged in both countries is therefore rather different: in Denmark health professionals predominantly provide elderly care and the welfare state guarantees sufficient availability of care, while in France the central state emphasises the role of the family in elderly care, supported by socio-cultural values favouring informal care over professional care.

\subsection{Significance of this study}

To conclude, the significance of this thesis is indicated for different audiences (Creswell, 2003 p. I49). Firstly, the the contribution of this study for the scientific audience of sociologists, health care scientists, and cultural scientists studying societal phenomena within a contextual environment is described. Especially, a contribution is hoped to be made to the sociological theory of professions. Secondly, 
implications of this study for policies regarding elderly and vulnerable people are indicated. The different modalities of home care within European countries have shown that some structures or practices seem to work in a better way than others. As European health care systems are now operating on a common European market that is increasingly harmonised, countries can learn from one another and develop 'best practices' regarding home care for the elderly and ways to balance professional and informal care. Thirdly, recommendations that are formulated to improve caring practices for the vulnerable within different European countries.

\subsubsection{Contribution to the sociology of the professions: beyond Abbott's network of professions to country-specific constellations in which home nursing is embedded}

In chapter two, several strands in the sociology of the professions were described. In this book, the perspective on professions of Abbott (I988) was taken as theoretical basis of this thesis (Abbott, I988a). According to this view, of central importance to professions is the concept of jurisdiction over areas of expertise, or in other words the way that professions organise and control their work by linking specific knowledge and expertise to certain tasks and responsibilities. They establish their own working domain by legitimising professional claims on specific task areas to other professions, the state and the public. This was linked to Benson (1975), who states that having an own working domain means that an organisation or profession has acquired legitimation of carrying out activities in a certain sphere and granted the right and responsibility deal with a broad problem area (Benson, I975).

Abbott (I988) deliberately does not precisely circumscribe the meaning of the concept 'profession', because he assumes that people generally understand what is meant by the term and therefore need no further specification of what a profession 'is' or represents nor an elaboration of their organisational structures. Instead, he argues to focus on their role and function in society and pleas to study professional practices. Next to the content of their work, the way in which professionals legitimise their claims on specific social problems should be studied as well as the relations between them in the system of interrelated professions. He states that these interprofessional relations are not stable or permanent, but make up a fluent and interacting system or 'ecology'. Within such a system, contests over jurisdictions would be of central importance and the way these generate a specific division of labour, for example by specialisation or subordination. Also, Abbott argues to take the influence of large-scale social developments and contextual factors on professions into account, such as rationalisation, bureaucracy, and the rise of multi-professional organisations. Influenced by such external forces, tasks are created, abolished, or reshaped, and relations between professions change. This thesis also departed from the assumption that the societal context is an important 
source to explain the significance and meaning of certain professional activities or characteristics. It has focused on the ways in which knowledge and expertise of specific social and cultural problems are organised and institutionalised in a particular domain of contemporary society, namely the domain of home nursing. Furthermore, the process of professionalisation has not been considered in this book as a uni-linear, evolutionary process or a simple collective action by a cohesive group as in earlier works on professions, but as a complex and dynamic process in which social and professional forces are interrelated (Abbott, I99I p. 380).

The findings in this book have shown that these ideas have been very fruitful as points of departure. Especially the country-specific relations between home nursing and other professions and domains have appeared to be of interest, such as GPS, physicians, hospitals, home helps, nursing homes and especially informal carers. In every country, a specific constellation or 'ecology' was found to exist in which home nursing is embedded, that shapes and influences the tasks of the home nursing profession and its social status. Also, the influence of large-scale social developments and differences in the specific historical and cultural backgrounds of countries have shown to be of particular value to explain and clarify the current modalities of home nursing in different European countries.

However, some refining of the original assumptions of Abbott (I988) seems to be in place. Abbott considers competition over tasks and contests over jurisdictions in the network of professions of central importance to professions. They are supposed to continuously strive for control over and recognition of their jurisdictional domain in front of other professions, the state and the public. This may certainly be the case in some professional fields, for example in eye care in the Netherlands (Stevens, Diederiks et al., 2007). However, this was not found for the home nursing profession in this book. In the countries studied, a clear home nursing domain could be discerned, focussing on providing nursing in the homes of vulnerable and (very) elderly people. Interprofessional relations and ways of co-operation were more important in some countries, especially the Netherlands, than in other countries. Active contests over jurisdictions and tasks only seem to play a minor role in home nursing. More central to this book is the way that home nursing has gradually been shaped and is currently still shaped by social and historical circumstances, the development of particular health care structures, and cultural and political views on the role of professionals and the family to care for the vulnerable. Bargaining over tasks and interprofessional competition between different health care professionals, managers and family members exists, but especially on the micro level between those working together in daily practice and not so much in the form of collective action of a united profession. More specifically, differences in the role and institutionalisation of home nursing between countries were not so much the result of interprofessional struggles over jurisdictional claims, but largely determined by contextual factors, such as the characteristics of health care systems, reimbursement structures, the bal- 
ance between cure and care, socio-cultural views on the role of professionals and the family in caring for elderly people, and the priority given to elderly policies by central states. Therefore, on the basis of the outcomes of this study can be concluded that interprofessional contests over jurisdictions are not always central to professions. I would conclude that country-specific constellations in which a profession is embedded shape its modalities.

More recently, Abbott refined his views on the social world surrounding professions by adding the concept of 'linked ecologies' (Abbott, 2004, 2005). This concept is more in line with the assumptions adhered to in this thesis. In I988, Abbott considered professions as fluent and contingent and shaped by interprofessional relations. The state and the public were still considered as quite fixed entities external to the system or ecology of the professions. However, these are now displayed as ecologies as well. Thus, for example states are no longer considered as a static force or fixed power block where professions can ask for legitimation of their claims, but as contingent and fluent entities as well. Also within states, subgroups and divisions exist and power relations change. Thus instead of only considering professions as fluent and contingent according to time, place and relations with others, also the ecologies surrounding professions are now considered as fluent and changing. The social world is now being perceived as existing of sets of linked ecologies that each act as a (flexible) environment for other ecologies. The conceptualisation of linked ecologies thereby adds a further layer of contingency as this particular environment can encourage or discourage the development of professional domains, in this case the home nursing domain.

The analysis of the domain and profession of home nursing in this book was performed on the basis of the comparative and contextual theoretical framework that was developed in chapter two. One can say that in this framework, the concept of linked ecologies was already included. After all, the different aspects included in the framework were not considered as fixed, but as country-specific. For example, the abstract concept of 'health care system' was characterised specifically for each country, showing that health care systems differ from country to country. Also the later added concept of the role of the central state was described for each country specifically. For example in Denmark, the welfare state formulates comprehensive public responsibilities and guarantees that care needs are provided for on the local level. In contrast, in France the state-periphery framework still plays a large role, meaning that facilities are often provided for in urbanised areas and especially in the Paris region, but the farther one travels from these urban centres, the more scarce these facilities become. Thus, states can also be considered as specific constellations having different characteristics in each country. All the aspects included in the theoretical framework can be considered as constellations or ecologies in themselves that are linked together in different ways. Thus, the outcomes of this study resulted in country-specific constellations of linked ecologies that were in 
some countries rather favourable to the development of home nursing, such as in Denmark and to a somewhat lesser extent in the Netherlands, and in other countries constellations that comprise barriers for the development of the home nursing sector and prevent many vulnerable and dependent clients from receiving the home care needed. The latter is the case in France and Germany. Thus, the framework of a contextual perspective has appeared to be useful in discerning essential differences in the characteristics of home nursing across Europe. It has been shown that societies are complex wholes of societal subsystems. The exact way in which these are related is too complex to unravel from the macro sociological point of view that was taken in this thesis and requires more detailed analysis, but the theoretical framework offered sufficient opportunities to depict the richness of the country-specific constellations of which home nursing is a part. The framework also provided backgrounds to explain the differences between the modalities of home nursing in each country and discern different degrees of institutionalisation of the home nursing domain.

\subsubsection{Implications for policy and future research}

A broad view on home nursing as a profession and as a societal sector as described in this book has shown that no simple conclusions can be drawn regarding the question what kind of future home care policies for elderly and vulnerable people should be developed. The four countries studied share many basic characteristics, such as parliamentary democracy, the existence of health care systems, welfare arrangements to provide for the needs of the poor and the vulnerable, EU membership, and the double ageing of their populations. However, these similarities do not predict the way that societies will deal with the current and future shift in care needs of elderly and vulnerable people. A closer look at these countries from the theoretical perspectives revealed that the socio-cultural background, the structure and way of financing of the health care system, and the constellations of care that have developed over many decades or even centuries all influence the perspectives in health policies and societies about the most adequate way to provide elderly care. The most important difficulty that all European societies have dealt with for a long time and will even more do so in the near future, is the affordability of their health care systems and how to sufficiently provide for the increasing demand for long-term elderly care facilities. A general trend is that European countries aim to substitute institutional care by community care and enable elderly people to remain living in their own homes or in adapted homes when possible, supported by ambulant home care facilities and informal care. The reason for this convergence within Europe is that common agreement exists that institution-based care is more expensive than community care, and that people should participate in social life as long as possible. However, the pace of substitution differs per country 
and some countries are more radical in this, for example Denmark, than others, such as France and Germany. This study shows that substitution is only possible and acceptable when sufficient alternative facilities in the form of ambulant home care are available. However, general accessibility is not guaranteed in France and Germany, where shortages of services and staff exist in several areas.

The following recommendations are proposed to improve home nursing and home care in the four countries studied. In France, although the home care domain has increased considerably in size during the last decades, home care is still not available in all regions. The home care domain should be further developed to guarantee a basic and appropriate level of community care across France. Also, the targeting of services needs to be improved, as the results showed that people who do not need care do receive it, while others with serious health problems remain deprived of care. Assessment of the care needs of elderly people and adequate availability of care should be improved in France. The current increase of voluntary care networks, developed by local municipalities since the severe heat waves some years ago, can be considered as an enormous improvement to monitor the health and social needs of very elderly and vulnerably people. However, this should be supported by adequate and sufficient professional home care services to provide for the care needs of these people. In France, the role of the family to care for their relatives is emphasised heavily by the government, that blames family members that are unable to provide informal care. Also, a cultural stigma exists regarding professional care that inhibits people from applying from home care, because it would indicate that they are not properly cared for by their family members. Less emphasis on the role of the family in health policies should stimulate a more open attitude of the population regarding professional caregiving and decrease the reluctancy of people to call in professional support when needed. Health policies in France should therefore seek a better balance between professional and informal care, because the burden and guilt placed on the family is currently too high and disguises the insufficient availability of home care sercives. Policies should therefore aim at general accessibility and availability of professional home in all regions, to compensate for those situations where the family is unable to care for their vulnerable family members. Together with the complex regulations for reimbursement, these tendencies prohibit the home care domain to develop and increase further in size to provide for the needs of elderly people. Regulations should be made less complex so that it would become easier for people to arrange ambulant care instead of institutionalise elderly people into a residential home or nursing home. The important role of independent nurses alongside the regular home care domain deserves further study.

In Germany, elderly care and the interests of elderly people in general should be put higher on the policy agenda. To ensure adequate care, the image of elderly people as a burden to their family and society in general should be diminished 
and more and better quality arrangements should be developed. Due to the very high numbers of elderly people in Germany (I8.6\% of the population is over 65 years of age, which is more than I3 million people), this cannot be left to the current fragmented way in which elderly care is provided in Germany. The princple of subsidiarity that underpins care provision already appears to be insufficient now to ensure adequate care to people in need of home care, while the demand for care will only increase in the near future. A stronger role of the central or federal governments is desirable in extending existing health care arrangements and making home care more accessible, so that more elderly people become entitled to home care. Now, only those with very high needs receive care, while others cannot afford it or are a heavy burden for their family. The current emphasis on medical and institutional care in Germany should be shifted to a higher priority of community care. Also, efforts should be undertaken to increase the social status of the caring professions and to improve and specialise the training of home care professionals to solve the current staffing problems and increase the quality of care.

In the Netherlands, home care has been of a very high standard for a very long time. Although provision is not public, such as in Denmark but private in the Netherlands, the cross organisations have succeeded in establishing a dense network of services across the country. The home nurse was considered as a local authority and her autonomy was large. Her allround profile foresaw in many needs of the families in her neighbourhood. However, since two or three decades, developments in society and health care affected the character of the home nursing profession. Budgetary measures, increased bureacracy, the introduction of many protocols and regulations, the emergence of other organs performing the indication procedure, and recently the introduction of the wmo, affected the professionalism of home nursing and resulted in many problems for this domain that are currently highly debated in the Netherlands and that render the home nursing profession far less attractive as a career. The developments in the Netherlands are not crystallised yet, which makes further research into the changes in this domain important. Recommendations that can be offered from the viewpoint of this research are that bureaucracy en protocolisation in home care practices should be reduced, and more leeway should be offered for independent decisionmaking by home nurses on the basis of their professional expertise. Also, assessment of the care needs and decision over the entitlement to care of clients should be made the responsibility of home nurses again. Their insight in the client's situation, based on their training and experiences is essential to provide adeqaute care of a high standard. Then they can also more easily adapt the care to changing care needs of their clients, which is difficult under the current bureaucratic way in which care needs are established. The government should reconsider the recent changes that took the indication out of the hands of the home nurses and re-estabish the degree of professionalism and autonomy that home nurses traditionally had in the Netherlands. This 
would make the home nursing profession more attractive as a career and would offer better opportunities to adequately provide for the needs of elderly people in the Netherlands.

The results of this book point to a quite favorable situation in home care in Denmark, especially when compared to other European countries. Danish home care is of a very high standard and a wide range of services is provided by the local municipalities to a relatively large number of elderly people. A major drawback of the current system is however its affordability. By alleviating family members from care to an extent that is unthinkable in the the Netherlands and even more so in Germany and France, the Danes pay a very high price in the form of high taxes to finance these extensive regulations. The role of family solidarity and the balance between professional care and informal care need further study. Considering these aspects, Denmark and France are direct opposites. Also, the role of managers and possible interference of bureaucracy in home care, that are striking in the Netherlands, have not accurately been revealed yet in Denmark. Nevertheless, health policies for elderly people that emphasis their growing old in the community, the general accessibility and affordibility of home care services by clients and the wide range of facilities that exist to support the vulnerable in Denmark may serve as examples for other European countries. 


\section{References}

(2005). BIG register. Beroepen in de Individuele Gezondheidszorg, www.bigregister.nl.

Abbott, A. (I98I). Status and Status Strain in the Professions. American Journal of Sociology 86(4): 819-835.

Abbott, A. (1988a). The System of Professions. An Essay on the Division of Expert Labor. Chicago/London: The University of Chicago Press.

(I988b). Introduction, pp. I-32.

(I988c). I - Work, Jurisdiction, and Competition. Chapter 2 : Professional Work, pp. 3358 .

(I988d). I - Work, Jurisdiction, and Competition. Chapter 3: The Claim of Jurisdiction, pp. $59-85$.

(I988e). I - Work, Jurisdiction, and Competition. Chapter 4: The System of Professions, pp. 86-II3.

(I988g). II - The systems Environment. Chapter 6: The Social Environment of Professional Development, pp. I43-I76.

(I988h). II - The systems Environment. Chapter 7: The Cultural Environment of Professional Development, pp. I77-2II.

(I988i). III - Three Case Studies. Chapter 8: The Information Professions, pp. 2I3246.

(I988j). III - Three Case Studies. Chapter 9: Lawyers and their Competitors, pp. 247279 .

(I988k). II - Three Case Studies. Chapter ıo: The Construction of the Personal Problems Jurisdiction, pp. 280-3I4.

(I9881). III - Three Case Studies. Conclusion, pp. 315-326.

Abbott, A. (1989). The New Occupational Structure. Work and Occupations 16(3): 273-29I.

Abbott, A. (I99I). The Order of Professionalization. An empirical Analysis. Work and Occupations 18(4): 355-384.

Abbott, A. (2004). Linked ecologies: State, Market and University as Environments for the Professions. Paper presented 22. September 2004 at the Fourth Interim Conference of the International Sociological Association "Knowledge Work and Organization" of the Research Committee 52 'Sociology of Professional Groups', University of Versailles, France.

Abbott, A. (2005). Linked ecologies: States and Universities as Environments for Professions. Sociological Theory 23(3): 245-247.

Abbott, P. and C. Wallace, Eds. (I990). The Sociology of the Caring Professions. London, The Falmer Press. 
Achterberg, T. v. (I997). Continuity of Care and Client Satisfaction in the Community. A Study of Professional and Non-Professional Care for the Chronically Ill. Thesis department of Medical Sociology. Maastricht, Maastricht University.

Adessa (2004). Vivre chez soi, www.federation-adessa.org/.

Adriaansen, M. and B. v. d. Laan (I994C). Kruisgewijs door de historie. In: Specifieke verpleegkunde: de wijkverpleging en thuiszorg. Bouwstenen voor verpleegkundig onderwijs. Houten: Bohn Stafleu Van Loghum, pp. 6-I2.

(I994d). De organisatie van het kruiswerk, pp. 13-26.

Adriaansens, H.P.M. and A.C. Zijderveld (I98I). Vrijwillig initiatief en de verzorgingsstaat: cultuursociologische analyse van een beleidsprobleem. Deventer: Van Loghum Slaterus.

Aiken, L.H. and D.M. Sloane (1997). Effects of Specialization and Client Differentiation on the Status of Nurses: The Case of AIDs. Journal of Health and Social Behavior 38(3): 203-222.

Alaszewski, A. (1995). Restructuring Health and welfare professions in the United Kingdom: the impact of internal markets on the medical, nursing and social work professions. In: Health Professions and the State in Europe. J. Johnson, G. Larkin and M. Saks (eds). London: Routledge, pp. 55-74.

Alber, J. (1996). The debate about long-term care reform in Germany. In: Caring for frail elderly people. Policies in evolution. OECD. Paris: OECD, pp. 26I-278.

Allen, D. (I997). The Nursing-medical Boundary. a negotiated Order? Sociology of Health and Illness 19(4): 498-520.

Ancenis (2007). Le C.C.A.s. et ses interventions, Mairie d'Ancenis (municipality of Ancenis). Retrieved I4-08-2007 from www.ancenis.fr/content.cfm?id=I88

Arend, A.J.G. v. d. (I992). Hoofdstuk 2: Professie en professionalisering: uitgangspunten en ontwikkelingen. In: Beroepscodes. Morele kanttekeningen bij een professionaliseringsaspect van de verpleging. Maastricht: Datawyse, pp. I9-57.

Artot, D. (I989). Hospitalization at home; the French system. In: Community nursing. Proceedings of the International Conference on Community Nursing, 16-17 March 1989. A. Kerkstra and R. Verheij (eds). Utrecht: Nivel, pp. 32-35.

Aublet-Cuvelier, B., P. Barberger-Gateau, et al. (I99I). Etude de l'adéquation des services d'aide-ménagère en Gironde à partir du programme PAQUID (Adequacy of homemaker services in Gironde according to the PAQUID program). Revue d'epidemiologie et de santé publique 39(2): I2I-I28.

Balslev Wingender, N. (1999). The Four-leaf Clover and the Fiery Souls. This history of the Danish Nurses' Organization 1899-1999. Copenhagen: Aarhuus Stiftsbogtrykkerie.

Bellanger, M. (2004). Chapter 4: Modernisation instead of privatisation in French health care. In: Privatisation in European health care. A comparative analysis in eight countries. H. Maarse (ed). Maarssen: Elsevier gezondheidszorg, pp. 63-77.

Benson, J.K. (I973). The Analysis of Bureaucratic-Professional Conflict: Functional versus Dialectical Approaches. The Sociological Quarterly 14(Summer): 376-394.

Benson, J.K. (I975). The Interorganizational Network as a Political Economy. Administrative Science Quarterly 20: 229-249.

Berg, M., K. Horstman, et al. (2000). Guidelines, professionals and the production of objectivity: standardisation and the professionalsim of insurance medicine. Sociology of Health Q Ilness 22(6): 765-79I. 
Berg, M. v. d., D.H. d. Bakker, et al. (2003). De werkdruk van de huisarts. Zorgvraag en arbeidsduur in 1987 en in 2001. Medisch Contact 58(26/27): 1054-1056.

Bertels, J. and W. Cocquyt (I995a). De sociale gezondheidszorgstelsels in de landen van de Europese Unie. Antwerpen/Apeldoorn: Maklu.

Bie, R.A. de. (2003). Natuurlijk Fysiotherapie. Inaugurele rede. Maastricht, Universitaire Pers Maastricht.

Bijsterveld, K. (I996). Ommuurde ouderdom: de ruimtelijke orde van verzorgingshuizen. In: Geen kwestie van leeftijd. Verzorgingstaat, wetenschap en discussies rond ouderen in Nederlands, 1945-1982. Amsterdam: Van Gennep, pp. I57-220.

Blaauwbroek, H.G. (I997). Chapter 22. Patient Organizations and Patients' Rights. In: Health and Health Care in the Netherlands. A critical Self-assessment by Dutch Experts in the Medical and Health Sciences. A.J.P. Schrijvers (ed). Maarssen: De Tijdstroom, pp. 223237.

Blau, P.M. (1967-68). The Hierarchy of Authority in Organisations. American Journal of Sociology: 453-467.

Boll Hansen, E. and M. Platz (I99I). Fra plejehjem til hjemmepleje. København, Amternes og Kommunernes Forskningsinstitut (The Counties and Municipalities Research Institute).

Boom, H.C.I. van der (1997). De geschiedenis van het ziekenhuiswezen in Nederland (19001940). Het ontstaan van het moderne ziekenhuis vanuit sociaal constructivistisch perspectief [The history of the hospital system in the Netherlands (I900-I940). A social constructivist perspective on the development of the modern hospital]. Afstudeerscriptie Cultuuren Wetenschapsstudies, Universiteit Maastricht.

Boom, H.C.I. van der, F.C.J. Stevens, H. Philipsen (2000). Cross-cultural comparison on the Institutionalisation and Professionalisation of Home Care in Six European Countries. Paper presented at the Interim Conference of the Research Committee RC 52 'Sociology of Professional Groups' of the International Sociological Association, titled "State Political Power and Professional Structures: New Patterns and New Challenges?" Lisbon, Portugal.

Boom, H.C.I. van der, F.C.J. Stevens, H. Philipsen (200I). Dilemmas and difficulties in home nursing; theoretical perspectives. Paper presented at the Fifth Conference of the European Sociological Association "Visions and divisions. Challenges to European Sociology", Helsinki, Finland.

Boom, H.C.I. van der, F.C.J. Stevens, H. Philipsen. (2004a). Caring for frail elderly people: the triad of home nursing, home help and family care in Denmark, the Netherlands and France. Paper presented at the Fourth Interim Conference of the International Sociological Association "Knowledge Work and Organization", University of Versailles, France.

Boom, H. van der, H. Philipsen, F. Stevens (2004b). Een schets van de professionalisering van de wijkvperleging in Nederland in de laatste vijftig jaar (I950-2004). Wijkverpleging - een uniek Nederlands verschijnsel: de autonomie van de wijkverpleegkundige. Gewina 27 Themanummer Zorgen als Professie: Ioo-II9.

Børgsen, I.-M. (2002). Interview with the manageress of the Dansk Sygeplejehistorisk Museum (Danish Museum of Nursing History), Kolding, Denmark.

Boven, P.F. van (RIVM) (2002). Consultatiebureaus per gemeente I999. In: Volksgezondheid Toekomst Verkenning, Nationale Atlas Volksgezondheid. Bilthoven: RIVM, retrieved 22-042003 from www.rivm.nl/vtv/data/atlas/opgz_okez_aoor.htm. 
Brannon, R.L. (I994). Professionalization and Work Identification. Nursing in the Cost Containment Era. Work and Occupations 21(2): 157-I78.

Brint, S. (I993). Eliot Freidson's Contribution to the Sociology of the Professions. Work and Occupations 20(3): 259-278.

Britannica (1992). Encyclopaedia Britannica. Chicago.

Brok, Y. de (200I). Werken met PGB-houders. Tijdschrift voor Verpleegkundigen 17(606-608).

Bundesministerium für Arbeit und Sozialordnung (1997). Arbeit und Sozialordnung. Pressemitteilung. Fakten zur Pflegeversicherung. Retrieved 25-10-2000 from www.bma. bund.de/alt.bmahome/aktuelle/aktuelle2/pressi67.html,.

Burau, V. (I999b). Occupational governance and the dynamics of change-a comparative analysis of nursing in Britain and Germany. Thesis University of Edinburgh.

Burau, V. (2000). Comparing professions - theoretical and methodological considerations. The first International Conference of the Research Network "Sociology of Professions" of the European Sociological Association, Jesi, Italy.

Busse, R. and A. Riesberg (2004). Health Care Systems in Transition - Germany. In: S. Thomson and E. Mossialos (eds), European Observatory on Health Care Systems, http://www. observatory.dk.

Caritas Soziale Berufe (2004a). Gesundheits- und Krankenpfleger(in). Retrieved I9-072004 from www.caritas-soziale-berufe.de/8263.html.

Caritas Soziale Berufe (2004b). Altenpfleger(in). Retrieved 19-07-2004 from www.caritassoziale-berufe.de/8263.html.

Caritas Soziale Berufe (2004c). Dorfhelfer(in). Retrieved 19-07-2004 from www.caritassoziale-berufe.de/8263.html.

Cars, O., S. Moelstad, A. Melander (200I). Variation in antibiotic use in the European Union. The Lancet 357(June 9): I85I-I853.

Champarnaud, J. (I996). Le projet santé de l'ADMr. Une approche globale de la santé [Home help. The health project of the Rural Home Help. A global approach to health]. Soins 160 (Nov): 2I-22.

Christiansen, T., U. Enemark, et al. (I999). Health Care and Cost Containment in Denmark. In: Health Care and Cost Containment in the European Union. E. Mossialos and J. Le Grand (eds). Aldershot: Ashgate Publishing Ltd, pp. 267-30I.

Clément, S. (I993). Des enfants pour la vieilesse. Forme de la fratrie et soutien aux parents agés dans le dus-ouest rural francais (Children for ageing. Sibling configuration and support to old parents in the rural south-western area of France). Social Science and Medicine 37(2): I39-I5I.

Colvez, A. and S. Ridez (I996). Déterminants objectifs en subjectifs du bénéfice de l'aide ménagère pour le maintien à domicile des personnes agées (Objective and subjective determinants in the benefits of home help services for the elderly. Revue d'epidemiologie et de santé publique 44(2): I63-I72.

Com-Ruelle, L., F. Midy, et al. (2000). La profession infirmière en mutation. Eléments de réflexion à partir d'exemples européens. Paris: CREDEs, Centre de Recherche d'Etude et de Documentation en Economie de la Santé.

Courtens, A.M. (I993). Kenmerken van zorg en kwaliteit van leven bij patiënten met kanker. Thesis Medical Sociology. Maastricht: Rijksuniversiteit Limburg.

Cox, K., S. Niemantsverdriet, et al. (1994). Discontinuiteiten in de thuiszorg: aantasting van de kwaliteit van zorg? Overzichtstudie Onderzoekprogramma Kwaliteit van Zorg. Den 
Haag: Nwo/wvc, Vakgroep medische sociologie en Verplegingswetenschap Rijksuniversiteit Limburg, Nivel.

CREDES (I998). Bulletin d'information en économie de la santé. Resultats. In: Les auxiliaires médicaux. Déterminants de la consommation médicale de ville en 1992. L. Com-Ruelle and S. Dumesnil (eds). Paris: CRedes, Centre de Rechrche d'Etude et de Documentation en Economie de la Sante. n. 4 - Janvier I998.

CREDES (2000). Bulletin d'information en économie de la santé. Analyses. In: La profession infirmière en mutation. Eléments de réflexion à partir d'exemples européens. L. Com-Ruelle, F. Midy and P. Ulmann (eds). Paris: Credes, Centre de Rechrche d'Etude et de Documentation en Economie de la Sante. n. 33 - Octobre 2000.

CREDES (2002). Bulletin d'information en économie de la santé. Analyses. In: Linfirmier libéral et la coordination avec les services d'aide au maintien à domicile. L. Com-Ruelle, P. Dourgnon and F. Midy (eds). Paris: Credes, Centre de Rechrche d'Etude et de Documentation en Economie de la Sante. n. 55 - Aout 2002.

Creswell, J.W. (2003). Research design. Qualitative, Quantitative, and Mixed Methods Approaches. London: Sage.

Curtis, S. and D. Bucquet (I987). Characteristics of elderly people receiving home-help in three regions of France. Revue d'epidemiologie et de santé publique 35(3-4): 318-322.

Dansk Sygeplejehistorisk Museum, (2004a). Picture of a Danish home nurse - from the exposition in the Museum. Retrieved 22-03-2004 from www.odensea.dk/dansk_sygeplejehistorisk_museum.htm.

Dansk Sygeplejeråd, D. (2004b). Danish Nurses Organization. Retrieved 05-03-2004 from www.sygeplejersken.dk/msite/msite.asp?id=ıo8.

Dautzenberg, M. (2000). Daughters caring for elderly parents. Thesis Medical Sociology. Maastricht, Universiteit Maastricht.

Davies, C. (I983). Professionals in bureaucracies: the conflict thesis revisited. In: The sociology of the professions. R. Dingwall and P. Lewis (eds). London: MacMillan Press, pp. I77-I94.

Davies, C. (I995a). Gender and the Professional Predicament in Nursing. Buckingham: Open University Press. (I995b). Professionalism and the Conundrum of Care, pp. I33-I54.

Delaunay, M., R. Teletchea, et al. (I996). Accompagner à domicile. Les acteurs de l'hospitalisation à domicile. Soins. Soins-la-revue-de-reference-infirmière Gog(Octobre): I3-I9.

Delnoij, D.M.J. (I996a). Home Care in Germany. In: Home Care in Europe. A Countryspecific Guide to its Organizations and Financing. J.B.F. Hutten and A. Kerkstra (eds). Aldershot: Arena, pp. I3I-I49.

Dent, M. (I993). Professionalism, educated labour and the state: hospital medicine and the new managerialism. Sociological Review 41(2): 244-73.

Derber, C. (I982). Professionals as Workers: Mental Labor in Advanced Capitalism. Boston: G.K. Hall \& Co.

Derksen, A.M.M. (I991а). Thuisverpleging in de Euregio. Overeenkomsten en verschillen. Doctoraalscriptie Gezondheidswetenschappen Maastricht, Rijksuniversiteit Limburg.

Derksen, A.M.M., J. van der Zee, F.C.J. Stevens. (I991b). Thuisverpleging in de Euregio. De eerste lijn in Limburg. Nu en straks. Verslag van het Lustrumcongres van de Rijksuniversiteit Limburg, Io oktober I991, Stein: Huntjens. 
Deschepper, R., R.H. v. Stichele, F.M. Haaijer-Ruskamp (2002). Cross-cultural differences in lay attitudes and utilisation of antibiotics in a Belgian and a Dutch city. Patient Education and Counselling 48: I6I-I69.

Diakonisches Werk der EKD (2004a). Menschen pflegen. Krankenschwester, Krankenpfleger. Retrieved I3-07-2004 from www.diakonie.de/de/html/fachforum/ı189_I20I.html.

Diakonisches Werk der EKD (2004b). Menschen pflegen. Altenpfleger/-in. Retrieved I6-072004 from www.diakonie.de/de/html/fachforum/ı189_ı20ı.html.

Dieck, M. (I99I). Home Care Services in the Federal Republic of Germany. In: Home Care for Older People in Europe. A. Jamieson (ed). Oxford: Oxford University Press, pp. II8I3O.

Diederiks, J.P.M., F.C.J. Stevens, J. van der Zee. (I99I). Medische consumptie en gezondheidsopvattingen in de Euregio. De eerste lijn in Limburg. Nu en straks. Verslag van het Lustrumcongres van de Rijksuniversiteit Limburg, Io oktober I99I, Stein: Huntjens.

Donker, M.C.H. (I997). 20. Professionaliteit of bureaucratie? Het nut van indicatieprotocollen. In: Vraaggestuurde zorg: dilemma's en nieuwe opties bij management, primair zorgproces, netwerken, automatisering, flexibele zorgprodukten, indicatiestelling. L. Boon (ed). Amstelveen: Stichting Sympoz, Platform voor ontwikkelingen in de gezondheidszorg, 24, pp. I08-II4.

Douste-Blazy, P. and H. Falco (2004). Plan canicule. Dossier de présentation. Paris, Ministère de la Santé et de la protection sociale. Retrieved I4-08-2007 from www.sante.gouv.fr/canicule/doc/dossier_presentation.pdf.

Drouet, M. (I995). Histoire de la profession d'infirmière et de la fonction d'aide-soignant. Revue-de-linfirmière 5(Mar): 64-65.

Dumons, B. and G. Pollet (I999). Healthcare. In: Modern France. Society in transition. M. Cook and G. Davie (eds). London: Routledge, pp. 216-234.

Eco-Santé (2003). Démographie, WWW.CREDES.fr.

Editor (2005). Été: Alès réactive son réseau de voisins-tuteurs. La service Solidarité du CCAs est à pied dóeuvre depuis plusieurs mois pour prémunir les personnes agées et isolées contre les effets d'une éventuelle canicule. Santé: 5 .

Elsevier (2007). Eerste thuiszorgbureau voor moslims, Elsevier gezondheidszorg. Retrieved 23-08-2007 from http://nieuws.elseviergezondheidszorg.nl/7I5I-eerste-thuiszorg bureau-voor-moslims.html.

Esping-Andersen, G. (I990). The Three Worlds of Welfare Capitalism. Cambridge: Polity Press.

European Communities and wно (1997). Highlights on Health in Denmark. Copenhagen, wH regional Office for Europe: European Commission.

European Observatory on Health Care Systems (2007a). Health Care Systems in Transition - France. European Observatory on Health Care Systems \& wHo Regional Office for Europe. Retrieved 02-0I-2007 from www.euro.who.int/document/e83126sum.pdf.

European Observatory on Health Care Systems (2007b). Health Care Systems in Transition - Denmark. European Observatory on Health Care Systems \& wно Regional Office for Europe. Retrieved 02-0I-2007 from www.euro.who.int/document/e83I26sum.pdf.

European Observatory on Health Care Systems (2007c). Health Care Systems in Transition - Germany. European Observatory on Health Care Systems \& wHo Regional Office for Europe. Retrieved 02-OI-2007 from www.euro.who.int/Document/E85472sum.pdf. 
European Observatory on Health Care Systems (2007d). Health Care Systems in Transition - the Netherlands. European Observatory on Health Care Systems \& w Ho Regional Office for Europe. Retrieved 02-OI-2007 from www.euro.who.int/Document/E84949sum. pdf.

Eurostat (1999). Demographic statistics. Data 1960-99. Theme 3: Population and social conditions. Luxembourg: Office for Official Publications of the European Communities.

Eurostat (200Ia). Eurostat Yearbook. The statistical guide to Europe. Data 1989-1999. Theme 1: general statistics. Luxembourg: Office for Official Publications of the European Communities.

Eurostat (2005). Europe in figures. Eurostat Yearbook 2005. Panorama of the European Union. Part 2: Population. Luxembourg: Eurostat, European Commission, theme General and regional statistics - Office for Official Publications of the European Communities, pp. 6I-I05.

Eurostat (2007a). Europe in figures. Eurostat Yearbook 2005. Panorama of the European Union. Tables. Luxembourg: Office for Official Publications of the European Communities. Retrieved 02-OI-2007 from http://epp.eurostat.ec.europa.eu/portal/page?_pageid=I33 4,49092079,I334_49092794\&_dad=portal\&_schema=PORTAL.

Eurostat (2007b). Chapter Health - Public Health. Luxembourg: Office for Official Publications of the European Communities. Retrieved 02-0I-2007 from http://epp.eurostat. ec.europa.eu/portal/page?_pageid=I996,45323734\&_dad=portal\&_schema=PORTAL\& screen=welcomeref\&open=/health/hlth/hlth_care/hlth_facil\&language=en\&product= EU_MASTER_health\&root=EU_MASTER_health\&scrollto=310.

Evers, A. (I995). Die Pflegeversicherung. Ein mixtum compositum im Prozeß der politischen Umsetzung. Sozialer Fortschritt 44(2): 23-28.

Evers, A. and I. Svetlik, Eds. (I99I). New Welfare mixes in care for the elderly. Eurosocial Reports vol. 40. Vienna: European Centre for Social Welfare Policy and Research.

Exter, A. den, H. Hermans, Dosljak, M., R. Busse (2004). Health Care Systems in Transition - Netherlands. S. Thomson and E. Mossialos (eds), European Observatory on Health Care Systems. Retrieved from www.observatory.dk.

Fнмд (2006). Visiedocument Faculty of Health, Medicine and Life Sciences, 28 maart 2006. Maastricht: Faculty of Health, Medicine and Life Sciences, Maastricht University.

Fнмд (2008). Programme Information Faculty of Health, Medicine and Life Sciences. Maastricht University. Retrieved 07-03-2008 from www.unimaas.nl.

Foets, M., F. v. d. Lucht, et al. (2005). Zijn er verschillen naar etniciteit? Zorg zonder verblijf - Marokkanen maken minder gebruik van thuiszorg. In: Volksgezondheid Toekomst Verkenning, Nationaal Kompas Volksgezondheid. Bilthoven: RIVM. Retrieved 23-08-2007 from www.rivm.nl/vtv/object_document/o2668n20327.html.

Fox, R.C. (1989). Chapter 2. The Professions of Medicine and Nursing. In: The Sociology of Medicine. A Participant Observer's View. Prentice Hall, pp. 38-7I.

Freidson, E. (I970a). Profession of Medicine. A Study of the Sociology of Applied Knowledge. New York: Dodd, Mead \& Company.

Freidson, E. (1983). The reorganization of the professions by regulation. Law and Human Behaviour(7): 279-290.

Freidson, E. (I986a). Professional Powers: a Study of the Institutionalization of Formal Knowledge. Chicago: University of Chicago Press. 
Galès, P.L. (I999). The regions. In: Modern France. Society in transition. M. Cook and G. Davie (eds). London: Routledge, pp. 9I-III.

Geertz, C. (1973). Chapter I: Thick description: Toward an Interpretive Theory of Culture. In: The Interpretation of cultures. New York: Basic Books, pp. 4-30.

Giarchi, G.G. (I996a). Caring for Older Europeans. Comparative studies in 29 countries. Arena Ashgate Publishing Ltd, Aldershot.

(I996b). Introduction: Setting the Scene, pp. I-37.

(I996g). France, pp. I93-2II.

(I996h). Germany, pp. 249-270.

Giesbers, H. (RIVM) (2002a). Reguliere thuiszorginstellingen I999. In: Volksgezondheid Toekomst Verkenning, Nationale Atlas Volksgezondheid. Bilthoven, RIVM.

$(2002 \mathrm{~b})$. Uren thuiszorg in relatie tot gewogen thuiswonenden.

(2002c). Uitleg berekening behoeftecriterium.

(2002d). Groei particuliere thuiszorgbureaus.

Giesbers, H. (2005). Locaties Centrum Indicatiestelling Zorg 2005. In: Volksgezondheid Toekomst Verkenning, Nationale Atlas Volksgezondheid. Bilthoven: RIVM.

Gilles, W., K. Kesteloot, Graeve, D. van, M. Jegers (200I). Zorgbekostigingssystemen in Europa. Acta Hospitalia 41: 5-II.

Goelabdien, R. (I996). Van roeping tot beroep. Verpleging en verzorging in historisch perspectief. Utrecht: De Tijdstroom.

Gradignan, Municipality of (2007). Plan canicule 2007 : quelques conseils pour supporter les grosses chaleurs. Retrieved I5-08-2007 from www.mairie-gradignan.com/index. php?id=392.

Greiner, W. and J.-M. Graf v.d. Schulenburg (I997). The Health System of Germany. In: Health Care and Reform in Industrialized Countries. M.W. Raffel(ed). Pennsylvania: Pennsylvania State University Press, pp. 77-IO4.

Gruyter, C. de (2003). De patiënt vraagt, de dokter schrijft voor. Bacteriën kosten elk jaar 2.800 levens in Belgische ziekenhuizen. NRC Handelsblad 30 juni.

Grypdonck, M. (2005). Zorg is geen product. In: Caleidoscoop van de thuiszorg. Met wenken en wensen voor de toekomst. J. Bastiaenen and C. Giezenaar (eds). Bunnik: Stichting Onderzoek en Ontwikkeling Maatschappelijke gezondheidszorg sтоом, pp. 42-46.

Habermas, J. (198I). Theorie des Kommunikativen Handelns. Frankfurt: Suhrkamp.

Hafferty, F.W. and J.B. McKinlay (Eds) (I993a). The Changing Medical Profession. Oxford: Oxford University Press.

Harismendy, P. (I999). Age: the life course. In: Modern France. Society in transition. M. Cook and G. Davie (eds). London: Routledge, pp. 73-90.

Hasenfeld, Y. (1983a). Human Service Organizations. London: Prentice Hall. (1983b). Chapter 5: Organizational Technology.

Hasenfeld, Y. (I992a). Human Services as Complex Organizations. London: Sage.

Henrard, J.C. (I996). Cultural problems of ageing especially regarding gender and intergenerational equity. Social Science and Medicine 43(5): 667-680.

Henrard, J.-C., J. Ankri, M-C. Isnard. (I99I). Home Care Services in France. In: Home Care for Older People in Europe. A. Jamieson (ed). Oxford: Oxford University Press, pp. 99II7. 
Henrard, J.-C., B. Cassou, D. le Disert (I990). The Effects of System Characteristics on Policy Implementation and Functioning of Care for the Elderly in France. International Journal of Health Services 20(I): I25-139.

Hermans, E. (2000). Hoofdstuk 8: beschouwing. In: In het verkeerde bed. Het zorgtraject van een cohort CVA-patiënten na ziekenhuisopname. Thesis Huisartsgeneeskunde. Maastricht: Datawyse, pp. III-I22.

Hofstede, G. (I980a). Culture's Consequences. London: Sage. (I980e). Chapter 6; Masculinity, pp 26I-298.

Horstman, K. (2004). Managers en uitvoerenden mogen geen neutrale vehikels zijn van overheidsbeleid. Nederlands tijdschrift voor jeugdzorg 8: I43-I49. Eerder gepresenteerd als lezing "De taal van publieke verantwoording: vertrouwen in standaarden of in pluraliteit” Lezing Jeugdzorg N-Brabant, I6-OI-2004.

Hutten, J.B.F. and A. Kerkstra (Eds). (I996a). Home Care in Europe. A Country-specific Guide to its Organizations and Financing. Aldershot, Arena.

IFSI (2003). Historique de la profession. Retrieved July 2003 from www.infirmiers.com/infirmiers/historique.php4.

Indenrigsministeriet (1998). Indenrigsministeriets kommunale nøgletal 1998. København: Indenrigsministeriet.

Jamieson, A. (I99IC). Home-Care Provision and Allocation. In: Home Care for Older People in Europe. Oxford: Oxford University Press, pp. 239-272.

Jamieson, A. (I996). Issues in Home Care Services. In: Caring for frail elderly people. Policies in evolution. OECD. Paris: OECD, pp. 67-79.

Jamin, H. (I999a). 125 jaar thuiszorg. Oude tradities en nieuwe ambities. Baarn: Tirion. (I999b). 5 Moeder- en kindzorg: de ontwikkeling van de professionele zorg voor kraamvrouwen, zuigelingen en kleuters, pp. I05-130.

Janse, A. (2006). Behoeften allochtone zorguragers in de provincie Limburg: interculturalisatie is zorg op maat. Doctoraalscriptie Maastricht: Universiteit Maastricht.

Jansen, P.G.M. (1996). Differentiated practice and specialization in community nursing. Thesis Nivel. Utrecht/Maastricht: Universiteit Maastricht.

Jansen, P.G.M. and A. Kerkstra (I993). Functiedifferentiatie binnen de thuiszorg. De functies van wijkverpleegkundige, wijkverpleegster en gezinsverzorgende nader omschreven. Utrecht: NIVEL.

Jansen, P.G.M. and A. Kerkstra (I997a). Effecten van functiedifferentiatie in de thuiszorg. Utrecht: De Tijdstroom.

Janssen, M. (I992). Personal networks of Chronic Patients. Thesis Medical Sociology. Maastricht: Rijksuniversiteit Limburg.

Johnson, J., G. Larkin, M. Saks (Eds) (1995a). Health Professions and the State in Europe. London: Routledge.

Kanters, H.W. and A.A. Smit (1994). Inventarisatie van functiedifferentiatie in de zorgsector: verslag van een verkennend onderzoek. Den Haag: Stichting Organisatie voor Strategisch Arbeidsmarktonderzoek.

Kemenade, Y.W. van (I997a). Health Care in Europe. The finance and reimbursement systems of 18 European Countries. Maarssen: Elsevier/De Tijdstroom. (1997e). 6 France, pp. 59-66.

Kerff, R.G.H.G. (I999). Persoonsgebonden budgetten in de ouderenzorg. Invoeringsmogelijkheden in de thuiszorg en de verzorgingshuizen. Thesis. Rotterdam: Erasmus Universiteit. 
Kerkstra, A. (I990). De toekomst van de wijkverpleging: all-round, gedifferentieerd of gespecialiseerd? Primary health care en internationale ervaringen. In: Minisymposium 'De toekomst van de wijkverpleging: all-round, gedifferentieerd, of gespecialiseerd?' Vereniging voor verpleegkundigen en verzorgenden in de MGZ, Pp. I-I3.

Kerkstra, A. (1996b). Home Care in the Netherlands. In: Home Care in Europe. A Countryspecific Guide to its Organizations and Financing. J.B.F. Hutten and A. Kerkstra (eds). Aldershot: Arena, pp. 219-244.

(I996a). A Cross-National Comparison on Home Care in Europe: Summary of the Findings, pp.I-40.

Keuning, D. (I997). I. Omgevingsinvloeden en trends in de omgeving. In: Organisaties, management en organiseren in de gezondheidszorg. Leiden: SMD, pp. 2I-53.

Krause, E.A. (1996a). Death of the Guilds. Professions, States, and the Advance of Capitalism, 1930 to the Present. New Haven/London: Yale University Press.

(I996e). France : Strong State, Cliental Professions, pp. I23-I7I.

Kremer, M. (2000). Geven en claimen. Burgerschap en informele zorg in Europees perspectief. Utrecht: NIZw.

Krogt, T.P.W.M. van der (I98Ic). Hoofdstuk 2: Professionalisering en theorie. In: Professionalisering en collectieve macht. 's Gravenhage: vUGA, pp. 36-56.

(I98Id). Hoofdstuk 3: De machts- of beheersingsbenadering van professionalisering, pp. 57-90.

Kroneman, M.W., H. Maarse and J. Van der Zee. (2006). Direct access in primary care and patient satisfaction: a European study. Health Policy 76(I): 72-79.

Kümpers, S., A. v. Raak, Hardy, B. and I. Mur. (2002). The influence of institutions and culture on health policies: different approaches to integrated care in England and the Netherlands. Public Administration, an international quarterly 80(2): 339-358.

Kunneman, H. (1995). Mantelzorg tussen systeem en leefwereld. Mantelzorg in de markt. Verslag provinciale middag Assen: Instituut voor de Zorg en Maatschappelijke Dienstverlening Drenthe.

Lammers, C.J., A.A. Meijs, W.J. van Noort. (2000a). Organisaties vergelijkenderwijs. Ontwikkeling en relevantie van het sociologisch denken over organisaties. Utrecht: Het Spectrum. (2000d). Interinstitutionele vergelijkingen, pp. 27I-3I8.

Lancry, P.-J. and S. Sandier (I999). Twenty years of Cures for the French Health Care System. In: Health Care and Cost Containment in the European Union. E. Mossialos and J. Le Grand (eds). Aldershot: Ashgate Publishing Ltd, pp. 443-470.

Landelijke Vereniging voor Thuiszorg (2004). De toekomstbestendige thuiszorg - In reactie op de Wet Maatschappelijke Ondersteuning. Retrieved I3-07-2005 from www.hetkenniscentrum.nl/kcgs/dossiers/Sociaal/Socialecohesie/maatschappelijkeopvang/Position_ paper_LTV_WMO_IO3O.html.

Leininger, M. (I994). Chapter I: Transcultural Nursing: a new and scientific subfield of study in Nursing. In: Transcultural Nursing: Concepts, Theories and Practices. Part I: Transcultural Nursing: Developments, Theories and Research approaches. Columbus: Greyden Press, pp. 7-30.

Lieburg, M.J. van (Ed) (200I). Een eeuw consultatiebureau in Nederland 1901-2001. Facetten van de medisch-preventieve zorg aan zuigelingen en peuters in verleden en heden. Rotterdam: Erasmus Publishing. 
Lieshout, M. van (2006). De ouderenhulplijn rinkelt zomers vaker. Nederlandse overheid laat bij hittegolf het initiatief om te helpen over aan particulieren. Vrijwel elke Franse gemeente heeft een coördinator canicule. de Volkskrant 22 Juli.

Lieshout, P. van (1996). Wat willen cliënten? In: Zorg aan huis. Een stand van zaken. H. Dokter, P. v. Lieshout and B. Schadé. Utrecht: De Tijdstroom.

Light, D. (I995). Countervailing powers: a framework for professions in transition. In: Health Professions and the State in Europe. J. Johnson, G. Larkin and M. Saks (eds). London: Routledge, pp. 25-4I.

Linden, B.A. van der (I997b). Chapter I7: Transmural Care. Facts and Future. In: Health and Health Care in the Netherlands. A critical Self-assessment by Dutch Experts in the Medical and Health Sciences. A.J.P. Schrijvers (ed). Maarssen: De Tijdstroom, pp. I78-I88.

Londen, J. van (2005). Over solidariteit in de thuiszorg. In: Caleidoscoop van de thuiszorg. Met wenken en wensen voor de toekomst. J. Bastiaenen and C. Giezenaar (eds). Bunnik: Stichting Onderzoek en Ontwikkeling Maatschappelijke gezondheidszorg sтоом, pp. I4-I8.

Los, T. (I997). Bureaucratisering, managementisme en de ondergang van het beroep gezinsverzorgster. Amsterdams Sociologisch Tijdschrift 23(4): 709-732.

Lyke, S.M. van der (2000a). Georganiseerde liefde : publieke bemoeienis met zorg in de privésfeer. Thesis Universiteit Maastricht. Utrecht: Van Arkel (2000b). 5. Georganiseerde liefde, pp. I34-I53.

Lyke, S.M. van der and P.H. Toren (I996). Een kwestie van vertrouwen. Procesevaluatie van indicatiesystemen in de thuiszorg. Verpleegkunde 11(I): 23-30.

Maarse, H. and K. Okma (2004b). Chapter 6: The privatisation paradox in Dutch health care. In: Privatisation in European health care. A comparative analysis in eight countries. H. Maarse (ed). Maarssen: Elsevier gezondheidszorg: 97-II6.

Maas, P.J. van der and J. P. Mackenbach (1995). De structuur van de Nederlandse gezondheidszorg. In: Volksgezondheid en gezondheidszorg. Utrecht: Bunge, pp. 229-280.

Made, F. van der, I. Jongerden, Hollands, L. and R. Halfens (I994). Kwaliteit van de verpleging en verzorging van Parkinsonpatiënten in het verpleeghuis. Maastricht: Rijksuniversiteit Limburg.

Marrée, J. and P.P. Groenewegen (I997a). Back to Bismarck. Eastern European Health Care Systems in Transition. Aldershot: Avebury. (I997b). Chapter I: Health Systems Reform in the CCEE, pp. 3-29.

Mead, N. and P. Bower (2000). Patient-centredness: a conceptual framework and review of the empirical literature. Social Science and Medicine Oct., 51(7): I087-IIIo.

Meershoek, A. (I999). Weer aan het werk. Verzekeringsgeneeskundige verzuimbegeleiding als onderhandeling over verantwoordelijkheden. Thesis Gezondheidsethiek en Wijsbegeerte. Maastricht: Universiteit Maastricht.

Mestheneos, E. and J. Triantafillou (I993). The Support of Family Carers of the Older Elderly in Greece. In: Informal Care in Europe. J. Twigg (Ed.). York: SPRU.

Meulen, R.H.J. ter and M. van der Burg (I998). Prioriteiten binnen de gezondheidszorg. Maastricht: Instituut voor Gezondheidsethiek.

Meurs, P. (I989). Organisatienetwerken in de gezondheidszorg. In: Veranderen door stilstaan. F. van der Berg and P. Meurs (eds), Eburon, pp. 45-62. 
Ministry of Health, Welfare and Sports (2006). Chapter I. Health Insurance Act. In: Health insurance in the Netherlands. The new health Insurance System from 2006. The Hague: Ministry of Health, Welfare and Sports.

Mintzberg, H. (1979b). The Machine Bureaucracy. In: The structuring of organizations: a synthesis of the research. Engelwood Cliffs: Prentice Hall.

(I979c). Chapter I9: The Professional Bureaucracy, pp. 348-379.

Mintzberg, H. (I993d). Structure in fives: designing effective organizations. Englewood Cliffs, Prentice Hall.

(I993c). The professional bureaucracy, pp. 348-379.

Mossialos, E. and J. Le Grand (1999a). Health Care and Cost Containment in the European Union. Aldershot: Ashgate Publishing Ltd.

(I999b). Cost Containment in the Eu: an overview, pp. I-62.

(I999d). The European Union: Health Care Spending, pp. 42-62.

Murphy, R. (I990). Prolerarianization of bureaucratization: the fall of the professional? In: The Formation of Professions. Knowledge, State and Strategy. R. Torstendahl and M. Burrage (eds). London: Sage, pp. 7I-96.

Murray, C.J.L. and J. Frenk (I999). A wHo framework for Health System Performance Assessment. Evidence and Information for Policy. World Health Organization, pp. I-27.

Murray, C.J.L. and J. Frenk (2000). A framework for assessing the performance of health systems. Bulletin of the World Health Organization 78(6): 7I7-731.

Navarro, V. (2000). Assessment of the World Health Report 2000. The Lancet 356(924I): I598-i6oi.

Nijenhuis, H. (I997a). Kunnen duizend bloemen bloeien? Discussie over integrale indicatiestelling voor verpleging en verzorging. vws Bulletin(24 april): 8-II.

Nijhuis, C., Vlaskamp, L. and M. Nieuwboer (200I). Het nieuwe opleidingsstelsel voor verpleging en verzorging. In: Gekwalificeerd voor nieuwe functies. Toelichting op het opleidingsstelsel en functiedifferentiatie in de verpleging en verzorging. Retrieved March 2003 from www.lcvv.nl/pub/kwal.htm. Utrecht: LCvv.

Nijkamp, P., Pacolet, J. Spinnewyn, H., Vollering, A., Wilderom, C. and S. Winters (I99I). Services for the Elderly in Europe. A Cross-National Comparative Study. Leuven/Amsterdam: HIV/vu.

Nivel, NZi, osa (I997a). 3 Verplegende en verzorgende beroepen. In: Rapportage Arbeidsmarkt Zorgsector. Rijswijk: Ministerie van Volksgezondheid, Welzijn \& Sport. 2 Tabellenrapport, pp. 79-80.

Novak, J.D. (I998). Learning, creating, and using knowledge: concept maps as facilitative tools in schools and corporations. Mahwah (N): Erlbaum Associates.

NRC Handelsblad (I999). Themanummer December Wachtlijsten. Retrieved from www. nrc.nl/W2/Lab/Profiel/Wachtlijsten/.

NVZ, VAZ, AVvv, (2000). Landelijke Regeling Verpleegkundige Vervolgopleidingen. Retrieved 05-07-2005 from www.lrvv.nl/pdf/lrvv-versier.pdf.

OECD (I994a). The Reform of health Care. A Comparative Analysis of Seven OECD Countries. Paris: OECD.

(I994d). Chapter 4. The Reform of the Health System in France, pp. 45-55.

OECD (I996a). Caring for frail elderly people. Policies in evolution. Paris: OECD.

(1996b). The policy context, pp. I3-3I.

(I996c). The provision of long-term care, pp. 47-65. 
(I996d). Denmark, pp. I2I-I30.

(I996e). France, pp. I3I-I4I.

OECD (I998). OECD Health Data 98. Comparative analysis of 29 countries. Paris.

OECD (2007). OECD Health Data 2006. Comparative analysis of 29 countries - Chapter

Health Policy and data. Paris. Retrieved 04-0I-2007 from www.oecd.org/document/I6/ 0,2340,en_2649-33929_2085200_I_I_I_I,00.html.

Payer, L. (I988). France: Cartesian Thinking and the Terrain. In: Medicine and Culture. Varieties of Treatment in the United States, England, West Germany, and France. New York: Penguin Books, pp. 35-73.

Philipsen, H. (I980). Internationale vergelijkingen van welvaart, gezondheidszorg en levensduur: het probleem van Galton. Gezondheid en Samenleving. Tijdschrift voor de gedragswetenschappen in de gezondheidszorg 1(I): 5-I7.

Philipsen, H. (I985). Rationaliteit en ons oordeel over de verdwijnende patiënt.Gezondheid en samenleving 6: I43-15I.

Philipsen, H. (1986). Zorgen maken over zorg. Gezond Limburg 39(3): I-7.

Philipsen, H. (I987). Beweging in de relatie tussen vraag en aanbod in de thuiszorg. In: Thuiszorg: emancipatie of nieuwe markt? Bunnik: Atis.

Philipsen, H. (1988a). Gezondheidszorg als project en bejegening. Waarden ten aanzien van ziekte, gezondheid en samenleving. Maastricht: Rijksuniversiteit Limburg.

Philipsen, H. (I997b). 4. Vraaggestuurde zorg: het belang van professionele fundering van de 'erkende vraag' naar zorg. In: Vraaggestuurde zorg: dilemma's en nieuwe opties bij management, primair zorgproces, netwerken, automatisering, flexibele zorgprodukten, indicatiestelling. L. Boon (ed). Amstelveen: Stichting Sympoz, Platform voor ontwikkelingen in de gezondheidszorg, 24, pp. 22-27.

Philipsen, H. (2000). Op zoek naar Zorgland. De wao, de thuiszorg en de Bijlmerramp: randgebieden rond het medisch centrum van de gezondheidszorg. In: Continuiteit in de Medische Sociologie. Redes gehouden op 30 juni 2000 ter gelegnheid van het aanvaarden van het ambt van Hoogleraar Medische Sociologie door Prof.dr. Th.M.van Eijk en het afscheid van Prof.dr. H. Philipsen. Maastricht: Universiteit Maastricht, pp. 29-54.

Philipsen, H. (2006). Evidence based health care: een kwestie van volksgezondheid. Verpleegkunde 21(I): IO-I5.

Philipsen, H. and F.C.J. Stevens (I997a). Modernization, Rationality and Continuity of Care: Theoretical Concepts and Empirical Findings. Sociological Focus 30(2): 189-204.

Piatkowski, M.C. (I994). Historique de la profession d'infirmiere. Revue-de-l'infirmière 7(Apr): 7-9.

Pitaud, P., R. Vercauteren, B. Dherbey, (I99I). France. New Welfare mixes in care for the elderly. Eurosocial Reports vol. 40. A. Evers and I. Svetlik (eds). Vienna: European Centre for Social Welfare Policy and Research. 3; Canada, France, Germany, Italy, United Kingdom, pp. 29-58.

Platz, M. (I990). Gamle I eget hjem. Bind 2: Hvordan klarer de sig? Copenhagen: The Danish National Institute of Social Research: Report 90, pp.Io.

Plovsing, J. (1992). Old. Home Care in Denmark. Copenhagen: The Danish National Institute of Social Research.

Polit, D.F. and C.T. Beck (2004). Nursing research: principles and methods. Philadelphia: Lippincott Wlliams \& Wilkins. 
Pomey, M.-P. and J.-P. Poullier (I997). France's Health Policy Conundrum. In: Health Care and Reform in Industrialized Countries. M.W. Raffel (ed). Pennsylvania: Pennsylvania State University Press, pp. 49-76.

Pool, A. (1997a). Chapter 20. Nursing. In: Health and Health Care in the Netherlands. A critical Self-assessment by Dutch Experts in the Medical and Health Sciences. A.J.P. Schrijvers (ed). Maarssen: De Tijdstroom, pp. 207-215.

Pool, A. (2005). Investeer in samenwerking en in kwaliteit van verpleegkundige zorg. In: Caleidoscoop van de thuiszorg. Met wenken en wensen voor de toekomst. J. Bastiaenen and C. Giezenaar (eds). Bunnik: Stichting Onderzoek en Ontwikkeling Maatschappelijke gezondheidszorg sтоом, pp. 90-93.

Potting, M.A.C.J. (200I). Van je familie...: zorg, familie en sekse in de mantelzorg. Thesis Department of Health Care Studies, section of Health Ethics and Philosophy, Maastricht University. Amsterdam: Aksant.

Poullier, J.-P. and S. Sandier (2000). France. Journal of Health Politics, Policy and Law 25(5, October): 899-905.

Powell, W.W. and P.J. DiMaggio, Eds. (I99I). The New Institutionalism in Organizational Analysis. Chicago/London: University of Chicago Press.

Raad voor de Volksgezondheid en Zorg (2002). Jaarverslag 200I. Zoetermeer, RvZ, Raad voor de Volksgezondheid en Zorg.

Raalte, A.W. van, R. Kuiperij, M. Warringa, (1999). Kiezen voor nieuw Elan. Strategisch plan. Utrecht, Stuurgroep Wijkverpleging met toekomst.

Raffel, M.W., (Ed). (I984). Comparative Health Systems. Descriptive Analysis of Fourteen National Health Systems. University Park/London: The Pennsylvania State University Press.

Raffel, M.W., (Ed). (I997a). Health Care and Reform in Industrialized Countries. Pennsylvania: Pennsylvania State University Press.

Ramakers, C. (I998a). Cliëntgebonden budget. Een experimenteel onderzoek. Nijmegen: ITs. (I998b). Achtergrond en vraagstelling van het onderzoek.

Ravensbergen, A. (I990). Intensieve thuiszorg in de praktijk. Medisch Contact(45): 748750.

Renckens, C.N.M. (2004). Dwaalwegen in de geneeskunde. Over alternatieve geneeswijzen, modeziekten en kwakzalverij. Thesis Nederlandse Vereniging voor Obstetrie en Gynaecologie. Amsterdam: Uitgeverij Bert Bakker, Universiteit van Amsterdam.

Ribbe, M.W., G. Ljunggren, et al. (1997). Nursing homes in Io nations: a comparison between countries and settings. Age and Ageing 26(Supplement 2): 3-I2.

Rogers, J. and P. Curtis (I980). The concept and measurement of continuity in primary care. American Journal of Public Health(70): I22-126.

Rostgaard, T. and T. Fridberg (1998). Caring for Older People - A Comparison of European Policies and Practices. Copenhagen: The Danish National Institute of Social Research. 98: 20.

(1998b). 3 Denmark, pp. 87-I63. (I998d). 9 Germany, pp. 485-55I.

Ruigrok, P.C.J. (2000). Recente transities in huwelijk, gezin en levenssyclus. In: Familie, Huwelijk en Gezin in West-Europa. Recente transities. Retrieved I8-II-2003 from www. geocities.com/pprevos/cultuur/cior2I/fghIV.htm: I-6. 
Ruwaard, D. and P.G.N. Kramers (Eds), (1993). Volksgezondheid Toekomst Verkenning. De gezondheidstoestand van de Nederlandse bevolking in de periode 1950-2010. Den Haag: Sdu.

Saltman, R.B. and J. Figueras (I997a). European Health Care Reform. Analysis of current strategies. Copenhagen: wHo - Regional Office for Europe.

Sandier, S., V. Paris, and D. Polton (2004). Health Care Systems in Transition - France. S. Thomson and E. Mossialos (eds), European Observatory on Health Care Systems. Retrieved from http://www.observatory.dk.

Scharf, T. (1998). Ageing and Ageing Policy in Germany. Oxford: Berg. (I998b). I Germany's Ageing Population: An Introduction, pp. I-22. (I998c). 2 Social Policies for Older People, pp. 23-50. (I998d). 4 Housing, pp. 93-II3. (I998e). 5 Meeting Health and Social Care Needs, pp. II4-I5I.

Schepers, R.M.J. and A.C. Nievaard (I990). Hoofdstuk 5. Beroepen in de gezondheidszorg. In: Ziekte en zorg. Inleiding in de medische sociologie. Leiden: Stenfert Kroese, pp. 87-95.

Schilstra, K. (2002). Primaat ligt niet bij de politiek. NRC Handelsblad 15 Januari.

Schleich, D. (2004a). Daten und Fakten zur Pflege. Interview mit prof.dr. Stefan Görres. Retrieved 20-07-2004 from www.br-online.de/umwelt-gesundheit/thema/pflege/daten. $\mathrm{xml}$.

Schleich, D. (2004b). Pflegenotstand. Interview mit prof.dr. Stefan Görres. Retrieved 20-072004 from www.br-online.de/umwelt-gesundheit/thema/pflege/notstand.xml.

Schleich, D. (2004c). Pflegekräfte. Interview mit prof.dr. Stefan Görres. Retrieved 20-072004 from www.br-online.de/umwelt-gesundheit/thema/pflege/pfleger.xml.

Schmitt, M.G. (1988). Sozialpolitik. Historische Entwicklung und internationaler Vergleich. Opladen: Leske und Budrich.

Schneider, M., Biene-Dietrich, P., Gabanyi, M., Hofmann, U., Huber, M., Köse, A., and J.H. Sommer (I994a). Gesundheitssysteme im internationalen Vergleich. Laufende Berichterstattung zu ausländischen Gesundheitssystemen. Augsburg: Basys. (I994C). Bundesrepublik Deutschland, pp. I93-225.

Schneider, M., Dennerlein, R.-H., Köse, A., and L. Scholtes (I992). Health Care in the EC Member States. Health Policy Health Policy Special Issue(I+2): II3-I23.

Schnepp, W. (200I). Familiale Sorge in der Gruppe der rußlanddeutschen Spätaussiedler - Funktion und Gestaltung. Gent: Wetenschappelijke uitgeverij Academia Press.

Schröer, C.A.P. (1998). Ziekte en sociale zekerheid. In: Medische sociologie. Sociologische perspectieven op ziekte en zorg. C.W. Aakster and J.W. Groothof. Groningen: Wolters Noordhoff, pp. 248-257.

Schuijt, K. (2003). Uitdijende rechtsstaat gevaar voor zichzelf. NRC Handelsblad 16 maart.

Scott, W.R. and J.W. Meyer (I99I). The Organization of Societal Sectors: Propositions and Early Evidence. In: The New Institutionalism in Organizational Analysis. W.W. Powell and P.J. DiMaggio (eds). London: Sage, pp. Io8-I40.

Scott, W.R. and J.W. Meyer (I992C). The Organization of Societal Sectors. In: Organizational Environments. Ritual and Rationality. J.W. Meyer and W.R. Scott (eds). London: Sage, pp. I29-I53.

Siegrist, H. (1990). Professionalization as a process: patterns, progression and discontinuity. In: Professions in Theory and History. Rethinking the Study of the Professions. M. Burrage and R. Torstendahl (eds). London: Sage, pp. 177-202. 
Siim, B. (I993). The Gendered Scandinavian Welfare State. Women and Social Policies in Europe - Work, Family and the State. In: J. Lewis (Ed.). Aldershot: Edward Elgar.

Simon, H. A. (1977). The New Science of Management Decision. Englewood Cliffs: Prentice Hall.

Smeenk, F.J.M. (1998). Transmural Care of Terminal Cancer Patients. An Evaluation Study in the Eindhoven Region. Thesis. Maastricht: Universiteit Maastricht.

Stallknecht, K. (I999). Hvor kommer du fra? En fortalling om Dansk Sygeplejehistorisk Museums samling af emblemer, der knytter sig til sygeplejerskeuddannelsen. Vanløse: Dansk Sygeplejeråd og Dansk Sygeplejehistorisk Museum.

Starmans, R. and H.A. Mostert (I989). Wie coördineert de zorg in de eerste lijn? Huisarts of wijkverpleegkundige. Medisch Contact(44): 87-89.

Stevens, F. and J. Diederiks (1995). Chapter 5: Health Culture in Europe - an Exploration of National, and Social Differences in Health-Related Values. In: Health Systems in the European Union. Diversity, Convergence and Integration. G. Lüschen, W. Cockerham, J. van der Zee (eds). München: Oldenbourg, pp. 75-88.

Stevens, F. and H. Philipsen (2003). Professionalisering van beroepen in de gezondheidszorg. In: Medische sociologie. C.W. Aakster and J.W. Groothoff. Groningen: WoltersNoordhoff, pp. 85-93.

Stevens, F.C.J. (2007). The Convergence and divergence of modern health care systems. In: Comparative Medical Sociology. W.C. Cockerham (ed), Blackwell Publishers, pp. I59-I76.

Stevens, F.C.J., J.P.M. Diederiks, Grit, F., and F. van der Horst (2007). Exclusive, idiosyncratic and cellective expertise in the interprofessional arena: the case of optomotery and eye care in the Netherlands. Sociology of Health Q Ilness 29(4): 48I-496.

Stevens, J.A.M., S.W. Mathijssen, and C.G.M. Pepels (200I). Brancherapport Volksgezondheid. Onderdeel Care. Deel A: Verpleging en Verzorging en Ouderenzorg. Den Haag: Prismant \& Ministerie van vws.

Stolk-van Delen, H.W. (1983). Wijkverpleging in historisch perspectief. Ontstaan en ontwikkeling van de wijkverpleging (1890-ca 1930) met aandacht voor aspekten van medicalisering en professionalisering. Amsterdam: Rodopi.

Straus, R. (I957). The nature and status of medical sociology. In: American Sociological Review(22): 200-204.

Stuurgroep Wijkverpleging met toekomst (I999a). 3 Profiel van de Wijkverpleegkundige. In: Kiezen voor nieuw Elan. Strategisch plan. A.W. v. Raalte, R. Kuiperij and M. Warringa. Utrecht: Stuurgroep Wijkverpleging met toekomst.

Svensson, R. (1996). The Interplay between Doctors and Nurses - a negotiated Order Perspective. Sociology of Health and Illness 18(3): 379-398.

Swaan, A. de (1988). In Care of the State, Health care, Education and Welfare in Europe and the UsA in the Modern Era. Cambridge: Polity Press.

Swaan, A. de (I989b). Hoofdstuk 6: Sociale zekerheid als de accumulatie van overdrachtsvermogen. In: Zorg en de staat: welzijn, onderwijs en gezondheidszorg in Europa en de Verenigde Staten in de nieuwe tijd. Amsterdam: Bakker, pp. I60-225.

Swagerman, Q.A.J. (I997). Het perspectief van de thuiszorg. In: Van revolutie naar evolutie. Tien jaar stelselwijziging in de Nederlandse gezondheidszorg. Y.W. v. Kemenade and E. Elsinga (eds). Maarssen: De Tijdstroom, pp. 78-103.

Theobald, H. (2003). Occupational control: New balances between state, market and family. Business consultancy and social care work in Germany during the I990s. In: Conceptual 
and Comparative Studies of Continental and Anglo-American Professions. L.G. Svensson and J. Evetts. Göteborg: Kompendiet, pp. I23-138.

Thiel, G. van and M. van den Hoven (I999). Als mensen aandacht tekort komen, smaakt het kopje thee ook niet. Ethische vragen in de Care sector. Utrecht: Centrum voor bio-ethiek en gezondheidsrecht, Universiteit Utrecht.

Thomas, P., S. Chanoin-Merlet, Hazif-Thomas, C., Belmin, J., Montagne, B., Clement, JP., Leburchec, M., and R. Billon. (2002). Complaints of informal caregivers providing home care for dementia patients: the Pixel Study. International Journal of Geriatric Psychiatry 17: I034-1047.

Tjadens, F. and M. Duijnstee (2000). Sectie C: de Landenrapportages - 4 Nederland. In: De ondersteuning van mantelzorgers en hun organisaties in zeven West-Europese landen. Stand van zaken in 1998. F. Tjadens and M. Pijl (eds). Utrecht: NIzw-Uitgeverij, pp. IO7-I55.

Torstendahl, R. and M. Burrage (Eds), (I990a). The Formation of Professions. Knowledge, State and Strategy. London: Sage.

Traynor, M. (I999). Managerialism and Nursing - beyond oppression and profession. London: Routledge.

Twigg, J. (I996). Issues in informal care. In: Caring for frail elderly people. Policies in evolution. Paris: OECD, pp. 8I-92.

UNFPA (2000). Wereld Bevolkingsrapport. Samen leven in een aparte wereld. Mannen en vrouwen in een tijd van verandering., UNFPA.

United Nations (2000). World Statistics Pocketbook. Economic \& Social Affairs. New York: United Nations Publication.

Vallgårda, S. (2002). The Danish health care system: A commentary. Euro Observer 4(2): I-2.

Vallgårda, S., A. Krasnik, et al. (200I). Health Care Systems in Transition - Denmark. S. Thomson and E. Mossialos (eds), European Observatory on Health Care Systems. Retrieved from www.observatory.dk.

Veer, A.J.E. de, A.L. Francke, and J.B. Hutten, (200I). Allochtonen en de thuiszorg: Bereik, verrichtingen en opbrengsten. Utrecht: Nivel/sтоом, pp. I-96.

Verheij, R. and A. Kerkstra (I993). Extramurale verpleging vergeleken in België, Canada, Duitsland, Engeland, Finland, Frankrijk, Nederland, Noorwegen en de Verenigde Staten. In: Handboek Thuiszorg. A.J.P. Schrijvers (ed). Utrecht: De Tijdstroom, pp. 5I-70.

Verheij, R.A. (I996). Home Care in France. In: Home Care in Europe. A Country-specific Guide to its Organizations and Financing. J.B.F. Hutten and A. Kerkstra (eds). Aldershot: Arena, pp. II6-I3O.

Verheij, R.A., Boven, P.F. van, and F. Kosterman, (200I). okz binnen bereik? De toegankelijkheid van ouder- en kindzorg in geografische perspectief. Houten: Bohn Stafleu Van Loghum.

Verheij, R.A. and A. Kerkstra (I992). International comparative study of community nursing. Aldershot: Avebury.

Verseput, J.M. (2005). We moeten terug naar een integrale, eerstelijns gezondheidszorg. In: Caleidoscoop van de thuiszorg. Met wenken en wensen voor de toekomst. J. Bastiaenen and C. Giezenaar (eds). Bunnik: Stichting Onderzoek en Ontwikkeling Maatschappelijke gezondheidszorg sтоом, pp. 26-29.

Voortman, I. (I999). Interculturele zorgverlening. Utrecht: Universiteit Utrecht. 
Vos, R., Houtepen, R. and K. Horstman, (2002). Evidence-Base Medicine and Power Shifts in health Care Systems. Health Care Analysis 10: 319-328.

Vos, R., Willems, D., and R. Houtepen, (2004). Coordinating the norms and values of medical research, medical practice and patient worlds - the ethics of evidence based medicine in orphaned fields of medicine. Journal of Medical Ethics 30: I66-I70.

Vrijhoef, H.J.M. (2002). Is it justifiable to treat chronic patients by nurse specialists? Evaluation of effects on quality of care. Thesis Department of Health Care Studies, section of Medical Sociology. Maastricht: Maastricht University.

Vulto, M. and M. Morée (I996). Thuisverzorging als professie. Een combinatie van hand, hoofd en hart. Utrecht: De Tijdstroom.

vws (2000). De Wet BIG. Hoofdlijnen van de wet Beroepen in de Individuele Gezondheidszorg. Den Haag, Ministerie van Volksgezondheid, Welzijn en Sport

vws and ocw (I996). Gekwalificeerd voor de toekomst. Kwalificatiestructuur en eindtermen voor Verpleging en Verzorging. C. Kwalificatiestructuur, A. Grotendorst and J. v. Londen (eds). Zoetermeer/Rijswijk: Ministerie van oc\&w, Ministerie van vws.

Walker, A. (1993). Age and Attitude - Main Results from a Eurobarometer Survey. DGv, Brussels: Commission of the European Communities.

Wever, J. (2002). Poldercultuur blokkeert doelgericht bestuur. NRC Handelsblad 15 Januari. w но (2000). The World Health Report 2000. Health Systems: Improving Performance. Geneva: wно.

Wiegman, N. (I996). 'Gij completeert zijn arbeid': over de professionalisering van het verpleegkundig beroep (I880-I925). Gewina 19(4): 96-II2.

Wiegman, N. (2005). Verpleegkundigen en verzorgenden: wees trots op je beroep! In: $\mathrm{Ca}$ leidoscoop van de thuiszorg. Met wenken en wensen voor de toekomst. J. Bastiaenen and C. Giezenaar (eds). Bunnik: Stichting Onderzoek en Ontwikkeling Maatschappelijke gezondheidszorg sтоом, pp. 30-33.

Wijers, H. and T. d. Graaf (2002). Primaat ligt niet bij de politiek. NRC Handelsblad 9 Januari.

Wilensky, H. (1964). The professionalization of everyone? American Journal of Sociology(70): I37-I58.

Williams, A. (200I). Science or marketing at wHo? A commentary on 'World Health 2000'. Health Economics 10(594): 93-100.

Windt, W. van der, Calsbeek, H. and L. Hingstmand, (I997). Verpleging en verzorging in kaart gebracht. Utrecht: De Tijdstroom/LCVv.

ZDF-online Ratgeber (2000). Pflegedienste - worauf Sie achten sollten. Praxis Q info Gesundheit. Pflegedienste. Retrieved 25-10-2000 from www.zdf.de/ratgeber/praxis/pflege/05376/index.html.

Zee, J. van der (1996). Home Care in Denmark. In: Home Care in Europe. A Country-specific Guide to its Organizations and Financing. J.B.F. Hutten and A. Kerkstra (eds). Aldershot: Arena, pp. 82-96.

Zee, J. van der, Kramer, K., Derksen, A., Kerkstra, A., and F.C.J. Stevens, (I994). Community Nursing in Belgium, Germany and the Netherlands. Journal of Advanced Nurs$\operatorname{ing}(20)$ : 79I-80I.

Zorgmediatheek (2006). Thuiszorg voor allochtonen in Deventer. De Thuiszorggids. Retrieved 23-08-2007 from http://thuiszorg-gids.nl/nieuws-archief-details.php?id=343\&offset= 480. 


\section{Summary}

This book describes the patterns of professionalisation and institutionalisation of home nursing in Denmark, France, the Netherlands and Germany. For this, a contextual and comparative perspective is developed on home care and informal care to frail elderly and vulnerable people, taking the specific national socio-cultural and political background into account as well as the differences in the health care systems of these countries.

In chapter one, the aim of this book is described, namely to gain insight into international differences in home nursing in Europe, as well as in the background of these differences. A macro sociological and cultural perspective is chosen, because earlier studies have pointed out that differences between countries on the level of health care systems, health and family policies, and social and cultural values regarding long-term care for frail and elderly people are reflected in international differences in micro-level community care practices. In order to find explanations and backgrounds for these differences, these are described within their broader context and related to differences in interprofessional relations, relations between health care sectors, the structures and way of financing of the overall health care system, the role of the family in the provision of care to elderly people, and differences in social, cultural and political norms and values between countries regarding health and illness and more specifically care to the vulnerable.

Then, the four different levels of analysis of the home nursing domain and profession are described, namely the micro level of the domain of home nursing, the intermediate level of the home nursing profession, the meso level of relations between different health professionals and sectors, and the macro level of the institutionalisation of home nursing. To analyse home nursing at these different levels, different theoretical perspectives are combined; a health sciences perspective, a sociological perspective on professions, a neo-institutional perspective on home nursing as a societal sector and cultural studies perspective taking different caring cultures within Europe into account.

Subsequently, reasons are provided for focussing on Denmark, France, the Netherlands and Germany. These countries are geographically close and thus have a number major features in common, but at the same time important societal, political and 
cultural differences exist that influence the conditions under which home nursing is provided. As these countries are positioned in the North West, Mid and South of Europe, these should provide more insight into different caring cultures from North to South and the different ways in which countries cope with an ageing population. Their different backgrounds are expected to result in a different constellation of health care arrangements, and consequently, a different position of home nursing in the health care systems of these countries. Finally, the research design is described, as well as the range and kind of sources used for this book. The methods of this book, in the form of use of theory, procedures of data collection, analysis, and writing, characterise this study as a comparative and institutional analysis of the home nursing domain and the way it is embedded in particular societies. The theoretical framework that is built in the second chapter functions as a 'theoretical lens' or overarching perspective, consisting of topics of interest that guide the data collection and is aimed at attaining a comprehensive view on home nursing.

Chapter two goes into the theoretical perspective of this book. First, several strands in the sociology of the professions are described, such as the trait perspective, that aims at defining characteristic criteria of professions to construct the ideal-type of a profession; the perspective that focuses on professional development, that is supposed to take place in a certain characteristic order of steps that successively raise the degree of professionalism; the hierarchical perspective that defines a successful profession as a gold standard to which other professions are compared in order to determine their degree of professionalism; and the perspective focussing on power relations between professions. Next to that, an influential view, the theoretical perspective of Abbott (I988) on professions, is described. This is taken as starting point for this thesis. Based on the assumptions of this view, a contextual and comparative framework is developed. Abbott argues that professions claim certain jurisdictions over areas of expertise that signify their domain, and advocates to study professions within the network or 'interacting system of professions' and to take large scale societal developments into account.

Then, the general characteristic features of the home nursing domain are described that form the core of the contextual theoretical framework that is developed in this chapter. Home nursing is considered as a special working domain and can be characterised as a broad sphere of action, with a large autonomy of home nurses because they are not supervised by professionals with a higher hierarchical status. The second layer of the theoretical framework goes into the system of professions, such as differentiation of tasks and interprofessional cooperation and co-ordination. Also, the training and education are considered important to gain insight in differences between home nursing professionals within the four countries studied, as well as the position of the patient and the impact of bureaucracy. The outer layer of the framework contains concepts that refer to the health care and societal context within which home nursing is 
provided, such as the kind of health care system in each country, the policies regarding health care and elderly people, cultural and political views on the role of the family regarding the provision of informal care to elderly people, the impact of the increasing cultural diversity of European societies, and the impact of managerial, instead of professional, perspectives on home nursing.

Chapter three functions as a background chapter for each of the following chapters, providing information on the general differences in home care provision within Europe, the main characteristics of the health care systems, as well as providing an overview of the demographic data and key characteristics of the four countries described. These are summarised in tables and graphs at the end of this chapter.

Chapter four to seven describe the role of home nursing in each country, thereby taking the health care context and the socio-cultural and political background into account. The concepts of the theoretical framework and the specific societal and health care situation are taken as points of departure for the descriptions.

In chapter four, the home nursing domain in Denmark is described. Denmark has a national health system (NHSs; National Health Security System) that is characteristic for Scandinavian, social-democratic regimes. Principles of universalism and state dominance prevail, meaning that rights, services and benefits are available for the entire population and are emancipated from tasks that were traditionally provided by family, members and the social network. As individuals are alleviated from family responsibilities, maximum individual independence can be attained. The general opinion in society and politics prevails that the welfare state should guarantee publicly provided health and social care and family members are not formally obliged to care for elderly or chronically ill people. Therefore, informal care plays no major role. Drawback of this system is the high taxes. This contrasts to countries such as France, Germany and the Netherlands where family solidarity is stressed and caregiving to elderly and vulnerable people by family members is encouraged in health policies and professional care is provided by non-governmental organisations. As state provision of professional services is seen as a right for all in Denmark, general accessibility and availability of health care services are main principles. The provision of health care is strongly decentralised; most services are provided and financed at the local level of counties or municipalities. The central government only provides an overall legal framework. The health care system is quite stable because major reforms have remained absent for several decades and only gradual adaptations have been introduced. There is hardly any market competition because this is not considered a desirable principle in health care provision in Scandinavian countries.

Characteristic regarding elderly care in Denmark is the radical change from institution-based care to community-based elderly care. Until the I970s, municipal obliga- 
tions to organise long-term home help and institutionalisation of elderly in nursing homes both had priority. However, in I987, new legislation was introduced separating the housing and service function to discourage nursing home care. Home care services could now be provided irrespective of where people lived. New nursing homes were not allowed to be built and many existing ones were closed. Since then, Danish health policies adhere to the ideology of 'ageing in place', which means that elderly people should be enabled to remain living in their own homes or in independent adapted homes for the elderly as long as possible. Institutionalisation should be prevented when possible and elderly people should be supported in their autonomy and self-help by public services provided at municipal level.

In Denmark, the tradition of home nursing dates back to the end of the nineteenth century. Currently, the home nurse still is a central person in case of illness of an elderly person. A generalist perception of care and continuity of care are important principles in Denmark. No specific nursing grades or levels of expertise are distinguished and the basic education of all caring professions is shared. Denmark is the only country where home nursing, home help and institutional elderly care are fully integrated. This community-based elderly care is financed and provided by the municipalities, where nurses are employed. These provide their care irrespective of where elderly people live: in their own homes, in nursing homes, and in special apartments for the old. Danish home nurses have more decision power and autonomy compared to their colleagues in other countries, where medical professionals (Germany, France) or 'objective assessment organisations' (the Netherlands) determine the need for home nursing and the tasks to be performed. The trust in health care professionals and their social status is quite high in Denmark, which can be recognised in the larger degree of professional autonomy and very low interference of managers or bureaucratic procedures. Home nurses and assistant nurses provide home nursing services, but no separate list of tasks is specified for each; both provide simple as well as complex tasks and earn the same salaries. This is an important difference compared to other countries, where differentiation of tasks is an important organisational principle. Almost all nurses are member of the Danish Nurses' Organisation DSR that exists since I899.

In chapter five, the home nursing domain in France is described. One of the most striking aspects regarding home nursing in France is the uneven availability of home nursing services across the country. Due to the uneven distribution of people over very densely populated areas such as Paris and its surroundings on the one hand, and very scarcely populated areas in the middle and south of France on the other hand, social, economical and health care services are unequally distributed regionally as well. Especially community-based services such as home nursing and home help are not spread evenly across the country and are sometimes even non-existent in remote areas. The tendency of older French people is however 'to stay where they are', even when health, housing and social facilities are not corresponding to their needs. This results in many 
elderly people living in social isolation and deprived housing circumstances in scarcely populated areas.

Other characteristics of the French health care system are its orientation towards medical and institution-based care. The French have a rather medical, cure-oriented attitude towards matters of health and disease and generally adhere to a biomedical definition of diseases, expecting to be cured by medical interference and drugs. The medical sector, comprising care provided by GPS, medical specialists and hospitals, clearly has priority over the nursing and caring sector in the health care system, which is organised such that medical and hospital care is easily accessible to patients. These are allowed to visit the physician of their choice as often as they wish and are free to consult several physicians about one complaint, and have direct access to private specialists and all types of hospitals. In contrast, ambulatory care services are not directly accessible, as a prescription of a physician or social worker is needed. Also, financial barriers regarding ambulant care exist, due to a very complex and overlapping set of provisions in which all kinds of schemes and levels of governments are involved. The (bio)medical and institution-based character of health care is also reflected in the relatively high number of GPS and specialists, hospital beds per I ooo population and very high pharmaceutical expenditure compared to other European countries.

A fourth aspect that is characteristic of France is that the state is an important centre of power regarding all aspects of French life, also regarding the health care system and health care professions. Elderly care in France is characterised by a dichotomy between the medical and institutional domain that are managed by the national government, and the social domain that is managed by local governments. This can also be recognised in home care where two kinds of home care exist. Hospitalisation à Domicile (HAD) is caring for patients with extremely serious and complex conditions in their homes, which is organised by hospitals, while Service de Soins Infirmiers à Domicile pour les Personnes Agées (SSIAD) is comparable to home care provided in other European countries, namely community-based home nursing and home help provided to vulnerable and elderly people in their own homes. A prescription by a physician is needed for reimbursement by health insurers for both kinds of home care.

The geographical scope of home care organisations varies considerably in France, as well as the kind of services provided; sometimes they only offer home nursing, sometimes a whole range of services are provided, including meals-on-wheels, téléalarm services and/or maternity care. A large problem in France is that many people in need of care do not receive services, so the informal support of the social network (family, friends, people from the neighbourhood) plays a large role. Older women living alone are a vulnerable group; especially when their family members have left the area they are prone to social isolation.

Since the I960s, policies for elderly people were developed. In most reports, the ideal was described of elderly people growing old in their own environments, supported by the family. Since the I980s, the home care domain has considerably increased in 
size, but the appeal to informal care is still large because the services available do not cover the needs of many elderly people. In most areas, so-called independent nurses provide a large amount of care from their private practices. Currently, discussions are going on about the role of the regular home nurses employed by the SSIAD services and independent nurses. The latter claim the role of co-ordinator of medical, nursing and social care for individual clients, but research pointed out their apparent lack of knowledge of the local situation and available professionals. A very large amount of home care is provided by nursing auxiliaries and by home helps.

From the literature, the image arose that the domain of home help is socially and culturally more accepted than the home nursing domain. Elderly people and their families generally consider home nurses as strangers intruding into their personal sphere. Due to the stigma associated with professional help, they are reluctant to apply for home nursing care; people are afraid it would indicate that the family is not able to properly care for their elderly relatives. Another difference compared to other countries is that home care professionals display a different attitude regarding clients and appreciate different professional values; personal contact and support of clients is stressed over values such as efficiency, rationality and keeping a professional distance, that are emphasised in professional caregiving in most other European countries.

In chapter six, home nursing in the Netherlands is described. The history of home nursing, in the form of non-governmental local and regional Cross Associations, dates back to about I880, as in Denmark. During the twentieth century, a dense network of home nursing services has developed across the country, ensuring the provision of home nursing in all parts of the Netherlands. Since the beginning of the twentieth century and especially since the I930s, the cross associations took a leading role in including mother and child care into the responsibilities of home nurses. They therefore developed an all-round expertise regarding all matters of health, disease and prevention to the families living in their neighbourhoods. Home nurses locally had societal authority alongside the GPS.

In general, the Dutch attitude towards caring is quite favourable. Many facilities exist for people in need of care and support, such as homes for the elderly, senior apartments with collective facilities for elderly people, nursing homes, and a rather dense network of home nursing and home help services. This is complemented by regulations that favour the provision of informal care by family members, neighbours and friends, such as the Personal Budget ( $\mathrm{PGB}$ ) with which elderly people can pay informal caregivers. Also, caring is considered as an independent professional activity with its own specified and circumscribed domain, and not only as supportive of the medical profession. This contrasts with countries such as France and Germany, where caring is more considered as being an extension of medical activities. Nevertheless, several problems in home care do exist in the Netherlands, which has increasingly become the case since the last few decades. 
Since the I970s onwards, many changes have been introduced into the Dutch health care system, also having large impacts on home nursing. Elements of market competition were introduced, as well as structural and budgetary measures. During recent decades, tendencies have become strong in the Netherlands to organise and pre-structure matters in a top down way, also called managerialism, and to deal with things in a bureaucratic way by designing and referring to rules and regulations and administrative procedures. Efficiency, rules and principles, universality and impersonality (in the form of neutral professional distance) are key concepts of managerialism. This is also strongly felt in health care and home nursing. Parts of the job that have always belonged to the core of the home nursing profession have been transferred to external bodies and actors, such as the RIos (regional assessment organs) and later CIz (centre for care assessment), thereby affecting the character of home nursing activities and the degree of professionalism. Since I997, these institutions decide on the entitlement to care by patients within the AWBz framework on the basis of objectified procedures. Formerly, home nurses performed the intake of clients themselves. It is argued that this has resulted in deprofessionalisation of the home nursing profession in the Netherlands. Also, during the last several decades, the leeway for home nurses in daily practice and the freedom to make independent decisions based on professional expertise, skills and experience have become more limited by an increasing use of guidelines for practices and standardised protocols. The current trend of Evidence Based health care practices, that is also becoming stronger in nursing, is criticised because the expertise of professionals that was based on their training and specific, contextbound experiences, has gradually made way for the 'average professional'. These apply standardised knowledge in a correct way and according to the latest insights and procedures, so that clients receive adequate treatment and care, regardless of their specific situation by no matter which professional. Also, organisational changes have made it easier for home care organisations to employ professionals working in shifts who no longer have responsibility over specific individual clients or families in their own neighbourhood. This implies for clients that continuity of care by one or few professionals cannot be guaranteed anymore and that many different professionals provide care to them. In addition, outsiders to the profession, especially managers and policy makers, can now exert more control over their job. These developments now tend to prevail over the professional and personal aspects of caregiving. From the comparison of the four countries in this book, these developments seem to be especially strong in the Netherlands.

In I997, a new educational system was introduced in nursing, resulting in a division in various levels and different responsibilities. Another major change was the integration of home help and home nursing into integrated home care in the I99os, provided by home care organisations that more and more increase in size due to subsequent mergers. At the same time, the appeal on informal carers to perform a large amount of care has become larger. The availability of informal carers is now also assessed 
during the intake procedure: elderly and vulnerable people with family members or spouses that can provide care are not entitled to home care anymore. Informal care has thus become a duty that is formalised in procedures. Thus, although home nursing in the Netherlands seems to have a rather favourable position in the health care system and provision of care to the vulnerable is guaranteed throughout the country, severe problems do exist in home care. An important conclusion in this chapter is that the character and professional authority of home nurses has fundamentally changed during the last decennia.

In chapter seven, home nursing in Germany is described. One of the findings was that elderly are considered as a problematic societal group. For a long time, they have been a somewhat neglected group that were to cope with their health and financial problems themselves and within their families. Compared to many other European countries, Germany was quite late with developing policies targeting on elderly people in the community. Current social and economic policies are largely responsible for a pessimistic view on elderly that refer to them as a burden to society. Doubts whether the national economy is able to provide sufficient resources to cope with the increasing health and social care needs of elderly people is most pronounced in Germany, as the number of elderly and the very old is highest in the EU. For many decades, however, caring and supporting activities that are performed in the 'periphery' of the health care system did not have a very high priority in Germany, compared to medical and cureoriented activities performed in the 'medical centre' of health care. The German health care system is quite medically and technically oriented, and traditionally, medical and hospital care have always had priority over other forms of care. Nursing is generally not considered as an independent activity in Germany, but primarily as an extension of medical activities and complement to medical or hospital care. Nursing tasks are more medical-technical in character than in other European countries, for example, home nurses often give injections.

The domain of professional caring activities, such as home nursing, has encountered financial as well as structural barriers for a long time. Physicians largely control procedures determining whether people are entitled to home nursing services: a medical referral is needed to receive reimbursement for nursing care. While medical treatment has already been an insurance-based and legally enforceable right for a long time, this is only the case with nursing care since the introduction of the Pflegegesetz in I995. Within the insurance framework there is a strict hierarchy of service entitlement, ranging from medical treatment, specialised nursing, basic nursing, and home help as a supplement to nursing. The health care system has inherent barriers for the provision of long-term ambulant care, while access to physicians, medical specialists and hospitals is easy.

Next to cure-oriented activities in health care, the reliance on the family to provide care and support is strong. Traditional family values are strong in Germany, and there has been a moral obligation (and until the Pflegeversicherung a legal obligation as well) for 
family members to provide informal care to older and indigent people. As in many European countries, the emphasis on family responsibilities regarding elderly is reflected in the role of the state in health care. The provision of welfare and social care is governed by the principle of subsidiarity. This means that the community should act first, and that the state should only intervene when there is no alternative. No services should be offered by the state when other organisations can perform those tasks satisfactorily on the state's behalf. The state should only function as a safety net. German policies for long-term care of frail elderly people reflect the belief in family solidarity. Public initiatives to support the provision of professional care have developed only slowly, and policies have primarily aimed at reinforcing family care. Thus, mostly, help and support is sought within families.

Since the I990s, health policies for elderly people gradually but reluctantly developed. Currently, older people's interests are still frequently ignored in the policy-making process in Germany, contrary to e.g. Denmark. Also, the shift from institutional care to community-based care in German elderly policies is fairly recent compared to other European countries. Several types of organisations are providing ambulatory care for the elderly and other categories of clients: the Ambulante sozialpflegerische Dienste (organisations for ambulatory social and nursing care). Of these, especially the Sozialstationen, established since the I970s, can be considered as very important in the provision of community care. Also, many organisations that fall under six national umbrella welfare organisations provide social and nursing care. Most of these are religious in character, such as the Protestant Diakonisches Werk and the Catholic Caritas. In these organisations, a range of different professionals provides help to elderly people. Cooperation between different kinds of professionals and organisations is however hardly taking place because of boundaries between the health and social domain, the influence of physicians who are no proponent of co-operation, and the fragmented way in which home care is provided by many different kinds of organisations and professionals.

In chapter eight, the conclusion, the four countries are compared from the theoretical perspective developed in the second chapter. The separate conclusions of home nursing and elderly care, drawn in chapters four to seven, were compared and integrated. On the basis of this, changes of the theoretical framework were proposed. Some aspects appeared to be less important, while some new aspects did appear to be important that had not been included into the framework in the first instance. For example, the period of establishment of home nursing appeared to be important for the development of home nursing and its establishment as a separate domain alongside the medical domain. The contrast between the rather strong position of home nursing in health care and society in Denmark and the Netherlands, where its history dates back to the nineteenth century, is large compared to the relative young establishment of regular home care organisations in France. Also a strong contrast was found between countries where 
home nurses are having an independent role and responsibility, such as in Denmark and the Netherlands, and countries where their activities are considered as an extension of or supportive of medical activities, such as in France and Germany.

General trends in Europe are the increasing focus on vulnerable and (very) elderly people as main client population, and the increasing task differentiation and specialisation of nurses at the expense of the all-round profile that is only still prominent in Denmark. The training and education of home nurses is different in the four countries studied. Denmark is the only country where nurses are trained as generalists and where different nursing levels do not exist. The aspects cooperation and co-ordination received rather limited attention in the literature, except from the Netherlands where these concepts are often discussed, apparently to attune the different professionals in case of care chains and care networks.

The domain size and availability of services appeared to be important characterising aspects for home care in each country. Both appeared to be problematic in Germany and especially in France, where home nursing is not available at all in some remote areas. In contrast, the home nursing domain is extensive in size in Denmark and the Netherlands, where the local availability of services is guaranteed. Also large differences were found in the degree and kind of autonomy of home nurses. The rather large autonomy home nurses used to have until recently in the Netherlands has been fundamentally affected by the system of indication and managers of home care organisations. In Denmark, their professional autonomy still is quite large, which is also the case for independent nurses in France who work from their own private practices. The attitude within countries towards (very) elderly people also varies; in Denmark, elderly people are encouraged to participate in social life and to remain living in independent households supported by all kinds of facilities, while German elderly are generally considered as a (financial) burden to society and caring burden for the family. French elderly that are still relatively young are encouraged to fully participate in social, leisure and family activities, such as cinema clubs, walking and luncheon clubs, while the very old are prone to social isolation and deprivation of family care in remote areas because their children have left these areas to work elsewhere. Currently, especially after the recent heat waves (canicules) in France, an entirely new network of volunteers is set up by municipalities who regularly check on the health situation of isolated elderly people. The attitude in the Netherlands lies in between that of the other countries. Care is available when people fit the requirements, but the family also plays a large role in providing care and is encouraged by health policies to do so even more.

Managerialism and bureaucracy appeared to be aspects that were especially dominant in the Netherlands, but not so much of a problematic issue in the other three countries. Tight working schedules, administrative tasks such as time-writing to literary account for every minute of care provided, the introduction of many protocols and the lengthy and bureaucratic indication procedure have changed the character of home nursing considerably in the Netherlands during the last ten to twenty years and 
affected its professionalism. In Germany and France, the influence of physicians and dominance of medical perspectives in health care are more prominent issues.

Cultural diversity did not seem to be a hotly debated issue in home nursing in each country, but this may be due to the broad perspective chosen in this study, that inhibited to shed light on possible local problems with the diverging cultural backgrounds of clients. However, the views of the central state regarding social relations and the role of the health care system in caring for vulnerable groups appeared to be very important. Both shape the health policies and health care structures that promote or guarantee the provision of home care on the one hand, or form barriers for the development of the home care domain. In some countries, the state transfers responsibilities to the local level, such as in Denmark, but in other countries, the role of the central state is felt in all aspects of health care and social life, such as in France. In the Netherlands, the role of the state is paradoxical, because it entices continuous changes in health care, while at the same time it promotes deregulation and increasing distance of the state and increased market regulation and competition.

Another aspect that emerged is the difference in health-related values between countries. The balance between medical-curative care provided by physicians and hospitals on the one hand, and nursing care and support provided in the domain of home care on the other hand, appeared to be highly influenced by the perspectives on how to deal with matters of health and disease in countries.

Inclusion of all the aspects mentioned above into the theoretical framework has resulted into a revised framework that can be used for further study. Then, the strengths and weaknesses of this study and its theoretical framework were discussed, actually concluding that the strengths are the weaknesses. Finally, conclusions were drawn on the degree of institutionalisation of home nursing in the four countries. 


\section{Samenvatting / Dutch summary}

In dit boek worden patronen van professionalisering en institutionalisering van de wijkverpleging beschreven in Denemarken, Frankrijk, Nederland en Duitsland. Er is een contextueel en vergelijkend perspectief ontwikkeld om thuiszorg en informele zorg aan kwetsbare en oudere mensen te bestuderen tegen de achtergrond van de specifieke nationale sociaal-culturele en politieke achtergrond en de verschillende stelsels van gezondheidszorg in deze landen.

In hoofdstuk een wordt het doel van het boek beschreven; het verkrijgen van inzicht in internationale verschillen in de wijkverpleging in Europa, evenals in de achtergrond van deze verschillen. Er is voor een macro sociologisch en cultureel perspectief gekozen, omdat uit eerdere studies is gebleken dat verschillen tussen landen op het niveau van gezondheidsstelsels, gezondheids- en gezinsbeleid, en sociale en culturele waarden ten aanzien van langdurige zorg voor kwetsbare en oudere mensen weerspiegeld wordt in internationale verschillen op het micro niveau van thuiszorgpraktijken. Om inzicht te krijgen in de achtergrond van deze verschillen en deze te kunnen verklaren worden deze beschreven binnen hun bredere context en gerelateerd aan verschillen in interprofessionele relaties, relaties tussen verschillende sectoren binnen de gezondheidszorg, de structuren en wijze van financiering van het gezondheidsstelsel, de rol van de familie in de verzorging van ouderen, en verschillen tussen landen in sociale, culturele en politieke normen en opvattingen ten aanzien van gezondheid en ziekte en ten aanzien van de zwakkeren in de samenleving in het bijzonder.

Er worden vier niveaus van analyse van het domein en de professie wijkverpleging beschreven; ten eerste het micro niveau van het domein van de wijkverpleging, ten tweede het intermediaire niveau van de professie wijkverpleging, ten derde het meso niveau van de relaties tussen verschillende professies en sectoren binnen de gezondheidszorg, en tenslotte het macro niveau van de institutionalisering van de wijkverpleging. Om de wijkverpleging op deze verschillende niveaus te kunnen analyseren, zijn verschillende theoretische perspectieven gecombineerd: een gezondheidswetenschappelijk perspectief, een professie-sociologisch perspectief, een neo-institutioneel perspectief op wijkverpleging als maatschappelijke sector, en een cultureel-wetenschappelijk perspectief dat verschillende zorgculturen binnen Europa veronderstelt. 
Vervolgens wordt de keuze van de landen beargumenteerd; namelijk Denemarken, Frankrijk, Nederland en Duitsland. Deze landen liggen geografisch dicht bij elkaar en delen daardoor een aantal belangrijke kenmerken, maar tegelijkertijd is er sprake van belangrijke maatschappelijke, politieke en culturele verschillen die de omstandigheden beïnvloeden waaronder wijkverpleging wordt verleend. Aangezien deze landen in Noordwest, Midden en Zuid Europa liggen, zouden zij meer inzicht moeten geven in verschillen in zorgculturen binnen Europa en de verschillende wijzen waarop landen omgaan met de verouderende bevolking. De verschillende achtergronden resulteren naar verwachting in verschillende constellaties van zorgarrangementen, en dus in verschillende posities van de wijkverpleging binnen de gezondheidsstelsels van deze landen.

Tenslotte wordt het onderzoeksdesign van deze studie beschreven, evenals de aard van de bronnen die gebruikt zijn en de gevolgde methoden. De wijze van gebruik van theorie, procedures van dataverzameling, analyse en het schrijfproces karakteriseren deze studie tot een vergelijkende, institutionele analyse van het domein wijkverpleging en de wijze waarop dit ingebed is in verschillende maatschappijen. Het theoretisch raamwerk dat in hoofdstuk twee wordt opgebouwd functioneert als een overkoepelend perspectief of 'theoretische lens' die uit een aantal richtinggevende concepten bestaat die de dataverzameling sturen en een generalistisch perspectief op de wijkverpleging mogelijk maakt.

In hoofdstuk twee komt het theoretische perspectief van dit boek aan bod. Allereerst worden verschillende tradities in de professiesociologie beschreven, zoals het kenmerkenperspectief, dat er op gericht is om criteria die professies zouden kenmerken te definiëren om het ideaaltype van een professie te kunnen construeren; ten tweede het professionaliseringsperspectief dat ervan uitgaat dat professionele ontwikkeling volgens een bepaalde volgorde van opeenvolgende stappen zou plaatsvinden, waarbij elke volgende stap tot een hogere graad van professionalisering zou leiden; het hiërarchische perspectief dat een bepaalde succesvolle professie als gouden standaard beschouwt waarmee andere professies vergeleken worden om hun mate van professionalisering te kunnen bepalen; en de machtsbenadering binnen de professiesociologie. Daarnaast wordt een invloedrijke visie op professies, het theoretische perspectief van Abbott (I988), besproken. Dit wordt vervolgens als vertrekpunt genomen voor deze studie. Op basis van de uitgangspunten van deze theorie wordt een contextueel en vergelijkend raamwerk ontwikkeld. Abbott gaat ervan uit dat professies bepaalde vormen van expertise claimen in de vorm van jurisdicties over hun domein, en beargumenteert dat professies bestudeerd moeten worden binnen een interacterend netwerk of 'systeem van professies', daarbij rekening houdend met grootschalige maatschappelijke ontwikkelingen.

Vervolgens worden de algemene kenmerken van het domein wijkverpleging beschreven, die de kern vormen van het theoretisch raamwerk dat in dit hoofdstuk wordt ontwikkeld. De wijkverpleging wordt als een speciaal arbeidsveld beschouwd 
waarbij wijkverpleegkundigen breed inzetbaar zijn en een grote autonomie hebben omdat ze niet onder supervisie werken van professionals met een hogere hiërarchische status. De tweede 'schil' van het theoretische raamwerk verwijst naar het systeem van professies, zoals taakdifferentiatie, interprofessionele samenwerking en coördinatie. De scholing en opleiding van wijkverpleegkundigen zijn ook van belang om inzicht te krijgen in verschillen tussen wijkverpleegkundigen in de vier landen, evenals de positie van de patiënt en de impact van bureaucratisering. De buitenste schil van het raamwerk bevat concepten die refereren aan de maatschappelijke context en de context van het gezondheidsstelsel waarbinnen wijkverpleging verleend wordt, zoals het type gezondheidsstelsel, beleid ten aanzien van gezondheidszorg en ouderen, de impact van de toenemende culturele diversiteit van Europese samenlevingen en de impact van perspectieven van managers, in plaats van professionele perspectieven, op wijkverpleging.

Hoofdstuk drie vormt een achtergrondhoofdstuk voor elk van de volgende hoofdstukken, en biedt informatie over de algemene verschillen in thuiszorg binnen Europa, de belangrijkste kenmerken van gezondheidsstelsels, en geeft een overzicht van demografische gegevens en de belangrijkste kenmerken van de vier landen. Deze worden samengevat in tabellen en grafieken aan het slot van dit hoofdstuk.

In hoofdstuk vier tot zeven wordt de rol van de wijkverpleging in elk land beschreven, binnen de context van de gezondheidszorg en de sociaal-culturele en politieke achtergrond van elk land. De concepten uit het theoretisch raamwerk en de specifieke maatschappelijke achtergrond en die van de gezondheidszorg worden als uitgangspunt voor de beschrijvingen genomen.

In hoofdstuk vier wordt het domein wijkverpleging in Denemarken beschreven. Denemarken heeft een nationaal gezondheidsstelsel (NHss; National Health Security System), dat kenmerkend is voor Scandinavische sociaal-democratische regimes. Universalistische principes en dominantie van de staat staan op de voorgrond, hetgeen betekent dat rechten, diensten en uitkeringen beschikbaar zijn voor de gehele bevolking en losgeweekt worden van taken die traditioneel door gezinsleden of het sociale netwerk werden vervuld. Doordat individuen ontheven worden van familiale verantwoordelijkheden kan maximale individuele onafhankelijkheid bereikt worden. De algemene opvatting in de maatschappij en in de politiek is dat de welvaartsstaat publiek georganiseerde gezondheidszorg en maatschappelijke zorg moet garanderen, en dat familieleden niet formeel verplicht zijn om zorg te verlenen aan ouderen en chronisch zieken. Daardoor speelt informele zorg geen belangrijke rol. De hoge belastingen vormen de keerzijde van dit systeem. Dit contrasteert sterk met Frankrijk, Duitsland en Nederland, waar familiesolidariteit wordt benadrukt en waar zorgverlening aan ouderen en kwetsbaren middels gezondheidsbeleid wordt aangemoedigd, en waar professionele zorg 
door niet-publieke organisaties wordt verleend. In Denemarken wordt professionele zorgverlening vanuit de overheid georganiseerd en als universeel recht beschouwd, waarbij algemene toegankelijkheid en beschikbaarheid van gezondheidszorgvoorzieningen centrale principes zijn. Het aanbod van gezondheidszorg is sterk gedecentraliseerd; de meeste voorzieningen worden op het lokale niveau verleend en gefinancierd. De centrale staat biedt enkel een overkoepelend wettelijk raamwerk. Het gezondheidsstelsel is vrij stabiel omdat er geen belangrijke hervormingen hebben plaatsgevonden in de laatste decennia en er enkel geleidelijke aanpassingen geïntroduceerd zijn. Er is nauwelijks sprake van marktwerking omdat dit niet als nastrevenswaardig principe wordt beschouwd in de Scandinavische gezondheidszorg.

Kenmerkend voor ouderenzorg in Denemarken is de radicale overgang van institutionele zorg naar ambulante ouderenzorg. Tot de jaren zeventig hadden gemeentelijke verplichtingen om zowel langdurige thuiszorg als institutionalisering van ouderen te organiseren prioriteit. In I987 werd echter nieuwe wetgeving geïntroduceerd om de woonfunctie en de zorgfunctie te scheiden, en verpleeghuiszorg te ontmoedigen. Thuiszorg kon vanaf dan onafhankelijk van waar mensen woonden verleend worden. Nieuwe verpleeghuizen mochten niet meer gebouwd worden en veel bestaande verpleeghuizen werden gesloten. Vanaf die periode is gezondheidsbeleid in Denmarken gebaseerd op de ideologie van 'ouder worden in de eigen omgeving', hetgeen betekent dat ouderen in staat gesteld moeten worden om zo lang mogelijk in hun eigen woning of in zelfstandige aangepaste ouderenwoningen te leven. Institutionalisering zou zoveel mogelijk voorkomen moeten worden en de autonomie en het zelfhulpvermogen van ouderen zouden ondersteund moeten worden door publieke voorzieningen die door gemeenten aangeboden worden.

De traditie van wijkverpleging in Denemarken ligt rond het eind van de negentiende eeuw. De wijkverpleegkundige is tegenwoordig nog steeds een centrale persoon ten tijde van ziekte van een oudere. Een generalistische zorgopvatting en continuïteit van zorg zijn belangrijke principes in Denemarken. Er worden geen specifieke verpleegkundige expertise-niveaus onderscheiden, en alle verzorgende beroepen hebben dezelfde basisopleiding. Denemarken is het enige land waar wijkverpleging, gezinszorg en institutionele ouderenzorg geheel geïntegreerd zijn. Deze ambulante ouderenzorg wordt aangeboden en gefinancierd de lokale gemeenten, waar de verpleegkundigen in dienst zijn. Zij verlenen hun zorg onafhankelijk van waar ouderen wonen; bij hen thuis, in verpleeghuizen, of in speciale ouderenappartementen. Deense wijkverpleegkundigen hebben meer beslissingmacht en autonomie in vergelijking met hun collega's in andere landen, waar medische professionals (zoals in Duitsland en Frankrijk), of 'objectieve indicatie-organisaties' (zoals in Nederland) de behoefte aan wijkverpleging en de te verlenen zorg bepalen. Het vertrouwen in professionals die in de gezondheidszorg werkzaam zijn en hun sociale status is tamelijk hoog in Denemarken, hetgeen tot uiting komt in de grotere mate van autonomie en zeer geringe bemoeienis van managers of bureaucratische procedures. Zowel wijkverpleegkundigen als 
wijkziekenverzorgenden verlenen wijkverpleging, maar er is geen aparte lijst van taken voor ieder gespecificeerd; beide verlenen eenvoudige en complexe taken en verdienen hetzelfde salaris. Dit is een belangrijk verschil ten opzichte van andere landen, waar taakdifferentiatie een belangrijk organisatorisch principe is. Bijna alle verpleegkundigen zijn lid van de Deense Organisatie voor Verpleging DSR die sinds I899 bestaat.

In hoofdstuk vijf wordt het domein wijkverpleging in Frankrijk beschreven. Een van de meest opvallende aspecten ten aanzien van de wijkverpleging in Frankrijk is de onevenwichtige beschikbaarheid van wijkverpleging in het land. Door de onevenwichtige verdeling van mensen over zeer dichtbevolkte gebieden zoals enerzijds Parijs en omgeving, en anderzijds zeer schaars bevolkte gebieden in het midden en zuiden van Frankrijk, zijn sociale, economische en gezondheidszorgvoorzieningen ook ongelijk verdeeld over de regio's. Met name ambulante diensten zoals wijkverpleging en gezinszorg zijn niet evenwichtig verdeeld over het land en zijn in afgelegen gebieden soms zelfs geheel afwezig. De tendens van oudere Fransen is echter om 'te blijven waar ze zijn', zelfs als gezondheids-, woon- en sociale voorzieningen niet overeenkomen met hun behoeften. Hierdoor leven veel ouderen in de schaars bevolkte gebieden met weinig voorzieningen sociaal geïsoleerd en in armoedige leefomstandigheden.

Andere kenmerken van het Franse gezondheidsstelsel zijn de oriëntatie op medische en institutionele zorg. Fransen hebben een vrij medische, curatief georiënteerde houding ten aanzien van zaken die met gezondheid en ziekte te maken hebben en geven over het algemeen blijk van een biomedische definitie van ziektes. Daarbij is de verwachting sterk om genezen te worden door medisch ingrijpen en medicijnen. De medische sector, die zowel huisartsenzorg als medisch specialisten en ziekenhuizen omvat, heeft duidelijk prioriteit ten opzichte van de verplegende en verzorgende sector in het gezondheidsstelsel, dat zo georganiseerd is dat medische zorg en ziekenhuiszorg gemakkelijk toegankelijk zijn voor patiënten. Deze mogen de arts van hun keuze zo vaak consulteren als zij willen en mogen verschillende artsen over een klacht consulteren, en hebben directe toegang tot privéspecialisten en alle typen ziekenhuizen. In tegenstelling daartoe is ambulante zorg niet direct toegankelijk, omdat een verwijzing van een arts of maatschappelijk werker vereist is. Ook bestaan er financiële barrières ten aanzien van ambulante zorg, door een aantal zeer complexe en overlappende voorzieningen waarbij allerlei vergoedingenstelsels en overheidsniveaus betrokken zijn. Het (bio)medische en institutionele karakter van de gezondheidszorg wordt ook weerspiegeld in het relatief hoge aantal huisartsen en specialisten, het aantal bedden per I 0 o inwoners en de zeer hoge uitgaven aan medicijnen in vergelijking met andere Europese landen.

Een vierde kenmerkend aspect van Frankrijk is dat de staat een belangrijk machtscentrum is dat merkbaar is in alle aspecten van het leven in Frankrijk, ook ten aanzien van het gezondheidsstelsel en professionals in de gezondheidszorg. Ouderenzorg in Frankrijk wordt gekenmerkt door een dichotomie tussen het medische en institutionele 
domein die onder verantwoordelijkheid van de nationale overheid vallen, en het sociale domein dat onder de lokale overheden valt. Dit wordt ook weerspiegeld in de thuiszorg, waar twee vormen van thuiszorg bestaan. Bij Hospitalisation à Domicile (HAD), te vertalen als ziekenhuiszorg in de thuissituatie, krijgen patiënten met zeer ernstige en complexe aandoeningen zorg in hun eigen huis. Dit wordt georganiseerd vanuit ziekenhuizen, terwijl Service de Soins Infirmiers à Domicile pour les Personnes Agées (SSIAD), te vertalen als de Verpleegkundige thuiszorgdienst voor ouderen, vergelijkbaar is met thuiszorg zoals deze in andere Europese landen wordt verleend, namelijk ambulante wijkverpleging en gezinszorg die verleend wordt aan kwetsbare en oudere mensen in hun eigen huis. Een verwijzing van een arts is noodzakelijk om voor vergoeding van beide typen thuiszorg door de ziektekostenverzekering in aanmerking te komen. De geografische reikwijdte van thuiszorgorganisaties varieert enorm in Frankrijk, evenals het aanbod van diensten; soms verlenen ze enkel wijkverpleging, soms is er sprake van een heel breed aanbod aan diensten, waaronder maaltijdverstrekking, alarmdiensten en/of moeder- en kindzorg. Een groot probleem in Frankrijk is dat veel mensen die zorgbehoeftig zijn geen zorg krijgen, zodat informele zorgverlening door het sociale netwerk (familie, vrienden, mensen uit de omgeving) een grote rol speelt. Oudere alleenstaande vrouwen vormen een kwetsbare groep; sociale isolatie is een wezenlijk probleem, vooral als hun familieleden de regio verlaten hebben.

Sinds de jaren zestig van de twintigste eeuw is ouderenbeleid ontwikkeld. In de meeste rapporten werd het ideaal beschreven van oudere mensen die in hun eigen omgeving ouder worden, daarbij ondersteund door hun familie. Sinds de jaren tachtig is het domein thuiszorg enorm in omvang toegenomen, maar het beroep dat op informele zorg wordt gedaan is nog steeds hoog, omdat de beschikbare zorg niet voldoende is om te voorzien in de zorgbehoeften van vele ouderen. In veel gebieden werken zelfstandige verpleegkundigen die een groot aandeel van de zorg op zich nemen vanuit hun privépraktijken. Tegenwoordig worden er discussies gevoerd over de rol van reguliere verpleegkundigen die in dienst zijn van de SSIAD thuiszorgorganisaties en de zelfstandige verpleegkundigen. Deze laatste claimen de rol van coördinator van medische, verpleegkundige en sociale zorg voor individuele cliënten, maar uit onderzoek blijkt dat zij onvoldoende kennis hebben over de lokale situatie en beschikbare professionals. Een zeer groot aandeel van thuiszorg wordt verleend door ziekenverzorgsters en gezinsverzorgsters.

Uit de literatuur ontstond het beeld dat het domein gezinszorg sociaal en cultureel meer geaccepteerd is dan het domein wijkverpleging. Ouderen en hun familieleden beschouwen wijkverpleegkundigen over het algemeen als 'vreemden' die in de persoonlijke levenssfeer binnendringen. Vanwege het stigma dat met professionele hulp verbonden is is men terughoudend om wijkverpleegkundige zorg aan te vragen; mensen zijn bang dat dit erop wijst dat hun familie niet in staat is om goed voor hun oudere familieleden te zorgen. Een ander verschil in vergelijking met andere landen is dat thuiszorgprofessionals een andere houding aannemen ten aanzien van hun cliënten 
en andere professionele waarden belangrijk te vinden dan elders; persoonlijk contact en het ondersteunen van cliënten wordt benadrukt boven waarden als efficiëntie, rationaliteit en het bewaren van een professionele afstand, die in veel andere landen voorop staan in professionele zorgverlening.

In hoofdstuk zes wordt de wijkverpleging in Nederland beschreven. De geschiedenis van de wijkverpleging, in de vorm van private, niet-gouvernementele, lokale en regionale Kruisorganisaties dateert van ongeveer I880, net als in Denemarken. Tijdens de twintigste eeuw heeft zich over het hele land een dicht netwerk van wijkverpleegkundige diensten ontwikkeld, waardoor wijkverpleging in alle delen van Nederland gegarandeerd werd. Sinds het begin van de twintigste eeuw, en in het bijzonder sinds de jaren dertig, namen kruisorganisaties een leidende rol op zich in het zich toe-eigenen van moeder- en kindzorg tot de verantwoordelijkheden van wijkverpleegkundigen. Ze ontwikkelden hiermee allround expertise ten aanzien van alle aangelegenheden rond gezondheid, ziekte en preventie aan gezinnen die in hun wijk of werkgebied woonden. Wijkverpleegkundigen golden lokaal als maatschappelijke autoriteit naast de huisartsen.

Over het algemeen is er in Nederland sprake van een gunstige houding ten aanzien van zorg (zorggerichtheid). Er bestaan veel voorzieningen voor mensen die behoefte hebben aan zorg en ondersteuning, zoals verzorgingshuizen, senior appartementen met collectieve voorzieningen voor ouderen, verpleeghuizen, en een vrij dicht netwerk van wijkverpleging en gezinszorg. Dit wordt aangevuld met regelingen die informele zorgverlening door familieleden, buren en vrienden aanmoedigen, zoals het Persoons Gebonden Budget (PGB) waarmee ouderen hun informele zorgverleners kunnen betalen. Daarnaast wordt zorgverlening beschouwd als een zelfstandige professionele activiteit, met een eigen specifiek en omschreven domein, en niet slechts als ondersteunende activiteit van de medische professie. Dit staat in contrast met landen als Frankrijk en Duitsland, waar verpleegkundige zorg meer beschouwd wordt als een verlengstuk van medische activiteiten. Desalniettemin bestaan er verschillende problemen in de thuiszorg in Nederland, in toenemende mate tijdens de laatste decennia.

Vanaf de jaren zeventig zijn er vele veranderingen geïntroduceerd in het Nederlandse gezondheidsstelsel, die ook grote effecten hadden op de wijkverpleging. Zo werden elementen van marktwerking geïntroduceerd en bezuinigingen doorgevoerd. In de laatste decennia is er in de Nederlandse maatschappij een sterke tendens om dingen op een topdown wijze te organiseren en voorstructureren, hetgeen ook wel managementisme genoemd wordt, en om op bureaucratische wijze met zaken om te gaan door regels en regelingen en administratieve procedures te ontwerpen en hiernaar te verwijzen. De belangrijkste concepten van managementisme omvatten efficiëntie, regels en principes, algemene geldigheid en onpersoonlijkheid (in de vorm van neutrale professionele distantie). Ook in de gezondheidszorg en wijkverpleging is dit duidelijk merkbaar. Delen van het werk die altijd tot de kern van de wijkverpleegkun- 
dige professie behoorden zijn overgedragen aan externe instanties en actoren, zoals in eerste instantie de RIo's (regionale indicatie organen) en later het CIz (centrum indicatiestelling zorg), waardoor het karakter van wijkverpleegkundige activiteiten en de mate van professionaliteit aangetast werd. Sinds I997 beslissen deze instellingen over het recht op zorg van patiënten binnen de AwBz op basis van geobjectiveerde procedures. Voorheen verrichten wijkverpleegkundigen de intake van cliënten zelf. Er wordt beargumenteerd dat dit geresulteerd heeft in verminderde professionalisering van de wijkverpleegkunde in Nederland. Ook is de speelruimte voor wijkverpleegkundigen in de dagelijkse praktijk verminderd en is de vrijheid beperkter geworden om zelfstandig beslissingen te nemen op basis van hun professionele expertise, vaardigheden en ervaring, door de toenemende toepassing van richtlijnen voor de praktijk en gestandaardiseerde protocollen. De huidige tendens van Evidence Based gezondheidszorg, die ook steeds sterker wordt in de verpleegkunde, wordt bekritiseerd omdat de professionele expertise, die gebaseerd was op hun opleiding en specifieke, contextgerelateerde ervaringen, geleidelijk plaats heeft gemaakt voor de 'gemiddelde professional'. Deze past gestandaardiseerde kennis op een juiste manier en volgens de meest recente procedures en inzichten toe, zodat cliënten adequate zorg en behandeling krijgen, onafhankelijk van hun specifieke situatie en door om het even welke professional. Organisatorische veranderingen hebben het ook gemakkelijker gemaakt voor thuiszorgorganisaties om professionals volgens dienstroosters in te plannen, zodat zij niet langer verantwoordelijk zijn voor specifieke individuele cliënten of gezinnen in hun eigen werkgebied. Voor cliënten heeft dit tot gevolg dat continuiteit van zorg door een of enkele professionals niet meer gewaarborgd kan worden en zij vaak met veel verschillende professionals te maken hebben. Daarnaast oefenen anderen dan wijkverpleegkundigen zelf, in het bijzonder managers en beleidsmakers, meer controle uit over hun werk. Deze ontwikkelingen lijken nu de overhand te hebben boven de professionele en persoonlijke aspecten van zorgverlening. Uit de vergelijking van de vier landen in dit boek lijken deze ontwikkelingen vooral in Nederland zeer sterk.

In 1997 werd een nieuw opleidingssysteem geïntroduceerd in de verpleging, dat geleid heeft tot een onderverdeling in verschillende niveaus met verschillende verantwoordelijkheden. Een andere belangrijke verandering was de samenvoeging van gezinszorg en wijkverpleging tot geïntegreerde thuiszorg in de jaren negentig, die door thuiszorgorganisaties verleend wordt die meer en meer in omvang toenemen door elkaar opeenvolgende fusies. Tegelijkertijd is het beroep op informele zorgverleners om een groot deel van de zorg op zich te nemen groter geworden. De beschikbaarheid van informele zorgverleners wordt nu geïnventariseerd tijdens de intake procedure: ouderen en kwetsbare mensen die familieleden of partners hebben die zorg kunnen verlenen kunnen niet langer aanspraak maken op zorg. Informele zorg is dus een plicht geworden die in procedures geformaliseerd is. Dus, ondanks dat wijkverpleging in Nederland een vrij gunstige positie inneemt in het gezondheidsstelsel en het aanbod aan zorg aan kwetsbare groepen gegarandeerd wordt in het hele land, is er sprake 
van ernstige problemen in de thuiszorg. Een belangrijke conclusie uit dit hoofdstuk is dat het professionele karakter en de autoriteit van wijkverpleegkundigen in de laatste decennia ingrijpend veranderd is.

In hoofdstuk zeven wordt de wijkverpleging in Duitsland beschreven. Een van de bevindingen was dat ouderen als een problematische maatschappelijke groep worden beschouwd. Lange tijd zijn ze een min of meer vergeten groep geweest die zich met gezondheids- en financiële problemen zelf en binnen hun familie moesten zien te redden. Vergeleken met veel andere Europese landen was Duitsland vrij laat met het ontwikkelen van beleid gericht op ouderen in de samenleving. Het huidige sociale en economische beleid is voor een groot deel verantwoordelijk voor een pessimistische visie ten aanzien van ouderen aangezien zij als een maatschappelijke last worden beschouwd. Twijfel of de nationale economie voldoende in staat is om te voorzien in de toenemende maatschappelijke en zorgbehoeften van ouderen staat in Duitsland op de voorgrond, vanwege het feit dat het aantal (zeer) ouderen het hoogste is binnen de EU. Gedurende veel decennia hebben zorgverlenende en ondersteunende activiteiten, die in de 'periferie' van het gezondheidsstelsel worden verleend, geen erg hoge prioriteit gehad in Duitsland, vergeleken met de medische en cure georiënteerde activiteiten die centraal staan in het 'medisch centrum' van de gezondheidszorg. Het Duitse gezondheidsstelsel is zeer medisch en technologisch georiënteerd, en medische en ziekenhuiszorg hebben traditioneel altijd prioriteit gehad boven andere vormen van zorg. Verpleging wordt over het algemeen niet als een onafhankelijke activiteit beschouwd in Duitsland, maar voornamelijk als een verlengstuk van medische activiteiten en aanvulling op medische zorg of ziekenhuiszorg. Verpleegkundige taken zijn meer medischtechnisch van karakter dan in andere Europese landen; wijkverpleegkundigen geven bijvoorbeeld ook veel injecties.

Het domein van professionele zorgverlening, zoals wijkverpleging, heeft lange tijd te maken gehad met financiële alsook structurele barrières. Procedures om te bepalen of mensen recht hebben op wijkverpleging zijn voor een groot deel in handen van artsen: een medische verwijzing is vereist om in aanmerking te komen voor vergoeding van verpleegkundige zorg. Terwijl medische behandeling al lange tijd een wettelijk afdwingbaar recht is dat gebaseerd is op verzekeringen, is dat met verpleegkundige zorg slechts het geval sinds de introductie van de Pflegegesetz in 1995. Binnen het kader van de verzekeringen is er een strikte hiërarchie van recht op zorgverlening, aflopend van medische behandeling, gespecialiseerde verpleging, basisverpleging, naar gezinszorg dat slechts als aanvulling op verpleging vergoed wordt. In het gezondheidsstelsel is sprake van inherente barrières ten aanzien van langdurige ambulante zorg, terwijl toegang tot artsen, medisch specialisten en ziekenhuizen gemakkelijk is.

Naast cure georiënteerde activiteiten in de gezondheidszorg wordt er in hoge mate gesteund op de familie om zorg en ondersteuning te bieden. Traditionele familiewaarden zijn sterk in Duitsland, en er geldt een morele verplichting (en tot de invoering van 
de Pflegeversicherung ook een wettelijke verplichting) voor familieleden om mantelzorg te verlenen aan oudere en behoeftige mensen. Zoals in veel Europese landen wordt de nadruk op de verantwoordelijkheden van familieleden weerspiegeld in de rol van de staat in de gezondheidszorg. Het verlenen van welzijns- en maatschappelijke zorg valt onder het subsidiariteitsbeginsel. Dit betekent dat de gemeenschap allereerst actie moet ondernemen, en dat de staat enkel ingrijpt als er geen alternatieven voorhanden zijn. Er zouden geen voorzieningen geboden moeten worden door de staat als andere organisaties die taken naar voldoening kunnen uitvoeren uit naam van de staat. Volgens dit principe moet de staat enkel als vangnet functioneren. Het beleid in Duitsland ten aanzien van langdurige zorg aan kwetsbare ouderen weerspiegelt het geloof in familiale solidariteit. Publieke initiatieven die professionele zorgverlening ondersteunen hebben zich slechts langzaam ontwikkeld, en beleid heeft zich voornamelijk gericht op het versterken van familiezorg. Daarom wordt hulp en steun voornamelijk binnen de familie gezocht.

Sinds de jaren negentig van de twintigste eeuw werd gezondheidsbeleid voor ouderen geleidelijk, maar aarzelend ontwikkeld. Tegenwoordig worden de belangen van ouderen in Duitsland nog steeds vaak genegeerd in het proces van beleidsvorming, in tegenstelling tot bijvoorbeeld in Denmarken. Ook is de verschuiving van institutionele zorg naar ambulante zorg in het Duitse ouderenbeleid vrij recent vergeleken met andere Europese landen. Verschillende typen organisaties verlenen ambulante zorg aan ouderen en andere patiëntencategorieën: de Ambulante sozialpflegerische Dienste (organisaties voor ambulante maatschappelijke en verpleegkundige zorg). Hiervan kunnen met name de Sozialstationen, die sinds de jaren zeventig zijn opgericht, als erg belangrijk beschouwd worden voor het verlenen van zorg bij mensen thuis. Ook wordt er veel maatschappelijke en verpleegkundige zorg geboden door organisaties die onder zes nationale koepels van welzijnsorganisaties vallen. De meeste van deze hebben een religieuze signatuur, zoals het Protestantse Diakonisches Werk en de Katholieke Caritas. Binnen deze organisaties verlenen een groot aantal verschillende professionals hulp aan ouderen. Samenwerking tussen verschillende soorten professionals en organisaties gebeurt echter nauwelijks, vanwege grenzen tussen het maatschappelijke domein en het gezondheidszorgdomein, de invloed van artsen die geen voorstander zijn van samenwerking, en de gefragmenteerde wijze waarop thuiszorg verleend wordt door veel verschillende soorten organisaties en professionals.

In hoofdstuk acht, de conclusie, worden de vier landen vergeleken middels het theoretisch perspectief dat in het tweede hoofdstuk ontwikkeld is. De afzonderlijke conclusies ten aanzien van de wijkverpleging en ouderenzorg uit hoofdstuk vier tot en met zeven worden vergeleken en geïntegreerd. Op basis hiervan worden aanpassingen van het theoretische perspectief voorgesteld. Sommige aspecten bleken minder belangrijk te zijn, terwijl enkele nieuwe aspecten die in eerste instantie niet waren opgenomen, wel belangrijk bleken te zijn. De periode waarin de wijkverpleging ontstond bleek bij- 
voorbeeld belangrijk te zijn voor de ontwikkeling van de wijkverpleging en de vestiging daarvan als zelfstandig domein naast het medische domein. Het contrast tussen de vrij sterke positie van de wijkverpleging in de gezondheidszorg en maatschappij in Denemarken en Nederland, waar de geschiedenis teruggaat tot de negentiende eeuw, is groot vergeleken met de relatief jonge vestiging van de reguliere thuiszorgorganisaties in Frankrijk. Ook werd er een sterk contrast gevonden tussen landen waar wijkverpleegkundigen een zelfstandige rol en verantwoordelijkheid hebben, zoals in Denemarken en Nederland, en in landen waar hun activiteiten als verlengstuk of ondersteuning van medische activiteiten beschouwd wordt, zoals in Frankrijk en Duitsland.

Algemene ontwikkelingen in Europa zijn de toenemende aandacht voor kwetsbare en (zeer) oudere mensen als belangrijkste cliëntenpopulatie, en de toenemende taakdifferentiatie en specialisatie van verpleegkundigen ten koste van het allround profiel, dat enkel nog prominent is in Denemarken. De opleiding en scholing van wijkverpleegkundigen zijn in elk land verschillend. Denemarken is het enige land waar verpleegkundigen als generalisten worden opgeleid en waar geen verschillende verpleegkundige niveaus bestaan. De aspecten samenwerking en coördinatie kregen relatief weinig aandacht in de literatuur, behalve in Nederland waar deze vaak bediscussieerd worden, om afstemming te bewerkstelligen tussen de verschillende professionals in het geval van zorgketens of zorgnetwerken.

De omvang van het domein en de beschikbaarheid van zorg bleken belangrijke kenmerkende aspecten te zijn voor de thuiszorg in elk land. Beide zijn problematisch in Duitsland en met name Frankrijk, waar wijkverpleging in sommige afgelegen gebieden helemaal niet beschikbaar is. In tegenstelling hiermee is het wijkverpleegkundige domein omvangrijk in Denemarken en Nederland, waar de lokale beschikbaarheid van diensten gegarandeerd wordt. Ook werden er grote verschillen gevonden in de mate van en het type autonomie van wijkverpleegkundigen. De vrij grote autonomie die wijkverpleegkundigen tot voor kort in Nederland hadden is fundamenteel aangetast door het indicatiestelsel en managers van thuiszorgorganisaties. In Denemarken is hun professionele autonomie nog steeds vrij groot, hetgeen ook het geval is bij zelfstandige verpleegkundigen in Frankrijk die vanuit hun eigen privépraktijk werken. De houding ten aanzien van (zeer) oudere mensen varieert ook per land: in Denemarken worden ouderen aangemoedigd om in het maatschappelijke leven te participeren en in zelfstandige huishoudens te blijven wonen, daarbij ondersteund door allerlei soorten gemeentelijke faciliteiten, terwijl Duitse ouderen over het algemeen als (financiële) last beschouwd worden voor de maatschappij en zorglast voor de familie. Franse ouderen die nog relatief jong zijn worden aangemoedigd om volledig in het maatschappelijke leven, in activiteiten in de vrije tijd en in het familieleven te participeren, zoals filmclubs, wandel- en lunchclubs. De zeer oude mensen in afgelegen gebieden zijn daarentegen kwetsbaar voor sociale isolatie en beschikken niet over familiezorg, omdat hun kinderen deze regio's verlaten hebben op zoek naar werk elders. Tegenwoordig, met name sinds de recente hittegolven (canicules) in Frankrijk, wordt een geheel nieuw 
netwerk van vrijwilligers opgezet door gemeenten die regelmatig de gezondheidssituatie van geïsoleerde ouderen controleert. De houding in Nederland ligt hier tussenin. Zorg is beschikbaar als mensen aan de vereisten doen, maar de familie speelt ook een grote rol in de zorgverlening en wordt door gezondheidsbeleid en indicatieregelingen aangemoedigd om dit nog meer te doen.

Managementisme en bureaucratie bleken aspecten te zijn die met name in Nederland dominant zijn, maar niet zozeer een problematische kwestie vormen in de andere drie landen. Krappe tijdsschema's, administratieve taken zoals tijdschrijven om letterlijk elke minuut zorg die verleend is te verantwoorden, de introductie van veel protocollen en de langdurige en bureaucratische indicatie-procedure hebben het karakter van de wijkverpleging behoorlijk veranderd in Nederland tijdens de laatste tien tot twintig jaar en de professionaliteit aangetast. In Duitsland en Frankrijk zijn de invloed van artsen en de dominantie van medische perspectieven in de gezondheidszorg meer prominente kwesties.

Culturele diversiteit leek niet zo'n sterk bediscussieerde kwestie te zijn in de wijkverpleging in elk land, maar dit zou kunnen komen door het brede perspectief van deze studie, waardoor geen inzicht verkregen is in potentiële lokale problemen ten aanzien van de verschillende culturele achtergrond van cliënten. Echter, de visie van de centrale overheid ten aanzien van sociale relaties en de rol van het gezondheidszorgstelsel in de verzorging van kwetsbare groepen bleek zeer belangrijk te zijn. Beide geven vorm aan gezondheidsbeleid en structuren van gezondheidszorg die enerzijds het aanbod van thuiszorg garanderen of aanmoedigen, of anderzijds barrières vormen voor de ontwikkeling van het thuiszorgdomein. In sommige landen hevelt de staat verantwoordelijkheden over naar het lokale niveau, zoals in Denemarken, terwijl in andere landen de rol van de centrale overheid gevoeld wordt in alle aspecten van de gezondheidszorg en het maatschappelijk leven, zoals in Frankrijk. In Nederland is de rol van de overheid paradoxaal, omdat het continue veranderingen in de gezondheidszorg in gang zet, terwijl het tegelijkertijd deregulering promoot, evenals een toenemende afstand van de overheid en toenemende marktregulering en -competitie.

Een ander aspect dat naar voren kwam is het verschil in gezondheidsgerelateerde waarden tussen landen. De balans tussen medisch-curatieve zorg die door artsen en ziekenhuizen verleend wordt aan de ene kant, en verpleegkundige thuiszorg aan de andere kant, bleek in hoge mate beïnvloed te zijn door de perspectieven die men inneemt ten aanzien van het omgaan met ziekte en gezondheid.

Het opnemen van al deze bovengenoemde aspecten in het theoretisch perspectief heeft geresulteerd in een herzien raamwerk dat voor verder onderzoek gebruikt kan worden. Daarna zijn de sterke en zwakke kanten van deze studie en het theoretisch perspectief aan bod gekomen, daarbij concluderend dat de sterke kanten de keerzijden van de zwakke kanten vormen. Tenslotte zijn er conclusies getrokken ten aanzien van de mate van institutionalisering van de wijkverpleging in de vier landen. 


\section{Dankwoord / Acknowledgements}

Later dan ik had kunnen vermoeden toen ik aan dit proefschrift begon, ligt het resultaat voor $\mathrm{u}$. Ik ben blij dat het nu zover is. De aard van het onderzoek, dat niet tevoren uitgestippeld was maar gaandeweg ontwikkeld is en waarbij vele zijpaden bewandeld zijn om een zeer omvattend beeld van de thuiszorg te kunnen schetsen, evenals mijn werk- en thuissituatie hebben ertoe bijgedragen dat het langer geduurd heeft dan verwacht. Tegelijkertijd met het schrijven van dit boek zijn in deze periode ook twee lieve en bijzondere kinderen geboren, is mijn man professor geworden en heb ik een moeizaam revalidatieproces doorlopen vanwege een complexe kniebreuk. Door al deze factoren was het niet altijd een gemakkelijk proces, maar doorzetten loont en ik ben trots op het resultaat.

Dit boek had niet geschreven kunnen zijn zonder mijn promotor Hans Philipsen en co-promotor Fred Stevens. Hun ideeën over professies lagen aan de basis van dit proefschrift en hun begeleiding en inspiratie zijn essentieel geweest voor de totstandkoming van dit boek. Beste Hans, ik waardeer het zeer dat u mijn promotor bent. Uw eruditie en onuitputtelijke ideeën hebben mij steeds geïnspireerd en weer van nieuwe inzichten voorzien. Uw kennis, ervaringen en anekdoten over de onderwerpen van het onderzoek hebben in hoge mate bijgedragen aan de inhoud van dit boek. Ik vind het heel speciaal dat ik heb mogen profiteren van uw enorme ervaring met promovendi en dat $u$ ook na uw officiële pensionering zeer nauw bij dit onderzoek betrokken bent geweest. Uw inhoudelijke kennis en steun tijdens het hele proces zijn zeer waardevol geweest voor dit boek en voor mij als persoon. U toonde altijd begrip en geduld voor de omstandigheden die dit boek omringd hebben en heeft mij door moeilijke perioden heen geloodst. Ik heb veel bewondering voor de wijze waarop u met mensen omgaat en heb hier veel van geleerd. Bejegening is niet alleen in de gezondheidszorg belangrijk, maar ook in de omgang met andere mensen in het algemeen.

Fred, je bent nauw betrokken geweest bij dit onderzoek en we hebben vele gesprekken gevoerd. Je kennis over de professiesociologie en over gezondheidsstelsels is zeer waardevol geweest voor dit boek. De congressen die we in Lissabon en Versailles bezocht hebben vond ik inspirerend en de gezamenlijke bezichtigingen en etentjes waren zeer aangenaam. Je was altijd bereid om stukken van mij te lezen en deze tot in detail te becommentariëren en behoedde mij samen met Hans voor te normatieve en 
geëngageerde standpunten. Dit heeft het sociologische perspectief in het proefschrift versterkt en ik denk dat dat goed is. Bedankt voor je inzet tijdens al deze jaren.

De periode die ik als AIo bij de vakgroep Medische Sociologie gewerkt heb was een speciale periode met speciale mensen. De sfeer bij Medische Sociologie, die vooral tot uiting kwam in de humor en conversaties bij de thee, was heel bijzonder. De grote diversiteit aan karakters gaf een specifieke dynamiek en er was sprake van een gemeenschappelijke band, ook al was ieder met soms zeer uiteenlopende onderwerpen of activiteiten bezig. Ik hoop dat de mensen die tot deze vakgroep behoorden ook vanuit hun huidige werkplek kunnen bijdragen aan een bijzondere sfeer die het tot fijn werken maakt. Enkele mensen wil ik graag noemen: Jos Diederiks, Kees Schröer, Ronald Knibbe, Paul Lemmens, Jan Joosten, Jouke van der Zee, Miriam Janssen, Susan Niemantsverdriet en natuurlijk het secretariaat, bemensd door Marjoke Arkenbout, Sylvia Nelk en Liliane Hesemans, en niet te vergeten de altijd-bereid-om-te-helpen Arnold van Alphen. Zij vormden voor mij de 'vaste kern' van Medische Sociologie die altijd belangstelling toonden voor mijn onderzoek en mijn gezin. Gelukkig spreek ik velen van hen nog regelmatig. Daarnaast zijn er nog vele anderen geweest bij Medische Sociologie waarmee ik aangenaam contact gehad heb, waaronder Ruud Kempen, Hans Bosma, Jacques van Eijk, en vele AIo's waarvan ik vooral Marja Veenstra, Rixt Zijlstra en Godelief Mars wil noemen.

De collega's van vakgroepen waar ik daarna gewerkt heb of nog werk, bij BEOz, Metamedica en International Health, wil ik graag bedanken voor hun interesse en collegialiteit. Rein Vos, Anja Krumeich en Agnes Meershoek (bij wie ik als studentassistente gewerkt heb) wil ik bedanken voor hun vertrouwen in mij, ook al was het proefschrift nog niet af. Een aantal bevriende collega's met wie ik regelmatig lunchgesprekken heb gehad wil ik bedanken voor hun interesse, gezelschap en mentale of inhoudelijke steun: Loes Kater, Susan Niemantsverdriet, Miriam Janssen, Marja Veenstra, Susanne Kümpers en Albine Moser. Met Ger Brouns heb ik vaak van gedachten gewisseld over het proces en we hebben steeds geprobeerd om elkaar een hart onder de riem te steken. Marja Veenstra en Nynke de Jong, bij wie ik regelmatig uit kon blazen, wil ik extra bedanken voor hun steun. Zij zullen ook mijn paranimfen zijn.

Tenslotte wil ik mij tot mijn gezin richten. Lieve Rob, we zijn in I987 samen naar Maastricht gekomen en kwamen als eerste in een appartementje in Rothem terecht. Wat was het daar koud en wat hebben we veel wakker gelegen van de vliegtuigen... We hebben inmiddels al heel veel samen meegemaakt en heel drukke periodes gekend waarin we beiden meerdere studies hebben gedaan, huizen hebben verbouwd en tuinen aangelegd, en waarin jouw carrière vele malen sneller ging dan de mijne. Het lief en leed dat we iedere keer weer delen is mij heel waardevol en je continue steun en aanmoediging hebben er in grote mate aan bijgedragen dat het boek er nu ligt. Je stimuleerde me altijd om me verder te ontwikkelen en geloofde in mijn capaciteiten, ook al begeven we ons op heel verschillende wetenschappelijke gebieden. We delen onze interesse in kamperen en Franse wijn, Engelse detectives en kookprogramma's, maar 
helaas niet in enge films... Het is fijn om samen met jou te proberen iets van het leven te maken. Sorry voor mijn regelmatige gemopper als er weer eens iets tegenzat.

Martijn en Eveline, onze lieve en bijzondere kinderen, het krijgen van jullie heeft mijn leven enorm verrijkt. Het opvoeden en begeleiden van jullie in het opgroeien kost veel energie en verantwoordelijkheid, maar ik heb ook vele dingen meer ervaren dan wanneer jullie er niet geweest zouden zijn. Ook al was de combinatie proefschrift en kinderen bij tijden zwaar en ging het een toch wel vaak ten koste van het ander, jullie zijn een verrijking van mijn leven. Ik kijk er iedere keer weer naar uit om jullie op te halen van school of bso en jullie samen te zien spelen (als jullie elkaar tenminste niet in de haren zitten...).

Pap en mam, ook al wonen jullie al enkele jaren in Zuid-Frankrijk, jullie zijn altijd geïnteresseerd geweest in mijn boek en mijn werk en uiteraard ons gezin en hebben ons vele malen van mentale en praktische steun voorzien, ook aan onze inmiddels bejaarde poes Brebise. Het is heel bijzonder om te ervaren dat geografische afstand geen belemmering is om een hechte band op te bouwen met je kleinkinderen. Ook andere familieleden, die eveneens niet om de hoek wonen, wil ik bedanken voor hun interesse in mijn boek en in ons gezin. 


\section{Curriculum Vitae}

Hannerieke van der Boom was born in Pernis, municipality of Rotterdam (the Netherlands) on July I7. I968. When she was four, she moved to the province of Overijssel in the north east of the Netherlands where she followed primary and secondary education. After completing secondary education (vwo) at the Thorbecke Scholengemeenschap in Zwolle in I986, she studied piano at the Utrecht Conservatory for one year and then moved to Maastricht, where she finished her Bachelor of Music in piano at the Maastricht Conservatory in I992. She then studied Arts and Sciences at Maastricht University and received her doctoral degree in I997. During her studies she followed additional courses in health ethics and participated in research at the Department of History on the history of hospital systems in Europe and the United States, and worked as a student assistant at the Department of Health Ethics \& Philosophy and shortly at the equal-rights commission of the University Council.

In 1997, she started research on her PhD thesis at the Department of Medical Sociology of Maastricht University. In 1998 and 2002 she had a son and a daughter. Since 2004, she has worked as a teacher and assistant professor at the Department of Medical Sociology, Department of Health Ethics \& Philosophy, and the Department of Health Organisation, Policy and Economics of the Faculty of Health, Medicine and Life Sciences. She is currently working in the Bachelor European Public Health of the Department of International Health, and the Bachelor and Master's programmes of Health Sciences at the Department of Health, Ethics \& Society of Maastricht University. 